THE CLARITY-BPA CORE

StUdY: A PERINATAL AND

Chronic EXTENDED-DOSE-

Range Study of BisphenOl A

IN RATS

NTP RR 9

SEPTEMBER 2018 


\section{NTP Research Report on the CLARITY-BPA Core Study: A Perinatal and Chronic Extended-Dose-Range Study of Bisphenol A in Rats}

Research Report 9

National Toxicology Program

September 2018

National Toxicology Program

Public Health Service

U.S. Department of Health and Human Services

ISSN: 2473-4756 


\section{Foreword}

This study was carried out under the auspices of the National Toxicology Program (NTP) as part of the Consortium Linking Academic and Regulatory Insights on Bisphenol A Toxicity (CLARITY-BPA), a consortium-based research program between the National Institute of Environmental Health Sciences (NIEHS) of the National Institutes of Health (NIH) and the National Center for Toxicological Research (NCTR) of the US Food and Drug Administration (FDA).

The aim of the CLARITY-BPA program was to bridge guideline-compliant research conducted at the FDA with hypothesis-based research investigations conducted by academia on the toxicity of bisphenol A (BPA). A detailed description of the CLARITY-BPA program is covered in Heindel and co-authors (https://www.ncbi.nlm.nih.gov/pubmed/26232693).

The CLARITY-BPA research program has two components: 1) A "core" guideline-compliant chronic study conducted at NCTR according to FDA Good Laboratory Practice (GLP) regulations (two-year perinatal only or chronic BPA exposure, including perinatal), and 2) CLARITY-BPA grantee studies of various health endpoints, conducted by NIEHS-funded researchers at academic institutions using animals born to the same exposed pregnant rats as the core GLP study.

This NTP Research Report only covers the core study and includes the narrative, tables, and figures reported in the GLP report. The core study GLP report had 34 appendices, which are listed in Appendix A and referred to as Supplemental Appendices throughout this report. The original GLP report for the core study is on file at NCTR.

The interpretation of biological and toxicological responses described in this report is based only on the results of the core GLP study. Integration of these data with other data from the grantee studies conducted as part of the CLARITY-BPA research program or extrapolation of the results to other species, including characterization of hazards and risks to humans, is outside of the scope of this report.

The core GLP study was designed to characterize and evaluate the toxicologic potential of BPA following perinatal only or chronic exposure in rats under the conditions of a chronic, extendeddose response design. The core GLP study was designed by NCTR and NIEHS scientists with substantial input from the CLARITY-BPA consortium members.

This study was funded via an interagency agreement between FDA and NIEHS. The study's conduct and progress were monitored by the Toxicology Study Selection and Review Committee (composed of representatives from NCTR, other FDA product centers, and NIEHS, ad hoc members of other federal government agencies, and academia), the CLARITY-BPA Steering Committee, and the CLARITY-BPA External Scientific Panel.

Animal care and use were in accordance with the Public Health Service Policy on Humane Care and Use of Animals. The core GLP study is subjected to retrospective quality assurance audits before being presented for public review. 


\section{Table of Contents}

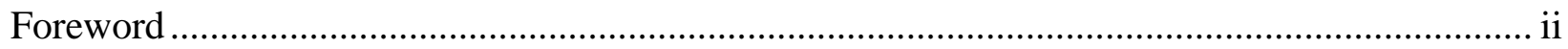

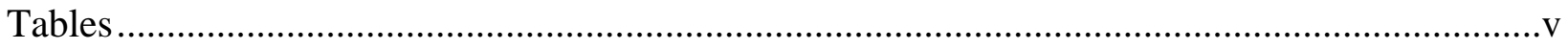

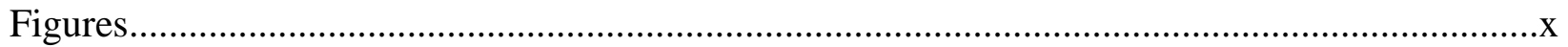

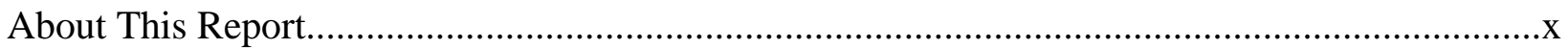

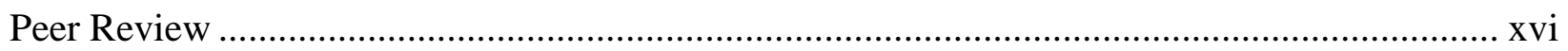

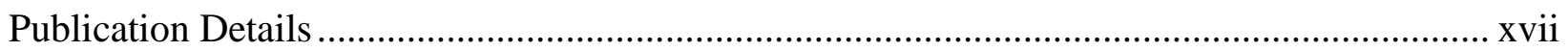

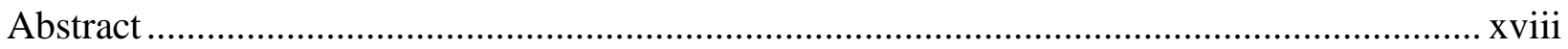

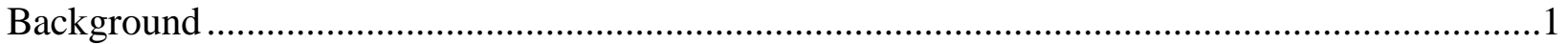

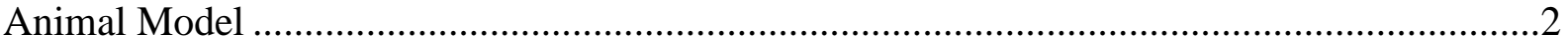

Rationale for Dose Selection for BPA and the Reference Estrogen, $\mathrm{EE}_{2} \ldots \ldots \ldots \ldots \ldots \ldots \ldots \ldots \ldots \ldots \ldots \ldots . . .2$

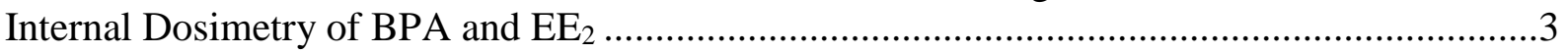

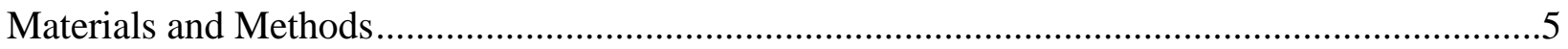

Procurement and Characterization of Bisphenol A..................................................................

Preparation and Analysis of Dose Formulations.................................................................

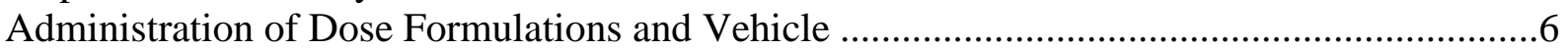

Diet Assessment: Nutrients and Contaminants, Including Background BPA ...........................6

Assessment of Background BPA in Study Materials Other than Diet.........................................

Animal Source and Microbiological Surveillance ...................................................................

Animal Welfare

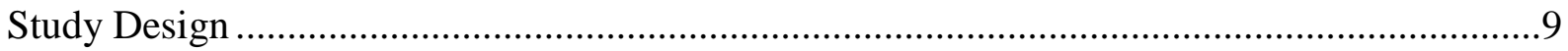

Animal Maintenance, Breeding, Randomized Allocation to Study, and Dosing ................9

Animal Identification ..............................................................................................11

Data Collected in Interim and Terminal Sacrifice Animals ..................................................11

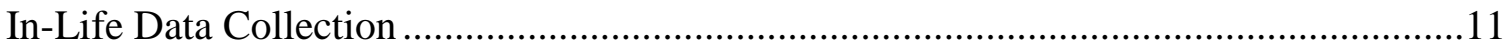

Clinical Chemistry and Hematology, Interim Sacrifice Animals ......................................12

Sperm Evaluations, Interim Sacrifice Animals ..............................................................13

Organ Weights and Histopathology ………………...............................................13

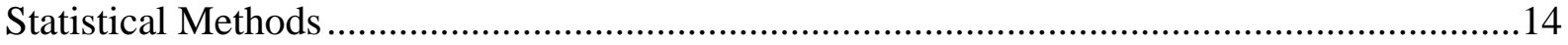

Survival Analyses (Supplemental Appendices XX, XXI, and XXII) ...............................15

Body Weight Analyses (Supplemental Appendices XVII, XXIII, XXIV, and

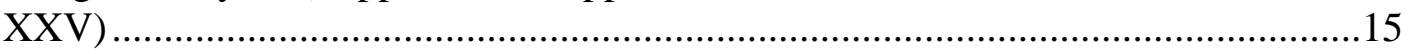

Implantation Sites and Litter Parameters (Supplemental Appendices XVIII and XIX) .......................................................................................................

Analyses of Vaginal Opening, Vaginal Cytology, and Onset of Aberrant Estrous

Cycles (Supplemental Appendices XXVI-XXVIII) …………………….................16

Organ Weight Analyses (Supplemental Appendix XXX) ……………….........................17

Clinical Chemistry and Hematology Analyses (Supplemental Appendix XXIX).............17

Sperm Parameter Analyses (Supplemental Appendix XXXI) ..........................................17

Analyses of Non-Neoplastic and Neoplastic Lesions, Interim and Terminal

Sacrifice (Supplemental Appendices XXXIII and XXXIV) ...................................17

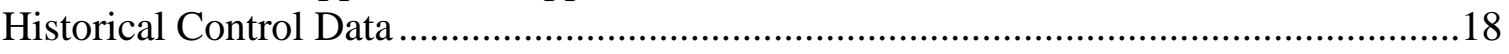




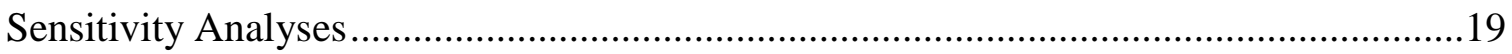

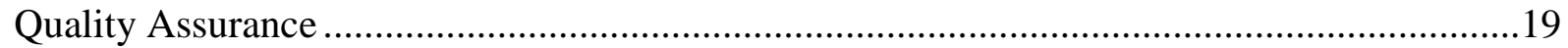

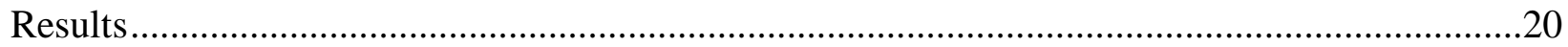

Gestational Body Weight, Fetal Implantation, and Litter Parameters ....................................20

Survival, Preweaning and Postweaning Study Phases .........................................................20

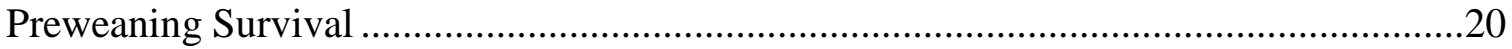

Postweaning Survival in Interim Sacrifice Animals ....................................................20

Postweaning Survival in Terminal Sacrifice Animals ..................................................21

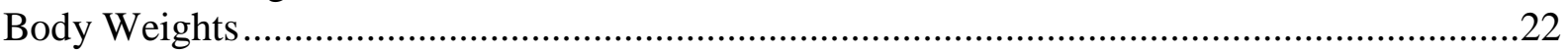

Body Weights in Preweaning Animals ...........................................................................22

Body Weights in Interim and Terminal Sacrifice Animals ............................................22

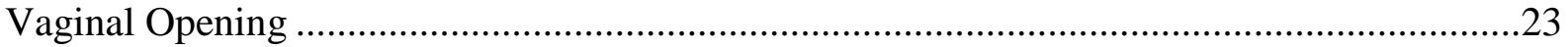

Vaginal Cytology - Estrous Cycle Analysis at Approximately 16 Weeks of Age ...................24

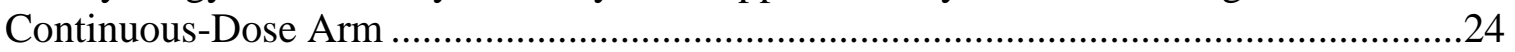

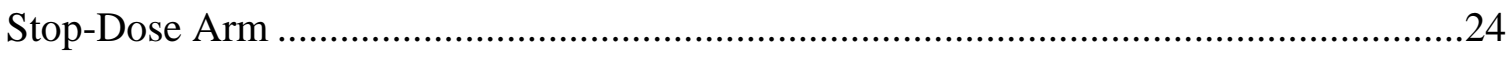

Vaginal Cytology - Onset of Aberrant Estrous Cycles in Aging Animals ...............................24

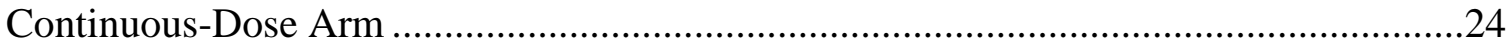

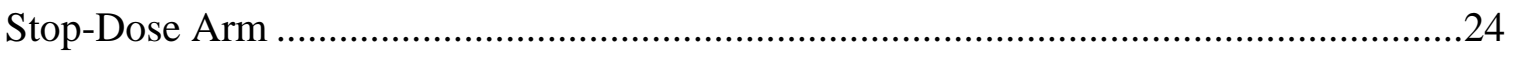

Hematology Endpoints in Interim Sacrifice Animals ............................................................25

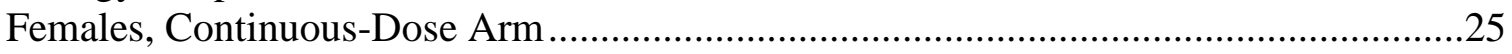

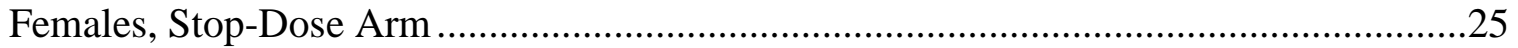

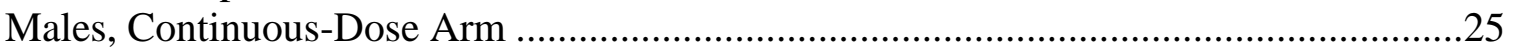

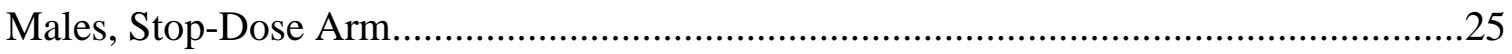

Serum Clinical Chemistry Endpoints in Interim Sacrifice Animals .......................................2.

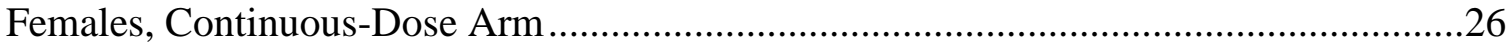

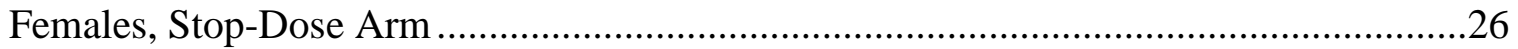

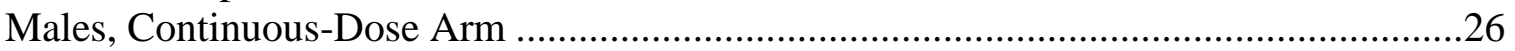

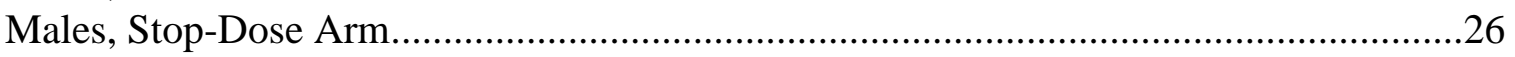

Organ Weights in Interim Sacrifice Animals .....................................................................26

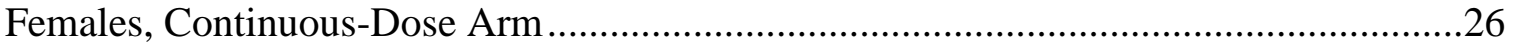

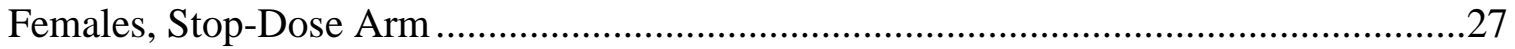

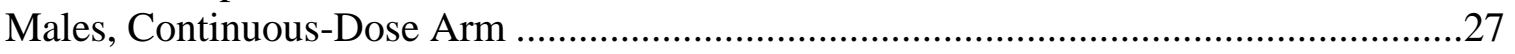

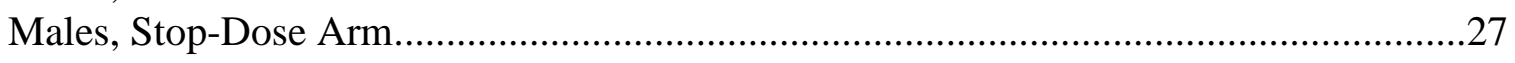

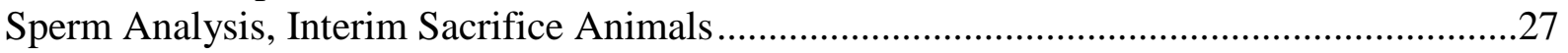

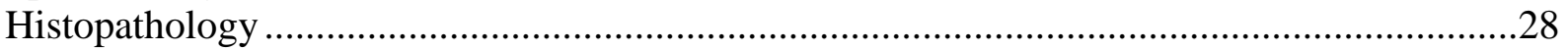

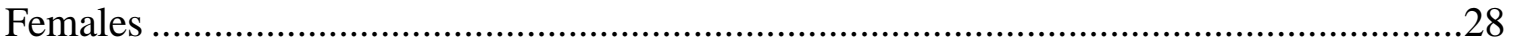

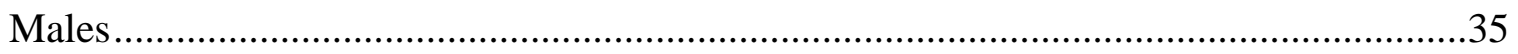

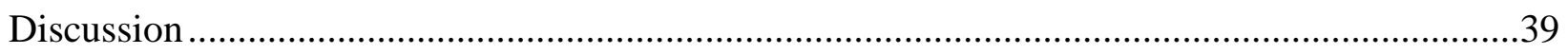

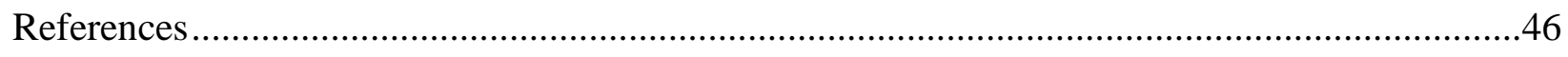

Appendix A $\quad$ List of Supplemental Appendices ………............................................. A-1 


\section{Tables}

Table 1. Summary of Endpoints Evaluated and Statistically Significant Treatment Effects of BPA and $\mathrm{EE}_{2}$ Relative to Vehicle Controls ................................................................. xxii

Table 2. Experimental Design and Materials and Methods in the Two-Year Chronic Gavage Toxicology Study of Bisphenol A (NCTR E0219001), Including Interim (One-Year) Assessment .....

Table 3. Numbers of $F_{0}$ Breeding Pairs Assigned to Study ......................................................56

Table 4. Number of Litters Produced Per Mating....................................................................56

Table 5. Number of Litters Contributing Pups to Interim and/or Terminal Assessments ............56

Table 6. Number of Male and Female Pups Represented in Interim (1-Year) Sacrifice from Each Mating, Continuous-Dose

Table 7. Number of Male and Female Pups Represented in Interim (1 Year) Sacrifice from Each Mating, Stop-Dose

Table 8. Number of Male and Female Pups Represented in Terminal (2 Year) Sacrifice from Each Mating, Continuous-Dose

Table 9. Number of Male and Female Pups Represented in Terminal (2 Year) Sacrifice from Each Mating, Stop-Dose

Table 10. Dam Body Weights from Time of Mating to Parturition in Vehicle, BPA, and $\mathrm{EE}_{2}$ Dose Groups (Mean \pm S.E.M.)

Table 11. Implantation Sites And Litter Parameters For Vehicle, BPA, And EE 2 Dose Groups (Mean \pm S.E.M).....

Table 12. Survival of Female Pups from PND 1 to Weaning in the Vehicle, BPA, and $\mathrm{EE}_{2}$ Dose Groups

Table 13. Survival of Male Pups from PND 1 to Weaning in the Vehicle, BPA, and $E_{2}$ Dose Groups.

Table 14. Survival of Female Pups from Weaning to Interim (1 Year) Sacrifice in the Continuous Vehicle, BPA, and $\mathrm{EE}_{2}$ Dose Groups....

Table 15. Survival of Female Pups from Weaning to Interim (1 Year) Sacrifice in the Stop-Dose Vehicle and BPA Groups

Table 16. Survival of Male Pups from Weaning to Interim (1 Year) Sacrifice in the Continuous Vehicle, BPA, and $\mathrm{EE}_{2}$ Dose Groups

Table 17. Survival of Male Pups from Weaning to Interim (1 Year) Sacrifice in the StopDose Vehicle and BPA Groups.

Table 18. Survival of Female Pups from Weaning to Terminal (2 Year) Sacrifice in the Continuous Vehicle, BPA, and $\mathrm{EE}_{2}$ Dose Groups.

Table 19. Survival of Female Pups from Weaning to Terminal (2 Year) Sacrifice in the Stop-Dose Vehicle and BPA Groups

Table 20. Survival of Male Pups from Weaning to Terminal (2 Year) Sacrifice in the Continuous Vehicle, BPA, and $\mathrm{EE}_{2}$ Dose Groups.

Table 21. Survival of Male Pups from Weaning to Terminal (2 Year) Sacrifice in the Stop-Dose Vehicle and BPA Groups

Table 22. Prewean Body Weights (g) of Female Pups in the Vehicle, BPA, and $\mathrm{EE}_{2}$ Dose Groups (Mean \pm S.E.M.).

Table 23. Prewean Body Weights (g) of Male Pups in the Vehicle, BPA, and $\mathrm{EE}_{2}$ Dose Groups (Mean \pm S.E.M.) 
Table 24. Female Postwean Body Weights (g), Vehicle, BPA, and $\mathrm{EE}_{2}$ Continuous-Dose, Interim (1 Year) Sacrifice (Mean \pm S.E.M.)

Table 25. Female Postwean Body Weights (g), Vehicle, BPA, and $\mathrm{EE}_{2}$ Continuous-Dose,

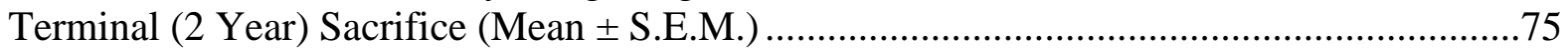

Table 26. Female Postwean Body Weights (g), Vehicle and BPA Stop-Dose, Interim (1 Year) Sacrifice (Mean \pm S.E.M.)

Table 27. Female Postwean Body Weights (g), Vehicle and BPA Stop-Dose, Terminal (2 Year) Sacrifice (Mean \pm S.E.M.)

Table 28. Male Postwean Body Weights (g), Vehicle, BPA, and EE 2 Continuous-Dose, Interim (1 Year) Sacrifice (Mean \pm S.E.M.)

Table 29. Male Postwean Body Weights (g), Vehicle, BPA, And EE 2 Continuous-Dose, Terminal (2 Year) Sacrifice (Mean \pm S.E.M.)

Table 30. Male Postwean Body Weights (g), Vehicle and BPA Stop-Dose, Interim (1 Year) Sacrifice (Mean \pm S.E.M.)

Table 31. Male Postwean Body Weights (g), Vehicle and BPA Stop-Dose, Terminal (2

Years) Sacrifice (Mean \pm S.E.M.)

Table 32. Vaginal Opening, Age and Body Weight (Means \pm S.E.M.) at Occurrence, Vehicle, BPA, and $\mathrm{EE}_{2}$ Continuous-Dose ....

Table 33. Vaginal Opening, Age and Body Weight (Means \pm S.E.M.) at Occurrence,

Vehicle and BPA Stop-Dose

Table 34. Estrous Cycle Analysis, Vehicle, BPA, and $\mathrm{EE}_{2}$ Continuous-Dose

Table 35. Estrous Cycle Analysis, Vehicle and BPA Stop-Dose.....

Table 36. Time to Onset of Aberrant Estrous Cycles in Vehicle, BPA, and $\mathrm{EE}_{2}$

Continuous-Dose Groups....

Table 37. Time to Onset of Aberrant Estrous Cycles in Vehicle and BPA Stop-Dose Groups

Table 38. Female Hematology, Vehicle, $\mathrm{BPA}$, and $\mathrm{EE}_{2}$ Continuous-Dose, Interim Sacrifice (Mean \pm S.E.M.)

Table 39. Female Hematology, Vehicle and BPA Stop-Dose, Interim Sacrifice (Mean \pm S.E.M.)

Table 40. Male Hematology, Vehicle, BPA, and $\mathrm{EE}_{2}$ Continuous-Dose, Interim Sacrifice (Mean \pm S.E.M.)

Table 41. Male Hematology, Vehicle and BPA Stop-Dose, Interim Sacrifice

(Mean \pm S.E.M.)

Table 42. Female Clinical Chemistry, Vehicle, BPA, and $\mathrm{EE}_{2}$ Continuous-Dose, Interim

Sacrifice (Mean \pm S.E.M.)

Table 43. Female Clinical Chemistry, Vehicle and BPA Stop-Dose, Interim Sacrifice

(Mean \pm S.E.M.)

Table 44. Male Clinical Chemistry, Vehicle, BPA, and EE2 Continuous-Dose, Interim

Sacrifice (Mean \pm S.E.M.)

Table 45. Male Clinical Chemistry, Vehicle and BPA Stop-Dose, Interim Sacrifice

(Mean \pm S.E.M.)

Table 46. Female Organ Weights, Vehicle, BPA, and $\mathrm{EE}_{2}$ Continuous-Dose, Interim

Sacrifice (Mean \pm S.E.M.)

Table 47. Female Organ Weights, Vehicle and BPA Stop-Dose, Interim Sacrifice

(Mean \pm S.E.M.) 
Table 48. Male Organ Weights, Vehicle, BPA, and $\mathrm{EE}_{2}$ Continuous-Dose, Interim

Sacrifice (Mean \pm S.E.M.)

Table 49. Male Organ Weights, Vehicle and BPA Stop-Dose, Interim Sacrifice

(Mean \pm S.E.M.)

Table 50. Sperm Analysis, Vehicle, BPA, and $\mathrm{EE}_{2}$ Continuous-Dose, Interim Sacrifice

(Mean \pm S.E.M.)

Table 51. Sperm Analysis, Vehicle and BPA Stop-Dose, Interim Sacrifice

(Mean \pm S.E.M.)

Table 52. Neoplastic Lesions in the Mammary Gland of Interim and Terminal Sacrifice

Female Rats: Continuous-Dose BPA

Table 53. Neoplastic Lesions in the Mammary Gland of Interim and Terminal Sacrifice

Female Rats: Continuous-Dose $\mathrm{EE}_{2}$

Table 54. Neoplastic Lesions in the Mammary Gland of Interim and Terminal Sacrifice

Female Rats: Stop-Dose BPA.

Table 55. Non-Neoplastic Lesions in the Mammary Gland of Interim and Terminal

Sacrifice Female Rats: Continuous-Dose BPA.

Table 56. Non-Neoplastic Lesions in the Mammary Gland of Interim and Terminal

Sacrifice Female Rats: Continuous-Dose $\mathrm{EE}_{2}$.

Table 57. Non-Neoplastic Lesions in the Mammary Gland of Interim and Terminal

Sacrifice Female Rats: Stop-Dose BPA.

Table 58. Neoplastic Lesions in the Uterus of Interim and Terminal Sacrifice Female

Rats: Continuous-Dose BPA.

Table 59. Neoplastic Lesions in the Uterus of Interim and Terminal Sacrifice Female

Rats: Continuous-Dose $\mathrm{EE}_{2}$

Table 60. Neoplastic Lesions in the Uterus of Interim and Terminal Sacrifice Female

Rats: Stop-Dose BPA

Table 61. Non-Neoplastic Lesions in the Uterus of Interim and Terminal Sacrifice

Female Rats: Continuous-Dose BPA

Table 62. Non-Neoplastic Lesions in the Uterus of Interim and Terminal Sacrifice

Female Rats: Continuous-Dose $\mathrm{EE}_{2}$

Table 63. Non-Neoplastic Lesions in the Uterus of Interim and Terminal Sacrifice

Female Rats: Stop-Dose BPA.

Table 64. Non-Neoplastic Lesions in the Ovary of Interim and Terminal Sacrifice Female

Rats: Continuous-Dose BPA.

Table 65. Non-Neoplastic Lesions in the Ovary of Interim and Terminal Sacrifice Female

Rats: Continuous-Dose $\mathrm{EE}_{2}$

Table 66. Non-Neoplastic Lesions in the Ovary of Interim and Terminal Sacrifice Female

Rats: Stop-Dose BPA

Table 67. Non-Neoplastic Lesions in the Vagina of Interim and Terminal Sacrifice

Female Rats: Continuous-Dose BPA.

Table 68. Non-Neoplastic Lesions in the Vagina of Interim and Terminal Sacrifice

Female Rats: Continuous-Dose $\mathrm{EE}_{2}$

Table 69. Non-Neoplastic Lesions in the Vagina of Interim and Terminal Sacrifice

Female Rats: Stop-Dose BPA

Table 70. Neoplastic Lesions in the Pituitary Gland of Interim and Terminal Sacrifice

Female Rats: Continuous-Dose BPA..... 
Table 71. Neoplastic Lesions in the Pituitary Gland of Interim and Terminal Sacrifice

Female Rats: Continuous-Dose $\mathrm{EE}_{2}$

Tabl 72. Neoplastic Lesions in the Pituitary Gland of Interim and Terminal Sacrifice

Female Rats: Stop-Dose BPA.

Table 73. Non-Neoplastic Lesions in the Pituitary of Interim and Terminal Sacrifice

Female Rats: Continuous-Dose BPA

Table 74. Non-Neoplastic Lesions in the Pituitary of Interim and Terminal Sacrifice

Female Rats: Continuous-Dose $\mathrm{EE}_{2}$

Table 75. Non-Neoplastic Lesions in the Pituitary of Interim and Terminal Sacrifice

Female Rats: Stop-Dose BPA.

Table 76. Non-Neoplastic Lesions in the Heart of Interim and Terminal Sacrifice Female

Rats: Continuous-Dose BPA.

Table 77. Non-Neoplastic Lesions in the Heart of Interim and Terminal Sacrifice Female

Rats: Continuous-Dose $\mathrm{EE}_{2}$.

Table 78. Non-Neoplastic Lesions in the Heart of Interim and Terminal Sacrifice Female

Rats: Stop-Dose BPA

Table 79. Non-Neoplastic Lesions in the Kidney of Interim and Terminal Sacrifice

Female Rats: Continuous-Dose BPA.

Table 80. Non-Neoplastic Lesions in the Kidney of Interim and Terminal Sacrifice

Female Rats: Continuous-Dose $\mathrm{EE}_{2}$

Table 81. Non-Neoplastic Lesions in the Kidney of Interim and Terminal Sacrifice

Female Rats: Stop-Dose BPA.

Table 82. Non-Neoplastic Lesions in the Liver of Interim and Terminal Sacrifice Female

Rats: Continuous-Dose BPA.

Table 83. Non-Neoplastic Lesions in the Liver of Interim and Terminal Sacrifice Female

Rats: Continuous-Dose $\mathrm{EE}_{2}$

Table 84. Non-Neoplastic Lesions in the Liver of Interim and Terminal Sacrifice Female Rats: Stop-Dose BPA

Table 85. Non-Neoplastic Lesions in the Thyroid of Interim and Terminal Sacrifice

Female Rats: Continuous-Dose BPA

Table 86. Non-Neoplastic Lesions in the Thyroid of Interim and Terminal Sacrifice

Female Rats: Continuous-Dose $\mathrm{EE}_{2}$

Table 87. Non-Neoplastic Lesions in the Thyroid of Interim and Terminal Sacrifice

Female Rats: Stop-Dose BPA.

Table 88. Non-Neoplastic Lesions in the Epididymis of Interim and Terminal Sacrifice

Male Rats: Continuous-Dose BPA .

Table 89. Non-Neoplastic Lesions in the Epididymis of Interim and Terminal Sacrifice

Male Rats: Continuous-Dose $\mathrm{EE}_{2}$.

Table 90. Non-Neoplastic Lesions in the Epididymis of Interim and Terminal Sacrifice

Male Rats: Stop-Dose BPA

Table 91. Non-Neoplastic Lesions in the Dorsal/Lateral Prostate of Interim and Terminal

Sacrifice Male Rats: Continuous-Dose BPA

Table 92. Non-Neoplastic Lesions in the Dorsal/Lateral Prostate of Interim and Terminal

Sacrifice Male Rats: Continuous-Dose $\mathrm{EE}_{2}$.

Table 93. Non-Neoplastic Lesions in the Dorsal/Lateral Prostate of Interim and Terminal

Sacrifice Male Rats: Stop-Dose BPA 
Table 94. Neoplastic Lesions in the Ventral Prostate of Interim and Terminal Sacrifice

Male Rats: Continuous-Dose BPA

Table 95. Neoplastic Lesions in the Ventral Prostate of Interim and Terminal Sacrifice

Male Rats: Continuous-Dose $\mathrm{EE}_{2}$.

Table 96. Neoplastic Lesions in the Ventral Prostate of Interim and Terminal Sacrifice

Male Rats: Stop-Dose BPA

Table 97. Non-Neoplastic Lesions in the Ventral Prostate of Interim and Terminal

Sacrifice Male Rats: Continuous-Dose BPA

Table 98. Non-Neoplastic Lesions in the Ventral Prostate of Interim and Terminal

Sacrifice Male Rats: Continuous-Dose $\mathrm{EE}_{2}$

Table 99. Non-Neoplastic Lesions in the Ventral Prostate of Interim and Terminal

Sacrifice Male Rats: Stop-Dose BPA

Table 100. Non-Neoplastic Lesions in the Pituitary of Interim and Terminal Sacrifice

Male Rats: Continuous-Dose BPA

Table 101. Non-Neoplastic Lesions in the Pituitary of Interim and Terminal Sacrifice

Male Rats: Continuous-Dose $\mathrm{EE}_{2}$.

Table 102. Non-Neoplastic Lesions in the Pituitary of Interim and Terminal Sacrifice

Male Rats: Stop-Dose BPA

Table 103. Non-Neoplastic Lesions in the Thyroid of Interim and Terminal Sacrifice

Male Rats: Continuous-Dose BPA

Table 104. Non-Neoplastic Lesions in the Thyroid of Interim and Terminal Sacrifice

Male Rats: Continuous-Dose $\mathrm{EE}_{2}$

Table 105. Non-Neoplastic Lesions in the Thyroid of Interim and Terminal Sacrifice

Male Rats: Stop-Dose BPA

Table 106. Non-Neoplastic Lesions in the Parathyroid Gland of Interim and Terminal

Sacrifice Male Rats: Continuous BPA

Table 107. Non-Neoplastic Lesions in the Parathyroid Gland of Interim and Terminal

Sacrifice Male Rats: Continuous-Dose $\mathrm{EE}_{2}$.

Table 108. Non-Neoplastic Lesions in the Parathyroid Gland of Interim and Terminal

Sacrifice Male Rats: Stop-Dose BPA

Table 109. Non-Neoplastic Lesions in the Kidney of Interim and Terminal Sacrifice Male

Rats: Continuous-Dose BPA.

Table 110. Non-Neoplastic Lesions in the Kidney of Interim and Terminal Sacrifice Male

Rats: Continuous-Dose $\mathrm{EE}_{2}$ 164

Table 111. Non-Neoplastic Lesions in the Kidney of Interim and Terminal Sacrifice Male

Rats: Stop-Dose BPA

Table 112. Non-Neoplastic Lesions in the Liver of Interim and Terminal Sacrifice Male

Rats: Continuous-Dose BPA.

Table 113. Non-Neoplastic Lesions in the Liver of Interim and Terminal Sacrifice Male

Rats: Continuous-Dose $\mathrm{EE}_{2}$

Table 114. Non-Neoplastic Lesions in the Liver of Interim and Terminal Sacrifice Male

Rats: Stop-Dose BPA 168 


\section{Figures}

Figure 1. Scheme for Chronic BPA Toxicity Study Design 169

Figure 2. Kaplan-Meier Survival Curve for Terminal Sacrifice Female BPA Continuous-

Dose Arm (Weeks 4-104)

Figure 3. Kaplan-Meier Survival Curve for Terminal Sacrifice Female EE2 Continuous-

Dose Arm (Weeks 4-104)

Figure 4. Kaplan-Meier Survival Curve for Terminal Sacrifice Female BPA Stop-Dose

Arm (Week 4-104)

Figure 5. Kaplan-Meier Survival Curve for Terminal Sacrifice Male BPA Continuous-

Dose Arm (Weeks 4-104)

Figure 6. Kaplan-Meier Survival Curve for Terminal Sacrifice Male EE 2 Continuous-

Dose Arm (Weeks 4-104)

Figure 7. Kaplan-Meier Survival Curve for Terminal Sacrifice Male BPA Stop-Dose Arm

(Week 4-104). 175

Figure 8. Body Weight for Interim Sacrifice Female BPA Continuous-Dose Arm ...................176

Figure 9. Body Weight for Interim Sacrifice Female $\mathrm{EE}_{2}$ Continuous-Dose Arm .....................177

Figure 10. Body Weight for Terminal Sacrifice Female BPA Continuous-Dose Arm ..............178

Figure 11. Body Weight for Terminal Sacrifice Female $\mathrm{EE}_{2}$ Continuous-Dose Arm .................179

Figure 12. Body Weight for Interim Sacrifice Female BPA Stop-Dose Arm ...........................180

Figure 13. Body Weight for Terminal Sacrifice Female BPA Stop-Dose Arm .........................181

Figure 14. Body Weight for Interim Sacrifice Male BPA Continuous-Dose Arm.....................182

Figure 15. Body Weight for Interim Sacrifice Male $\mathrm{EE}_{2}$ Continuous-Dose Arm......................183

Figure 16. Body Weight for Terminal Sacrifice Male BPA Continuous-Dose Arm..................184

Figure 17. Body Weight for Terminal Sacrifice Male EE 2 Continuous-Dose Arm....................185

Figure 18. Body Weight for Interim Sacrifice Male BPA Stop-Dose Arm.............................186

Figure 19. Body Weight for Terminal Sacrifice Male BPA Stop-Dose Arm ............................187

Figure 20. Kaplan-Meier Time to Aberrant Cycling Curve for BPA Continuous-Dose

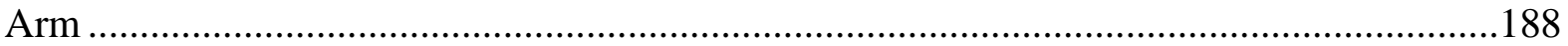

Figure 21. Kaplan-Meier Time to Aberrant Cycling Curve for $\mathrm{EE}_{2}$ Continuous-Dose Arm ......189

Figure 22. Kaplan-Meier Time to Aberrant Cycling Curve for BPA Stop-Dose Arm................190 


\section{About This Report}

National Toxicology Program ${ }^{1}$

${ }^{1}$ Division of the National Toxicology Program, National Institute of Environmental Health

Sciences, Research Triangle Park, North Carolina, USA

\section{Collaborators}

K. Barry Delclos, Luísa Camacho, Sherry M. Lewis, Michelle M. Vanlandingham, Frederick A. Beland, Matthew S. Bryant, Kathy A. Carroll, Robert P. Felton, Gonçalo Gamboa da Costa, Paul C. Howard, Greg R. Olson, Ralph Patton, Nigel J. Walker

\section{U.S. Food and Drug Administration, National Center for Toxicological Research,} Jefferson, Arkansas, USA

Study conduct, evaluation, and reporting of findings

Frederick A. Beland, Ph.D.

Matthew S. Bryant, Ph.D.

Kathy A. Carroll

Luísa Camacho, Ph.D., Co-Principal Investigator

K. Barry Delclos, Ph.D., Principal Investigator

Robert P. Felton, M.S.

Gonçalo Gamboa da Costa, Ph.D.

Paul C. Howard, Ph.D. (now retired)

Sherry M. Lewis, Ph.D., Co-Principal Investigator (now retired)

Michelle M. Vanlandingham, Co-Principal Investigator

Toxicologic Pathology Associates, Frederick, Maryland, USA

Study conduct, evaluation and reporting of findings

Greg R. Olson, D.V.M., Ph.D., Study Pathologist

Ralph Patton, B.S.

National Institute of Environmental Health Sciences, Research Triangle Park, North Carolina, USA

Study conduct, evaluation and reporting of findings

Nigel J. Walker, Ph.D.

\section{Contributors}

U.S. Food and Drug Administration, National Center for Toxicological Research, Jefferson, Arkansas, USA

Microbiology surveillance and diagnostics

Sung-Guk Kim, Ph.D.

Lillie M. Sims, B.S.

Roger S. Steel, B.S.

Christine V. Summage-West, B.S. 
Chemical analysis and dose certifications

Matthew S. Bryant, Ph.D.

Mani Chidambaram, Ph.D.

Gonçalo Gamboa da Costa, Ph.D.

F. Michelle McLellan, M.S.

Kellie A. Woodling, Ph.D.

Computer support

Kathy A. Carroll

Patricia Shores

Quality assurance

Joe M. Fowler, B.S.

Russell D. Smith, B.S.

Statistical support

Robert P. Felton, M.S.

Beth E. Juliar,* M.S. (now retired)

Mackean P. Maisha, M.S.

*Beth E. Juliar, M.Sc., did much of the analysis of non-histopathology data from this study. She retired from NCTR prior to the completion of the report.

Veterinary services*

Jeff Carraway, D.V.M, M.S. (now retired)

Neera Gopee, D.V.M., Ph.D. (now at the Office of Laboratory Animal Welfare, National

Institutes of Health)

Pamela Mack, D.V.M., M.S.

*During the in-life portion of the study, Dr. Jeff Carraway and Dr. Neera Gopee were the veterinarians involved in the study. Dr. Pamela Mack became Director of Veterinary Services after the in-life portion of the study was completed.

\section{Priority One Services, Alexandria, Virginia, USA}

Diet and dose preparation, animal care

Florene Lewis

Andy Matson, B.S.

Mark Moore, B.S.

Felita Reynolds

Toxicologic Pathology Associates, Frederick, Maryland, USA

Histopathology and clinical chemistry evaluations and reporting

Kelly J. Davis, D.V.M.

Greg R. Olson, D.V.M., Ph.D.

Ralph E. Patton, B.S.

Betty P. Raiford, B.S.

Kristie L. Voris-Acuff

Alan R. Warbritton

Lisa P. Wiley, B.S. 
William M. Witt, D.V.M., Ph.D.

NTP Pathology Working Group, National Institute of Environmental Health Sciences, Research Triangle Park, North Carolina, USA

Evaluation of pathology findings

Kelly J. Davis, D.V.M., Toxicologic Pathology Associates

Darlene Dixon, D.V.M., Ph.D., National Institute of Environmental Health Sciences

Susan A. Elmore, M.S., D.V.M., National Institute of Environmental Health Sciences

Jerry F. Hardisty, D.V.M., Experimental Pathology Laboratories, Inc.

David Malarkey, D.V.M., Ph.D., National Institute of Environmental Health Sciences

Steven Mog, D.V.M., Center for Food Safety and Applied Nutrition, U.S. Food and Drug

Administration

Greg R. Olson, D.V.M., Ph.D., Toxicologic Pathology Associates

Erin Quist, D.V.M., M.S., Experimental Pathology Laboratories, Inc.

Karen S. Regan, D.V.M., Regan Path Tox Services, Inc.

Thomas J. Steinbach, D.V.M., Experimental Pathology Laboratories, Inc.

National Institute of Environmental Health Sciences, Research Triangle Park, North

Carolina, USA

Management of peer review of draft document

Mary S. Wolfe, Ph.D.

Oversight for data management

Jennifer M. Fostel, Ph.D.

ICF, Durham, North Carolina, USA

Preparation of NTP Research Report from the National Center for Toxicological Research GLP report, peer-review support

Susan Blaine, B.A.

Natalie Blanton, M.P.H.

Canden Byrd, B.S.

Tara Hamilton, M.S.

Katherine Helmick, M.P.H.

Penelope Kellar, M.S.

Jeanne Luh, Ph.D.

Whitney Mitchell, B.S.

Cara Peyser, B.S.

Kelly Shipkowski, Ph.D.

Anna Stamatogiannakis, B.S.

ASRC Vistronix, Beltsville, Maryland, USA

Support for data management and reporting

Marcus A. Jackson, M.S.

Isabel A. Lea, Ph.D.

Carolyn R. Martini, B.S.

Jamie F. Moose, M.S. 
National Institute of Environmental Health Sciences/U.S. Food and Drug Administration, National Center for Toxicological Research Interagency Agreement Project Officers, Research Triangle Park, North Carolina, USA

Oversight and coordination of all Interagency Agreement aspects of the CLARITY-BPA research program

Gonçalo Gamboa da Costa,* Ph.D., National Center for Toxicological Research, U.S. Food and Drug Administration

Paul C. Howard, Ph.D., National Center for Toxicological Research, U.S. Food and Drug Administration (now retired)

Nigel J. Walker, Ph.D., National Institute of Environmental Health Sciences

*Dr. Gonçalo Gamboa da Costa became the FDA Interagency Agreement Project Officer after the in-life portion of the study was complete.

\section{Consortium Linking Academic and Regulatory Insights on Bisphenol A Toxicity (CLARITY-BPA)}

Participating members of the consortium involved in design of the core study and monitoring of study progress

Luísa Camacho, Ph.D., National Center for Toxicological Research, U.S. Food and Drug Administration

K. Barry Delclos, Ph.D., National Center for Toxicological Research, U.S. Food and Drug Administration

Gonçalo Gamboa da Costa, Ph.D., National Center for Toxicological Research, U.S. Food and Drug Administration

Paul C. Howard, Ph.D., National Center for Toxicological Research, U.S. Food and Drug Administration (now retired)

Jason Aungst, Ph.D., Center for Food Safety and Applied Nutrition, U.S. Food and Drug Administration

John R. Bucher, Ph.D., National Institute of Environmental Health Sciences

Jerry J. Heindel, Ph.D., National Institute of Environmental Health Sciences (now retired)

Retha R. Newbold, M.S., National Institute of Environmental Health Sciences

Thaddeus T. Schug, Ph.D., National Institute of Environmental Health Sciences

Nigel J. Walker, Ph.D., National Institute of Environmental Health Sciences

Scott M. Belcher, Ph.D., North Carolina State University

Nira Ben-Jonathan, Ph.D., University of Cincinnati

Kim Boekelheide, M.D., Ph.D., Brown University

Jodi A. Flaws, Ph.D., University of Illinois at Urbana-Champaign

Nestor Gonzalez-Cadavid, Ph.D., University of California, Los Angeles

Andrew S. Greenberg, M.D., Tufts University

Shuk-Mei Ho, Ph.D., University of Cincinnati

Norbert E. Kaminski, Ph.D., Michigan State University

Heather B. Patisaul, Ph.D., North Carolina State University

Gail S. Prins, Ph.D., University of Illinois at Chicago

Cheryl S. Rosenfeld, D.V.M., Ph.D., University of Missouri

Ana M. Soto, M.D., Tufts University

Frederick S. vom Saal, Ph.D., University of Missouri

R. Thomas Zoeller, Ph.D., University of Massachusetts, Amherst 


\section{CLARITY-BPA External Scientific Panel}

Input and guidance on CLARITY-BPA research program's progress

Daniel A. Casciano, Ph.D., University of Arkansas at Little Rock

Michael A. Gallo, Ph.D., Rutgers University

Martin A. Philbert, Ph.D., University of Michigan 


\section{Peer Review}

NTP convened an expert panel on April 26, 2018, to peer review the NTP Research Report on the CLARITY-BPA Core Study: A Perinatal and Chronic Extended-Dose-Range Study of

Bisphenol A in Rats. The panel members are listed below. These reviewers served as independent scientists, not as representatives of any institution, company, or governmental agency. In this capacity, the panel provided input on the scientific and technical elements of the CLARITY-BPA Core Study and ensured that this NTP Research Report presented the experimental results and conclusions fully and clearly.

Meeting materials, the peer review report, and presentation recordings can be found on the NTP website: https://ntp.niehs.nih.gov/events/past/index.html.

\section{Peer Reviewers}

David Dorman, D.V.M., Ph.D. (Chair)

Professor of Toxicology, Department of Molecular Biomedical Sciences

College of Veterinary Medicine

North Carolina State University

Raleigh, NC

Tracie Bunton, D.V.M., Ph.D.

Founder

Eicarte LLC

Gettysburg, PA

Michael Conner, D.V.M.

Vice President, Toxicology

Global Blood Therapeutics, Inc.

San Francisco, CA

Thomas Rosol, D.V.M., Ph.D.

Professor and Chair, Department of Biomedical Sciences, Heritage College of Osteopathic

Medicine, Ohio University

Adjunct Professor, Division of Urology, Department of Medicine, The Ohio State University Marietta, $\mathrm{OH}$

Janet Tooze, Ph.D.

Professor, Department of Biostatistical Sciences

Wake Forest School of Medicine

Wake Forest University

Winston-Salem, NC

Kimberley Treinen, Ph.D.

Executive Director, Discovery and Preclinical Research

Sunovion Pharmaceuticals, Inc.

Hopkinton, MA 


\section{Publication Details}

Publisher: National Toxicology Program

Publishing Location: Research Triangle Park, NC

ISSN: 2473-4756

DOI: https://doi.org/10.22427/NTP-RR-9

Report Series: NTP Research Report Series

Report Series Number: 9

Official citation: National Toxicology Program. 2018. NTP Research Report on the CLARITYBPA Core Study: A Perinatal and Chronic Extended-Dose-Range Study of Bisphenol A in Rats. NTP RR 9. Research Triangle Park, NC. National Toxicology Program (9): 1-221. 


\section{Abstract}

Bisphenol A (BPA, CAS \#80-05-7) is a high-production-volume industrial chemical used as a monomer for polycarbonate plastics and epoxy resins that have broad applications in consumer products, including storage containers for foods and beverages and medical devices. The potential toxicity resulting from chronic exposure to BPA as an indirect food additive is the concern addressed in this study.

This study is part of the Consortium Linking Academic and Regulatory Insights on Bisphenol A Toxicity (CLARITY-BPA), a research program between the National Institute of Environmental Health Sciences (NIEHS) and the National Center for Toxicological Research (NCTR) of the Food and Drug Administration (FDA), developed to bridge guideline-compliant research conducted at the FDA with hypothesis-based research investigations conducted by academia on the toxicity of BPA. The CLARITY-BPA research program has two components: 1) A "core" guideline-compliant chronic study conducted at NCTR according to FDA Good Laboratory Practice (GLP) regulations and 2) studies of various endpoints, conducted by NIEHS-funded researchers at academic institutions using animals born to the same exposed pregnant rats as the core GLP study. The purpose of this research program was to evaluate chronic exposure to BPA over a broad dose range using traditional and non-traditional endpoints. It aimed to determine if non-traditional endpoints reveal toxicity not detected by traditional guideline study endpoints and provide mechanistic support for observations made in the guideline study. The current research report covers only data from the "core" guideline-compliant chronic study.

The toxicity of BPA administered by oral gavage from gestation day (GD) 6 through the start of labor and then by oral gavage to pups from postnatal day (PND) 1 (day of birth = PND 0) until termination at one year or two years was examined in Sprague-Dawley rats from the NCTR breeding colony (Sprague-Dawley/CD23/NctrBR). BPA doses were 2.5, 25, 250, 2,500, and $25,000 \mu \mathrm{g} / \mathrm{kg}$ body weight (bw)/day. A vehicle ( $0.3 \%$ carboxymethylcellulose (CMC)) control group was also included. In addition to animals that were dosed daily throughout the study, a stop-dose study arm was included with animals dosed daily until PND 21 and then held without further treatment until termination to assess any effects that were due to early exposure only. Because many of the effects of BPA reported in the literature are associated with estrogen signaling pathways, two doses $(0.05$ and $0.5 \mu \mathrm{g} / \mathrm{kg}$ bw/day) of the orally active estrogen ethinyl estradiol $\left(\mathrm{EE}_{2}\right)$ were also included in the continuous-dose arm to assess the sensitivity of the test system to low doses of an estrogen. Reference estrogen groups were not included in the stopdose study arm of the core study due to resource constraints, primarily lack of animal facility space. Rats were obtained as weanlings from the NCTR breeding colony and placed under study conditions (soy- and alfalfa-free diet (5K96, LabDiet, Purina Mills), polysulfone cages, hardwood chip bedding, glass water bottles, and food-grade silicone stoppers) until mating. Study materials were monitored for background BPA levels; the only material with detectable levels of BPA was the diet, which had less than $3 \mathrm{ppb}$ BPA. Prior to mating to males that were not siblings or first cousins, female rats were stratified by body weight and were randomized to treatment groups to give approximately equivalent mean starting body weights in each group. Each morning after pairing, females were examined for evidence of mating (presence of an in situ vaginal plug or sperm-positive vaginal smear). Upon evidence of mating, the females were separated from the males and individually housed; this day was considered GD 0. On GD 6, daily dosing of the dam with $\mathrm{BPA}, \mathrm{EE}_{2}$, or vehicle began and was based on the body weight 
measured immediately prior to the administration of these compounds. Direct gavage dosing of the pups was started on PND 1, with the same dose and agent that was administered to their dams. At weaning on PND 21, no more than one animal per sex per litter was assigned to the following study arms: 1) continuous dosing to sacrifice at two years (terminal sacrifice, 46-50 animals per sex per vehicle control or BPA treatment group and 26 animals per sex per $\mathrm{EE}_{2}$ group); 2) continuous dosing to sacrifice at one year (interim sacrifice, 20-26 animals per sex for all groups); 3) no further treatment after PND 21 until sacrifice at two years (stop-dose terminal sacrifice, 46-50 animals per sex per preweaning vehicle control or BPA group); and 4) no further treatment after PND 21 until sacrifice at one year (stop-dose interim sacrifice, 20-26 animals per sex for preweaning vehicle control and BPA groups). The stop-dose study arms for which gavage dosing was not continued beyond weaning were included to assess the potential of permanent effects induced by exposure to hormonally active compounds during developmental stages. The interim (one-year) sacrifice group was included to allow evaluation of long-term exposure effects with less confounding due to background lesions of aging than would be expected at two years, and to allow assessment of any precursors of any treatment-related lesions observed at two years.

Data collected included body weights, litter parameters, age at vaginal opening, vaginal cytology, clinical pathology (interim sacrifice only), sperm parameters (interim sacrifice only), organ weights (interim sacrifice only), and histopathology (both interim and terminal sacrifices). Vaginal cytology data were collected for 14 consecutive days at approximately 16 weeks of age from the same subset of females in the terminal sacrifice arm that had been monitored for vaginal opening; these same animals were then monitored for five consecutive days monthly to estimate the time at which they began having aberrant estrous cycles. In addition to the summary tables provided in this report and appendices, all individual animal data are available online (https://doi.org/10.22427/NTP-DATA-018-00015-0001-000-6).

Table 1 lists all non-histopathology endpoints analyzed and associated statistical findings. For histopathology data, Table 1 only lists the endpoints where a statistically significant difference was found by the primary statistical tests applied (Cochran-Armitage/Fisher's Exact Test for interim sacrifice animals; survival-adjusted Poly-3 test for terminal sacrifice animals). Results from all statistical tests applied to the histopathology data, which further included JonckheereTerpstra/Shirley-Williams (JT/SW) and relative treatment effect (RTE) tests for non-neoplastic lesions assigned severity scores, are included in the text of this abstract and in the report text and tables. Statistically significant results are indicated regardless of biological significance.

There were few significant effects of BPA treatment in the in-life data collected. In the late stages of the study (weeks 96-104), mean female body weights in the $250 \mu \mathrm{g} \mathrm{BPA} / \mathrm{kg}$ bw/day continuous-dose group were significantly higher than the mean vehicle control body weights. For clinical pathology endpoints and organ weights, some statistically significant effects of continuous- or stop-dose BPA treatments were observed. These effects were of questionable relevance to BPA toxicity given that they were seen only in single-dose groups, in several cases differed from the vehicle control by less than 10\%, and, in the case of organ weights, were not significant when adjusted for body weight.

In the stop-dose BPA study arm at two years, there was a statistically significant increase in the incidence of female mammary gland adenocarcinoma (22\% versus $6 \% ; p=0.016)$ and the combination of adenoma and adenocarcinoma $(24 \%$ versus $8 \% ; p=0.018)$ in the $2.5 \mu \mathrm{g} \mathrm{BPA} / \mathrm{kg}$ bw/day dose group. No increase in female mammary gland neoplasms was observed in the 
continuous BPA dose arm at two years. There were no significant treatment-related nonneoplastic lesions in the mammary gland of interim or terminal sacrifice stop-dose BPA groups. In the interim and terminal BPA continuous dosing arm, there was an increase, significant by the secondary RTE test only, in female mammary gland atypical foci at $2.5 \mu \mathrm{g} \mathrm{BPA} / \mathrm{kg}$ bw/day (14\% versus $0 \%$ and $15 \%$ versus $4 \%$ for the interim and terminal dose group animals, respectively). Increased adenoma/adenocarcinoma incidence observed only in the stop-dose animals, lack of a dose response, absence of non-neoplastic lesions in interim or terminal sacrifice stop-dose animals, and comparison to limited historical control data for this strain of rats in experiments conducted at NCTR bring into question the biological plausibility of this lesion as a BPA treatment-related effect. In addition to mammary gland neoplasms, a significant trend $(p=0.037)$ for uterine stromal polyps in the interim sacrifice animals in the continuous BPA dose arm was observed; this was not observed in the terminal sacrifice animals.

In the histopathological evaluations, there were many non-neoplastic lesions associated with aging in this strain of rats in both males and females that were variable across control and BPA treatment levels. In the interim stop-dose sacrifice BPA females, there was a significant dose trend with a significant increase in follicular cysts in the ovary at $25,000 \mu \mathrm{g} \mathrm{BPA} / \mathrm{kg} \mathrm{bw} /$ day dose group. The secondary statistical tests, which incorporated both incidence and severity scores, indicated an increase in cystic endometrial hyperplasia and squamous metaplasia in the uterus at $25,000 \mu \mathrm{g} \mathrm{BPA} / \mathrm{kg}$ bw/day in the interim stop-dose females. In the terminal stop-dose animals, secondary tests indicated an increase in cystic endometrial hyperplasia at 2,500 and 25,000 $\mu \mathrm{g}$ $\mathrm{BPA} / \mathrm{kg}$ bw/day, although severity was similar in the vehicle control and the BPA-treated groups. Cardiomyopathy was increased in the terminal stop-dose females at 2.5, 250, 2,500, and $25,000 \mu \mathrm{g} \mathrm{BPA} / \mathrm{kg}$ bw/day, as assessed by statistical tests that incorporated incidence and severity scores, although background incidence was high at this age and severity score differences across dose groups were similar. In interim continuous-dose females, uterine apoptosis and vaginal epithelial hyperplasia were elevated at 25,000 $\mu \mathrm{g} \mathrm{BPA} / \mathrm{kg} \mathrm{bw} /$ day. Vaginal epithelial hyperplasia was also increased in terminal continuous-dose animals at doses from 25 to $25,000 \mu \mathrm{g} \mathrm{BPA} / \mathrm{kg}$ bw/day, with a similar response across each of those dose levels.

There were no significant differences between treatment groups and vehicle controls in the incidences of neoplastic lesions in stop-dose or continuous-dose interim or terminal sacrifice males. There were also no apparent treatment-related non-neoplastic effects in interim stop-dose males; in terminal stop-dose BPA males, an increase of hyperplasia in the pars distalis of the pituitary at $25,000 \mu \mathrm{g} \mathrm{BPA} / \mathrm{kg}$ bw/day was noted. In interim, but not terminal, continuous-dose males there was an increase in exfoliated germ cells and an increase in lymphocyte infiltration in the epididymis at $25,000 \mu \mathrm{g}$ BPA/kg bw/day. No significant effects on sperm parameters or testicular histopathology were noted in the BPA dose groups. In the terminal continuous-dose males, hyperplasia of the pars distalis of the pituitary was increased at 25 and $25,000 \mu \mathrm{g} \mathrm{BPA} / \mathrm{kg}$ bw/day. Increases in dorsal/lateral prostate inflammation in most BPA dose groups were variable across a high background in both interim and terminal sacrifice animals.

In the $\mathrm{EE}_{2}$ reference estrogen dose groups, there were multiple significant treatment-related effects at the $0.5 \mu \mathrm{g} / \mathrm{kg}$ bw/day exposure level in females. At the time of estrous cycle evaluation at 16 weeks, more than $90 \%$ of the females in the $0.5 \mu \mathrm{g} \mathrm{EE} / \mathrm{kg}$ bw/day dose group were exhibiting prolonged estrus. At the interim sacrifice, mean weights of the adrenal glands, heart, kidney, liver, and pituitary gland were higher in the $0.5 \mu \mathrm{g} \mathrm{EE}_{2} / \mathrm{kg}$ bw/day dose group than the vehicle control means. Ovarian/parametrial fat pad and ovary weights were significantly lower 
than mean vehicle control weights in the high $\mathrm{EE}_{2}$ dose group. At the interim sacrifice, lobular hyperplasia and ductal dilatation were elevated in the mammary glands of the $0.5 \mu \mathrm{g} \mathrm{EE} / \mathrm{kg}$ bw/day dose group. Increases in apoptosis, cystic endometrial hyperplasia, and squamous metaplasia were observed in the uterus of the interim high dose $\mathrm{EE}_{2}$ females. Atrophy and cystic follicles were increased in the ovaries, the incidence of vaginal hyperplasia was increased, and increases in hyperplasia of the pars distalis and angiectasis were observed in the pituitary at 0.5 $\mu \mathrm{g} \mathrm{EE} / \mathrm{kg}$ bw/day. The incidences of cardiomyopathy and nephropathy were also increased in the high dose $\mathrm{EE}_{2}$ females at one year. At terminal sacrifice, there were significant increases in the incidence of mammary gland adenocarcinomas and combined adenomas/carcinomas of the pituitary pars distalis in the $0.5 \mu \mathrm{g} \mathrm{EE} / \mathrm{kg}$ bw/day dose group. There was a trend toward increasing uterine metaplasia at two years, and the incidence of nephropathy was increased in both the 0.05 and $0.5 \mu \mathrm{g} \mathrm{EE} 2 / \mathrm{kg}$ bw/day dose groups.

Few statistically significant effects of $\mathrm{EE}_{2}$ in males were observed. In the high dose $\mathrm{EE}_{2}$ group, there was an elevated incidence of lymphocyte infiltration observed in the epididymis in interim sacrifice animals and an increase in hyperplasia in the pars distalis of the pituitary at two years.

In conclusion, in the CLARITY-BPA core study, statistical differences between BPA treatment groups, particularly below $25,000 \mu \mathrm{g} / \mathrm{kg}$ bw/day, and the vehicle control group detected by the low-stringency statistical tests applied to histopathology lesions, were not dose responsive, sometimes occurring in only one low or intermediate dose group, and did not demonstrate a clear pattern of consistent responses within or across organs within the stop- and continuous-dose arms and sacrifice times. In contrast, the high $\mathrm{EE}_{2}$-dose elicited several estrogenic effects in females in a clearly interpretable and biologically plausible manner. Several observations at $25,000 \mu \mathrm{g} \mathrm{BPA} / \mathrm{kg}$ bw/day may be treatment related, including effects mentioned above in the female reproductive tract (ovary, uterus, and vagina) and in the male pituitary. 
Table 1. Summary of Endpoints Evaluated and Statistically Significant Treatment Effects of BPA and $\mathrm{EE}_{2}$ Relative to Vehicle Controls ${ }^{\mathrm{a}, \mathrm{b}}$

\begin{tabular}{|c|c|c|c|}
\hline Endpoint & $\begin{array}{l}\text { Stop-Dose } \\
\text { BPA }\end{array}$ & $\begin{array}{c}\text { Continuous-Dose } \\
\text { BPA }\end{array}$ & $\begin{array}{l}\text { Continuous- } \\
\text { Dose EE } 2\end{array}$ \\
\hline Gestational weight & NA & - & - \\
\hline Implantation sites & NA & - & - \\
\hline Litter size & NA & - & - \\
\hline Sex ratio & NA & - & - \\
\hline Litter weight & NA & - & - \\
\hline Male group pup weight, PND 1 & NA & - & - \\
\hline Female group pup weight, PND 1 & NA & - & - \\
\hline Preweaning pup survival, male & NA & - & - \\
\hline Preweaning pup survival, female & NA & - & $\downarrow(0.05)$ \\
\hline Preweaning pup body weight, male & NA & - & - \\
\hline Preweaning pup body weight, female & NA & - & $\begin{array}{c}\downarrow(0.05) \\
\text { PND } 4 \& 7\end{array}$ \\
\hline Age at vaginal opening & - & - & - \\
\hline Body weight at vaginal opening & Not analyzed ${ }^{\mathrm{c}}$ & - & - \\
\hline Postweaning survival, male, 1 year & - & - & - \\
\hline Postweaning survival, female, 1 year & - & - & - \\
\hline Postweaning body weight, male, 1 year & - & - & - \\
\hline Postweaning body weight, female, 1 year & - & - & - \\
\hline Postweaning survival, male, 2 years & - & - & - \\
\hline Postweaning survival, female, 2 years & - & - & - \\
\hline Postweaning body weight, male, 2 years & $\downarrow(\mathrm{T})$, wk 4 & - & - \\
\hline Postweaning body weight, female, 2 years & $\downarrow(\mathrm{T})$, wk 4 & $\begin{array}{c}\uparrow(250) \\
\text { wks } 96-104\end{array}$ & $\begin{array}{c}\uparrow(0.5) \\
\text { wks } 4 \text { and } 8\end{array}$ \\
\hline Abnormal estrous cycles at 16 weeks of age & - & - & $\uparrow(0.5)$ \\
\hline Early onset of aberrant estrous cycles & $\downarrow(2,500)$ & - & $\uparrow(0.5)$ \\
\hline \multicolumn{4}{|l|}{ Female organ weights, 1 year ${ }^{d}$} \\
\hline Adrenal gland & - & - & $\uparrow(0.5)^{\mathrm{e}}$ \\
\hline Fat pad, ovarian/parametrial & - & - & $\downarrow(0.5)$ \\
\hline Fat pad, retroperitoneal & - & $-{ }^{f}$ & - \\
\hline Heart & - & - & $\uparrow(0.5)$ \\
\hline Kidney & - & - & $\uparrow(0.5)^{\mathrm{e}}$ \\
\hline Liver & - & $\uparrow(\mathrm{T})$ & $\uparrow(0.5)^{\mathrm{e}}$ \\
\hline Ovary & $\downarrow(\mathrm{T})^{\mathrm{g}}$ & - & $\downarrow(0.5)^{\mathrm{e}}$ \\
\hline Pituitary gland & - & - & $\uparrow(0.5)^{\mathrm{e}}$ \\
\hline Spleen & - & - & - \\
\hline Thymus & - & - & - \\
\hline Thyroid gland & - & - & - \\
\hline Uterus & - & - & - \\
\hline \multicolumn{4}{|l|}{ Male organ weights, 1 year } \\
\hline Adrenal gland & - & - & - \\
\hline Epididymis & - & - & - \\
\hline Fat pad, epididymal & - & - & - \\
\hline Fat pad, retroperitoneal & - & - & - \\
\hline Heart & - & - & - \\
\hline Kidney & - & - & - \\
\hline Liver & $\uparrow(\mathrm{T})$ & $\downarrow(2.5)$ & - \\
\hline Pituitary gland & - & - & - \\
\hline
\end{tabular}


CLARITY-BPA Core Study

\begin{tabular}{lcccc}
\hline & Endpoint & Stop-Dose & Continuous-Dose & $\begin{array}{c}\text { Continuous- } \\
\text { BPAse EE2 }\end{array}$ \\
\hline Spleen & BPA & - & - \\
Testes & - & - & - \\
Thymus & - & - & - \\
Thyroid gland & - & - & -
\end{tabular}

Female hematology and clinical chemistry, 1 year

\begin{tabular}{|c|c|c|c|}
\hline \\
\hline Hematocrit & - & - & - \\
\hline Hemoglobin concentration & - & $\uparrow(\mathrm{T})$ & - \\
\hline Red blood cells & $\uparrow(\mathrm{T})$ & - & - \\
\hline \% Reticulocytes & - & - & - \\
\hline Packed cell volume & - & - & - \\
\hline Mean corpuscular volume & - & - & - \\
\hline Mean corpuscular hemoglobin & $\downarrow(\mathrm{T})$ & - & - \\
\hline Mean corpuscular hemoglobin concentration & - & $\uparrow(25)$ & - \\
\hline Platelets & - & $\downarrow(25,000, \mathrm{~T})$ & $\downarrow(0.5)$ \\
\hline White blood cells & - & - & - \\
\hline Neutrophils & - & - & - \\
\hline$\%$ Neutrophils & - & - & - \\
\hline Lymphocytes & - & - & - \\
\hline$\%$ Lymphocytes & - & - & - \\
\hline Monocytes & - & $\uparrow(\mathrm{T})$ & - \\
\hline$\%$ Monocytes & - & - & - \\
\hline Basophils & - & - & - \\
\hline$\%$ Basophils & $\uparrow(\mathrm{T})$ & - & - \\
\hline Eosinophils & - & $\downarrow(250)$ & $\downarrow(0.5)$ \\
\hline$\%$ Eosinophils & - & - & $\downarrow(0.5)$ \\
\hline Blood urea nitrogen (BUN) & - & - & - \\
\hline Creatinine & - & - & - \\
\hline Total protein & - & - & - \\
\hline Albumin & $(\mathrm{T})^{\mathrm{h}}$ & - & - \\
\hline Alkaline phosphatase & - & $\uparrow(250)$ & $\uparrow(0.05)$ \\
\hline Alanine aminotransferase & - & - & - \\
\hline Aspartate aminotransferase & - & - & - \\
\hline Sorbitol dehydrogenase & - & - & - \\
\hline Gamma-glutamyl transferase & - & - & - \\
\hline Total bile acids & - & - & - \\
\hline Cholesterol & - & - & - \\
\hline Glucose & - & - & - \\
\hline Triglycerides & - & - & - \\
\hline Insulin & - & - & - \\
\hline Leptin & - & - & - \\
\hline Triiodothyronine (T3) & - & - & - \\
\hline Thyroxine (T4) & - & - & - \\
\hline Thyroid-stimulating hormone (TSH) & - & - & $\uparrow(0.5)$ \\
\hline \multicolumn{4}{|c|}{ Male hematology, clinical chemistry, and sperm analyses, 1 year } \\
\hline Hematocrit & - & $\uparrow(\mathrm{T})$ & - \\
\hline Hemoglobin concentration & - & $\uparrow(25,000, \mathrm{~T})$ & $\uparrow(0.05)$ \\
\hline Red blood cells & - & - & - \\
\hline$\%$ Reticulocytes & - & - & - \\
\hline Packed cell volume & - & $\uparrow(\mathrm{T})$ & - \\
\hline Mean corpuscular volume & - & $\uparrow(\mathrm{T})$ & - \\
\hline
\end{tabular}




\begin{tabular}{|c|c|c|c|}
\hline Endpoint & $\begin{array}{c}\text { Stop-Dose } \\
\text { BPA }\end{array}$ & $\begin{array}{c}\text { Continuous-Dose } \\
\text { BPA }\end{array}$ & $\begin{array}{l}\text { Continuous- } \\
\text { Dose EE } 2\end{array}$ \\
\hline Mean corpuscular hemoglobin & - & $\uparrow(\mathrm{T})$ & - \\
\hline Mean corpuscular hemoglobin concentration & & - & - \\
\hline Platelets & & $\downarrow(\mathrm{T})$ & \\
\hline White blood cells & - & - & - \\
\hline Neutrophils & - & - & - \\
\hline$\%$ Neutrophils & $\downarrow(\mathrm{T})$ & - & - \\
\hline Lymphocytes & - & - & - \\
\hline$\%$ Lymphocytes & - & - & - \\
\hline Monocytes & - & - & - \\
\hline$\%$ Monocytes & - & - & - \\
\hline Basophils & - & - & - \\
\hline$\%$ Basophils & - & - & - \\
\hline Eosinophils & - & - & - \\
\hline$\%$ Eosinophils & - & $\downarrow(250)$ & - \\
\hline Blood urea nitrogen (BUN) & - & - & - \\
\hline Creatinine & - & - & - \\
\hline Total protein & $\downarrow(25)$ & - & - \\
\hline Albumin & - & $(\mathrm{T})^{\mathrm{h}}$ & - \\
\hline Alkaline phosphatase & - & - & - \\
\hline Alanine aminotransferase & - & - & - \\
\hline Aspartate aminotransferase & - & - & - \\
\hline Sorbitol dehydrogenase & - & - & - \\
\hline Gamma-glutamyl transferase & - & - & - \\
\hline Total bile acids & $\downarrow(25, \mathrm{~T})$ & $\downarrow(\mathrm{T})$ & - \\
\hline Cholesterol & - & - & - \\
\hline Glucose & - & - & - \\
\hline Triglycerides & - & - & $\uparrow(0.5)$ \\
\hline Insulin & - & - & $\downarrow(0.05)$ \\
\hline Leptin & - & - & - \\
\hline Troponin $\mathrm{T}$ & & $\uparrow(\mathrm{T})$ & \\
\hline $\mathrm{T} 3$ & - & - & - \\
\hline $\mathrm{T} 4$ & $\downarrow(\mathrm{T})$ & $(\mathrm{T})^{\mathrm{h}}$ & - \\
\hline $\mathrm{TSH}$ & - & - & - \\
\hline Testicular spermatid head counts & - & - & - \\
\hline Cauda sperm counts & - & - & - \\
\hline Cauda sperm, $\%$ motility & - & - & - \\
\hline Cauda sperm, abnormal & - & - & - \\
\hline
\end{tabular}

Females, neoplastic lesions, 1 year

Uterus, stromal polyps $--\uparrow(\mathrm{T})$

\section{Females, neoplastic lesions, 2 years}

Mammary gland, adenocarcinoma

Adrenal, medulla, pheochromocytoma, benign

Thyroid gland, adenoma, C-cell

$\begin{array}{ccc}\uparrow(2.5) & - & \uparrow(0.5, \mathrm{~T}) \\ - & - & \uparrow(\mathrm{T}) \\ - & - & \uparrow(\mathrm{T})\end{array}$

Females, non-neoplastic lesions, 1 year ${ }^{\mathrm{i}, \mathrm{j}}$

Mammary gland, dilatation, duct

Mammary gland, hyperplasia, lobular

Mammary gland, galactocele

Uterus, apoptosis

\begin{tabular}{c}
$\uparrow(0.5, \mathrm{~T})$ \\
$\uparrow(0.5, \mathrm{~T})$ \\
$\uparrow(\mathrm{T})$ \\
$\uparrow(0.5, \mathrm{~T})$ \\
\hline
\end{tabular}




\begin{tabular}{|c|c|c|c|}
\hline Endpoint & $\begin{array}{c}\text { Stop-Dose } \\
\text { BPA }\end{array}$ & $\begin{array}{c}\text { Continuous-Dose } \\
\text { BPA }\end{array}$ & $\begin{array}{c}\text { Continuous- } \\
\text { Dose } \mathrm{EE}_{2}\end{array}$ \\
\hline Uterus, hyperplasia, cystic, endometrium & - & - & $\uparrow(0.5, \mathrm{~T})$ \\
\hline Uterus, metaplasia, squamous & - & $\uparrow(\mathrm{T})$ & $\uparrow(0.5, \mathrm{~T})$ \\
\hline Ovary, atrophy & - & - & $\uparrow(0.5, \mathrm{~T})$ \\
\hline Ovary, cyst, follicle & $\uparrow(25,000, T)$ & - & $\uparrow(0.5, \mathrm{~T})$ \\
\hline Ovary, cyst, bursa & - & - & $\uparrow(\mathrm{T})$ \\
\hline Ovary, cyst, corpora lutea & - & $\uparrow(\mathrm{T})$ & - \\
\hline Ovary, depletion, corpora lutea & - & $\uparrow(\mathrm{T})$ & $\uparrow(0.5, \mathrm{~T})$ \\
\hline Ovary, hypertrophy, interstitial cell & - & $\uparrow(\mathrm{T})$ & $\uparrow(0.5, \mathrm{~T})$ \\
\hline Vagina, hyperplasia, epithelium & - & $\uparrow(\mathrm{T})$ & $\uparrow(0.5, \mathrm{~T})$ \\
\hline Vagina, mucification, epithelium & - & - & $\uparrow(\mathrm{T})$ \\
\hline Pituitary, angiectasis & - & - & $\uparrow(\mathrm{T})$ \\
\hline Heart, cardiomyopathy & - & - & $\uparrow(0.5, \mathrm{~T})$ \\
\hline Kidney, mineralization & - & $\uparrow(\mathrm{T})$ & - \\
\hline Kidney, cyst, renal tubule & - & $\uparrow(2.5)$ & $\uparrow(0.05)$ \\
\hline Kidney, nephropathy & - & - & $\uparrow(0.5, \mathrm{~T})$ \\
\hline Liver, infiltration, mononuclear cells & $\uparrow(2.5,25,000)$ & - & - \\
\hline \multicolumn{4}{|l|}{ Females, non-neoplastic lesions, 2 years ${ }^{\mathrm{i}, \mathrm{k}}$} \\
\hline Mammary gland, dilatation, duct & - & - & $\uparrow(0.5, \mathrm{~T})$ \\
\hline Mammary gland, dilatation, alveolus & - & - & $\uparrow(0.5, \mathrm{~T})$ \\
\hline Mammary gland, galoctocele & - & - & $\uparrow(\mathrm{T})$ \\
\hline Ovary, cyst & - & - & $\uparrow(\mathrm{T})$ \\
\hline Ovary, cyst, bursa & - & - & $\uparrow(\mathrm{T})$ \\
\hline Uterus, dilatation, lumen & - & $\uparrow(\mathrm{T})$ & - \\
\hline Uterus, cyst, endometrium & - & - & $\uparrow(\mathrm{T})$ \\
\hline Uterus, hyperplasia, endometrium & - & - & $\uparrow(0.05)$ \\
\hline Uterus, metaplasia, squamous & - & - & $\uparrow(\mathrm{T})$ \\
\hline Uterus, atrophy & - & - & $\uparrow(0.5, \mathrm{~T})$ \\
\hline Vagina, hyperplasia, epithelium & - & $\begin{array}{c}\uparrow(25,2,500, \\
25,000, T)\end{array}$ & - \\
\hline Vagina, degeneration, epithelium & - & $\uparrow(2,500)$ & - \\
\hline Pituitary, angiectasis & - & - & $\uparrow(0.5, \mathrm{~T})$ \\
\hline Pituitary, hemorrhage & - & - & $\uparrow(0.5, \mathrm{~T})$ \\
\hline Kidney, nephropathy & - & $\uparrow(2.5)$ & $\uparrow(\mathrm{T})$ \\
\hline Kidney, cyst, renal tubule & $\uparrow(2.5)$ & - & - \\
\hline Kidney, cyst, cortex & - & - & $\uparrow(0.05)$ \\
\hline Liver, cystic degeneration & $\uparrow(\mathrm{T})$ & - & - \\
\hline Liver, basophilic focus & - & - & $\uparrow(\mathrm{T})$ \\
\hline Liver, vacuolization, cytoplasmic & - & - & $\uparrow(0.05)$ \\
\hline Thyroid, hyperplasia, follicular cells & - & - & $\uparrow(0.05)$ \\
\hline Thyroid, ultimobranchial cyst & $\uparrow(250,2,500)$ & - & $\uparrow(\mathrm{T})$ \\
\hline Thymus, atrophy & - & $\uparrow(\mathrm{T})$ & - \\
\hline Pancreas, hyperplasia, acinar cell & - & - & $\uparrow(\mathrm{T})$ \\
\hline Adrenal cortex, degeneration, cystic & - & - & $\uparrow(0.5, \mathrm{~T})$ \\
\hline Adrenal cortex, pigmentation & - & - & $\uparrow(\mathrm{T})$ \\
\hline Bone marrow, hyperplasia, myeloid cell & - & - & $\uparrow(\mathrm{T})$ \\
\hline Spleen, pigmentation & - & - & $\uparrow(0.5, \mathrm{~T})$ \\
\hline Brain stem, compression & - & - & $\uparrow(0.5, \mathrm{~T})$ \\
\hline Brain stem, hemorrhage & - & - & $\uparrow(0.5, \mathrm{~T})$ \\
\hline
\end{tabular}




\begin{tabular}{lccc}
\hline \multicolumn{1}{c}{ Endpoint } & $\begin{array}{c}\text { Stop-Dose } \\
\text { BPA }\end{array}$ & $\begin{array}{c}\text { Continuous-Dose } \\
\text { BPA }\end{array}$ & $\begin{array}{c}\text { Continuous- } \\
\text { Dose EE } 2\end{array}$ \\
\hline Males, neoplastic lesions, 1 year & & & \\
\hline None & - & - & -
\end{tabular}

\author{
Males, neoplastic lesions, 2 years \\ Malignant lymphoma, systemic
}

$\uparrow(\mathrm{T})^{1}$

$-$

$-$

\begin{tabular}{lccc}
\hline Males, non-neoplastic lesions, $\mathbf{1}$ year $\mathbf{i}, \mathbf{j}$ & & & \\
\hline Epididymis, exfoliated germ cells & - & $\uparrow(25,000, \mathrm{~T})$ & - \\
Epididymis, infiltration cellular, lymphocyte & - & $\uparrow(25,000, \mathrm{~T})$ & - \\
Liver, hepatodiaphragmatic nodule & - & $\uparrow(2,500)$ & - \\
Liver, infiltration, mononuclear cells & - & $\uparrow(250,2,500)$ & $\uparrow(0.05)$ \\
Liver, fatty change & - & - & $\uparrow(\mathrm{T})$ \\
Spleen, hematopoietic cell proliferation & $\uparrow(250, \mathrm{~T})$ & $\uparrow(\mathrm{T})$ & - \\
Spleen, pigmentation & & - & -
\end{tabular}

\begin{tabular}{lccc}
\hline Males, non-neoplastic, 2 years $\mathbf{i}, \mathbf{k}$ & & & \\
\hline Dorsal/lateral prostate, suppurative inflammation & - & $\uparrow(2.5)$ & - \\
Mammary gland, dilatation, alveolus & - & $\uparrow(2.5)$ & - \\
Kidney, hyperplasia, transitional epithelium & $\uparrow(\mathrm{T})$ & $\uparrow(25)$ & - \\
Kidney, cyst, renal tubule & $\uparrow(\mathrm{T})$ & $\uparrow(250,2,500)$ & $\uparrow(0.05)$ \\
Pituitary gland, hyperplasia, pars distalis & $\uparrow(25,000, \mathrm{~T})$ & $\uparrow(25,000, \mathrm{~T})$ & $\uparrow(0.5, \mathrm{~T})$ \\
Pituitary, cyst, pars distalis & $\uparrow(250)$ & $\uparrow(\mathrm{T})$ & - \\
& & $($ multilocular $)$ & \\
Thyroid gland, hyperplasia, C-cell & - & $\uparrow(2,500, \mathrm{~T})$ & $\uparrow(0.05)$ \\
Parathyroid gland, hyperplasia & $\uparrow(\mathrm{T})$ & $\uparrow(25)$ & - \\
Pancreas, pigmentation & $\uparrow(2.5)$ & - & - \\
Pancreas, polyarteritis & $\uparrow(2,500, \mathrm{~T})$ & - & - \\
Heart, cardiomyopathy & $\uparrow(\mathrm{T})$ & - & - \\
Heart, metaplasia, osseus & - & - & $\uparrow(\mathrm{T})$ \\
Adrenal medulla, hyperplasia & $\uparrow(2,500, \mathrm{~T})$ & - & - \\
Adrenal cortex, hypertrophy & - & - & $\uparrow(0.05)$ \\
Testes, polyarteritis & $\uparrow(2,500, \mathrm{~T})$ & - & - \\
Bone marrow, hypocellularity & $\uparrow(250,25,000)$ & - & - \\
Spleen, hyperplasia, lymphoid & $\uparrow(250, \mathrm{~T})$ & - & - \\
Liver, angiectasis & - & $\uparrow(2.5)$ & - \\
Liver, vacuolization, cytoplasmic & - & - & $\uparrow(0.5, \mathrm{~T})$ \\
\hline
\end{tabular}

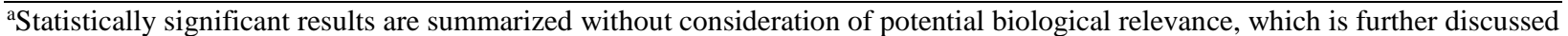
in the text. Results for the sensitivity analyses that excluded a subset of animals (see text) are not included in this summary table. NA, not applicable; $\uparrow$ or $\downarrow$, significant increase or decrease relative to controls at the exposure concentration ( $\mu \mathrm{g} / \mathrm{kg}$ bw/day) indicated in parentheses; "--"= no significant treatment effect; "T" = trend.

${ }^{b}$ For $\mathrm{EE}_{2}$ dose groups, trend analyses were only conducted for the histopathology data.

${ }^{c}$ Endpoint was not analyzed since body weight at vaginal opening was not recorded for many pups.

${ }^{\mathrm{d}}$ Results for organ weights adjusted for body weights are summarized in this table.

${ }^{\mathrm{e}}$ Absolute organ weight and organ weight adjusted for brain weight were also significantly different from vehicle control. ${ }^{\mathrm{f}} \mathrm{Absolute}$ organ weight and organ weight adjusted for brain weight in the $2.5 \mu \mathrm{g} \mathrm{BPA} / \mathrm{kg}$ bw/day dose group were significantly higher than the vehicle control.

gThere were also significant dose trends for the absolute organ weight and organ weight adjusted for brain weight, and the $25,000 \mu \mathrm{g}$ BPA $/ \mathrm{kg}$ bw/day dose group was significantly lower than the vehicle control weight in both cases.

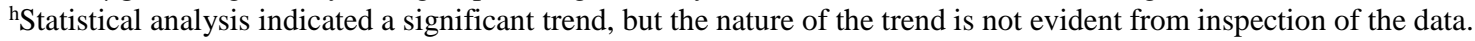

${ }^{i}$ For histopathology data, statistically significant effects in a negative, or potentially beneficial, direction relative to controls are not included in this summary table, but are presented in the Supplemental Appendices and, in some cases, in the body of the report. Some diagnoses included in the statistical tables (Supplemental Appendices XXXIII and XXXIV), such as stages of the 


\section{CLARITY-BPA Core Study}

estrous cycle in females or changes noted that would not be considered of pathological significance (e.g., tension lipidosis), are not tabulated in this summary table.

${ }^{j}$ For interim non-neoplastic lesions, only Cochran-Armitage with Fisher's exact (CAFE) test results are summarized in this table. Results of additional statistical tests are presented and discussed in the text.

${ }^{k}$ For terminal non-neoplastic lesions, only Poly-3 test results are summarized in this table. Results of additional statistical tests are presented and discussed in the text.

${ }^{1}$ A significant trend for systemic lymphoma was noted in liver, bone marrow, spleen, kidney, and dorsal/lateral prostate. In addition, the incidence in the dorsal/lateral prostate at 25,000 $\mu \mathrm{g} \mathrm{BPA} / \mathrm{kg}$ bw/day was significantly higher than the incidence in vehicle control. 


\section{Background}

Bisphenol A (BPA) is a high-production-volume industrial chemical that is used as a monomer in the production of polycarbonate plastics and epoxy resins that have broad applications in consumer products, including storage containers for foods and beverages and in medical devices. BPA has undergone extensive toxicological evaluations in laboratories around the world, but the conclusions derived from the aggregate results of the studies remain under debate. The current safety assessments by the preponderance of international regulatory agencies conclude that BPA at current exposure levels (upper $95^{\text {th }}$ percentile of typical daily aggregate exposure $<0.5 \mu \mathrm{g} / \mathrm{kg}$ body weight (bw)/day) does not pose a risk to humans via dietary exposure ${ }^{1-4}$. In contrast, others have concluded that the overall body of evidence from BPA investigations indicates that BPA is likely to be a human health hazard ${ }^{5-7}$. France has banned the use of BPA in food contact materials based on the assessment of the French Agency for Food, Environmental Health and Safety ${ }^{8}$. The Office of Environmental Health Hazard Assessment of the California Environmental Protection Agency has listed BPA as a reproductive toxicant under California's Proposition $65^{9}$.

The National Toxicology Program (NTP) previously assessed BPA in two-year dietary administration studies in both sexes of F344 rats at feed concentrations of 1,000 and 2,000 ppm (approximately 50,000 and 100,000 $\mathrm{g} \mathrm{BPA} / \mathrm{kg}$ bw/day), in male $\mathrm{B} 6 \mathrm{C} 3 \mathrm{~F}_{1}$ mice at feed concentrations of 1,000 and 5,000 ppm (approximately 150,000 and 750,000 $\mu \mathrm{g} \mathrm{BPA} / \mathrm{kg}$ bw/day), and in female $\mathrm{B}_{6} \mathrm{C} 3 \mathrm{~F}_{1}$ mice at feed concentrations of 5,000 and 10,000 ppm (approximately 750,000 and 1,500,000 $\mu \mathrm{g} \mathrm{BPA} / \mathrm{kg}$ bw/day) ${ }^{10}$. The NTP conclusions from these studies were that there was no convincing evidence of carcinogenesis. The earlier NTP studies did not address the current issues of toxicities resulting from developmental exposures at levels closer to human exposure levels. Given the current public controversy, the NTP and the National Institute of Environmental Health Sciences (NIEHS) Division of Extramural Research and Training (DERT) agreed to sponsor a study that would involve exposures to a broad range of BPA doses, include developmental exposure, and, in addition to evaluating endpoints typically used for regulatory decision-making, provide animals or tissues from this study to a group of NIEHS-funded academic scientists to pursue hypothesis-driven studies in various organ systems. The purpose of this research program was to evaluate chronic exposure to BPA over a broad dose range using traditional and non-traditional endpoints. It aimed to determine if the nontraditional endpoints could reveal toxicity not detected by traditional guideline study endpoints and provide mechanistic support for observations made in the guideline study. For most of the hypothesis-driven studies, the academic scientists were provided by the Food and Drug Administration (FDA)'s National Center for Toxicological Research (NCTR) with tissues or serum from animals of various ages. The exceptions were behavioral and erectile dysfunction studies, where staff from the academic laboratories conducted the studies with animals at NCTR with the assistance of NCTR staff. Detailed descriptions of the general plan of this project, termed the Consortium Linking Academic and Regulatory Insights on BPA Toxicity (CLARITY-BPA), have been published ${ }^{11 ; 12}$. This report focuses only on the description of the conduct of and results from the core chronic study, which focused on standard toxicological endpoints. 


\section{Animal Model}

The animal model used in these studies was the Sprague-Dawley rat maintained at NCTR. This colony had its origins in the late 1970s from Charles River Sprague-Dawley founders and has been used in toxicology studies with hormonally active agents for over a decade at NCTR ${ }^{13-20}$. A comprehensive evaluation of the pharmacokinetics of BPA in this rat strain across life stages, including upon oral exposure by gavage, has been conducted ${ }^{21-23}$. Dietary ethinyl estradiol $\left(\mathrm{EE}_{2}\right)$ exposure studies in this strain ${ }^{15 ; 16 ; 18 ; 20}$ resulted in peak blood levels of $\mathrm{EE}_{2}$ in adult animals below $10 \mathrm{pg} / \mathrm{mL}(30 \mathrm{pM})$ at the highest dose tested (50-ppb feed concentration, or approximately 4 and $5-6 \mu \mathrm{g} / \mathrm{kg}$ bw/day in males and females, respectively $)^{24}$. Stimulation of male mammary hyperplasia was observed at 2, 10, and $50 \mathrm{ppb}$ dietary $\mathrm{EE}_{2}$ at postnatal day (PND) 140, but not in older animals (two years of age). Effects on vaginal opening, body weight gain, food efficiency, and estrous cyclicity were observed at the higher doses of 10 and $50 \mathrm{ppb}$, approximately 1 and 6 $\mu \mathrm{g} / \mathrm{kg}$ bw/day, respectively. Similar, but not identical, effects were observed with dietary genistein over a dose range of 5 to $500 \mathrm{ppm}$ (approximately 300 to $50,000 \mu \mathrm{g} / \mathrm{kg}$ bw/day) ${ }^{18}$. Taken together with the more recent gavage BPA and $\mathrm{EE}_{2}$ studies $^{19 ; 25}$, these data demonstrate the relative sensitivity of this rat strain to estrogenic compounds.

\section{Rationale for Dose Selection for BPA and the Reference Estrogen, $\mathrm{EE}_{2}$}

Prior to the design of this chronic toxicity study and the conception of the CLARITY-BPA project, a subchronic study was conducted at NCTR using the same animal model and dosing regimen used in the current chronic study ${ }^{19}$. BPA in $0.3 \%$ carboxymethylcellulose (CMC) was administered by oral gavage to pregnant female Sprague-Dawley rats from gestation day (GD) 6 through the start of parturition, with seven doses between 2.5 and 2,700 $\mu \mathrm{g} \mathrm{BPA} / \mathrm{kg}$ bw/day spaced at half-log intervals. Two high doses of BPA $(100,000$ and 300,000 $\mu \mathrm{g} / \mathrm{kg}$ bw/day) were added as doses expected to produce adverse effects based on published guideline studies ${ }^{26 ; 27}$. Vehicle and naïve (not dosed by gavage) controls were included. Dams were not dosed after their litters were born, but pups were directly dosed by oral gavage from PND 1 until termination (PND $90 \pm 5$ ). Pups were directly dosed because of the demonstrated low transmission of BPA to pups via milk ${ }^{28}$. BPA showed clearly adverse effects in $F_{1}$ females at the highest doses of 100,000 and 300,000 $\mu \mathrm{g} \mathrm{BPA} / \mathrm{kg}$ bw/day. Statistically significant differences at lower BPA doses were few and sporadic and were judged not to provide evidence of BPA-induced toxicity ${ }^{19}$. There were no BPA effects observed in the males.

The design of the CLARITY-BPA core study was discussed and finalized at a series of meetings in late 2011 and early 2012. These meetings included NTP, NCTR, NIEHS/DERT, and FDA product center representatives, as well as NIEHS-funded CLARITY-BPA grantees. It was proposed that, because of the literature concerning permanent adverse effects resulting from developmental exposures to hormonally active agents, the study would include groups of animals for which exposure would terminate at weaning. For discussion of dose selection, a summary of data obtained from the NCTR BPA subchronic study ${ }^{19}$ was presented and discussed. It was initially agreed that a vehicle control and six log-spaced doses between 2.5 and 250,000 $\mu \mathrm{g}$ $\mathrm{BPA} / \mathrm{kg}$ bw/day would provide an adequate dose range from reasonably close to human exposure on the low end to a dose expected to produce clear adverse effects at the high end. Serum measurements of BPA across the postnatal life span of animals in the subchronic study ${ }^{25}$ showed 
that the high dose of $300,000 \mu \mathrm{g} \mathrm{BPA} / \mathrm{kg}$ bw/day gave rise to approximately $50 \mu \mathrm{M}$ active unconjugated BPA in PND 4 animals and approximately $1 \mu \mathrm{M}$ in PND 80 animals, which were clearly out of the range of attainable human internal exposure from dietary sources, estimated to be in the low to sub-pM range ${ }^{29}$. There was general agreement that the current concern was restricted to a lower dose range, below the previously reported no-observed-adverse-effect level in guideline-compliant regulatory toxicity assays, which was $5,000 \mu \mathrm{g} / \mathrm{kg} \mathrm{bw} /$ day $^{26 ; 27}$. The $250,000 \mu \mathrm{g} \mathrm{BPA} / \mathrm{kg}$ bw/day dose group would provide little additional information to influence regulatory action. A high dose of $25,000 \mu \mathrm{g} \mathrm{BPA} / \mathrm{kg}$ bw/day was viewed as providing an adequate margin of human exposure, greater than 25,000-fold based on the aggregate human exposure estimates of $<0.5 \mu \mathrm{g}$ BPA $/ \mathrm{kg}$ bw/day mentioned above. The low dose selected, $2.5 \mu \mathrm{g} \mathrm{BPA} / \mathrm{kg}$ bw/day, provided a margin of exposure at least 10-fold higher than the maximum allowed background dietary exposure (see below).

Much of the research on BPA, particularly early in the investigations of the potential toxicity of low doses of BPA, focused on its estrogenic activity, although the involvement of mechanisms other than classical estrogen receptors have been increasingly implicated in BPA actions ${ }^{30-33}$. Both doses of $\mathrm{EE}_{2}$ used in the NCTR BPA subchronic study (0.5 and $5 \mu \mathrm{g} \mathrm{EE} / \mathrm{kg}$ bw/day) produced strong effects in female reproductive organs and on estrous cyclicity. In males, the low dose of $0.5 \mu \mathrm{g} / \mathrm{kg} \mathrm{bw} /$ day had little effect, with an increase in male mammary hyperplasia as the only apparent effect at PND $90^{19}$. The $5 \mu \mathrm{g} \mathrm{EE} / \mathrm{kg}$ bw/day dose also stimulated male mammary gland hyperplasia at PND 90, and increased hyperplasia in the coagulating gland, increased degeneration in the testicular germinal epithelium, and increased exfoliated germ cells and hypospermia in the epididymis. Based on the observed effects in the NCTR BPA subchronic study, two levels of $\mathrm{EE}_{2}$, one of which was lower than the doses used in the subchronic study, were selected for use in the current study to expand information on the sensitivity of the animal model to $\mathrm{EE}_{2}$. In the absence of data from this exposure model to guide the selection of the $\mathrm{EE}_{2}$ lower dose, a 10-fold lower dose was chosen. The CLARITY-BPA consortium consensus for the two doses of $\mathrm{EE}_{2}$ used in the current study was 0.05 and $0.5 \mu \mathrm{g} / \mathrm{kg}$ bw/day. Resource limitations did not allow for the inclusion of stop-dose $\mathrm{EE}_{2}$ groups. Likewise, although the NCTR BPA subchronic study had included a naïve control group that was not dosed by gavage, this group could not be included in the chronic study. The responses of the naïve and vehicle control groups in the NCTR BPA subchronic study were similar ${ }^{19}$.

\section{Internal Dosimetry of BPA and $\mathrm{EE}_{2}$}

In the previous NCTR BPA subchronic study, blood levels of conjugated (biologically inactive) and unconjugated (biologically active) BPA (dose levels of 2.5, 8, 25, 80, 260, 840, 2,700, 100,000 , and $300,000 \mu \mathrm{g} / \mathrm{kg}$ bw/day) and $\mathrm{EE}_{2}(0.5$ and $5.0 \mu \mathrm{g} / \mathrm{kg}$ bw/day) were measured. The previous study used identical conditions to the current chronic study, including the same animal model, feed, housing materials, dosing formulation, and dosing regimen. Blood was collected at approximate maximal concentration time $\left(\mathrm{C}_{\max }, 15-30\right.$ minutes after dosing) at PND 4, 21, and $80^{10}$. For both BPA and $\mathrm{EE}_{2}$, percentages of unconjugated compound were highest on PND 4 and decreased with age, reflective of increasing capacity for metabolism with age ${ }^{17}$. Although the percentage of unconjugated BPA at doses lower than $100,000 \mu \mathrm{g} / \mathrm{kg}$ bw/day were approximately $4 \%$ at PND 4 and 1-2\% at PNDs 21 and 80, $\mathrm{EE}_{2}$ was $100 \%, 50 \%$, and $10 \%$ unconjugated on PNDs 4, 21, and 80, respectively. For BPA, there was evidence of saturation of metabolism at the higher doses, with $\geq 10 \%$ unconjugated at 100,000 and $300,000 \mu \mathrm{g} / \mathrm{kg}$ bw/day. Based on these 
data and an earlier single-dose pharmacokinetic study in this rat strain ${ }^{21}$, peak serum levels of unconjugated BPA across the $2.5-25,000 \mu \mathrm{g} / \mathrm{kg}$ bw/day dose span tested in the present study were anticipated to range from $<0.5 \mathrm{nM}$ to approximately $2.5-3 \mu \mathrm{M}$ at PND 4 and from approximately $0.01 \mathrm{nM}$ (below detectable limits) to approximately $30 \mathrm{nM}$ after weaning. In the case of $0.5 \mu \mathrm{g} \mathrm{EE} / \mathrm{kg} /$ day, equivalent to the high $\mathrm{EE}_{2}$ dose of in this chronic study, peak measured serum levels were approximately 500 and 10 pM on PND 4 and 21, respectively, and below the limit of detection of $5 \mathrm{pM}$ in adults (PND 80) ${ }^{25}$. Estimates of the potency of BPA relative to $\mathrm{EE}_{2}$ from in vitro transcriptional and in vivo uterotrophic assays are in the range of $10^{-}$ ${ }^{4}-10^{-5}$ (see, for example, Conley et al. ${ }^{34}$ ). 


\section{Materials and Methods}

\section{Procurement and Characterization of Bisphenol A}

Bisphenol A (BPA, CAS \#80-05-7, synonyms: 2,2-bis(4-hydroxyphenyl)propane; 4,4'-isopropylidenediphenol) was supplied by TCI America (Portland, OR; product \#B0494) as lot \#6052012, with a purity assessment on the Certificate of Analysis by Battelle, Inc.

(Columbus, $\mathrm{OH}$ ) of $99.9 \%$. This lot of BPA (original lot designation AOHOK) was purchased by the NTP in 2009 and was characterized for identity and purity by proton nuclear magnetic resonance (NMR) and high performance liquid chromatography with photodiode array detection (HPLC-PDA) at NCTR prior to the start of the BPA subchronic study (NCTR E0217601). Battelle Laboratories extensively characterized this lot of BPA and reported the analysis to be consistent with the manufacturer's stated purity of 99.9\% (Bulk Chemical Limited Analysis Report: Bisphenol A, Battelle Project No. G005430-DSU, October 29, 2010, which was included as part of the NCTR Technical Report for E0217601, July 15, 2013). For the present study, Battelle air milled this same lot of BPA prior to shipment to NCTR and provided a current statement of purity. Purity assessment was conducted at NCTR prior to the study start, at intervals during the study, and after study completion using the technique of HPLC-PDA (spectral purity). A sample of the test article was subjected to HPLC separation at least in triplicate and the PDA was scanned from 200 to $400 \mathrm{~nm}$. A single major peak was obtained at the expected HPLC retention time for BPA and showed 99-100\% purity (Supplemental Appendix VII). ${ }^{\mathrm{a}}$

The reference estrogen test article ethinyl estradiol (EE 2 , CAS \#57-63-6, product \#E4876, lot \#071M1492V) was purchased from Sigma-Aldrich Corporation (St. Louis, MO). The stated purity of the $\mathrm{EE}_{2}$ was $>98 \%$. A sample of this test article was evaluated by HPLC with PDA and electrospray mass spectrometry and found to contain a single peak that contained fragment ions consistent with and matching a reference sample of $\mathrm{EE}_{2}$ from Steraloids, Inc. (Newport, RI). Analysis in the same manner as described for BPA showed purity $>99 \%$ (Supplemental Appendix VII).

CMC (sodium salt; product \#C5013, lot \#041M0105V) was obtained from Sigma-Aldrich (St. Louis, MO). Aqueous CMC solutions were prepared for use as the vehicle for BPA and $\mathrm{EE}_{2}$ by the Diet Preparation Support Group at NCTR as described in Supplemental Appendix IX.

\section{Preparation and Analysis of Dose Formulations}

Dosing solutions or suspensions were prepared by the Diet Preparation Support Group at NCTR (Supplemental Appendix IX). For BPA, the target concentrations of the dose preparations were $0.5,5,50,500$, and $5000 \mu \mathrm{g} / \mathrm{mL} 0.3 \% \mathrm{CMC}$ for the $2.5,25,250,2,500$, and $25,000 \mu \mathrm{g} / \mathrm{kg}$ bw/day dose groups, respectively. For $\mathrm{EE}_{2}$, the target concentrations were 0.01 and $0.1 \mu \mathrm{g} / \mathrm{mL}$ $0.3 \% \mathrm{CMC}$ for the 0.05 and $0.5 \mu \mathrm{g} / \mathrm{kg}$ bw/day dose groups, respectively. The two high BPA dosing suspensions were mixed by directly adding BPA solid to the vehicle with sonication, and the suspensions were stirred constantly. The three low BPA and the two $\mathrm{EE}_{2}$ dosing solutions were mixed by serial dilutions of stock solutions prepared and certified by the NCTR Chemistry Support Group. Stabilities ( $\pm 10 \%$ of the target) of the low and high dose levels of both BPA and

aSupplemental Appendices at: https://doi.org/10.22427/NTP-DATA-018-00015-0001-000-6. 
$\mathrm{EE}_{2}$ were established for up to 50 days. Homogeneity ( $\pm 10 \%$ of the target) of the high BPA suspension and both $\mathrm{EE}_{2}$ dosing solutions was established (Supplemental Appendix VII).

At the start of the study and approximately every 8-10 weeks over the course of the study, all dose level preparations were assayed by the Chemistry Support Group prior to delivery to the animal rooms and certified to be within $10 \%$ of the target concentration with a $\% \mathrm{CV}$ of $\leq 10 \%$. In addition, at intervals spaced 4-7 months apart, BPA and $\mathrm{EE}_{2}$ dosing preparations from the animal rooms were assayed at the end of their use to verify the dose concentrations. One batch of $0.3 \% \mathrm{CMC}$ vehicle was found to have a detectable concentration of BPA $(0.11 \mu \mathrm{g} / \mathrm{mL})$ that was approximately $20 \%$ of the low BPA dose level (Table 13 in Supplemental Appendix VII). One container of this batch of vehicle was used to dose 16 cages (31 animals; 6 males and 6 females continuous-dose one-year sacrifice and 9 males and 10 females continuous-dose two-year sacrifice) of vehicle control animals ranging in age from PND 139 to PND 141 on a single day before this batch of vehicle was discarded (Supplemental Appendix III, note to study file October 13, 2017). Given the short half-live of BPA and age of the animals, the impact of this event was considered minimal. All affected animals were from the first mating group. Thus, these animals were excluded from the sensitivity analysis described in the Statistical Methods section below. Following the detection of this contaminated batch of vehicle, all batches of vehicle that had been previously used in the study and all subsequent batches of vehicle were assayed and none contained BPA detectable above the analytical background.

\section{Administration of Dose Formulations and Vehicle}

Doses were administered by gavage with modified Hamilton Microlab® ML511C programmable 115 V pumps (Hamilton Co., Reno, NV). Dosing containers were constantly stirred and dose volume calculation and dispensing were automated ${ }^{35}$. Four separate dosing stations were used in each animal room: (1) vehicle control; (2) 2.5, 25, and $250 \mu \mathrm{g}$ BPA/kg bw/day; (3) 2,500 and $25,000 \mu \mathrm{g} \mathrm{BPA} / \mathrm{kg}$ bw/day; and (4) 0.05 and $0.5 \mu \mathrm{g} \mathrm{EE} / \mathrm{kg}$ bw/day. Dosing was conducted from the lowest to highest dose on any given pump and the Teflon tubing was flushed between dose levels. At the end of each day, each pump was flushed sequentially with water, $70 \%$ ethanol, and water. Verification of the accuracy of dose delivery from these pumps had been demonstrated for BPA dosing solutions ranging from 0.5 to $60,000 \mu \mathrm{g} / \mathrm{mL} 0.3 \% \mathrm{CMC}$ and for 0.1 and $1 \mu \mathrm{g}$ $\mathrm{EE}_{2} / \mathrm{mL}$ 0.3\% CMC for the prior NCTR BPA subchronic study (NCTR E0217601); accuracy was verified before the start of this study by chemical analysis for the low dose of $\mathrm{EE}_{2}$, which was 10-fold lower than the lowest dose used in the previous experiment (Table 8C in Supplemental Appendix VII). Subsequently, the accuracy of liquid delivery from the pumps was assessed every three months and established to be within $10 \%$ of the target volume (Supplemental Appendix XI).

\section{Diet Assessment: Nutrients and Contaminants, Including Background BPA}

Pelleted rodent chow, verified casein diet 10 IF, irradiated, 5K96 (product \#1810069, TestDiet, Purina Mills, Richmond, IN) was the diet used in the study. The manufacturer provided analyses for selected nutritive quality attributes (including protein, fat, crude fiber, calcium, phosphorous, and vitamins $\mathrm{A}, \mathrm{B}_{1}$, and $\mathrm{E}$ ) and contaminants (including nitrosamines, fumonisins, arsenic, cadmium, lead, mercury, aflatoxins, organochlorine and organophosphate pesticides, butylated 
hydroxyanisole, butylated hydroxytoluene, and tert-butyl hydroquinone). These analysis reports are found in Supplemental Appendix XII.

On arrival at NCTR, each lot of diet used in the present study was sampled and assayed by the Chemistry Support Group for background BPA and selected phyto- and myco-estrogens (genistein, daidzein, coumestrol, and zearalenone). These results are found in Supplemental Appendix VII. Low (ppb) background levels of BPA above the limit of blank (LOB) had previously been reported in all lots of 5K96 diet used in the prior NCTR BPA subchronic study and in other rodent diets ${ }^{19 ; 36}$ and a rejection limit of 5 ppb BPA in feed was set for both that and the present study. Ten of the 11 lots of diet used in the present study contained detectable levels of BPA, with an average (using 0 for the lot with no BPA detectable above the LOB) of $1.3 \pm 0.9$ (standard deviation, S.D.) ng/g diet (range 0-3.0). Based on estimates of food consumption (Supplemental Appendix XIIIa and XIIIb) for interim and terminal sacrifice animals, respectively, this resulted in an average consumption over the course of the study of approximately $0.05-0.06 \mu \mathrm{g} \mathrm{BPA} / \mathrm{kg}$ bw/day (2-2.5\% of the lowest BPA dose tested) at the mean dietary concentration of BPA and approximately $0.12-0.15 \mu \mathrm{g} \mathrm{BPA} / \mathrm{kg}$ bw/day $(5-6 \%$ of the lowest BPA dose tested) at the maximum measured dietary concentration (calculations summarized in Supplemental Appendix XIV). Since younger animals consume higher quantities of food per unit of body weight, younger animals consumed higher background levels of dietary BPA. For example, estimates of food consumption for the week after weaning (week 4) indicate that the amount of BPA consumed per kg bw was approximately 2- to 3-fold higher than the mean value calculated over the entire study (Supplemental Appendix XIV).

The 5K96 diet, which is low in soy-derived phytoestrogens, was selected to ensure a consistent and low level of these phytoestrogens to minimize any impact on the endpoints measured in this study. A goal of 2 ppm total genistein and daidzein was stated in the protocol based on our prior experience with the isoflavone levels attainable in this diet. Although the diet manufacturer indicated that the levels of isoflavones were less than $10 \mathrm{ppm}$ in this diet (page 6 of Supplemental Appendix IX), less than 2 ppm combined genistein and daidzein (measured after hydrolysis of glucosides in the diet) was typical in studies conducted with this diet at NCTR ${ }^{13-16 \text {; }}$ 25;36. Samples of each lot of diet were acid hydrolyzed to convert the glucosides of genistein and daidzein to aglycones that were quantified using HPLC-electrospray ionization-multiple reaction monitoring mass spectrometry. There was variation from lot to lot (Supplemental Appendix VII), but values of genistein and daidzein reported over the course of the study were consistent with the expectation of combined levels less than $2 \mathrm{ppm}$. After the study concluded and during the preparation of the Chemistry Support Report, however, it was discovered that a calculation error was made and that values of genistein and daidzein were 10-fold higher than those reported during the study. The values reported during the study are reflected in the Diet Preparation Report, Supplemental Appendix IX and the corrected values are found in the Chemistry Support Report, Supplemental Appendix VII. Mean values for genistein and daidzein in the 11 diet lots tested were $1.79 \pm 1.94$ (S.D.) and $1.66 \pm 2.06$ (S.D.) ppm, respectively. Although the values of isoflavones in the diet were higher than anticipated based on prior experience with this diet ${ }^{13-16 \text {; }}$ $25 ; 36$, the measured levels are low relative to other commonly used chow diets that have been reported to have values ranging from 100 to greater than $600 \mathrm{ppm}^{37-40}$. The phytoestrogen coumestrol and the mycoestrogen zearalenone were also measured in the diets used in the present study. No rejection level was set for these compounds, although any lot of diet with greater than $0.5 \mathrm{ppm}$ of coumestrol or zearelonone required approval prior to use. Two of the 11 diet lots 
tested were positive for coumestrol $(0.05$ and $0.08 \mathrm{ppm})$ and two lots were positive for zearalenone (0.01 and 0.05 ppm) (Supplemental Appendix VII).

\section{Assessment of Background BPA in Study Materials Other than Diet}

In addition to the dosing vehicle and diet, the rodent drinking water and extracts of animal cages and bedding (hardwood chip and cellulose) were assayed for background BPA levels. These results are reported in Supplemental Appendix VII. For the polysulfone cages (Ancare Corporation, Bellmore, NY) used in this study, previous results from NCTR BPA subchronic study (NCTR E0217601) indicated no BPA detectable above the analytical background (NCTR Technical Report for E0217601, July 15, 2013). For the present study, three previously used polysulfone cages and three newly purchased cages were extracted and the extracts confirmed to have no detectable BPA above the analytical background. Similarly, all drinking water samples and bedding extracts had BPA levels that were less than or close to the analytical limits of the BPA assay.

\section{Animal Source and Microbiological Surveillance}

The NCTR Multigeneration Support System (MGSS), an operator-prompted database system, was used to track the genealogy of all animals in the current study and to collect in-life animal data. For the parental $\left(\mathrm{F}_{0}\right)$ generation, 600 male and 600 female weanling (circa PND 21) Sprague-Dawley/CD23/NctrBR rats were obtained from the NCTR breeding colony in five groups of 120 of each sex. While in the breeding colony, these $\mathrm{F}_{0}$ breeders were maintained with their dams under the standard conditions used in the NCTR colony (fed NIH-41 irradiated pellets and housed in polycarbonate cages with hardwood chip bedding and water from polycarbonate bottles). Once assigned to the study at weaning on PND 21, the $\mathrm{F}_{0}$ breeders were fed pelleted irradiated TestDiet 5K96 feed, housed two to three per cage in polysulfone cages with hardwood chip bedding, and given water from glass water bottles with silicone stoppers. The rats were held under these conditions until they were individually housed prior to mating for one to two weeks for females, or for 48 hours for males. Animals were 10-15 weeks of age at mating. The animal rooms, water, feed, and health of the animals were monitored during the study in accordance with NCTR's Sentinel Animal Program; the results are reported in Supplemental Appendix VIII. As noted in a protocol deviation (Supplemental Appendix II), one room was without a sentinel for approximately the last month of the study as the sentinel animal had to be removed as moribund. All 46 sentinel animals evaluated periodically over the course of the study were determined to be free of pathogenic organisms.

\section{Animal Welfare}

Animal care and use were in accordance with the Public Health Service Policy on Humane Care and Use of Animals (https://grants.nih.gov/grants/olaw/references/phspolicylabanimals.pdf). The study was conducted in an animal facility accredited by the Association for the Assessment and Accreditation of Laboratory Animal Care International. The study was approved by the NCTR Animal Care and Use Committee and conducted in accordance with all relevant NIH and NTP animal care and use policies and applicable federal, state, and local regulations and guidelines. NCTR Veterinary Services Staff monitored the health of the animals throughout the study and made recommendations as to the timing of animal removal from the study based on evident 
distress or discomfort, decreased mobility, or inability to eat or drink. The Veterinary Staff also recommended the transfer of a subset of animals with pododermatitis or ventral masses from hardwood chip bedding that was irritating these lesions to Alpha-Dri cellulose bedding (Shepherd Specialty Papers, Watertown, TN; Supplemental Appendix II and Supplemental Appendix XV, Animals transferred to Alpha-Dri bedding).

\section{Study Design}

A sacrifice was conducted at one year of age (PND $365 \pm 20)$ and the terminal sacrifice was conducted at two years of age (PND $730 \pm 20$ ). The interim (one-year) sacrifice group was included to allow evaluation of long-term exposure effects with less confounding due to background lesions of aging than would be expected at two years. Organ weights and clinical pathology (clinical chemistry and hematology) data were also collected from interim, but not terminal, sacrifice animals given the expected increased moribund removal later in the study and the level of variability in older animals due to age-related disease. A summary of the experimental design and data collected is found in Table 2, and a scheme is shown in Figure 1. The study protocol and all amendments are found in Supplemental Appendix I. Protocol deviations are included in Supplemental Appendix II. Many of these deviations document missing data collections or missed or under- or over-dosing of specific animals on specific days. Cases where missing data affected data analyses are noted in the data summary tables and in the statistical report appendices (Supplemental Appendices XVII-XXXI, XXXIII, and XXXIV).

An important component of this study, shown in Figure 1, was the provision of animals from the core study to academic investigators for studies funded by NIEHS as part of the CLARITY-BPA consortium $^{11 ; 12}$. Results from these academic grantee-conducted studies are not reported here, but are or will be reported in the open literature. All the data collected by the academic grantees as part of the CLARITY-BPA-funded studies will be made available to the public via the NTP website (https://ntp.niehs.nih.gov) and in the NTP's Chemical Effects in Biological Systems (CEBS) database $\mathrm{e}^{\mathrm{b}}$. The allocation of animals to these studies did impact the allocation of animals to the study reported here, particularly regarding adjustment of the sex distribution for PND 1 culling and the number of animals assigned to the interim sacrifice.

\section{Animal Maintenance, Breeding, Randomized Allocation to Study, and Dosing}

Animals were housed in rooms with a 12-hour light cycle (lights on at 6 AM and off at 6 PM) and 10 room air changes per hour. For all animals, cages were changed twice weekly. Glass water bottles were changed weekly or as often as necessary to maintain a constant supply of drinking water. Throughout the study, cage racks were changed every two weeks and cage locations on those racks were rotated every two weeks. The study definition documents and startup memos that describe the cage and rack arrangements in the animal rooms are included as Supplemental Appendices IV and V. Animal rooms were maintained at $23 \pm 3^{\circ} \mathrm{C}$ and $50 \pm 20 \%$ humidity and were monitored so that corrective action could be taken if values went outside these limits. Summary temperature and humidity reports for all animal rooms are in

$\mathrm{b}_{\text {https://doi.org/10.22427/NTP-DATA-018-00015-0001-000-6. }}$ 
Supplemental Appendix X. In one case, a malfunction of a humidity sensor required movement of animals to another animal room for approximately 11 weeks (Supplemental Appendix II).

Approximately two weeks prior to mating, female breeders were randomized to treatment groups, stratified by body weight to produce approximately equivalent mean body weights in each group. The weight ranking and pairing information provided by the NCTR Statistical Support Group for each mating are in Supplemental Appendix VI. Male breeders were assigned to breeding pairs with the stipulation that no sibling or first cousin mating was permitted. Rats were mated at 10-14 weeks of age for females and 11-15 weeks of age for males. Females were placed in solid-bottomed polysulfone cages with hardwood chip bedding with the assigned males and were assessed daily for up to 10 days for sperm-positive vaginal smears or a copulation plug that precluded a vaginal smear. In three cases, males intended for mating with females in BPA or $\mathrm{EE}_{2}$ dose groups died prior to mating and, in two of these cases, males from the vehicle control group that had already been mated with control females were re-mated with these females (Table 3). Dams were separated from the males on the day of sperm/plug detection, which was designated GD 0. If no sperm-positive vaginal smear or copulation plug was detected after 10 days, the pair was removed from the study and euthanized. Mating was conducted in five cohorts spaced four weeks apart. The number of pairs assigned to treatment groups in each mating group is shown in Table 3. While equivalent numbers of breeding pairs were assigned to the vehicle control and all BPA dose groups in the first three cohorts, adjustments were made in the fourth and fifth mating assignments based on the number of pups that had been allocated to the study from the earlier matings. The number of litters produced in each dose group in each mating and the number of litters contributing pups to the study are shown in Table 4 and Table 5, respectively. Mating was conducted prior to the start of dosing on GD 6, and thus breeding success was not an endpoint for analysis in this study.

Daily gavage dosing of the dams began on GD 6 (GD $0=$ sperm- or plug-positive day) and continued until the initiation of parturition. Pups were not dosed on the day of birth (PND 0). Pups without evident malformations were randomly culled to a maximum of five males and five females on PND 1. While the post-culling sex ratio was generally balanced, the sex distribution was skewed toward males later in the study because the hypothesis-driven studies conducted by academic investigators required a culling strategy to provide more males than females. Litters with fewer than three pups/sex and live litters born to dams earlier than GD 20 were excluded from the core study, except in the cases of three females in the $25,000 \mu \mathrm{g} / \mathrm{kg}$ bw/day continuous BPA dose group that came from litters that did not have three pups/sex (Supplemental Appendix II). Direct gavage dosing of the pups started on PND 1 after the litter was culled. For animals younger than PND 5, the gavage needle was inserted to the opening of the esophagus, but did not enter the esophagus ${ }^{41}$. This dosing method for young pups had also been used in the BPA subchronic study conducted at NCTR, where serum levels were measured after dosing in PND 4 animals demonstrating the effectiveness of this dosing procedure ${ }^{19 ; 25}$. Pups were weighed and dosed daily until weaning at PND 21. After weaning, same-sex pups were housed two per cage and either dosed by gavage with vehicle, $\mathrm{BPA}$, or $\mathrm{EE}_{2}$ daily until termination (continuous-dose arm) or maintained without further dosing (stop-dose arm).

At weaning, up to a maximum of three pups/sex/litter were assigned to the chronic study. Samesex littermates were not assigned to the same combination of study dose arm and time of sacrifice so that litter of origin was not a factor to be considered in the statistical analysis of endpoints collected after weaning. Twenty to $26 \mathrm{pups} / \mathrm{sex} /$ dose group were assigned to the one- 
year interim continuous-dose assessment (Table 6); 19 to 22 pups/sex/dose group were assigned to the one-year interim stop-dose assessment (Table 7); 46 to 50 pups/sex/BPA dose group/dose arm and $26 \mathrm{pups} / \mathrm{sex} / \mathrm{EE}_{2}$ dose group were assigned to the two-year study continuous-dose assessment (Table 8); and 46 to 50 pups/sex/BPA dose group/dose arm were assigned to the twoyear study stop-dose assessment (Table 9). The remaining pups from those litters with more than three same-sex pups were assigned to the hypothesis-driven studies of academic investigators. The reason that stop-dose $\mathrm{EE}_{2}$ groups were not included in the study was solely an animal facility space and resource consideration, given the number of animals that needed to be provided and housed for both this study and the NIEHS-funded academic CLARITY-BPA grantee studies.

\section{Animal Identification}

Prior to mating, all $\mathrm{F}_{0}$ females were identified with their unique cage number by tail tattoo (Animal Identification and Marking Systems, Inc., Hornell, NY). F1 pups were initially numbered on their backs with an indelible marker after culling on PND 1 and then shortly thereafter with a standard 4-paw tattoo pattern. The paw tattoo pattern and dam identification number (cage number) provided unique identification for preweaning pups. Retained $\mathrm{F}_{1}$ pups were marked by tail tattoo with their unique identification number (cage number and an additional digit to distinguish cage mates) after weaning on PND 21.

\section{Data Collected in Interim and Terminal Sacrifice Animals}

\section{In-Life Data Collection}

All activities conducted by animal care technicians in the animal rooms were monitored by the MGSS. Morbidity/mortality checks were performed twice daily and clinical observations were recorded weekly or when a significant clinical observation was noted. Starting at six months of age, animals were palpated weekly to detect the presence and progress of tissue masses. Body weights were obtained prior to dosing for dams from GD 6 through parturition and similarly for the pups from PND 1, as described above. Feed consumption was measured weekly from the start of dosing for approximately the next 13 weeks and monthly afterward. These data were not analyzed beyond summary statistics that were used to estimate consumption of background dietary BPA as a result of the low $(<5 \mathrm{ppb})$ level of BPA in the feed (Supplemental Appendices XIII a and b, XIV). On the day of birth, PND 0, the number of pups alive and dead was recorded. On PND 1, the number of pups alive and dead, sex ratio, and live litter weight by sex were determined prior to culling. Individual body weights for all retained pups were recorded daily from PND 1 until weaning at PND 21. Animals in the continuous-dose arm were weighed daily through PND $90 \pm 3$ and weekly thereafter. Weights of animals in the stop-dose arm were recorded weekly after weaning.

All dams that were sperm- or plug-positive were euthanized after litters were weaned, on the litter's day of birth if the litter had less than three pups/sex, or on GD 26 if no litter was produced. The uterus was removed and stained with $10 \%$ ammonium sulfide for enumeration of implantation sites.

Females (26 animals from 13 randomly selected cages per dose group per dose arm from the two-year study arm) were monitored daily for vaginal opening from PND 22. Vaginal smears were collected for 14 consecutive days from these same animals beginning at $16 \pm 2$ weeks of 
age. One month after these vaginal smears were completed, the same animals had vaginal smears collected for five consecutive days monthly until the animal did not show evidence of cycling, that is, that showed three or more consecutive days of estrus (including estrus, estrus/diestrus, or proestrus/estrus intermediate stages) or five consecutive days that did not include an estrus smear for two consecutive months.

\section{Clinical Chemistry and Hematology, Interim Sacrifice Animals}

For the one-year interim sacrifice, food, but not water, was removed from cages on the evening before the scheduled necropsy. Animals were anesthetized with gaseous carbon dioxide and blood was collected from the retro-orbital sinus. Hematology and clinical chemistry endpoints evaluated are listed in Table 2. All blood was collected into serum and ethylenediaminetetraacetic acid (EDTA) tubes between 7:00 AM and 12:00 PM. Blood in the serum tubes was allowed to clot and centrifuged at $1000 \times g$ for 10 minutes at room temperature. The serum was removed and aliquoted into two tubes. One tube was used for immediate testing (see below) and the other was frozen at $-60^{\circ} \mathrm{C}$ until additional testing was performed. The EDTA tube was used for hematology testing performed the same day as collection.

Clinical chemistry analyses were conducted on an Alfa Wassermann ALERA (West Caldwell, NJ). Alfa Wassermann reagents were used to quantify glucose, alkaline phosphatase, alanine aminotransferase, aspartate aminotransferase, $\gamma$-glutamyl transpeptidase, total protein, albumin, cholesterol, triglycerides, blood urea nitrogen, and creatinine. Catachem (Bridgeport, CT) reagents were used to quantify sorbitol dehydrogenase and total bile acids. All testing was completed on the day of collection. The instrument was calibrated daily and two levels of assayed controls were included in daily analyses as internal controls.

Rat-specific troponin I, troponin T (Life Diagnostics, West Chester, PA), and leptin (Millipore, St. Charles, MO) were quantified using ELISA methods. The plates were read on an ELx800 Universal Microplate Reader (Bio-Tek, Winooski, VT). A standard curve was run with each batch of samples and results were calculated by the instrument's software. Insulin, triiodothyronine (T3), and thyroxine (T4) were quantified using Siemens (Los Angeles, CA) RIA "Coat-a-Count" method and thyroid-stimulating hormone (TSH) with rat-specific TSH radioimmune assay (Alpco, Salem, $\mathrm{NH}$ ). The tubes were then counted on a Wizard2 gamma counter (PerkinElmer, Shelton, CT). A standard curve was run with each batch of samples and results were calculated by the instrument's software. Two levels of assayed controls were included in daily analyses as internal controls.

Complete blood counts were determined on an Pentra $60 \mathrm{C}+$ analyzer (ABX, Irvine, CA). Maintenance and calibration were done per the manufacturer's recommendations. Three levels of assayed controls were included in daily analyses as internal controls. Packed cell volume (PCV) analysis was performed by centrifugation in a CritSpin centrifuge (Beckman Coulter, Inc, Indianapolis, IN) and PCV determined by manual read. For determining the percentage of reticulocytes, 1,000 cells per animal were counted on slides prepared from blood collected in EDTA tubes and stained with New Methylene Blue Reticulocyte Stain (Volu-Sol, Salt Lake City, UT). 


\section{Sperm Evaluations, Interim Sacrifice Animals}

For sperm motility assessment, the left epididymis was dissected from the testis and weighed. If gross lesions or abnormalities were noted at necropsy with either the left testis or epididymis, the right organ was used for motility studies and the left was sent for histology. The cauda section was dissected and immediately placed in a petri dish containing $40 \mathrm{~mL}$ of a solution consisting of $1 \%$ bovine serum albumin dissolved in phosphate buffered saline. The solution was prewarmed to a temperature of approximately $37^{\circ} \mathrm{C}$. A minimum 2-minute period was allowed for the sperm to swim out. Following the swim-out period, a sperm sample was obtained using an $80 \mu \mathrm{m}$ deep slide. The slide was immediately loaded into the prewarmed stage of the Hamilton Thorne IVOS automated sperm analyzer. Five fields were automatically selected by the analyzer, and each motion image was recorded and stored on an optical disk. The images were subsequently analyzed and the percent motility determined for each animal. Two eosin-stained slides were also prepared for each animal from the caudal epididymis suspension for evaluation of morphological development; a minimum of 200 sperm cells/animal was examined.

After the motility and morphology samples were collected, the cauda was minced with scissors and mixed. Approximately $1 \mathrm{~mL}$ of the suspension was placed in a prelabeled tube and frozen on dry ice for subsequent determination of the total caudal sperm count. The left testis was weighed, placed on dry ice, and stored frozen until evaluation for testicular spermatid head counts. Each frozen epididymis suspension and testis was thawed. The tunic was removed from the testis, and each testis was weighed and homogenized. The suspension was transferred to a vial containing a dye (IDENT, bis-benzimide trihydrochloride, Hamilton Thorne, Inc., Beverly, MA) that uniquely stains the head of sperm. A sample of the stained sperm was placed into a $20 \mu \mathrm{m}$ deep glass slide that was loaded into the analyzer. Twenty fields were automatically selected by the instrument for each animal, and total sperm counts were determined. The counts were reported adjusted for testis weight (million sperm/g tissue).

\section{Organ Weights and Histopathology}

All animals maintained on study after weaning that survived to the scheduled sacrifice dates or were removed early as dead or moribund were subjected to a full necropsy. All gross lesions were processed for histological evaluation. Selected organs were collected and weighed at the interim sacrifice (Table 2). Weights of the dorsal/lateral and ventral prostate lobes were not collected, because the dissection and trimming procedure necessary to obtain these weights would interfere with the processing of the organ for histopathology (Supplementary Appendix II, Protocol Amendment \#7). Organs were not weighed at the terminal sacrifice. Tissues not specified for microscopic evaluation, which are listed in Table 2, were processed to paraffin block and held for potential later evaluation. For tissues specified for evaluation by the study pathologist (Table 2), tissues from all dose groups were evaluated for both the interim and terminal sacrifice animals. Tissues were processed in accordance with NTP specifications (https://ntp.niehs.nih.gov/ntp/test_info/finalntp_reprospecsmay2011_508.pdf, https://ntp.niehs.nih.gov/ntp/test_info/finalntp_toxcarspecsjan2011.pdf), except that six step sections of each prostate were examined. The International Harmonization of Nomenclature and Diagnostic Criteria (INHAND) guidelines (https://www.toxpath.org/inhand.asp) were used as diagnostic criteria for the microscopic evaluations. For the female reproductive tissues, mammary gland, and male reproductive tissues, the diagnostic criteria outlined in Dixon et al. (2014), Rudmann et al. (2012), and Creasy et al. (2012) ${ }^{42-44}$, respectively, were used. 
Approximately 9\% of the animals in the study, represented in all dose groups, were observed to have seizures over the course of the study, mostly during handling procedures (e.g., dosing, cage changes). The brains, spinal cords, and peripheral nerves of these animals were examined by the study pathologist for any histological abnormalities (Supplemental Appendix XXXII). For the two-year sacrifice, animals were not fasted, and no clinical chemistry, hematology, organ weights, or sperm evaluations were conducted.

Upon completion of the microscopic evaluations, the data were entered into the NTP's Toxicology Data Management System Enterprise. Slides, paraffin blocks, and residual wet tissues were sent to the Block and Slide Laboratory for inventory, slide/block match, and wet tissue audit. Individual animal data records and pathology tables were evaluated by an independent quality assessment (QA) group, and QA pathologists evaluated selected histopathology slides. The reviewed slides, along with the diagnoses made by the study pathologist and QA pathologists, were reviewed by the Pathology Working Group (PWG). The QA pathologists served as coordinators of the PWG. Representative histopathology slides containing examples of lesions potentially related to chemical administration, examples of disagreements in diagnoses between the laboratory and QA pathologists, or lesions of general interest were presented by the coordinator to the PWG for review. While tissues from multiple organ systems were selected by the QA pathologists for review by the PWG to allow evaluation and confirmation of the broad spectrum of lesions observed in control and treatment groups, female and male reproductive tissues were emphasized in the review. The PWG consisted of the QA pathologists, the study pathologist, and other pathologists experienced in rodent toxicological pathology. This group examined the tissues with no knowledge of dose groups. When the PWG consensus differed from the opinion of the study pathologist, the diagnosis was changed. Final diagnoses for reviewed lesions represent a consensus of the PWG.

\section{Statistical Methods}

The full statistical analysis reports for all protocol-specified endpoints, including detailed methods and results for each analysis, including the omnibus tests, are found in Supplemental Appendices XVII-XXXI, XXXIII, and XXXIV. The pairwise comparisons to the vehicle control and trend tests are the comparisons of interest that are presented in the tables in this report. The statistical methodology for each endpoint is summarized below. Statistical comparisons were conducted within sex and, for data collected after weaning, within dosing arm (continuous-dose or stop-dose). For pairwise comparisons, the five BPA dose groups were compared to the vehicle control group. Similarly, the two $\mathrm{EE}_{2}$ reference estrogen dose groups were compared to the vehicle control. Tests were conducted at the 0.05 significance level and, in most cases, were twosided. Exceptions were one-sided tests for the pairwise comparisons of histopathology lesion incidence and severity to vehicle controls and trend tests for abnormal estrous cycles. Although a $p$-value of $<0.05$ was used to flag a result as significant, the actual $p$-values are included in some of the tables in this report and in all the statistical report appendices (Supplemental Appendices XVII-XXXI, XXXIII, and XXXIV) to aid in the further evaluation of the statistical and biological significance of each result. Trend tests for treatment effect (either increased or decreased relative to vehicle control) with increasing dose were conducted only for vehicle control and BPA treatment groups, except for non-neoplastic and neoplastic lesions, where trend tests were also conducted within the vehicle control and $\mathrm{EE}_{2}$ groups. Because pups within litter 
and sex were assigned at weaning to different dosing arms and sacrifice times, litter correlation was not a consideration for endpoints evaluated after weaning in this study.

\section{Survival Analyses (Supplemental Appendices XX, XXI, and XXII)}

Animals with a disposition of dead or moribund were treated as uncensored observations, while those reaching PND 21, one year, or two years were considered censored for the preweaning, interim, and terminal survival analyses, respectively. To compare survival of treatment groups to the control group, Cox proportional hazards regression analysis was performed. For the interim sacrifice survival analysis, several groups had $100 \%$ survival. For this situation, a modified Cox proportional hazards regression analysis was performed after adjusting the number of uncensored observations by adding one for each treatment group and sex to allow estimability. Multiple comparisons of treatments to the vehicle control group were adjusted using Holm's (step-down Bonferroni) method.

\section{Body Weight Analyses (Supplemental Appendices XVII, XXIII, XXIV, and XXV)}

Gestational weight at parturition was analyzed using analysis of covariance (ANOCOVA) with terms for treatment group, dam weight at baseline, and litter size as covariates, and the interaction between treatment and litter size. Data were collected at baseline on GD 0 or GD 1 prior to dosing and daily from GD 6 to parturition. Gestational weight at parturition was defined as the last dam body weight prior to delivery. For preweaning pup body weight data, the analysis was performed using contrasts within sex and PND. The experimental unit was the litter, and a stratified one-way repeated measures, mixed model analysis of variance (ANOVA) was used to test for treatment effect and to account for litter correlation assuming a compound symmetric correlation structure. The cross-sectional analysis was performed on selected PNDs (1, 4, 7, 14, and 21) so that the intra-litter correlation could be accounted for accurately.

For the interim sacrifice and terminal sacrifice postweaning analyses, there were no littermates among the males or females in any dose group within each dosing arm and sacrifice time, so intra-litter correlation was not considered. Body weight data collected from four to 52 weeks (interim sacrifice animals) or four to 104 weeks (terminal sacrifice animals) were analyzed using the last weekly observation for each animal, with PND 21 defined as the first day of week four. Although no formal comparisons were made between the continuous- and stop-dose study arms, for females and males in both the interim and terminal sacrifice phases, the week 4 body weights in the continuous-dose groups appeared to be higher than the week 4 body weights in the stopdose groups (compare Table 24 and Table 25 with Table 26 and Table 27 for females; compare Table 28 and Table 29 with Table 30 and Table 31 for males). This apparent difference was an artifact of the experimental design and the statistical analysis. After weaning, the continuousdose groups were weighed daily until PND 90, so that the last body weight of week 4 was PND 27. For the stop-dose groups, weekly body weights were taken after weaning, so the last body weight recorded in week 4 was generally earlier than PND 27. Outliers were identified by comparing observed body weight to predicted body weight using a five-point running median smoother and nearest neighbor interpolation. A threshold for outlier exclusion was set at a difference between observed and predicted weights greater than $35 \mathrm{~g}$ for both sexes in the interim analysis, and 60 and $65 \mathrm{~g}$ for females and males, respectively, in the terminal analysis. Lists of the outliers are found in Supplemental Appendices XXIV and XXV. Pairwise 
comparisons of means were performed using contrasts within a two-way repeated measures, mixed model ANOVA for females and males separately. Model terms were treatment group, weeks, and the interaction. Within-group correlations were modeled using a heterogeneous firstorder autoregressive (ARH(1)) correlation structure that allows for correlated differences in variability across time points. Pairwise comparisons of each treatment mean to the vehicle control group mean were performed using contrasts with Dunnett's method of adjustment for multiple comparisons.

\section{Implantation Sites and Litter Parameters (Supplemental Appendices XVIII and XIX)}

Implantation site counts and litter weight data were analyzed using a one-way ANOVA and litter mean pup weights were analyzed using contrasts within an ANOCOVA, with litter size as a covariate, to test for treatment effect. Dunnett's test was used for comparisons to the vehicle control group to adjust for multiple comparisons. Sex ratios of pups within litters were analyzed for treatment effects using logistic regression. Pup counts (number alive, males, females, number unsexed (i.e., pups that could not be definitively assigned as male or female), and number born dead) were analyzed using Poisson regression. For analyses of sex proportions and female and male counts, unsexed pups were assigned either as male sex or female sex in separate runs with comparable results (Supplemental Appendix XIX).

\section{Analyses of Vaginal Opening, Vaginal Cytology, and Onset of Aberrant Estrous Cycles (Supplemental Appendices XXVI-XXVIII)}

Analyses of age and body weight at occurrence of vaginal opening were performed using contrasts within a one-way ANOVA to test for treatment effect. Comparisons of dosed groups to vehicle control for age and body weight were performed with Dunnett's method to adjust for multiple comparisons.

Summary statistics were calculated for proportions of days spent in estrus, diestrus, and proestrus for each animal and for estrous cycle length. Cycle length in days was defined from the first day of estrus in one sequence of contiguous days to the first day of estrus in the following sequence of stages. Cycles were considered censored if the last stage of data collection was either diestrus or proestrus.

Analyses were conducted on proportions of animals with abnormal cycles. The endpoints evaluated were any abnormal cycling, extended estrus, extended diestrus, and excessive proestrus. Extended estrus was defined as three or more consecutive days of estrus; extended diestrus was defined as four or more consecutive days of diestrus; and excessive proestrus was defined as two or more consecutive days of proestrus in a cycle. For abnormal cycling defined by animal, the Cochran-Armitage method for binomial proportions was used to evaluate the pairwise differences in proportions. The two-sided $p$-value for the Fisher's exact test is reported for comparisons of dosed groups to control, and the one-sided Cochran-Armitage trend test was performed. Holm's (step-down Bonferroni) method was used to adjust for multiple pairwise comparisons of dosed groups to control.

An accelerated failure time model assuming a lognormal distribution was used for onset of aberrant cycling, which was defined as occurring at first swab date of two consecutive months of aberrant estrous cycle data. An aberrant estrous cycle was defined as three or more consecutive 
days of estrus or five consecutive days without estrus. The data for this endpoint contained left-, right-, and interval-censored data, which can all be accommodated by the accelerated failure time model used. Left censoring occurred because some animals had begun to show aberrant cycles prior to the start of monitoring, while right censoring occurred because some animals died or reached the end of the study without showing aberrant cycles. The intermittent nature of the data collection, one 5-day period every month, makes it impossible to determine the exact time of onset of aberrant cycles, so the data are interval censored. Multiple comparisons were adjusted using Holm's (step-down Bonferroni) method for treatment group comparisons to the control group.

\section{Organ Weight Analyses (Supplemental Appendix XXX)}

Statistical analyses were performed separately for the BPA study arms, stop-dose and continuous-dose, and for the $\mathrm{EE}_{2}$ continuous-dose arm in one-year interim sacrifice rats. Weights of paired organs were analyzed as combined weight. ANOVA was performed for each sex and organ to determine the effect of treatment on organ weight. ANOCOVA was performed to determine the effect of treatment on organ weight adjusted for body weight at necropsy or brain weight. Separate analyses were performed with each covariate. Comparisons of dosed groups versus vehicle control were performed using Dunnett's method for adjusted contrasts. Tests of trend, increasing treatment effect with increasing dose, were performed for the BPA and vehicle control groups. Organ weight exclusions based on the observation of gross cysts or consideration of statistical distributions are listed in Supplemental Appendix XXX.

\section{Clinical Chemistry and Hematology Analyses (Supplemental Appendix XXIX)}

A non-parametric ANOVA method based on mid-ranks was used to evaluate the effect of treatment on clinical chemistry and hematology assessments assuming an unstructured covariance $^{45}$. The average of the left and right ranks was used for ties. Dunnett's adjustment was used for pairwise multiple comparisons relative to the control. Orthogonal contrasts were used to test for trends over increasing BPA dose concentrations. Any measurements below the limit of detection (LOD) were evaluated at half the LOD level.

\section{Sperm Parameter Analyses (Supplemental Appendix XXXI)}

Analysis of sperm morphology data was performed using a generalized linear model with a Poisson distribution and a log link function. Each treatment was compared to the vehicle control group, and adjustment for multiple comparisons was performed using Dunnett's method. Percent sperm motility, testes sperm counts, and caudal sperm counts were analyzed using an ANOVA model with Kenward-Roger estimated degrees of freedom ${ }^{46}$. Each treatment was compared to the control group, and adjustment for multiple comparisons was performed using Dunnett's method. Tests of trends, increasing treatment effect with increasing dose, were performed for each compound and dosing arm.

\section{Analyses of Non-Neoplastic and Neoplastic Lesions, Interim and Terminal Sacrifice (Supplemental Appendices XXXIII and XXXIV)}

Microscopic findings were recorded in NTP's automated Toxicology Data Management System Enterprise. For the statistical analyses of groups with 20-26 animals (all interim sacrifice groups 
and $\mathrm{EE}_{2}$ terminal sacrifice groups), any lesion present in at least two animals in any dose group was included in the analyses. In groups with 46-50 animals (all terminal sacrifice vehicle control and BPA groups), any lesion present in at least four animals in any dose group was included in the analyses.

The NTP-preferred approach to assess neoplastic and non-neoplastic lesion prevalence is a survival-adjusted quantal-response procedure (see description of Poly-3 test below) that modifies the Cochran-Armitage linear trend test to take survival differences into account (https://ntp.niehs.nih.gov/testing/types/stats/index.html). For neoplasm and non-neoplasm incidence for interim sacrifice animals, where early removals or deaths were few, the CochranArmitage test, without survival adjustment, was used to test for a linear dose trend, with the Fisher's exact test used to compare dosed groups to the vehicle control. This combination of tests is referred to as CAFE. For neoplasm and non-neoplasm incidence for terminal sacrifice animals, the Poly-3 method of Bailer and Portier ${ }^{47}$, as modified by Bieler and Williams ${ }^{48}$, and the NIEHS continuity-correction, discussed in Peddada and Kissling ${ }^{49}$, was used to analyze age-adjusted incidence for linear dose trend and for pairwise comparisons to the vehicle control. For both the analysis of interim and terminal neoplasm and non-neoplasm incidences, tests were one-sided for treatment comparisons to the vehicle control group with no adjustment for multiple comparisons to the vehicle control, while the trend test was two-sided.

Lesion severities, ordinal scores provided by the Study Pathologist, were available for many of the non-neoplastic lesions. Although these scores are more subjective than the lesion incidences, statistical tests were run to include this additional information with the incidence data. The Jonckheere-Terpstra test ${ }^{50 ; 51}$ was run to test for monotonic dose trends, followed by Shirley's test $^{52 ; 53}$ to compare to controls. This combination of tests is referred to as JT/SW, and it presumes a monotonic dose response. One aspect of this study was to consider potential nonmonotonic effects, which would be detected by the pairwise comparisons. JT/SW is blind to this effect. Therefore, a test not typically used in NTP studies, the non-parametric relative treatment effect (RTE) method ${ }^{45}$ was used to test for non-monotonic dose effects. While the results of the JT/SW and RTE tests are presented in the summary tables and in the statistical appendices (Supplemental Appendices XXXIII and XXXIV), the CAFE and Poly-3 tests are the primary tests to be considered both because of the nature of the severity scores and, in the case of terminal sacrifice, because the JT/SW and RTE tests are not mortality adjusted.

A subset of lesions is discussed and tabulated in the body of this report. Comprehensive tabulations of all statistical results, including the JT/SW and RTE test results, for all lesions diagnosed in the study are found in Supplemental Appendices XXXIII and XXXIV.

\section{Historical Control Data}

The concurrent control group represents the most valid comparison to the treated groups and is the only control group analyzed statistically in NTP bioassays. Historical control data, however, are often helpful in interpreting potential treatment-related effects. Although these historical control data are generally most useful for uncommon or rare neoplasms, they can be useful for assessing the range of background lesions in the animal strain used as part of the weight-ofevidence consideration of whether an observed lesion is treatment related. For meaningful comparisons, the conditions for studies used for historical control comparisons generally should be similar. In the case of the present study, two two-year studies conducted with the SpragueDawley/CD23/Nctr BR rat using the same diet used in the present study have been conducted ${ }^{14 ;}$ 
16. These were multigenerational studies in which animals in the $F_{1}$ and $F_{3}$ generations were evaluated at two years of age. Thus, each study had two sets of control animals of both sexes. These were dietary administration studies, rather than gavage studies, and were conducted outside the five-year period generally used for such comparisons, although drift in neoplasm incidence over time can vary among strain and neoplasm type ${ }^{54 ; 55}$. Historical incidences of neoplasms (mean percentages and ranges) from these studies are referenced in the Results and Discussion sections.

\section{Sensitivity Analyses}

In the present study, approximately $20 \%$ of the animals were housed for a short period early in the study in the same room as animals dosed with $250,000 \mu \mathrm{g} \mathrm{BPA} / \mathrm{kg}$ bw/day exclusively for a CLARITY-BPA grantee study. These animals were potentially exposed to low levels of BPA, leading to blood levels of BPA metabolites above the LOD and similar to those resulting from the $2.5 \mu \mathrm{g} \mathrm{BPA} / \mathrm{kg}$ bw/day dose ${ }^{12}$ (Supplemental Appendix XVI). Animals co-housed only with the high dose from the two-year study $(25,000 \mu \mathrm{g}$ BPA/kg bw/day) had no detectable BPA metabolites in their blood ${ }^{12}$. As a conservative approach to determine if this low-level exposure to a subset of animals impacted the interpretation of study results, a sensitivity analysis was conducted for each endpoint. In the sensitivity analysis, all animals that for any portion of their lives were co-housed in the same room as the subset of animals treated with the $250,000 \mu \mathrm{g}$ $\mathrm{BPA} / \mathrm{kg}$ bw/day dose were excluded. Any significant effects found in the sensitivity analysis that were not found in the analysis that included all animals are noted in data tables and in the statistical appendices (Supplemental Appendices XVII-XXXI, XXXIII, and XXXIV). Only those animals involved in or resulting from the first mating were co-housed at any point in their lives with the animals dosed with $250,000 \mu \mathrm{g} \mathrm{BPA} / \mathrm{kg}$ bw/day. Thus, the sensitivity analyses that were conducted after exclusion of all animals in the first mating removed all animals that potentially had exposure to BPA above that present in the diet. This included the subset of animals that had received a single dose of contaminated vehicle as adults (see above under "Preparation and Analysis of Dose Formulations" and Supplemental Appendix III, note to study file October 13, 2017).

\section{Quality Assurance}

This study was conducted in compliance with the Food and Drug Administration (FDA) Good Laboratory Practice (GLP) for the conduct of nonclinical laboratory studies (United States Code of Federal Regulations Title 21, Part 58). The Quality Assurance Unit at NCTR performed audits and inspections of the protocols, procedures, data, and reports throughout the course of the study. Separate audits covering completeness and accuracy of the pathology data, pathology specimens, and final pathology tables, and a draft of this technical report were conducted. Audit procedures and findings are on file at NCTR. The audit findings were reviewed and assessed by the NCTR staff, and all comments were resolved or otherwise addressed either before or during the preparation of the technical report. Raw data sheets from the study are archived by NCTR's Record Management Unit. Histopathology samples collected during the study are stored in the archives of Toxicologic Pathology Associates at NCTR. Backup computer data are maintained by the Computer Support Group at the NCTR. All records and samples are stored in accordance with FDA GLP Regulations. 


\section{Results}

\section{Gestational Body Weight, Fetal Implantation, and Litter Parameters}

Dam body weights during pregnancy were not affected by BPA or $\mathrm{EE}_{2}$ treatment (Table 10). The number of implantation sites in mated dams did not differ across BPA or $\mathrm{EE}_{2}$ treatment groups and control groups as expected given that treatment did not begin until after implantation. There were no treatment effects on litter size, sex ratio, litter weight by sex, or mean pup weight at birth by sex (Table 11).

\section{Survival, Preweaning and Postweaning Study Phases}

\section{Preweaning Survival}

\section{Females}

The survival of female pups between PND 1 and PND 21 ranged from 91-95\% in vehicle control and BPA dose groups (Table 12). There were no significant BPA treatment effects. The survival of female pups in the 0.05 and $0.5 \mu \mathrm{g} \mathrm{EE} / \mathrm{kg}$ bw/day groups was $85 \%$ and $91 \%$, respectively. Survival in the lower $\mathrm{EE}_{2}$ group was significantly lower than that in the vehicle control group. The study protocol did not call for detailed evaluation of the cause of morbidity/death in pups removed from the study prior to weaning.

\section{Males}

The survival of male pups between PND 1 and PND 21 ranged from 91-95\% in vehicle control and BPA dose groups (Table 13). There were no significant BPA treatment effects. The survival

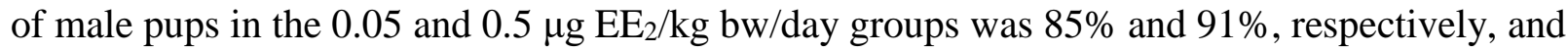
did not differ significantly from the vehicle control. The study protocol did not call for detailed evaluation of the cause of morbidity/death in pups removed from the study prior to weaning.

\section{Postweaning Survival in Interim Sacrifice Animals}

\section{Females, Continuous-Dose Arm}

The survival of females dosed daily with vehicle or BPA until the scheduled one-year sacrifice ranged from 91-100\% and there were no treatment effects (Table 14). Over the same period, the survival of females in the low and high $\mathrm{EE}_{2}$ dose groups was 92 and 100\%, respectively, and did not differ from vehicle controls. Causes of morbidity/death in animals that did not survive to the interim sacrifice, when known, are noted in footnotes to Table 14.

\section{Females, Stop-Dose Arm}

In the stop-dose study arm, there was $100 \%$ survival in all dose groups of females scheduled for sacrifice at one year, except for the $25,000 \mu \mathrm{g}$ BPA/kg bw/day dose group where survival was $91 \%$ (Table 15). There was no treatment effect. Causes of morbidity/death in animals that did not survive to the interim sacrifice, when known, are noted in footnotes to Table 15. 


\section{Males, Continuous-Dose Arm}

Survival of males dosed daily with vehicle or various BPA doses until the scheduled one-year sacrifice ranged from $82-100 \%$, with the lowest percent survival seen in the vehicle control group (Table 16). There were no significant treatment effects on survival after this one-year exposure. Over the same period, the survival of males in the low and high $\mathrm{EE}_{2}$ dose groups was 85 and $88 \%$, respectively, and did not differ significantly from vehicle controls. Causes of morbidity/death in animals that did not survive to the interim sacrifice, when known, are noted in footnotes to Table 16.

\section{Males, Stop-Dose Arm}

In the stop-dose study arm, there was $100 \%$ survival in males of all BPA dose groups sacrificed at one year, except for the $25 \mu \mathrm{g} \mathrm{BPA} / \mathrm{kg}$ bw/day dose group where survival was 95\% (Table 17). There was no treatment effect. The cause of death in the one animal in the $25 \mu \mathrm{g} \mathrm{BPA} / \mathrm{kg}$ bw/day dose group that died early was uncertain (Supplemental Appendix XXXII, Subappendix VI).

\section{Postweaning Survival in Terminal Sacrifice Animals}

\section{Females, Continuous-Dose Arm}

The Kaplan-Meier survival curves for females dosed daily with the vehicle or BPA, or with the vehicle or $\mathrm{EE}_{2}$, until sacrifice at two years are shown in Figure 2 and Figure 3, respectively. Data and analysis results are shown in Table 18. Survival at the end of the study in the BPA dose groups ranged from 17-40\%. Survival in the low and high $\mathrm{EE}_{2}$ dose groups was 27 and 15\%, respectively. No significant treatment effects were seen for this chronic exposure to BPA or $\mathrm{EE}_{2}$ at the doses administered. Most of the animals that did not survive until the terminal sacrifice were removed as moribund between one and two years of age. Causes of death/morbidity are listed in an appendix to the pathology report (Supplemental Appendix XXXII, Subappendix VI); mammary gland fibroadenomas and pituitary adenomas accounted for most of the early removals.

\section{Females, Stop-Dose Arm}

The Kaplan-Meier survival curves for females in the vehicle control or BPA two-year stop-dose arm are shown in Figure 4. Data and analysis results are shown in Table 19. Survival at the end of the study in the BPA dose groups ranged from 24-34\%; survival in the vehicle control group was $22 \%$. There were no significant treatment effects in female rats after only gestational and preweaning exposure to BPA. Most animals that did not survive until the terminal sacrifice were removed as moribund between one and two years of age. Causes of death/morbidity are listed in an appendix to the pathology report (Supplemental Appendix XXXII, Subappendix VI); as was the case in the two-year continuous-dose arm females, mammary gland fibroadenomas and pituitary adenomas accounted for most of the early removals.

\section{Males, Continuous-Dose Arm}

The Kaplan-Meier survival curves for males dosed daily with vehicle or BPA, or with vehicle or $\mathrm{EE}_{2}$, until sacrifice at two years are shown in Figure 5 and Figure 6, respectively. Data and analysis results are shown in Table 20. Survival at the end of the study in the BPA dose groups ranged from 24-35\%. Survival in the low and high $\mathrm{EE}_{2}$ dose groups was 35 and $46 \%$, respectively. There were no significant treatment effects. Most animals that did not survive until the terminal sacrifice were removed as moribund between one and two years of age. Causes of 
death/morbidity are listed in an appendix to the pathology report (Supplemental Appendix XXXII, Subappendix VI). Many primary and contributory conditions in various organs were diagnosed, with pituitary adenomas, nephropathy, preputial gland carcinoma, and malignant lymphoma indicated as primary causes of death/morbidity in multiple animals across all dose groups.

\section{Males, Stop-Dose Arm}

The Kaplan-Meier survival curves for males in the vehicle control or BPA two-year stop-dose arm are shown in Figure 7. Data and analysis results are shown in Table 21. Survival at the end of the study in the BPA dose groups ranged from $20-33 \%$ in comparison to the $34 \%$ survival seen in the vehicle controls. There were no significant treatment effects. Most animals that did not survive until the terminal sacrifice were removed as moribund between one and two years of age. Causes of death/morbidity are listed in an appendix to the pathology report (Supplemental Appendix XXXII, Subappendix VI) and were similar to those diagnosed for the continuous-dose arm males.

\section{Body Weights}

\section{Body Weights in Preweaning Animals}

\section{Females}

Body weights on PNDs 1, 4, 7, 14, and 21 in female pups treated daily with vehicle, BPA, or $\mathrm{EE}_{2}$ are shown in Table 22. There were no BPA treatment-related effects on female pup body weights at these time points. On PNDs 4 and 7, the low $\mathrm{EE}_{2}$ dose group females had significantly lower mean body weight than vehicle controls, with body weights in the treated animals approximately $5 \%$ lower than controls on both days. At PND 4, both the $2.5 \mu \mathrm{g} \mathrm{BPA} / \mathrm{kg}$ bw/day and the $0.05 \mu \mathrm{g}$ $\mathrm{EE}_{2} / \mathrm{bw} /$ day dose groups had identical means and standard errors, but only the latter was statistically significant, suggesting that the smaller sample size in that group contributed to this marginal difference.

\section{Males}

Body weights on PNDs 1, 4, 7, 14, and 21 in male pups treated daily with vehicle, BPA, or $\mathrm{EE}_{2}$ are shown in Table 23. There were no BPA or $\mathrm{EE}_{2}$ treatment-related effects on male pup body weights at these time points.

\section{Body Weights in Interim and Terminal Sacrifice Animals}

\section{Females, Continuous-Dose Arm}

Body weights of females in the continuous vehicle control and BPA groups scheduled for the interim sacrifice are shown in Figure 8 and Table 24. The data for the $\mathrm{EE}_{2}$ groups are also tabulated in Table 24 and are shown graphically in Figure 9. There were no significant differences from the vehicle controls in any of the BPA or $\mathrm{EE}_{2}$ dose groups. The mean body weights in the $2.5 \mu \mathrm{g} \mathrm{BPA} / \mathrm{kg}$ bw/day group in weeks 36-52 were $10-13 \%$ higher than vehicle control means; however, these differences were not statistically significant.

Body weights of females in the continuous vehicle control, $\mathrm{BPA}$, and $\mathrm{EE}_{2}$ dose groups scheduled for the terminal sacrifice are shown in Table 25 and in Figure 10 and Figure 11 for BPA and $\mathrm{EE}_{2}$, 
respectively. Mean body weights of females in the $250 \mu \mathrm{g}$ BPA/kg bw/day dose group were significantly higher by $16-18 \%$ than those of the vehicle control group for weeks $96-104$. In this same period, the mean body weights of females in the 2.5 and $25 \mu \mathrm{g}$ BPA/kg bw/day dose groups were $11-16 \%$ higher than those of vehicle controls, but these differences were not significant. Animals in the $2.5 \mu \mathrm{g}$ BPA/ $\mathrm{kg}$ bw/day dose group did not have higher mean body weights than vehicle controls in the earlier weeks noted above for interim sacrifice animals. In the terminal sacrifice high $\mathrm{EE}_{2}$ dose group, transiently higher mean body weights were observed at 4 and 8 weeks ( $7 \%$ and $8 \%$, respectively). The same tendency in the high $\mathrm{EE}_{2}$ dose group was seen in the interim sacrifice animals at 4 and 8 weeks, although the differences in mean body weights were not statistically significant.

\section{Females, Stop-Dose Arm}

Body weights of females in the vehicle control and BPA stop-dose groups scheduled for the interim sacrifice are shown in Table 26 and Figure 12. There were no significant treatment effects. In the stop-dose females scheduled for terminal sacrifice (Table 27 and Figure 13), there was a significant decreasing trend at week 4, but no other treatment effects. Although statistical comparisons were not conducted between continuous- and stop-dose arms, the stop-dose arm females had higher mean weights over the course of the study (vehicle controls, mean stop-dose compared to continuous-dose: interim, 104\%, range 98-109\% from weeks 8-52; terminal, 107\%, range $97-114 \%$ from weeks $8-104)$.

\section{Males, Continuous-Dose Arm}

Body weights of the continuously dosed males in the vehicle control, BPA, and $\mathrm{EE}_{2}$ groups for interim and terminal sacrifices are shown in Table 28 and Table 29, respectively. The growth curves for interim BPA and $\mathrm{EE}_{2}$ dose groups are shown in Figure 14 and Figure 15, respectively, and the growth curves for terminal BPA and $\mathrm{EE}_{2}$ dose groups are shown in Figure 16 and Figure 17, respectively. There were no significant treatment effects for either compound, although means were $10-16 \%$ higher than vehicle control means in the $250 \mu \mathrm{g} \mathrm{BPA} / \mathrm{kg}$ bw/day dose group from weeks 92 through 104.

\section{Males, Stop-Dose Arm}

Mean body weights of the stop-dose arm vehicle control and BPA groups of male rats for interim and terminal sacrifices are shown in Table 30 and Table 31 and graphically depicted in Figure 18 and Figure 19, respectively. The sole statistically significant treatment effect was a decreasing dose trend at week 4 in the terminal sacrifice animals (Table 31). Although statistical comparisons were not conducted between continuous- and stop-dose arms, the stop-dose arm males had higher mean weights over the course of the study (vehicle controls, mean stop-dose compared to continuous-dose: interim, 102\%, range 92-105\% from weeks 8-52; terminal, 107\%, range $92-113 \%$ from weeks $8-104)$.

\section{Vaginal Opening}

Female pups were evaluated for vaginal opening starting on PND 22. Mean age and body weight at vaginal opening for continuous-dose vehicle control, BPA, and $\mathrm{EE}_{2}$ dose groups are shown in Table 32. There were no treatment-related effects on these endpoints for any dose of either compound. For stop-dose vehicle control and BPA groups, mean age at vaginal opening is shown in Table 33. No treatment effects were observed in the stop-dose BPA groups. Body weight at 
vaginal opening could not be analyzed for the stop-dose animals because weight at vaginal opening was not recorded for many of the animals due to a technical error (Supplemental Appendix II, deviations 72-74). While no formal analysis was conducted comparing vehicle controls in the continuous- and stop-dose arms, the mean vaginal opening date appears to be later regardless of BPA treatment in the stop-dose groups.

\section{Vaginal Cytology - Estrous Cycle Analysis at Approximately 16 Weeks of Age}

\section{Continuous-Dose Arm}

Data and results of analysis of the 14 consecutive daily vaginal smears collected from animals in the continuous-dose arm at $16 \pm 2$ weeks of age are summarized in Table 34. The individual animal data are found in the statistical report in Supplemental Appendix XXVII. There were no significant differences from the vehicle control among the continuous BPA dose groups. The high $\mathrm{EE}_{2}$ dose had a highly significant effect on the estrous cycle, with $96 \%$ of the animals showing extended estrus as compared to $12 \%$ of the vehicle controls (Table 34). When all types of abnormal cycles were considered, $100 \%$ of the high $\mathrm{EE}_{2}$ dose group animals showed abnormal cycles compared to $27 \%$ of the vehicle controls.

\section{Stop-Dose Arm}

There were no BPA treatment-related effects on the estrous cycle in the stop-dose animals (Table 35). The individual animal data are found in the statistical report in Supplemental Appendix XXVII.

\section{Vaginal Cytology - Onset of Aberrant Estrous Cycles in Aging Animals}

\section{Continuous-Dose Arm}

The time of onset of aberrant estrous cycles in aging females was estimated by evaluating five consecutive vaginal smears every month (see Materials and Methods and legend to Table 36). The data for the animals in the continuous-dose arm are summarized in Table 36, and the complete statistical report is found in Supplemental Appendix XXVIII. The Kaplan-Meier survival curves related to the onset of aberrant cycles for the continuous vehicle control and BPA and vehicle control and $\mathrm{EE}_{2}$ groups are shown in Figure 20 and Figure 21, respectively. There was no treatment effect of BPA in the continuous-dose arm. None of the dose groups differed significantly in the median onset time of 56.8 weeks in the vehicle control group. As expected based on the previously mentioned analysis of estrous cycle data at 16 weeks, the onset of aberrant cycles occurred significantly earlier in the $0.5 \mu \mathrm{g} \mathrm{EE} / 2 \mathrm{~kg}$ bw/day dose group.

\section{Stop-Dose Arm}

The time of onset of aberrant estrous cycles in the stop-dose BPA females is shown in Table 37. The Kaplan-Meier survival curves related to the onset of aberrant cycles for the stop-dose vehicle control and BPA groups are shown in Figure 22, and the complete statistical report is found in Supplemental Appendix XXVIII. The sole significant effect was a delay in the median 
time of onset in the $2,500 \mu \mathrm{g}$ BPA/kg bw/day dose group (57 weeks versus 42 weeks in vehicle controls). While no formal analysis was conducted to compare the continuous-dose vehicle control group with the stop-dose vehicle control group, the estimated median time of onset of aberrant cycling appeared shorter in the stop-dose vehicle control group.

\section{Hematology Endpoints in Interim Sacrifice Animals}

\section{Females, Continuous-Dose Arm}

Hematology endpoints examined at the interim sacrifice in females dosed continuously with $\mathrm{BPA}$ or $\mathrm{EE}_{2}$ are shown in Table 38. Platelet counts were significantly lower $(\sim 10 \%)$ than vehicle controls in the $25,000 \mu \mathrm{g} \mathrm{BPA} / \mathrm{kg}$ bw/day dose group. Eosinophils were decreased $(\sim 25 \%)$ in the $250 \mu \mathrm{g} \mathrm{BPA} / \mathrm{kg}$ bw/day dose group relative to the vehicle control group, and mean corpuscular hemoglobin concentration was significantly higher $(\sim 1.4 \%)$ than vehicle controls in the $25 \mu \mathrm{g}$ $\mathrm{BPA} / \mathrm{kg}$ bw/day dose group. There were significant trends over increasing levels of BPA dose concentrations for hemoglobin concentration $(p=0.023)$, monocytes $(p=0.045)$, and platelet counts $(p=0.008)$. In female rats continuously dosed with $0.5 \mu \mathrm{g} \mathrm{EE} / \mathrm{kg}$ bw/day, lower eosinophil counts $(\sim 25 \%), \%$ eosinophils $(\sim 21 \%)$, and platelet counts $(\sim 8 \%)$ were observed.

\section{Females, Stop-Dose Arm}

Hematology endpoints examined at the interim sacrifice in stop-dose BPA female rats are shown in Table 39. No statistically significant differences in values were observed in pairwise comparisons between BPA dose groups and vehicle controls. Significant trends were noted for $\%$ basophils $(\mathrm{p}=0.031)$, mean corpuscular hemoglobin $(p=0.013)$, and red blood cells $(p=0.044)$.

\section{Males, Continuous-Dose Arm}

Hematology endpoints examined at the interim sacrifice in males dosed continuously with BPA or $\mathrm{EE}_{2}$ are shown in Table 40. Hemoglobin levels were significantly higher ( 4\%) in the $25,000 \mu \mathrm{g} \mathrm{BPA} / \mathrm{kg}$ bw/day group and the percentage of eosinophils lower $(\sim 28 \%)$ in the $250 \mu \mathrm{g}$ $\mathrm{BPA} / \mathrm{kg}$ bw/day group relative to vehicle controls. Significant trends were observed for hematocrit $(p=0.006)$, hemoglobin concentration $(p=0.016), \operatorname{PCV}(p=0.008)$, mean corpuscular hemoglobin $(p=0.018)$, mean corpuscular hemoglobin volume $(p=0.016)$, and platelet counts $(p=0.011)$. The sole observed statistically significant effect in the $\mathrm{EE}_{2}$ groups was an elevated hemoglobin concentration $(\sim 3 \%)$ relative to the vehicle control level in the $0.05 \mu \mathrm{g} \mathrm{EE} 2 / \mathrm{kg}$ bw/day group.

\section{Males, Stop-Dose Arm}

Hematology endpoints examined at the interim sacrifice in stop-dose BPA male rats are shown in Table 41. No statistically significant effects were observed in pairwise comparisons between BPA dose groups and vehicle controls. A significant trend for $\%$ neutrophils $(p=0.045)$ was noted over the levels of BPA dose concentrations in the stop-dose arm. 


\section{Serum Clinical Chemistry Endpoints in Interim Sacrifice Animals}

\section{Females, Continuous-Dose Arm}

Clinical chemistry endpoints examined at the interim sacrifice in females dosed continuously with BPA or $\mathrm{EE}_{2}$ are shown in Table 42. Alkaline phosphatase levels were significantly higher $(\sim 31 \%)$ in the $250 \mu \mathrm{g} \mathrm{BPA} / \mathrm{kg}$ bw/day group than levels in the vehicle control group. Although mean levels of alkaline phosphatase were higher than controls in most of the BPA groups, none of the others were statistically significant. There were no other statistically significant treatment effects on clinical chemistry endpoints in any continuous BPA dose group. The female rats in the continuous $0.5 \mu \mathrm{g} \mathrm{EE} 2 / \mathrm{kg}$ bw/day dose group had higher $(\sim 38 \%)$ mean levels of TSH, while rats in the $0.05 \mu \mathrm{g} \mathrm{EE}_{2} / \mathrm{kg}$ bw/day dose group had higher ( 24\%) mean levels of alkaline phosphatase than the vehicle control group. There were no $\mathrm{EE}_{2}$ treatment effects on T3 or T4.

\section{Females, Stop-Dose Arm}

Clinical chemistry endpoints examined at the interim sacrifice in stop-dose BPA female rats are shown in Table 43. No statistically significant differences were noted in pairwise comparisons between stop-dose BPA-treated female rats and the vehicle controls. There was a significant trend over levels of BPA dose concentrations for albumin $(p=0.004)$.

\section{Males, Continuous-Dose Arm}

Clinical chemistry endpoints examined at the interim sacrifice in males dosed continuously with BPA or $\mathrm{EE}_{2}$ are shown in Table 44. No statistically significant differences were noted in pairwise comparisons between any BPA treatment group and vehicle controls. Significant trends were noted for albumin $(p=0.007), \mathrm{T} 4(p=0.002)$, total bile acids $(p=0.026)$, and troponin T $(p=0.003)$. For $\mathrm{EE}_{2}$, mean insulin levels were significantly lower $(\sim 35 \%, p=0.047)$ in the $0.05 \mu \mathrm{g} \mathrm{EE}_{2} / \mathrm{kg}$ bw/day dose group and mean triglyceride levels were significantly higher $(\sim 26 \%)$ in the $0.5 \mu \mathrm{g} \mathrm{EE} 2 / \mathrm{kg}$ bw/day dose group than those in vehicle controls.

\section{Males, Stop-Dose Arm}

Clinical chemistry endpoints examined at the interim sacrifice in stop-dose BPA male rats are shown in Table 45 . In the $25 \mu \mathrm{g}$ BPA/kg bw/day dose group, decreases in the mean levels of total protein $(\sim 4 \%)$ and total bile acids $(\sim 31 \%)$ relative to vehicle controls were observed.

Significant trends were noted over the levels of BPA dose concentrations for T4 $(p=0.046)$ and for total bile acids $(p=0.024)$.

\section{Organ Weights in Interim Sacrifice Animals}

\section{Females, Continuous-Dose Arm}

Summary statistics for organ weights collected from females in the continuous-dose BPA and $\mathrm{EE}_{2}$ dose groups are shown in Table 46. Organ weights were analyzed as absolute weights, and relative weights with brain and body weight at necropsy as covariates. There were few sporadic significant differences between BPA groups and the vehicle control group. In the $2.5 \mu \mathrm{g} \mathrm{BPA} / \mathrm{kg}$ bw/day dose group, the mean absolute retroperitoneal fat pad weight was significantly higher $(40 \%)$ than the mean weight in the vehicle control group. The brain weight-adjusted 
retroperitoneal fat pad weight was similarly significantly greater than the vehicle control. The mean retroperitoneal fat pad weight adjusted for body weight was higher (23\%) than the vehicle control group, but this was not a statistically significant difference. The only other significant BPA treatment effect was a dose trend for liver adjusted for body weight.

Multiple organ weights were significantly affected by the $0.5 \mu \mathrm{g} \mathrm{EE} / \mathrm{kg}$ bw/day treatment. Mean absolute adrenal weights, as well as adrenal weights adjusted for brain and body weights, were increased by $27 \%, 28 \%$, and $22 \%$, respectively. Mean ovarian/parametrial fat pad weight was decreased, with mean weight adjusted for body weight significantly decreased $(\sim 17 \%)$ relative to the vehicle control group mean. Mean heart weight was also increased in the high $\mathrm{EE}_{2}$ dose group, with mean heart weight adjusted for body weight significantly increased $(\sim 6 \%)$ relative to the vehicle control group mean. Mean absolute kidney weights, as well as kidney weights adjusted for brain and body weights, were increased relative to the vehicle control mean by $15 \%$, $16 \%$, and $13 \%$, respectively. Mean absolute liver weights, as well as liver weights adjusted for brain and body weights, were increased relative to the vehicle control mean by $20 \%, 20 \%$, and $18 \%$, respectively. Mean absolute ovary weights, as well as ovary weights adjusted for brain and body weights, were decreased relative to the vehicle control mean by $18 \%, 16 \%$, and $15 \%$, respectively. Mean absolute pituitary weights, as well as pituitary weights adjusted for brain and body weights, were increased by $31 \%, 32 \%$, and $20 \%$, respectively.

\section{Females, Stop-Dose Arm}

Summary statistics for organ weights collected from females in the BPA stop-dose groups are shown in Table 47. There were significant dose trends for absolute ovary weight and ovary weight adjusted for brain and body weight. The mean absolute ovary weight, as well as ovary weight adjusted for brain weight, in the $25,000 \mu \mathrm{g} \mathrm{BPA} / \mathrm{kg}$ bw/day dose group were significantly lower than the vehicle control group by approximately $13 \%$ and $12 \%$, respectively. Mean ovary weight adjusted for body weight was also $9 \%$ lower than the controls, but this difference was not statistically different.

\section{Males, Continuous-Dose Arm}

Summary statistics for organ weights collected from males in the continuous-dose BPA and $\mathrm{EE}_{2}$ dose groups are shown in Table 48. The sole significant BPA treatment effect was a lower $(\sim 7 \%)$ mean liver weight adjusted for body weight relative to the vehicle control in the $2.5 \mu \mathrm{g} \mathrm{BPA} / \mathrm{kg}$ bw/day dose group. There were no significant treatment effects of either $\mathrm{EE}_{2}$ dose.

\section{Males, Stop-Dose Arm}

Summary statistics for organ weights collected from males in the BPA stop-dose groups are shown in Table 49. The sole significant treatment effect was a significant dose trend for liver weight adjusted for body weight.

\section{Sperm Analysis, Interim Sacrifice Animals}

Testicular spermatid head counts, caudal sperm counts, and caudal sperm motility and morphology data are shown in Table 50 for continuous BPA and $\mathrm{EE}_{2}$ dose groups and in Table 51 for BPA stop-dose groups. There were no significant treatment effects observed for either compound. 


\section{Histopathology}

The pathology report, which has tabulations of organs assessed and all lesions noted in all animals in both the interim (one-year) and terminal (two-year) phases of the study, along with the Study Pathologist's narrative report, is found in Supplemental Appendix XXXII. Neoplastic lesions that showed any statistically significant increased incidence for either BPA or the reference estrogen, $\mathrm{EE}_{2}$, are discussed below. As noted in the pathology narrative, many nonneoplastic lesions common to aging animals in this strain of rat were found in both sexes at one and two years of age. Incidences were highly variable across dose groups and BPA treatment effects were not evident to the Study Pathologist. After the finalization of the pathology report, statistical analyses, described in Materials and Methods, were conducted for any lesion that had an incidence of two or more in the interim sacrifice animals or in the $\mathrm{EE}_{2}$ terminal sacrifice animals and four or more in the BPA-treated terminal sacrifice animals. All statistical results are shown in Supplemental Appendix XXXIII for interim sacrifice animals and Supplemental Appendix XXXIV for terminal sacrifice animals and selected lesions with statistically significant increased incidences in BPA or $\mathrm{EE}_{2}$ dose groups are discussed below. Lesions for which there was significantly lower incidence in treatment groups relative to vehicle controls are generally not discussed, although a few of these cases are presented. For statistical analyses of microscopic lesions, one-sided $p$-values with no correction for multiple comparisons are reported for pairwise comparisons with the vehicle control, while two-sided results are reported for trends. In the text, the unadjusted incidence (i.e., lesions observed/animals examined) is reported, while in the tables both the unadjusted and survival-adjusted (Poly-3) incidences are shown for terminal sacrifice animals.

\section{Females}

Neoplastic lesions showing statistically significant differences in the BPA dose groups versus the vehicle control group were limited to the female mammary gland and uterus. For $\mathrm{EE}_{2}$, there were significant trends and significant differences between the high dose group and vehicle control for adenocarcinomas in the mammary gland and combined adenoma/carcinoma in the pituitary pars distalis. Significant dose trends were also noted in terminal sacrifice animals for benign pheochromocytoma in the adrenal medulla and $\mathrm{C}$-cell adenomas in the thyroid gland with incidences in the high dose $\mathrm{EE}_{2}$ group of $8 \%$ versus $0 \%$ in controls for both cases (Supplemental Appendix XXXII and XXXIV).

\section{Mammary gland, neoplastic lesions}

Neoplastic lesions in the mammary glands of interim and terminal sacrifice females for continuous BPA, continuous $\mathrm{EE}_{2}$ and stop-dose BPA treatments are shown in Table 52, Table 53, and Table 54, respectively. Fibroadenomas are a common high-incidence lesion in this strain of rats, with a historical control incidence at two years of 132/210 (63\%, range 59-69\%). Fibroadenomas were observed in 4-25\% of interim sacrifice females and 54-90\% of terminal sacrifice animals with no significant treatment effects. Fibroadenoma counts in each affected animal were recorded and are found in an appendix to the pathology report (Supplemental Appendix XXXII, Subappendix VII).

Adenocarcinomas and/or adenomas were observed in the continuous-dose BPA-treated animals (Table 52). In interim sacrifice animals, there were no treatment-related increases in neoplasm incidence, but both the 2.5 and $25 \mu \mathrm{g} \mathrm{BPA} / \mathrm{kg}$ bw/day continuous-dose groups had single 
adenocarcinomas in 22 animals examined (4\% incidence). In the terminal sacrifice continuousdose vehicle control animals, $8 \%$ had adenocarcinoma and $12 \%$ had adenoma or adenocarcinoma. In the continuous BPA dose groups, the incidence of adenocarcinoma varied between 6 and 18\%, and the incidence of adenocarcinoma or adenoma varied between 9 and $20 \%$. None of these incidences were significant compared to the vehicle control group. The historical control incidence of combined adenoma/adenocarcinomas was 32/210 (15.2\%, range $12-17 \%)$.

In interim sacrifice animals, 2 of 26 animals (8\% incidence) from the $0.05 \mu \mathrm{g} \mathrm{EE} / \mathrm{kg}$ bw/day dose group had adenocarcinomas (Table 53). For the terminal sacrifice $\mathrm{EE}_{2}$ treatment groups, there was a significant dose trend $(p<0.001)$ and a significant increase in adenocarcinomas in the $0.5 \mu \mathrm{g} \mathrm{EE} / 2 / \mathrm{kg}$ bw/day dose group (38\% versus $8 \%, p<0.001)$.

Adenocarcinomas and/or adenomas were also observed in the terminal stop-dose females (Table 54). In the terminal sacrifice stop-dose females, vehicle controls had $6 \%$ animals with adenocarcinomas and $8 \%$ with adenomas or adenocarcinomas. The $2.5 \mu \mathrm{g} \mathrm{BPA} / \mathrm{kg}$ bw/day stopdose group had a significantly higher incidence of adenocarcinomas ( $22 \%$ versus $6 \%, p=0.016)$ or adenomas and adenocarcinomas combined $(24 \%$ versus $8 \%, p=0.018)$.

\section{Mammary gland, non-neoplastic lesions}

Non-neoplastic lesions in the mammary glands of interim and terminal sacrifice females for continuous BPA and $\mathrm{EE}_{2}$ and stop-dose BPA treatments are shown in Table 55, Table 56, and Table 57, respectively.

In the continuous-dose BPA groups, in both the interim and terminal sacrifice females, the incidences of atypical foci were higher in some treatment groups than in vehicle controls, and this was significant (by the RTE test only) for the $2.5 \mu \mathrm{g} \mathrm{BPA} / \mathrm{kg}$ bw/day dose group in both the interim (14\% versus $0 \%$ ) and terminal (15\% versus $4 \%$ ) females (Table 55). In the interim sacrifice animals, there was a significantly increased incidence (RTE test only) of ductal dilatation (32\% versus $9 \%$ ) in the $25 \mu \mathrm{g}$ BPA/kg bw/day dose group, but this was not the case in the terminal sacrifice females, where the incidence in the dose group was decreased relative to the vehicle controls (15\% versus $30 \%)$.

In the continuous-dose $\mathrm{EE}_{2}$ treatments, there were several significant trends and high dose treatment effects observed by all statistical tests applied (Table 56). In both interim and terminal animals, there was a significant trend and a significant pairwise comparison of the $0.5 \mu \mathrm{g} \mathrm{EE} / \mathrm{kg}$ bw/day dose group and vehicle control for ductal dilatation (85\% versus $9 \%$, interim; $81 \%$ versus $30 \%$, terminal). In interim sacrifice animals, there was a significant trend and a significant pairwise comparison of the $0.5 \mu \mathrm{g} \mathrm{EE} / \mathrm{kg}$ bw/day dose group and vehicle control for lobular hyperplasia ( $88 \%$ versus $44 \%$ ). In terminal sacrifice animals, there was a significant trend and a significant pairwise comparison of the $0.5 \mu \mathrm{g} \mathrm{EE} / \mathrm{kg}$ bw/day dose group and vehicle control for alveolar dilatation ( $85 \%$ versus $18 \%)$.

In the stop-dose BPA treatments, there were no statistically significant increased mammary gland non-neoplastic lesion incidences in BPA dose groups relative to vehicle controls, although multiple cases of decreased incidences in BPA groups relative to vehicle were observed (Table 57). 


\section{Uterus, neoplastic lesions}

Stromal polyps were found in interim and terminal females and their incidences are shown for continuous BPA and EE2 and stop-dose BPA in Table 58, Table 59, and Table 60, respectively.

There was a significant dose trend in the interim sacrifice females treated continuously with BPA (Table 58). The incidence for the vehicle control group was $1 / 23(4 \%)$ compared to 3/20 (15\%) for the 2,500 $\mu \mathrm{g} \mathrm{BPA} / \mathrm{kg}$ bw/day group and 3/24 (12\%) for the 25,000 $\mu \mathrm{g}$ BPA/kg bw/day group, but these differences were not statistically significant. This trend toward increased incidence at the higher BPA dose groups was not observed in the terminal sacrifice animals, where the control unadjusted incidence was 5/50 (10\%) and incidences in BPA treatment groups varied from 4-16\%, with 4/48 (8\%) and 3/46 (6\%) in the 2,500 and 25,000 $\mu \mathrm{g} \mathrm{BPA/kg} \mathrm{bw/day} \mathrm{groups,}$ respectively.

There were no significant effects of continuous $\mathrm{EE}_{2}$ treatment (Table 59).

In the stop-dose terminal females, the vehicle control incidence of stromal polyps was $14 \%$, and a negative trend was observed along with a reduced incidence in the $25,000 \mu \mathrm{g} \mathrm{BPA} / \mathrm{kg}$ bw/day dose group relative to the vehicle control (14\% versus $2 \%$, Table 60$)$. There were no other statistically significant neoplastic effects observed in interim or terminal sacrifice BPA continuous or stop-dose treatments in the uterus.

\section{Uterus, non-neoplastic lesions}

Non-neoplastic lesions in the uteri of interim and terminal sacrifice females for continuous BPA and $\mathrm{EE}_{2}$ and stop-dose BPA treatments are shown in Table 61, Table 62, and Table 63, respectively.

In interim sacrifice, continuous-dose BPA females, there was a significant dose trend for apoptosis in the luminal epithelial cells of the endometrium for all statistical analyses applied, with the incidence in the $25,000 \mu \mathrm{g} \mathrm{BPA} / \mathrm{kg}$ bw/day dose group significantly higher than the vehicle controls (38\% versus $9 \%$, Table 61$)$. Endometrial hyperplasia was significantly increased in the interim continuous-dose 2.5 and $250 \mu \mathrm{g} \mathrm{BPA} / \mathrm{kg}$ bw/day dose groups (RTE test only, 32\% versus $9 \%$ and $29 \%$ versus $9 \%$, respectively). There were also significant dose trends for squamous metaplasia and dilatation of the lumen in the interim and terminal sacrifice continuous-dose BPA females, respectively, with no significant pairwise comparisons for any dose group to vehicle controls (Table 61).

In the continuous $\mathrm{EE}_{2}$-treated interim sacrifice females, there were increased trends and significant pairwise comparisons of the $0.5 \mu \mathrm{g} \mathrm{EE} 2 / \mathrm{kg}$ bw/day dose group to the vehicle control detected in all statistical tests applied for uterine apoptosis (69\% versus $9 \%)$, cystic endometrial hyperplasia (54\% versus 22\%), and squamous metaplasia (54\% versus $4 \%$ ) (Table 62). In the terminal animals, a trend was detected for squamous metaplasia in both the Poly-3 and JT/SW tests (incidences of $4 \%, 8 \%$, and $15 \%$ in the vehicle control, low and high $\mathrm{EE}_{2}$ dose groups, respectively).

In the stop-dose females, the incidence of apoptosis in the $25,000 \mu \mathrm{g} \mathrm{BPA} / \mathrm{kg}$ bw/day dose group was higher than that in vehicle controls (27\% versus $10 \%)$, but this difference was not statistically significant (Table 63). In stop-dose BPA-treated females, there was a significant increase in cystic endometrial hyperplasia relative to vehicle controls in the $25,000 \mu \mathrm{g} \mathrm{BPA} / \mathrm{kg}$ bw/day dose group (32\% versus 10\%, JT/SW and RTE tests) at interim sacrifice, while in the terminal sacrifice females there was a significant dose trend and the incidences in the 2,500 and 
$25,000 \mu \mathrm{g} \mathrm{BPA} / \mathrm{kg}$ bw/day dose groups were significantly higher than that in the vehicle control (57\% and 52\%, respectively, versus 37\%). Additional statistically significant differences in BPA stop-dose interim sacrifice animals versus controls were an increased incidence of squamous metaplasia in the $25,000 \mu \mathrm{g} \mathrm{BPA} / \mathrm{kg}$ bw/day dose group (JT/SW and RTE tests, $18 \%$ versus $0 \%$ ) and an increased incidence of dilatation of the lumen in the $250 \mu \mathrm{g} \mathrm{BPA} / \mathrm{kg}$ bw/day dose group (RTE test only, $18 \%$ versus 5\%) (Table 63).

\section{Ovary, non-neoplastic lesions}

Non-neoplastic lesions in the interim and terminal sacrifice animals for continuous BPA and $\mathrm{EE}_{2}$ and stop-dose BPA are shown in Table 64, Table 65, and Table 66, respectively.

There were no statistically significant BPA treatment-related effects in the continuous-dose terminal sacrifice females. Females in the interim sacrifice continuous BPA dose arm showed significant dose trends for depletion of corpora lutea and interstitial cell hypertrophy (Table 64). For interstitial cell hypertrophy, the $2,500 \mu \mathrm{g}$ BPA/kg bw/day dose group was significantly different from the vehicle control incidence (RTE test only, 40\% versus $17 \%$ ). The $25,000 \mu \mathrm{g}$ $\mathrm{BPA} / \mathrm{kg}$ bw/day dose group had an incidence of $38 \%(p=0.068)$.

In the ovaries of continuous $\mathrm{EE}_{2}$-treated interim sacrifice females, there were significant dose trends and significant pairwise comparisons of the $0.5 \mu \mathrm{gEE}_{2} / \mathrm{kg}$ bw/day dose group to the vehicle control detected in all statistical tests applied for atrophy (100\% versus $44 \%)$, follicular cysts $(100 \%$ versus $35 \%)$, depleted corpora lutea (100\% versus $17 \%)$, and interstitial cell hypertrophy (100\% versus $17 \%$ ) (Table 65 ). In the terminal sacrifice females, there was a $94 \%$ incidence of ovarian atrophy in vehicle controls and $100 \%$ atrophy in the high $\mathrm{EE}_{2}$ dose group, with greater severity in the $\mathrm{EE}_{2}$-treated group that was significantly greater than controls by both tests that incorporate severity scores (JT/SW and RTE, $p<0.001$ for both).

There were no statistically significant BPA treatment-related effects in the stop-dose terminal sacrifice females. In the stop-dose BPA-treated interim sacrifice animals, there was a significant dose trend $(p<0.001)$ for follicular cysts and the $25,000 \mu \mathrm{g} \mathrm{BPA} / \mathrm{kg}$ bw/day dose group had a higher incidence than vehicle controls (82\% versus $25 \%$ ) (Table 66). The $2,500 \mu \mathrm{g} \mathrm{BPA} / \mathrm{kg}$ bw/day dose group had an incidence of $55 \%(p=0.053)$. There were no continuous-dose BPA groups with significantly higher follicular cyst incidence than the vehicle controls (Table 64).

\section{Vagina, non-neoplastic lesions}

Non-neoplastic lesions in the vaginas of interim and terminal sacrifice females for continuous BPA and $\mathrm{EE}_{2}$ and stop-dose BPA are shown in Table 67, Table 68, and Table 69, respectively.

Statistically significant effects were observed in both the interim and terminal sacrifice BPAtreated animals for epithelial hyperplasia for the continuous-dose arm (Table 67). For the interim sacrifice animals, there was a significant dose trend (all statistical tests) and the 25,000 $\mu \mathrm{g}$ $\mathrm{BPA} / \mathrm{kg}$ bw/day dose group had a significantly higher incidence of hyperplasia than the vehicle controls (JT/SW and RTE tests, $33 \%$ versus $13 \%$ ). The incidence in the $2,500 \mu \mathrm{g} \mathrm{BPA} / \mathrm{kg}$ bw/day continuous-dose group was $30 \%$ ( $p=0.074$ and 0.067 for the JT/SW and RTE tests, respectively). In the terminal sacrifice females, there was a significant dose trend (all statistical tests) and significant pairwise comparisons to control for $25-25,000 \mu \mathrm{g} \mathrm{BPA} / \mathrm{kg} \mathrm{bw} /$ day dose groups, although the response was similar across dose groups (incidences of $8 \%$ in vehicle controls and $27 \%, 20 \%, 22 \%$, and $26 \%$ for the $25,250,2,500$, and 25,000 $\mu \mathrm{g} \mathrm{BPA} / \mathrm{kg}$ bw/day 
dose groups, respectively). The Poly-3 and RTE tests were not significant for the $250 \mu \mathrm{g} \mathrm{BPA} / \mathrm{kg}$ bw/day dose group.

In the vaginas of females treated continuously with $\mathrm{EE}_{2}$, statistically significant effects were observed in the interim sacrifice animals, but not in terminal sacrifice animals. For all statistical tests applied, there was a dose trend for epithelial hyperplasia and a significant pairwise comparison for the $0.5 \mu \mathrm{g} \mathrm{EE} / \mathrm{kg}$ bw/day dose group relative to vehicle control ( $77 \%$ versus $13 \%$ ) (Table 68). There was also a trend toward increased epithelial mucification (all statistical tests) and a significant pairwise comparison for the $0.5 \mu \mathrm{g} \mathrm{EE} / \mathrm{kg}$ bw/day dose group relative to vehicle control (69\% versus $44 \%$, JT/SW and RTE tests).

In the stop-dose arm, there were no statistically significant BPA effects in the interim or terminal sacrifice females, although the incidence of epithelial hyperplasia was $27 \%$ in the $25,000 \mu \mathrm{g}$ $\mathrm{BPA} / \mathrm{kg}$ bw/day dose group versus $10 \%$ in the vehicle control $(p=0.064)$ in the interim sacrifice animals (Table 69).

\section{Pituitary, neoplastic and non-neoplastic lesions}

Neoplastic lesions in the pituitaries of interim and terminal sacrifice females for continuous BPA and $\mathrm{EE}_{2}$ and stop-dose BPA are shown in Table 70, Table 71, and Table 72, respectively.

Adenomas or carcinomas of the pars distalis were observed at low incidence in interim animals, and higher incidences, primarily of adenomas, were observed in terminal animals. There were no statistically significant effects in BPA continuous- or stop-dose interim or terminal sacrifice animals (Table 70 and Table 72). In the terminal $\mathrm{EE}_{2}$ females, the incidence of combined adenomas and carcinomas of the pars distalis was increased in the $0.5 \mu \mathrm{g} / \mathrm{kg}$ bw/day dose group (77\% versus $44 \%$, Table 71$)$.

Non-neoplastic lesions in the pituitaries of interim and terminal sacrifice females for continuous BPA and $\mathrm{EE}_{2}$ and stop-dose BPA are shown in Table 73, Table 74, and Table 75, respectively.

There were no statistically significant treatment effects in the pituitaries of continuous BPA dose arm interim or terminal sacrifice females (Table 73).

In the pituitaries of females treated continuously with $\mathrm{EE}_{2}$, increased lesion incidences relative to vehicle controls were observed in the interim and terminal sacrifice animals (Table 74). In the interim sacrifice animals, there was a significant increasing trend for hyperplasia in the pars distalis and a significant pairwise comparison for the $0.5 \mu \mathrm{g} \mathrm{EE} / 2 \mathrm{~kg}$ bw/day dose group relative to the high incidence in the vehicle control (96\% versus $78 \%$, JT/SW and RTE tests). There was also a significant trend for angiectasis in the interim sacrifice females (all statistical tests) and a significant pairwise comparison for the $0.5 \mu \mathrm{g} \mathrm{EE} / \mathrm{kg}$ bw/day dose group relative to vehicle control (23\% versus 4\%, JT/SW and RTE tests). Similar trends and pairwise comparisons of the high $\mathrm{EE}_{2}$ dose group to vehicle control were seen in the terminal sacrifice females $(65 \%$ versus $20 \%$, all statistical tests).

There were few statistically significant increased incidences over vehicle control in the stop-dose BPA arm, all indicated only by the RTE test (Table 75). In the interim sacrifice females, there was an increase in angiectasis (dilated vasculature) in the $2.5 \mu \mathrm{g}$ BPA/kg bw/day stop-dose group (9\% versus $0 \%)$. In the stop-dose terminal sacrifice females, the incidence of hyperplasia in the pars distalis was increased in the 2.5 and $25 \mu \mathrm{g}$ BPA/kg bw/day stop-dose groups (64\% and $71 \%$, respectively, versus 51\%) (Table 75). In the terminal sacrifice continuous-dose females, the incidences of the same lesion in the vehicle control, 2.5 and $25 \mu \mathrm{g}$ BPA/kg bw/day stop-dose 
groups were $54 \%, 46 \%$, and $70 \%$, respectively, with no statistically significant comparisons (Table 73).

\section{Heart, non-neoplastic lesions}

Cardiomyopathy is a high-incidence background lesion in this rat strain, which increases with aging in both sexes. The lower incidences in females than in males allowed for a better evaluation of any potential treatment effects. Incidences and severity scores for cardiomyopathy in interim and terminal sacrifice females for continuous BPA and $\mathrm{EE}_{2}$ and stop-dose BPA are shown in Table 76, Table 77, and Table 78, respectively.

There were no statistically significant positive effects observed in female rats dosed continuously with BPA (Table 76). In the terminal sacrifice continuous-dose females, the vehicle control incidence of cardiomyopathy was $70 \%$, with a reduced incidence $(52 \%)$ in the $25 \mu \mathrm{g} \mathrm{BPA} / \mathrm{kg}$ bw/day dose group.

In interim sacrifice females dosed continuously with $\mathrm{EE}_{2}$, all three statistical tests applied indicated a significant dose trend and a significant increase in the incidence of cardiomyopathy in the $0.5 \mu \mathrm{g} \mathrm{EE}_{2} / \mathrm{kg}$ bw/day dose group relative to vehicle control (65\% versus $30 \%$ ) (Table 77). The JT/SW and RTE tests also detected an increase in the terminal sacrifice females ( $85 \%$ in the $0.5 \mu \mathrm{g} \mathrm{EE} / 2 \mathrm{~kg}$ bw/day dose group versus $70 \%$ in the vehicle control group). The RTE test also indicated a significant increasing trend in terminal sacrifice $\mathrm{EE}_{2}$ females.

In stop-dose BPA animals, no effects were observed in interim sacrifice females (Table 78). In terminal sacrifice stop-dose females, the statistical tests that incorporate severity scores along with incidence (JT/SW and RTE) detected a dose trend, and there were significant pairwise comparisons for $2.5,250,2,500$, and $25,000 \mu \mathrm{g}$ BPA $/ \mathrm{kg}$ bw/day dose groups relative to the vehicle control (incidences of $74 \%, 74 \%, 70 \%$, and $76 \%$, respectively, versus $64 \%$ ). The mean severity scores varied from 1.5 to 1.7 in the significantly different BPA groups versus 1.3 in the vehicle control, mostly due to an increased percentage of lesions diagnosed as mild rather than minimal in those groups.

\section{Kidney, non-neoplastic lesions}

Non-neoplastic lesions in the kidneys of interim and terminal sacrifice females for continuous BPA, EE 2 , and stop-dose BPA are shown in Table 79, Table 80, and Table 81, respectively.

The incidence of nephropathy was high and highly variable between continuous- and stop-dose vehicle controls (continuous interim and terminal controls, 26\% and 38\% (Table 79), respectively; stop-dose interim and terminal controls, 50\% and 57\% (Table 81), respectively).

For interim sacrifice females dosed continuously with BPA, the RTE test indicated an increased incidence of nephropathy in the 25 and $2,500 \mu \mathrm{g}$ BPA/ $\mathrm{kg}$ bw/day dose groups relative to the vehicle control (50\% and 55\%, respectively, versus 26\%) (Table 79). In the terminal sacrifice females in the continuous BPA dose arm, both the Poly-3 and RTE tests detected increased incidences of nephropathy in the $2.5 \mu \mathrm{g}$ BPA/kg bw/day group (58\% versus $38 \%$ ). In addition, the JT/SW and RTE tests detected an increased incidence in the 25,000 $\mu \mathrm{g} \mathrm{BPA} / \mathrm{kg}$ bw/day dose group relative to vehicle control (54\% versus $38 \%)$. Additional statistically significant effects in the continuous BPA dose groups in the interim sacrifice animals were an increase in renal tubular cysts in the $2.5 \mu \mathrm{g} \mathrm{BPA} / \mathrm{kg}$ bw/day group relative to vehicle controls (32\% versus $0 \%$, CAFE test; the control incidence in stop-dose group was 20\% (Table 81) and an increased dose 
trend (all statistical tests applied) of mineralization, with a greater incidence in the $25,000 \mu \mathrm{g}$ $\mathrm{BPA} / \mathrm{kg}$ bw/day group relative to the vehicle control group (67\% versus $48 \%, \mathrm{JT} / \mathrm{SW}$ and RTE tests only).

In females dosed continuously with $\mathrm{EE}_{2}$, statistically significant increased incidences of kidney lesions relative to vehicle controls were also observed (Table 80). In interim sacrifice females, all statistical tests applied indicated a dose trend and a significant pairwise comparison for the $0.5 \mu \mathrm{g} \mathrm{EE}_{2} / \mathrm{kg}$ bw/day dose group relative to vehicle control for nephropathy (58\% versus $26 \%$ ). This trend was also evident in the terminal sacrifice females (all statistical tests applied), and the JT/SW and/or RTE tests indicated significantly increased incidences in both the low and high $\mathrm{EE}_{2}$ dose groups (54\% and 58\%, respectively, versus 38\%). In the interim female $0.05 \mu \mathrm{g} \mathrm{EE} / \mathrm{kg}$ bw/day dose group, there was an increase in renal tubule cysts (19\% versus $0 \%)$ and mineralization (RTE test only, $65 \%$ versus $48 \%$ ).

In terminal stop-dose females, renal tubular cysts were increased in the $2.5 \mu \mathrm{g} \mathrm{BPA} / \mathrm{kg}$ bw/day group relative to the vehicle control group (43\% versus 21\%) (Table 81 ).

\section{Liver, non-neoplastic lesions}

Non-neoplastic lesions in the livers of interim and terminal sacrifice females for continuous BPA and $\mathrm{EE}_{2}$ and stop-dose BPA are shown in Table 82, Table 83, and Table 84, respectively.

No statistically significant positive treatment-related effects were observed in continuous BPA or $\mathrm{EE}_{2}$ treatment groups (Table 82 and Table 83).

In the stop-dose BPA treatments in interim sacrifice females, mononuclear cell infiltration showed an increased incidence in several dose groups that was statistically significant for the 2.5 (CAFE and RTE tests) and 25,000 $\mu \mathrm{g} \mathrm{BPA} / \mathrm{kg}$ bw/day stop-dose groups (all statistical tests applied; $46 \%$ and $36 \%$, respectively, versus 10\%) (Table 84). In the terminal sacrifice females, there was a trend toward an increased incidence of cystic degeneration (all statistical tests applied) and significant pairwise comparisons (JT/SW and RTE tests) for 2,500 and 25,000 $\mu \mathrm{g}$ $\mathrm{BPA} / \mathrm{kg}$ bw/day stop-dose groups (16\% and 15\%, respectively, versus $4 \%$ ).

\section{Thyroid, non-neoplastic lesions}

Non-neoplastic lesions in the thyroid glands of interim and terminal sacrifice females for continuous BPA and $\mathrm{EE}_{2}$ and stop-dose BPA are shown in Table 85, Table 86, and Table 87, respectively.

In the continuous-dose BPA terminal sacrifice females, the sole significant effect was an elevated incidence (RTE test only) of follicular cell hyperplasia in the $2.5 \mu \mathrm{g} \mathrm{BPA} / \mathrm{kg}$ bw/day dose group relative to the vehicle control (12\% versus $2 \%$ ) (Table 85$)$. The vehicle control incidence of follicular cell hyperplasia in the terminal sacrifice stop-dose vehicle control was $8 \%$ (Table 87).

The only statistically significant effects in females treated continuously with $\mathrm{EE}_{2}$ were an elevation (Poly-3 and RTE tests) of the incidence of follicular cell hyperplasia in the $0.05 \mu \mathrm{g}$ $\mathrm{EE}_{2} / \mathrm{kg}$ bw/day dose group relative to vehicle control (15\% versus $2 \%$ ) and an increasing trend for ultimobranchial cysts in terminal sacrifice animals (Table 86).

In stop-dose BPA interim sacrifice females, the $2.5 \mu \mathrm{g}$ BPA/kg bw/day stop-dose group had a higher incidence of C-cell hyperplasia than controls (RTE test only; $73 \%$ versus $50 \%$ ) (Table 87). In stop-dose BPA terminal sacrifice females, the incidence of ultimobranchial cysts 
was elevated in the 250 and $2,500 \mu \mathrm{g} \mathrm{BPA} / \mathrm{kg}$ bw/day stop-dose groups relative to vehicle controls (19\% and 22\%, respectively, versus 4\%) (Table 87).

\section{Males}

No statistically significant differences versus control were found regarding organ-specific neoplasms in males in any BPA treatment group. There was an increased trend ( $p$-values ranging from 0.002 to 0.009 ) for systemic lymphoma that presented in multiple organs (liver, dorsal/lateral prostate, bone marrow, spleen, and kidney) in terminal sacrifice animals of the stop-dose BPA arm (Supplemental Appendix XXXII and XXXIV). The incidence of lymphoma in the dorsal/lateral prostate was increased in the $25,000 \mu \mathrm{g} \mathrm{BPA} / \mathrm{kg}$ bw/day stop-dose group $(9 \%$ versus 0\%, Supplemental Appendix XXXIV). Selected neoplastic and non-neoplastic lesions are presented below.

\section{Epididymis, non-neoplastic lesions}

Non-neoplastic lesions in the epididymides of interim and terminal sacrifice males for continuous BPA and $\mathrm{EE}_{2}$ and stop-dose BPA are shown in Table 88, Table 89, and Table 90, respectively.

In the continuous-dose BPA interim sacrifice males, there were significant trends (all statistical tests applied) for exfoliated germ cells and lymphocyte infiltration (Table 88). With both lesions, the incidence in the $25,000 \mu \mathrm{g} \mathrm{BPA} / \mathrm{kg}$ bw/day dose group was significantly higher than that in the vehicle control group (all statistical tests applied; $27 \%$ versus $4 \%$ for exfoliated germ cells, $23 \%$ versus $0 \%$ for lymphocyte infiltration). There were no significant BPA treatment effects in the terminal sacrifice males in the continuous BPA dose groups.

In males dosed continuously with $\mathrm{EE}_{2}$, there were increased trends for lymphocyte infiltration in interim (JT/SW and RTE tests) and terminal (RTE test only) sacrifice animals and significant pairwise comparisons for the $0.5 \mu \mathrm{g} \mathrm{EE}_{2} / \mathrm{kg}$ bw/day dose group relative to vehicle control (12\% versus $0 \%$ for interim animals, $38 \%$ versus $20 \%$ for terminal animals) (Table 89 ).

The sole statistically significant effect in the stop BPA dose males was an increase in exfoliated germ cells in the $2.5 \mu \mathrm{g} \mathrm{BPA} / \mathrm{kg}$ bw/day dose group (RTE test only; 15\% versus 0\%) (Table 90).

\section{Dorsal/Lateral and ventral prostate, neoplastic and non-neoplastic lesions}

Non-neoplastic lesions in the dorsal/lateral prostates of interim and terminal sacrifice males for continuous BPA and $\mathrm{EE}_{2}$ and stop-dose BPA are shown in Table 91, Table 92, and Table 93, respectively.

There were statistically significant increased incidences relative to vehicle controls of lymphocyte infiltration and suppurative inflammation in continuous-dose BPA groups, primarily in the interim sacrifice males (Table 91). Lymphocyte infiltration was increased (RTE test only) in the $2.5 \mu \mathrm{g} \mathrm{BPA} / \mathrm{kg}$ bw/day dose group (46\% versus 18\%). The incidence of suppurative inflammation was increased (RTE and/or JT/SW tests) over a high background ( $82 \%$ in vehicle control) in the $2.5,250,2,500$, and $25,000 \mu \mathrm{g}$ BPA/kg bw/day dose groups $(91 \%, 92 \%, 90 \%$, and $86 \%$, respectively) in interim sacrifice continuous-dose males (Table 91). The incidence of suppurative inflammation was increased (Poly-3 test) in the $2.5 \mu \mathrm{g} \mathrm{BPA} / \mathrm{kg}$ bw/day dose group in terminal sacrifice animals (96\% versus $82 \%$; Table 91$)$. 
There were no statistically significant differences in lesion incidences in continuous-dose $\mathrm{EE}_{2}$ (Table 92) or stop-dose BPA (Table 93) groups relative to their respective vehicle control group.

The incidences of ventral prostate adenomas in terminal sacrifice males were discussed in the narrative pathology report (Supplemental Appendix XXXII) and are shown for continuous BPA and $\mathrm{EE}_{2}$ and stop-dose BPA in Table 94, Table 95, and Table 96, respectively. The continuousand stop-dose vehicle control incidences were $12 \%$ and $8 \%$, respectively, and there were no treatment-related differences in any exposure group relative to vehicle controls. Regarding nonneoplastic lesions in the ventral prostate, lower incidences of suppurative inflammation were seen in some treatment groups in continuous BPA (Table 97) or $\mathrm{EE}_{2}$ (Table 98), but not stopdose BPA (Table 99), relative to the vehicle control group.

\section{Pituitary, non-neoplastic lesions}

Non-neoplastic lesions in the pituitaries of interim and terminal sacrifice males for continuous BPA and $\mathrm{EE}_{2}$ and stop-dose BPA are shown in Table 100, Table 101, and Table 102, respectively.

There were no significant treatment effects in interim sacrifice animals in the continuous (Table 100) or stop-dose (Table 102) BPA arms or in the continuous $\mathrm{EE}_{2}$ treatments (Table 101).

In terminal sacrifice males treated continuously with BPA, there was a significant dose trend (all statistical tests applied) for hyperplasia in the pars distalis and a significant increase relative to vehicle controls in the $25 \mu \mathrm{g}$ BPA/kg bw/day dose group (RTE test only, $40 \%$ versus $23 \%$ ) and the $25,000 \mu \mathrm{g} \mathrm{BPA} / \mathrm{kg}$ bw/day dose group (all statistical tests applied, incidence $42 \%$ versus 23\%) (Table 100).

In males continuously dosed with $\mathrm{EE}_{2}$, there was an increased trend (all statistical tests applied) for pars distalis hyperplasia in terminal sacrifice animals and a significant pairwise comparison for the $0.5 \mu \mathrm{g} \mathrm{EE} / \mathrm{kg}$ bw/day dose group relative to vehicle control (50\% versus $23 \%$ ) (Table 101).

In stop-dose BPA terminal sacrifice males, the Poly-3 test indicated a significant dose trend for pars distalis hyperplasia and a significant pairwise comparison between the $25,000 \mu \mathrm{g} \mathrm{BPA} / \mathrm{kg}$ bw/day dose group and vehicle controls (incidences, 44\% versus 26\%) (Table 102).

\section{Thyroid, non-neoplastic lesions}

Non-neoplastic lesions in the thyroid glands of interim and terminal sacrifice males for continuous BPA and $\mathrm{EE}_{2}$ and stop-dose BPA are shown in Table 103, Table 104, and Table 105, respectively.

There were no statistically significant treatment effects in the thyroid gland of continuous-dose interim sacrifice males (Table 103). In terminal sacrifice males continuously dosed with BPA, there was a significant dose trend (Poly-3 test), and the 2,500 $\mu \mathrm{g}$ BPA/kg bw/day dose group had a higher hyperplasia incidence than vehicle controls (Poly-3 and RTE tests, 46\% versus 20\%). Follicular cell hyperplasia was increased at $25 \mu \mathrm{g} \mathrm{BPA} / \mathrm{kg}$ bw/day relative to the vehicle control group (19\% versus 6\%, RTE test only).

In males continuously dosed with $\mathrm{EE}_{2}$, the incidence of $\mathrm{C}$-cell hyperplasia was significantly higher in the $0.05 \mu \mathrm{g} \mathrm{EE}_{2} / \mathrm{kg}$ bw/day dose group relative to vehicle control (Poly-3 and RTE tests, $48 \%$ versus $20 \%$ ) (Table 104). 
There were no statistically significant effects in the thyroid of stop-dose BPA interim or terminal sacrifice males (Table 105).

\section{Parathyroid, non-neoplastic lesions}

Non-neoplastic lesions in the parathyroid glands of interim and terminal sacrifice males for continuous BPA and $\mathrm{EE}_{2}$ and stop-dose BPA are shown in Table 106, Table 107, and Table 108, respectively.

Statistically significant treatment effects were seen in the terminal sacrifice animals, but not in the interim sacrifice animals. In the males dosed continuously with BPA, the incidence of hyperplasia in the parathyroid gland was increased at $25 \mu \mathrm{g} \mathrm{BPA} / \mathrm{kg}$ bw/day (Poly-3 and RTE tests, $49 \%$ versus $22 \%$ ) and at $250 \mu \mathrm{g}$ BPA/kg bw/day (RTE test only, 36\% versus $22 \%$ ) (Table 106).

In males continuously dosed with $\mathrm{EE}_{2}$, there was a significant dose trend (JT/SW and RTE tests) in parathyroid gland hyperplasia, and the incidence in the $0.5 \mu \mathrm{g} \mathrm{EE} 2 / \mathrm{kg}$ bw/day dose group was significantly higher than in the vehicle control group (44\% versus $22 \%$ ) (Table 107).

In terminal sacrifice BPA stop-dose males, there was a significant dose trend (Poly-3 test) in the incidence of parathyroid gland hyperplasia (Table 108).

\section{Kidney, non-neoplastic lesions}

Non-neoplastic lesions in the kidneys of interim and terminal sacrifice males for continuous BPA and $\mathrm{EE}_{2}$ and stop-dose BPA are shown in Table 109, Table 110, and Table 111, respectively.

Hyperplasia of the transitional epithelium of the kidney was significantly increased in terminal sacrifice males relative to vehicle controls in the $25 \mu \mathrm{g} \mathrm{BPA} / \mathrm{kg}$ bw/day dose group of the continuous BPA dose arm (Poly-3 and RTE tests, 25\% versus 6\%) (Table 109).

In males continuously dosed with $\mathrm{EE}_{2}$, there were no observed statistically significant effects in the kidney (Table 110).

In the BPA stop-dose males in the terminal sacrifice arm, there was a significant dose trend for hyperplasia of the transitional epithelium of the kidney (Poly-3 test) (Table 111). The only dose group that had an apparent, although not statistically significant, increased incidence relative to controls was the $2,500 \mu \mathrm{g} \mathrm{BPA} / \mathrm{kg}$ bw/day dose group (40\% versus $24 \%$ ).

\section{Liver, non-neoplastic lesions}

Non-neoplastic lesions in the livers of interim and terminal sacrifice males for continuous BPA and $\mathrm{EE}_{2}$ and stop-dose BPA are shown in Table 112, Table 113, and Table 114, respectively.

In the livers of continuous BPA dose males, statistically significant pairwise comparisons to vehicle controls were noted for fatty change, hepatodiaphragmatic nodules, and mononuclear cell infiltration (Table 112). Two of 20 animals in the $25 \mu \mathrm{g}$ BPA $/ \mathrm{kg}$ bw/day interim sacrifice dose group were diagnosed with fatty change; these were the only animals in the interim sacrifice continuous BPA dose group diagnosed with this change, which was statistically significant by the RTE test only. The diagnosis occurred in 4-17\% of the terminal sacrifice continuous BPA dose males (vehicle control incidence, $8 \%$ ), but there was no significant treatment effect. 
Stop-dose BPA interim sacrifice males had variable incidences (4-10\%) of fatty liver diagnosed in some dose groups, but none were significant and there were similar findings in the terminal sacrifice stop-dose males (Table 114).

Fatty liver was also diagnosed in the interim sacrifice males treated continuously with $\mathrm{EE}_{2}$, and there was a significant dose trend (all tests) and a significantly higher incidence in the $0.5 \mu \mathrm{g}$ $\mathrm{EE}_{2} / \mathrm{kg}$ bw/day dose group relative to vehicle control (15\% versus $0 \%, \mathrm{JT} / \mathrm{SW}$ and RTE tests only) (Table 113).

Hepatodiaphragmatic nodule, growth of the median lobe into the diaphragm, is a congenital background lesion. As with fatty liver, there were variable incidences of this lesion diagnosed across treatment groups in both interim and terminal sacrifice males for continuous- and stopdose BPA and continuous-dose $\mathrm{EE}_{2}$. In one case, there was a significantly higher incidence (CAFE test) in the interim sacrifice 2,500 $\mu \mathrm{g} \mathrm{BPA} / \mathrm{kg}$ bw/day continuous-dose group relative to control (21\% versus $0 \%)$ (Table 112).

Mononuclear cell infiltration was diagnosed in all dose groups in both interim and terminal sacrifice groups for continuous- and stop-dose BPA and continuous-dose $\mathrm{EE}_{2}$. Significant increases over control were noted in multiple continuous BPA dose treatment groups $(2.5,250$, 2,500 , and 25,000 $\mu \mathrm{g} \mathrm{BPA} / \mathrm{kg}$ bw/day) relative to vehicle control in interim sacrifice males (Table 112). There were no statistically significant treatment effects in terminal sacrifice BPAtreated males. Mononuclear cell infiltration was increased relative to the vehicle controls in the $0.05 \mu \mathrm{g} \mathrm{EE}_{2} / \mathrm{kg}$ bw/day terminal sacrifice males (85\% versus 70\%, Table 113). 


\section{Discussion}

BPA is a high-production-volume chemical to which there is ubiquitous sub-microgram $/ \mathrm{kg}$ bw/day human exposure, primarily through the diet. A large body of data has been published on the effects of BPA in in vitro and in vivo systems, including epidemiological studies investigating the association of early life exposures to BPA with a variety of diseases ${ }^{7 ; 56-59}$. After considering these data, the levels of exposure, and the extent of metabolic inactivation of BPA upon ingestion, most international regulatory agencies have concluded that current nonoccupational BPA exposures do not pose a credible risk to humans. However, this conclusion is not without controversy. Given the large body of data available on BPA and the level of human exposure that is prompting the debate, the present study was designed not as a high dose hazard identification study, but rather to examine a broad dose range from reasonably close to human dietary exposure levels to levels $>25,000$-fold higher than current estimated aggregate nonoccupational exposure. The dose spacing of 10-fold used over the broad dose range in the study is typically the maximum dose range covered in a bioassay.

Yoshida et al. ${ }^{60}$ originally raised the issue of the presence of BPA in commercial rodent diets when they reported approximately $40 \mathrm{ppb}$ in the diet as measured by an HPLC-fluorescence method. The background diet analyses that were conducted for this study and for previous studies using a more specific HPLC-MS/MS method ${ }^{19 ; 36}$ confirmed that commercial rodent diets contain trace levels (low ppb) of BPA. The ingested levels resulting from this dietary background did not lead to measurable levels of BPA or its metabolites in blood or tissues, but the presence of this background places a lower limit on the dose of BPA that can be tested. For the present study, a rejection level of $5 \mathrm{ppb}$ BPA in the diet was established. This level of dietary BPA would have resulted in ingestion of approximately $0.25 \mu \mathrm{g} / \mathrm{kg}$ bw/day, a 10 -fold lower exposure, on a $\mu \mathrm{g} / \mathrm{kg}$ bw/day basis, over the course of the study than the exposure in the lowest chosen BPA dose group of $2.5 \mu \mathrm{g} / \mathrm{kg}$ bw/day. None of the lots of diet used in the study had a background level of BPA $>3 \mathrm{ppb}$.

Survival of the interim sacrifice animals was $82-100 \%$, and there were no effects of BPA on survival of the interim or terminal sacrifice animals. However, $\geq 60 \%$ of the terminal sacrifice animals did not survive to the scheduled sacrifice. Most were removed as moribund for animal welfare considerations and provided a full set of data for evaluation. The Poly-3 test applied for the analysis of the two-year pathology data is adjusted for mortality.

Female body weights in the continuous $250 \mu \mathrm{g}$ BPA/kg bw/day dose group were significantly higher than the mean vehicle control body weights in the final weeks of the study (weeks 96104). There were no other statistically significant body weight differences in pairwise comparisons of BPA dose groups to the vehicle controls. There were few statistically significant effects of BPA treatment, in either the continuous- or stop-dose arms, on clinical pathology endpoints or organ weights, and these effects could not be clearly defined as treatment-related as they were observed only in single-dose groups, in several cases differed from the vehicle control by less than $10 \%$, and, in the case of organ weights, were not significant when adjusted for body weight.

The approach used by the NTP to determine if neoplasms are likely related to treatment has always focused on a weight-of-evidence approach, encompassing a range of factors, including statistical analysis, consideration of consistency of responses within the study, and historical 
controls $^{61 ; 62}$. This approach was followed in the present study to evaluate the histopathology data for the neoplastic and non-neoplastic lesions. There were relatively few neoplastic lesions that showed potential treatment effects. There were many non-neoplastic lesions in both males and females that were variable across control and BPA treatment levels. One issue that contributed to the challenge of clearly establishing effects as treatment related was that most diagnosed lesions, whether neoplastic or non-neoplastic, were not rare lesions, but rather common lesions associated with aging. To assess these lesions for any potential treatment effects, the CAFE test was applied for lesion incidence in the one-year study, and the survival-adjusted Poly-3 test was used for lesion incidence in the two-year study. The pairwise comparisons conducted as part of these analyses do not assume a monotonic dose response, but do not incorporate the severity scores assigned to many of the non-neoplastic lesions during the microscopic evaluation. The JT/SW test, which assumes monotonicity across dose levels, was applied to incorporate severity scores along with the incidence data. Because non-monotonic dose responses have been central to the discussion of the effects of BPA and other potential hormonally active agents, another test was applied that incorporates both incidence and severity scores and does not assume monotonicity ${ }^{45}$. This test (RTE) has not been widely used in toxicology studies, although it was used in the previous BPA subchronic study conducted at NCTR ${ }^{19}$. Neither the JT/SW nor the RTE tests adjust for survival. Although the use of multiple statistical tests coupled with the multitude of endpoints examined and lack of correction for multiple comparisons can lead to erroneous inferences in the interpretation of histopathology data based on $p$-values alone, the results of all statistical tests are presented in the Results section and Supplemental Appendices XXXIII and XXXIV, primarily to guide the selection of histopathological lesions that required evaluation.

At the terminal sacrifice of females in the stop-dose BPA study arm, there was a statistically significant increase in the incidence of mammary gland adenocarcinoma (22\% versus $6 \%$, $p=0.016)$ and the combination of adenoma/adenocarcinoma ( $24 \%$ versus $8 \%, p=0.018)$ in the $2.5 \mu \mathrm{g} \mathrm{BPA} / \mathrm{kg}$ bw/day dose group. This incidence is higher than the limited data available for historical controls of this rat strain at NCTR utilizing the same diet, ${ }^{14 ; 15}$ which indicate a background rate of 11-16\% for mammary gland adenocarcinoma in two-year-old control females. In the continuous-dose BPA study arm, the incidence of female mammary gland adenocarcinoma and the combination of adenoma/adenocarcinoma was $8 \%$ and $12 \%$, respectively, in the control group. The incidence in each of the BPA continuous-dose groups varied between 6 and 18\% for adenocarcinoma and between 9 and 20\% for combined adenoma/adenocarcinoma, none of which was significant.

There were no treatment-related non-neoplastic changes in the mammary gland of interim or terminal sacrifice female stop-dose animals. In the interim and terminal continuous-dose arm, there were some differences in incidence and severity between BPA groups and the vehicle control group indicated by the secondary RTE statistical test. There was an increase in atypical foci in the mammary gland at $2.5 \mu \mathrm{g} \mathrm{BPA} / \mathrm{kg}$ bw/day (14\% versus $0 \%$ and $15 \%$ versus $4 \%$ for the interim and terminal dose group animals, respectively). There was also an increase in ductal dilatation at the interim sacrifice in animals continuously administered $25 \mu \mathrm{g} \mathrm{BPA} / \mathrm{kg}$ bw/day (32\% versus $9 \%$ ).

Evaluation of the totality of the evidence regarding the elevated incidence of mammary adenocarcinomas or combined adenomas and adenocarcinomas in the stop-dose females exposed to $2.5 \mu \mathrm{g} \mathrm{BPA} / \mathrm{kg}$ bw/day makes it unlikely that this is a plausible BPA treatment-related lesion. 
The elevated incidence occurs in a single dose group of the stop-dose arm and was not observed in the continuous-dose arm. In addition, there were no treatment-related non-neoplastic lesions in the mammary gland of interim or terminal sacrifice stop-dose animals. Comparison of the incidences of mammary gland adenoma/adenocarcinoma in control and treatment groups to the limited historical control data for this strain of rats further questions the biological plausibility of this lesion as a treatment-related effect. In the continuous-dose, but not stop-dose, arm animals, there was an elevation of atypical foci in the $2.5 \mu \mathrm{g}$ BPA/kg bw/day group in interim and terminal sacrifice animals, but no increase in adenomas or adenocarcinomas. It is difficult to envision a mechanism whereby the stop-dose animals would develop mammary adenocarcinomas, while continuous-dose animals would not. Yoshizawa et al. ${ }^{63}$ reported a gavage study of 2, 3', 4, 4', 5-pentachlorobiphenyl (PCB 118) in rats where the highest dose group was either exposed from 8 weeks of age through two years (continuous exposure group), or from 8 weeks of age through 32 weeks and then dosed with vehicle on the same schedule as the continuously exposed animals through two years (stop exposure group). Uterine carcinoma was elevated only in the stop exposure group. A plausible hypothesis proposed by the authors was that the body weight depression of greater than $10 \%$ in the continuously exposed group may have suppressed development of the uterine carcinomas, and cited that mammary fibroadenomas, a spontaneous neoplasm known to be suppressed by decreased body weight gain, was also lower in the continuously dosed animals. In the present study, although there were no statistical comparisons conducted across dosing arms, there was an apparently higher body weight in the stop-dose females (average 7\% and 5\% higher than continuous-dose females for vehicle control and $2.5 \mu \mathrm{g} \mathrm{BPA} / \mathrm{kg}$ bw/day groups, respectively). The development of spontaneous mammary gland fibroadenomas, however, was not suppressed in the BPA continuous-dose females nor was the development of mammary gland adenocarcinomas in high dose $\mathrm{EE}_{2}$ animals (discussed below).

In contrast to BPA treatments, the reference estrogen $\mathrm{EE}_{2}$ had a clearly interpretable impact on the female mammary gland. As indicated earlier, although targets for BPA other than classical estrogen receptors have been proposed ${ }^{30-33}$, many of the reported effects of BPA involve estrogen signaling pathways. Thus, sensitivity of the animal model to exogenous estrogen was considered important, and $\mathrm{EE}_{2}$ was included for this purpose rather than necessarily to compare effects of the two agents. The high dose $(0.5 \mu \mathrm{g} \mathrm{EE} / \mathrm{kg}$ bw/day) induced an increased incidence of adenocarcinoma ( $38 \%$ versus $8 \%$ in controls) and dilatation of ducts and alveoli in the mammary glands of terminal animals. In the mammary glands of interim females, the incidences of lobular hyperplasia and dilatation of ducts were increased in the high $\mathrm{EE}_{2}$ dose group. In addition, increased incidence of combined adenomas and carcinomas in the pituitary pars distalis at $0.5 \mu \mathrm{g}$ $\mathrm{EE}_{2} / \mathrm{kg}$ bw/day may be related to increased mammary neoplasm incidence. Exogenous estrogen exposure in the rat can lead to decreased activity of dopaminergic neurons in the hypothalamus leading to prolactinomas in the pituitary that contribute to mammary adenocarcinoma development ${ }^{64 ; 65}$. The production of prolactin from the pituitary neoplasms was not investigated in this study, although serum prolactin was reported to be elevated in adult rats at $0.5 \mu \mathrm{g} \mathrm{EE} / \mathrm{kg}$ bw/day in the previous NCTR subchronic BPA study ${ }^{19}$.

The only other statistically significant neoplastic lesion in BPA-treated females was a significant trend $(p=0.037)$ for uterine stromal polyps in the interim continuous-dose arm animals. This trend was driven by incidences of $3 / 20(15 \%)$ and $3 / 24(12 \%)$ in the 2,500 and $25,000 \mu \mathrm{g} \mathrm{BPA} / \mathrm{kg}$ bw/day dose groups, respectively, which were not statistically different in pairwise comparisons with the control incidence of $1 / 23$ (4\%). No trend toward higher 
incidences in the higher BPA dose groups was evident in the terminal sacrifice continuous-dose animals, nor were there any significant pairwise comparisons between any BPA dose group and controls in the terminal sacrifice animals. Likewise, stromal polyps were not induced in the stopdose BPA or in the interim or terminal sacrifice $\mathrm{EE}_{2}$ animals. The relatively small increased incidences leading to the significant trend in the interim sacrifice animals together with the lack of any effects in the terminal sacrifice animals indicate that uterine stromal polyps are not likely to be a consequence of BPA treatment.

In the uterus, cystic endometrial hyperplasia was elevated in interim and terminal sacrifice animals in the stop-dose study arm at 25,000 $\mu \mathrm{g} \mathrm{BPA} / \mathrm{kg}$ bw/day (interim) and at 2,500 and $25,000 \mu \mathrm{g} \mathrm{BPA} / \mathrm{kg}$ bw/day (terminal). The incidence in the terminal stop-dose vehicle control was low compared to all other terminal sacrifice groups. An increase in this lesion was not observed in the continuous-dose BPA animals, but did occur at the interim sacrifice with the high dose $\mathrm{EE}_{2}$ animals. Uterine squamous metaplasia was also increased in interim stop-dose animals at $25,000 \mu \mathrm{g} \mathrm{BPA} / \mathrm{kg}$ bw/day. None of these uterine BPA effects were significant using the CAFE or Poly- 3 tests that were considered the primary statistical tests in the study and, in the case of the cystic endometrial hyperplasia, severity was not increased in the treated groups relative to the vehicle control. An increase in squamous metaplasia of the uterus also occurred at the interim sacrifice with the high dose $\mathrm{EE}_{2}$ animals. In interim continuous-dose females, there were significant trends for increased apoptosis in luminal epithelial cells of the endometrium and squamous metaplasia, with a statistically significant elevation of the apoptosis at $25,000 \mu \mathrm{g} \mathrm{BPA} / \mathrm{kg}$ bw/day. Apoptosis of the endometrial luminal epithelial cells also was significantly increased by the high $\mathrm{EE}_{2}$ dose in interim sacrifice animals. Taken together, these data, particularly at the interim sacrifice, suggest that the $25,000 \mu \mathrm{g} \mathrm{BPA} / \mathrm{kg}$ bw/day dose may exert weak estrogen-like effects on the uterus.

In the ovary, there were trends in interim sacrifice animals for depletion of corpora lutea and interstitial cell hypertrophy in the continuous-dose arm and a trend and increase in follicular cysts at 25,000 $\mu \mathrm{g} \mathrm{BPA} / \mathrm{kg}$ bw/day in stop-dose BPA groups. The magnitude of the increase in follicular cysts at the high BPA dose and the trend evident in the two highest BPA groups suggests that this is a treatment-related effect, although the lack of effect on this endpoint in the continuous-dose animals cannot be readily explained. In the ovaries of interim sacrifice high dose $\mathrm{EE}_{2}$ females, there was a $100 \%$ incidence of cystic follicles.

There was an increasing trend for vaginal epithelial hyperplasia in interim sacrifice continuous BPA dose females with an increased incidence at 25,000 $\mu \mathrm{g} \mathrm{BPA} / \mathrm{kg}$ bw/day, although not in the primary CAFE test pairwise comparison. Vaginal epithelial hyperplasia was increased to nearly the same magnitude at all doses from $25-25,000 \mu \mathrm{g} \mathrm{BPA} / \mathrm{kg}$ bw/day in terminal continuous-dose BPA animals. There were no significant BPA effects on vaginal epithelial hyperplasia in stopdose animals. A more pronounced increased incidence of vaginal epithelial hyperplasia was also indicated by primary and secondary statistical tests with the interim sacrifice $0.5 \mu \mathrm{g} \mathrm{EE} / \mathrm{kg}$ bw/day dose group. This was accompanied by an increased incidence of epithelial mucification in the $\mathrm{EE}_{2}$ animals. When considered together with the observations mentioned above on the uterus in continuous-dose animals, the increased vaginal epithelial hyperplasia observed at 25,000 $\mu \mathrm{g} \mathrm{BPA} / \mathrm{kg}$ bw/day may be a plausible estrogenic effect. The sporadic and inconsistent responses observed at lower BPA doses in the female reproductive tract suggest that they are not of toxicological relevance. 
BPA treatment did not have adverse effects on the estrous cycle. In the high dose $\mathrm{EE}_{2}$ females, the estrous cycle was disrupted by the time of evaluation at approximately 16-weeks of age and the one-year sacrifice animals showed multiple organ weight changes and histological changes expected of estrogenic stimulation in the ovary, uterus, and vagina. We did not observe an effect of $\mathrm{EE}_{2}$ on the time of vaginal opening in the present study. In the previous BPA subchronic study conducted at $\mathrm{NCTR}^{19}$, a delay in the time of vaginal opening was observed with $0.5 \mu \mathrm{g} \mathrm{EE} / / \mathrm{kg}$ bw/day, with a more pronounced effect at $5 \mu \mathrm{g} \mathrm{EE} / \mathrm{kg}$ bw/day. Variable effects on this endpoint depending on timing and dose have been reported in the literature ${ }^{66 ; 67}$. Disruption of the estrous cycle, as observed in the present study, may be a more sensitive response to early estrogen treatment, as has been reported with a different exposure regimen. Shirota et al. ${ }^{68}$ administered five consecutive daily subcutaneous doses of $\mathrm{EE}_{2}(0.4$ and $2.0 \mu \mathrm{g} / \mathrm{kg}$ bw/day $)$ to female Sprague-Dawley rat pups starting on PND 1 and found no effect on vaginal opening at either dose level, but a disruption of the estrous cycle at later times.

By the secondary statistical tests that include severity scores and incidence, but do not adjust for survival, cardiomyopathy was increased in the stop-dose BPA terminal sacrifice females at 2.5, $250,2,500$, and $25,000 \mu \mathrm{gPA} / \mathrm{kg}$ bw/day, although background incidence was high at this age. Mean severity scores in the higher treated groups varied from 1.5-1.7, while the vehicle control severity score was 1.3. The percentage of lesions evaluated as mild rather than minimal in these BPA stop-dose groups relative to the controls appears to account for the modest difference in scores, and the percentage of mild diagnoses in the terminal stop-dose controls is lower than the continuous-dose controls or any other terminal sacrifice group. Cardiomyopathy was also increased in the high dose $\mathrm{EE}_{2}$ females at both the interim and terminal sacrifices, with all statistical tests indicating a significant increase in the interim sacrifice animals (65\% versus $30 \%$, with mean severity of 1.2 versus 1.1 ). The high background incidence of this lesion at terminal sacrifice and variability in incidence and severity scores across groups make the toxicological significance of these results questionable, with the possible exception of the acceleration of development of the lesion by high dose $\mathrm{EE}_{2}$.

For males, there were no significant differences in the incidences of neoplasms in treatment groups versus vehicle controls in any organ in stop-dose or continuous-dose interim or terminal sacrifice males. There were also no treatment-related non-neoplastic effects in stop-dose interim or terminal sacrifice males. An increase in exfoliated germ cells and lymphocyte cellular infiltration in the epididymis at one year in the continuous-dose study arm and hyperplasia in the pars distalis of the pituitary at two years in both continuous- and stop-dose study arms at $25,000 \mu \mathrm{g}$ BPA/kg bw/day were notable effects in males. Hyperplasia of the pituitary pars distalis was also observed in $0.5 \mu \mathrm{g} \mathrm{EE} 2 / \mathrm{kg}$ bw/day terminal sacrifice males. For the epididymal lesions, there were no potentially related lesions noted in the testes to increase confidence in this observation as an effect of toxicological significance.

Other non-neoplastic lesions in males showed variable increases in some dose groups without a pattern in dose response. In the thyroid gland of continuous-dose BPA males at terminal sacrifice, an increase in C-cell hyperplasia was noted at 2,500 $\mu \mathrm{g} \mathrm{BPA} / \mathrm{kg}$ bw/day, with high variability across dose groups for this endpoint. In addition, TSH levels were not elevated in any BPA dose group. Multiple step sections of the prostate lobes were examined in this study, since prostate has been a target organ identified in the BPA literature. Increases in dorsal/lateral prostate inflammation were variable across a high background in both interim and terminal sacrifice animals. Inflammation in the aging rat prostate is common and has been associated with 
increasing prolactin levels ${ }^{44}$ and there were no differences between treated groups and vehicle control for hyperplasia or other non-neoplastic lesions observed in the prostate lobes.

As expected from the previous NCTR BPA subchronic study conducted under identical conditions to those reported here ${ }^{19}$, males were less responsive to $\mathrm{EE}_{2}$ than the females. This is consistent with other rat studies (e.g., Howdeshell et al., 2008; Ryan et al., 2010, and references therein $)^{69 ; 70}$. The only significant effect observed in males in the NCTR BPA subchronic study at $0.5 \mu \mathrm{g} \mathrm{EE} / 2 \mathrm{~kg}$ bw/day was an increase in mammary gland hyperplasia at 90 days of age ${ }^{19}$. Our previous work with $\mathrm{EE}_{2}$ administered in the diet indicated increased mammary gland hyperplasia in young male animals (140 days of age) that ingested a dose approximating the low dose in this study (estimated between 0.5 and $1.0 \mu \mathrm{g} / \mathrm{kg}$ bw/day). An attenuated response was observed in two-year-old animals ${ }^{20}$. Hence, the lack of an increase of male mammary hyperplasia by $\mathrm{EE}_{2}$ in the current study is likely due to the advanced age of animals at examination. In addition, measurements of serum $\mathrm{EE}_{2}$ levels at approximate maximum concentration $\left(\mathrm{C}_{\max }\right)$ were made at various ages in animals from the NCTR BPA subchronic study ${ }^{25}$. Like BPA, serum levels of EE $\mathrm{E}_{2}$ at a given dose level declined with age; that is, peak serum levels after an oral dose of $0.5 \mu \mathrm{g}$ $\mathrm{EE}_{2} / \mathrm{kg}$ bw were $\sim 500 \mathrm{pM}, \sim 10 \mathrm{pM}$, and below the LOD of $5 \mathrm{pM}$ on PND 4, 21, and 80, respectively ${ }^{25}$. Thus, in the present study, although the $\mathrm{EE}_{2}$ dosing was continuous throughout the study, the highest internal exposures occurred in preweaning animals. In the case of the low dose, $0.05 \mu \mathrm{g} \mathrm{EE} / \mathrm{kg}$ bw/day, serum levels would be expected to be less than $1 \mathrm{pM}$ by the time of weaning.

After the present study began, analysis of samples from a separate study conducted under identical conditions (NCTR BPA subchronic study) ${ }^{19}$ indicated that vehicle controls housed in the same room as animals dosed with $\geq 100,000 \mu \mathrm{g}$ BPA/kg bw/day had blood levels of BPAglucuronide consistent with the lowest exposure dose in the study, which was $2.5 \mu \mathrm{g} / \mathrm{kg}$ bw/day ${ }^{25}$. Thus, evaluation of treatment effects observed in the subchronic study needed to consider the background exposure to BPA above the dietary exposure of $\leq 0.25 \mu \mathrm{g} / \mathrm{kg}$ bw/day. Since clear adverse effects occurred only at BPA doses $\geq 100,000 \mu \mathrm{g} / \mathrm{kg}$ bw/day and there were robust effects of the reference estrogen $\mathrm{EE}_{2}$ at doses of 0.5 and $5 \mu \mathrm{g} / \mathrm{kg} \mathrm{bw} /$ day, the background BPA exposure was considered to have no impact on the study ${ }^{19 ; 71}$. It should also be noted that given the presence of variable levels of BPA in commercial diets as discussed earlier, all BPA studies should account for the margin of exposure between the BPA dose levels showing effects and the background level of BPA exposure from the diet.

In the present study, a subset of the animals was housed for a short period early in the study in the same room as animals dosed with $250,000 \mu \mathrm{g}$ BPA/kg bw/day for a CLARITY-BPA academic grantee study. As discussed in the Statistical Methods section, a conservative approach was taken in this study that assumed all animals that were housed for any period of their lives in the same rooms as the animals dosed with $250,000 \mu \mathrm{g}$ BPA/kg bw/day were potentially exposed to low levels of BPA above the dietary exposure. An additional statistical analysis excluding these animals, referred to throughout the text as a sensitivity analysis, was conducted for each endpoint. It was reasoned that if inadvertent exposure of vehicle controls to environmental BPA approximately 10-fold higher (i.e., $2.5 \mu \mathrm{g}$ BPA/kg bw/day) than had been anticipated was masking robust effects of BPA at the lower end of the dose range, this would be detected with the sensitivity analysis. The results of the sensitivity analyses did not show any consistency with respect to tissues or doses in revealing treatment effects not evident in the inclusive analysis and 
indicated that any inadvertent BPA exposure early in the study had minimal impact on the conclusions derived from the statistical tests.

In conclusion, in the CLARITY-BPA core study, differences between BPA treatment groups, particularly below $25,000 \mu \mathrm{g}$ BPA/kg bw/day, and the vehicle control group detected by the lowstringency statistical tests applied to histopathology lesions, were not dose responsive, sometimes occurring in only one low or intermediate dose group, and did not demonstrate a clear pattern of consistent responses within or across organs within the stop- and continuous-dose arms and the interim and terminal sacrifices. In contrast, the high $\mathrm{EE}_{2}$ dose elicited several strong effects in females in a clearly interpretable and biologically plausible as estrogenic effects. At $25,000 \mu \mathrm{g} \mathrm{BPA} / \mathrm{kg}$ bw/day, several observations may be treatment related, including the effects mentioned above in the female reproductive tract (ovary, uterus, and vagina) and in the male pituitary. 


\section{References}

1. U.S. FDA (U.S. Food and Drug Administration). 2014. Bisphenol A Joint Emerging Science Working Group: Final report for the review of literature and data on BPA (draft). http://www.fda.gov/downloads/Food/IngredientsPackagingLabeling/FoodAdditivesIngredients/U CM424011.pdf.

2. EFSA (European Food Safety Authority). Scientific Opinion on the risks to public health related to the presence of bisphenol A (BPA) in foodstuffs: PART II - Toxicological assessment and risk characterization. EFSA Journal. 2015; 13(1):3978.

http://dx.doi.org/10.2903/j.efsa.2015.3978

3. Health Canada. 2012. Health Canada's updated assessment of bisphenol A (BPA) exposure from food sources. http://www.hc-sc.gc.ca/fn-an/alt_formats/pdf/securit/packagemball/bpa/bpa_hra-ers-2012-09-eng.pdf.

4. U.S. FDA (U.S. Food and Drug Administration). 2014. Updated safety assessment of bisphenol A (BPA) for use in food contact applications. . https://www.fda.gov/downloads/NewsEvents/PublicHealthFocus/UCM424266.pdf.

5. Zoeller RT, Brown TR, Doan LL, Gore AC, Skakkebaek NE, Soto AM, Woodruff TJ, Vom Saal FS. Endocrine-disrupting chemicals and public health protection: a statement of principles from The Endocrine Society. Endocrinology. 2012; 153(9):4097-4110.

http://dx.doi.org/10.1210/en.2012-1422

6. Gore AC, Chappell VA, Fenton SE, Flaws JA, Nadal A, Prins GS, Toppari J, Zoeller RT. EDC-2: The Endocrine Society's second scientific statement on endocrine-disrupting chemicals. Endocr Rev. 2015; 36(6):E1-E150. http://dx.doi.org/10.1210/er.2015-1010

7. Rochester JR. Bisphenol A and human health: a review of the literature. Reprod Toxicol. 2013; 42:132-155. http://dx.doi.org/10.1016/j.reprotox.2013.08.008

8. ANSES (Administración Nacional de la Seguridad Social). 2013. Opinion of the French Agency for Food, Environmental and Occupational Health \& Safety on the assessment of the risks associated with bisphenol $\mathrm{A}$ for human health, and on toxicological data and data on the use of bisphenols S, F, M, B, AP, AF and BADGE. https://www.anses.fr/en/system/files/CHIM2009sa0331Ra-0EN.PDF.

9. Proposition 65: Bisphenol A listed as known to the state of California to cause reproductive toxicity. http://oehha.ca.gov/prop65/CRNR_notices/list_changes/051115listBPA.html; 2015.

10. NTP (National Toxicology Program). 1982. Carcinogenesis bioassay of bisphenol A (CAS No. 80-05-7) in F344 rats and B6C3F1 mice (feed study).

http://www.ncbi.nlm.nih.gov/pubmed/12778220.

11. Schug TT, Heindel JJ, Camacho L, Delclos KB, Howard P, Johnson AF, Aungst J, Keefe D, Newbold R, Walker NJ et al. A new approach to synergize academic and guideline-compliant research: the CLARITY-BPA research program. Reprod Toxicol. 2013; 40:35-40. http://dx.doi.org/10.1016/j.reprotox.2013.05.010 
12. Heindel JJ, Newbold RR, Bucher JR, Camacho L, Delclos KB, Lewis SM, Vanlandingham M, Churchwell MI, Twaddle NC, McLellen M et al. NIEHS/FDA CLARITY-BPA research program update. Reprod Toxicol. 2015; 58:33-44.

http://dx.doi.org/10.1016/j.reprotox.2015.07.075

13. NTP (National Toxicology Program). 2008. Multigenerational reproductive study of genistein (Cas No. 446-72-0) in Sprague-Dawley rats (feed study).

http://www.ncbi.nlm.nih.gov/pubmed/18685713.

14. NTP (National Toxicology Program). 2008. Toxicology and carcinogenesis studies of genistein (Cas No. 446-72-0) in Sprague-Dawley rats (feed study).

http://www.ncbi.nlm.nih.gov/pubmed/18685716.

15. NTP (National Toxicology Program). 2010. Toxicology and carcinogenesis study of ethinyl estradiol (CAS No. 57-63-6) in Sprague-Dawley rats (feed study).

http://www.ncbi.nlm.nih.gov/pubmed/21031006.

16. NTP (National Toxicology Program). 2010. Multigenerational reproductive toxicology study of ethinyl estradiol (CAS No. 57-63-6) in Sprague-Dawley rats.

http://www.ncbi.nlm.nih.gov/pubmed/21031005.

17. Delclos KB, Bucci TJ, Lomax LG, Latendresse JR, Warbritton A, Weis CC, Newbold RR. Effects of dietary genistein exposure during development on male and female CD (SpragueDawley) rats. Reprod Toxicol. 2001; 15(6):647-663. http://dx.doi.org/10.1016/S0890$\underline{6238(01) 00177-0}$

18. Delclos KB, Weis CC, Bucci TJ, Olson G, Mellick P, Sadovova N, Latendresse JR, Thorn B, Newbold RR. Overlapping but distinct effects of genistein and ethinyl estradiol (EE(2)) in female Sprague-Dawley rats in multigenerational reproductive and chronic toxicity studies. Reprod Toxicol. 2009; 27(2):117-132. http://dx.doi.org/10.1016/j.reprotox.2008.12.005

19. Delclos KB, Camacho L, Lewis SM, Vanlandingham MM, Latendresse JR, Olson GR, Davis KJ, Patton RE, Gamboa da Costa G, Woodling KA et al. Toxicity evaluation of bisphenol A administered by gavage to Sprague Dawley rats from gestation day 6 through postnatal day 90. Toxicol Sci. 2014; 139(1):174-197. http://dx.doi.org/10.1093/toxsci/kfu022

20. Latendresse JR, Bucci TJ, Olson G, Mellick P, Weis CC, Thorn B, Newbold RR, Delclos KB. Genistein and ethinyl estradiol dietary exposure in multigenerational and chronic studies induce similar proliferative lesions in mammary gland of male Sprague-Dawley rats. Reprod Toxicol. 2009; 28(3):342-353. http://dx.doi.org/10.1016/j.reprotox.2009.04.006

21. Doerge DR, Twaddle NC, Vanlandingham M, Fisher JW. Pharmacokinetics of bisphenol A in neonatal and adult Sprague-Dawley rats. Toxicol Appl Pharmacol. 2010; 247(2):158-165. http://dx.doi.org/10.1016/j.taap.2010.06.008

22. Doerge DR, Twaddle NC, Vanlandingham M, Brown RP, Fisher JW. Distribution of bisphenol A into tissues of adult, neonatal, and fetal Sprague-Dawley rats. Toxicol Appl Pharmacol. 2011; 255(3):261-270. http://dx.doi.org/10.1016/j.taap.2011.07.009 
23. Twaddle NC, Churchwell MI, Vanlandingham M, Doerge DR. Quantification of deuterated bisphenol A in serum, tissues, and excreta from adult Sprague-Dawley rats using liquid chromatography with tandem mass spectrometry. Rapid Commun Mass Spectrom. 2010; 24(20):3011-3020. http://dx.doi.org/10.1002/rcm.4733

24. Twaddle NC, Churchwell MI, Newbold RR, Delclos KB, Doerge DR. Determination using liquid-chromatography-electrospray tandem mass spectroscopy of ethinylestradiol serum pharmacokinetics in adult Sprague-Dawley rats. J Chromatogr B Analyt Technol Biomed Life Sci. 2003; 793(2):309-315. http://dx.doi.org/10.1016/S1570-0232(03)00331-3

25. Churchwell MI, Camacho L, Vanlandingham MM, Twaddle NC, Sepehr E, Delclos KB, Fisher JW, Doerge DR. Comparison of life-stage-dependent internal dosimetry for bisphenol A, ethinyl estradiol, a reference estrogen, and endogenous estradiol to test an estrogenic mode of action in Sprague Dawley rats. Toxicol Sci. 2014; 139(1):4-20.

http://dx.doi.org/10.1093/toxsci/kfu021

26. Tyl RW, Myers CB, Marr MC, Sloan CS, Castillo NP, Veselica MM, Seely JC, Dimond SS, Van Miller JP, Shiotsuka RN et al. Two-generation reproductive toxicity study of dietary bisphenol A in CD-1 (Swiss) mice. Toxicol Sci. 2008; 104(2):362-384.

http://dx.doi.org/10.1093/toxsci/kfn084

27. Tyl RW, Myers CB, Marr MC, Thomas BF, Keimowitz AR, Brine DR, Veselica MM, Fail PA, Chang TY, Seely JC et al. Three-generation reproductive toxicity study of dietary bisphenol A in CD Sprague-Dawley rats. Toxicol Sci. 2002; 68(1):121-146.

http://dx.doi.org/10.1093/toxsci/68.1.121

28. Doerge DR, Vanlandingham M, Twaddle NC, Delclos KB. Lactational transfer of bisphenol A in Sprague-Dawley rats. Toxicol Lett. 2010; 199(3):372-376.

http://dx.doi.org/10.1016/j.toxlet.2010.09.022

29. Yang X, Doerge DR, Teeguarden JG, Fisher JW. Development of a physiologically based pharmacokinetic model for assessment of human exposure to bisphenol A. Toxicol Appl Pharmacol. 2015; 289(3):442-456. http://dx.doi.org/10.1016/j.taap.2015.10.016

30. Wetherill YB, Akingbemi BT, Kanno J, McLachlan JA, Nadal A, Sonnenschein C, Watson CS, Zoeller RT, Belcher SM. In vitro molecular mechanisms of bisphenol A action. Reprod Toxicol. 2007; 24(2):178-198. http://dx.doi.org/10.1016/j.reprotox.2007.05.010

31. Acconcia F, Pallottini V, Marino M. Molecular mechanisms of action of BPA. Dose Response. 2015; 13(4):1-9. http://dx.doi.org/10.1177/1559325815610582

32. Nadal A, Fuentes E, Ripoll C, Villar-Pazos S, Castellano-Munoz M, Soriano S, MartinezPinna J, Quesada I, Alonso-Magdalena P. Extranuclear-initiated estrogenic actions of endocrine disrupting chemicals: Is there toxicology beyond paracelsus? J Steroid Biochem Mol Biol. 2018; 176:16-22. http://dx.doi.org/10.1016/j.jsbmb.2017.01.014

33. Villar-Pazos S, Martinez-Pinna J, Castellano-Munoz M, Alonso-Magdalena P, Marroqui L, Quesada I, Gustafsson JA, Nadal A. Molecular mechanisms involved in the non-monotonic effect of bisphenol-a on ca2+ entry in mouse pancreatic beta-cells. Sci Rep. 2017; 7(1):11770. http://dx.doi.org/10.1038/s41598-017-11995-3 
34. Conley JM, Hannas BR, Furr JR, Wilson VS, Gray LE, Jr. A demonstration of the uncertainty in predicting the estrogenic activity of individual chemicals and mixtures from an in vitro estrogen receptor transcriptional activation assay (T47D-KBluc) to the in vivo uterotrophic assay using oral exposure. Toxicol Sci. 2016; 153(2):382-395.

http://dx.doi.org/10.1093/toxsci/kfw134

35. Lewis SM, Lee FW, Ali AA, Allaben WT, Weis CC, Leakey JE. Modifying a displacement pump for oral gavage dosing of solution and suspension preparations to adult and neonatal mice. Lab Anim (NY). 2010; 39(5):149-154. http://dx.doi.org/10.1038/laban0510-149

36. Camacho L, Lewis SM, Vanlandingham MM, Juliar BE, Olson GR, Patton RE, Gamboa da Costa G, Woodling K, Sepehr E, Bryant MS et al. Comparison of endpoints relevant to toxicity assessments in 3 generations of CD-1 mice fed irradiated natural and purified ingredient diets with varying soy protein and isoflavone contents. Food Chem Toxicol. 2016; 94:39-56. http://dx.doi.org/10.1016/j.fct.2016.05.014

37. Brown NM, Setchell KDR. Animal models impacted by phytoestrogens in commercial chow: Implications for pathways induced by hormones. Laboratory Investigation. 2001; 81:735-747. http://dx.doi.org/10.1038/labinvest.3780282

38. Thigpen JE, Setchell KD, Padilla-Banks E, Haseman JK, Saunders HE, Caviness GF, Kissling GE, Grant MG, Forsythe DB. Variations in phytoestrogen content between different mill dates of the same diet produces significant differences in the time of vaginal opening in CD1 mice and F344 rats but not in CD Sprague-Dawley rats. Environ Health Perspect. 2007; 115(12):1717-1726. http://dx.doi.org/10.1289/ehp.10165

39. Thigpen JE, Setchell KDR, Kissling GE, Locklear J, Caviness GF, Whteside T, Belcher SM, Brown NM, Collins BJ, Lih FB et al. The estrogenic content of rodent diets, bedding, cages, and water bottles and its effect on bisphenol A studies. J Am Assoc Lab Anim Sci. 2013; 52(2):130141.

40. Thigpen JE, Setchell KDR, Saunders HE, Haseman JK, Grant MG, Forsythe DB. Selecting the appropriate rodent diet for endocrine disruptor research and testing studies. ILAR Journal. 2004; 45(4):401-416. http://dx.doi.org/10.1093/ilar.45.4.401

41. Butchbach ME, Edwards JD, Schussler KR, Burghes AH. A novel method for oral delivery of drug compounds to the neonatal SMNDelta7 mouse model of spinal muscular atrophy. J Neurosci Methods. 2007; 161(2):285-290. http://dx.doi.org/10.1016/j.jneumeth.2006.11.002

42. Dixon D, Alison R, Bach U, Colman K, Foley GL, Harleman JH, Haworth R, Herbert R, Heuser A, Long $\mathrm{G}$ et al. Nonproliferative and proliferative lesions of the rat and mouse female reproductive system. J Toxicol Pathol. 2014; 27(3-4 Suppl):1S-107S. http://dx.doi.org/10.1293/tox.27.1S

43. Rudmann D, Cardiff R, Chouinard L, Goodman D, Kuttler K, Marxfeld H, Molinolo A, Treumann S, Yoshizawa K, Inhand Mammary ZsP et al. Proliferative and nonproliferative lesions of the rat and mouse mammary, Zymbal's, preputial, and clitoral glands. Toxicol Pathol. 2012; 40(6 Suppl):7S-39S. http://dx.doi.org/10.1177/0192623312454242 
44. Creasy D, Bube A, de Rijk E, Kandori H, Kuwahara M, Masson R, Nolte T, Reams R, Regan $\mathrm{K}$, Rehm S et al. Proliferative and nonproliferative lesions of the rat and mouse male reproductive system. Toxicol Pathol. 2012; 40(6_suppl):40S-121S. http://dx.doi.org/10.1177/0192623312454337

45. Brunner E, Domhof S, Langer F. Nonparametric Analysis of Longitudinal Data in Factorial Experiments. New York, New York: John Wiley and Sons; 2002.

46. Kenward MG, Roger JH. Small sample inference for fixed effects from restricted maximum likelihood. Biometrics. 1997; 53(3):983-997. http://dx.doi.org/10.2307/2533558

47. Bailer AJ, Portier CJ. Effects of treatment-induced mortality and tumor-induced mortality on tests for carcinogenicity in small samples. Biometrics. 1988; 44(2):417-431.

http://dx.doi.org/10.2307/2531856

48. Bieler GS, Williams RL. Ratio estimates, the delta method, and quantal response tests for increased carcinogenicity. Biometrics. 1993; 49(3):793-801. http://dx.doi.org/10.2307/2532200

49. Peddada SD, Kissling GE. A Survival-Adjusted Quantal-Response Test for Analysis of Tumor Incidence Rates in Animal Carcinogenicity Studies. Environmental Health Perspectives. 2005; 114(4):537-541. http://dx.doi.org/10.1289/ehp.8590

50. Jonckheere AR. A distribution-free k-sample test against ordered alternatives. Biometrika. 1954; 41:133-145. http://dx.doi.org/10.1093/biomet/41.1-2.133

51. Terpstra TJ. The asymptotic normality and consistency of Kendall's test against trend when ties are present in one ranking. Indagationes Mathematicae (Proceedings). 1952; 55:327-333. http://dx.doi.org/10.1016/S1385-7258(52)50043-X

52. Shirley E. A non-parametric equivalent of Williams' test for contrasting increasing dose levels of a treatment. Biometrics. 1977; 33:386-389. http://dx.doi.org/10.2307/2529789

53. Williams DA. A note on Shirley's nonparametric test for comparing several dose levels with a zero-dose control. Biometrics. 1986; 42:183-186. http://dx.doi.org/10.2307/2531254

54. Tennekes H, Gembardt C, Dammann M, van Ravenzwaay B. The stability of historical control data for common neoplasms in laboratory rats: adrenal gland (medulla), mammary gland, liver, endocrine pancreas, and pituitary gland. Regul Toxicol Pharmacol. 2004; 40(1):18-27. http://dx.doi.org/10.1016/j.yrtph.2004.04.003

55. Tennekes H, Kaufmann W, Dammann M, van Ravenzwaay B. The stability of historical control data for common neoplasms in laboratory rats and the implications for carcinogenic risk assessment. Regul Toxicol Pharmacol. 2004; 40(3):293-304.

http://dx.doi.org/10.1016/j.yrtph.2004.07.007

56. Peretz J, Vrooman L, Ricke WA, Hunt PA, Ehrlich S, Hauser R, Padmanabhan V, Taylor HS, Swan SH, VandeVoort CA et al. Bisphenol a and reproductive health: update of experimental and human evidence, 2007-2013. Environ Health Perspect. 2014; 122(8):775-786. http://dx.doi.org/10.1289/ehp.1307728 
57. Le Corre L, Besnard P, Chagnon MC. BPA, an energy balance disruptor. Crit Rev Food Sci Nutr. 2015; 55(6):769-777. http://dx.doi.org/10.1080/10408398.2012.678421

58. Seachrist DD, Bonk KW, Ho SM, Prins GS, Soto AM, Keri RA. A review of the carcinogenic potential of bisphenol A. Reprod Toxicol. 2016; 59:167-182. http://dx.doi.org/10.1016/j.reprotox.2015.09.006

59. Braun JM. Early-life exposure to EDCs: role in childhood obesity and neurodevelopment. Nat Rev Endocrinol. 2017; 13(3):161-173. http://dx.doi.org/10.1038/nrendo.2016.186

60. Yoshida M, Shimomoto T, Katashima S, Watanabe G, Taya K, Maekawa A. Maternal exposure to low doses of bisphenol A has no effects on development of female reproductive tract and uterine carcinogenesis in Donryu rats. J Reprod Dev. 2004; 50(3):349-360.

http://dx.doi.org/10.1262/jrd.50.349

61. Haseman JK. Use of statistical decision rules for evaluating laboratory animal carcinogenicity studies. Fund Appl Toxicol. 1990; 14(637-648). http://dx.doi.org/10.1016/0272$\underline{0590(90) 90289-\mathrm{V}}$

62. Kissling GE, Haseman JK, Zeiger E. Proper interpretation of chronic toxicity studies and their statistics: A critique of "Which level of evidence does the US National Toxicology Program provide? Statistical considerations using the Technical Report 578 on Ginkgo biloba as an example". Toxicol Lett. 2015; 237(2):161-164. http://dx.doi.org/10.1016/j.toxlet.2014.09.016

63. Yoshizawa K, Brix AE, Sells DM, Jokinen MP, Wyde M, Orzech DP, Kissling GE, Walker NJ, Nyska A. Reproductive lesions in female Harlan Sprague-Dawley rats following two-year oral treatment with dioxin and dioxin-like compounds. Toxicol Pathol. 2009; 37(7):921-937. http://dx.doi.org/10.1177/0192623309351721

64. Sarkar DK, Gottschall PE, Meites J. Damage to hypothalamic dopaminergic neurons is associated with development of prolactin-secreting pituitary tumors. Science. 1982; 218:684686. http://dx.doi.org/10.1126/science.7134966

65. Blankenstein MA, Broerse JJ, van Zweiten MJ, van der Molen HJ. Prolactin concentration in plasma and susceptibility to mammary tumors in female rats from different strains treated chronically with estradiol-17beta. Breast Cancer Res Treat. 1984; 4:137-141. http://dx.doi.org/10.1007/BF01806396

66. Ferguson SA, Law CD, Kissling GE. Developmental treatment with ethinyl estradiol, but not bisphenol A, causes alterations in sexually dimorphic behaviors in male and female Sprague Dawley rats. Toxicol Sci. 2014; 140(2):374-392. http://dx.doi.org/10.1093/toxsci/kfu077

67. Zaccaroni M, Massolo A, Della Seta D, Farabollini F, Giannelli G, Fusani L, Dessi-Fulgheri F. Developmental exposure to low levels of ethinylestradiol affects play behavior in juvenile female rats. Neurotox Res. 2018; 33(4):876-886. http://dx.doi.org/10.1007/s12640-017-9852-4

68. Shirota M, Kawashima J, Nakamura T, Kamiie J, Shirota K, Yoshida M. Dose-dependent acceleration in the delayed effects of neonatal oral exposure to low-dose 17 alphaethynylestradiol on reproductive functions in female Sprague-Dawley rats. J Toxicol Sci. 2015; 40(6):727-738. http://dx.doi.org/10.2131/jts.40.727 
69. Howdeshell KL, Furr J, Lambright CR, Wilson VS, Ryan BC, Gray LE, Jr. Gestational and lactational exposure to ethinyl estradiol, but not bisphenol A, decreases androgen-dependent reproductive organ weights and epididymal sperm abundance in the male long evans hooded rat. Toxicol Sci. 2008; 102(2):371-382. http://dx.doi.org/10.1016/j.reprotox.2015.07.075

70. Ryan BC, Hotchkiss AK, Crofton KM, Gray LE, Jr. In utero and lactational exposure to bisphenol A, in contrast to ethinyl estradiol, does not alter sexually dimorphic behavior, puberty, fertility, and anatomy of female LE rats. Toxicol Sci. 2010; 114(1):133-148.

http://dx.doi.org/10.1093/toxsci/kfp266

71. Delclos KB, Doerge DR. Response to Hunt et al., invalid controls undermine conclusions of FDA studies. Toxicol Sci. 2014; 141(1):iii-iv. http://dx.doi.org/10.1093/toxsci/kfu102 
Table 2. Experimental Design and Materials and Methods in the Two-Year Chronic Gavage Toxicology Study of Bisphenol A (NCTR E0219001), Including Interim (One-Year) Assessment

\begin{tabular}{|c|c|}
\hline & Experimental Design and Materials and Methods \\
\hline Study Laboratory & National Center for Toxicological Research (NCTR); Jefferson, AR \\
\hline Test Article & $\begin{array}{l}\text { Bisphenol A (BPA), >99\% (CAS \#80-05-7), TCI America, Portland, OR } \\
\text { [Catalogue \#B0494, Lot \#6052012, ground to a fine powder by Batelle, Inc., } \\
\text { Columbus, OH] }\end{array}$ \\
\hline Control Article, Reference & Ethinyl estradiol ( $\left.\mathrm{EE}_{2}\right),>98 \%$ (CAS \#57-63-6), Sigma-Aldrich Corporation, St. \\
\hline Estrogen & Louis, MO [Catalogue \#E4876, Lot \#071M1492V] \\
\hline Control Article, Vehicle & $\begin{array}{l}\text { Carboxymethylcellulose, sodium salt, Sigma-Aldrich Corporation, St. Louis, MO, } \\
\text { [Catalogue \#C-5013, Lot \#041M0105V] used as a } 0.3 \%(\mathrm{w} / \mathrm{w}) \text { aqueous solution }\end{array}$ \\
\hline Strain and Species & Rats: Sprague-Dawley/CD23/Nctr BR \\
\hline Animal Source & NCTR breeding colony (Jefferson, AR) \\
\hline Time Held Before Study & $\begin{array}{l}\text { Breeder animals for the study were obtained from the breeding colony at weaning } \\
\text { (approximately PND 21) and placed under study conditions (TestDiet low } \\
\text { phytoestrogen 5K96 diet, polysulfone rat cages, hardwood chip bedding, glass } \\
\text { water bottles with silicone stoppers) until mated at PND 70-100 for females and } \\
\text { PND 77- } 105 \text { for males. }\end{array}$ \\
\hline Age When Exposure Began & $\begin{array}{l}\text { Sperm- or in situ vaginal plug-positive females were dosed from GD } 6 \text { (sperm or } \\
\text { plug detection = GD 0) }\end{array}$ \\
\hline \multirow{5}{*}{$\begin{array}{l}\text { Date of First Exposure for } \mathrm{F}_{0} \\
(\mathrm{GD} 6)\end{array}$} & $09 / 08 / 2012$ \\
\hline & $10 / 06 / 2012$ \\
\hline & $11 / 03 / 2012$ \\
\hline & $12 / 01 / 2012$ \\
\hline & $12 / 29 / 2012$ \\
\hline Route of Exposure & Oral gavage (for pups <PND 5, gavage needle did not enter esophagus) \\
\hline Duration of Exposure & $\begin{array}{l}\text { Dams were dosed daily until start of parturition. There was no dosing on the day of } \\
\text { birth (PND 0), and pups were dosed directly from PND } 1 \text { until PND } 21 \text { for stop- } \\
\text { dose group animals and from PND } 1 \text { until the day prior to termination for } \\
\text { continuous-dose group animals }\end{array}$ \\
\hline Date of Last Exposure & $01 / 14 / 2015$ \\
\hline \multirow[t]{3}{*}{ Age at Necropsy } & Scheduled interim necropsy at 1 year $(365 \pm 20$ days of age $)$ \\
\hline & Scheduled terminal necropsy at 2 years $(104 \pm 3$ weeks of age $)$ \\
\hline & Moribund and dead animals necropsied on removal \\
\hline Size of Study Groups & $\begin{array}{l}\text { For vehicle and BPA dose groups: } 20-26 \text { litters in the interim necropsy groups, and } \\
46-50 \text { litters in the terminal necropsy groups } \\
\text { For } \mathrm{EE}_{2} \text { dose groups: } 26 \text { litters (interim and terminal necropsy groups) }\end{array}$ \\
\hline Method of Animal Allocation & $\begin{array}{l}\mathrm{F}_{0} \text { females from the NCTR breeding colony were randomly allocated to dose } \\
\text { groups prior to mating to give approximately equal mean body weights per dose } \\
\text { group. Sires were selected randomly with the specification that there would be no } \\
\text { brother/sister or first cousin matings. After the first mating, the numbers of mating } \\
\text { pairs assigned to dose groups were adjusted to meet any deficits in pups available } \\
\text { in a particular dose group. Pups were randomly culled to a maximum of } 5 \text { males } \\
\text { and } 5 \text { females on PND 1, no fostering was conducted. The minimum litter size for } \\
\text { keeping a litter on study was } 3 \text { males and } 3 \text { females. Up to } 3 \text { pups per sex per litter } \\
\text { were assigned to the study at weaning; additional pups were assigned to studies } \\
\text { conducted by CLARITY-BPA academic investigators and reported elsewhere. The } \\
\text { rotating order of assignment of pups of a given sex to the study was as follows: } 1 \text { ) } \\
\text { continuous-dose, 2-year sacrifice; } 2 \text { ) stop-dose, } 2 \text {-year sacrifice; } 3 \text { ) continuous- } \\
\text { dose, } 1 \text {-year sacrifice; } 4 \text { ) continuous-dose, } 2 \text {-year sacrifice; 5) stop-dose, 2-year } \\
\text { sacrifice; and 6) stop-dose, } 1 \text {-year sacrifice. }\end{array}$ \\
\hline Animals per Cage & $\begin{array}{l}\text { Pregnant females were housed singly and litters were kept with their dams until } \\
\text { weaning at PND } 21 \text {. Animals were pair housed after weaning in same-sex pairs } \\
\text { within dose groups. If an animal died or was removed as moribund, the cage mate } \\
\text { remained single housed. }\end{array}$ \\
\hline
\end{tabular}




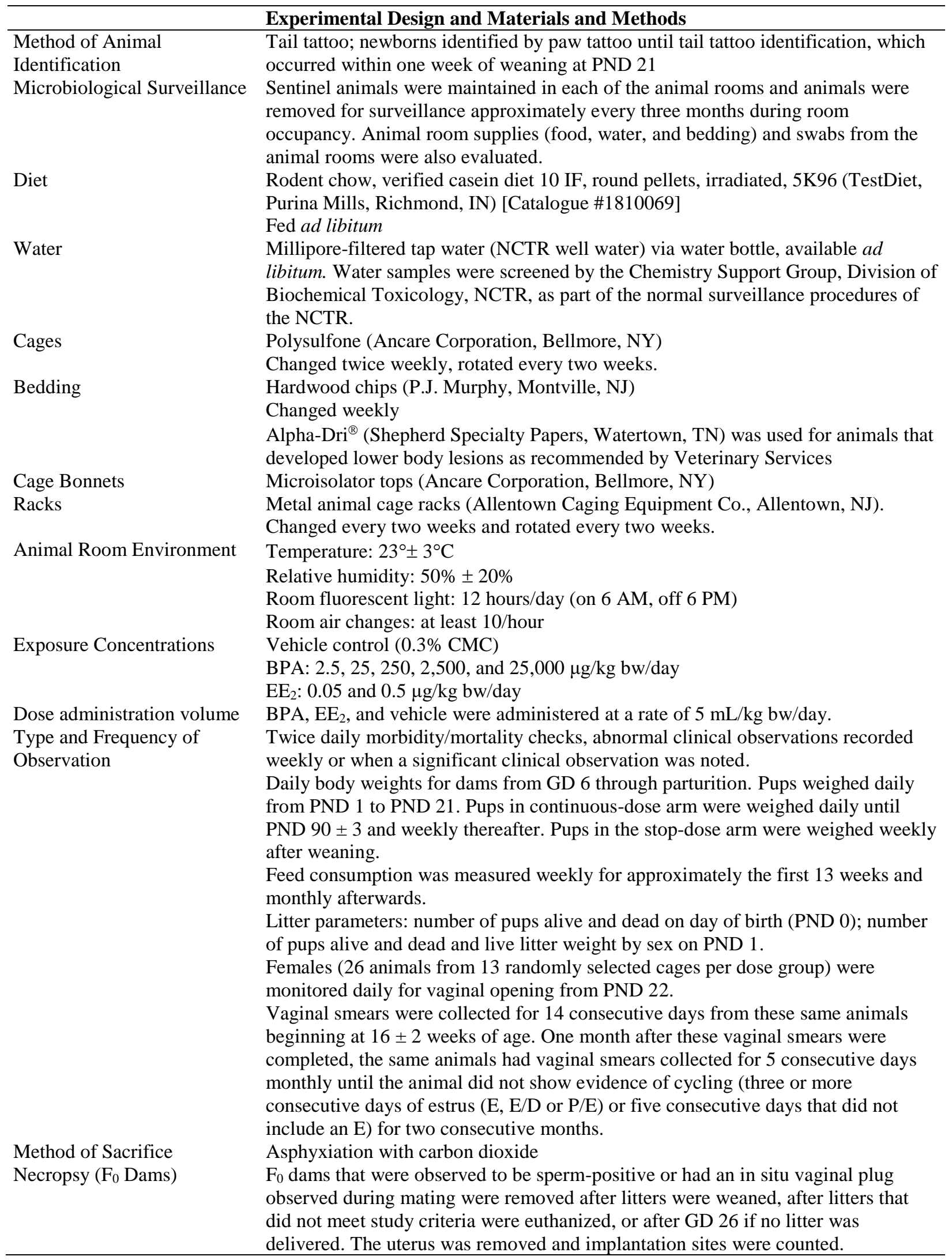




\section{Experimental Design and Materials and Methods}

Necropsy (Dead or moribund $\mathrm{F}_{0}$ dams and all postweaning $\mathrm{F}_{1}$ animals)

Necropsy (Interim sacrifice, PND $365 \pm 20$ )

Hematology (Interim sacrifice, PND $365 \pm 20$ )

Clinical Chemistry (Interim sacrifice, PND $365 \pm 20$ )

Sperm analysis (Interim sacrifice, PND $365 \pm 20$ ) Necropsy and histopathology (Terminal sacrifice, $104 \pm 3$ weeks)

Histopathology (Interim sacrifice, PND $365 \pm 20$ and terminal sacrifice, $104 \pm 3$ weeks)
Animals underwent a gross examination and complete necropsy as described for interim and terminal sacrifice animals (see below). In addition to tissues designated for processing and/or histopathology in the protocol, the following tissues were examined microscopically to assess possible cause of death in all dead or moribund animals: esophagus, colon, ileum, lung, nose, stomach, trachea, and any gross lesions.

Animals were fasted overnight. Animals were anesthetized with carbon dioxide and blood was collected from the retro-orbital sinus prior to euthanasia and complete necropsy.

Weighed organs (males): adrenals, brain, epididymides, heart, kidneys, liver, pituitary (after 48-hour fixation), seminal vesicles with coagulating gland, spleen, testes, thymus, thyroid with parathyroid (after 48-hour fixation), epididymal, and retroperitoneal fat pads.

Weighed organs (females): adrenals, brain, heart, kidneys, liver, ovaries (with oviducts), pituitary (after 48-hour fixation), spleen, thymus, thyroid with parathyroid (after 48-hour fixation), uterus (blotted), ovarian and parametrial (combined), and retroperitoneal fat pads.

The following endpoints were evaluated in an aliquot of whole blood: hematocrit, hemoglobin concentration, erythrocyte, leukocyte, reticulocyte, and platelet counts, leukocyte differential count, mean corpuscular volume, and mean corpuscular hemoglobin.

The following endpoints were measured in serum: total protein, albumin, urea nitrogen, creatinine, alanine aminotransferase, gamma glutamyl transpeptidase, sorbitol dehydrogenase, aspartate aminotransferase, alkaline phosphatase, total bile acids, glucose, cholesterol, triglycerides, insulin, leptin, cardiac troponins T and I, T3, T4, and TSH.

Testicular spermatid head counts (left testis); epididymal sperm counts, morphology, and motility evaluations (left epididymis).

Procedures were similar to those used for the interim sacrifice, except that the animals were not fasted, blood was not collected, and there were no hematology, clinical chemistry, sperm, or organ weight data collected.

The following organs, as well as all gross lesions, were evaluated microscopically: Males - adrenals, aorta (thoracic), bone marrow (femur), brain, right epididymis, heart, kidneys, liver, $5^{\text {th }}$ left mammary gland (inguinal), pancreas, parathyroid, pituitary, prostate (dorsal/lateral and ventral), seminal vesicles with coagulating gland, spleen, right testis, thymus, and thyroid. For the dorsal/lateral prostate, 6 step sections cut at $100 \mu \mathrm{m}$ intervals were evaluated. Subsets of intermediate sections were collected and stored unstained for potential additional evaluation.

Females - adrenals, aorta (thoracic), bone marrow (femur), brain, heart, kidneys, liver, $5^{\text {th }}$ left mammary gland (inguinal), ovaries, oviduct, pancreas, parathyroid, pituitary, spleen, thymus, thyroid, uterus, and vagina.

All tissues, except testes and eyes, were fixed in $10 \% \mathrm{NBF}$ and stained with H\&E for microscopic evaluation. Testes and eyes were fixed in modified Davidson's fixative and testes were stained with periodic acid-Schiff (PAS) stain. Fixation times for the tissues listed for histopathology, except the brain, were limited to 96120 hours. Brain remained in fixative until processing.

Tissues removed, fixed in Clitoral gland, esophagus, epididymal fat pad, ovarian/parametrial fat pad, $10 \%$ NBF, processed to block retroperitoneal fat pad, Harderian gland, cecum, colon, rectum, duodenum, ileum, and stored (Interim sacrifice, jejunum, lung with bronchi, lymph nodes (mandibular and mesenteric), nose, penis, PND $365 \pm 20$ and terminal sacrifice, $104 \pm 3$ weeks) preputial gland, salivary glands, skin, forestomach, glandular stomach, trachea, and urinary bladder. 
Table 3. Numbers of $F_{0}$ Breeding Pairs Assigned to Study ${ }^{a}$

\begin{tabular}{|c|c|c|c|c|c|c|c|c|c|}
\hline Matings & Vehicle & $2.5 \mathrm{BPA}$ & 25 BPA & 250 BPA & 2500 BPA & 25000 BPA & $0.05 \mathrm{EE}_{2}$ & $0.50 \mathrm{EE}_{2}$ & Total \\
\hline Mating 1 & 16 & 16 & $16^{\mathrm{b}}$ & 16 & 16 & 16 & 10 & 14 & 120 \\
\hline Mating 2 & 17 & 17 & 17 & 17 & 17 & 17 & 9 & 9 & 120 \\
\hline Mating 3 & 17 & 17 & 17 & 17 & 17 & 17 & 9 & 9 & 120 \\
\hline Mating 4 & 15 & 15 & 17 & 16 & $14^{\mathrm{c}}$ & 14 & $13^{\mathrm{c}}$ & 14 & 118 \\
\hline Mating 5 & 15 & 16 & 20 & 18 & 15 & 11 & 10 & 15 & 120 \\
\hline Total, Matings $1-5^{d}$ & 80 & 81 & 87 & 84 & 79 & 75 & 51 & 61 & 598 \\
\hline
\end{tabular}

${ }^{a}$ Doses of BPA and $\mathrm{EE}_{2}$ are $\mu \mathrm{g} / \mathrm{kg}$ bw/day.

${ }^{\mathrm{b}}$ Only 15 pairs were mated due to the death of a male prior to breeding.

${ }^{\mathrm{c}}$ Due to the deaths of male breeders, one female in each of these groups was mated with a male that had previously mated with a control female.

${ }^{\mathrm{d}}$ The numbers of resulting sperm-positive dams were as follows: vehicle, 78; $2.5 \mathrm{BPA}, 74 ; 25 \mathrm{BPA}, 76 ; 250 \mathrm{BPA}, 78 ; 2500 \mathrm{BPA}, 76 ; 25000 \mathrm{BPA}, 71 ; 0.05 \mathrm{EE} 2,51 ; 0.50 \mathrm{EE} 2$, 60.

Table 4. Number of Litters Produced Per Mating ${ }^{\mathrm{a}}$

\begin{tabular}{|c|c|c|c|c|c|c|c|c|c|}
\hline Matings & Vehicle & $2.5 \mathrm{BPA}$ & 25 BPA & 250 BPA & 2500 BPA & 25000 BPA & $0.05 \mathrm{EE}_{2}$ & $0.50 \mathrm{EE}_{2}$ & Total \\
\hline Mating 1 & 16 & 15 & 8 & 9 & 13 & 12 & 9 & 12 & 94 \\
\hline Mating 2 & 14 & 13 & 10 & 14 & 15 & 16 & 8 & 8 & 98 \\
\hline Mating 3 & 15 & 13 & 13 & 13 & 15 & 16 & 6 & 8 & 99 \\
\hline Mating 4 & 13 & 13 & 13 & 12 & 12 & 11 & 10 & 10 & 94 \\
\hline Mating 5 & 15 & 11 & 17 & 16 & 9 & 10 & 8 & 13 & 99 \\
\hline Total, Matings 1-5 & 73 & 65 & 61 & 64 & 64 & 65 & 41 & 51 & 484 \\
\hline
\end{tabular}

${ }^{\mathrm{a}}$ Doses of BPA and $\mathrm{EE}_{2}$ are $\mu \mathrm{g} / \mathrm{kg}$ bw/day.

Table 5. Number of Litters Contributing Pups to Interim and/or Terminal Assessments ${ }^{\mathrm{a}}$

\begin{tabular}{|c|c|c|c|c|c|c|c|c|c|}
\hline Matings & Vehicle & 2.5 BPA & 25 BPA & 250 BPA & 2500 BPA & 25000 BPA & $0.05 \mathrm{EE}_{2}$ & $0.50 \mathrm{EE}_{2}$ & Total \\
\hline Mating 1 & 16 & 14 & 8 & 7 & 13 & 12 & 6 & 10 & 86 \\
\hline Mating 2 & 13 & 13 & 10 & 14 & 15 & 14 & 7 & 8 & 94 \\
\hline Mating 3 & 15 & 12 & 12 & 11 & 14 & 14 & 4 & 6 & 88 \\
\hline Mating 4 & 12 & 12 & 13 & 11 & 11 & 11 & 8 & 4 & 82 \\
\hline Mating 5 & 15 & 11 & 16 & 16 & 8 & 9 & 2 & 3 & 80 \\
\hline Total, Matings 1-5 & 71 & 62 & 59 & 59 & 61 & 60 & 27 & 31 & 430 \\
\hline
\end{tabular}

Doses of BPA and $\mathrm{EE}_{2}$ are $\mu \mathrm{g} / \mathrm{kg}$ bw/day. 
Table 6. Number of Male and Female Pups Represented in Interim (1-Year) Sacrifice from Each Mating, Continuous-Dose ${ }^{a}$

\begin{tabular}{|c|c|c|c|c|c|c|c|c|c|}
\hline Continuous-Dose & Vehicle & 2.5 BPA & 25 BPA & 250 BPA & 2500 BPA & 25000 BPA & $0.05 \mathrm{EE}_{2}$ & $0.50 \mathrm{EE}_{2}$ & Total \\
\hline Males, Mating 1 & 6 & 6 & 4 & 2 & 4 & 4 & 6 & 8 & 40 \\
\hline Males, Mating 2 & 4 & 4 & 4 & 6 & 6 & 6 & 6 & 8 & 44 \\
\hline Males, Mating 3 & 4 & 6 & 4 & 4 & 6 & 6 & 4 & 4 & 38 \\
\hline Males, Mating 4 & 6 & 4 & 4 & 4 & 2 & 4 & 8 & 4 & 36 \\
\hline Males, Mating 5 & 2 & 2 & 4 & 8 & 2 & 2 & 2 & 2 & 24 \\
\hline Total males, Matings 1-5 & 22 & 22 & 20 & 24 & 20 & 22 & 26 & 26 & 182 \\
\hline Females, Mating 1 & 8 & 6 & 4 & 4 & 6 & 4 & 6 & 8 & 46 \\
\hline Females, Mating 2 & 5 & 4 & 4 & 6 & 6 & 6 & 6 & 8 & 45 \\
\hline Females, Mating 3 & 6 & 4 & 4 & 4 & 4 & 6 & 4 & 6 & 38 \\
\hline Females, Mating 4 & 2 & 2 & 4 & 4 & 4 & 4 & 8 & 2 & 30 \\
\hline Females, Mating 5 & 2 & 6 & 6 & 6 & 0 & 4 & 2 & 2 & 28 \\
\hline Total females, Matings 1-5 & 23 & 22 & 22 & 24 & 20 & 24 & 26 & 26 & 187 \\
\hline
\end{tabular}

${ }^{\mathrm{a}}$ Doses of BPA and $\mathrm{EE}_{2}$ are $\mu \mathrm{g} / \mathrm{kg}$ bw/day.

Table 7. Number of Male and Female Pups Represented in Interim (1 Year) Sacrifice from Each Mating, Stop-Dose ${ }^{a}$

\begin{tabular}{|c|c|c|c|c|c|c|c|}
\hline Stop-Dose & Vehicle & 2.5 BPA & 25 BPA & 250 BPA & 2500 BPA & 25000 BPA & Total \\
\hline Males, Mating 1 & 4 & 4 & 2 & 2 & 6 & 6 & 24 \\
\hline Males, Mating 2 & 6 & 4 & 2 & 6 & 6 & 4 & 28 \\
\hline Males, Mating 3 & 6 & 4 & 6 & 4 & 6 & 6 & 32 \\
\hline Males, Mating 4 & 2 & 4 & 6 & 4 & 2 & 4 & 22 \\
\hline Males, Mating 5 & 2 & 4 & 4 & 4 & 0 & 2 & 16 \\
\hline Total males, Matings 1-5 & 20 & 20 & 20 & 20 & 20 & 22 & 122 \\
\hline Females, Mating 1 & 4 & 6 & 2 & 2 & 4 & 6 & 24 \\
\hline Females, Mating 2 & 4 & 6 & 4 & 4 & 6 & 6 & 30 \\
\hline Females, Mating 3 & 6 & 6 & 2 & 4 & 6 & 6 & 30 \\
\hline Females, Mating 4 & 4 & 4 & 6 & 4 & 2 & 4 & 24 \\
\hline Females, Mating 5 & 2 & 0 & 6 & 8 & 2 & 0 & 18 \\
\hline Total females, Matings 1-5 & 20 & 22 & 20 & 22 & 20 & 22 & 126 \\
\hline
\end{tabular}

a Doses of BPA are $\mu \mathrm{g} / \mathrm{kg}$ bw/day. 
Table 8. Number of Male and Female Pups Represented in Terminal (2 Year) Sacrifice from Each Mating, Continuous-Dose ${ }^{\mathrm{a}}$

\begin{tabular}{|c|c|c|c|c|c|c|c|c|c|}
\hline Continuous-Dose & Vehicle & $2.5 \mathrm{BPA}$ & 25 BPA & 250 BPA & 2500 BPA & 25000 BPA & $0.05 \mathrm{EE}_{2}$ & $0.50 \mathrm{EE}_{2}$ & Total \\
\hline Males, Mating 1 & 13 & 12 & 6 & 6 & 10 & 10 & 6 & 10 & 73 \\
\hline Males, Mating 2 & 11 & 10 & 8 & 12 & 12 & 12 & 6 & 8 & 79 \\
\hline Males, Mating 3 & 12 & 10 & 10 & 8 & 12 & 12 & 4 & 4 & 72 \\
\hline Males, Mating 4 & 12 & 8 & 12 & 10 & 8 & 8 & 8 & 2 & 68 \\
\hline Males, Mating 5 & 2 & 8 & 12 & 14 & 8 & 4 & 2 & 2 & 52 \\
\hline Total males, Matings 1-5 & 50 & 48 & 48 & 50 & 50 & 46 & 26 & 26 & 344 \\
\hline Females, Mating 1 & 14 & 12 & 6 & 6 & 10 & 10 & 6 & 10 & 74 \\
\hline Females, Mating 2 & 10 & 10 & 8 & 12 & 12 & 10 & 6 & 8 & 76 \\
\hline Females, Mating 3 & 12 & 10 & 10 & 8 & 12 & 10 & 4 & 4 & 70 \\
\hline Females, Mating 4 & 10 & 8 & 10 & 10 & 8 & 8 & 8 & 2 & 64 \\
\hline Females, Mating 5 & 4 & 8 & 12 & 14 & 8 & 8 & 2 & 2 & 58 \\
\hline Total females, Matings 1-5 & 50 & 48 & 46 & 50 & 50 & 46 & 26 & 26 & 342 \\
\hline
\end{tabular}

${ }^{\mathrm{a}}$ Doses of BPA and $\mathrm{EE}_{2}$ are $\mu \mathrm{g} / \mathrm{kg}$ bw/day.

Table 9. Number of Male and Female Pups Represented in Terminal (2 Year) Sacrifice from Each Mating, Stop-Dose ${ }^{\text {a }}$

\begin{tabular}{|c|c|c|c|c|c|c|c|}
\hline Stop-Dose & Vehicle & 2.5 BPA & 25 BPA & 250 BPA & 2500 BPA & 25000 BPA & Total \\
\hline Males, Mating 1 & 14 & 12 & 6 & 6 & 10 & 10 & 58 \\
\hline Males, Mating 2 & 10 & 10 & 8 & 12 & 12 & 12 & 64 \\
\hline Males, Mating 3 & 12 & 10 & 10 & 8 & 12 & 12 & 64 \\
\hline Males, Mating 4 & 12 & 8 & 12 & 10 & 10 & 8 & 60 \\
\hline Males, Mating 5 & 2 & 8 & 12 & 14 & 6 & 4 & 46 \\
\hline Total males, Matings 1-5 & 50 & 48 & 48 & 50 & $\mathbf{5 0}$ & 46 & 292 \\
\hline Females, Mating 1 & 14 & 12 & 6 & 6 & 10 & 10 & 58 \\
\hline Females, Mating 2 & 10 & 10 & 8 & 12 & 12 & 12 & 64 \\
\hline Females, Mating 3 & 12 & 10 & 10 & 8 & 12 & 12 & 64 \\
\hline Females, Mating 4 & 10 & 10 & 12 & 10 & 10 & 8 & 60 \\
\hline Females, Mating 5 & 4 & 8 & 12 & 14 & 6 & 4 & 48 \\
\hline Total females, Matings 1-5 & 50 & 50 & 48 & 50 & 50 & 46 & 294 \\
\hline
\end{tabular}

${ }^{2}$ Doses of BPA are $\mu \mathrm{g} / \mathrm{kg}$ bw/day. 
CLARITY-BPA Core Study

Table 10. Dam Body Weights from Time of Mating to Parturition in Vehicle, BPA, and $\mathrm{EE}_{2}$ Dose Groups $\left(\right.$ Mean \pm S.E.M.) ${ }^{\mathrm{a}}$

\begin{tabular}{|c|c|c|c|c|c|c|c|c|}
\hline Body Weight & $\begin{array}{l}\text { Vehicle } \\
\mathrm{n}=72 \\
\end{array}$ & $\begin{array}{c}2.5 \text { BPA } \\
n=65\end{array}$ & $\begin{array}{c}25 \text { BPA } \\
n=61\end{array}$ & $\begin{array}{c}250 \text { BPA } \\
n=63 \\
\end{array}$ & $\begin{array}{c}2500 \text { BPA } \\
n=63\end{array}$ & $\begin{array}{c}25000 \text { BPA } \\
n=64\end{array}$ & $\begin{array}{c}0.05 \mathrm{EE}_{2} \\
\mathrm{n}=41\end{array}$ & $\begin{array}{c}0.5 \mathrm{EE}_{2} \\
\mathrm{n}=51\end{array}$ \\
\hline Baseline (GD 0/ GD 1), g & $244 \pm 3$ & $248 \pm 3$ & $248 \pm 4$ & $244 \pm 3$ & $246 \pm 3$ & $252 \pm 4$ & $247 \pm 4$ & $253 \pm 4$ \\
\hline GD 6, g & $275 \pm 3$ & $281 \pm 4$ & $278 \pm 4$ & $274 \pm 4$ & $278 \pm 3$ & $283 \pm 4$ & $278 \pm 5$ & $284 \pm 4$ \\
\hline Parturition, $\mathrm{g}$ & $393 \pm 4$ & $406 \pm 5$ & $397 \pm 6$ & $394 \pm 5$ & $402 \pm 5$ & $396 \pm 5$ & $398 \pm 6$ & $402 \pm 6$ \\
\hline
\end{tabular}

${ }^{a}$ Number of dams producing litters given under dose group column headings. Doses of BPA and $\mathrm{EE}_{2}$ are $\mu \mathrm{g} / \mathrm{kg}$ bw/day.

Gestational weight at parturition was analyzed separately for the BPA and EE2 dose groups using ANOCOVA, with terms for treatment group, dam weight at baseline as a covariate, litter size as a covariate, and the interaction between treatment and litter size. Data were collected at baseline on GD 0 or GD 1 and daily from GD 6 to parturition.

Gestational weight at parturition was defined as the last dam body weight prior to delivery. Pairwise comparisons of treatment means to the control group were performed using contrasts with Dunnett's adjustment for multiple comparisons. Tests of trend, increasing treatment effect with increasing dose, were performed for the BPA and vehicle control groups only. All tests were performed as two-sided tests at the 0.05 significance level. There were no statistically significant results. There were also no significant treatment effects in the sensitivity analysis that excluded all animals that overlapped with animals treated with 250,000 $\mu \mathrm{g}$ BPA/kg bw/day (see Materials and Methods, Statistical Methods). Full results of the statistical analyses are found in Supplemental Appendix XVII. 
CLARITY-BPA Core Study

Table 11. Implantation Sites And Litter Parameters For Vehicle, BPA, And EE 2 Dose Groups (Mean \pm S.E.M) ${ }^{\mathrm{a}}$

\begin{tabular}{|c|c|c|c|c|c|c|c|c|}
\hline Endpoint & Vehicle & $2.5 \mathrm{BPA}$ & 25 BPA & 250 BPA & 2500 BPA & 25000 BPA & $0.05 \mathrm{EE}_{2}$ & $0.5 \mathrm{EE}_{2}$ \\
\hline Implantation sites & $\begin{array}{c}12.8 \pm 0.5 \\
(78)\end{array}$ & $\begin{array}{c}12.4 \pm 0.6 \\
(74)\end{array}$ & $\begin{array}{c}11.2 \pm 0.7 \\
(74)\end{array}$ & $\begin{array}{c}11.2 \pm 0.6 \\
(78)\end{array}$ & $\begin{array}{c}11.6 \pm 0.6 \\
(74)\end{array}$ & $\begin{array}{c}12.2 \pm 0.6 \\
(70)\end{array}$ & $\begin{array}{c}11.7 \pm 0.8 \\
(49)\end{array}$ & $\begin{array}{c}12.1 \pm 0.7 \\
(59)\end{array}$ \\
\hline Litter size ${ }^{b}$ & $\begin{array}{c}11.8 \pm 0.4 \\
(73)\end{array}$ & $\begin{array}{c}12.6 \pm 0.3 \\
(65)\end{array}$ & $\begin{array}{c}11.9 \pm 0.5 \\
(61)\end{array}$ & $\begin{array}{c}11.6 \pm 0.5 \\
(64)\end{array}$ & $\begin{array}{c}12.3 \pm 0.4 \\
(64)\end{array}$ & $\begin{array}{c}11.5 \pm 0.4 \\
(64)\end{array}$ & $\begin{array}{l}11.8 \pm 0.6 \\
(41)\end{array}$ & $\begin{array}{l}12.2 \pm 0.4 \\
\quad(51)\end{array}$ \\
\hline Males $^{\mathrm{b}}$ & $\begin{array}{c}5.8 \pm 0.2 \\
(73)\end{array}$ & $\begin{array}{c}6.4 \pm 0.3 \\
(65)\end{array}$ & $\begin{array}{c}6.2 \pm 0.3 \\
\quad(61)\end{array}$ & $\begin{array}{c}5.7 \pm 0.2 \\
(64)\end{array}$ & $\begin{array}{c}6.2 \pm 0.3 \\
(64)\end{array}$ & $\begin{array}{c}5.5 \pm 0.3 \\
(64)\end{array}$ & $\begin{array}{c}6.1 \pm 0.4 \\
(41)\end{array}$ & $\begin{array}{c}5.8 \pm 0.3 \\
(51)\end{array}$ \\
\hline Females $^{\mathrm{b}}$ & $\begin{array}{c}5.8 \pm 0.2 \\
(73)\end{array}$ & $\begin{array}{c}6.0 \pm 0.3 \\
(65)\end{array}$ & $\begin{array}{c}5.6 \pm 0.3 \\
(61)\end{array}$ & $\begin{array}{c}5.7 \pm 0.4 \\
(64)\end{array}$ & $\begin{array}{c}5.8 \pm 0.3 \\
(64)\end{array}$ & $\begin{array}{c}5.8 \pm 0.3 \\
(64)\end{array}$ & $\begin{array}{l}5.4 \pm 0.4 \\
\quad(41)\end{array}$ & $\begin{array}{c}6.1 \pm 0.3 \\
(51)\end{array}$ \\
\hline Unsexed $^{c}$ & $\begin{array}{l}0.2 \pm 0.1 \\
(73)\end{array}$ & $\begin{array}{c}0.2 \pm 0.1 \\
(65)\end{array}$ & $\begin{array}{c}0.1 \pm 0 \\
(61)\end{array}$ & $\begin{array}{l}0.2 \pm 0.1 \\
\quad(64)\end{array}$ & $\begin{array}{c}0.3 \pm 0.1 \\
(64)\end{array}$ & $\begin{array}{l}0.2 \pm 0.1 \\
\quad(64)\end{array}$ & $\begin{array}{l}0.3 \pm 0.2 \\
\quad(41)\end{array}$ & $\begin{array}{c}0.3 \pm 0.1 \\
(51)\end{array}$ \\
\hline Born dead & $\begin{array}{c}0 \pm 0 \\
(73)\end{array}$ & $\begin{array}{c}0.08 \pm 0.05 \\
(65)\end{array}$ & $\begin{array}{c}0.02 \pm 0.02 \\
(61)\end{array}$ & $\begin{array}{c}0 \pm 0 \\
(64)\end{array}$ & $\begin{array}{c}0.02 \pm 0.02 \\
(64)\end{array}$ & $\begin{array}{c}0.02 \pm 0.02 \\
(64)\end{array}$ & $\begin{array}{c}0 \pm 0 \\
(41)\end{array}$ & $\begin{array}{c}0.04 \pm 0.04 \\
(51)\end{array}$ \\
\hline$\%$ Males $^{\mathrm{d}}$ & $\begin{array}{l}49.4 \pm 1.5 \\
\quad(73)\end{array}$ & $\begin{array}{c}51.5 \pm 2.3 \\
(65)\end{array}$ & $\begin{array}{c}52.2 \pm 2.2 \\
(61)\end{array}$ & $\begin{array}{c}50.6 \pm 2.0 \\
(64)\end{array}$ & $\begin{array}{c}50.9 \pm 1.8 \\
(64)\end{array}$ & $\begin{array}{c}47.3 \pm 2.3 \\
(64)\end{array}$ & $\begin{array}{c}53.2 \pm 2.4 \\
\quad(41)\end{array}$ & $\begin{array}{l}48.0 \pm 2.1 \\
\quad(51)\end{array}$ \\
\hline$\%$ Females $^{\mathrm{d}}$ & $\begin{array}{c}49.5 \pm 1.5 \\
(73)\end{array}$ & $\begin{array}{c}47.0 \pm 2.3 \\
\quad(65)\end{array}$ & $\begin{array}{c}47.0 \pm 2.2 \\
(61)\end{array}$ & $\begin{array}{l}47.9 \pm 2.1 \\
\quad(64)\end{array}$ & $\begin{array}{c}46.7 \pm 1.7 \\
(64)\end{array}$ & $\begin{array}{c}51.5 \pm 2.3 \\
(64)\end{array}$ & $\begin{array}{c}44.8 \pm 2.2 \\
(41)\end{array}$ & $\begin{array}{c}49.6 \pm 2.1 \\
(51)\end{array}$ \\
\hline$\%$ Unsexed & $\begin{array}{c}1.1 \pm 0.4 \\
(73)\end{array}$ & $\begin{array}{c}1.6 \pm 0.6 \\
(65)\end{array}$ & $\begin{array}{c}0.8 \pm 0.3 \\
(61)\end{array}$ & $\begin{array}{c}1.5 \pm 0.7 \\
(64)\end{array}$ & $\begin{array}{c}2.3 \pm 0.9 \\
(64)\end{array}$ & $\begin{array}{c}1.1 \pm 0.6 \\
(64)\end{array}$ & $\begin{array}{c}1.9 \pm 1.1 \\
(41)\end{array}$ & $\begin{array}{c}2.4 \pm 1.0 \\
(51)\end{array}$ \\
\hline Litter weight, total ${ }^{\mathrm{e}}$ & $\begin{array}{c}78.6 \pm 2.3 \\
(73)\end{array}$ & $\begin{array}{c}80.7 \pm 2.6 \\
(62)\end{array}$ & $\begin{array}{c}80.2 \pm 2.8 \\
(61)\end{array}$ & $\begin{array}{c}75.6 \pm 2.9 \\
(64)\end{array}$ & $\begin{array}{c}78.7 \pm 2.4 \\
(63)\end{array}$ & $\begin{array}{c}75.3 \pm 2.6 \\
(64)\end{array}$ & $\begin{array}{c}76.7 \pm 3.9 \\
(39)\end{array}$ & $\begin{array}{c}78.6 \pm 2.9 \\
(50)\end{array}$ \\
\hline Litter weight, males ${ }^{\mathrm{e}}$ & $\begin{array}{c}40.1 \pm 1.7 \\
(73)\end{array}$ & $\begin{array}{c}43.6 \pm 2.3 \\
(62)\end{array}$ & $\begin{array}{c}43.5 \pm 2.2 \\
(61)\end{array}$ & $\begin{array}{c}39.1 \pm 1.6 \\
(64)\end{array}$ & $\begin{array}{c}41.6 \pm 1.8 \\
(63)\end{array}$ & $\begin{array}{c}38.1 \pm 2.0 \\
(64)\end{array}$ & $\begin{array}{c}41.8 \pm 2.9 \\
(39)\end{array}$ & $\begin{array}{c}38.6 \pm 2.0 \\
(50)\end{array}$ \\
\hline Litter weight, females ${ }^{\mathrm{e}}$ & $\begin{array}{l}38.5 \pm 1.6 \\
(73)\end{array}$ & $\begin{array}{c}37.2 \pm 2.3 \\
\quad(62)\end{array}$ & $\begin{array}{c}36.7 \pm 2.0 \\
(61)\end{array}$ & $\begin{array}{c}36.4 \pm 2.2 \\
\quad(64)\end{array}$ & $\begin{array}{c}37.0 \pm 1.7 \\
(63)\end{array}$ & $\begin{array}{c}37.2 \pm 1.7 \\
(64)\end{array}$ & $\begin{array}{c}34.9 \pm 2.4 \\
(39)\end{array}$ & $\begin{array}{c}40 \pm 2.3 \\
(50)\end{array}$ \\
\hline Mean pup weight ${ }^{\mathrm{f}}$ & $\begin{array}{c}7.0 \pm 0.1 \\
(73)\end{array}$ & $\begin{array}{c}6.8 \pm 0.1 \\
(62)\end{array}$ & $\begin{array}{c}7.0 \pm 0.1 \\
(61)\end{array}$ & $\begin{array}{c}6.9 \pm 0.1 \\
(64)\end{array}$ & $\begin{array}{c}6.9 \pm 0.1 \\
(63)\end{array}$ & $\begin{array}{c}7.0 \pm 0.1 \\
(64)\end{array}$ & $\begin{array}{c}6.8 \pm 0.1 \\
(39)\end{array}$ & $\begin{array}{c}7.0 \pm 0.1 \\
(50)\end{array}$ \\
\hline Mean male pup weight ${ }^{\mathrm{f}}$ & $\begin{array}{c}7.1 \pm 0.1 \\
(73)\end{array}$ & $\begin{array}{l}7.0 \pm 0.1 \\
(62)\end{array}$ & $\begin{array}{l}7.2 \pm 0.1 \\
\quad(61)\end{array}$ & $\begin{array}{c}7.1 \pm 0.1 \\
(64)\end{array}$ & $\begin{array}{c}7.0 \pm 0.1 \\
(63)\end{array}$ & $\begin{array}{l}7.2 \pm 0.1 \\
(64)\end{array}$ & $\begin{array}{l}7.1 \pm 0.1 \\
(39)\end{array}$ & $\begin{array}{l}7.2 \pm 0.1 \\
\quad(50)\end{array}$ \\
\hline Mean female pup weight ${ }^{f}$ & $\begin{array}{c}6.8 \pm 0.1 \\
(73)\end{array}$ & $\begin{array}{c}6.6 \pm 0.1 \\
(62)\end{array}$ & $\begin{array}{c}6.8 \pm 0.1 \\
(61)\end{array}$ & $\begin{array}{c}6.8 \pm 0.1 \\
(64)\end{array}$ & $\begin{array}{c}6.7 \pm 0.1 \\
(63)\end{array}$ & $\begin{array}{c}6.9 \pm 0.1 \\
(64)\end{array}$ & $\begin{array}{c}6.6 \pm 0.1 \\
(39)\end{array}$ & $\begin{array}{c}6.9 \pm 0.1 \\
(50)\end{array}$ \\
\hline
\end{tabular}

${ }^{a} \mathrm{BPA}$ and $\mathrm{EE}_{2}$ doses are $\mu \mathrm{g} / \mathrm{kg}$ bw/day. Numbers in parentheses are numbers of dams (for implantation sites) or litters from which data were collected. All analyses and

adjustments for multiple comparisons were performed separately for the BPA and $\mathrm{EE}_{2}$ treatments. Dunnett's method was used to adjust for multiple comparisons. Tests of trend, for increasing treatment effect with increasing dose, were performed for the vehicle and BPA groups. All tests were performed as two-sided tests at the 0.05 significance level.

There were no statistically significant treatment effects for any endpoint. There were also no significant treatment effects in the sensitivity analysis that excluded all animals that overlapped with animals treated with 250,000 $\mu \mathrm{g}$ BPA/kg bw/day (see Materials and Methods, Statistical Methods). Full results of the analyses are found in Supplemental Appendices XVIII (implantation sites) and XIX (all other endpoints).

${ }^{b}$ Litter size (number alive) and numbers of males and females were analyzed using Poisson regression.

'Unsexed pups (i.e., pups that could not be definitively assigned as male or female) were assigned as males for analysis of sex proportions and of female and male counts.

${ }^{\mathrm{d}}$ Sex proportions were analyzed using logistic regression. 


\section{CLARITY-BPA Core Study}

e Litter weight data (g), across and by sex, were analyzed using contrasts within a one-way ANOVA to test for treatment effects. ${ }^{\mathrm{f}}$ Litter mean pup weights $(\mathrm{g})$ were analyzed using ANOCOVA, with litter size as a covariate, to test for treatment effects. 
Table 12. Survival of Female Pups from PND 1 to Weaning in the Vehicle, BPA, and $\mathrm{EE}_{2}$ Dose Groups $^{\mathrm{a}}$

\begin{tabular}{|c|c|c|c|c|c|c|c|c|}
\hline Preweaning Females & Vehicle & $\begin{array}{c}2.5 \\
\text { BPA }\end{array}$ & $\begin{array}{c}25 \\
\text { BPA }\end{array}$ & $\begin{array}{r}250 \\
\text { BPA }\end{array}$ & $\begin{array}{l}2500 \\
\text { BPA }\end{array}$ & $\begin{array}{l}25000 \\
\text { BPA }\end{array}$ & $\begin{array}{l}0.05 \\
\mathrm{EE}_{2}\end{array}$ & $\begin{array}{c}0.5 \\
\mathrm{EE}_{2}\end{array}$ \\
\hline $\begin{array}{l}\text { Number of female pups } \\
\text { after PND } 1 \text { culling }\end{array}$ & 311 & 266 & 259 & 250 & 260 & 244 & 153 & 180 \\
\hline Dead & 5 & 11 & 7 & 6 & 2 & 3 & 8 & 5 \\
\hline Missing & 3 & 5 & 2 & 5 & 13 & 6 & 5 & 3 \\
\hline Moribund & 1 & 1 & 5 & 4 & 1 & 2 & 2 & 0 \\
\hline Reallocated $^{\mathrm{b}}$ & 8 & 8 & 8 & 8 & 8 & 8 & 8 & 8 \\
\hline $\begin{array}{l}\text { Pups surviving to } \\
\text { weaning }\end{array}$ & 294 & 241 & 237 & 227 & 236 & 225 & 130 & 164 \\
\hline $\begin{array}{l}\text { Percent survival at } \\
\text { weaning }\end{array}$ & 95 & 91 & 92 & 91 & 91 & 92 & 85 & 91 \\
\hline $\begin{array}{l}\text { Survival analysis, } \\
p \text {-value }\end{array}$ & $0.361^{\mathrm{c}}$ & 0.245 & 0.280 & 0.245 & 0.245 & 0.313 & $0.005^{* *}$ & 0.369 \\
\hline
\end{tabular}

${ }^{\mathrm{a}} \mathrm{BPA}$ and $\mathrm{EE}_{2}$ doses are $\mu \mathrm{g} / \mathrm{kg}$ bw/day. Cox proportional hazard analyses were performed separately for the BPA and $\mathrm{EE}_{2}$ dose groups. Female pups removed as dead or moribund were considered uncensored, while pups surviving to weaning were considered censored. Multiple comparisons of treatments to the vehicle control were adjusted using Holm's method. All tests were performed as two-sided tests at the 0.05 significance level. Statistically significant effects are indicated by asterisks $(* *$, $p<0.01$ ). There were no additional significant treatment effects in the sensitivity analysis that excluded all animals that overlapped with animals treated with $250,000 \mu \mathrm{g}$ BPA $/ \mathrm{kg}$ bw/day (see Materials and Methods, Statistical Methods). The full statistical report for this endpoint is found in Supplemental Appendix XX.

${ }^{\mathrm{b}}$ The reallocated pups were removed by design on PND 15 for the associated CLARITY-BPA study that will be reported separately.

${ }^{\mathrm{c}} \mathrm{A}$ test of dose trend, increasing treatment effect with increasing dose, was performed for vehicle and BPA groups only. The $p$-value for the trend analysis is given in the vehicle column; $p$-values in BPA and $\mathrm{EE}_{2}$ columns are for pairwise comparisons to the vehicle control. 
Table 13. Survival of Male Pups from PND 1 to Weaning in the Vehicle, BPA, and EE Dose $_{2}$ Groups $^{\mathrm{a}}$

\begin{tabular}{|c|c|c|c|c|c|c|c|c|}
\hline Preweaning Males & Vehicle & $\begin{array}{c}2.5 \\
\text { BPA }\end{array}$ & $\begin{array}{c}25 \\
\text { BPA } \\
\end{array}$ & $\begin{aligned} 250 \\
\text { BPA }\end{aligned}$ & $\begin{array}{l}2500 \\
\text { BPA }\end{array}$ & $\begin{array}{l}25000 \\
\text { BPA }\end{array}$ & $\begin{array}{l}0.05 \\
\mathbf{E E}_{2} \\
\end{array}$ & $\begin{array}{c}0.5 \\
\mathrm{EE}_{2} \\
\end{array}$ \\
\hline $\begin{array}{l}\text { Number of male pups } \\
\text { after PND } 1 \text { culling }\end{array}$ & 338 & 300 & 281 & 292 & 292 & 275 & 156 & 208 \\
\hline Dead & 5 & 1 & 4 & 8 & 4 & 2 & 8 & 5 \\
\hline Missing & 9 & 14 & 5 & 9 & 6 & 1 & 5 & 6 \\
\hline Moribund & 1 & 3 & 1 & 0 & 1 & 4 & 2 & 0 \\
\hline Reallocated ${ }^{\mathrm{b}}$ & 8 & 8 & 8 & 8 & 8 & 8 & 8 & 8 \\
\hline $\begin{array}{l}\text { Pups surviving to } \\
\text { weaning }\end{array}$ & 315 & 274 & 263 & 267 & 273 & 260 & 133 & 189 \\
\hline $\begin{array}{l}\text { Percent survival at } \\
\text { weaning }\end{array}$ & 93 & 91 & 94 & 91 & 93 & 95 & 85 & 91 \\
\hline $\begin{array}{l}\text { Survival analysis, } \\
p \text {-value }\end{array}$ & $0.143^{c}$ & 1.000 & 1.000 & 1.000 & 1.000 & 1.000 & 0.062 & 0.656 \\
\hline \multicolumn{9}{|c|}{$\begin{array}{l}\text { BPA and } \mathrm{EE}_{2} \text { doses are } \mu \mathrm{g} / \mathrm{kg} \text { bw/day. Cox proportional hazard analyses were performed separately for the BPA and } \mathrm{EE}_{2} \text { dose } \\
\text { groups. Male pups removed as dead or moribund were considered uncensored, while pups surviving to weaning were considered } \\
\text { censored. Multiple comparisons of treatments to the vehicle control were adjusted using Holm's method. All tests were } \\
\text { performed as two-sided tests at the } 0.05 \text { significance level. There were no statistically significant treatment effects. There were } \\
\text { also no significant treatment effects in the sensitivity analysis that excluded all animals that overlapped with animals treated with } \\
250,000 \mu \mathrm{g} \text { BPA/kg bw/day (see Materials and Methods, Statistical Methods). The full statistical report for this endpoint is found } \\
\text { in Supplemental Appendix XX. } \\
\text { bThe reallocated pups were removed by design on PND } 15 \text { for the associated CLARITY-BPA study that will be reported } \\
\text { separately. } \\
{ }^{c} \text { A test of dose trend, increasing treatment effect with increasing dose, was performed for vehicle and BPA groups only. The } \\
p \text {-value for the trend analysis is given in the vehicle column; } p \text {-values in BPA and } \mathrm{EE}_{2} \text { columns are for pairwise comparisons to } \\
\text { the vehicle control. }\end{array}$} \\
\hline
\end{tabular}


Table 14. Survival of Female Pups from Weaning to Interim (1 Year) Sacrifice in the Continuous Vehicle, BPA, and EE 2 Dose Groups ${ }^{\mathrm{a}}$

\begin{tabular}{|c|c|c|c|c|c|c|c|c|}
\hline Interim Sacrifice Females & Vehicle & $\begin{array}{r}2.5 \\
\text { BPA } \\
\end{array}$ & $\begin{array}{c}25 \\
\text { BPA } \\
\end{array}$ & $\begin{aligned} 250 \\
\text { BPA }\end{aligned}$ & $\begin{array}{l}2500 \\
\text { BPA }\end{array}$ & $\begin{array}{l}\text { 25000 } \\
\text { BPA }\end{array}$ & $\begin{array}{l}\mathbf{0 . 0 5} \\
\mathbf{E E}_{2}\end{array}$ & $\begin{array}{l}\mathbf{0 . 5 0} \\
\mathrm{EE}_{2}\end{array}$ \\
\hline $\begin{array}{l}\text { Females initially allocated } \\
\text { for interim evaluation }\end{array}$ & 23 & 22 & 22 & 24 & 20 & 24 & 26 & 26 \\
\hline Moribund & $1^{\mathrm{b}}$ & 0 & 0 & $2^{\mathrm{d}}$ & 0 & 0 & $1^{\mathrm{e}}$ & 0 \\
\hline Natural deaths & $1^{\mathrm{b}}$ & 0 & $1^{\mathrm{c}}$ & 0 & 0 & 0 & $1^{\mathrm{e}}$ & 0 \\
\hline $\begin{array}{l}\text { Animals surviving to } \\
\text { scheduled termination }\end{array}$ & 21 & 22 & 21 & 22 & 20 & 24 & 24 & 26 \\
\hline $\begin{array}{l}\text { Percent survival at end of } \\
\text { study }\end{array}$ & 91 & 100 & 95 & 92 & 100 & 100 & 92 & 100 \\
\hline Survival analysis, $p$-value & $0.470^{\mathrm{f}}$ & 1.000 & 1.000 & 1.000 & 1.000 & 1.000 & 0.921 & 0.605 \\
\hline
\end{tabular}

${ }^{\mathrm{a} B P A}$ and $\mathrm{EE}_{2}$ doses are $\mu \mathrm{g} / \mathrm{kg}$ bw/day. Cox proportional hazard analyses were performed separately for the BPA and $\mathrm{EE}_{2} \mathrm{dose}$ groups for all female pups assigned to the continuously dosed interim sacrifice group at weaning. Female pups removed as dead or moribund were considered uncensored, while animals surviving to scheduled removal were considered censored. Since there was $100 \%$ survival in both $\mathrm{BPA}$ and $\mathrm{EE}_{2}$ dose groups, a modified analysis in which one was added to the number of all uncensored observations was conducted to allow estimability. Multiple comparisons of treatments to the vehicle control were adjusted using Holm's method. All tests were performed as two-sided tests at the 0.05 significance level. There were no statistically significant treatment effects. There were also no significant treatment effects in the sensitivity analysis that excluded all animals that overlapped with animals treated with 250,000 $\mu \mathrm{g} \mathrm{BPA} / \mathrm{kg}$ bw/day (see Materials and Methods, Statistical Methods). The full statistical report for this endpoint is found in Supplemental Appendix XXI.

${ }^{b}$ One animal had nephropathy, while the cause of death/morbidity of the second animal was uncertain (Subappendix VI in Supplemental Appendix XXXII).

'Nephropathy was the cause of death (Subappendix VI in Supplemental Appendix XXXII).

${ }^{\mathrm{d}}$ Nephropathy was the cause of death/morbidity in one animal and a mammary fibroadenoma in the second animal (Subappendix VI in Supplemental Appendix XXXII).

${ }^{e}$ One animal had a mammary adenocarcinoma, while the cause of death/morbidity in the second animal was uncertain (Subappendix VI in Supplemental Appendix XXXII).

${ }^{\mathrm{f}} \mathrm{A}$ test of dose trend, increasing treatment effect with increasing dose, was performed for vehicle and BPA groups only. The $p$-value for the trend analysis is given in the vehicle column; $p$-values in BPA and $\mathrm{EE}_{2}$ columns are for pairwise comparisons to the vehicle control. 
Table 15. Survival of Female Pups from Weaning to Interim (1 Year) Sacrifice in the Stop-Dose Vehicle and BPA Groups ${ }^{a}$

\begin{tabular}{lcccccc}
\hline \multicolumn{1}{c}{ Interim Sacrifice Females } & Vehicle & 2.5 BPA & 25 BPA & 250 BPA & 2500 BPA & 25000 BPA \\
\hline Females initially allocated for & 20 & 22 & 20 & 22 & 20 & 22 \\
interim evaluation & 0 & 0 & 0 & 0 & 0 & $2^{\mathrm{b}}$ \\
Moribund & 0 & 0 & 0 & 0 & 0 & 0 \\
Natural deaths & 20 & 22 & 20 & 22 & 20 & 20 \\
$\begin{array}{l}\text { Animals surviving to scheduled } \\
\text { termination }\end{array}$ & 100 & 100 & 100 & 100 & 100 & 91 \\
Percent survival at end of study & $0.455^{\mathrm{c}}$ & 1.000 & 1.000 & 1.000 & 1.000 & 1.000 \\
Survival analysis, $p$-value & & & & & & \\
\hline
\end{tabular}

${ }^{a}$ BPA doses are $\mu \mathrm{g} / \mathrm{kg}$ bw/day. Cox proportional hazard analyses were performed for the BPA dose groups for all female pups assigned to the stop-dose interim sacrifice group at weaning. Female pups removed as dead or moribund were considered uncensored, while animals surviving to scheduled removal were considered censored. Since there was $100 \%$ survival in most dose groups, a modified analysis in which one was added to the number of all uncensored observations was conducted to allow estimability. Multiple comparisons of treatments to the vehicle control were adjusted using Holm's method. All tests were performed as two-sided tests at the 0.05 significance level. There were no statistically significant treatment effects. There were also no significant treatment effects in the sensitivity analysis that excluded all animals that overlapped with animals treated with $250,000 \mu \mathrm{g} \mathrm{BPA} / \mathrm{kg}$ bw/day (see Materials and Methods, Statistical Methods). The full statistical report for this endpoint is found in Supplemental Appendix XXI.

${ }^{\mathrm{b}}$ One animal had nephropathy and the second animal had a malignant meningioma of the cerebellum as the primary cause of morbidity/death (Subappendix VI in Supplemental Appendix XXXII).

${ }^{\mathrm{c}}$ A test of dose trend, increasing treatment effect with increasing dose, was performed and the $p$-value for the trend analysis is given in the vehicle column; $p$-values in BPA columns are for pairwise comparisons to the vehicle control. 
Table 16. Survival of Male Pups from Weaning to Interim (1 Year) Sacrifice in the Continuous Vehicle, BPA, and EE 2 Dose Groups ${ }^{\mathrm{a}}$

\begin{tabular}{|c|c|c|c|c|c|c|c|c|}
\hline Interim Sacrifice Males & Vehicle & $\begin{array}{c}2.5 \\
\text { BPA } \\
\end{array}$ & $\begin{array}{c}25 \\
\text { BPA } \\
\end{array}$ & $\begin{array}{r}250 \\
\text { BPA }\end{array}$ & $\begin{array}{l}2500 \\
\text { BPA }\end{array}$ & $\begin{array}{c}25000 \\
\text { BPA }\end{array}$ & $\begin{array}{l}0.05 \\
\mathbf{E E}_{2}\end{array}$ & $\begin{array}{r}\mathbf{0 . 5} \\
\mathrm{EE}_{2} \\
\end{array}$ \\
\hline $\begin{array}{l}\text { Males initially allocated } \\
\text { for interim evaluation }\end{array}$ & 22 & 22 & 20 & 24 & 20 & 22 & 26 & 26 \\
\hline Moribund & $4^{\mathrm{b}}$ & 0 & $1^{\mathrm{c}}$ & 0 & 0 & 0 & $2^{\mathrm{e}}$ & 0 \\
\hline Natural deaths & 0 & 0 & $1^{\mathrm{c}}$ & 0 & $2^{\mathrm{d}}$ & $1^{\mathrm{d}}$ & $2^{\mathrm{e}}$ & $3^{\mathrm{d}}$ \\
\hline $\begin{array}{l}\text { Animals surviving to } \\
\text { scheduled termination }\end{array}$ & 18 & 22 & 18 & 24 & 18 & 21 & 22 & 23 \\
\hline $\begin{array}{l}\text { Percent survival at end of } \\
\text { study }\end{array}$ & 82 & 100 & 90 & 100 & 90 & 95 & 85 & 88 \\
\hline Survival analysis, $p$-value & $0.666^{\mathrm{f}}$ & 0.597 & 1.000 & 0.597 & 1.000 & 0.789 & 1.000 & 1.000 \\
\hline
\end{tabular}

${ }^{\mathrm{a}} \mathrm{BPA}$ and $\mathrm{EE}_{2}$ doses are $\mu \mathrm{g} / \mathrm{kg}$ bw/day. Cox proportional hazard analyses were performed separately for the $\mathrm{BPA}_{\text {and }} \mathrm{EE}_{2}$ dose groups for all male pups assigned to the continuously dosed interim sacrifice group at weaning. Animals removed as dead or moribund were considered uncensored, while animals surviving to scheduled removal were considered censored. Since there was $100 \%$ survival in some of the BPA dose groups, a modified analysis in which one was added to the number of all uncensored observations was conducted to allow estimability. Multiple comparisons of treatments to the vehicle control were adjusted using Holm's method. All tests were performed as two-sided tests at the 0.05 significance level. There were no statistically significant treatment effects. There were also no significant treatment effects in the sensitivity analysis that excluded all animals that overlapped with animals treated with 250,000 $\mu \mathrm{g}$ BPA/kg bw/day (see Materials and Methods, Statistical Methods). The full statistical report for this endpoint is found in Supplemental Appendix XXI.

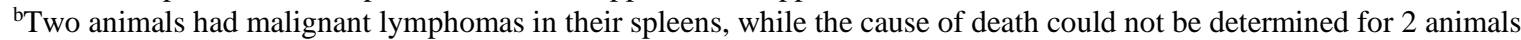
(Subappendix VI in Supplemental Appendix XXXII).

${ }^{\mathrm{c}}$ One animal had a perforated esophagus and another had a hemorrhaged lung (Subappendix VI in Supplemental Appendix XXXII).

${ }^{\mathrm{d}}$ Cause of death/morbidity was uncertain (Subappendix VI in Supplemental Appendix XXXII).

${ }^{e}$ One animal had nephropathy, one animal was removed due to an abscessed skin wound, and the cause of death/morbidity for two animals was uncertain (Subappendix VI in Supplemental Appendix XXXII).

${ }^{\mathrm{f}} \mathrm{A}$ test of dose trend, increasing treatment effect with increasing dose, was performed for vehicle and BPA groups only. The $p$-value for the trend analysis is given in the vehicle column; $p$-values in BPA and $\mathrm{EE}_{2}$ columns are for pairwise comparisons to the vehicle control. 
Table 17. Survival of Male Pups from Weaning to Interim (1 Year) Sacrifice in the Stop-Dose Vehicle and BPA Groups ${ }^{\mathrm{a}}$

\begin{tabular}{lcccccc}
\hline \multicolumn{1}{c}{ Interim Sacrifice Males } & Vehicle & 2.5 BPA & 25 BPA & 250 BPA & 2500 BPA & 25000 BPA \\
\hline $\begin{array}{l}\text { Males initially allocated for interim } \\
\text { evaluation }\end{array}$ & 20 & 20 & 20 & 19 & 20 & 22 \\
Moribund & 0 & 0 & 0 & 0 & 0 & 0 \\
Natural deaths & 0 & 0 & $1^{\mathrm{b}}$ & 0 & 0 & 0 \\
$\begin{array}{l}\text { Animals surviving to scheduled } \\
\text { termination }\end{array}$ & 20 & 20 & 19 & 19 & 20 & 22 \\
$\begin{array}{l}\text { Percent survival at end of study } \\
\text { Survival analysis, } p \text {-value }\end{array}$ & 100 & 100 & 95 & 100 & 100 & 100 \\
\hline
\end{tabular}

${ }^{a}$ BPA doses are $\mu \mathrm{g} / \mathrm{kg}$ bw/day. Cox proportional hazard analyses were performed for the BPA dose groups for all male pups assigned to the stop-dose interim sacrifice group at weaning. Animals removed as dead or moribund were considered uncensored, while animals surviving to scheduled removal were considered censored. Since there was $100 \%$ survival in some dose groups, a modified analysis in which one was added to the number of all uncensored observations was conducted to allow estimability. Multiple comparisons of treatments to the vehicle control were adjusted using Holm's method. All tests were performed as twosided tests at the 0.05 significance level. There were no statistically significant treatment effects. There were also no significant treatment effects in the sensitivity analysis that excluded all animals that overlapped with animals treated with $250,000 \mu \mathrm{g}$ BPA $/ \mathrm{kg}$ bw/day (see Materials and Methods, Statistical Methods). The full statistical report for this endpoint is found in Supplemental Appendix XXI.

${ }^{\mathrm{b}}$ Cause of death/morbidity was uncertain (Subappendix VI in Supplemental Appendix XXXII).

${ }^{\mathrm{c}} \mathrm{A}$ test of dose trend, increasing treatment effect with increasing dose, was performed and the $p$-value for the trend analysis is given in the vehicle column; $p$-values in BPA columns are for pairwise comparisons to the vehicle control. 
Table 18. Survival of Female Pups from Weaning to Terminal (2 Year) Sacrifice in the Continuous Vehicle, BPA, and EE 2 Dose Groups ${ }^{\mathrm{a}}$

\begin{tabular}{|c|c|c|c|c|c|c|c|c|}
\hline $\begin{array}{c}\text { Terminal Sacrifice } \\
\text { Females } \\
\end{array}$ & Vehicle & $\begin{array}{c}2.5 \\
\text { BPA } \\
\end{array}$ & $\begin{array}{c}25 \\
\text { BPA } \\
\end{array}$ & $\begin{array}{r}250 \\
\text { BPA } \\
\end{array}$ & $\begin{array}{l}2500 \\
\text { BPA } \\
\end{array}$ & $\begin{array}{c}25000 \\
\text { BPA }\end{array}$ & $\begin{array}{l}\mathbf{0 . 0 5} \\
\mathbf{E E}_{2} \\
\end{array}$ & $\begin{array}{l}0.50 \\
\mathbf{E E}_{2}\end{array}$ \\
\hline $\begin{array}{l}\text { Females initially allocated } \\
\text { for terminal evaluation }\end{array}$ & 50 & 48 & 46 & 49 & 50 & 46 & 26 & 26 \\
\hline Moribund & 28 & 28 & 31 & 31 & 33 & 35 & 18 & 18 \\
\hline Natural deaths & 6 & 1 & 1 & 5 & 7 & 3 & 1 & 4 \\
\hline $\begin{array}{l}\text { Animals surviving to } \\
\text { scheduled termination }\end{array}$ & 16 & 19 & 14 & 13 & 10 & 8 & 7 & 4 \\
\hline $\begin{array}{l}\text { Percent survival at end of } \\
\text { study }\end{array}$ & 32 & 40 & 30 & 27 & 20 & 17 & 27 & 15 \\
\hline Survival analysis, $p$-value & $0.071^{\mathrm{b}}$ & 1.000 & 1.000 & 1.000 & 0.502 & 1.000 & 0.396 & 0.188 \\
\hline \multicolumn{9}{|c|}{$\begin{array}{l}\text { BPA and } \mathrm{EE}_{2} \text { doses are } \mu \mathrm{g} / \mathrm{kg} \text { bw/day. Cox proportional hazard analyses were performed separately for the BPA and EE } \mathrm{B}_{2} \mathrm{dose}^{\mathrm{B}} \\
\text { groups for all female pups assigned to the continuously dosed terminal sacrifice group at weaning. Animals removed as dead or } \\
\text { moribund were considered uncensored, while animals surviving to scheduled removal were considered censored. Multiple } \\
\text { comparisons of treatments to the vehicle control were adjusted using Holm's method. All tests were performed as two-sided tests } \\
\text { at the } 0.05 \text { significance level. There were no statistically significant treatment effects. There were also no significant treatment } \\
\text { effects in the sensitivity analysis that excluded all animals that overlapped with animals treated with } 250,000 \mu \mathrm{g} \text { BPA/kg bw/day } \\
\text { (see Materials and Methods, Statistical Methods). The full statistical report for this endpoint is found in Supplemental Appendix } \\
\text { XXII. } \\
\text { bA test of dose trend, increasing treatment effect with increasing dose, was performed for vehicle and BPA groups only. The } \\
p \text {-value for the trend analysis is given in the vehicle column; } p \text {-values in BPA and EE columns are for pairwise comparisons to } \\
\text { the vehicle control. }\end{array}$} \\
\hline
\end{tabular}


Table 19. Survival of Female Pups from Weaning to Terminal (2 Year) Sacrifice in the Stop-Dose Vehicle and BPA Groups ${ }^{a}$

\begin{tabular}{lcccccc}
\hline \multicolumn{1}{c}{ Terminal Sacrifice Females } & Vehicle & 2.5 BPA & 25 BPA & 250 BPA & 2500 BPA & 25000 BPA \\
\hline Females initially allocated for & 50 & 50 & 48 & 50 & 50 & 46 \\
terminal evaluation & 36 & 32 & 32 & 35 & 30 & 31 \\
Moribund & 3 & 6 & 3 & 2 & 3 & 2 \\
Natural deaths & 11 & 12 & 13 & 13 & 17 & 13 \\
Animals surviving to scheduled & & & & & & \\
termination & 22 & 24 & 27 & 26 & 34 & 28 \\
Percent survival at end of study & $0.203^{\mathrm{b}}$ & 1.000 & 1.000 & 1.000 & 1.000 & 1.000 \\
Survival analysis, $p$-value & & &
\end{tabular}

${ }^{a}$ BPA doses are $\mu \mathrm{g} / \mathrm{kg}$ bw/day. Cox proportional hazard analyses were performed for the BPA dose groups for all female pups assigned to the stop-dose interim sacrifice group at weaning. Animals removed as dead or moribund were considered uncensored, while animals surviving to scheduled removal were considered censored. Multiple comparisons of treatments to the vehicle control were adjusted using Holm's method. All tests were performed as two-sided tests at the 0.05 significance level. There were no statistically significant treatment effects. There were also no significant treatment effects in the sensitivity analysis that excluded all animals that overlapped with animals treated with 250,000 $\mu \mathrm{g}$ BPA/kg bw/day (see Materials and Methods, Statistical Methods). The full statistical report for this endpoint is found in Supplemental Appendix XXII.

${ }^{\mathrm{b}} \mathrm{A}$ test of dose trend, increasing treatment effect with increasing dose, was performed and the $p$-value for the trend analysis is given in the vehicle column; $p$-values in BPA columns are for pairwise comparisons to the vehicle control. 
Table 20. Survival of Male Pups from Weaning to Terminal (2 Year) Sacrifice in the Continuous Vehicle, BPA, and EE 2 Dose Groups ${ }^{\mathrm{a}}$

\begin{tabular}{|c|c|c|c|c|c|c|c|c|}
\hline Terminal Sacrifice Males & Vehicle & $\begin{array}{c}2.5 \\
\text { BPA } \\
\end{array}$ & $\begin{array}{c}25 \\
\text { BPA }\end{array}$ & $\begin{array}{r}250 \\
\text { BPA } \\
\end{array}$ & $\begin{array}{l}2500 \\
\text { BPA }\end{array}$ & $\begin{array}{c}25000 \\
\text { BPA }\end{array}$ & $\begin{array}{l}\mathbf{0 . 0 5} \\
\mathbf{E E}_{2}\end{array}$ & $\begin{array}{l}\mathbf{0 . 5 0} \\
\mathbf{E E}_{2}\end{array}$ \\
\hline $\begin{array}{l}\text { Males initially allocated } \\
\text { for terminal evaluation }\end{array}$ & 50 & 48 & 48 & 50 & 50 & 46 & 26 & 26 \\
\hline Moribund & 24 & 16 & 27 & 21 & 24 & 27 & 14 & 10 \\
\hline Natural deaths & 11 & 16 & 4 & 15 & 10 & 8 & 3 & 4 \\
\hline $\begin{array}{l}\text { Animals surviving to } \\
\text { scheduled termination }\end{array}$ & 15 & 16 & 17 & 14 & 16 & 11 & 9 & 12 \\
\hline $\begin{array}{l}\text { Percent survival at end of } \\
\text { study }\end{array}$ & 30 & 33 & 35 & 28 & 32 & 24 & 35 & 46 \\
\hline Survival analysis, $p$-value & $0.327^{\mathrm{b}}$ & 1.000 & 1.000 & 1.000 & 1.000 & 1.000 & 0.879 & 0.419 \\
\hline
\end{tabular}

${ }^{\mathrm{a} B P A}$ and $\mathrm{EE}_{2}$ doses are $\mu \mathrm{g} / \mathrm{kg}$ bw/day. Cox proportional hazard analyses were performed separately for the $\mathrm{BPA}_{\text {and }} \mathrm{EE}_{2} \mathrm{dose}$ groups for all male pups assigned to the continuously dosed terminal sacrifice group at weaning. Animals removed as dead or moribund were considered uncensored, while animals surviving to scheduled removal were considered censored. Multiple comparisons of treatments to the vehicle control were adjusted using Holm's method. All tests were performed as two-sided tests at the 0.05 significance level. There were no statistically significant treatment effects. There were also no significant treatment effects in the sensitivity analysis that excluded all animals that overlapped with animals treated with $250,000 \mu \mathrm{g}$ BPA/kg bw/day (see Materials and Methods, Statistical Methods). The full statistical report for this endpoint, including is found in Supplemental Appendix XXII.

${ }^{\mathrm{b}} \mathrm{A}$ test of dose trend, increasing treatment effect with increasing dose, was performed for vehicle and BPA groups only. The $p$-value for the trend analysis is given in the vehicle column; $p$-values in BPA and $\mathrm{EE}_{2}$ columns are for pairwise comparisons to the vehicle control. 
Table 21. Survival of Male Pups from Weaning to Terminal (2 Year) Sacrifice in the Stop-Dose Vehicle and BPA Groups ${ }^{\mathrm{a}}$

\begin{tabular}{lcccccc}
\hline \multicolumn{1}{c}{ Terminal Sacrifice Males } & Vehicle & 2.5 BPA & 25 BPA & 250 BPA & 2500 BPA & 25000 BPA \\
\hline Males initially allocated for & 50 & 48 & 48 & 50 & 50 & 46 \\
terminal evaluation & 20 & 20 & 24 & 29 & 27 & 29 \\
Moribund & 13 & 12 & 8 & 8 & 8 & 8 \\
Natural deaths & 17 & 16 & 16 & 13 & 15 & 9 \\
Animals surviving to scheduled & & & & & & \\
termination & 34 & 33 & 33 & 26 & 30 & 20 \\
Percent survival at end of study & $0.053^{\mathrm{b}}$ & 1.000 & 1.000 & 0.424 & 1.000 & 0.209 \\
Survival analysis, $p$-value & & &
\end{tabular}

${ }^{a}$ BPA doses are $\mu \mathrm{g} / \mathrm{kg}$ bw/day. Cox proportional hazard analyses were performed for the BPA dose groups for all male pups assigned to the stop-dose interim sacrifice group at weaning. Animals removed as dead or moribund were considered uncensored, while animals surviving to scheduled removal were considered censored. Multiple comparisons of treatments to the vehicle control were adjusted using Holm's method. All tests were performed as two-sided tests at the 0.05 significance level. There were no statistically significant treatment effects. There were also no significant treatment effects in the sensitivity analysis that excluded all animals that overlapped with animals treated with 250,000 $\mu \mathrm{g}$ BPA/kg bw/day (see Materials and Methods, Statistical Methods). The full statistical report for this endpoint is found in Supplemental Appendix XXII.

${ }^{\mathrm{b}} \mathrm{A}$ test of dose trend, increasing treatment effect with increasing dose, was performed and the $p$-value for the trend analysis is given in the vehicle column; $p$-values in BPA columns are for pairwise comparisons to the vehicle control. 
Table 22. Prewean Body Weights (g) of Female Pups in the Vehicle, BPA, and $\mathrm{EE}_{2}$ Dose Groups

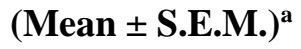

\begin{tabular}{|c|c|c|c|c|c|c|c|c|}
\hline $\begin{array}{c}\text { Postnatal } \\
\text { Day }\end{array}$ & Vehicle & 2.5 BPA & 25 BPA & 250 BPA & 2500 BPA & 25000 BPA & $0.05 \mathrm{EE}_{2}$ & $0.5 \mathbf{E E}_{2}$ \\
\hline 1 & $\begin{array}{c}6.8 \pm 0.1 \\
(71)\end{array}$ & $\begin{array}{c}6.7 \pm 0.1 \\
(60)\end{array}$ & & & & & & \\
\hline 4 & $\begin{array}{c}10.6 \pm 0.1 \\
\quad(71)\end{array}$ & $\begin{array}{c}10.1 \pm 0.2 \\
\quad(59)\end{array}$ & $\begin{array}{c}10.5 \pm 0.2 \\
\quad(57)\end{array}$ & $\begin{array}{c}10.4 \pm 0.1 \\
\quad(58)\end{array}$ & $\begin{array}{c}10.3 \pm 0.2 \\
\quad(58)\end{array}$ & $\begin{array}{c}10.4 \pm 0.2 \\
\quad(59)\end{array}$ & $\begin{array}{c}10.1 \pm 0.2^{*} \\
(34)\end{array}$ & $\begin{array}{c}10.9 \pm 0.2 \\
\quad(47)\end{array}$ \\
\hline 7 & $\begin{array}{c}15.8 \pm 0.2 \\
(71)\end{array}$ & $\begin{array}{c}15.2 \pm 0.2 \\
(59)\end{array}$ & $\begin{array}{c}15.6 \pm 0.2 \\
(57)\end{array}$ & $\begin{array}{c}15.3 \pm 0.2 \\
(58)\end{array}$ & $\begin{array}{c}15.1 \pm 0.3 \\
(58)\end{array}$ & $\begin{array}{c}15.4 \pm 0.2 \\
(59)\end{array}$ & $\begin{array}{r}15.0= \\
(3=\end{array}$ & $\begin{array}{c}16.0 \pm 0.3 \\
(47)\end{array}$ \\
\hline 14 & $\begin{array}{r}30.6 \\
(7\end{array}$ & 30.1 & $\begin{array}{r}30.9 \\
(5\end{array}$ & $\begin{array}{r}30.0 \\
(5\end{array}$ & 29.6 & $\begin{array}{r}29.9 \\
(5\end{array}$ & $\begin{array}{r}29.9 \\
(3\end{array}$ & $\begin{array}{c}31.4 \pm 0.4 \\
\quad(47)\end{array}$ \\
\hline 21 & $\begin{array}{c}50.1 \pm 0.6 \\
(70)\end{array}$ & $\begin{array}{c}49.5 \pm 0.7 \\
(56)\end{array}$ & $\begin{array}{r}50.6 \\
(5 \\
\end{array}$ & $\begin{array}{c}49.2 \pm 0.7 \\
(56)\end{array}$ & $\begin{array}{r}48.6 \\
\quad 5 \\
\end{array}$ & $\begin{array}{c}49.1 \pm 0.6 \\
(57)\end{array}$ & $\begin{array}{c}50.2 \pm 0.9 \\
(33)\end{array}$ & $\begin{array}{c}51.9 \pm 0.7 \\
(47)\end{array}$ \\
\hline . & 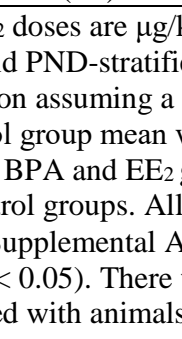 & 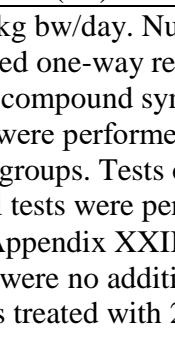 & 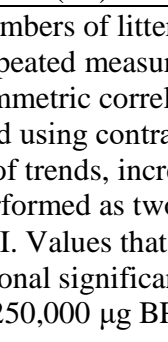 & & & - & & $\begin{array}{l}\text { ntrasts } \\
\text { ting for } \\
\text { ans to the } \\
\text { isons } \\
\text { for the }\end{array}$ \\
\hline
\end{tabular}


Table 23. Prewean Body Weights (g) of Male Pups in the Vehicle, BPA, and EE 2 Dose Groups

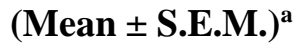

\begin{tabular}{|c|c|c|c|c|c|c|c|c|}
\hline $\begin{array}{c}\text { Postnatal } \\
\text { Day }\end{array}$ & Vehicle & 2.5 BPA & 25 BPA & 250 BPA & 2500 BPA & 25000 BPA & $0.05 \mathrm{EE}_{2}$ & $0.5 \mathrm{EE}_{2}$ \\
\hline 1 & & & & & & & & \\
\hline 4 & 11 & $11 \pm$ & 0.2 & 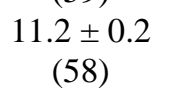 & 0 & 11 & ) & $11.3 \pm 0.2$ \\
\hline 7 & 169 & 16.4 & 16.8 & 0.2 & 16. & & 0.4 & 0.3 \\
\hline 14 & 32.3 & 31.7 & 32.4 & 4 & 31.3 & 31.7 & 0.6 & 0.5 \\
\hline 21 & $\begin{array}{c}53.0 \pm 0.6 \\
(70)\end{array}$ & $\begin{array}{c}52.2 \pm 0.6 \\
(58)\end{array}$ & $\begin{array}{c}53.6 \pm 0.6 \\
(56)\end{array}$ & $\begin{array}{r}51.8 \\
(5\end{array}$ & $\begin{array}{r}51.5 \\
(5\end{array}$ & 52.4 & 1.0 & $\begin{array}{l}4.0 \pm 0.8 \\
(47)\end{array}$ \\
\hline \multicolumn{9}{|c|}{$\begin{array}{l}\text { BPA and } \mathrm{EE}_{2} \text { doses are } \mu \mathrm{g} / \mathrm{kg} \text { bw/day. Numbers of litters are shown in parentheses. Analysis was performed using contrasts } \\
\text { within sex- and PND-stratified one-way repeated measures, mixed model ANOVA to test for treatment effects accounting for } \\
\text { itter correlation assuming a compound symmetric correlation structure. Pairwise comparisons of treatment group means to the } \\
\text { vehicle control group mean were performed using contrasts with Dunnett's method of adjustment for multiple comparisons } \\
\text { separately for BPA and } \mathrm{EE}_{2} \text { groups. Tests of trends, increasing treatment effect with increasing dose, were performed for the } \\
\text { BPA and control groups. All tests were performed as two-sided tests at the } 0.05 \text { significance level. Full results of the analyses are } \\
\text { resented in Supplemental Appendix XXIII. There were no statistically significant trends or pairwise comparisons to controls. } \\
\text { There were also no significant treatment effects in the sensitivity analysis that excluded all animals that overlapped with animals } \\
\text { reated with } 250,000 \mu \mathrm{g} \text { BPA/kg bw/day (see Materials and Methods, Statistical Methods). }\end{array}$} \\
\hline
\end{tabular}


Table 24. Female Postwean Body Weights (g), Vehicle, BPA, and EE 2 Continuous-Dose, Interim (1 Year) Sacrifice (Mean \pm S.E.M.) ${ }^{\mathrm{a}}$

\begin{tabular}{ccccccccc}
\hline Week & Vehicle & 2.5 BPA & 25 BPA & 250 BPA & 2500 BPA & 25000 BPA & $\mathbf{0 . 0 5 ~ E E}_{2}$ & $\mathbf{0 . 5 ~ E E}_{2}$ \\
\hline 4 & $76 \pm 1$ & $77 \pm 2$ & $76 \pm 2$ & $74 \pm 2$ & $73 \pm 1$ & $75 \pm 2$ & $75 \pm 2$ & $81 \pm 2$ \\
& $(23)$ & $(22)$ & $(22)$ & $(24)$ & $(20)$ & $(24)$ & $(26)$ & $(26)$ \\
8 & $196 \pm 5$ & $203 \pm 4$ & $205 \pm 4$ & $196 \pm 4$ & $196 \pm 4$ & $197 \pm 4$ & $201 \pm 4$ & $209 \pm 4$ \\
& $(23)$ & $(22)$ & $(22)$ & $(24)$ & $(20)$ & $(24)$ & $(26)$ & $(26)$ \\
12 & $268 \pm 6$ & $277 \pm 6$ & $278 \pm 6$ & $266 \pm 6$ & $262 \pm 5$ & $262 \pm 6$ & $273 \pm 7$ & $262 \pm 5$ \\
& $(22)$ & $(22)$ & $(22)$ & $(24)$ & $(20)$ & $(24)$ & $(26)$ & $(26)$ \\
16 & $302 \pm 8$ & $314 \pm 7$ & $317 \pm 7$ & $299 \pm 7$ & $293 \pm 5$ & $293 \pm 7$ & $308 \pm 7$ & $291 \pm 5$ \\
& $(22)$ & $(22)$ & $(22)$ & $(24)$ & $(20)$ & $(24)$ & $(26)$ & $(26)$ \\
20 & $322 \pm 8$ & $342 \pm 9$ & $345 \pm 8$ & $316 \pm 8$ & $311 \pm 6$ & $316 \pm 10$ & $332 \pm 8$ & $312 \pm 6$ \\
& $(22)$ & $(22)$ & $(22)$ & $(24)$ & $(20)$ & $(24)$ & $(26)$ & $(26)$ \\
24 & $340 \pm 10$ & $363 \pm 12$ & $362 \pm 9$ & $331 \pm 9$ & $331 \pm 6$ & $332 \pm 11$ & $352 \pm 9$ & $330 \pm 8$ \\
& $(22)$ & $(22)$ & $(22)$ & $(24)$ & $(20)$ & $(24)$ & $(26)$ & $(26)$ \\
28 & $354 \pm 10$ & $382 \pm 13$ & $380 \pm 11$ & $344 \pm 10$ & $346 \pm 7$ & $351 \pm 12$ & $365 \pm 9$ & $348 \pm 8$ \\
& $(22)$ & $(22)$ & $(22)$ & $(24)$ & $(20)$ & $(24)$ & $(26)$ & $(26)$ \\
32 & $370 \pm 13$ & $403 \pm 14$ & $395 \pm 11$ & $360 \pm 11$ & $362 \pm 8$ & $366 \pm 13$ & $384 \pm 10$ & $367 \pm 10$ \\
& $(22)$ & $(22)$ & $(22)$ & $(24)$ & $(20)$ & $(24)$ & $(26)$ & $(26)$ \\
36 & $384 \pm 14$ & $422 \pm 16 \mathrm{~b}$ & $410 \pm 12$ & $374 \pm 12$ & $376 \pm 9$ & $378 \pm 14$ & $398 \pm 10$ & $384 \pm 10$ \\
& $(22)$ & $(22)$ & $(22)$ & $(24)$ & $(20)$ & $(24)$ & $(26)$ & $(26)$ \\
40 & $396 \pm 15$ & $439 \pm 18^{\mathrm{b}}$ & $422 \pm 12$ & $377 \pm 10$ & $394 \pm 10$ & $396 \pm 14$ & $417 \pm 11$ & $397 \pm 10$ \\
& $(22)$ & $(22)$ & $(22)$ & $(23)$ & $(20)$ & $(24)$ & $(25)$ & $(26)$ \\
44 & $410 \pm 16$ & $455 \pm 19^{\mathrm{b}}$ & $434 \pm 13$ & $389 \pm 11$ & $409 \pm 12$ & $409 \pm 16$ & $434 \pm 11$ & $412 \pm 11$ \\
& $(22)$ & $(22)$ & $(22)$ & $(23)$ & $(20)$ & $(24)$ & $(25)$ & $(26)$ \\
48 & $421 \pm 18$ & $476 \pm 20^{\mathrm{b}}$ & $447 \pm 14$ & $408 \pm 13$ & $424 \pm 12$ & $428 \pm 17$ & $449 \pm 13$ & $427 \pm 12$ \\
& $(21)$ & $(22)$ & $(21)$ & $(22)$ & $(20)$ & $(24)$ & $(24)$ & $(26)$ \\
52 & $436 \pm 19$ & $494 \pm 22^{\mathrm{b}}$ & $460 \pm 15$ & $427 \pm 14$ & $436 \pm 13$ & $441 \pm 18$ & $468 \pm 14$ & $440 \pm 12$ \\
& $(21)$ & $(22)$ & $(21)$ & $(22)$ & $(20)$ & $(24)$ & $(24)$ & $(26)$ \\
\hline
\end{tabular}

${ }^{\mathrm{a} B P A}$ and $\mathrm{EE}_{2}$ doses are $\mu \mathrm{g} / \mathrm{kg}$ bw/day. Numbers of litters are shown in parentheses. Outlier identification and exclusion are described in Materials and Methods and Supplemental Appendix XXIV. Analyses were conducted separately for BPA and EE 2 dose groups. Pairwise comparisons of means were performed using contrasts within a two-way repeated measures, mixed model ANOVA. Model terms were treatment group, weeks, and the interaction. Within-group correlations were modeled using a heterogeneous first-order autoregressive (ARH(1)) correlation structure, which allows for correlated differences in variability across time points. Tests of trends, increasing treatment effect with increasing dose, were performed for the BPA and vehicle control groups. Pairwise comparisons of treatment groups to the vehicle control group were conducted with Dunnett's method to adjust for multiple comparisons. All tests were performed as two-sided tests at the 0.05 significance level. Full results of the analyses are presented in Supplemental Appendix XXIV. There were no statistically significant trends or pairwise comparisons to controls. There were also no significant treatment effects in the sensitivity analysis that excluded all animals that overlapped with animals treated with 250,000 $\mu \mathrm{g} \mathrm{BPA} / \mathrm{kg}$ bw/day (see Materials and Methods, Statistical Methods).

${ }^{b}$ Mean body weights at the later time points, weeks 36 to 52 , were $10-13 \%$ higher than control means in the $2.5 \mu \mathrm{g}$ BPA $/ \mathrm{kg}$ bw/day. The Dunnett-corrected $p$-values were $0.080,0.058,0.071,0.065$, and 0.062 , for weeks $36,40,44,48$, and 52, respectively. 
Table 25. Female Postwean Body Weights (g), Vehicle, BPA, and EE 2 Continuous-Dose, Terminal $(2 \text { Year) Sacrifice (Mean } \pm \text { S.E.M. })^{\mathrm{a}}$

\begin{tabular}{|c|c|c|c|c|c|c|c|c|}
\hline Week & Vehicle & 2.5 BPA & 25 BPA & 250 BPA & 2500 BPA & 25000 BPA & $0.05 \mathrm{EE}_{2}$ & $0.5 \mathrm{EE}_{2}$ \\
\hline 4 & $\begin{array}{c}75 \pm 1 \\
(50)\end{array}$ & $\begin{array}{c}76 \pm 1 \\
(48)\end{array}$ & $\begin{array}{c}78 \pm 1 \\
(46)\end{array}$ & $\begin{array}{c}76 \pm 1 \\
(49)\end{array}$ & $\begin{array}{c}74 \pm 1 \\
(50)\end{array}$ & $\begin{array}{c}76 \pm 1 \\
(46)\end{array}$ & $\begin{array}{c}76 \pm 1 \\
(26)\end{array}$ & $\begin{array}{c}80 \pm 2^{*} \\
(26)\end{array}$ \\
\hline 8 & $\begin{array}{c}196 \pm 3 \\
(50)\end{array}$ & $\begin{array}{c}201 \pm 3 \\
(48)\end{array}$ & $\begin{array}{c}202 \pm 2 \\
(46)\end{array}$ & $\begin{array}{c}199 \pm 2 \\
(49)\end{array}$ & $\begin{array}{c}198 \pm 3 \\
(50)\end{array}$ & $\begin{array}{c}204 \pm 3^{b} \\
(45)\end{array}$ & $\begin{array}{c}200 \pm 4 \\
(26)\end{array}$ & $\begin{array}{c}212 \pm 4^{*} \\
(26)\end{array}$ \\
\hline 12 & $\begin{array}{c}266 \pm 4 \\
(50)\end{array}$ & $\begin{array}{c}269 \pm 4 \\
(48)\end{array}$ & $\begin{array}{c}270 \pm 4 \\
(46)\end{array}$ & $\begin{array}{c}267 \pm 3 \\
(49)\end{array}$ & $\begin{array}{c}266 \pm 4 \\
(50)\end{array}$ & $\begin{array}{c}269 \pm 3 \\
(45)\end{array}$ & $\begin{array}{c}269 \pm 6 \\
(26)\end{array}$ & $\begin{array}{c}268 \pm 6 \\
(26)\end{array}$ \\
\hline 16 & $\begin{array}{c}302 \pm 5 \\
(50)\end{array}$ & $\begin{array}{c}305 \pm 5 \\
(47)\end{array}$ & $\begin{array}{c}303 \pm 5 \\
(46)\end{array}$ & $\begin{array}{c}299 \pm 3 \\
(49)\end{array}$ & $\begin{array}{c}300 \pm 4 \\
(50)\end{array}$ & $\begin{array}{c}302 \pm 4 \\
(45)\end{array}$ & $\begin{array}{c}299 \pm 6 \\
(26)\end{array}$ & $\begin{array}{c}299 \pm 7 \\
(26)\end{array}$ \\
\hline 20 & $\begin{array}{c}324 \pm 6 \\
(50)\end{array}$ & $\begin{array}{c}327 \pm 6 \\
(47)\end{array}$ & $\begin{array}{c}325 \pm 6 \\
(46)\end{array}$ & $\begin{array}{c}319 \pm 4 \\
(49)\end{array}$ & $\begin{array}{c}319 \pm 5 \\
(50)\end{array}$ & $\begin{array}{c}321 \pm 4 \\
(45)\end{array}$ & $\begin{array}{c}320 \pm 7 \\
(26)\end{array}$ & $\begin{array}{c}320 \pm 9 \\
(26)\end{array}$ \\
\hline 24 & $\begin{array}{c}342 \pm 6 \\
(50)\end{array}$ & $\begin{array}{c}345 \pm 7 \\
(46)\end{array}$ & $\begin{array}{c}346 \pm 6 \\
(45)\end{array}$ & $\begin{array}{c}336 \pm 5 \\
(49)\end{array}$ & $\begin{array}{c}336 \pm 5 \\
(50)\end{array}$ & $\begin{array}{c}338 \pm 5 \\
(45)\end{array}$ & $\begin{array}{c}339 \pm 8 \\
(26)\end{array}$ & $\begin{array}{c}338 \pm 10 \\
(26)\end{array}$ \\
\hline 28 & $\begin{array}{c}359 \pm 7 \\
(50)\end{array}$ & $\begin{array}{c}360 \pm 7 \\
(46)\end{array}$ & $\begin{array}{c}362 \pm 7 \\
(45)\end{array}$ & $\begin{array}{c}354 \pm 5 \\
(49)\end{array}$ & $\begin{array}{c}352 \pm 6 \\
(50)\end{array}$ & $\begin{array}{c}356 \pm 5 \\
(45)\end{array}$ & $\begin{array}{c}351 \pm 8 \\
(26)\end{array}$ & $\begin{array}{c}354 \pm 11 \\
(26)\end{array}$ \\
\hline 32 & $\begin{array}{c}372 \pm 8 \\
(50)\end{array}$ & $\begin{array}{c}376 \pm 8 \\
(46)\end{array}$ & $\begin{array}{c}376 \pm 8 \\
(45)\end{array}$ & $\begin{array}{c}366 \pm 6 \\
(49)\end{array}$ & $\begin{array}{c}366 \pm 6 \\
(50)\end{array}$ & $\begin{array}{c}369 \pm 6 \\
(45)\end{array}$ & $\begin{array}{c}366 \pm 9 \\
(26)\end{array}$ & $\begin{array}{c}373 \pm 12 \\
(26)\end{array}$ \\
\hline 36 & $\begin{array}{c}387 \pm 8 \\
(50)\end{array}$ & $\begin{array}{c}392 \pm 9 \\
(46)\end{array}$ & $\begin{array}{c}393 \pm 8 \\
(45)\end{array}$ & $\begin{array}{c}384 \pm 6 \\
(49)\end{array}$ & $\begin{array}{c}375 \pm 7 \\
(50)\end{array}$ & $\begin{array}{c}379 \pm 6 \\
(45)\end{array}$ & $\begin{array}{c}375 \pm 9 \\
(26)\end{array}$ & $\begin{array}{c}390 \pm 13 \\
(26)\end{array}$ \\
\hline 40 & $\begin{array}{c}402 \pm 9 \\
(50)\end{array}$ & $\begin{array}{c}407 \pm 10 \\
(46)\end{array}$ & $\begin{array}{c}407 \pm 10 \\
(45)\end{array}$ & $\begin{array}{c}396 \pm 7 \\
(48)\end{array}$ & $\begin{array}{c}388 \pm 8 \\
(49)\end{array}$ & $\begin{array}{c}394 \pm 7 \\
(45)\end{array}$ & $\begin{array}{c}392 \pm 10 \\
(26)\end{array}$ & $\begin{array}{c}406 \pm 13 \\
(26)\end{array}$ \\
\hline 44 & $\begin{array}{c}417 \pm 10 \\
(50)\end{array}$ & $\begin{array}{c}421 \pm 11 \\
\quad(45)\end{array}$ & $\begin{array}{c}419 \pm 10 \\
(45)\end{array}$ & $\begin{array}{c}410 \pm 8 \\
(47)\end{array}$ & $\begin{array}{c}403 \pm 8 \\
(48)\end{array}$ & $\begin{array}{c}410 \pm 8 \\
(45)\end{array}$ & $\begin{array}{c}403 \pm 11 \\
(26)\end{array}$ & $\begin{array}{c}421 \pm 14 \\
\quad(26)\end{array}$ \\
\hline 48 & $\begin{array}{c}431 \pm 10 \\
(50)\end{array}$ & $\begin{array}{c}438 \pm 12 \\
\quad(44)\end{array}$ & $\begin{array}{c}437 \pm 12 \\
(44)\end{array}$ & $\begin{array}{c}426 \pm 8 \\
(48)\end{array}$ & $\begin{array}{c}419 \pm 8 \\
(48)\end{array}$ & $\begin{array}{c}424 \pm 8 \\
(45)\end{array}$ & $\begin{array}{c}418 \pm 11 \\
(26)\end{array}$ & $\begin{array}{c}439 \pm 15 \\
(26)\end{array}$ \\
\hline 52 & $\begin{array}{c}447 \pm 10 \\
(50)\end{array}$ & $\begin{array}{c}456 \pm 13 \\
(44)\end{array}$ & $\begin{array}{c}451 \pm 12 \\
(44)\end{array}$ & $\begin{array}{c}448 \pm 8 \\
(48)\end{array}$ & $\begin{array}{c}434 \pm 9 \\
(48)\end{array}$ & $\begin{array}{c}442 \pm 9 \\
(45)\end{array}$ & $\begin{array}{c}433 \pm 12 \\
(26)\end{array}$ & $\begin{array}{c}450 \pm 15 \\
(26)\end{array}$ \\
\hline 56 & $\begin{array}{c}463 \pm 11 \\
(50)\end{array}$ & $\begin{array}{c}474 \pm 14 \\
(44)\end{array}$ & $\begin{array}{c}463 \pm 13 \\
(44)\end{array}$ & $\begin{array}{c}466 \pm 9 \\
(47)\end{array}$ & $\begin{array}{c}449 \pm 10 \\
(48)\end{array}$ & $\begin{array}{c}457 \pm 9 \\
(45)\end{array}$ & $\begin{array}{c}450 \pm 13 \\
(26)\end{array}$ & $\begin{array}{c}459 \pm 16 \\
(25)\end{array}$ \\
\hline 60 & $\begin{array}{c}478 \pm 12 \\
(50)\end{array}$ & $\begin{array}{c}492 \pm 15 \\
\quad(44)\end{array}$ & $\begin{array}{c}484 \pm 14 \\
(43)\end{array}$ & $\begin{array}{c}487 \pm 10 \\
(47)\end{array}$ & $\begin{array}{c}468 \pm 11 \\
(48)\end{array}$ & $\begin{array}{c}474 \pm 10 \\
(45)\end{array}$ & $\begin{array}{c}462 \pm 13 \\
(26)\end{array}$ & $\begin{array}{c}475 \pm 18 \\
(25)\end{array}$ \\
\hline 64 & $\begin{array}{c}495 \pm 12 \\
\quad(49)\end{array}$ & $\begin{array}{c}510 \pm 16 \\
\quad(44)\end{array}$ & $\begin{array}{c}502 \pm 15 \\
\quad(42)\end{array}$ & $\begin{array}{c}504 \pm 10 \\
(47)\end{array}$ & $\begin{array}{c}477 \pm 11 \\
\quad(44)\end{array}$ & $\begin{array}{c}491 \pm 10 \\
\quad(45)\end{array}$ & $\begin{array}{c}477 \pm 14 \\
(26)\end{array}$ & $\begin{array}{c}485 \pm 20 \\
(25)\end{array}$ \\
\hline 68 & $\begin{array}{c}506 \pm 12 \\
\quad(47)\end{array}$ & $\begin{array}{c}532 \pm 17 \\
(44)\end{array}$ & $\begin{array}{c}519 \pm 16 \\
(41)\end{array}$ & $\begin{array}{c}528 \pm 11 \\
(46)\end{array}$ & $\begin{array}{c}489 \pm 13 \\
\text { (39) }\end{array}$ & $\begin{array}{c}506 \pm 11 \\
\quad(44)\end{array}$ & $\begin{array}{c}497 \pm 15 \\
(24)\end{array}$ & $\begin{array}{c}492 \pm 23 \\
(24)\end{array}$ \\
\hline 72 & $\begin{array}{c}520 \pm 14 \\
(44)\end{array}$ & $\begin{array}{c}544 \pm 18 \\
\quad(43)\end{array}$ & $\begin{array}{c}544 \pm 18 \\
\quad(38)\end{array}$ & $\begin{array}{c}547 \pm 11 \\
(44)\end{array}$ & $\begin{array}{c}497 \pm 12 \\
(36)\end{array}$ & $\begin{array}{c}520 \pm 12 \\
(42)\end{array}$ & $\begin{array}{c}521 \pm 16 \\
\quad(21)\end{array}$ & $\begin{array}{c}506 \pm 24 \\
(22)\end{array}$ \\
\hline 76 & $\begin{array}{c}531 \pm 16 \\
(40)\end{array}$ & $\begin{array}{c}549 \pm 19 \\
(39)\end{array}$ & $\begin{array}{c}564 \pm 21 \\
\quad(34)\end{array}$ & $\begin{array}{c}564 \pm 13 \\
(41)\end{array}$ & $\begin{array}{c}500 \pm 12 \\
(33)\end{array}$ & $\begin{array}{c}522 \pm 12 \\
(38)\end{array}$ & $\begin{array}{c}530 \pm 19 \\
(17)\end{array}$ & $\begin{array}{c}521 \pm 26 \\
(21)\end{array}$ \\
\hline 80 & $\begin{array}{c}542 \pm 16 \\
(39)\end{array}$ & $\begin{array}{c}562 \pm 22 \\
(34)\end{array}$ & $\begin{array}{c}560 \pm 19 \\
(26)\end{array}$ & $\begin{array}{c}571 \pm 13 \\
\quad(40)\end{array}$ & $\begin{array}{c}514 \pm 13 \\
(31)\end{array}$ & $\begin{array}{c}537 \pm 12 \\
(33)\end{array}$ & $\begin{array}{c}544 \pm 18 \\
\quad(15)\end{array}$ & $\begin{array}{c}531 \pm 34 \\
(17)\end{array}$ \\
\hline 84 & $\begin{array}{c}533 \pm 15 \\
(35)\end{array}$ & $\begin{array}{c}577 \pm 24 \\
(33)\end{array}$ & $\begin{array}{c}574 \pm 20 \\
\quad(26)\end{array}$ & $\begin{array}{c}579 \pm 15 \\
(38)\end{array}$ & $\begin{array}{c}521 \pm 14 \\
(27)\end{array}$ & $\begin{array}{l}542 \pm 14 \\
(29)\end{array}$ & $\begin{array}{c}552 \pm 20 \\
\quad(14)\end{array}$ & $\begin{array}{c}551 \pm 40 \\
\quad(15)\end{array}$ \\
\hline 88 & $\begin{array}{c}534 \pm 18 \\
\quad(32)\end{array}$ & $\begin{array}{c}584 \pm 26 \\
\quad(31)\end{array}$ & $\begin{array}{c}585 \pm 22 \\
\quad(24)\end{array}$ & $\begin{array}{c}588 \pm 20 \\
(27)\end{array}$ & $\begin{array}{c}527 \pm 17 \\
(24)\end{array}$ & $\begin{array}{c}556 \pm 15 \\
\quad(28)\end{array}$ & $\begin{array}{c}539 \pm 23 \\
\quad(13)\end{array}$ & $\begin{array}{c}564 \pm 51 \\
\quad(11)\end{array}$ \\
\hline 92 & $\begin{array}{c}530 \pm 15 \\
(26)\end{array}$ & $\begin{array}{c}584 \pm 22 \\
(26)\end{array}$ & $\begin{array}{c}565 \pm 21 \\
\quad(18)\end{array}$ & $\begin{array}{c}608 \pm 23 \\
(22)\end{array}$ & $\begin{array}{c}526 \pm 19 \\
(21)\end{array}$ & $\begin{array}{c}564 \pm 16 \\
(27)\end{array}$ & $\begin{array}{l}561 \pm 22 \\
\quad(12)\end{array}$ & $\begin{array}{c}512 \pm 27 \\
(8)\end{array}$ \\
\hline 96 & $\begin{array}{c}531 \pm 17 \\
(23)\end{array}$ & $\begin{array}{c}604 \pm 23 \\
(22)\end{array}$ & $\begin{array}{c}594 \pm 20 \\
(16)\end{array}$ & $\begin{array}{c}616 \pm 25^{*} \\
(19)\end{array}$ & $\begin{array}{c}540 \pm 23 \\
\quad(18)\end{array}$ & $\begin{array}{c}569 \pm 19 \\
(21)\end{array}$ & $\begin{array}{c}563 \pm 26 \\
\quad(10)\end{array}$ & $\begin{array}{c}525 \pm 34 \\
\text { (7) }\end{array}$ \\
\hline 100 & $\begin{array}{c}537 \pm 21 \\
(17)\end{array}$ & $\begin{array}{c}621 \pm 26 \\
\quad(21)\end{array}$ & $\begin{array}{c}594 \pm 16 \\
\quad(14)\end{array}$ & $\begin{array}{c}634 \pm 32^{*} \\
(16)\end{array}$ & $\begin{array}{c}528 \pm 17 \\
(13)\end{array}$ & $\begin{array}{l}584 \pm 18 \\
\quad(17)\end{array}$ & $\begin{array}{c}597 \pm 34 \\
(7)\end{array}$ & $\begin{array}{c}542 \pm 36 \\
(6)\end{array}$ \\
\hline 104 & $\begin{array}{c}534 \pm 22 \\
(17)\end{array}$ & $\begin{array}{c}619 \pm 28 \\
(18)\end{array}$ & $\begin{array}{c}607 \pm 18 \\
(13)\end{array}$ & $\begin{array}{c}622 \pm 36^{*} \\
(12)\end{array}$ & $\begin{array}{c}524 \pm 22 \\
(11)\end{array}$ & $\begin{array}{c}597 \pm 34 \\
(9)\end{array}$ & $\begin{array}{c}602 \pm 35 \\
(7)\end{array}$ & $\begin{array}{c}562 \pm 50 \\
(4)\end{array}$ \\
\hline
\end{tabular}

${ }^{\mathrm{a}} \mathrm{BPA}$ and $\mathrm{EE}_{2}$ doses are $\mu \mathrm{g} / \mathrm{kg}$ bw/day. Numbers of litters are shown in parentheses. Outlier identification and exclusion are described in Materials and Methods and Supplemental Appendix XXV. Pairwise comparisons of means were performed using contrasts within a two-way repeated measures, mixed model ANOVA. Model terms were treatment group, weeks, and the interaction. Within-group correlations were modeled using a heterogeneous first-order autoregressive (ARH(1)) correlation structure, which allows for correlated differences in variability across time points. Tests of trends, increasing treatment effect with increasing dose, were performed for the BPA and vehicle control groups. Pairwise comparisons of treatment groups to the 


\section{CLARITY-BPA Core Study}

vehicle control group were conducted with Dunnett's method to adjust for multiple comparisons. All tests were performed as two-sided tests at the 0.05 significance level. Full results of the analyses are presented in Supplemental Appendix XXV.

Significant effects are indicated with asterisks (*, $p<0.05)$.

bIn the sensitivity analysis that excluded all animals that overlapped with animals treated with 250,000 $\mu \mathrm{g}$ BPA/kg bw/day (see Materials and Methods, Statistical Methods), the mean for week 8, 25,000 $\mu \mathrm{g} \mathrm{BPA} / \mathrm{kg}$ bw/day was significantly different ( $p=0.031)$ from the vehicle control mean. 
Table 26. Female Postwean Body Weights (g), Vehicle and BPA Stop-Dose, Interim (1 Year) Sacrifice (Mean \pm S.E.M.) ${ }^{\mathrm{a}}$

\begin{tabular}{ccccccc}
\hline Week & Vehicle & 2.5 BPA & 25 BPA & 250 BPA & 2500 BPA & 25000 BPA \\
\hline 4 & $58 \pm 3$ & $54 \pm 3$ & $55 \pm 3$ & $54 \pm 2$ & $55 \pm 3$ & $51 \pm 2$ \\
& $(20)$ & $(22)$ & $(20)$ & $(22)$ & $(20)$ & $(22)$ \\
8 & $192 \pm 5$ & $184 \pm 3$ & $190 \pm 4$ & $190 \pm 5$ & $188 \pm 5$ & $184 \pm 4$ \\
& $(20)$ & $(22)$ & $(20)$ & $(22)$ & $(20)$ & $(21)$ \\
12 & $270 \pm 7$ & $263 \pm 5$ & $270 \pm 7$ & $266 \pm 6$ & $272 \pm 8$ & $262 \pm 6$ \\
& $(20)$ & $(20)$ & $(20)$ & $(22)$ & $(20)$ & $(21)$ \\
16 & $304 \pm 11$ & $302 \pm 5$ & $305 \pm 9$ & $305 \pm 7$ & $310 \pm 8$ & $303 \pm 8$ \\
& $(18)$ & $(22)$ & $(20)$ & $(22)$ & $(20)$ & $(21)$ \\
20 & $331 \pm 11$ & $329 \pm 7$ & $328 \pm 10$ & $330 \pm 8$ & $338 \pm 10$ & $326 \pm 8$ \\
& $(20)$ & $(22)$ & $(20)$ & $(22)$ & $(20)$ & $(21)$ \\
24 & $351 \pm 12$ & $350 \pm 8$ & $344 \pm 11$ & $348 \pm 8$ & $358 \pm 12$ & $344 \pm 9$ \\
& $(20)$ & $(22)$ & $(20)$ & $(22)$ & $(20)$ & $(21)$ \\
28 & $369 \pm 12$ & $366 \pm 9$ & $360 \pm 12$ & $368 \pm 10$ & $373 \pm 14$ & $361 \pm 9$ \\
& $(20)$ & $(22)$ & $(20)$ & $(22)$ & $(20)$ & $(21)$ \\
32 & $386 \pm 14$ & $391 \pm 10$ & $374 \pm 13$ & $381 \pm 11$ & $392 \pm 15$ & $374 \pm 10$ \\
& $(20)$ & $(20)$ & $(20)$ & $(22)$ & $(20)$ & $(21)$ \\
36 & $400 \pm 14$ & $399 \pm 10$ & $389 \pm 15$ & $399 \pm 13$ & $404 \pm 15$ & $386 \pm 10$ \\
& $(20)$ & $(22)$ & $(20)$ & $(22)$ & $(20)$ & $(21)$ \\
40 & $410 \pm 16$ & $419 \pm 12$ & $404 \pm 16$ & $418 \pm 15$ & $421 \pm 17$ & $407 \pm 12$ \\
& $(18)$ & $(22)$ & $(20)$ & $(20)$ & $(20)$ & $(20)$ \\
44 & $439 \pm 17$ & $433 \pm 12$ & $419 \pm 16$ & $431 \pm 16$ & $438 \pm 18$ & $420 \pm 12$ \\
& $(20)$ & $(22)$ & $(20)$ & $(22)$ & $(20)$ & $(20)$ \\
48 & $459 \pm 18$ & $453 \pm 14$ & $436 \pm 17$ & $449 \pm 18$ & $452 \pm 18$ & $440 \pm 13$ \\
& $(20)$ & $(22)$ & $(20)$ & $(22)$ & $(20)$ & $(20)$ \\
52 & $477 \pm 19$ & $468 \pm 14$ & $450 \pm 19$ & $468 \pm 20$ & $466 \pm 19$ & $455 \pm 14$ \\
& $(20)$ & $(22)$ & $(20)$ & $(22)$ & $(20)$ & $(20)$ \\
\hline
\end{tabular}

${ }^{\mathrm{a} B P A}$ doses are $\mu \mathrm{g} / \mathrm{kg}$ bw/day. Numbers of litters are shown in parentheses. Outlier identification and exclusion are described in Materials and Methods and Supplemental Appendix XXIV. Pairwise comparisons of means were performed using contrasts within a two-way repeated measures, mixed model ANOVA. Model terms were treatment group, weeks, and the interaction. Within-group correlations were modeled using a heterogeneous first-order autoregressive (ARH(1)) correlation structure, which allows for correlated differences in variability across time points. Tests of trends, increasing treatment effect with increasing dose, were performed for the BPA and vehicle control groups. Pairwise comparisons of treatment groups to the vehicle control group were conducted with Dunnett's method to adjust for multiple comparisons. All tests were performed as two-sided tests at the 0.05 significance level. Full results of the analyses are presented in Supplemental Appendix XXIV. There were no statistically significant trends or pairwise comparisons to controls. There were also no significant treatment effects in the sensitivity analysis that excluded all animals that overlapped with animals treated with $250,000 \mu \mathrm{g} \mathrm{BPA} / \mathrm{kg}$ bw/day (see Materials and Methods, Statistical Methods). 
Table 27. Female Postwean Body Weights (g), Vehicle and BPA Stop-Dose, Terminal (2 Year) Sacrifice (Mean \pm S.E.M.) ${ }^{\mathrm{a}}$

\begin{tabular}{|c|c|c|c|c|c|c|}
\hline Week & Vehicle & $2.5 \mathrm{BPA}$ & 25 BPA & 250 BPA & 2500 BPA & 25000 BPA \\
\hline 4 & $\begin{array}{c}57 \pm 2^{*} \\
(50)\end{array}$ & $\begin{array}{c}56 \pm 2 \\
(50)\end{array}$ & $\begin{array}{c}54 \pm 2 \\
(48)\end{array}$ & $\begin{array}{c}53 \pm 1 \\
(50)\end{array}$ & $\begin{array}{c}52 \pm 2^{b} \\
(50)\end{array}$ & $\begin{array}{c}54 \pm 1 \\
(46)\end{array}$ \\
\hline 8 & $\begin{array}{c}190 \pm 4 \\
(50)\end{array}$ & $\begin{array}{c}190 \pm 2 \\
(50)\end{array}$ & $\begin{array}{c}189 \pm 2 \\
(48)\end{array}$ & $\begin{array}{c}193 \pm 2 \\
(50)\end{array}$ & $\begin{array}{c}187 \pm 4 \\
(50)\end{array}$ & $\begin{array}{c}190 \pm 2 \\
(46)\end{array}$ \\
\hline 12 & $\begin{array}{c}270 \pm 5 \\
(50)\end{array}$ & $\begin{array}{c}270 \pm 3 \\
(50)\end{array}$ & $\begin{array}{c}268 \pm 3 \\
(48)\end{array}$ & $\begin{array}{c}271 \pm 4 \\
(50)\end{array}$ & $\begin{array}{c}267 \pm 5 \\
(50)\end{array}$ & $\begin{array}{c}270 \pm 4 \\
(46)\end{array}$ \\
\hline 16 & $\begin{array}{c}312 \pm 7 \\
(50)\end{array}$ & $\begin{array}{c}312 \pm 4 \\
(50)\end{array}$ & $\begin{array}{c}307 \pm 5 \\
(48)\end{array}$ & $\begin{array}{c}310 \pm 5 \\
(50)\end{array}$ & $\begin{array}{c}305 \pm 6 \\
(50)\end{array}$ & $\begin{array}{c}311 \pm 5 \\
(46)\end{array}$ \\
\hline 20 & $\begin{array}{c}338 \pm 8 \\
(50)\end{array}$ & $\begin{array}{c}339 \pm 5 \\
(50)\end{array}$ & $\begin{array}{c}330 \pm 5 \\
(46)\end{array}$ & $\begin{array}{c}334 \pm 5 \\
(50)\end{array}$ & $\begin{array}{c}330 \pm 7 \\
(50)\end{array}$ & $\begin{array}{c}338 \pm 5 \\
(46)\end{array}$ \\
\hline 24 & $\begin{array}{c}359 \pm 8 \\
(50)\end{array}$ & $\begin{array}{c}361 \pm 5 \\
(50)\end{array}$ & $\begin{array}{c}349 \pm 6 \\
(48)\end{array}$ & $\begin{array}{c}353 \pm 6 \\
(50)\end{array}$ & $\begin{array}{c}350 \pm 7 \\
(49)\end{array}$ & $\begin{array}{c}358 \pm 6 \\
(46)\end{array}$ \\
\hline 28 & $\begin{array}{c}379 \pm 10 \\
(50)\end{array}$ & $\begin{array}{c}375 \pm 6 \\
(50)\end{array}$ & $\begin{array}{c}365 \pm 7 \\
(48)\end{array}$ & $\begin{array}{c}370 \pm 7 \\
(50)\end{array}$ & $\begin{array}{c}366 \pm 8 \\
(49)\end{array}$ & $\begin{array}{c}376 \pm 6 \\
(46)\end{array}$ \\
\hline 32 & $\begin{array}{c}393 \pm 10 \\
(50)\end{array}$ & $\begin{array}{c}393 \pm 6 \\
(48)\end{array}$ & $\begin{array}{c}380 \pm 7 \\
(48)\end{array}$ & $\begin{array}{c}384 \pm 8 \\
(50)\end{array}$ & $\begin{array}{c}381 \pm 8 \\
(49)\end{array}$ & $\begin{array}{c}392 \pm 7 \\
(46)\end{array}$ \\
\hline 36 & $\begin{array}{c}409 \pm 10 \\
(50)\end{array}$ & $\begin{array}{c}409 \pm 7 \\
(50)\end{array}$ & $\begin{array}{c}397 \pm 8 \\
(48)\end{array}$ & $\begin{array}{c}403 \pm 9 \\
(50)\end{array}$ & $\begin{array}{c}394 \pm 9 \\
(49)\end{array}$ & $\begin{array}{c}409 \pm 8 \\
(46)\end{array}$ \\
\hline 40 & $\begin{array}{c}420 \pm 11 \\
(43)\end{array}$ & $\begin{array}{c}428 \pm 8 \\
(47)\end{array}$ & $\begin{array}{c}414 \pm 9 \\
(44)\end{array}$ & $\begin{array}{c}418 \pm 10 \\
(44)\end{array}$ & $\begin{array}{c}411 \pm 10 \\
(47)\end{array}$ & $\begin{array}{c}427 \pm 9 \\
(44)\end{array}$ \\
\hline 44 & $\begin{array}{c}436 \pm 11 \\
(47)\end{array}$ & $\begin{array}{c}446 \pm 9 \\
(50)\end{array}$ & $\begin{array}{c}426 \pm 9 \\
(48)\end{array}$ & $\begin{array}{c}435 \pm 10 \\
(50)\end{array}$ & $\begin{array}{c}426 \pm 10 \\
(48)\end{array}$ & $\begin{array}{c}445 \pm 9 \\
(46)\end{array}$ \\
\hline 48 & $\begin{array}{c}457 \pm 12 \\
(49)\end{array}$ & $\begin{array}{c}466 \pm 10 \\
(50)\end{array}$ & $\begin{array}{c}446 \pm 10 \\
(48)\end{array}$ & $\begin{array}{c}451 \pm 10 \\
(50)\end{array}$ & $\begin{array}{c}444 \pm 11 \\
(49)\end{array}$ & $\begin{array}{c}463 \pm 10 \\
(46)\end{array}$ \\
\hline 52 & $\begin{array}{c}479 \pm 12 \\
(49)\end{array}$ & $\begin{array}{c}489 \pm 10 \\
(49)\end{array}$ & $\begin{array}{c}462 \pm 10 \\
(47)\end{array}$ & $\begin{array}{c}474 \pm 11 \\
(50)\end{array}$ & $\begin{array}{c}459 \pm 11 \\
(49)\end{array}$ & $\begin{array}{c}477 \pm 11 \\
(46)\end{array}$ \\
\hline 56 & $\begin{array}{c}497 \pm 13 \\
(49)\end{array}$ & $\begin{array}{c}506 \pm 10 \\
(49)\end{array}$ & $\begin{array}{c}482 \pm 11 \\
(47)\end{array}$ & $\begin{array}{c}496 \pm 12 \\
(50)\end{array}$ & $\begin{array}{c}480 \pm 12 \\
(49)\end{array}$ & $\begin{array}{c}495 \pm 12 \\
(45)\end{array}$ \\
\hline 60 & $\begin{array}{c}516 \pm 14 \\
(48)\end{array}$ & $\begin{array}{c}524 \pm 11 \\
(46)\end{array}$ & $\begin{array}{c}502 \pm 11 \\
(47)\end{array}$ & $\begin{array}{c}515 \pm 12 \\
(50)\end{array}$ & $\begin{array}{c}496 \pm 12 \\
(48)\end{array}$ & $\begin{array}{c}513 \pm 14 \\
(44)\end{array}$ \\
\hline 64 & $\begin{array}{c}536 \pm 15 \\
(48)\end{array}$ & $\begin{array}{c}549 \pm 13 \\
(45)\end{array}$ & $\begin{array}{c}520 \pm 12 \\
(47)\end{array}$ & $\begin{array}{c}537 \pm 12 \\
(50)\end{array}$ & $\begin{array}{c}517 \pm 13 \\
\quad(48)\end{array}$ & $\begin{array}{c}533 \pm 15 \\
(43)\end{array}$ \\
\hline 68 & $\begin{array}{c}558 \pm 16 \\
(47)\end{array}$ & $\begin{array}{c}573 \pm 13 \\
(44)\end{array}$ & $\begin{array}{c}540 \pm 13 \\
(43)\end{array}$ & $\begin{array}{c}546 \pm 13 \\
(46)\end{array}$ & $\begin{array}{c}537 \pm 14 \\
(47)\end{array}$ & $\begin{array}{c}550 \pm 15 \\
(39)\end{array}$ \\
\hline 72 & $\begin{array}{c}571 \pm 18 \\
(44)\end{array}$ & $\begin{array}{c}580 \pm 13 \\
(39)\end{array}$ & $\begin{array}{c}552 \pm 14 \\
(41)\end{array}$ & $\begin{array}{c}561 \pm 14 \\
(44)\end{array}$ & $\begin{array}{c}556 \pm 16 \\
\quad(45)\end{array}$ & $\begin{array}{c}552 \pm 14 \\
(35)\end{array}$ \\
\hline 76 & $\begin{array}{c}570 \pm 19 \\
(36)\end{array}$ & $\begin{array}{c}598 \pm 14 \\
(40)\end{array}$ & $\begin{array}{c}568 \pm 15 \\
(39)\end{array}$ & $\begin{array}{c}574 \pm 15 \\
\quad(41)\end{array}$ & $\begin{array}{c}568 \pm 16 \\
\quad(41)\end{array}$ & $\begin{array}{c}575 \pm 16 \\
(35)\end{array}$ \\
\hline 80 & $\begin{array}{c}576 \pm 18 \\
(34)\end{array}$ & $\begin{array}{c}619 \pm 15 \\
(39)\end{array}$ & $\begin{array}{c}575 \pm 15 \\
(35)\end{array}$ & $\begin{array}{c}586 \pm 18 \\
(36)\end{array}$ & $\begin{array}{c}580 \pm 17 \\
(38)\end{array}$ & $\begin{array}{c}589 \pm 16 \\
(35)\end{array}$ \\
\hline 84 & $\begin{array}{c}588 \pm 21 \\
(31)\end{array}$ & $\begin{array}{c}633 \pm 18 \\
(32)\end{array}$ & $\begin{array}{c}601 \pm 18 \\
(30)\end{array}$ & $\begin{array}{c}605 \pm 19 \\
(34)\end{array}$ & $\begin{array}{c}591 \pm 19 \\
(36)\end{array}$ & $\begin{array}{c}604 \pm 19 \\
(31)\end{array}$ \\
\hline 88 & $\begin{array}{c}595 \pm 22 \\
(28)\end{array}$ & $\begin{array}{c}624 \pm 20 \\
(26)\end{array}$ & $\begin{array}{c}599 \pm 18 \\
(26)\end{array}$ & $\begin{array}{c}614 \pm 22 \\
(31)\end{array}$ & $\begin{array}{c}606 \pm 21 \\
\quad(33)\end{array}$ & $\begin{array}{c}613 \pm 20 \\
(29)\end{array}$ \\
\hline 92 & $\begin{array}{c}591 \pm 28 \\
(23)\end{array}$ & $\begin{array}{c}626 \pm 23 \\
(20)\end{array}$ & $\begin{array}{c}590 \pm 21 \\
(20)\end{array}$ & $\begin{array}{c}626 \pm 24 \\
(28)\end{array}$ & $\begin{array}{c}609 \pm 24 \\
(31)\end{array}$ & $\begin{array}{c}625 \pm 22 \\
(26)\end{array}$ \\
\hline 96 & $\begin{array}{c}600 \pm 33 \\
(20)\end{array}$ & $\begin{array}{c}608 \pm 20 \\
\quad(16)\end{array}$ & $\begin{array}{c}595 \pm 25 \\
(16)\end{array}$ & $\begin{array}{c}651 \pm 29 \\
(23)\end{array}$ & $\begin{array}{c}633 \pm 28 \\
(24)\end{array}$ & $\begin{array}{c}616 \pm 24 \\
(22)\end{array}$ \\
\hline 100 & $\begin{array}{c}600 \pm 30 \\
(17)\end{array}$ & $\begin{array}{c}625 \pm 21 \\
(16)\end{array}$ & $\begin{array}{c}614 \pm 28 \\
(15)\end{array}$ & $\begin{array}{c}650 \pm 37 \\
\quad(18)\end{array}$ & $\begin{array}{c}614 \pm 34 \\
(19)\end{array}$ & $\begin{array}{c}610 \pm 29 \\
(17)\end{array}$ \\
\hline 104 & $\begin{array}{c}608 \pm 45 \\
(11)\end{array}$ & $\begin{array}{c}631 \pm 23 \\
(13)\end{array}$ & $\begin{array}{c}595 \pm 25 \\
(13)\end{array}$ & $\begin{array}{c}630 \pm 39 \\
(13)\end{array}$ & $\begin{array}{c}641 \pm 35 \\
(17)\end{array}$ & $\begin{array}{c}623 \pm 35 \\
(13)\end{array}$ \\
\hline
\end{tabular}

${ }^{\mathrm{a} B P A}$ doses are $\mu \mathrm{g} / \mathrm{kg}$ bw/day. Numbers of litters are shown in parentheses. Outlier identification and exclusion are described in Materials and Methods and Supplemental Appendix XXV. Pairwise comparisons of means were performed using contrasts within a two-way repeated measures, mixed model ANOVA. Model terms were treatment group, weeks, and the interaction. Within-group correlations were modeled using a heterogeneous first-order autoregressive $(\mathrm{ARH}(1))$ correlation structure, which allows for correlated differences in variability across time points. Tests of trends, increasing treatment effect with increasing dose, were performed for the BPA and vehicle control groups. Pairwise comparisons of treatment groups to the vehicle control 


\section{CLARITY-BPA Core Study}

group were conducted with Dunnett's method to adjust for multiple comparisons. All tests were performed as two-sided tests at the 0.05 significance level. Full results of the analyses are presented in Supplemental Appendix XXV. The only significant effect is indicated with an asterisk (dose trend, week $4, p=0.037$ ).

bIn the sensitivity analysis that excluded all animals that overlapped with animals treated with $250,000 \mu \mathrm{g}$ BPA $/ \mathrm{kg}$ bw/day (see Materials and Methods, Statistical Methods), the mean for week 4, 2,500 $\mu \mathrm{g} \mathrm{BPA} / \mathrm{kg}$ bw/day was significantly different

(approximately $12 \%$ lower, $p=0.016$ ) from the vehicle control mean. 
Table 28. Male Postwean Body Weights (g), Vehicle, BPA, and EE 2 Continuous-Dose, Interim (1 Year) Sacrifice (Mean \pm S.E.M.) $)^{\mathrm{a}}$

\begin{tabular}{ccccccccc}
\hline Week & Vehicle & $\mathbf{2 . 5}$ BPA & 25 BPA & 250 BPA & 2500 BPA & 25000 BPA & $\mathbf{0 . 0 5 ~ E E}_{2}$ & $\mathbf{0 . 5 ~ E E}_{2}$ \\
\hline 4 & $84 \pm 2$ & $83 \pm 2$ & $85 \pm 2$ & $81 \pm 2$ & $83 \pm 2$ & $80 \pm 1$ & $84 \pm 2$ & $88 \pm 2$ \\
& $(22)$ & $(22)$ & $(20)$ & $(24)$ & $(20)$ & $(22)$ & $(26)$ & $(26)$ \\
8 & $298 \pm 7$ & $299 \pm 7$ & $296 \pm 7$ & $288 \pm 7$ & $296 \pm 8$ & $290 \pm 5$ & $298 \pm 7$ & $304 \pm 5$ \\
& $(22)$ & $(22)$ & $(20)$ & $(24)$ & $(20)$ & $(21)$ & $(26)$ & $(26)$ \\
12 & $424 \pm 9$ & $429 \pm 8$ & $429 \pm 10$ & $416 \pm 8$ & $426 \pm 11$ & $417 \pm 8$ & $431 \pm 9$ & $437 \pm 6$ \\
& $(22)$ & $(22)$ & $(20)$ & $(24)$ & $(20)$ & $(21)$ & $(26)$ & $(26)$ \\
16 & $494 \pm 10$ & $505 \pm 9$ & $500 \pm 11$ & $490 \pm 8$ & $496 \pm 12$ & $484 \pm 9$ & $504 \pm 10$ & $505 \pm 8$ \\
& $(22)$ & $(22)$ & $(20)$ & $(24)$ & $(20)$ & $(21)$ & $(26)$ & $(26)$ \\
20 & $540 \pm 10$ & $554 \pm 11$ & $553 \pm 13$ & $541 \pm 10$ & $542 \pm 13$ & $532 \pm 10$ & $551 \pm 12$ & $550 \pm 10$ \\
& $(22)$ & $(22)$ & $(19)$ & $(24)$ & $(20)$ & $(21)$ & $(25)$ & $(24)$ \\
24 & $578 \pm 12$ & $590 \pm 12$ & $586 \pm 14$ & $576 \pm 12$ & $579 \pm 14$ & $568 \pm 12$ & $588 \pm 13$ & $584 \pm 9$ \\
& $(22)$ & $(22)$ & $(19)$ & $(24)$ & $(20)$ & $(21)$ & $(25)$ & $(26)$ \\
28 & $604 \pm 13$ & $621 \pm 12$ & $612 \pm 15$ & $607 \pm 12$ & $610 \pm 16$ & $596 \pm 13$ & $613 \pm 14$ & $611 \pm 10$ \\
& $(21)$ & $(22)$ & $(19)$ & $(24)$ & $(20)$ & $(21)$ & $(25)$ & $(26)$ \\
32 & $627 \pm 14$ & $643 \pm 13$ & $636 \pm 16$ & $633 \pm 12$ & $635 \pm 18$ & $620 \pm 14$ & $636 \pm 14$ & $634 \pm 10$ \\
& $(21)$ & $(22)$ & $(19)$ & $(24)$ & $(20)$ & $(21)$ & $(25)$ & $(26)$ \\
36 & $645 \pm 15$ & $667 \pm 14$ & $653 \pm 17$ & $653 \pm 13$ & $656 \pm 19$ & $639 \pm 14$ & $660 \pm 15$ & $652 \pm 12$ \\
& $(21)$ & $(22)$ & $(19)$ & $(24)$ & $(20)$ & $(21)$ & $(25)$ & $(25)$ \\
40 & $664 \pm 17$ & $690 \pm 16$ & $673 \pm 18$ & $674 \pm 14$ & $675 \pm 21$ & $659 \pm 15$ & $681 \pm 16$ & $673 \pm 13$ \\
& $(20)$ & $(22)$ & $(19)$ & $(24)$ & $(19)$ & $(21)$ & $(24)$ & $(25)$ \\
44 & $688 \pm 16$ & $707 \pm 17$ & $689 \pm 19$ & $691 \pm 14$ & $693 \pm 21$ & $676 \pm 17$ & $697 \pm 17$ & $687 \pm 13$ \\
& $(19)$ & $(22)$ & $(19)$ & $(24)$ & $(19)$ & $(21)$ & $(25)$ & $(25)$ \\
48 & $702 \pm 18$ & $724 \pm 20$ & $715 \pm 20$ & $712 \pm 15$ & $706 \pm 24$ & $693 \pm 17$ & $712 \pm 19$ & $715 \pm 14$ \\
& $(19)$ & $(22)$ & $(18)$ & $(24)$ & $(18)$ & $(21)$ & $(24)$ & $(23)$ \\
52 & $720 \pm 20$ & $742 \pm 21$ & $729 \pm 22$ & $732 \pm 16$ & $724 \pm 26$ & $714 \pm 19$ & $730 \pm 21$ & $724 \pm 16$ \\
& $(18)$ & $(22)$ & $(18)$ & $(24)$ & $(18)$ & $(21)$ & $(22)$ & $(23)$ \\
\hline
\end{tabular}

${ }^{\mathrm{a}} \mathrm{BPA}$ and $\mathrm{EE}_{2}$ doses are $\mu \mathrm{g} / \mathrm{kg}$ bw/day. Numbers of litters are shown in parentheses. Outlier identification and exclusion are described in Materials and Methods and Supplemental Appendix XXIV. Analyses were conducted separately for BPA and EE 2 dose groups. Pairwise comparisons of means were performed using contrasts within a two-way repeated measures, mixed model ANOVA. Model terms were treatment group, weeks, and the interaction. Within-group correlations were modeled using a heterogeneous first-order autoregressive (ARH(1)) correlation structure, which allows for correlated differences in variability across time points. Tests of trends, increasing treatment effect with increasing dose, were performed for the BPA and vehicle control groups. Pairwise comparisons of treatment groups to the vehicle control group were conducted with Dunnett's method to adjust for multiple comparisons. All tests were performed as two-sided tests at the 0.05 significance level. Full results of the analyses are presented in Supplemental Appendix XXIV. There were no statistically significant trends or pairwise comparisons to controls. There were also no significant treatment effects in the sensitivity analysis that excluded all animals that overlapped with animals treated with 250,000 $\mu \mathrm{g}$ BPA/kg bw/day (see Materials and Methods, Statistical Methods). 
Table 29. Male Postwean Body Weights (g), Vehicle, BPA, And EE $E_{2}$ Continuous-Dose, Terminal (2 Year) Sacrifice (Mean \pm S.E.M.) ${ }^{\mathrm{a}}$

\begin{tabular}{|c|c|c|c|c|c|c|c|c|}
\hline Week & Vehicle & $2.5 \mathrm{BPA}$ & 25 BPA & 250 BPA & 2500 BPA & 25000 BPA & $0.05 \mathrm{EE}_{2}$ & $0.5 \mathrm{EE}_{2}$ \\
\hline 4 & $\begin{array}{c}84 \pm 1 \\
(50)\end{array}$ & $\begin{array}{c}84 \pm 1 \\
(48)\end{array}$ & $\begin{array}{c}86 \pm 1 \\
(48)\end{array}$ & $\begin{array}{c}84 \pm 1 \\
(50)\end{array}$ & $\begin{array}{c}82 \pm 1 \\
(50)\end{array}$ & $\begin{array}{c}85 \pm 1 \\
(46)\end{array}$ & $\begin{array}{c}82 \pm 2 \\
(26)\end{array}$ & $\begin{array}{c}88 \pm 2 \\
(26)\end{array}$ \\
\hline 8 & $\begin{array}{c}295 \pm 4 \\
(50)\end{array}$ & $\begin{array}{c}302 \pm 4 \\
(48)\end{array}$ & $\begin{array}{c}297 \pm 4 \\
(48)\end{array}$ & $\begin{array}{c}290 \pm 4 \\
(50)\end{array}$ & $\begin{array}{c}292 \pm 4 \\
(50)\end{array}$ & $\begin{array}{c}305 \pm 4 \\
(46)\end{array}$ & $\begin{array}{c}297 \pm 5 \\
(26)\end{array}$ & $\begin{array}{c}306 \pm 5 \\
(26)\end{array}$ \\
\hline 12 & $\begin{array}{c}429 \pm 7 \\
(49)\end{array}$ & $\begin{array}{c}434 \pm 5 \\
(47)\end{array}$ & $\begin{array}{c}425 \pm 5 \\
(48)\end{array}$ & $\begin{array}{c}419 \pm 5 \\
(50)\end{array}$ & $\begin{array}{c}420 \pm 5 \\
(50)\end{array}$ & $\begin{array}{c}435 \pm 5 \\
(46)\end{array}$ & $\begin{array}{c}428 \pm 8 \\
(26)\end{array}$ & $\begin{array}{c}431 \pm 8 \\
(26)\end{array}$ \\
\hline 16 & $\begin{array}{c}503 \pm 10 \\
(49)\end{array}$ & $\begin{array}{c}506 \pm 6 \\
(47)\end{array}$ & $\begin{array}{c}496 \pm 6 \\
(48)\end{array}$ & $\begin{array}{c}492 \pm 6 \\
(50)\end{array}$ & $\begin{array}{c}496 \pm 6 \\
(50)\end{array}$ & $\begin{array}{c}505 \pm 6 \\
(46)\end{array}$ & $\begin{array}{c}504 \pm 10 \\
(26)\end{array}$ & $\begin{array}{c}502 \pm 10 \\
\quad(26)\end{array}$ \\
\hline 20 & $\begin{array}{c}550 \pm 11 \\
(49)\end{array}$ & $\begin{array}{c}554 \pm 7 \\
(45)\end{array}$ & $\begin{array}{c}546 \pm 8 \\
(48)\end{array}$ & $\begin{array}{c}543 \pm 6 \\
(50)\end{array}$ & $\begin{array}{c}543 \pm 7 \\
(50)\end{array}$ & $\begin{array}{c}552 \pm 6 \\
(46)\end{array}$ & $\begin{array}{c}553 \pm 11 \\
(26)\end{array}$ & $\begin{array}{c}549 \pm 12 \\
(26)\end{array}$ \\
\hline 24 & $\begin{array}{c}586 \pm 12 \\
\quad(49)\end{array}$ & $\begin{array}{c}591 \pm 7 \\
(47)\end{array}$ & $\begin{array}{c}583 \pm 8 \\
(48)\end{array}$ & $\begin{array}{c}581 \pm 7 \\
(50)\end{array}$ & $\begin{array}{c}577 \pm 8 \\
(50)\end{array}$ & $\begin{array}{c}586 \pm 7 \\
(46)\end{array}$ & $\begin{array}{c}586 \pm 13 \\
(26)\end{array}$ & $\begin{array}{l}581 \pm 11 \\
\quad(26)\end{array}$ \\
\hline 28 & $\begin{array}{c}614 \pm 14 \\
(49)\end{array}$ & $\begin{array}{c}620 \pm 8 \\
(46)\end{array}$ & $\begin{array}{c}609 \pm 9 \\
(48)\end{array}$ & $\begin{array}{c}612 \pm 7 \\
(50)\end{array}$ & $\begin{array}{c}603 \pm 9 \\
(50)\end{array}$ & $\begin{array}{c}614 \pm 8 \\
(46)\end{array}$ & $\begin{array}{c}614 \pm 13 \\
(26)\end{array}$ & $\begin{array}{c}608 \pm 13 \\
(24)\end{array}$ \\
\hline 32 & $\begin{array}{c}639 \pm 16 \\
(49)\end{array}$ & $\begin{array}{c}643 \pm 9 \\
(46)\end{array}$ & $\begin{array}{c}631 \pm 10 \\
(48)\end{array}$ & $\begin{array}{c}641 \pm 8 \\
(50)\end{array}$ & $\begin{array}{c}625 \pm 9 \\
(50)\end{array}$ & $\begin{array}{c}637 \pm 8 \\
(46)\end{array}$ & $\begin{array}{c}637 \pm 14 \\
(26)\end{array}$ & $\begin{array}{c}632 \pm 13 \\
(26)\end{array}$ \\
\hline 36 & $\begin{array}{c}647 \pm 10 \\
(48)\end{array}$ & $\begin{array}{c}663 \pm 10 \\
(46)\end{array}$ & $\begin{array}{c}654 \pm 10 \\
(47)\end{array}$ & $\begin{array}{c}661 \pm 9 \\
(50)\end{array}$ & $\begin{array}{c}643 \pm 9 \\
(50)\end{array}$ & $\begin{array}{c}656 \pm 9 \\
(46)\end{array}$ & $\begin{array}{c}658 \pm 16 \\
(26)\end{array}$ & $\begin{array}{c}655 \pm 14 \\
(26)\end{array}$ \\
\hline 40 & $\begin{array}{c}667 \pm 10 \\
\quad(48)\end{array}$ & $\begin{array}{c}685 \pm 10 \\
(46)\end{array}$ & $\begin{array}{c}672 \pm 11 \\
(48)\end{array}$ & $\begin{array}{c}682 \pm 10 \\
(49)\end{array}$ & $\begin{array}{c}660 \pm 9 \\
(50)\end{array}$ & $\begin{array}{c}674 \pm 9 \\
(46)\end{array}$ & $\begin{array}{c}678 \pm 16 \\
(26)\end{array}$ & $\begin{array}{c}673 \pm 15 \\
\quad(26)\end{array}$ \\
\hline 44 & $\begin{array}{c}684 \pm 11 \\
\quad(47)\end{array}$ & $\begin{array}{c}700 \pm 10 \\
(45)\end{array}$ & $\begin{array}{c}691 \pm 12 \\
(47)\end{array}$ & $\begin{array}{c}703 \pm 11 \\
(49)\end{array}$ & $\begin{array}{c}678 \pm 10 \\
(49)\end{array}$ & $\begin{array}{c}687 \pm 10 \\
\quad(46)\end{array}$ & $\begin{array}{c}696 \pm 17 \\
(26)\end{array}$ & $\begin{array}{c}692 \pm 16 \\
\quad(26)\end{array}$ \\
\hline 48 & $\begin{array}{c}700 \pm 12 \\
(47)\end{array}$ & $\begin{array}{c}717 \pm 11 \\
(45)\end{array}$ & $\begin{array}{c}707 \pm 13 \\
(47)\end{array}$ & $\begin{array}{c}729 \pm 11 \\
(48)\end{array}$ & $\begin{array}{c}696 \pm 10 \\
(49)\end{array}$ & $\begin{array}{c}707 \pm 10 \\
(44)\end{array}$ & $\begin{array}{c}712 \pm 18 \\
(26)\end{array}$ & $\begin{array}{c}710 \pm 17 \\
(26)\end{array}$ \\
\hline 52 & $\begin{array}{c}719 \pm 13 \\
(47)\end{array}$ & $\begin{array}{c}732 \pm 12 \\
(45)\end{array}$ & $\begin{array}{c}719 \pm 12 \\
(44)\end{array}$ & $\begin{array}{c}746 \pm 10 \\
(47)\end{array}$ & $\begin{array}{c}712 \pm 11 \\
(48)\end{array}$ & $\begin{array}{c}727 \pm 11 \\
(43)\end{array}$ & $\begin{array}{c}726 \pm 20 \\
(26)\end{array}$ & $\begin{array}{c}726 \pm 18 \\
(26)\end{array}$ \\
\hline 56 & $\begin{array}{c}736 \pm 13 \\
(47)\end{array}$ & $\begin{array}{c}750 \pm 13 \\
(45)\end{array}$ & $\begin{array}{c}745 \pm 14 \\
(46)\end{array}$ & $\begin{array}{c}765 \pm 11 \\
(46)\end{array}$ & $\begin{array}{c}726 \pm 12 \\
(47)\end{array}$ & $\begin{array}{c}750 \pm 12 \\
(41)\end{array}$ & $\begin{array}{c}754 \pm 20 \\
(24)\end{array}$ & $\begin{array}{c}742 \pm 18 \\
\text { (26) }\end{array}$ \\
\hline 60 & $\begin{array}{c}748 \pm 13 \\
(47)\end{array}$ & $\begin{array}{c}768 \pm 13 \\
(45)\end{array}$ & $\begin{array}{c}756 \pm 16 \\
(45)\end{array}$ & $\begin{array}{c}784 \pm 12 \\
(45)\end{array}$ & $\begin{array}{c}743 \pm 12 \\
(46)\end{array}$ & $\begin{array}{c}770 \pm 13 \\
(40)\end{array}$ & $\begin{array}{c}783 \pm 19 \\
(23)\end{array}$ & $\begin{array}{c}760 \pm 18 \\
(26)\end{array}$ \\
\hline 64 & $\begin{array}{c}765 \pm 14 \\
(46)\end{array}$ & $\begin{array}{c}791 \pm 14 \\
(44)\end{array}$ & $\begin{array}{c}779 \pm 17 \\
(44)\end{array}$ & $\begin{array}{c}803 \pm 13 \\
(45)\end{array}$ & $\begin{array}{c}755 \pm 14 \\
(46)\end{array}$ & $\begin{array}{c}791 \pm 14 \\
\quad(40)\end{array}$ & $\begin{array}{c}803 \pm 21 \\
(23)\end{array}$ & $\begin{array}{c}771 \pm 20 \\
(25)\end{array}$ \\
\hline 68 & $\begin{array}{c}783 \pm 15 \\
(45)\end{array}$ & $\begin{array}{c}805 \pm 14 \\
(42)\end{array}$ & $\begin{array}{c}800 \pm 19 \\
\quad(44)\end{array}$ & $\begin{array}{c}818 \pm 14 \\
(43)\end{array}$ & $\begin{array}{c}784 \pm 15 \\
(43)\end{array}$ & $\begin{array}{c}815 \pm 16 \\
(37)\end{array}$ & $\begin{array}{c}828 \pm 22 \\
(22)\end{array}$ & $\begin{array}{c}788 \pm 26 \\
(24)\end{array}$ \\
\hline 72 & $\begin{array}{c}792 \pm 17 \\
(42)\end{array}$ & $\begin{array}{c}821 \pm 15 \\
(41)\end{array}$ & $\begin{array}{c}822 \pm 20 \\
(40)\end{array}$ & $\begin{array}{c}838 \pm 15 \\
(40)\end{array}$ & $\begin{array}{c}799 \pm 16 \\
(42)\end{array}$ & $\begin{array}{c}832 \pm 17 \\
(37)\end{array}$ & $\begin{array}{c}848 \pm 24 \\
(22)\end{array}$ & $\begin{array}{c}806 \pm 24 \\
(22)\end{array}$ \\
\hline 76 & $\begin{array}{c}801 \pm 19 \\
\quad(41)\end{array}$ & $\begin{array}{c}840 \pm 16 \\
\quad(40)\end{array}$ & $\begin{array}{c}842 \pm 20 \\
(39)\end{array}$ & $\begin{array}{c}858 \pm 15 \\
\quad(39)\end{array}$ & $\begin{array}{c}817 \pm 17 \\
(40)\end{array}$ & $\begin{array}{c}848 \pm 19 \\
(34)\end{array}$ & $\begin{array}{c}864 \pm 24 \\
\quad(22)\end{array}$ & $\begin{array}{c}832 \pm 24 \\
\quad(21)\end{array}$ \\
\hline 80 & $\begin{array}{c}819 \pm 19 \\
(38)\end{array}$ & $\begin{array}{c}858 \pm 18 \\
(38)\end{array}$ & $\begin{array}{c}846 \pm 22 \\
(36)\end{array}$ & $\begin{array}{c}868 \pm 17 \\
\quad(39)\end{array}$ & $\begin{array}{c}828 \pm 17 \\
(37)\end{array}$ & $\begin{array}{c}862 \pm 20 \\
\quad(31)\end{array}$ & $\begin{array}{c}873 \pm 27 \\
(20)\end{array}$ & $\begin{array}{c}855 \pm 25 \\
(20)\end{array}$ \\
\hline 84 & $\begin{array}{c}827 \pm 21 \\
(34)\end{array}$ & $\begin{array}{c}867 \pm 19 \\
(37)\end{array}$ & $\begin{array}{c}858 \pm 25 \\
(29)\end{array}$ & $\begin{array}{c}890 \pm 19 \\
(36)\end{array}$ & $\begin{array}{c}833 \pm 16 \\
(33)\end{array}$ & $\begin{array}{c}875 \pm 21 \\
(29)\end{array}$ & $\begin{array}{c}889 \pm 29 \\
(18)\end{array}$ & $\begin{array}{c}854 \pm 25 \\
\text { (19) }\end{array}$ \\
\hline 88 & $\begin{array}{c}826 \pm 23 \\
(30)\end{array}$ & $\begin{array}{c}865 \pm 19 \\
(35)\end{array}$ & $\begin{array}{c}867 \pm 25 \\
(27)\end{array}$ & $\begin{array}{c}894 \pm 20 \\
\quad(31)\end{array}$ & $\begin{array}{c}829 \pm 18 \\
(30)\end{array}$ & $\begin{array}{c}871 \pm 21 \\
(25)\end{array}$ & $\begin{array}{c}917 \pm 28 \\
\quad(15)\end{array}$ & $\begin{array}{c}870 \pm 27 \\
(16)\end{array}$ \\
\hline 92 & $\begin{array}{c}823 \pm 27 \\
(26)\end{array}$ & $\begin{array}{c}858 \pm 22 \\
(31)\end{array}$ & $\begin{array}{c}873 \pm 26 \\
(25)\end{array}$ & $\begin{array}{c}905 \pm 24 \\
\quad(24)\end{array}$ & $\begin{array}{c}819 \pm 17 \\
(27)\end{array}$ & $\begin{array}{c}868 \pm 22 \\
(24)\end{array}$ & $\begin{array}{c}925 \pm 31 \\
\quad(14)\end{array}$ & $\begin{array}{c}872 \pm 27 \\
\quad(17)\end{array}$ \\
\hline 96 & $\begin{array}{c}831 \pm 30 \\
(23)\end{array}$ & $\begin{array}{c}864 \pm 24 \\
(25)\end{array}$ & $\begin{array}{c}883 \pm 23 \\
(22)\end{array}$ & $\begin{array}{c}914 \pm 24 \\
\quad(24)\end{array}$ & $\begin{array}{c}834 \pm 19 \\
(24)\end{array}$ & $\begin{array}{c}860 \pm 27 \\
\quad(16)\end{array}$ & $\begin{array}{c}925 \pm 34 \\
\text { (13) }\end{array}$ & $\begin{array}{c}873 \pm 28 \\
(16)\end{array}$ \\
\hline 100 & $\begin{array}{c}823 \pm 36 \\
(20)\end{array}$ & $\begin{array}{c}854 \pm 26 \\
(21)\end{array}$ & $\begin{array}{c}856 \pm 25 \\
(19)\end{array}$ & $\begin{array}{c}904 \pm 29 \\
\quad(22)\end{array}$ & $\begin{array}{c}846 \pm 22 \\
(20)\end{array}$ & $\begin{array}{c}894 \pm 23 \\
(14)\end{array}$ & $\begin{array}{c}926 \pm 42 \\
\quad(10)\end{array}$ & $\begin{array}{c}888 \pm 30 \\
(15)\end{array}$ \\
\hline 104 & $\begin{array}{c}818 \pm 40 \\
\quad(18)\end{array}$ & $\begin{array}{c}877 \pm 25 \\
\quad(16)\end{array}$ & $\begin{array}{c}847 \pm 30 \\
(17)\end{array}$ & $\begin{array}{c}946 \pm 36 \\
(14)\end{array}$ & $\begin{array}{c}842 \pm 22 \\
(16)\end{array}$ & $\begin{array}{c}864 \pm 12 \\
(12)\end{array}$ & $\begin{array}{c}901 \pm 53 \\
(9)\end{array}$ & $\begin{array}{c}853 \pm 23 \\
(13)\end{array}$ \\
\hline
\end{tabular}

${ }^{\mathrm{a} B P A}$ and $\mathrm{EE}_{2}$ doses are $\mu \mathrm{g} / \mathrm{kg}$ bw/day. Numbers of litters are shown in parentheses. Outlier identification and exclusion are described in Materials and Methods and Supplemental Appendix XXV. Pairwise comparisons of means were performed using contrasts within a two-way repeated measures, mixed model ANOVA. Model terms were treatment group, weeks, and the interaction. Within-group correlations were modeled using a heterogeneous first-order autoregressive (ARH(1)) correlation structure, which allows for correlated differences in variability across time points. Tests of trends, increasing treatment effect with increasing dose, were performed for the BPA and vehicle control groups. Pairwise comparisons of treatment groups to the 


\section{CLARITY-BPA Core Study}

vehicle control group were conducted with Dunnett's method to adjust for multiple comparisons. All tests were performed as two-sided tests at the 0.05 significance level. Full results of the analyses are presented in Supplemental Appendix XXV. There were no statistically significant trends or pairwise comparisons to controls. There were also no significant treatment effects in the sensitivity analysis that excluded all animals that overlapped with animals treated with $250,000 \mu \mathrm{g}$ BPA/kg bw/day (see Materials and Methods, Statistical Methods). 
Table 30. Male Postwean Body Weights (g), Vehicle and BPA Stop-Dose, Interim (1 Year) Sacrifice $(\text { Mean } \pm \text { S.E.M. })^{\mathrm{a}}$

\begin{tabular}{ccccccc}
\hline Week & Vehicle & 2.5 BPA & 25 BPA & 250 BPA & 2500 BPA & 25000 BPA \\
\hline 4 & $61 \pm 2$ & $58 \pm 3$ & $59 \pm 3$ & $60 \pm 3$ & $57 \pm 3$ & $61 \pm 2$ \\
& $(20)$ & $(20)$ & $(20)$ & $(19)$ & $(20)$ & $(22)$ \\
8 & $274 \pm 7$ & $269 \pm 8$ & $271 \pm 6$ & $270 \pm 9$ & $261 \pm 6$ & $277 \pm 4$ \\
& $(20)$ & $(20)$ & $(20)$ & $(19)$ & $(20)$ & $(22)$ \\
12 & $424 \pm 11$ & $432 \pm 9$ & $426 \pm 7$ & $430 \pm 12$ & $413 \pm 8$ & $436 \pm 8$ \\
& $(20)$ & $(20)$ & $(20)$ & $(19)$ & $(20)$ & $(22)$ \\
16 & $505 \pm 14$ & $529 \pm 12$ & $512 \pm 9$ & $515 \pm 14$ & $503 \pm 11$ & $522 \pm 9$ \\
& $(20)$ & $(20)$ & $(20)$ & $(19)$ & $(20)$ & $(22)$ \\
20 & $561 \pm 15$ & $587 \pm 14$ & $572 \pm 11$ & $566 \pm 15$ & $560 \pm 12$ & $581 \pm 10$ \\
& $(20)$ & $(20)$ & $(19)$ & $(19)$ & $(20)$ & $(22)$ \\
24 & $597 \pm 16$ & $628 \pm 16$ & $610 \pm 12$ & $603 \pm 16$ & $593 \pm 11$ & $618 \pm 11$ \\
& $(20)$ & $(20)$ & $(19)$ & $(19)$ & $(20)$ & $(22)$ \\
28 & $627 \pm 18$ & $658 \pm 17$ & $642 \pm 14$ & $630 \pm 17$ & $623 \pm 14$ & $645 \pm 12$ \\
& $(20)$ & $(20)$ & $(18)$ & $(19)$ & $(20)$ & $(22)$ \\
32 & $652 \pm 19$ & $685 \pm 18$ & $671 \pm 15$ & $655 \pm 18$ & $651 \pm 15$ & $667 \pm 12$ \\
& $(20)$ & $(20)$ & $(19)$ & $(19)$ & $(20)$ & $(22)$ \\
36 & $675 \pm 21$ & $710 \pm 19$ & $701 \pm 18$ & $677 \pm 19$ & $674 \pm 17$ & $685 \pm 13$ \\
& $(20)$ & $(20)$ & $(17)$ & $(19)$ & $(20)$ & $(22)$ \\
40 & $684 \pm 22$ & $737 \pm 21$ & $717 \pm 18$ & $687 \pm 18$ & $692 \pm 18$ & $709 \pm 14$ \\
& $(18)$ & $(19)$ & $(19)$ & $(18)$ & $(20)$ & $(22)$ \\
44 & $717 \pm 23$ & $761 \pm 21$ & $738 \pm 19$ & $716 \pm 20$ & $712 \pm 19$ & $726 \pm 15$ \\
& $(20)$ & $(20)$ & $(19)$ & $(19)$ & $(20)$ & $(22)$ \\
48 & $731 \pm 25$ & $783 \pm 23$ & $758 \pm 22$ & $735 \pm 22$ & $735 \pm 20$ & $743 \pm 16$ \\
& $(20)$ & $(19)$ & $(19)$ & $(19)$ & $(20)$ & $(22)$ \\
52 & $753 \pm 26$ & $803 \pm 23$ & $775 \pm 23$ & $743 \pm 23$ & $751 \pm 22$ & $759 \pm 17$ \\
& $(20)$ & $(20)$ & $(19)$ & $(18)$ & $(20)$ & $(22)$ \\
\hline
\end{tabular}

${ }^{\mathrm{a} B P A}$ doses are $\mu \mathrm{g} / \mathrm{kg}$ bw/day. Numbers of litters are shown in parentheses. Outlier identification and exclusion are described in Materials and Methods and Supplemental Appendix XXIV. Pairwise comparisons of means were performed using contrasts within a two-way repeated measures, mixed model ANOVA. Model terms were treatment group, weeks, and the interaction. Within-group correlations were modeled using a heterogeneous first-order autoregressive $(\mathrm{ARH}(1))$ correlation structure, which allows for correlated differences in variability across time points. Tests of trends, increasing treatment effect with increasing dose, were performed for the BPA and vehicle control groups. Pairwise comparisons of treatment groups to the vehicle control group were conducted with Dunnett's method to adjust for multiple comparisons. All tests were performed as two-sided tests at the 0.05 significance level. Full results of the analyses are presented in Supplemental Appendix XXIV. There were no statistically significant trends or pairwise comparisons to controls. There were also no significant treatment effects in the sensitivity analysis that excluded all animals that overlapped with animals treated with $250,000 \mu \mathrm{g} \mathrm{BPA} / \mathrm{kg}$ bw/day (see Materials and Methods, Statistical Methods). 
Table 31. Male Postwean Body Weights (g), Vehicle and BPA Stop-Dose, Terminal (2 Years) Sacrifice (Mean \pm S.E.M.) ${ }^{\mathrm{a}}$

\begin{tabular}{|c|c|c|c|c|c|c|}
\hline Week & Vehicle & $2.5 \mathrm{BPA}$ & 25 BPA & $250 \mathrm{BPA}$ & 2500 BPA & 25000 BPA \\
\hline 4 & $\begin{array}{c}61 \pm 2^{*} \\
(50)\end{array}$ & $\begin{array}{c}60 \pm 2 \\
(48)\end{array}$ & $\begin{array}{c}59 \pm 2 \\
(48)\end{array}$ & $\begin{array}{c}56 \pm 2 \\
(50)\end{array}$ & $\begin{array}{c}58 \pm 2 \\
(50)\end{array}$ & $\begin{array}{c}57 \pm 2 \\
(46)\end{array}$ \\
\hline 8 & $\begin{array}{c}270 \pm 4 \\
(49)\end{array}$ & $\begin{array}{c}273 \pm 5 \\
(48)\end{array}$ & $\begin{array}{c}281 \pm 4 \\
(48)\end{array}$ & $\begin{array}{c}279 \pm 4 \\
(50)\end{array}$ & $\begin{array}{c}271 \pm 4 \\
(50)\end{array}$ & $\begin{array}{c}268 \pm 5 \\
(46)\end{array}$ \\
\hline 12 & $\begin{array}{c}428 \pm 5 \\
(49)\end{array}$ & $\begin{array}{c}425 \pm 6 \\
(48)\end{array}$ & $\begin{array}{c}437 \pm 6 \\
(48)\end{array}$ & $\begin{array}{c}433 \pm 5 \\
(50)\end{array}$ & $\begin{array}{c}426 \pm 6 \\
(50)\end{array}$ & $\begin{array}{c}428 \pm 7 \\
(46)\end{array}$ \\
\hline 16 & $\begin{array}{c}509 \pm 6 \\
(49)\end{array}$ & $\begin{array}{c}508 \pm 7 \\
(48)\end{array}$ & $\begin{array}{c}522 \pm 8 \\
(48)\end{array}$ & $\begin{array}{c}515 \pm 7 \\
(50)\end{array}$ & $\begin{array}{c}511 \pm 6 \\
(50)\end{array}$ & $\begin{array}{c}515 \pm 8 \\
(46)\end{array}$ \\
\hline 20 & $\begin{array}{c}567 \pm 6 \\
(49)\end{array}$ & $\begin{array}{c}564 \pm 8 \\
(48)\end{array}$ & $\begin{array}{c}582 \pm 10 \\
\quad(48)\end{array}$ & $\begin{array}{c}572 \pm 8 \\
(50)\end{array}$ & $\begin{array}{c}569 \pm 7 \\
(50)\end{array}$ & $\begin{array}{c}574 \pm 9 \\
(43)\end{array}$ \\
\hline 24 & $\begin{array}{c}606 \pm 7 \\
(49)\end{array}$ & $\begin{array}{c}602 \pm 9 \\
(48)\end{array}$ & $\begin{array}{c}619 \pm 10 \\
(48)\end{array}$ & $\begin{array}{c}613 \pm 9 \\
(50)\end{array}$ & $\begin{array}{c}608 \pm 8 \\
(49)\end{array}$ & $\begin{array}{c}612 \pm 10 \\
(45)\end{array}$ \\
\hline 28 & $\begin{array}{c}637 \pm 8 \\
(48)\end{array}$ & $\begin{array}{c}630 \pm 10 \\
(48)\end{array}$ & $\begin{array}{c}648 \pm 11 \\
(45)\end{array}$ & $\begin{array}{c}642 \pm 10 \\
(50)\end{array}$ & $\begin{array}{c}635 \pm 8 \\
(49)\end{array}$ & $\begin{array}{c}639 \pm 10 \\
(45)\end{array}$ \\
\hline 32 & $\begin{array}{c}664 \pm 9 \\
(47)\end{array}$ & $\begin{array}{c}655 \pm 10 \\
(48)\end{array}$ & $\begin{array}{c}673 \pm 12 \\
(48)\end{array}$ & $\begin{array}{c}665 \pm 10 \\
(50)\end{array}$ & $\begin{array}{c}661 \pm 9 \\
(49)\end{array}$ & $\begin{array}{c}664 \pm 11 \\
(45)\end{array}$ \\
\hline 36 & $\begin{array}{c}688 \pm 10 \\
(49)\end{array}$ & $\begin{array}{c}681 \pm 11 \\
(48)\end{array}$ & $\begin{array}{c}698 \pm 12 \\
(48)\end{array}$ & $\begin{array}{c}687 \pm 11 \\
(50)\end{array}$ & $\begin{array}{c}683 \pm 9 \\
(49)\end{array}$ & $\begin{array}{c}688 \pm 12 \\
(45)\end{array}$ \\
\hline 40 & $\begin{array}{c}704 \pm 10 \\
(47)\end{array}$ & $\begin{array}{c}695 \pm 11 \\
(44)\end{array}$ & $\begin{array}{c}724 \pm 14 \\
(44)\end{array}$ & $\begin{array}{c}705 \pm 14 \\
(44)\end{array}$ & $\begin{array}{c}700 \pm 10 \\
(46)\end{array}$ & $\begin{array}{c}710 \pm 14 \\
(42)\end{array}$ \\
\hline 44 & $\begin{array}{c}732 \pm 12 \\
(46)\end{array}$ & $\begin{array}{c}724 \pm 12 \\
(48)\end{array}$ & $\begin{array}{c}746 \pm 14 \\
(47)\end{array}$ & $\begin{array}{c}731 \pm 14 \\
(50)\end{array}$ & $\begin{array}{c}723 \pm 10 \\
(48)\end{array}$ & $\begin{array}{c}732 \pm 15 \\
(43)\end{array}$ \\
\hline 48 & $\begin{array}{c}752 \pm 12 \\
(49)\end{array}$ & $\begin{array}{c}745 \pm 13 \\
\quad(48)\end{array}$ & $\begin{array}{c}763 \pm 15 \\
(48)\end{array}$ & $\begin{array}{c}749 \pm 15 \\
(50)\end{array}$ & $\begin{array}{c}744 \pm 11 \\
(49)\end{array}$ & $\begin{array}{c}755 \pm 16 \\
(43)\end{array}$ \\
\hline 52 & $\begin{array}{c}772 \pm 13 \\
(49)\end{array}$ & $\begin{array}{c}763 \pm 14 \\
(47)\end{array}$ & $\begin{array}{c}781 \pm 15 \\
(48)\end{array}$ & $\begin{array}{c}757 \pm 14 \\
(49)\end{array}$ & $\begin{array}{c}756 \pm 12 \\
(49)\end{array}$ & $\begin{array}{c}770 \pm 17 \\
(43)\end{array}$ \\
\hline 56 & $\begin{array}{c}789 \pm 14 \\
(49)\end{array}$ & $\begin{array}{c}778 \pm 15 \\
(47)\end{array}$ & $\begin{array}{c}800 \pm 16 \\
\quad(48)\end{array}$ & $\begin{array}{c}773 \pm 15 \\
(49)\end{array}$ & $\begin{array}{c}778 \pm 13 \\
(49)\end{array}$ & $\begin{array}{c}792 \pm 18 \\
(42)\end{array}$ \\
\hline 60 & $\begin{array}{c}811 \pm 15 \\
\quad(48)\end{array}$ & $\begin{array}{c}799 \pm 17 \\
(46)\end{array}$ & $\begin{array}{c}813 \pm 17 \\
(46)\end{array}$ & $\begin{array}{c}798 \pm 16 \\
(46)\end{array}$ & $\begin{array}{c}793 \pm 14 \\
(49)\end{array}$ & $\begin{array}{c}810 \pm 19 \\
(42)\end{array}$ \\
\hline 64 & $\begin{array}{c}831 \pm 16 \\
\quad(48)\end{array}$ & $\begin{array}{c}815 \pm 16 \\
\quad(44)\end{array}$ & $\begin{array}{c}832 \pm 18 \\
\quad(46)\end{array}$ & $\begin{array}{c}816 \pm 17 \\
\quad(45)\end{array}$ & $\begin{array}{c}815 \pm 15 \\
\quad(47)\end{array}$ & $\begin{array}{c}826 \pm 20 \\
(42)\end{array}$ \\
\hline 68 & $\begin{array}{c}855 \pm 16 \\
(46)\end{array}$ & $\begin{array}{c}834 \pm 18 \\
\quad(43)\end{array}$ & $\begin{array}{c}846 \pm 19 \\
(46)\end{array}$ & $\begin{array}{c}837 \pm 17 \\
(45)\end{array}$ & $\begin{array}{c}836 \pm 16 \\
(45)\end{array}$ & $\begin{array}{c}848 \pm 22 \\
(39)\end{array}$ \\
\hline 72 & $\begin{array}{c}874 \pm 17 \\
(46)\end{array}$ & $\begin{array}{c}851 \pm 19 \\
(42)\end{array}$ & $\begin{array}{c}861 \pm 22 \\
\quad(43)\end{array}$ & $\begin{array}{c}837 \pm 19 \\
(42)\end{array}$ & $\begin{array}{c}858 \pm 18 \\
(44)\end{array}$ & $\begin{array}{c}866 \pm 22 \\
(35)\end{array}$ \\
\hline 76 & $\begin{array}{c}884 \pm 17 \\
\quad(44)\end{array}$ & $\begin{array}{c}870 \pm 21 \\
(39)\end{array}$ & $\begin{array}{c}896 \pm 22 \\
\quad(40)\end{array}$ & $\begin{array}{c}874 \pm 19 \\
(37)\end{array}$ & $\begin{array}{c}869 \pm 18 \\
(41)\end{array}$ & $\begin{array}{c}878 \pm 24 \\
(34)\end{array}$ \\
\hline 80 & $\begin{array}{c}888 \pm 18 \\
\quad(43)\end{array}$ & $\begin{array}{c}883 \pm 23 \\
(39)\end{array}$ & $\begin{array}{c}913 \pm 25 \\
(38)\end{array}$ & $\begin{array}{c}892 \pm 22 \\
(33)\end{array}$ & $\begin{array}{c}875 \pm 20 \\
(40)\end{array}$ & $\begin{array}{c}893 \pm 30 \\
(29)\end{array}$ \\
\hline 84 & $\begin{array}{c}896 \pm 17 \\
\quad(39)\end{array}$ & $\begin{array}{c}873 \pm 22 \\
(35)\end{array}$ & $\begin{array}{c}920 \pm 25 \\
(36)\end{array}$ & $\begin{array}{c}895 \pm 24 \\
(30)\end{array}$ & $\begin{array}{c}879 \pm 18 \\
(37)\end{array}$ & $\begin{array}{c}915 \pm 32 \\
(26)\end{array}$ \\
\hline 88 & $\begin{array}{c}906 \pm 18 \\
\quad(37)\end{array}$ & $\begin{array}{c}885 \pm 26 \\
(31)\end{array}$ & $\begin{array}{c}932 \pm 23 \\
(33)\end{array}$ & $\begin{array}{c}908 \pm 30 \\
(26)\end{array}$ & $\begin{array}{c}874 \pm 21 \\
(34)\end{array}$ & $\begin{array}{c}918 \pm 34 \\
(23)\end{array}$ \\
\hline 92 & $\begin{array}{c}903 \pm 16 \\
(32)\end{array}$ & $\begin{array}{c}879 \pm 25 \\
(27)\end{array}$ & $\begin{array}{c}911 \pm 22 \\
(31)\end{array}$ & $\begin{array}{c}935 \pm 35 \\
(21)\end{array}$ & $\begin{array}{c}887 \pm 23 \\
(30)\end{array}$ & $\begin{array}{c}955 \pm 28 \\
(20)\end{array}$ \\
\hline 96 & $\begin{array}{c}903 \pm 19 \\
(29)\end{array}$ & $\begin{array}{c}874 \pm 28 \\
(25)\end{array}$ & $\begin{array}{c}901 \pm 26 \\
(27)\end{array}$ & $\begin{array}{c}941 \pm 44 \\
\quad(17)\end{array}$ & $\begin{array}{c}884 \pm 27 \\
(27)\end{array}$ & $\begin{array}{c}925 \pm 30 \\
(16)\end{array}$ \\
\hline 100 & $\begin{array}{c}928 \pm 20 \\
\quad(21)\end{array}$ & $\begin{array}{c}876 \pm 29 \\
(18)\end{array}$ & $\begin{array}{c}893 \pm 30 \\
(21)\end{array}$ & $\begin{array}{c}953 \pm 49 \\
(13)\end{array}$ & $\begin{array}{c}878 \pm 26 \\
(25)\end{array}$ & $\begin{array}{c}932 \pm 31 \\
(14)\end{array}$ \\
\hline 104 & $\begin{array}{c}908 \pm 23 \\
(16)\end{array}$ & $\begin{array}{c}845 \pm 30 \\
(16)\end{array}$ & $\begin{array}{c}908 \pm 33 \\
(16)\end{array}$ & $\begin{array}{c}941 \pm 45 \\
(13)\end{array}$ & $\begin{array}{c}863 \pm 33 \\
(16)\end{array}$ & $\begin{array}{c}894 \pm 37 \\
(10) \\
\end{array}$ \\
\hline
\end{tabular}

${ }^{\mathrm{a} B P A}$ doses are $\mu \mathrm{g} / \mathrm{kg}$ bw/day. Numbers of litters are shown in parentheses. Outlier identification and exclusion are described in Materials and Methods and Supplemental Appendix XXV. Pairwise comparisons of means were performed using contrasts within a two-way repeated measures, mixed model ANOVA. Model terms were treatment group, weeks, and the interaction. Within-group correlations were modeled using a heterogeneous first-order autoregressive $(\mathrm{ARH}(1))$ correlation structure, which allows for correlated differences in variability across time points. Tests of trends, increasing treatment effect with increasing dose, were performed for the BPA and vehicle control groups. Pairwise comparisons of treatment groups to the vehicle control 


\section{CLARITY-BPA Core Study}

group were conducted with Dunnett's method to adjust for multiple comparisons. All tests were performed as two-sided tests at the 0.05 significance level. Full results of the analyses are presented in Supplemental Appendix XXV. The only significant effect is indicated with an asterisk (dose trend, week $4, p=0.043$ ). There were no additional significant treatment effects in the sensitivity analysis that excluded all animals that overlapped with animals treated with $250,000 \mu \mathrm{g}$ BPA/kg bw/day (see Materials and Methods, Statistical Methods). 
Table 32. Vaginal Opening, Age and Body Weight (Means \pm S.E.M.) at Occurrence, Vehicle, BPA, and EE Continuous-Dose $^{\text {a }}$

\begin{tabular}{|c|c|c|c|c|c|c|c|c|}
\hline Endpoint & $\begin{array}{c}\text { Vehicle } \\
(26)^{b}\end{array}$ & $\begin{array}{c}2.5 \text { BPA } \\
(25)\end{array}$ & $\begin{array}{c}25 \text { BPA } \\
(24)\end{array}$ & $\begin{array}{c}250 \text { BPA } \\
(25)\end{array}$ & $\begin{array}{c}2500 \text { BPA } \\
(25)\end{array}$ & $\begin{array}{c}25000 \text { BPA } \\
(24)\end{array}$ & $\begin{array}{c}0.05 \mathrm{EE}_{2} \\
(25)\end{array}$ & $\begin{array}{c}0.5 \text { EE2 } \\
(21)\end{array}$ \\
\hline Age, days & $35.9 \pm 1.1$ & $35.2 \pm 0.7$ & $36.5 \pm 0.8$ & $37.8 \pm 1.4$ & $34.1 \pm 0.5$ & $35.4 \pm 0.6$ & $35.5 \pm 0.7$ & $34.8 \pm 2.8$ \\
\hline Body weight, $g$ & $120.5 \pm 4.6$ & $117.1 \pm 5.0$ & $128.6 \pm 4.6$ & $131.1 \pm 5.8$ & $109.6 \pm 2.6$ & $121.0 \pm 4.3$ & $123.0 \pm 4.8$ & $117.1 \pm 11.8$ \\
\hline
\end{tabular}

${ }^{\mathrm{a}} \mathrm{BPA}$ and $\mathrm{EE}_{2}$ doses are $\mu \mathrm{g} / \mathrm{kg}$ bw/day. Twenty-six females (13 cages) from the 2-year continuous-dose groups were scheduled to be assessed for age and weight at vaginal opening and undergo vaginal cytology later in the study to evaluate estrous cyclicity. There were no litter mates among these animals. Several dose groups have less than 26 animals due to either failure to record the information or delayed start of monitoring for vaginal opening. These incidents were documented in protocol deviations. BPA and EE 2 dose groups were analyzed separately. Analyses were performed using contrasts within a one-way ANOVA to test for treatment effect. Tests of trends, increasing treatment effect with increasing dose, were performed for the BPA and vehicle control groups. Comparisons of dosed groups to vehicle control for age and body weight were performed with Dunnett's method for adjusted contrasts. All tests were performed as two-sided tests at the 0.05 significance level. The full statistical report is found in Supplemental Appendix XXVI. There were no statistically significant treatment effects. There were no additional significant treatment effects in the sensitivity analysis that excluded all animals that overlapped with animals treated with $250,000 \mu \mathrm{g}$ BPA/kg bw/day (see Materials and Methods, Statistical Methods).

bNumbers in parentheses are the number of animals examined.

Table 33. Vaginal Opening, Age and Body Weight (Means \pm S.E.M.) at Occurrence, Vehicle and BPA Stop-Dose ${ }^{\mathrm{a}}$

\begin{tabular}{|c|c|c|c|c|c|c|}
\hline Endpoint & $\begin{array}{c}\text { Vehicle } \\
(26)^{b}\end{array}$ & $\begin{array}{c}2.5 \text { BPA } \\
(26)\end{array}$ & $\begin{array}{c}25 \text { BPA } \\
(25)\end{array}$ & $\begin{array}{c}250 \mathrm{BPA} \\
(26)\end{array}$ & $\begin{array}{c}2500 \text { BPA } \\
(26)\end{array}$ & $\begin{array}{c}25000 \text { BPA } \\
(26)\end{array}$ \\
\hline Age, days & $41.1 \pm 1.8$ & $42.1 \pm 2.5$ & $40.0 \pm 1.5$ & $39.6 \pm 1.2$ & $42.4 \pm 1.2$ & $38.0 \pm 1.3$ \\
\hline Body weight, g & - & - & - & - & - & - \\
\hline
\end{tabular}

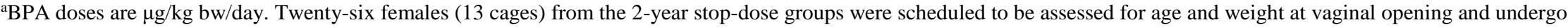
vaginal cytology later in the study to evaluate estrous cyclicity. There were no litter mates among these animals. The $25 \mu \mathrm{g}$ BPA/kg bw/day dose group had one animal for which the date of vaginal opening was not recorded. Due to a technical error, 31 animals in the stop-dose arm did not have body weights recorded on the day of vaginal opening and thus this endpoint was not analyzed (Supplemental Appendix II, protocol deviations \#72-74). Analysis was performed using contrasts within a one-way ANOVA to test for treatment effect. Tests of trends, increasing treatment effect with increasing dose, were performed for the BPA and vehicle control groups. Comparisons of dosed groups to vehicle control for age and body weight were performed with Dunnett's method for adjusted contrasts. All tests were performed as two-sided tests at the 0.05 significance level. The full statistical report is found in Supplemental Appendix XXVI. There were no statistically significant treatment effects. There were no additional significant treatment effects in the sensitivity analysis that excluded all animals that overlapped with animals treated with 250,000 $\mu \mathrm{g} \mathrm{BPA} / \mathrm{kg}$ bw/day (see Materials and Methods, Statistical Methods).

${ }^{b}$ Numbers in parentheses are the number of animals examined. 
CLARITY-BPA Core Study

Table 34. Estrous Cycle Analysis, Vehicle, BPA, and $\mathrm{EE}_{2}$ Continuous-Dose $^{\mathrm{a}}$

\begin{tabular}{|c|c|c|c|c|c|c|c|c|}
\hline Endpoint & $\begin{array}{c}\text { Vehicle } \\
(26)^{b} \\
\end{array}$ & $\begin{array}{c}2.5 \text { BPA } \\
(25)\end{array}$ & $\begin{array}{c}25 \text { BPA } \\
(26)\end{array}$ & $\begin{array}{c}250 \text { BPA } \\
(25)\end{array}$ & $\begin{array}{c}2500 \text { BPA } \\
(26)\end{array}$ & $\begin{array}{c}25000 \text { BPA } \\
(25)\end{array}$ & $\begin{array}{c}0.05 \mathrm{EE}_{2} \\
(26)\end{array}$ & $\begin{array}{c}0.5 \mathrm{EE}_{2} \\
(26)\end{array}$ \\
\hline \# readable smears & 362 & 348 & 359 & 348 & 362 & 348 & 363 & 361 \\
\hline$\%$ Diestrus & 57.7 & 54.0 & 55.2 & 49.4 & 59.9 & 55.7 & 54.3 & 15.5 \\
\hline$\%$ Proestrus & 13.8 & 14.9 & 11.1 & 17.2 & 13.8 & 12.6 & 11.0 & 0.8 \\
\hline$\%$ Estrus & 28.5 & 31.0 & 33.7 & 33.3 & 26.2 & 31.6 & 34.7 & 83.7 \\
\hline Cycle length, days ${ }^{c}$ & $4.37 \pm 0.18^{\mathrm{d}}$ & $4.56 \pm 0.29^{\mathrm{d}}$ & $4.47 \pm 0.21^{\mathrm{e}}$ & $5.20 \pm 0.51^{\mathrm{e}}$ & $4.33 \pm 0.15^{\mathrm{e}}$ & $4.84 \pm 0.31^{\mathrm{f}}$ & $4.72 \pm 0.33^{\mathrm{d}}$ & $5.58 \pm 0.64^{\mathrm{g}}$ \\
\hline \multicolumn{9}{|l|}{ Abnormal Diestrus } \\
\hline Abnormal & 4 & 2 & 4 & 1 & 4 & 5 & 6 & 2 \\
\hline Normal & 22 & 23 & 22 & 24 & 22 & 20 & 20 & 24 \\
\hline$\%$ Abnormal & 15.4 & 8.0 & 15.4 & 4.0 & 15.4 & 20.0 & 23.1 & 7.7 \\
\hline \multicolumn{9}{|l|}{ Abnormal Estrus ${ }^{h}$} \\
\hline Abnormal & 3 & 3 & 4 & 4 & 2 & 4 & 4 & 25 \\
\hline Normal & 23 & 22 & 22 & 21 & 24 & 21 & 22 & 1 \\
\hline$\%$ Abnormal & 11.5 & 12.0 & 15.4 & 16.0 & 7.7 & 16.0 & 15.4 & $96.2^{* * * *}$ \\
\hline \multicolumn{9}{|l|}{ Abnormal Proestrus ${ }^{\text {h }}$} \\
\hline Abnormal & 0 & 0 & 0 & 1 & 1 & 1 & 0 & 0 \\
\hline Normal & 26 & 25 & 26 & 24 & 25 & 24 & 26 & 26 \\
\hline$\%$ Abnormal & 0 & 0 & 0 & 4.0 & 3.8 & 4.0 & 0 & 0 \\
\hline \multicolumn{9}{|l|}{ Combined Abnormal } \\
\hline Abnormal & 7 & 5 & 8 & 5 & 7 & 9 & 10 & 26 \\
\hline Normal & 19 & 20 & 18 & 20 & 19 & 16 & 16 & 0 \\
\hline$\%$ Abnormal & 26.9 & 20.0 & 30.8 & 20.0 & 26.9 & 36.0 & 38.5 & $100^{* * *}$ \\
\hline
\end{tabular}

${ }^{\mathrm{a}} \mathrm{BPA}$ and $\mathrm{EE}_{2}$ doses are $\mu \mathrm{g} / \mathrm{kg}$ bw/day. At $16 \pm 2$ weeks, daily vaginal smears were collected for 14 consecutive days from 26 animals (13 cages) assigned to the continuous-dose study arm, two-year sacrifice. There were no litter mates among the animals.

bNumbers in parentheses are the number of animals examined. Any deviations from this number due to exclusions are indicated by footnotes.

${ }^{c}$ Cycle length: first day of estrus in one sequence of contiguous days to the first day of estrus in the following sequence of stages. For cycle length, cycle days were defined from the first day of estrus in one sequence of contiguous days to the first day of estrus in the following sequence of stages. Cycles were considered censored if the last stage of data collection was either diestrus or proestrus. The number of animals, $\mathrm{n}$, for this endpoint includes all animals with at least one uncensored cycle.

$\mathrm{d}_{\mathrm{n}}=23$.

$\mathrm{e} n=24$.

$\mathrm{f} n=22$.

$\mathrm{g}_{\mathrm{n}}=12$.

${ }^{\mathrm{h}}$ Abnormal (extended) diestrus was defined as four or more consecutive days of diestrus; abnormal estrus was defined as three or more consecutive days of estrus; and extended proestrus was defined as two or more consecutive days of proestrus. The Cochran-Armitage trend test (one-sided) was performed, and Fisher's exact test (two-sided) was conducted for comparisons of dosed groups to control. $P$-values for pairwise comparisons were corrected using Holm's method, and unadjusted $p$-values are also presented in the full statistical report (Supplemental Appendix XXVII). BPA and $E_{2}$ dose groups were analyzed separately. Statistically significant treatment effects are shown with asterisks and were confined to the high $\mathrm{EE}_{2}$ dose group; $* * *, p<0.001$. There were no additional significant effects in the sensitivity analysis that excluded all animals that overlapped with animals treated with 250,000 $\mu \mathrm{g} \mathrm{BPA} / \mathrm{kg}$ bw/day (see Materials and Methods, Statistical Methods). 
CLARITY-BPA Core Study

Table 35. Estrous Cycle Analysis, Vehicle and BPA Stop-Dose ${ }^{\mathrm{a}}$

\begin{tabular}{|c|c|c|c|c|c|c|}
\hline Endpoint & $\begin{array}{c}\text { Vehicle } \\
(26)^{b}\end{array}$ & $\begin{array}{c}2.5 \text { BPA } \\
(26)\end{array}$ & $\begin{array}{c}25 \text { BPA } \\
\text { (26) }\end{array}$ & $\begin{array}{c}250 \text { BPA } \\
\text { (26) }\end{array}$ & $\begin{array}{c}2500 \text { BPA } \\
(26)\end{array}$ & $\begin{array}{c}25000 \text { BPA } \\
(26)\end{array}$ \\
\hline \# readable smears & 360 & 360 & 361 & 363 & 362 & 359 \\
\hline$\%$ Diestrus & 56.4 & 60.3 & 52.4 & 51.2 & 58.3 & 58.8 \\
\hline$\%$ Proestrus & 13.1 & 9.2 & 14.4 & 15.2 & 13.8 & 10.3 \\
\hline$\%$ Estrus & 30.6 & 30.6 & 33.2 & 33.6 & 27.9 & 30.9 \\
\hline Cycle length, days ${ }^{\mathrm{c}}$ & $4.08 \pm 0.12^{\mathrm{d}}$ & $4.23 \pm 0.13^{\mathrm{e}}$ & $4.17 \pm 0.12^{\mathrm{f}}$ & $4.47 \pm 0.23^{\mathrm{g}}$ & $4.42 \pm 0.15$ & $4.38 \pm 0.17^{\mathrm{h}}$ \\
\hline \multicolumn{7}{|l|}{ Abnormal Diestrus ${ }^{i}$} \\
\hline Abnormal & 5 & 5 & 2 & 1 & 4 & 5 \\
\hline Normal & 21 & 21 & 24 & 25 & 22 & 21 \\
\hline$\%$ Abnormal & 19.2 & 19.2 & 7.7 & 3.8 & 15.4 & 19.2 \\
\hline \multicolumn{7}{|l|}{ Abnormal Estrus ${ }^{i}$} \\
\hline Abnormal & 5 & 2 & 3 & 5 & 2 & 3 \\
\hline Normal & 21 & 24 & 23 & 21 & 24 & 23 \\
\hline$\%$ Abnormal & 19.2 & 7.7 & 11.5 & 19.2 & 7.7 & 11.5 \\
\hline \multicolumn{7}{|l|}{ Abnormal Proestrusi } \\
\hline Abnormal & 2 & 1 & 1 & 0 & 0 & 0 \\
\hline Normal & 24 & 25 & 25 & 26 & 26 & 26 \\
\hline$\%$ Abnormal & 7.7 & 3.8 & 3.8 & 0 & 0 & 0 \\
\hline \multicolumn{7}{|l|}{ Combined Abnormal } \\
\hline Abnormal & 10 & 7 & 5 & 6 & 6 & 8 \\
\hline Normal & 16 & 19 & 21 & 20 & 20 & 18 \\
\hline$\%$ Abnormal & 38.5 & 26.9 & $19.2^{\mathrm{j}}$ & 23.1 & 23.1 & 30.8 \\
\hline
\end{tabular}

${ }^{a}$ BPA doses are $\mu \mathrm{g} / \mathrm{kg}$ bw/day. At $16 \pm 2$ weeks, daily vaginal smears were collected for 14 consecutive days from 26 animals (13 cages) assigned to the stop-dose study arm, twoyear sacrifice. There were no litter mates among the animals.

${ }^{b}$ Numbers in parentheses are the number of animals examined. Any deviations from this number due to exclusions are indicated by footnotes.

${ }^{c}$ Cycle length: first day of estrus in one sequence of contiguous days to the first day of estrus in the following sequence of stages. For cycle length, cycle days were defined from the first day of estrus in one sequence of contiguous days to the first day of estrus in the following sequence of stages. Cycles were considered censored if the last stage of data collection was either diestrus or proestrus. The number of animals, $\mathrm{n}$, for this endpoint includes all animals with at least one uncensored cycle.

${ }^{\mathrm{d}} \mathrm{n}=20$.

$\mathrm{e}_{\mathrm{n}}=22$.

$\mathrm{f}_{\mathrm{n}}=23$.

$\mathrm{g}_{\mathrm{n}}=25$.

$\mathrm{h}_{\mathrm{n}}=24$.

iAbnormal (extended) diestrus was defined as four or more consecutive days of diestrus; abnormal estrus was defined as three or more consecutive days of estrus; and extended proestrus was defined as two or more consecutive days of proestrus. The Cochran-Armitage trend test (one-sided) was performed, and Fisher's exact test (two-sided) was

conducted for comparisons of dosed groups to control. $P$-values for pairwise comparisons were corrected using Holm's method, and unadjusted $p$-values are also presented in the full statistical report (Supplemental Appendix XXVII). There were no statistically significant treatment effects.

In the sensitivity analysis that excluded all animals that overlapped with animals treated with $250,000 \mu \mathrm{g} \mathrm{BPA} / \mathrm{kg}$ bw/day (see Materials and Methods, Statistical Methods), there was a significant difference for combined abnormal for BPA stop-dose $25 \mu \mathrm{g} / \mathrm{kg}$ bw/day compared to the vehicle control group ( $p=0.038$ ). The proportion of total $\%$ abnormal in the $25 \mu \mathrm{g} \mathrm{BPA} / \mathrm{kg}$ bw/day stop-dose group was lower than in the vehicle control (5.0\% abnormal in the dosed group compared to $33.3 \%$ abnormal in the control). 
CLARITY-BPA Core Study

Table 36. Time to Onset of Aberrant Estrous Cycles in Vehicle, BPA, and $\mathrm{EE}_{2}$ Continuous-Dose Groups $^{\mathrm{a}}$

\begin{tabular}{|c|c|c|c|c|c|c|c|c|}
\hline Statistic & $\begin{array}{l}\text { Vehicle } \\
(26)^{b}\end{array}$ & $\begin{array}{l}2.5 \text { BPA } \\
\text { (25) }\end{array}$ & $\begin{array}{c}25 \text { BPA } \\
(25)\end{array}$ & $\begin{array}{l}250 \text { BPA } \\
\text { (25) }\end{array}$ & $\begin{array}{c}2500 \text { BPA } \\
(26)\end{array}$ & $\begin{array}{c}25000 \text { BPA } \\
\text { (25) }\end{array}$ & $\begin{array}{l}\mathbf{0 . 0 5} \mathbf{E E}_{2} \\
(26)\end{array}$ & $\begin{array}{l}0.5 \mathrm{EE}_{2} \\
(26)\end{array}$ \\
\hline$\%$ uncensored & 88 & 96 & 100 & 84 & 81 & 96 & 88 & 23 \\
\hline $\begin{array}{l}\% \text { aberrant at start (left } \\
\text { censored) }\end{array}$ & 4 & 0 & 0 & 8 & 4 & 4 & 4 & 77 \\
\hline $\begin{array}{l}\% \text { with normal cycles at } \\
\text { removal (right censored) }\end{array}$ & 8 & 4 & 0 & 8 & 15 & 0 & 8 & 0 \\
\hline $\begin{array}{l}\text { Median onset of aberrant } \\
\text { cycles, weeks (lower and } \\
\text { upper } 95 \% \text { confidence } \\
\text { intervals) }\end{array}$ & $\begin{array}{c}56.8 \\
(42.0,66.9)\end{array}$ & $\begin{array}{c}47.0 \\
(36.9,52.0)\end{array}$ & $\begin{array}{c}51.9 \\
(42.1,56.9)\end{array}$ & $\begin{array}{c}56.9 \\
(46.9,61.9)\end{array}$ & $\begin{array}{c}52.0 \\
(46.9,56.7)\end{array}$ & $\begin{array}{c}46.9 \\
(41.7,56.9)\end{array}$ & $\begin{array}{c}51.8 \\
(37,62.1)\end{array}$ & $\begin{array}{c}21.9^{* * *} \\
(21.7,22)\end{array}$ \\
\hline$p$-value & - & 0.74 & 0.80 & 0.79 & 0.80 & 0.79 & 0.36 & $<0.001^{* * * *}$ \\
\hline
\end{tabular}

${ }^{a} \mathrm{BPA}$ and $\mathrm{EE}_{2}$ doses are $\mu \mathrm{g} / \mathrm{kg}$ bw/day. One month after the collection of the 14 consecutive vaginal smears to evaluate the estrous cycle (Table 34 ), the same animals from the continuous-dose, two-year study arm were monitored monthly for cycling status with five consecutive daily vaginal smears. The criteria for declaring an animal as having an aberrant estrous cycle were 3 or more consecutive days of estrus (E, E/D, or P/E) or five consecutive days that did not include an estrus. The animal was no longer monitored after two consecutive months with an aberrant cycle, and the time of onset of aberrant cycling was defined as having occurred at the first swab date of two consecutive months of

aberrant estrous cycle data. Separate analyses were conducted for the BPA and $\mathrm{EE}_{2}$ dose groups. An accelerated failure time model assuming a lognormal distribution was used for analysis, and multiple comparisons were adjusted using Holm's method for treatment comparisons to the control. All tests were performed as two-sided tests at the 0.05

significance level. The full statistical report is found in Supplemental Appendix XXVIII. Statistically significant results are marked with asterisks (***, $p<0.001$ ). There were no additional significant effects in the sensitivity analysis that excluded all animals that overlapped with animals treated with 250,000 $\mu \mathrm{g}$ BPA/kg bw/day (see Materials and Methods, Statistical Methods).

${ }^{b}$ Numbers in parentheses are the number of animals examined. 
CLARITY-BPA Core Study

Table 37. Time to Onset of Aberrant Estrous Cycles in Vehicle and BPA Stop-Dose Groups ${ }^{\mathrm{a}}$

\begin{tabular}{|c|c|c|c|c|c|c|}
\hline Statistic & $\begin{array}{c}\text { Vehicle } \\
(26)^{b}\end{array}$ & $\begin{array}{c}2.5 \text { BPA } \\
(26)\end{array}$ & $\begin{array}{c}25 \text { BPA } \\
(26)\end{array}$ & $\begin{array}{c}250 \text { BPA } \\
(26)\end{array}$ & $\begin{array}{c}2500 \text { BPA } \\
(25)\end{array}$ & $\begin{array}{c}25000 \mathrm{BPA} \\
(26)\end{array}$ \\
\hline$\%$ uncensored & 88 & 77 & 96 & 88 & 80 & 92 \\
\hline$\%$ aberrant at start (left censored) & 0 & 8 & 0 & 8 & 4 & 0 \\
\hline $\begin{array}{l}\% \text { with normal cycles at removal } \\
\text { (right censored) }\end{array}$ & 12 & 15 & 4 & 4 & 16 & 8 \\
\hline $\begin{array}{l}\text { Median onset of aberrant cycles, } \\
\text { weeks (lower and upper } 95 \% \\
\text { confidence intervals) }\end{array}$ & $\begin{array}{c}41.9 \\
(41.3,51.7)\end{array}$ & $\begin{array}{c}51.7 \\
(36.9,57.0)\end{array}$ & $\begin{array}{c}46.8 \\
(41.9,56.9)\end{array}$ & $\begin{array}{c}51.9 \\
(41.9,56.9)\end{array}$ & $\begin{array}{c}56.9^{*} \\
(51.7,66.6)\end{array}$ & $\begin{array}{c}52.1 \\
(41.9,61.9)\end{array}$ \\
\hline$p$-value & - & 1.00 & 0.83 & 1.00 & $0.03^{*}$ & 0.52 \\
\hline
\end{tabular}

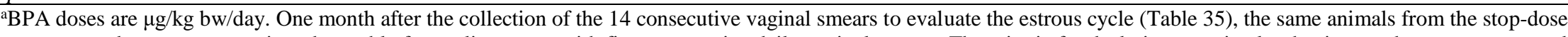
two-year study arm were monitored monthly for cycling status with five consecutive daily vaginal smears. The criteria for declaring an animal as having an aberrant estrous cycle were 3 or more consecutive days of estrus (E, E/D, or P/E) or five consecutive days that did not include an estrus. The animal was no longer monitored after two consecutive months with an aberrant cycle, and the time of onset of aberrant cycling was defined as having occurred at the first swab date of two consecutive months of aberrant estrous cycle data. An accelerated failure time model assuming a lognormal distribution was used for analysis, and multiple comparisons were adjusted using Holm's method for treatment comparisons to the control. All tests were performed as two-sided tests at the 0.05 significance level. The full statistical report is found in Supplemental Appendix XXVIII.

Statistically significant results are marked with an asterisk $\left(^{*}, p<0.05\right)$. There were no additional significant effects in the sensitivity analysis that excluded all animals that overlapped with animals treated with 250,000 $\mu \mathrm{g}$ BPA/kg bw/day (see Materials and Methods, Statistical Methods).

bumber in parentheses are the number of animals examined. 
CLARITY-BPA Core Study

Table 38. Female Hematology, Vehicle, BPA, and $\mathrm{EE}_{2}$ Continuous-Dose, Interim Sacrifice (Mean \pm S.E.M.) ${ }^{\mathrm{a}}$

\begin{tabular}{|c|c|c|c|c|c|c|c|c|}
\hline Endpoint, Units & $\begin{array}{c}\text { Vehicle } \\
(21)^{b} \\
\end{array}$ & $\begin{array}{c}2.5 \text { BPA } \\
(22)\end{array}$ & $\begin{array}{c}25 \text { BPA } \\
(21)\end{array}$ & $\begin{array}{c}250 \text { BPA } \\
(22) \\
\end{array}$ & $\begin{array}{l}2500 \text { BPA } \\
(20) \\
\end{array}$ & $\begin{array}{c}25000 \text { BPA } \\
(24) \\
\end{array}$ & $\begin{array}{c}0.05 \mathrm{EE}_{2} \\
(24) \\
\end{array}$ & $\begin{array}{c}0.5 \mathrm{EE}_{2} \\
(26) \\
\end{array}$ \\
\hline Hematocrit, \% & $47.3 \pm 0.4$ & $46.0 \pm 0.4$ & $45.7 \pm 0.5$ & $47.4 \pm 0.5$ & $46.8 \pm 0.5$ & $47.5 \pm 0.3$ & $47.1 \pm 0.5$ & $46.7 \pm 0.8$ \\
\hline Hemoglobin, g/dL & $16.4 \pm 0.1^{*}$ & $16.0 \pm 0.1$ & $16.0 \pm 0.2$ & $16.5 \pm 0.2$ & $16.3 \pm 0.2$ & $16.5 \pm 0.1$ & $16.4 \pm 0.2$ & $16.3 \pm 0.2$ \\
\hline Red Blood Cells, $10^{6} / \mathrm{mm}^{3}$ & $8.3 \pm 0.1$ & $8.2 \pm 0.1$ & $8.1 \pm 0.1$ & $8.5 \pm 0.1$ & $8.3 \pm 0.1$ & $8.4 \pm 0.1$ & $8.3 \pm 0.1$ & $8.2 \pm 0.1$ \\
\hline$\%$ Reticulocytes & $1.3 \pm 0.1$ & $1.3 \pm 0$ & $1.2 \pm 0$ & $1.4 \pm 0.1$ & $1.3 \pm 0.1$ & $1.2 \pm 0.0$ & $1.2 \pm 0.0$ & $1.2 \pm 0.1$ \\
\hline Packed Cell Volume, \% & $47.3 \pm 0.4$ & $46.0 \pm 0.4$ & $45.9 \pm 0.5$ & $47.4 \pm 0.5$ & $46.9 \pm 0.5$ & $47.5 \pm 0.3$ & $47.2 \pm 0.5$ & $46.9 \pm 0.7$ \\
\hline $\begin{array}{l}\text { Mean Corpuscular } \\
\text { Volume, } \mu \mathrm{m}^{3}\end{array}$ & $57.0 \pm 0.4$ & $56.5 \pm 0.3$ & $56.9 \pm 0.4$ & $56.0 \pm 0.5$ & $56.4 \pm 0.4$ & $56.6 \pm 0.3$ & $56.5 \pm 0.3$ & $56.8 \pm 0.3$ \\
\hline $\begin{array}{l}\text { Mean Corpuscular } \\
\text { Hemoglobin, pg }\end{array}$ & $19.7 \pm 0.2$ & $19.6 \pm 0.1$ & $19.9 \pm 0.2$ & $19.4 \pm 0.2$ & $19.6 \pm 0.1$ & $19.7 \pm 0.1$ & $19.7 \pm 0.1$ & $19.8 \pm 0.1$ \\
\hline $\begin{array}{l}\text { Mean Corpuscular } \\
\text { Hemoglobin } \\
\text { Concentration, g/dL }\end{array}$ & $34.6 \pm 0.1$ & $34.8 \pm 0.1$ & $35.1 \pm 0.2^{* *}$ & $34.8 \pm 0.1$ & $34.7 \pm 0.1$ & $34.9 \pm 0.1$ & $34.8 \pm 0.1$ & $34.9 \pm 0.1$ \\
\hline Platelets, $10^{3} / \mathrm{mm}^{3}$ & $650.4 \pm 21.3^{* *}$ & $651.5 \pm 28.5$ & $651.0 \pm 22.8$ & $635.1 \pm 21.5$ & $633.1 \pm 16.2$ & $585.9 \pm 20.5^{*}$ & $598.1 \pm 17.4^{\mathrm{c}}$ & $597.7 \pm 18.9$ \\
\hline $\begin{array}{l}\text { White Blood Cells, } \\
10^{3} / \mathrm{mm}^{3}\end{array}$ & $8.4 \pm 0.3$ & $7.5 \pm 0.3$ & $7.9 \pm 0.4$ & $7.8 \pm 0.4$ & $8.1 \pm 0.4$ & $7.6 \pm 0.3$ & $8.0 \pm 0.4$ & $7.8 \pm 0.4$ \\
\hline Neutrophils, $10^{3} / \mathrm{mm}^{3}$ & $2.1 \pm 0.2$ & $2.1 \pm 0.1$ & $2.1 \pm 0.2$ & $2.0 \pm 0.2$ & $2.1 \pm 0.2$ & $1.8 \pm 0.2$ & $2.1 \pm 0.2$ & $2.0 \pm 0.1$ \\
\hline$\%$ Neutrophils & $24.9 \pm 2.3$ & $29.6 \pm 2.6$ & $26.0 \pm 1.4$ & $25.6 \pm 1.7$ & $26.1 \pm 1.6$ & $23.8 \pm 1.2$ & $25.7 \pm 1.5$ & $25.6 \pm 1.0$ \\
\hline Lymphocytes, $10^{3} / \mathrm{mm}^{3}$ & $5.5 \pm 0.3$ & $4.6 \pm 0.3$ & $4.9 \pm 0.3$ & $5.0 \pm 0.3$ & $5.2 \pm 0.3$ & $5.1 \pm 0.2$ & $5.2 \pm 0.3$ & $5.1 \pm 0.2$ \\
\hline$\%$ Lymphocytes & $66.1 \pm 2.2$ & $61.0 \pm 2.5$ & $62.9 \pm 1.8$ & $64.7 \pm 1.8$ & $64.6 \pm 1.5$ & $67.6 \pm 1.5$ & $65.4 \pm 1.6$ & $66.5 \pm 1.0$ \\
\hline Monocytes, $10^{3} / \mathrm{mm}^{3}$ & $0.6 \pm 0.1^{*}$ & $0.6 \pm 0.1$ & $0.8 \pm 0.1$ & $0.7 \pm 0.1$ & $0.7 \pm 0.1$ & $0.6 \pm 0.1$ & $0.6 \pm 0$ & $0.5 \pm 0$ \\
\hline$\%$ Monocytes & $7.4 \pm 0.4$ & $7.9 \pm 0.6$ & $9.5 \pm 0.9^{\mathrm{d}}$ & $8.4 \pm 0.8$ & $8.0 \pm 0.6$ & $7.1 \pm 0.5$ & $7.2 \pm 0.4$ & $6.6 \pm 0.4$ \\
\hline Basophils, $10^{3} / \mathrm{mm}^{3}$ & $0.01 \pm 0$ & $0.01 \pm 0$ & $0.02 \pm 0$ & $0.01 \pm 0$ & $0.01 \pm 0$ & $0.01 \pm 0$ & $0.01 \pm 0$ & $0.01 \pm 0$ \\
\hline$\%$ Basophils & $0.2 \pm 0$ & $0.2 \pm 0$ & $0.3 \pm 0.1$ & $0.2 \pm 0$ & $0.1 \pm 0$ & $0.2 \pm 0$ & $0.2 \pm 0$ & $0.2 \pm 0$ \\
\hline Eosinophils, $10^{3} / \mathrm{mm}^{3}$ & $0.12 \pm 01$ & $0.09 \pm 0.01$ & $0.11 \pm 0.01$ & $0.09 \pm 0.01^{*}$ & $0.10 \pm 0.01$ & $0.10 \pm 0.01$ & $0.12 \pm 0.01$ & $0.09 \pm 0.01^{*}$ \\
\hline$\%$ Eosinophils & $1.4 \pm 0.1$ & $1.3 \pm 0.1$ & $1.4 \pm 0.1$ & $1.1 \pm 0.1$ & $1.2 \pm 0.1$ & $1.3 \pm 0.1$ & $1.5 \pm 0.2$ & $1.1 \pm 0.1^{*}$ \\
\hline
\end{tabular}

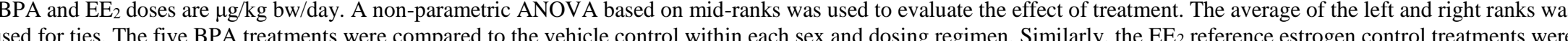
compared to the vehicle control. Dunnett's adjustment was used for pairwise multiple comparisons relative to the control. Orthogonal contrasts were used to test for trend over increasing BPA dose concentrations. All statistical tests are two-sided. Statistical significance was assessed at the 0.05 level. Statistically significant effects are indicated by asterisks $(*, p<0.05 ; * *, p<0.01)$. Asterisks in the vehicle column indicate a significant trend in the BPA dose groups versus the vehicle control group. Full results of the analyses are presented in Supplemental Appendix XXIX.

bumbers in parentheses are the number of animals examined.

cPlatelet numbers in $0.05 \mathrm{EE}_{2}$ dose group significantly different from vehicle control $(p=0.002)$ in the sensitivity analysis that excluded all animals that overlapped with animals treated with $250,000 \mu \mathrm{g} \mathrm{BPA} / \mathrm{kg}$ bw/day.

d\% monocytes in 25 BPA dose group significantly different from vehicle control $(p=0.033)$ in the sensitivity analysis that excluded all animals that overlapped with animals treated with $250,000 \mu \mathrm{g} \mathrm{BPA} / \mathrm{kg}$ bw/day. 
CLARITY-BPA Core Study

Table 39. Female Hematology, Vehicle and BPA Stop-Dose, Interim Sacrifice (Mean \pm S.E.M.) ${ }^{\mathrm{a}}$

\begin{tabular}{|c|c|c|c|c|c|c|}
\hline Endpoint, Units & $\begin{array}{c}\text { Vehicle } \\
(20)^{b}\end{array}$ & $\begin{array}{c}2.5 \text { BPA } \\
(22)\end{array}$ & $\begin{array}{c}25 \text { BPA } \\
(20)\end{array}$ & $\begin{array}{c}250 \text { BPA } \\
(22) \\
\end{array}$ & $\begin{array}{c}2500 \text { BPA } \\
(20) \\
\end{array}$ & $\begin{array}{c}25000 \text { BPA } \\
(19)\end{array}$ \\
\hline Hematocrit, \% & $46.9 \pm 0.4$ & $47.1 \pm 0.4$ & $47.3 \pm 0.6$ & $46.9 \pm 0.5$ & $46.6 \pm 0.7$ & $47.4 \pm 0.4$ \\
\hline Hemoglobin, g/dL & $16.2 \pm 0.1$ & $16.4 \pm 0.1$ & $16.4 \pm 0.2$ & $16.2 \pm 0.2$ & $16.1 \pm 0.2$ & $16.3 \pm 0.2$ \\
\hline Red Blood Cells, $10^{6} / \mathrm{mm}^{3}$ & $8.3 \pm 0.1^{*}$ & $8.3 \pm 0.1$ & $8.4 \pm 0.1$ & $8.4 \pm 0.1$ & $8.4 \pm 0.1$ & $8.5 \pm 0.1$ \\
\hline$\%$ Reticulocytes & $1.3 \pm 0.1$ & $1.3 \pm 0.1$ & $1.2 \pm 0.1$ & $1.3 \pm 0.1$ & $1.3 \pm 0.1$ & $1.4 \pm 0.1$ \\
\hline Packed Cell Volume, \% & $47.0 \pm 0.4$ & $47.2 \pm 0.4$ & $47.3 \pm 0.6$ & $47.0 \pm 0.5$ & $46.6 \pm 0.7$ & $47.4 \pm 0.4$ \\
\hline Mean Corpuscular Volume, $\mu \mathrm{m}^{3}$ & $56.2 \pm 0.3$ & $57.0 \pm 0.4$ & $56.3 \pm 0.4$ & $55.7 \pm 0.5$ & $55.7 \pm 0.4$ & $55.6 \pm 0.3$ \\
\hline Mean Corpuscular Hemoglobin, pg & $19.5 \pm 0.1^{*}$ & $19.9 \pm 0.1$ & $19.5 \pm 0.2$ & $19.3 \pm 0.2$ & $19.2 \pm 0.2$ & $19.1 \pm 0.1$ \\
\hline Mean Corpuscular Hemoglobin & $34.6 \pm 0.1$ & $34.8 \pm 0.1$ & $34.6 \pm 0.1$ & $34.6 \pm 0.1$ & $34.5 \pm 0.1$ & $34.4 \pm 0.1$ \\
\hline Concentration, g/dL & & & & & & \\
\hline Platelets, $10^{3} / \mathrm{mm}^{3}$ & $645.1 \pm 32.6$ & $586.7 \pm 21.6$ & $592.6 \pm 29.1$ & $594.0 \pm 26.2$ & $646.1 \pm 36.2$ & $621.1 \pm 19.5$ \\
\hline White Blood Cells, $10^{3} / \mathrm{mm}^{3}$ & $8.0 \pm 0.4$ & $7.3 \pm 0.4$ & $8.2 \pm 0.5$ & $8.3 \pm 0.4$ & $8.7 \pm 1.2$ & $7.1 \pm 0.4$ \\
\hline Neutrophils, $10^{3} / \mathrm{mm}^{3}$ & $2.0 \pm 0.1$ & $1.7 \pm 0.2$ & $1.9 \pm 0.2$ & $2.1 \pm 0.2$ & $2.5 \pm 0.7$ & $1.7 \pm 0.1$ \\
\hline$\%$ Neutrophils & $24.8 \pm 1.1$ & $23.5 \pm 1.3$ & $23.4 \pm 1.2$ & $25.1 \pm 1.8$ & $25.5 \pm 1.7$ & $24.1 \pm 1.3$ \\
\hline Lymphocytes, $10^{3} / \mathrm{mm}^{3}$ & $5.3 \pm 0.3$ & $4.9 \pm 0.3$ & $5.4 \pm 0.3$ & $5.3 \pm 0.3$ & $5.4 \pm 0.4$ & $4.6 \pm 0.3$ \\
\hline$\%$ Lymphocytes & $66.3 \pm 1.3$ & $67.0 \pm 1.6$ & $66.3 \pm 1.3$ & $64.9 \pm 1.8$ & $65.6 \pm 2.1$ & $66.1 \pm 1.7$ \\
\hline Monocytes, $10^{3} / \mathrm{mm}^{3}$ & $0.6 \pm 0$ & $0.6 \pm 0$ & $0.7 \pm 0.1$ & $0.7 \pm 0.1$ & $0.8 \pm 0.2$ & $0.6 \pm 0.1$ \\
\hline$\%$ Monocytes & $7.4 \pm 0.5$ & $8.0 \pm 0.6$ & $8.8 \pm 0.8$ & $8.4 \pm 0.8$ & $7.6 \pm 0.8$ & $8.5 \pm 0.7$ \\
\hline Basophils, $10^{3} / \mathrm{mm}^{3}$ & $0.01 \pm 0$ & $0.01 \pm 0$ & $0.02 \pm 0$ & $0.02 \pm 0.01$ & $0.01 \pm 0$ & $0.01 \pm 0$ \\
\hline$\%$ Basophils & $0.1 \pm 0^{*}$ & $0.2 \pm 0$ & $0.2 \pm 0$ & $0.3 \pm 0.1$ & $0.1 \pm 0$ & $0.1 \pm 0$ \\
\hline Eosinophils, $10^{3} / \mathrm{mm}^{3}$ & $0.11 \pm 0.01$ & $0.1 \pm 0.01$ & $0.1 \pm 0.01$ & $0.12 \pm 0.01$ & $0.09 \pm 0.01$ & $0.09 \pm 0.01$ \\
\hline$\%$ Eosinophils & $1.35 \pm 0.11$ & $1.34 \pm 0.13$ & $1.28 \pm 0.08$ & $1.37 \pm 0.09$ & $1.17 \pm 0.12$ & $1.27 \pm 0.1$ \\
\hline
\end{tabular}

${ }^{a} \mathrm{BPA}$ doses are $\mu \mathrm{g} / \mathrm{kg}$ bw/day. A non-parametric ANOVA based on mid-ranks was used to evaluate the effect of treatment. The five BPA treatments were compared to the vehicle control, and Dunnett's adjustment was used for pairwise multiple comparisons relative to the control. Orthogonal contrasts were used to test for trend over increasing BPA dose concentrations. All statistical tests are two-sided. Asterisks in the vehicle column indicate a significant trend $(*, p<0.05)$. Statistical significance was assessed at the 0.05 level. Full results of the analyses are presented in Supplemental Appendix XXIX.

${ }^{b}$ Numbers in parentheses are the number of animals examined. 
CLARITY-BPA Core Study

Table 40. Male Hematology, Vehicle, BPA, and $\mathrm{EE}_{2}$ Continuous-Dose, Interim Sacrifice (Mean \pm S.E.M.) ${ }^{\mathrm{a}}$

\begin{tabular}{|c|c|c|c|c|c|c|c|c|}
\hline Endpoint, Units & $\begin{array}{c}\text { Vehicle } \\
(18)^{b}\end{array}$ & $\begin{array}{l}2.5 \mathrm{BPA} \\
(22)\end{array}$ & $\begin{array}{c}25 \text { BPA } \\
(18)\end{array}$ & $\begin{array}{c}250 \text { BPA } \\
\text { (24) }\end{array}$ & $\begin{array}{l}2500 \text { BPA } \\
\text { (18) }\end{array}$ & $\begin{array}{c}25000 \text { BPA } \\
\text { (21) }\end{array}$ & $\begin{array}{l}0.05 \mathbf{E E}_{2} \\
(22)\end{array}$ & $\begin{array}{c}\mathrm{O.5} \mathrm{EE}_{2} \\
(23)\end{array}$ \\
\hline Hematocrit, \% & $47.7 \pm 0.4^{* *}$ & $47.7 \pm 0.5$ & $46.9 \pm 0.6$ & $47.9 \pm 0.4$ & $47.8 \pm 0.5$ & $49.0 \pm 0.5$ & $48.8 \pm 0.4$ & $48.6 \pm 0.3$ \\
\hline Hemoglobin, g/dL & $16.1 \pm 0.1^{*}$ & $16.3 \pm 0.2$ & $16.0 \pm 0.2$ & $16.3 \pm 0.1$ & $16.3 \pm 0.2$ & $16.7 \pm 0.2^{*}$ & $16.6 \pm 0.1^{*}$ & $16.5 \pm 0.1$ \\
\hline Red Blood Cells, $10^{6} / \mathrm{mm}^{3}$ & $9.3 \pm 0.1$ & $9.3 \pm 0.1$ & $9.3 \pm 0.1$ & $9.4 \pm 0.1$ & $9.4 \pm 0.1$ & $9.4 \pm 0.1$ & $9.4 \pm 0.1$ & $9.5 \pm 0.1$ \\
\hline$\%$ Reticulocytes & $1.3 \pm 0.1$ & $1.3 \pm 0.1$ & $1.3 \pm 0.1$ & $1.3 \pm 0$ & $1.3 \pm 0.1$ & $1.3 \pm 0.1$ & $1.2 \pm 0.1$ & $1.2 \pm 0.1$ \\
\hline Packed Cell Volume, \% & $47.7 \pm 0.4^{* *}$ & $47.7 \pm 0.5$ & $47.1 \pm 0.6$ & $47.9 \pm 0.4$ & $47.8 \pm 0.5$ & $49.0 \pm 0.5$ & $48.7 \pm 0.4$ & $48.6 \pm 0.3$ \\
\hline $\begin{array}{l}\text { Mean Corpuscular } \\
\text { Volume, } \mu \mathrm{m}^{3}\end{array}$ & $51.2 \pm 0.2^{*}$ & $51.5 \pm 0.3$ & $50.5 \pm 0.3$ & $51.1 \pm 0.3$ & $51.0 \pm 0.4$ & $52.3 \pm 0.5$ & $51.7 \pm 0.3$ & $51.4 \pm 0.3$ \\
\hline $\begin{array}{l}\text { Mean Corpuscular } \\
\text { Hemoglobin, pg }\end{array}$ & $17.3 \pm 0.1^{*}$ & $17.6 \pm 0.1$ & $17.2 \pm 0.1$ & $17.4 \pm 0.1$ & $17.5 \pm 0.2$ & $17.8 \pm 0.2$ & $17.6 \pm 0.1$ & $17.4 \pm 0.1$ \\
\hline $\begin{array}{l}\text { Mean Corpuscular } \\
\text { Hemoglobin } \\
\text { Concentration, g/dL }\end{array}$ & $33.9 \pm 0.1$ & $34.1 \pm 0.1$ & $34.1 \pm 0.1$ & $34.1 \pm 0.1$ & $34.2 \pm 0.1$ & $34.0 \pm 0.1$ & $34.0 \pm 0.1$ & $34.0 \pm 0.1$ \\
\hline Platelets, $10^{3} / \mathrm{mm}^{3}$ & $751.3 \pm 32.6^{*}$ & $716.2 \pm 25.1^{\mathrm{c}}$ & $749.1 \pm 45.3^{\mathrm{d}}$ & $702.3 \pm 37.3$ & $754.3 \pm 18.1$ & $671 \pm 28.4$ & $709.7 \pm 26.7$ & $740.6 \pm 22.3$ \\
\hline $\begin{array}{l}\text { White Blood Cells, } \\
10^{3} / \mathrm{mm}^{3}\end{array}$ & $9.8 \pm 0.5$ & $10.3 \pm 0.3$ & $9.8 \pm 0.4$ & $10.5 \pm 0.3$ & $9.9 \pm 0.6$ & $9.4 \pm 0.5$ & $10.5 \pm 0.5$ & $11.1 \pm 0.5$ \\
\hline Neutrophils, $10^{3} / \mathrm{mm}^{3}$ & $2.3 \pm 0.2$ & $2.4 \pm 0.2$ & $2.2 \pm 0.2$ & $2.5 \pm 0.2$ & $2.1 \pm 0.2$ & $2.2 \pm 0.2$ & $2.2 \pm 0.2$ & $2.5 \pm 0.1$ \\
\hline$\%$ Neutrophils & $22.9 \pm 0.8$ & $23.4 \pm 1.4$ & $22.5 \pm 1.2$ & $23.5 \pm 1.0$ & $22.1 \pm 1.8$ & $23.3 \pm 1.2$ & $21.4 \pm 1.2$ & $22.4 \pm 0.9$ \\
\hline Lymphocytes, $10^{3} / \mathrm{mm}^{3}$ & $6.6 \pm 0.4$ & $6.8 \pm 0.3$ & $6.5 \pm 0.3$ & $6.9 \pm 0.2$ & $6.9 \pm 0.5$ & $6.2 \pm 0.4$ & $7.1 \pm 0.4$ & $7.5 \pm 0.4$ \\
\hline$\%$ Lymphocytes & $67.0 \pm 1.1$ & $66.0 \pm 1.5$ & $66.4 \pm 1.8$ & $66.0 \pm 1.5$ & $69.0 \pm 1.8$ & $65.9 \pm 1.3$ & $67.8 \pm 1.4$ & $67.5 \pm 1.3$ \\
\hline Monocytes, $10^{3} / \mathrm{mm}^{3}$ & $0.8 \pm 0.1$ & $0.9 \pm 0.1$ & $0.9 \pm 0.1$ & $1.0 \pm 0.1$ & $0.7 \pm 0.1$ & $0.9 \pm 0.1$ & $1.0 \pm 0.1$ & $1.0 \pm 0.1$ \\
\hline$\%$ Monocytes & $8.2 \pm 0.9$ & $8.6 \pm 0.7$ & $9.5 \pm 0.9$ & $9.0 \pm 0.8$ & $7.5 \pm 0.7$ & $9.1 \pm 0.6$ & $9.3 \pm 0.7$ & $8.6 \pm 0.6$ \\
\hline Basophils, $10^{3} / \mathrm{mm}^{3}$ & $0.02 \pm 0$ & $0.02 \pm 0$ & $0.02 \pm 0$ & $0.02 \pm 0$ & $0.01 \pm 0$ & $0.02 \pm 0$ & $0.02 \pm 0$ & $0.03 \pm 0.01$ \\
\hline$\%$ Basophils & $0.2 \pm 0$ & $0.2 \pm 0$ & $0.2 \pm 0$ & $0.2 \pm 0$ & $0.1 \pm 0$ & $0.2 \pm 0$ & $0.2 \pm 0$ & $0.2 \pm 0.1$ \\
\hline Eosinophils, $10^{3} / \mathrm{mm}^{3}$ & $0.17 \pm 0.02$ & $0.18 \pm 0.02$ & $0.15 \pm 0.01$ & $0.13 \pm 0.01$ & $0.13 \pm 0.01$ & $0.14 \pm 0.02$ & $0.14 \pm 0.01$ & $0.15 \pm 0.01$ \\
\hline$\%$ Eosinophils & $1.71 \pm 0.14$ & $1.74 \pm 0.23$ & $1.53 \pm 0.13$ & $1.23 \pm 0.1^{*}$ & $1.29 \pm 0.09$ & $1.44 \pm 0.16$ & $1.37 \pm 0.11$ & $1.33 \pm 0.1$ \\
\hline
\end{tabular}

${ }^{\mathrm{B} P A}$ and $\mathrm{EE}_{2}$ doses are $\mu \mathrm{g} / \mathrm{kg}$ bw/day. A non-parametric ANOVA based on mid-ranks was used to evaluate the effect of treatment. The average of the left and right ranks was used for ties. The five BPA treatments were compared to the vehicle control within each sex and dosing regimen. Similarly, the EE 2 reference estrogen control treatments were compared to the vehicle control. Dunnett's adjustment was used for pairwise multiple comparisons relative to the control. Orthogonal contrasts were used to test for trend over increasing BPA dose concentrations. All statistical tests are two-sided. Statistical significance was assessed at the 0.05 level. Statistically significant effects are indicated by asterisks $(*, p<0.05 ; * *, p<0.01)$. Asterisks in the vehicle column indicate a significant trend. Asterisks in the BPA or EE 2 dose group columns indicate significant differences in pairwise comparisons to the vehicle group. Full results of the analyses are presented in Supplemental Appendix XXIX.

${ }^{b}$ Numbers in parentheses are the number of animals examined. Any deviations from this number due to exclusions or missing data are indicated by footnotes. ${ }^{c} \mathrm{n}=21$ in $2.5 \mu \mathrm{g} \mathrm{BPA} / \mathrm{kg}$ bw/day platelet count due to missing data (no result reported) for one animal.

${ }^{\mathrm{d}} \mathrm{n}=17$ in $25 \mu \mathrm{g} \mathrm{BPA} / \mathrm{kg}$ bw/day platelet count due to missing data (no result reported) for one animal. 
CLARITY-BPA Core Study

Table 41. Male Hematology, Vehicle and BPA Stop-Dose, Interim Sacrifice (Mean \pm S.E.M.) ${ }^{\mathrm{a}}$

\begin{tabular}{|c|c|c|c|c|c|c|}
\hline Endpoint, Units & $\begin{array}{c}\text { Vehicle } \\
(19)^{b}\end{array}$ & $\begin{array}{c}2.5 \text { BPA } \\
(20)\end{array}$ & $\begin{array}{c}25 \text { BPA } \\
(19)\end{array}$ & $\begin{array}{c}250 \text { BPA } \\
(19) \\
\end{array}$ & $\begin{array}{c}2500 \text { BPA } \\
(20) \\
\end{array}$ & $\begin{array}{c}25000 \text { BPA } \\
(22)\end{array}$ \\
\hline Hematocrit, \% & $48.5 \pm 0.5$ & $48.2 \pm 0.4$ & $47.5 \pm 0.6$ & $48.0 \pm 0.6$ & $47.0 \pm 0.8$ & $46.8 \pm 1.0$ \\
\hline Hemoglobin, g/dL & $16.4 \pm 0.2$ & $16.3 \pm 0.1$ & $16.1 \pm 0.2$ & $16.2 \pm 0.2$ & $15.8 \pm 0.3$ & $15.9 \pm 0.3$ \\
\hline Red Blood Cells, $10^{6} / \mathrm{mm}^{3}$ & $9.5 \pm 0.1$ & $9.4 \pm 0.1$ & $9.3 \pm 0.13$ & $9.5 \pm 0.1$ & $9.2 \pm 0.2$ & $9.1 \pm 0.2$ \\
\hline$\%$ Reticulocytes & $1.3 \pm 0.1$ & $1.2 \pm 0.1$ & $1.3 \pm 0.1$ & $1.2 \pm 0.1$ & $1.2 \pm 0.1$ & $1.2 \pm 0.1$ \\
\hline Packed Cell Volume, \% & $48.5 \pm 0.5$ & $48.2 \pm 0.4$ & $47.6 \pm 0.6$ & $48.0 \pm 0.6$ & $46.9 \pm 0.8$ & $46.8 \pm 1.0$ \\
\hline Mean Corpuscular Volume, $\mu \mathrm{m}^{3}$ & $51.3 \pm 0.3$ & $51.2 \pm 0.4$ & $51.2 \pm 0.3$ & $50.7 \pm 0.4$ & $51.7 \pm 1.0$ & $51.4 \pm 0.5$ \\
\hline Mean Corpuscular Hemoglobin, pg & $17.3 \pm 0.1$ & $17.3 \pm 0.1$ & $17.4 \pm 0.1$ & $17.1 \pm 0.2$ & $17.4 \pm 0.3$ & $17.5 \pm 0.2$ \\
\hline Mean Corpuscular Hemoglobin & $33.7 \pm 0.1^{\mathrm{c}}$ & $33.8 \pm 0.1$ & $33.9 \pm 0.1$ & $33.6 \pm 0.1$ & $33.7 \pm 0.1$ & $33.9 \pm 0.1$ \\
\hline Concentration, g/dL & & & & & & \\
\hline Platelets, $10^{3} / \mathrm{mm}^{3}$ & $753.5 \pm 24.6$ & $785.7 \pm 24.1$ & $769.0 \pm 37.7$ & $712.8 \pm 35.2$ & $742.3 \pm 12.2$ & $764.3 \pm 30.3$ \\
\hline White Blood Cells, $10^{3} / \mathrm{mm}^{3}$ & $10.5 \pm 0.4$ & $11.7 \pm 1.0$ & $10.3 \pm 0.4$ & $10.9 \pm 0.6$ & $10.8 \pm 0.5$ & $11.4 \pm 0.8$ \\
\hline Neutrophils, $10^{3} / \mathrm{mm}^{3}$ & $2.4 \pm 0.2$ & $3.5 \pm 0.6$ & $2.7 \pm 0.3$ & $2.8 \pm 0.3$ & $2.3 \pm 0.1$ & $2.4 \pm 0.2$ \\
\hline$\%$ Neutrophils & $23.9 \pm 2.0 *$ & $28.4 \pm 2.3$ & $25.9 \pm 2.2$ & $25.2 \pm 2.1$ & $21.0 \pm 0.8$ & $21.8 \pm 1.6$ \\
\hline Lymphocytes, $10^{3} / \mathrm{mm}^{3}$ & $7.1 \pm 0.45$ & $6.9 \pm 0.4$ & $6.4 \pm 0.3$ & $7.0 \pm 0.5$ & $7.5 \pm 0.35$ & $7.7 \pm 0.6$ \\
\hline$\%$ Lymphocytes & $66.2 \pm 2.2$ & $60.9 \pm 2.3$ & $62.7 \pm 2.2$ & $63.5 \pm 2.1$ & $69.5 \pm 0.9$ & $67.5 \pm 1.7$ \\
\hline Monocytes, $10^{3} / \mathrm{mm}^{3}$ & $0.9 \pm 0.1$ & $1.1 \pm 0.1$ & $1.0 \pm 0.1$ & $1.0 \pm 0.1$ & $0.9 \pm 0.1$ & $1.1 \pm 0.2$ \\
\hline$\%$ Monocytes & $8.3 \pm 1.0$ & $9.1 \pm 0.8$ & $9.6 \pm 0.7$ & $9.8 \pm 1.0$ & $8.1 \pm 0.6$ & $8.9 \pm 0.9$ \\
\hline Basophils, $10^{3} / \mathrm{mm}^{3}$ & $0.02 \pm 0$ & $0.02 \pm 0$ & $0.02 \pm 0$ & $0.02 \pm 0$ & $0.02 \pm 0$ & $0.02 \pm 0$ \\
\hline$\%$ Basophils & $0.2 \pm 0$ & $0.2 \pm 0$ & $0.2 \pm 0$ & $0.2 \pm 0$ & $0.2 \pm 0$ & $0.2 \pm 0$ \\
\hline Eosinophils, $10^{3} / \mathrm{mm}^{3}$ & $0.15 \pm 0.01$ & $0.16 \pm 0.01$ & $0.16 \pm 0.02$ & $0.15 \pm 0.02$ & $0.13 \pm 0.01$ & $0.17 \pm 0.01$ \\
\hline$\%$ Eosinophils & $1.46 \pm 0.12$ & $1.42 \pm 0.09$ & $1.58 \pm 0.13$ & $1.39 \pm 0.13$ & $1.23 \pm 0.12$ & $1.54 \pm 0.11$ \\
\hline
\end{tabular}

${ }^{a}$ BPA doses are $\mu \mathrm{g} / \mathrm{kg}$ bw/day. A non-parametric ANOVA based on mid-ranks was used to evaluate the effect of treatment. The five BPA treatments were compared to the vehicle control, and Dunnett's adjustment was used for pairwise multiple comparisons relative to the control. Orthogonal contrasts were used to test for trend over increasing BPA dose concentrations. All statistical tests are two-sided. Statistical significance was assessed at the 0.05 level. An asterisk in the vehicle column indicates a significant trend $(*, p<0.05)$ versus the vehicle control. Full results of the analyses are presented in Supplemental Appendix XXIX.

${ }^{b}$ Numbers in parentheses are the number of animals examined.

cSignificant trend $(p=0.04)$ for mean corpuscular hemoglobin concentration in sensitivity analysis that excluded all animals that overlapped with animals treated with $250,000 \mu \mathrm{g}$ BPA/kg bw/day. 


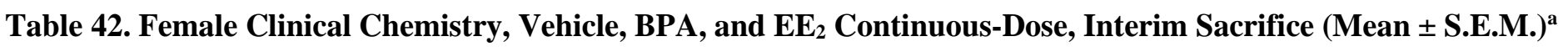

\begin{tabular}{|c|c|c|c|c|c|c|c|c|}
\hline Endpoint, Units & $\begin{array}{c}\text { Vehicle } \\
(21)^{b}\end{array}$ & $\begin{array}{c}2.5 \text { BPA } \\
(22)\end{array}$ & $\begin{array}{c}25 \text { BPA } \\
(21)\end{array}$ & $\begin{array}{c}250 \text { BPA } \\
(22) \\
\end{array}$ & $\begin{array}{c}2500 \text { BPA } \\
(20) \\
\end{array}$ & $\begin{array}{c}25000 \text { BPA } \\
(24) \\
\end{array}$ & $\begin{array}{c}0.05 \mathrm{EE}_{2} \\
(24)\end{array}$ & $\begin{array}{c}0.5 \mathrm{EE}_{2} \\
(26)\end{array}$ \\
\hline Urea nitrogen, $\mathrm{mg} / \mathrm{dL}$ & $14.4 \pm 0.6$ & $13.5 \pm 0.3$ & $14.0 \pm 0.4$ & $15.5 \pm 0.6$ & $14.5 \pm 0.7$ & $14.4 \pm 0.4$ & $14.5 \pm 0.5$ & $15.6 \pm 0.6$ \\
\hline Creatinine, $\mathrm{mg} / \mathrm{dL}$ & $0.5 \pm 0$ & $0.5 \pm 0$ & $0.5 \pm 0$ & $0.5 \pm 0$ & $0.5 \pm 0$ & $0.5 \pm 0$ & $0.5 \pm 0$ & $0.5 \pm 0$ \\
\hline Total protein, mg/dL & $7.5 \pm 0.1$ & $7.6 \pm 0.1$ & $7.8 \pm 0.1$ & $7.4 \pm 0.1$ & $7.7 \pm 0.1$ & $7.6 \pm 0.1$ & $7.6 \pm 0.1$ & $7.7 \pm 0.1$ \\
\hline Albumin, $\mathrm{g} / \mathrm{dL}$ & $4.1 \pm 0.1$ & $4.0 \pm 0.1$ & $4.1 \pm 0.1$ & $4.0 \pm 0.1$ & $4.2 \pm 0.1$ & $4.2 \pm 0.1$ & $4.2 \pm 0.1$ & $4.2 \pm 0.0$ \\
\hline Alkaline phosphatase, U/L & $54.3 \pm 2.8$ & $62.9 \pm 3.6$ & $72.1 \pm 7.3$ & $71.0 \pm 5.5^{*}$ & $63.9 \pm 3.6$ & $58.1 \pm 3.6$ & $67.6 \pm 3.8^{*}$ & $68.1 \pm 5.5$ \\
\hline Alanine aminotransferase, U/L & $31.0 \pm 3.0$ & $37.5 \pm 6.8$ & $37.6 \pm 4.8$ & $34.2 \pm 2.5$ & $32.0 \pm 2.3$ & $29.0 \pm 1.8$ & $30.5 \pm 1.6$ & $37.0 \pm 2.6$ \\
\hline Aspartate aminotransferase, U/L & $90.8 \pm 8.0$ & $82.5 \pm 4.7$ & $86.7 \pm 10.6$ & $76.2 \pm 3.7$ & $79.2 \pm 4.5$ & $78.9 \pm 6.0$ & $78.5 \pm 3.7$ & $80.3 \pm 3.6$ \\
\hline Sorbitol dehydrogenase, U/L & $27.4 \pm 4.2$ & $30.5 \pm 5.7$ & $33.2 \pm 5.5$ & $24.1 \pm 2.1$ & $22.3 \pm 2.1$ & $24.2 \pm 3.1$ & $26.8 \pm 2.0$ & $19.4 \pm 2.0$ \\
\hline Gamma-glutamyl transferase, U/L & $4.4 \pm 0.3$ & $4.1 \pm 0.3$ & $4.6 \pm 0.3$ & $4.0 \pm 0.3$ & $4.2 \pm 0.3$ & $4.2 \pm 0.3$ & $3.8 \pm 0.2^{\mathrm{c}}$ & $3.9 \pm 0.3$ \\
\hline Total bile acids, $\mu \mathrm{mol} / \mathrm{L}$ & $47.0 \pm 5.8$ & $53.5 \pm 9.9$ & $49.7 \pm 5.3$ & $53.0 \pm 5.3$ & $51.4 \pm 5.7$ & $42.3 \pm 5.6$ & $50.6 \pm 7.1$ & $72.0 \pm 9.1$ \\
\hline Cholesterol, mg/dL & $109.5 \pm 4.4$ & $111.7 \pm 7.2$ & $128.9 \pm 11.1$ & $107.6 \pm 4.6$ & $111.3 \pm 7.6$ & $107.7 \pm 8.4$ & $127.5 \pm 6.6$ & $121.5 \pm 4.9$ \\
\hline Glucose, mg/dL & $130.0 \pm 6.0$ & $126.0 \pm 4.8$ & $130.2 \pm 5$ & $124.8 \pm 4.8$ & $122.1 \pm 4.3$ & $136.1 \pm 6.6$ & $128.8 \pm 4.5$ & $120.6 \pm 3.3$ \\
\hline Triglycerides, ng/mL & $266 \pm 24.3$ & $258.6 \pm 24.3$ & $299.6 \pm 41.5$ & $237.5 \pm 30.3$ & $330.7 \pm 40.3$ & $331.6 \pm 67.7$ & $282.9 \pm 34.5$ & $369.4 \pm 39.2$ \\
\hline Insulin, $\mathrm{mg} / \mathrm{mL}$ & $1.5 \pm 0.2$ & $2.2 \pm 0.4$ & $2.0 \pm 0.3$ & $1.6 \pm 0.2$ & $1.6 \pm 0.2$ & $2.1 \pm 0.5$ & $2.0 \pm 0.3$ & $1.3 \pm 0.1$ \\
\hline Leptin, ng/mL & $19.3 \pm 2.4$ & $27.8 \pm 3.5$ & $24.5 \pm 3.1$ & $18.5 \pm 2.5$ & $19.8 \pm 2.1$ & $20.7 \pm 2.7$ & $23.7 \pm 2.9$ & $17.9 \pm 1.8$ \\
\hline \multirow[t]{2}{*}{ Troponin $\mathrm{T}, \mathrm{pg} / \mathrm{mL}^{\mathrm{d}}$} & $10.3 \pm 1.6$ & $8.0 \pm 1.7$ & $10.2 \pm 2.2$ & $6.6 \pm 1.9$ & $5.9 \pm 1.5$ & $6.5 \pm 1.9$ & $6.3 \pm 1.4$ & $6.9 \pm 1.9$ \\
\hline & $18 / 21(86 \%)^{e, f}$ & $13 / 22(59 \%)$ & $14 / 21(67 \%)$ & $12 / 22(55 \%)$ & $12 / 20(60 \%)$ & $13 / 24(54 \%)$ & $15 / 24(62 \%)$ & $13 / 26(50 \%)$ \\
\hline T3, ng/dL & $71.6 \pm 3.5$ & $72.1 \pm 4.1$ & $79.2 \pm 4.3$ & $70.8 \pm 3$ & $78.2 \pm 3.5$ & $73.3 \pm 2.5$ & $79.3 \pm 2.6$ & $78.0 \pm 3.5$ \\
\hline $\mathrm{T} 4, \mu \mathrm{g} / \mathrm{dL}$ & $3.8 \pm 0.2^{\mathrm{g}}$ & $3.9 \pm 0.2$ & $3.8 \pm 0.2$ & $3.8 \pm 0.2$ & $3.9 \pm 0.2$ & $4.1 \pm 0.2$ & $4.2 \pm 0.2$ & $3.8 \pm 0.2$ \\
\hline $\mathrm{TSH}, \mathrm{ng} / \mathrm{mL}$ & $3.7 \pm 0.7$ & $4.4 \pm 0.9$ & $3.6 \pm 0.4$ & $4.2 \pm 0.4$ & $4.9 \pm 0.7$ & $4.5 \pm 0.4$ & $3.4 \pm 0.3$ & $5.1 \pm 0.5^{*}$ \\
\hline
\end{tabular}

${ }^{\mathrm{a}} \mathrm{BPA}$ and $\mathrm{EE}_{2}$ doses are $\mu \mathrm{g} / \mathrm{kg}$ bw/day. A non-parametric ANOVA based on mid-ranks was used to evaluate the effect of treatment. The average of the left and right ranks was used for ties. The five BPA treatments were compared to the vehicle control within each sex and dosing regimen. Similarly, the EE 2 reference estrogen control treatments were compared to the vehicle control. Dunnett's adjustment was used for pairwise multiple comparisons relative to the control. Orthogonal contrasts were used to test for trend over increasing BPA dose concentrations. All statistical tests are two-sided. Statistical significance was assessed at the 0.05 level. Statistically significant effects are indicated by asterisks. Asterisks in the BPA or $\mathrm{EE}_{2}$ dose group columns indicate significant differences in pairwise comparisons to the vehicle group ( $*, p<0.05$ ). Full results of the analyses are presented in Supplemental Appendix XXIX.

${ }^{b}$ Numbers in parentheses are the number of animals examined. Any deviations from this number due to exclusions or missing data are indicated by footnotes.

${ }^{c}$ Gamma-glutamyl transferase in $0.05 \mu \mathrm{g} \mathrm{EE} / \mathrm{kg}$ bw/day group significantly different from vehicle control $(p=0.018)$ in the sensitivity analysis that excluded all animals that overlapped with animals treated with $250,000 \mu \mathrm{g}$ BPA $/ \mathrm{kg}$ bw/day.

${ }^{\mathrm{d}}$ Means were calculated using $1 / 2$ the limit of detection $(\mathrm{LOD}, 1.0 \mathrm{pg} / \mathrm{mL})$ for samples below $L O D$. Troponin I was also measured, but the overall percentage of samples above the limit of detection was less than $5 \%$, so no statistical analysis was conducted.

${ }^{\mathrm{e}}$ Number of samples with troponin T levels above LOD/total number of samples. Percentage of detects is given in parenthesis. Because of the high percentage of non-detects, the proportion of samples with detectable troponin $T$ were statistically analyzed rather than means or medians. No significant trends across BPA dose groups or pairwise comparisons to vehicle control were detected.

${ }^{E}$ ERRATUM: An error was identified in the NTP Research Report on the CLARITY-BPA Core Study: A Perinatal and Chronic Extended-Dose-Range Study of Bisphenol A in Rats (RR-9). The method of calculation of mean Troponin T, number of samples with detectable levels of Troponin T, and statistical analysis approach were not originally included in the Table. This error has been corrected; the new information is italicized.

$\mathrm{g}_{\mathrm{n}}=20$ in vehicle $\mathrm{T} 4$ assay due to insufficient quantity of serum for assay for one animal. 
CLARITY-BPA Core Study

Table 43. Female Clinical Chemistry, Vehicle and BPA Stop-Dose, Interim Sacrifice (Mean \pm S.E.M.) ${ }^{\mathrm{a}}$

\begin{tabular}{|c|c|c|c|c|c|c|}
\hline Endpoint, Units & $\begin{array}{c}\text { Vehicle } \\
(20)^{b}\end{array}$ & $\begin{array}{c}2.5 \text { BPA } \\
(22)\end{array}$ & $\begin{array}{c}25 \text { BPA } \\
(20)\end{array}$ & $\begin{array}{c}250 \text { BPA } \\
(22)\end{array}$ & $\begin{array}{c}2500 \text { BPA } \\
(20)\end{array}$ & $\begin{array}{c}25000 \text { BPA } \\
(19)\end{array}$ \\
\hline Urea nitrogen, $\mathrm{mg} / \mathrm{dL}$ & $13.9 \pm 0.5$ & $14.0 \pm 0.5$ & $14.5 \pm 0.5$ & $14.1 \pm 0.5$ & $14.3 \pm 0.5$ & $14.3 \pm 0.5$ \\
\hline Creatinine, $\mathrm{mg} / \mathrm{dL}$ & $0.5 \pm 0.0$ & $0.5 \pm 0.0$ & $0.5 \pm 0.0$ & $0.4 \pm 0.0$ & $0.5 \pm 0.0$ & $0.5 \pm 0.0$ \\
\hline Total protein, $\mathrm{mg} / \mathrm{dL}$ & $7.4 \pm 0.1$ & $7.7 \pm 0.1$ & $7.6 \pm 0.1$ & $7.6 \pm 0.1$ & $7.5 \pm 0.1$ & $7.7 \pm 0.1$ \\
\hline Albumin, g/dL & $4.1 \pm 0.1 * *$ & $4.2 \pm 0.1$ & $4.0 \pm 0.0$ & $4.0 \pm 0.1$ & $4.0 \pm 0.1$ & $4.2 \pm 0.0$ \\
\hline Alkaline phosphatase, U/L & $67.2 \pm 3.8$ & $75.4 \pm 5.3$ & $72.9 \pm 3.8$ & $62.5 \pm 3.0$ & $69.0 \pm 6.0$ & $83.1 \pm 7.2$ \\
\hline Alanine aminotransferase, U/L & $29.2 \pm 2.0$ & $33.3 \pm 2.9$ & $35.2 \pm 3.8$ & $29.5 \pm 2.0$ & $29.7 \pm 2.8$ & $35.4 \pm 3.6$ \\
\hline Aspartate aminotransferase, U/L & $77.5 \pm 3.1$ & $81.5 \pm 5.7$ & $82.8 \pm 6.5$ & $72.4 \pm 3.3$ & $76.5 \pm 4.0$ & $86.5 \pm 7.9$ \\
\hline Sorbitol dehydrogenase, U/L & $24.5 \pm 3.0^{c}$ & $29.1 \pm 3.5$ & $31.8 \pm 4.6$ & $28.0 \pm 1.9$ & $22.3 \pm 2.4$ & $31.2 \pm 2.7$ \\
\hline $\begin{array}{l}\text { Gamma-glutamyl transferase, } \\
\text { U/L }\end{array}$ & $4.4 \pm 0.3$ & $4.3 \pm 0.3$ & $3.6 \pm 0.4$ & $3.8 \pm 0.3$ & $3.8 \pm 0.3$ & $3.9 \pm 0.3$ \\
\hline Total bile acids, $\mu \mathrm{mol} / \mathrm{L}$ & $43.3 \pm 4.1$ & $48.9 \pm 5.8$ & $56.5 \pm 6.1$ & $44.8 \pm 4.2$ & $46.0 \pm 4.7$ & $59.2 \pm 7.5$ \\
\hline Cholesterol, mg/dL & $116.9 \pm 6.7$ & $116.0 \pm 7.4$ & $108.6 \pm 6.7$ & $111.9 \pm 5.1$ & $113.4 \pm 4.4$ & $125.8 \pm 12.5$ \\
\hline Glucose, $\mathrm{mg} / \mathrm{dL}$ & $129.8 \pm 3.8$ & $137.2 \pm 5.9$ & $128.2 \pm 4.5$ & $128.4 \pm 4.2$ & $125.6 \pm 5.4$ & $125.4 \pm 5.5$ \\
\hline Triglycerides, ng/mL & $261.2 \pm 21.4$ & $373.7 \pm 68.4$ & $253.2 \pm 38.4$ & $274.6 \pm 55.8$ & $342.2 \pm 49.8$ & $315.2 \pm 27.7$ \\
\hline Insulin, $\mathrm{mg} / \mathrm{mL}$ & $2.0 \pm 0.3$ & $2.6 \pm 0.4$ & $1.5 \pm 0.2$ & $2.3 \pm 0.5$ & $2.0 \pm 0.3$ & $2.1 \pm 0.3$ \\
\hline Leptin, $\mathrm{ng} / \mathrm{mL}$ & $24.2 \pm 3.1$ & $28.1 \pm 3.4$ & $22.2 \pm 2.9$ & $22.4 \pm 2.5$ & $22.2 \pm 2.7$ & $24.2 \pm 2.8$ \\
\hline $\mathrm{T} 3, \mathrm{ng} / \mathrm{dL}$ & $68.1 \pm 3.3$ & $72.1 \pm 3.6$ & $75.8 \pm 3.2$ & $71.0 \pm 2.8$ & $71.4 \pm 4.1$ & $78.7 \pm 4.7$ \\
\hline \multirow[t]{2}{*}{ Troponin $\mathrm{T}, \mathrm{pg} / \mathrm{mL}^{\mathrm{d}}$} & $7.0 \pm 1.8$ & $6.3 \pm 1.5$ & $3.6 \pm 1.0$ & $5.3 \pm 1.0$ & $6.5 \pm 2.2$ & $8.7 \pm 2.9$ \\
\hline & $10 / 20(50 \%)^{e, f}$ & $13 / 22(59 \%)$ & $10 / 20(50 \%)$ & $12 / 22(55 \%)$ & $12 / 20(60 \%)$ & $10 / 19(53 \%)$ \\
\hline $\mathrm{T} 4, \mu \mathrm{g} / \mathrm{dL}$ & $3.7 \pm 0.2$ & $3.6 \pm 0.2$ & $4.1 \pm 0.2$ & $3.7 \pm 0.1$ & $3.7 \pm 0.2$ & $3.7 \pm 0.2$ \\
\hline $\mathrm{TSH}, \mathrm{ng} / \mathrm{mL}$ & $4.6 \pm 0.8$ & $3.5 \pm 0.4$ & $3.8 \pm 0.5$ & $4.8 \pm 0.7$ & $4.3 \pm 0.5$ & $4.7 \pm 0.5$ \\
\hline
\end{tabular}

${ }^{\mathrm{a}} \mathrm{BPA}$ doses are $\mu \mathrm{g} / \mathrm{kg}$ bw/day. A non-parametric ANOVA based on mid-ranks was used to evaluate the effect of treatment. The five BPA treatments were compared to the vehicle control, and Dunnett's adjustment was used for pairwise multiple comparisons relative to the control. Orthogonal contrasts were used to test for trend over increasing BPA dose concentrations. All statistical tests are two-sided. Statistical significance was assessed at the 0.05 level. Asterisks in the vehicle column indicates a significant trend $(* *, p<0.01)$ versus the vehicle control. Full results of the analyses are presented in Supplemental Appendix XXIX.

bNumbers in parentheses are the number of animals examined.

cSignificant trend $(p=0.002)$ for sorbitol dehydrogenase in the sensitivity analysis that excluded all animals that overlapped with animals treated with $250,000 \mu \mathrm{g}$ BPA $/ \mathrm{kg}$ bw/day. ${ }^{\mathrm{d}}$ Means were calculated using $1 / 2$ the limit of detection $(L O D, 1.0 \mathrm{pg} / \mathrm{mL})$ for samples below LOD. Troponin I was also measured, but the overall percentage of samples above the limit of detection was less than $5 \%$, so no statistical analysis was conducted.

${ }^{\mathrm{e}}$ Number of samples with troponin T levels above LOD/total number of samples. Percentage of detects is given in parenthesis. Because of the high percentage of non-detects, the proportion of samples with detectable troponin T were statistically analyzed rather than means or medians. No significant trends across BPA dose groups or pairwise comparisons to vehicle control were detected.

fERRATUM: An error was identified in the NTP Research Report on the CLARITY-BPA Core Study: A Perinatal and Chronic Extended-Dose-Range Study of Bisphenol A in Rats (RR-9). The method of calculation of mean Troponin T, number of samples with detectable levels of Troponin T, and statistical analysis approach were not originally included in the Table. This error has been corrected; the new information is italicized. 
Table 44. Male Clinical Chemistry, Vehicle, BPA, and EE2 Continuous-Dose, Interim Sacrifice (Mean \pm S.E.M.) ${ }^{\mathrm{a}}$

\begin{tabular}{|c|c|c|c|c|c|c|c|c|}
\hline Endpoint, Units & $\begin{array}{c}\text { Vehicle } \\
(\mathbf{1 8})^{\mathrm{b}}\end{array}$ & $\begin{array}{c}2.5 \mathrm{BPA} \\
(22)\end{array}$ & $\begin{array}{c}25 \text { BPA } \\
\text { (18) }\end{array}$ & $\begin{array}{c}250 \text { BPA } \\
(24)\end{array}$ & $\begin{array}{c}2500 \text { BPA } \\
(18)\end{array}$ & $\begin{array}{c}25000 \mathrm{BPA} \\
(21)\end{array}$ & $\begin{array}{c}\mathrm{O.05}_{\mathrm{EE}} \\
(22)\end{array}$ & $\begin{array}{c}0.5 \mathrm{EE}_{2} \\
(23)\end{array}$ \\
\hline Urea nitrogen, $\mathrm{mg} / \mathrm{dL}$ & $14.2 \pm 0.4$ & $14.0 \pm 0.3$ & $14.3 \pm 0.5$ & $14.5 \pm 0.4$ & $14.3 \pm 0.6$ & $14.1 \pm 0.3$ & $14.4 \pm 0.3$ & $14.6 \pm 0.4$ \\
\hline Creatinine, $\mathrm{mg} / \mathrm{dL}$ & $0.4 \pm 0$ & $0.4 \pm 0$ & $0.4 \pm 0$ & $0.4 \pm 0$ & $0.4 \pm 0$ & $0.4 \pm 0$ & $0.4 \pm 0$ & $0.4 \pm 0$ \\
\hline Total protein, mg/dL & $7.4 \pm 0.1$ & $7.3 \pm 0.1$ & $7.3 \pm 0.1$ & $7.2 \pm 0.1$ & $7.3 \pm 0.1$ & $7.3 \pm 0.1$ & $7.3 \pm 0.1$ & $7.2 \pm 0.1$ \\
\hline Albumin, $\mathrm{g} / \mathrm{dL}$ & $3.7 \pm 0^{* *}$ & $3.7 \pm 0$ & $3.6 \pm 0$ & $3.6 \pm 0^{\mathrm{c}}$ & $3.7 \pm 0.1$ & $3.7 \pm 0$ & $3.7 \pm 0$ & $3.7 \pm 0$ \\
\hline Alkaline phosphatase, U/L & $130.6 \pm 24.1$ & $107.2 \pm 5.5$ & $99.9 \pm 5.4$ & $98.7 \pm 3.9$ & $107.6 \pm 7.7$ & $99.7 \pm 4.9$ & $107.3 \pm 4.5$ & $104.2 \pm 5.9$ \\
\hline Alanine aminotransferase, U/L & $30.1 \pm 1.6$ & $33.8 \pm 2.4$ & $27.3 \pm 2.0$ & $29.6 \pm 1.3$ & $32.0 \pm 2.3$ & $31.0 \pm 1.9$ & $30.6 \pm 2.7$ & $33.7 \pm 2.0$ \\
\hline Aspartate aminotransferase, U/L & $69.7 \pm 3.2$ & $73.4 \pm 4.8$ & $73.5 \pm 6.7$ & $68.7 \pm 2.4$ & $76.7 \pm 5.7$ & $76.3 \pm 6.4$ & $79.9 \pm 8.5$ & $71.2 \pm 3.0$ \\
\hline Sorbitol dehydrogenase, U/L & $24.5 \pm 2.4$ & $26.8 \pm 2.7$ & $26.6 \pm 2.7$ & $27.4 \pm 3.0$ & $24.4 \pm 2.7$ & $25.9 \pm 2.8$ & $31.5 \pm 4.8$ & $22.8 \pm 2.0$ \\
\hline Gamma-glutamyl transferase, U/L & $4.1 \pm 0.3$ & $4.6 \pm 0.3$ & $4.2 \pm 0.3$ & $4.4 \pm 0.3$ & $4.8 \pm 0.4$ & $4.4 \pm 0.3$ & $4.2 \pm 0.2$ & $3.9 \pm 0.2$ \\
\hline Total bile acids, $\mu \mathrm{mol} / \mathrm{L}$ & $32.8 \pm 2.7^{*}$ & $33.3 \pm 4.1$ & $32.5 \pm 2.4$ & $35.8 \pm 3.4$ & $42.0 \pm 4.7$ & $28.1 \pm 2.7$ & $38.6 \pm 4.3$ & $35.6 \pm 3.2$ \\
\hline Cholesterol, mg/dL & $118.0 \pm 6.4$ & $116.9 \pm 4.8$ & $118.3 \pm 6.7$ & $107.4 \pm 4.4$ & $127.3 \pm 6.7$ & $107.2 \pm 5.2$ & $120.4 \pm 6.4$ & $117.5 \pm 7.1$ \\
\hline Glucose, mg/dL & $126.1 \pm 4.8$ & $125.9 \pm 3.9$ & $127.1 \pm 4.4$ & $127.3 \pm 6.1$ & $136.3 \pm 5.8$ & $119.0 \pm 3.4$ & $125.4 \pm 3.8$ & $123.7 \pm 4.1$ \\
\hline Triglycerides, ng/mL & $267.9 \pm 23.4$ & $278.2 \pm 19.5$ & $276.1 \pm 20.6$ & $252.9 \pm 18.7$ & $304.6 \pm 24.3$ & $283.0 \pm 13.5$ & $266.8 \pm 19.1$ & $338.4 \pm 21.8^{*}$ \\
\hline Insulin, $\mathrm{mg} / \mathrm{mL}$ & $2.0 \pm 0.2$ & $1.6 \pm 0.1$ & $1.5 \pm 0.1$ & $1.4 \pm 0.1$ & $1.7 \pm 0.3$ & $1.8 \pm 0.1$ & $1.3 \pm 0.1^{*}$ & $1.6 \pm 0.1$ \\
\hline Leptin, ng/mL & $26 \pm 2.5$ & $29.2 \pm 3.0$ & $23.8 \pm 2.3$ & $26.3 \pm 2.6$ & $26.7 \pm 3.0$ & $29.4 \pm 2.8$ & $25.4 \pm 2.6$ & $27.2 \pm 1.9$ \\
\hline \multirow[t]{2}{*}{ Troponin $\mathrm{T}, \mathrm{pg} / \mathrm{mL}^{\mathrm{d}}$} & $7.4 \pm 2.5$ & $5.9 \pm 1.4$ & $9.8 \pm 2.4$ & $8.5 \pm 2.2$ & $6.4 \pm 1.5$ & $11.8 \pm 2.2$ & $5.1 \pm 1.3$ & $7.2 \pm 1.6$ \\
\hline & $11 / 18(61 \%)^{e, f * *}$ & $10 / 22(46 \%)$ & $11 / 18(61 \%)$ & $14 / 24(58 \%)$ & $12 / 18(67 \%)$ & $17 / 20(85 \%)$ & $9 / 22(41 \%)$ & $18 / 23(78 \%)$ \\
\hline T3, ng/dL & $62.8 \pm 2.7$ & $57.8 \pm 3.4$ & $63.3 \pm 2.3$ & $60.5 \pm 3.0$ & $67.3 \pm 4.1$ & $67.0 \pm 4.0^{\mathrm{g}}$ & $70.5 \pm 4.1$ & $66.4 \pm 3.5$ \\
\hline $\mathrm{T} 4, \mu \mathrm{g} / \mathrm{dL}$ & $5.0 \pm 0.3^{*}$ & $4.3 \pm 0.3$ & $5.0 \pm 0.2$ & $4.9 \pm 0.2$ & $4.7 \pm 0.2$ & $5.5 \pm 0.2$ & $5.1 \pm 0.2$ & $4.7 \pm 0.2$ \\
\hline $\mathrm{TSH}, \mathrm{ng} / \mathrm{mL}$ & $3.6 \pm 0.3$ & $3.4 \pm 0.3$ & $3.4 \pm 0.3$ & $3.5 \pm 0.4$ & $3.5 \pm 0.4$ & $3.5 \pm 0.4$ & $4.1 \pm 0.3$ & $3.2 \pm 0.4$ \\
\hline
\end{tabular}

${ }^{\mathrm{a}} \mathrm{BPA}$ and $\mathrm{EE}_{2}$ doses are $\mu \mathrm{g} / \mathrm{kg}$ bw/day. A non-parametric ANOVA based on mid-ranks was used to evaluate the effect of treatment. The average of the left and right ranks was used for ties. The five BPA treatments were compared to the vehicle control within each sex and dosing regimen. Similarly, the EE 2 reference estrogen control treatments were compared to the vehicle control. Dunnett's adjustment was used for pairwise multiple comparisons relative to the control. Orthogonal contrasts were used to test for trend over increasing BPA dose concentrations. All statistical tests are two-sided. Statistical significance was assessed at the 0.05 level. Statistically significant effects are indicated by asterisks $(*, p<0.05 ; * *, p<0.01)$. Asterisks in the vehicle column indicate a significant trend in the BPA dose groups versus the vehicle control. Asterisks in the BPA or EE 2 dose group columns indicate significant differences in pairwise comparisons to the vehicle group. Full results of the analyses are presented in Supplemental Appendix XXIX.

${ }^{b}$ Numbers in parentheses are the number of animals examined.

${ }^{c}$ Albumin in $250 \mu \mathrm{g} \mathrm{BPA} / \mathrm{kg}$ bw/day group significantly different from vehicle control $(p=0.012)$ in the sensitivity analysis that excluded all animals that overlapped with animals treated with $250,000 \mu \mathrm{g} \mathrm{BPA} / \mathrm{kg}$ bw/day.

${ }^{\mathrm{d}}$ Means were calculated using $1 / 2$ the limit of detection $(L O D, 1.0 \mathrm{pg} / \mathrm{mL})$ for samples below LOD. Troponin I was also measured, but the overall percentage of samples above the limit of detection was less than $5 \%$, so no statistical analysis was conducted.

${ }^{\mathrm{e}}$ Number of samples with troponin T levels above LOD/total number of samples. Percentage of detects is given in parenthesis. Because of the high percentage of non-detects, the proportion of samples with troponin T were statistically analyzed rather than means or medians. The asterisks indicate a significant trend across BPA dose groups for the proportion of samples with detectable troponin $T$.

fERRATUM: An error was identified in the NTP Research Report on the CLARITY-BPA Core Study: A Perinatal and Chronic Extended-Dose-Range Study of Bisphenol A in Rats (RR-9). The method of calculation of mean Troponin T, number of samples with detectable levels of Troponin T, and statistical analysis approach were not originally included in the Table. This error has been corrected; the new information is italicized.

$\mathrm{g}_{\mathrm{n}}=20$ in 2,500 BPA T3 assay due to insufficient quantity of serum for assay for one animal. 
CLARITY-BPA Core Study

Table 45. Male Clinical Chemistry, Vehicle and BPA Stop-Dose, Interim Sacrifice (Mean \pm S.E.M.) ${ }^{\mathrm{a}}$

\begin{tabular}{|c|c|c|c|c|c|c|}
\hline Endpoint, Units & $\begin{array}{c}\text { Vehicle } \\
(20)^{b}\end{array}$ & $\begin{array}{c}2.5 \text { BPA } \\
(20)\end{array}$ & $\begin{array}{c}25 \text { BPA } \\
(19)\end{array}$ & $\begin{array}{c}250 \text { BPA } \\
(19) \\
\end{array}$ & $\begin{array}{c}2500 \text { BPA } \\
(20)\end{array}$ & $\begin{array}{c}25000 \text { BPA } \\
(22)\end{array}$ \\
\hline Urea nitrogen, $\mathrm{mg} / \mathrm{dL}$ & $14.1 \pm 0.4$ & $13.7 \pm 0.3$ & $15.0 \pm 1.0$ & $13.8 \pm 0.6$ & $14.4 \pm 0.4$ & $19.6 \pm 3.7$ \\
\hline Creatinine, $\mathrm{mg} / \mathrm{dL}$ & $0.4 \pm 0$ & $0.4 \pm 0$ & $0.5 \pm 0$ & $0.5 \pm 0$ & $0.4 \pm 0$ & $0.6 \pm 0.1$ \\
\hline Total protein, $\mathrm{mg} / \mathrm{dL}$ & $7.3 \pm 0.1$ & $7.1 \pm 0.1$ & $7.0 \pm 0.1^{*}$ & $7.2 \pm 0.1$ & $7.2 \pm 0.1$ & $7.2 \pm 0.1$ \\
\hline Albumin, g/dL & $3.6 \pm 0$ & $3.6 \pm 0$ & $3.5 \pm 0.1$ & $3.6 \pm 0.1$ & $3.6 \pm 0.1$ & $3.6 \pm 0.1$ \\
\hline Alkaline phosphatase, U/L & $108.8 \pm 7.9$ & $100.4 \pm 4.2$ & $104.5 \pm 5.7$ & $103.5 \pm 11.3$ & $100.5 \pm 5.3$ & $102.9 \pm 6.0$ \\
\hline Alanine aminotransferase, U/L & $32.4 \pm 3.1$ & $32.3 \pm 2.0$ & $28.5 \pm 1.5$ & $32.6 \pm 5.5$ & $26.7 \pm 1.2$ & $29.0 \pm 2.6$ \\
\hline Aspartate aminotransferase, U/L & $84.6 \pm 8.8$ & $85.3 \pm 4.5$ & $82.7 \pm 7.4$ & $77.2 \pm 7.1$ & $68.8 \pm 3.1$ & $69.9 \pm 3.6$ \\
\hline Sorbitol dehydrogenase, U/L & $25.0 \pm 2.8$ & $27.4 \pm 2.8$ & $32.0 \pm 3.2$ & $28.9 \pm 2.8$ & $26.7 \pm 2.3$ & $28.6 \pm 2.9$ \\
\hline Gamma-glutamyl transferase, U/L & $4.3 \pm 0.4$ & $4.1 \pm 0.3$ & $4.2 \pm 0.4$ & $3.7 \pm 0.3$ & $4.8 \pm 0.2$ & $4.3 \pm 0.3$ \\
\hline Total bile acids, $\mu \mathrm{mol} / \mathrm{L}$ & $36.4 \pm 3.1^{*}$ & $34.6 \pm 3.2$ & $25.0 \pm 2.7^{*}$ & $32.6 \pm 6.5$ & $33.7 \pm 4.3$ & $35.0 \pm 2.5$ \\
\hline Cholesterol, mg/dL & $123.5 \pm 5.6$ & $115.5 \pm 5.9$ & $115.3 \pm 6.7$ & $117.8 \pm 8.9$ & $132.7 \pm 4.7$ & $147.7 \pm 14.2$ \\
\hline Glucose, $\mathrm{mg} / \mathrm{dL}$ & $128.7 \pm 5.3$ & $121.9 \pm 3.2$ & $130.8 \pm 5.9$ & $120.1 \pm 4.1$ & $131.3 \pm 4.7$ & $125.2 \pm 3.4$ \\
\hline Triglycerides, ng/mL & $285.2 \pm 20.0$ & $252.3 \pm 15.4$ & $282.3 \pm 26.1$ & $279.9 \pm 16.5$ & $355.2 \pm 22$ & $329.8 \pm 24.2$ \\
\hline Insulin, $\mathrm{mg} / \mathrm{mL}$ & $1.5 \pm 0.2$ & $1.5 \pm 0.2$ & $1.9 \pm 0.2$ & $1.5 \pm 0.1$ & $1.7 \pm 0.2$ & $1.6 \pm 0.2$ \\
\hline Leptin, ng/mL & $26.8 \pm 3.5$ & $26.8 \pm 1.7$ & $32.9 \pm 3.7$ & $25.5 \pm 3.4$ & $28.0 \pm 3.0$ & $28.1 \pm 2.8$ \\
\hline \multirow[t]{2}{*}{ Troponin $\mathrm{T}, \mathrm{pg} / \mathrm{mL}^{\mathrm{d}}$} & $8.0 \pm 1.9$ & $5.9 \pm 1.7$ & $4.2 \pm 1.3$ & $7.7 \pm 2.3$ & $4.8 \pm 1.6$ & $5.1 \pm 1.8$ \\
\hline & $12 / 20(60 \%)^{e, f}$ & $8 / 20(40 \%)$ & $7 / 19(37 \%)$ & $12 / 19(63 \%)$ & $9 / 20(45 \%)$ & $8 / 22(36 \%)$ \\
\hline $\mathrm{T} 3, \mathrm{ng} / \mathrm{dL}$ & $65.8 \pm 3.1$ & $63.3 \pm 3.3$ & $56.7 \pm 3.0$ & $60.6 \pm 4.5$ & $69.4 \pm 2.9$ & $66.6 \pm 2.8$ \\
\hline $\mathrm{T} 4, \mu \mathrm{g} / \mathrm{dL}$ & $4.9 \pm 0.2^{*}$ & $4.8 \pm 0.2$ & $4.7 \pm 0.2$ & $4.7 \pm 0.2$ & $4.7 \pm 0.3$ & $4.2 \pm 0.2^{c}$ \\
\hline $\mathrm{TSH}, \mathrm{ng} / \mathrm{mL}$ & $4.0 \pm 0.4$ & $4.2 \pm 0.4$ & $3.8 \pm 0.5$ & $4.5 \pm 0.5$ & $5.0 \pm 0.6$ & $3.9 \pm 0.4$ \\
\hline
\end{tabular}

${ }^{\mathrm{a} B P A}$ doses are $\mu \mathrm{g} / \mathrm{kg}$ bw/day. A non-parametric ANOVA based on mid-ranks was used to evaluate the effect of treatment. The five BPA treatments were compared to the vehicle control, and Dunnett's adjustment was used for pairwise multiple comparisons relative to the control. Orthogonal contrasts were used to test for trend over increasing BPA dose concentrations. All statistical tests are two-sided. Statistical significance was assessed at the 0.05 level. Statistically significant effects are indicated by asterisks $(*, p<0.05)$. Asterisks in the vehicle column indicate a significant trend, while asterisks in BPA dose group columns indicate significant differences in pairwise comparisons to the vehicle group. Full results of the analyses are presented in Supplemental Appendix XXIX.

${ }^{b}$ Numbers in parentheses are the number of animals examined.

${ }^{c} 25,000 \mu \mathrm{g} \mathrm{BPA} / \mathrm{kg}$ bw/day T4 significantly different from vehicle control $(p=0.015)$ in the sensitivity analysis that excluded all animals that overlapped with animals treated with $250,000 \mu \mathrm{g} \mathrm{BPA} / \mathrm{kg}$ bw/day.

$\mathrm{d}$ Means were calculated using $1 / 2$ the limit of detection $(L O D, 1.0 \mathrm{pg} / \mathrm{mL})$ for samples below LOD. Troponin I was also measured, but the overall percentage of samples above the limit of detection was less than $5 \%$, so no statistical analysis was conducted.

${ }^{\mathrm{e}}$ Number of samples with troponin T levels above LOD/total number of samples. Percentage of detects is given in parenthesis. Because of the high percentage of non-detects, the proportion of samples with detectable troponin T were statistically analyzed rather than means or medians. No significant trends across BPA dose groups or pairwise comparisons to vehicle control were detected.

fERRATUM: An error was identified in the NTP Research Report on the CLARITY-BPA Core Study: A Perinatal and Chronic Extended-Dose-Range Study of Bisphenol A in Rats (RR-9). The method of calculation of mean Troponin T, number of samples with detectable levels of Troponin T, and statistical analysis approach were not originally included in the Table. This error has been corrected; the new information is italicized. 
CLARITY-BPA Core Study

Table 46. Female Organ Weights, Vehicle, BPA, and $\mathrm{EE}_{2}$ Continuous-Dose, Interim Sacrifice (Mean \pm S.E.M.) ${ }^{\mathrm{a}}$

\begin{tabular}{|c|c|c|c|c|c|c|c|c|}
\hline Endpoint, Units & $\begin{array}{c}\text { Vehicle } \\
(21)^{b} \\
\end{array}$ & $\begin{array}{c}2.5 \text { BPA } \\
(22) \\
\end{array}$ & $\begin{array}{c}25 \text { BPA } \\
(21) \\
\end{array}$ & $\begin{array}{c}250 \text { BPA } \\
(22) \\
\end{array}$ & $\begin{array}{c}2500 \text { BPA } \\
(20) \\
\end{array}$ & $\begin{array}{c}25000 \text { BPA } \\
(24) \\
\end{array}$ & $\begin{array}{c}0.05 \mathrm{EE}_{2} \\
(24) \\
\end{array}$ & $\begin{array}{c}0.5 \mathrm{EE}_{2} \\
(26)\end{array}$ \\
\hline \multicolumn{9}{|l|}{ Body Weight at Necropsy } \\
\hline $\mathrm{g}$ & $420 \pm 19$ & $477 \pm 22$ & $445 \pm 15$ & $411 \pm 14$ & $422 \pm 13$ & $425 \pm 17$ & $453 \pm 13$ & $423 \pm 12$ \\
\hline \multicolumn{9}{|l|}{ Adrenal } \\
\hline Absolute, mg & $72.4 \pm 2.0$ & $76.4 \pm 2.8$ & $74.9 \pm 3.8$ & $75.4 \pm 4.7$ & $76.5 \pm 3.8$ & $73.3 \pm 3.1$ & $75.3 \pm 4.2$ & $92.2 \pm 3.8^{* * * *}$ \\
\hline Ratio to Brain, mg/g & $34.8 \pm 1.0$ & $36.2 \pm 1.4$ & $35.6 \pm 1.7$ & $36.2 \pm 2.2$ & $37.4 \pm 1.9$ & $35.4 \pm 1.5$ & $36.0 \pm 1.9$ & $44.7 \pm 1.9^{* * *}$ \\
\hline Ratio to $\mathrm{bw}, \mathrm{mg} / \mathrm{g}$ & $0.18 \pm 0.01$ & $0.16 \pm 0.01$ & $0.17 \pm 0.01$ & $0.19 \pm 0.01$ & $0.18 \pm 0.01$ & $0.18 \pm 0.01$ & $0.17 \pm 0.01$ & $0.22 \pm 0.01^{* * *}$ \\
\hline \multicolumn{9}{|l|}{ Brain } \\
\hline Absolute, g & $2.09 \pm 0.02$ & $2.11 \pm 0.03$ & $2.10 \pm 0.02$ & $2.08 \pm 0.02$ & $2.05 \pm 0.03$ & $2.08 \pm 0.03$ & $2.09 \pm 0.02$ & $2.07 \pm 0.02$ \\
\hline Ratio to bw, mg/g & $5.16 \pm 0.23$ & $4.60 \pm 0.19$ & $4.82 \pm 0.16$ & $5.18 \pm 0.17$ & $4.93 \pm 0.13$ & $5.04 \pm 0.18$ & $4.71 \pm 0.14$ & $4.98 \pm 0.13$ \\
\hline \multicolumn{9}{|c|}{ Ovarian/Parametrial Fat Pad } \\
\hline Absolute, $\mathrm{g}$ & $14.1 \pm 1.2$ & $17.1 \pm 1.0$ & $14.4 \pm 0.8$ & $14.4 \pm 0.8$ & $14.8 \pm 1.0$ & $14.2 \pm 0.9$ & $15.5 \pm 0.8$ & $11.4 \pm 0.6$ \\
\hline Ratio to Brain, g/g & $6.72 \pm 0.54$ & $8.13 \pm 0.47$ & $6.83 \pm 0.35$ & $6.93 \pm 0.40$ & $7.21 \pm 0.44$ & $6.83 \pm 0.40$ & $7.42 \pm 0.35$ & $5.50 \pm 0.30$ \\
\hline Ratio to bw, mg/g & $32.3 \pm 1.8$ & $36.3 \pm 1.7$ & $32.2 \pm 1.2$ & $34.8 \pm 1.2$ & $34.7 \pm 1.6$ & $33.2 \pm 1.3$ & $34.1 \pm 1.0$ & $26.7 \pm 1.0^{* * * *}$ \\
\hline \multicolumn{9}{|l|}{ Retroperitoneal Fat Pad } \\
\hline Absolute, g & $14.1 \pm 1.5$ & $19.8 \pm 2.0^{*}$ & $16.1 \pm 1.4$ & $14.1 \pm 1.1$ & $14.1 \pm 1.2$ & $14.7 \pm 1.2$ & $15.6 \pm 1.2$ & $13.0 \pm 0.9$ \\
\hline Ratio to Brain, g/g & $6.72 \pm 0.70$ & $9.41 \pm 0.97^{*}$ & $7.64 \pm 0.67$ & $6.76 \pm 0.52$ & $6.84 \pm 0.55$ & $7.09 \pm 0.56$ & $7.41 \pm 0.58$ & $6.26 \pm 0.44$ \\
\hline Ratio to bw, mg/g & $32.4 \pm 2.7$ & $40.0 \pm 2.8$ & $35.0 \pm 2.1$ & $33.5 \pm 1.7$ & $32.9 \pm 2.5$ & $33.7 \pm 1.6$ & $33.7 \pm 2.0$ & $30.0 \pm 1.5$ \\
\hline \multicolumn{9}{|l|}{ Heart } \\
\hline Absolute, $\mathrm{g}$ & $1.31 \pm 0.05$ & $1.44 \pm 0.04$ & $1.42 \pm 0.04$ & $1.29 \pm 0.03$ & $1.31 \pm 0.04$ & $1.38 \pm 0.04$ & $1.40 \pm 0.04$ & $1.42 \pm 0.04$ \\
\hline Ratio to Brain, g/g & $0.63 \pm 0.02$ & $0.68 \pm 0.02$ & $0.67 \pm 0.02$ & $0.62 \pm 0.01$ & $0.64 \pm 0.02$ & $0.66 \pm 0.02$ & $0.67 \pm 0.02$ & $0.68 \pm 0.02$ \\
\hline Ratio to bw, mg/g & $3.16 \pm 0.08$ & $3.06 \pm 0.08$ & $3.21 \pm 0.10$ & $3.18 \pm 0.09$ & $3.13 \pm 0.08$ & $3.30 \pm 0.08$ & $3.13 \pm 0.09$ & $3.36 \pm 0.04^{*}$ \\
\hline \multicolumn{9}{|l|}{ Kidney } \\
\hline Absolute, $\mathrm{g}$ & $2.27 \pm 0.07$ & $2.54 \pm 0.08$ & $2.51 \pm 0.10$ & $2.23 \pm 0.06$ & $2.31 \pm 0.08$ & $2.30 \pm 0.07^{\mathrm{c}}$ & $2.42 \pm 0.08^{c}$ & $2.61 \pm 0.08^{* *}$ \\
\hline Ratio to Brain, g/g & $1.09 \pm 0.03$ & $1.21 \pm 0.04$ & $1.20 \pm 0.05$ & $1.07 \pm 0.03$ & $1.12 \pm 0.03$ & $1.11 \pm 0.03$ & $1.15 \pm 0.04$ & $1.26 \pm 0.04^{* *}$ \\
\hline Ratio to bw, mg/g & $5.50 \pm 0.17$ & $5.43 \pm 0.17$ & $5.69 \pm 0.22$ & $5.47 \pm 0.12$ & $5.51 \pm 0.17$ & $5.59 \pm 0.13$ & $5.34 \pm 0.10$ & $6.19 \pm 0.10^{* * *}$ \\
\hline \multicolumn{9}{|l|}{ Liver } \\
\hline Absolute, $\mathrm{g}$ & $10.7 \pm 0.5$ & $12.4 \pm 0.7$ & $12.2 \pm 0.5$ & $10.8 \pm 0.3$ & $11.3 \pm 0.5$ & $11.6 \pm 0.7$ & $12.1 \pm 0.4$ & $12.8 \pm 0.5^{* *}$ \\
\hline Ratio to Brain, g/g & $5.12 \pm 0.24$ & $5.90 \pm 0.36$ & $5.81 \pm 0.25$ & $5.18 \pm 0.16$ & $5.49 \pm 0.21$ & $5.60 \pm 0.30$ & $5.77 \pm 0.20$ & $6.17 \pm 0.21^{* *}$ \\
\hline Ratio to bw, mg/g & $25.5 \pm 0.6^{*}$ & $25.9 \pm 0.8$ & $27.3 \pm 0.6$ & $26.4 \pm 0.6$ & $26.7 \pm 0.7$ & $27.2 \pm 0.7$ & $26.7 \pm 0.7$ & $30.1 \pm 0.5^{* * *}$ \\
\hline \multicolumn{9}{|l|}{ Ovary } \\
\hline Absolute, mg & $140 \pm 8^{\mathrm{d}}$ & $147 \pm 7$ & $142 \pm 6^{\mathrm{d}}$ & $138 \pm 5^{\mathrm{d}}$ & $140 \pm 7^{\mathrm{d}}$ & $140 \pm 5^{\mathrm{d}}$ & $143 \pm 4^{\mathrm{d}}$ & $115 \pm 8^{*}, \mathrm{~d}$ \\
\hline Ratio to Brain, mg/g & $67 \pm 3$ & $70 \pm 4$ & $68 \pm 3$ & $66 \pm 2$ & $68 \pm 3$ & $68 \pm 3$ & $68 \pm 2$ & $56 \pm 4^{*}$ \\
\hline Ratio to bw, mg/g & $0.33 \pm 0.02$ & $0.32 \pm 0.02$ & $0.33 \pm 0.01$ & $0.34 \pm 0.01$ & $0.33 \pm 0.01$ & $0.34 \pm 0.02$ & $0.32 \pm 0.01$ & $0.28 \pm 0.02^{*}$ \\
\hline \multicolumn{9}{|l|}{ Pituitary } \\
\hline Absolute, mg & $20.6 \pm 1.1$ & $21.2 \pm 1.3$ & $23.5 \pm 1.8$ & $20.4 \pm 1.5$ & $20.2 \pm 1.4$ & $21.6 \pm 1.1$ & $22.7 \pm 1.7$ & $26.9 \pm 1.2^{* *}$ \\
\hline Ratio to Brain, mg/g & $9.9 \pm 0.5$ & $10.0 \pm 0.6$ & $11.2 \pm 0.9$ & $9.8 \pm 0.7$ & $9.9 \pm 0.7$ & $10.4 \pm 0.5$ & $10.8 \pm 0.8$ & $13.1 \pm 0.6^{* *}$ \\
\hline Ratio to bw, mg/g & $0.05 \pm 0$ & $0.05 \pm 0$ & $0.05 \pm 0$ & $0.05 \pm 0$ & $0.05 \pm 0$ & $0.05 \pm 0$ & $0.05 \pm 0$ & $0.06 \pm 0^{* *}$ \\
\hline
\end{tabular}


CLARITY-BPA Core Study

\begin{tabular}{|c|c|c|c|c|c|c|c|c|}
\hline Endpoint, Units & $\begin{array}{c}\text { Vehicle } \\
(21)^{b}\end{array}$ & $\begin{array}{c}2.5 \text { BPA } \\
(22)\end{array}$ & $\begin{array}{c}25 \text { BPA } \\
(21)\end{array}$ & $\begin{array}{c}250 \text { BPA } \\
(22)\end{array}$ & $\begin{array}{c}2500 \text { BPA } \\
(20)\end{array}$ & $\begin{array}{c}25000 \text { BPA } \\
(24)\end{array}$ & $\begin{array}{c}0.05 \text { EE2 } \\
(24)\end{array}$ & $\begin{array}{c}0.5 \mathrm{EE}_{2} \\
(26)\end{array}$ \\
\hline \multicolumn{9}{|l|}{ Spleen } \\
\hline Absolute, mg & $598 \pm 25$ & $648 \pm 27$ & $638 \pm 17$ & $600 \pm 20$ & $642 \pm 23$ & $638 \pm 26$ & $645 \pm 21$ & $646 \pm 25$ \\
\hline Ratio to Brain, $\mathrm{mg} / \mathrm{g}$ & $286 \pm 11$ & $307 \pm 13$ & $304 \pm 8$ & $288 \pm 9$ & $314 \pm 10$ & $307 \pm 11$ & $308 \pm 10$ & $311 \pm 11$ \\
\hline Ratio to bw, $\mathrm{mg} / \mathrm{g}$ & $1.44 \pm 0.05$ & $1.37 \pm 0.04$ & $1.45 \pm 0.05$ & $1.48 \pm 0.05$ & $1.54 \pm 0.05$ & $1.52 \pm 0.05$ & $1.43 \pm 0.04$ & $1.52 \pm 0.04$ \\
\hline \multicolumn{9}{|l|}{ Thymus } \\
\hline Absolute, mg & $150 \pm 11$ & $185 \pm 16$ & $155 \pm 12$ & $135 \pm 12$ & $142 \pm 9$ & $151 \pm 10$ & $137 \pm 10$ & $137 \pm 9$ \\
\hline Ratio to Brain, $\mathrm{mg} / \mathrm{g}$ & $72 \pm 5$ & $88 \pm 8$ & $74 \pm 6$ & $65 \pm 6$ & $70 \pm 5$ & $73 \pm 5$ & $65 \pm 5$ & $66 \pm 4$ \\
\hline Ratio to bw, mg/g & $0.37 \pm 0.03$ & $0.39 \pm 0.03$ & $0.35 \pm 0.02$ & $0.33 \pm 0.03$ & $0.34 \pm 0.02$ & $0.36 \pm 0.02$ & $0.30 \pm 0.02$ & $0.32 \pm 0.02$ \\
\hline \multicolumn{9}{|l|}{ Thyroid } \\
\hline Absolute, mg & $38.2 \pm 1.7$ & $37.4 \pm 1.6$ & $37.2 \pm 1.5$ & $35.8 \pm 1.2$ & $37.8 \pm 2.0$ & $38.5 \pm 1.5$ & $39.0 \pm 1.8$ & $36.5 \pm 1.2$ \\
\hline Ratio to Brain, $\mathrm{mg} / \mathrm{g}$ & $18.3 \pm 0.8$ & $17.8 \pm 0.8$ & $17.8 \pm 0.7$ & $17.2 \pm 0.6$ & $18.5 \pm 1.0$ & $18.6 \pm 0.7$ & $18.6 \pm 0.8$ & $17.7 \pm 0.6$ \\
\hline Ratio to bw, $\mathrm{mg} / \mathrm{g}$ & $0.09 \pm 0$ & $0.08 \pm 0$ & $0.08 \pm 0$ & $0.09 \pm 0$ & $0.09 \pm 0$ & $0.09 \pm 0$ & $0.09 \pm 0$ & $0.09 \pm 0$ \\
\hline \multicolumn{9}{|l|}{ Uterus } \\
\hline Absolute, mg & $773 \pm 45$ & $742 \pm 52$ & $872 \pm 60^{\mathrm{e}}$ & $757 \pm 58$ & $831 \pm 51$ & $832 \pm 61$ & $777 \pm 38$ & $827 \pm 37$ \\
\hline Ratio to Brain, $\mathrm{mg} / \mathrm{g}$ & $371 \pm 22$ & $354 \pm 26$ & $414 \pm 26$ & $364 \pm 28$ & $411 \pm 29$ & $403 \pm 30$ & $372 \pm 19$ & $399 \pm 16$ \\
\hline Ratio to bw, mg/g & $1.92 \pm 0.15$ & $1.64 \pm 0.14$ & $1.98 \pm 0.15$ & $1.89 \pm 0.16$ & $2.05 \pm 0.17$ & $2.07 \pm 0.21$ & $1.76 \pm 0.11$ & $1.97 \pm 0.09$ \\
\hline
\end{tabular}

${ }^{a} \mathrm{BPA}$ and $\mathrm{EE}_{2}$ doses are $\mu \mathrm{g} / \mathrm{kg}$ bw/day. The indicated organs were collected from animals at the interim (one-year) necropsy and weights recorded. BPA and EE 2 groups were analyzed separately. Paired organs are presented and were analyzed as combined weights. ANOVA was performed for absolute organ weights to determine the effect of treatment on organ weight. Separate ANOCOVA were performed to determine the effect of treatment on organ weight adjusted for brain weight or receiving weight. Comparisons of dosed groups versus vehicle control were performed using Dunnett's method to adjust for multiple comparisons. Tests of trends, increasing treatment effect with increasing dose, were also performed for vehicle and BPA groups. Tests were conducted as two-sided at the 0.05 significance level. Statistically significant effects are indicated by asterisks $(*, p<0.05$; $\left.*^{*}, p<0.01 ; * *, p<0.001\right)$; asterisks in the vehicle column indicate a significant trend, while asterisks in BPA or EE 2 dose group columns indicate significant differences in pairwise comparisons to the vehicle group. There were no additional statistically significant effects in the sensitivity analysis that excluded all animals that overlapped with animals treated with 250,000 $\mu \mathrm{g} \mathrm{BPA} / \mathrm{kg}$ bw/day (see Statistical Methods). The complete statistical report is found in Supplemental Appendix XXX.

${ }^{b}$ Numbers in parentheses are the number of animals examined. Any deviations from this number due to exclusions are indicated by footnotes.

'For kidneys, $\mathrm{n}=23$ for both the 25,000 BPA and $0.05 \mathrm{EE}_{2}$ dose groups.

${ }^{\mathrm{d}}$ For ovaries, $\mathrm{n}=20$ for vehicle group, 17 for $25 \mathrm{BPA}, 21$ for $250 \mathrm{BPA}, 18$ for 2,500 BPA, 21 for 25,000 BPA, and 23 for both 0.05 and $0.5 \mathrm{EE} 2$ dose groups

${ }^{e}$ For uterus, $\mathrm{n}=20$ for 25 BPA dose group. 
CLARITY-BPA Core Study

Table 47. Female Organ Weights, Vehicle and BPA Stop-Dose, Interim Sacrifice (Mean \pm S.E.M.) ${ }^{\mathrm{a}}$

\begin{tabular}{|c|c|c|c|c|c|c|}
\hline Endpoint, Units & $\begin{array}{c}\text { Vehicle } \\
(20)^{b} \\
\end{array}$ & $\begin{array}{c}2.5 \text { BPA } \\
(22)\end{array}$ & $\begin{array}{c}25 \text { BPA } \\
(20)\end{array}$ & $\begin{array}{c}250 \text { BPA } \\
(22) \\
\end{array}$ & $\begin{array}{c}2500 \text { BPA } \\
(20)\end{array}$ & $\begin{array}{c}25000 \text { BPA } \\
(20)\end{array}$ \\
\hline \multicolumn{7}{|l|}{ Body Weight } \\
\hline $\mathrm{g}$ & $465 \pm 19$ & $455 \pm 14$ & $437 \pm 19$ & $453 \pm 19$ & $454 \pm 19$ & $444 \pm 14$ \\
\hline \multicolumn{7}{|l|}{ Adrenal } \\
\hline Absolute, mg & $73.1 \pm 2.9$ & $70.0 \pm 1.6$ & $71.6 \pm 2.7$ & $71.2 \pm 2.4$ & $68.5 \pm 2.9$ & $75.6 \pm 3.1$ \\
\hline Ratio to Brain, mg/g & $34.9 \pm 1.3$ & $33.4 \pm 0.7$ & $34.3 \pm 1.3$ & $34.8 \pm 1.2$ & $32.8 \pm 1.3$ & $36.5 \pm 1.4$ \\
\hline Ratio to $\mathrm{bw}, \mathrm{mg} / \mathrm{g}$ & $0.16 \pm 0.01$ & $0.16 \pm 0.01$ & $0.17 \pm 0$ & $0.16 \pm 0.01$ & $0.16 \pm 0.01$ & $0.17 \pm 0.01$ \\
\hline \multicolumn{7}{|l|}{ Brain } \\
\hline Absolute, $\mathrm{g}$ & $2.09 \pm 0.02$ & $2.10 \pm 0.02$ & $2.09 \pm 0.03$ & $2.05 \pm 0.02$ & $2.09 \pm 0.02$ & $2.07 \pm 0.02$ \\
\hline Ratio to bw, mg/g & $4.60 \pm 0.14$ & $4.68 \pm 0.13$ & $4.93 \pm 0.20$ & $4.68 \pm 0.18$ & $4.75 \pm 0.20$ & $4.75 \pm 0.14$ \\
\hline \multicolumn{7}{|c|}{ Ovarian/Parametrial Fat Pad } \\
\hline Absolute, g & $17.2 \pm 1.2$ & $16.2 \pm 0.7$ & $14.3 \pm 1.0^{\mathrm{c}}$ & $15.3 \pm 1.1$ & $16.3 \pm 1.1$ & $15.0 \pm 0.9$ \\
\hline Ratio to Brain, g/g & $8.17 \pm 0.53$ & $7.72 \pm 0.33$ & $6.84 \pm 0.45^{\mathrm{c}}$ & $7.47 \pm 0.51$ & $7.79 \pm 0.53$ & $7.22 \pm 0.41$ \\
\hline Ratio to bw, mg/g & $36.3 \pm 1.3$ & $35.4 \pm 1.0$ & $32.4 \pm 1.1^{\mathrm{c}}$ & $33.3 \pm 1.6$ & $35.6 \pm 1.7$ & $33.3 \pm 1.1$ \\
\hline \multicolumn{7}{|l|}{ Retroperitoneal Fat Pad } \\
\hline Absolute, g & $18.2 \pm 1.6$ & $15.4 \pm 1.5$ & $17.0 \pm 1.8$ & $17.1 \pm 1.5$ & $16.2 \pm 1.3$ & $16.4 \pm 1.4$ \\
\hline Ratio to Brain, g/g & $8.66 \pm 0.67$ & $7.36 \pm 0.73$ & $8.10 \pm 0.87$ & $8.28 \pm 0.69$ & $7.71 \pm 0.61$ & $7.93 \pm 0.68$ \\
\hline Ratio to bw, mg/g & $38.1 \pm 1.8$ & $33.3 \pm 2.6$ & $37.0 \pm 2.7$ & $36.3 \pm 2.1$ & $34.8 \pm 2.0$ & $36.0 \pm 2.3$ \\
\hline \multicolumn{7}{|l|}{ Heart } \\
\hline Absolute, $\mathrm{g}$ & $1.43 \pm 0.05$ & $1.36 \pm 0.02$ & $1.35 \pm 0.04$ & $1.37 \pm 0.04$ & $1.38 \pm 0.04$ & $1.35 \pm 0.04$ \\
\hline Ratio to Brain, g/g & $0.68 \pm 0.02$ & $0.65 \pm 0.01$ & $0.65 \pm 0.02$ & $0.67 \pm 0.02$ & $0.66 \pm 0.02$ & $0.65 \pm 0.02$ \\
\hline Ratio to bw, mg/g & $3.11 \pm 0.08$ & $3.02 \pm 0.07$ & $3.13 \pm 0.09$ & $3.07 \pm 0.10$ & $3.06 \pm 0.07$ & $3.06 \pm 0.08$ \\
\hline \multicolumn{7}{|l|}{ Kidney } \\
\hline Absolute, $\mathrm{g}$ & $2.47 \pm 0.11$ & $2.30 \pm 0.09^{d}$ & $2.28 \pm 0.09$ & $2.41 \pm 0.1^{\mathrm{d}}$ & $2.35 \pm 0.09$ & $2.38 \pm 0.08$ \\
\hline Ratio to Brain, g/g & $1.18 \pm 0.04$ & $1.10 \pm 0.04$ & $1.09 \pm 0.04$ & $1.17 \pm 0.04$ & $1.12 \pm 0.04$ & $1.15 \pm 0.04$ \\
\hline Ratio to bw, mg/g & $5.34 \pm 0.12$ & $5.17 \pm 0.23$ & $5.24 \pm 0.12$ & $5.38 \pm 0.15$ & $5.22 \pm 0.14$ & $5.39 \pm 0.15$ \\
\hline \multicolumn{7}{|l|}{ Liver } \\
\hline Absolute, $\mathrm{g}$ & $12.3 \pm 0.7$ & $11.5 \pm 0.4$ & $11.2 \pm 0.6$ & $12.1 \pm 0.6$ & $12.0 \pm 0.6$ & $11.6 \pm 0.4$ \\
\hline Ratio to Brain, g/g & $5.86 \pm 0.28$ & $5.52 \pm 0.19$ & $5.38 \pm 0.27$ & $5.89 \pm 0.26$ & $5.70 \pm 0.25$ & $5.63 \pm 0.20$ \\
\hline Ratio to bw, mg/g & $26.4 \pm 0.8$ & $25.4 \pm 0.6$ & $25.7 \pm 0.6$ & $26.8 \pm 0.8$ & $26.4 \pm 0.9$ & $26.3 \pm 0.6$ \\
\hline \multicolumn{7}{|l|}{ Ovary } \\
\hline Absolute, mg & $157 \pm 6^{*}$ & $149 \pm 4$ & $148 \pm 6$ & $147 \pm 4$ & $147 \pm 6$ & $136 \pm 5^{*}$ \\
\hline Ratio to Brain, mg/g & $75 \pm 3^{*}$ & $71 \pm 2$ & $71 \pm 3$ & $72 \pm 2$ & $70 \pm 3$ & $66 \pm 3^{*}$ \\
\hline Ratio to bw, mg/g & $0.34 \pm 0.02^{*}$ & $0.33 \pm 0.01$ & $0.35 \pm 0.02$ & $0.33 \pm 0.02$ & $0.33 \pm 0.01$ & $0.31 \pm 0.01$ \\
\hline \multicolumn{7}{|l|}{ Pituitary } \\
\hline Absolute, mg & $21.1 \pm 1$ & $20.3 \pm 0.8$ & $19.9 \pm 1.3$ & $20.1 \pm 0.7$ & $19 \pm 0.9$ & $20.3 \pm 1.1$ \\
\hline Ratio to Brain, mg/g & $10.0 \pm 0.4$ & $9.7 \pm 0.4$ & $9.6 \pm 0.7$ & $9.8 \pm 0.3$ & $9.1 \pm 0.4$ & $9.8 \pm 0.5$ \\
\hline Ratio to bw, mg/g & $0.05 \pm 0$ & $0.04 \pm 0$ & $0.05 \pm 0$ & $0.05 \pm 0$ & $0.04 \pm 0$ & $0.05 \pm 0$ \\
\hline
\end{tabular}


CLARITY-BPA Core Study

\begin{tabular}{|c|c|c|c|c|c|c|}
\hline Endpoint, Units & $\begin{array}{c}\text { Vehicle } \\
(20)^{b}\end{array}$ & $\begin{array}{c}2.5 \text { BPA } \\
(22)\end{array}$ & $\begin{array}{c}25 \text { BPA } \\
(20)\end{array}$ & $\begin{array}{c}250 \text { BPA } \\
(22)\end{array}$ & $\begin{array}{c}2500 \text { BPA } \\
(20)\end{array}$ & $\begin{array}{c}25000 \text { BPA } \\
(20)\end{array}$ \\
\hline \multicolumn{7}{|l|}{ Spleen } \\
\hline Absolute, mg & $678 \pm 30$ & $618 \pm 16$ & $612 \pm 24^{\mathrm{e}}$ & $693 \pm 24$ & $659 \pm 30$ & $611 \pm 17$ \\
\hline Ratio to Brain, $\mathrm{mg} / \mathrm{g}$ & $323 \pm 11$ & $295 \pm 7^{e}$ & $293 \pm 11^{\mathrm{e}}$ & $339 \pm 12$ & $315 \pm 14$ & $295 \pm 8$ \\
\hline Ratio to bw, mg/g & $1.47 \pm 0.05$ & $1.37 \pm 0.04$ & $1.41 \pm 0.04$ & $1.56 \pm 0.06$ & $1.48 \pm 0.07$ & $1.40 \pm 0.05$ \\
\hline \multicolumn{7}{|l|}{ Thymus } \\
\hline Absolute, mg & $154 \pm 7$ & $139 \pm 6^{f}$ & $142 \pm 10$ & $164 \pm 14$ & $150 \pm 11$ & $167 \pm 12$ \\
\hline Ratio to Brain, $\mathrm{mg} / \mathrm{g}$ & $74 \pm 3$ & $67 \pm 3$ & $68 \pm 5$ & $80 \pm 7$ & $72 \pm 5$ & $80 \pm 6$ \\
\hline Ratio to bw, $\mathrm{mg} / \mathrm{g}$ & $0.34 \pm 0.02$ & $0.31 \pm 0.02$ & $0.32 \pm 0.02$ & $0.37 \pm 0.03$ & $0.34 \pm 0.03$ & $0.38 \pm 0.02$ \\
\hline \multicolumn{7}{|l|}{ Thyroid } \\
\hline Absolute, mg & $37.8 \pm 2.2$ & $36.0 \pm 1.6$ & $36.3 \pm 1.7$ & $36.3 \pm 1.3$ & $38.2 \pm 2.3$ & $36.9 \pm 1.7$ \\
\hline Ratio to Brain, $\mathrm{mg} / \mathrm{g}$ & $18.0 \pm 1$ & $17.2 \pm 0.8$ & $17.4 \pm 0.8$ & $17.7 \pm 0.6$ & $18.3 \pm 1.1$ & $17.8 \pm 0.8$ \\
\hline Ratio to bw, mg/g & $0.08 \pm 0$ & $0.08 \pm 0$ & $0.08 \pm 0$ & $0.08 \pm 0$ & $0.08 \pm 0$ & $0.08 \pm 0$ \\
\hline \multicolumn{7}{|l|}{ Uterus } \\
\hline Absolute, mg & $744 \pm 46$ & $699 \pm 45$ & $795 \pm 80$ & $843 \pm 76^{\mathrm{g}}$ & $747 \pm 54$ & $789 \pm 51$ \\
\hline Ratio to Brain, $\mathrm{mg} / \mathrm{g}$ & $356 \pm 22$ & $334 \pm 22$ & $382 \pm 40$ & $410 \pm 35$ & $360 \pm 28$ & $381 \pm 24$ \\
\hline Ratio to bw, mg/g & $1.67 \pm 0.14$ & $1.58 \pm 0.12$ & $1.87 \pm 0.19$ & $1.93 \pm 0.20$ & $1.74 \pm 0.16$ & $1.83 \pm 0.14$ \\
\hline
\end{tabular}

${ }^{a}$ BPA doses are $\mu \mathrm{g} / \mathrm{kg}$ bw/day. The indicated organs were collected from animals at the interim (one-year) necropsy and weights recorded. Paired organs are presented and were analyzed as combined weights. ANOVA was performed for absolute organ weights to determine the effect of treatment on organ weight. Separate ANOCOVA were performed to determine the effect of treatment on organ weight adjusted for brain weight or receiving body weight. Comparisons of dosed groups versus vehicle control were performed using Dunnett's method to adjust for multiple comparisons. Tests of trends, increasing treatment effect with increasing dose, were also performed. Tests were conducted as two-sided at the 0.05 significance level. Statistically significant effects are indicated by asterisks $(*, p<0.05)$; asterisks in the vehicle column indicate a significant trend, while asterisks in BPA dose group columns indicate significant differences in pairwise comparisons to the vehicle group. The complete statistical report is found in Supplemental Appendix XXX. ${ }^{b}$ Numbers in parentheses are the number of animals examined. Any deviations from this number due to exclusions are indicated by footnotes.

25 BPA ovarian/parametrial fat pad weight significantly different from vehicle control in sensitivity analysis (see Statistical Methods) that excluded all animals that overlapped with animals treated with $250,000 \mu \mathrm{g} \mathrm{BPA} / \mathrm{kg}$ bw/day: absolute weight, $25.7 \%$ lower than control $(p=0.010)$; brain as covariate, $25 \%$ lower than control $(p=0.010)$; body weight as covariate, $13.7 \%$ lower than control $(p=0.035)$.

$\mathrm{d}_{\mathrm{n}}=21$ for kidneys in the 2.5 and 250 BPA dose groups. One kidney weight in each indicated dose group was excluded because kidneys had grossly observable cysts.

eSpleen weight statistically different from vehicle control in sensitivity analysis that excluded all animals that overlapped with animals treated with $250,000 \mu \mathrm{g}$ BPA/kg bw/day (see Statistical Methods): 2.5 BPA, brain covariate, $13.1 \%$ lower than control ( $p=0.039$ ); 25 BPA absolute and brain covariate, $14.2 \%$ and $13.7 \%$ lower than control, respectively $(p=0.023$ for both).

$\mathrm{f}=21$ for thymus in the 2.5 BPA dose group, one $(627 \mathrm{mg})$ excluded as a statistical outlier.

$\mathrm{g}_{\mathrm{n}}=21$ for uterus in the 250 BPA dose group, one $(4,680 \mathrm{mg})$ excluded as it was the only uterus noted to be fluid filled. 
CLARITY-BPA Core Study

Table 48. Male Organ Weights, Vehicle, BPA, and $\mathrm{EE}_{2}$ Continuous-Dose, Interim Sacrifice (Mean \pm S.E.M.) ${ }^{\mathrm{a}}$

\begin{tabular}{|c|c|c|c|c|c|c|c|c|}
\hline Endpoint, Units & $\begin{array}{c}\text { Vehicle } \\
(\mathbf{1 8})^{\mathrm{b}}\end{array}$ & $\begin{array}{c}2.5 \text { BPA } \\
(22)\end{array}$ & $\begin{array}{c}25 \text { BPA } \\
(18)\end{array}$ & $\begin{array}{c}250 \text { BPA } \\
(24) \\
\end{array}$ & $\begin{array}{c}2500 \text { BPA } \\
(18) \\
\end{array}$ & $\begin{array}{c}25000 \text { BPA } \\
(21) \\
\end{array}$ & $\begin{array}{c}0.05 \mathrm{EE}_{2} \\
(22)\end{array}$ & $\begin{array}{c}0.5 \mathrm{EE}_{2} \\
(23)\end{array}$ \\
\hline \multicolumn{9}{|c|}{ Body Weight at Necropsy } \\
\hline $\mathrm{g}$ & $701 \pm 19$ & $722 \pm 20$ & $709 \pm 21$ & $713 \pm 15$ & $704 \pm 26$ & $695 \pm 18$ & $712 \pm 21$ & $704 \pm 16$ \\
\hline \multicolumn{9}{|l|}{ Adrenal } \\
\hline Absolute, mg & $60.1 \pm 2.8$ & $64.2 \pm 2.8$ & $68.0 \pm 2.4$ & $62.5 \pm 2.1$ & $61.7 \pm 2.6$ & $57.4 \pm 1.7$ & $62.6 \pm 2.2^{c}$ & $62.9 \pm 1.8$ \\
\hline Ratio to Brain, $\mathrm{mg} / \mathrm{g}$ & $26.1 \pm 1.2$ & $27.8 \pm 1.1$ & $29.7 \pm 1.1$ & $27.8 \pm 0.8$ & $27.0 \pm 1.0$ & $25.7 \pm 0.8$ & $27.4 \pm 0.9$ & $27.1 \pm 0.7$ \\
\hline Ratio to bw, mg/g & $0.09 \pm 0$ & $0.09 \pm 0$ & $0.10 \pm 0$ & $0.09 \pm 0$ & $0.09 \pm 0$ & $0.08 \pm 0$ & $0.09 \pm 0$ & $0.09 \pm 0$ \\
\hline \multicolumn{9}{|l|}{ Brain } \\
\hline Absolute, $\mathrm{g}$ & $2.30 \pm 0.02$ & $2.31 \pm 0.03$ & $2.30 \pm 0.04$ & $2.24 \pm 0.03$ & $2.28 \pm 0.03$ & $2.24 \pm 0.02$ & $2.29 \pm 0.02$ & $2.32 \pm 0.02$ \\
\hline Ratio to bw, mg/g & $3.32 \pm 0.07$ & $3.25 \pm 0.10$ & $3.27 \pm 0.08$ & $3.18 \pm 0.08$ & $3.30 \pm 0.10$ & $3.26 \pm 0.08$ & $3.27 \pm 0.09$ & $3.32 \pm 0.07$ \\
\hline \multicolumn{9}{|l|}{ Epididymides } \\
\hline Absolute, $g$ & $1.28 \pm 0.04$ & $1.21 \pm 0.04$ & $1.32 \pm 0.02$ & $1.27 \pm 0.04$ & $1.27 \pm 0.04$ & $1.25 \pm 0.02$ & $1.24 \pm 0.03$ & $1.34 \pm 0.03$ \\
\hline Ratio to Brain, g/g & $0.56 \pm 0.02$ & $0.53 \pm 0.02$ & $0.58 \pm 0.01$ & $0.56 \pm 0.02$ & $0.56 \pm 0.01$ & $0.56 \pm 0.01$ & $0.54 \pm 0.01$ & $0.58 \pm 0.01$ \\
\hline Ratio to bw, mg/g & $1.85 \pm 0.06$ & $1.70 \pm 0.07$ & $1.89 \pm 0.06$ & $1.79 \pm 0.06$ & $1.83 \pm 0.06$ & $1.82 \pm 0.06$ & $1.76 \pm 0.06$ & $1.90 \pm 0.04$ \\
\hline \multicolumn{9}{|l|}{ Epididymal Fat Pad } \\
\hline Absolute, $\mathrm{g}$ & $13.0 \pm 0.7$ & $14.5 \pm 0.8$ & $13.6 \pm 0.6$ & $14.1 \pm 0.8$ & $14.3 \pm 1.2$ & $14.2 \pm 1.0$ & $14.2 \pm 0.8$ & $12.8 \pm 0.8$ \\
\hline Ratio to Brain, g/g & $5.62 \pm 0.26$ & $6.28 \pm 0.37$ & $5.91 \pm 0.23$ & $6.29 \pm 0.39$ & $6.24 \pm 0.50$ & $6.35 \pm 0.45$ & $6.18 \pm 0.32$ & $5.54 \pm 0.33$ \\
\hline Ratio to bw, mg/g & $18.5 \pm 0.8$ & $19.8 \pm 0.7$ & $19.2 \pm 0.7$ & $19.5 \pm 1.0$ & $20.0 \pm 1.1$ & $20.2 \pm 1.2$ & $19.8 \pm 0.7$ & $18.0 \pm 0.8$ \\
\hline \multicolumn{9}{|l|}{ Retroperitoneal Fat Pad } \\
\hline Absolute, $\mathrm{g}$ & $22.2 \pm 1.8$ & $22.8 \pm 1.6$ & $24.5 \pm 2.5$ & $22.9 \pm 1.9$ & $21.8 \pm 2.7$ & $23.6 \pm 2.4$ & $22.6 \pm 2.3$ & $21.0 \pm 2.3$ \\
\hline Ratio to Brain, g/g & $9.59 \pm 0.74$ & $9.88 \pm 0.69$ & $10.57 \pm 1.02$ & $10.19 \pm 0.81$ & $9.56 \pm 1.21$ & $10.59 \pm 1.09$ & $9.79 \pm 0.97$ & $9.04 \pm 1.01$ \\
\hline Ratio to bw, mg/g & $31.3 \pm 2.0$ & $30.9 \pm 1.5$ & $33.7 \pm 2.6$ & $31.5 \pm 2.3$ & $29.7 \pm 2.7$ & $33.2 \pm 2.9$ & $30.9 \pm 2.5$ & $29.0 \pm 2.7$ \\
\hline \multicolumn{9}{|l|}{ Heart } \\
\hline Absolute, $\mathrm{g}$ & $2.04 \pm 0.06$ & $2.14 \pm 0.05$ & $2.26 \pm 0.08$ & $2.21 \pm 0.07$ & $2.19 \pm 0.07$ & $1.99 \pm 0.04$ & $2.11 \pm 0.06$ & $2.12 \pm 0.05$ \\
\hline Ratio to Brain, g/g & $0.89 \pm 0.02$ & $0.93 \pm 0.02$ & $0.99 \pm 0.04$ & $0.98 \pm 0.03$ & $0.96 \pm 0.03$ & $0.89 \pm 0.02$ & $0.92 \pm 0.02$ & $0.91 \pm 0.02$ \\
\hline Ratio to bw, mg/g & $2.92 \pm 0.07$ & $3.00 \pm 0.09$ & $3.20 \pm 0.10$ & $3.11 \pm 0.10$ & $3.14 \pm 0.09$ & $2.88 \pm 0.05$ & $2.99 \pm 0.08$ & $3.02 \pm 0.08$ \\
\hline \multicolumn{9}{|l|}{ Kidney } \\
\hline Absolute, $\mathrm{g}$ & $4.08 \pm 0.15^{\mathrm{d}}$ & $4.20 \pm 0.12$ & $4.15 \pm 0.17^{\mathrm{d}}$ & $4.05 \pm 0.11$ & $4.00 \pm 0.17^{\mathrm{d}}$ & $3.88 \pm 0.08$ & $4.01 \pm 0.13^{\mathrm{d}}$ & $4.17 \pm 0.12$ \\
\hline Ratio to Brain, g/g & $1.77 \pm 0.05$ & $1.82 \pm 0.05$ & $1.80 \pm 0.06$ & $1.80 \pm 0.04$ & $1.75 \pm 0.07$ & $1.73 \pm 0.04$ & $1.75 \pm 0.05$ & $1.80 \pm 0.05$ \\
\hline Ratio to bw, mg/g & $5.83 \pm 0.14$ & $5.85 \pm 0.14$ & $5.87 \pm 0.15$ & $5.70 \pm 0.12$ & $5.67 \pm 0.16$ & $5.62 \pm 0.11$ & $5.63 \pm 0.10$ & $5.91 \pm 0.09$ \\
\hline \multicolumn{9}{|l|}{ Liver } \\
\hline Absolute, $\mathrm{g}$ & $22.1 \pm 0.9$ & $21.1 \pm 0.7$ & $22.6 \pm 1.0$ & $22.4 \pm 0.6$ & $22.1 \pm 1.0$ & $20.6 \pm 0.6$ & $22.2 \pm 0.7$ & $23.1 \pm 0.6$ \\
\hline Ratio to Brain, g/g & $9.58 \pm 0.34$ & $9.15 \pm 0.29$ & $9.84 \pm 0.34$ & $9.97 \pm 0.26$ & $9.71 \pm 0.43$ & $9.21 \pm 0.26$ & $9.66 \pm 0.29$ & $9.96 \pm 0.29$ \\
\hline Ratio to bw, mg/g & $31.4 \pm 0.6$ & $29.3 \pm 0.6^{*}$ & $31.9 \pm 0.8$ & $31.4 \pm 0.5$ & $31.4 \pm 0.6$ & $29.7 \pm 0.5$ & $31.1 \pm 0.5$ & $32.7 \pm 0.4$ \\
\hline \multicolumn{9}{|l|}{ Pituitary } \\
\hline Absolute, mg & $14.6 \pm 0.7$ & $14.6 \pm 0.6$ & $15.2 \pm 0.6$ & $16.1 \pm 1.2$ & $15.4 \pm 0.8$ & $15.1 \pm 0.6$ & $15.6 \pm 0.6$ & $16.0 \pm 0.5$ \\
\hline Ratio to Brain, $\mathrm{mg} / \mathrm{g}$ & $6.3 \pm 0.2$ & $6.3 \pm 0.3$ & $6.7 \pm 0.3$ & $7.1 \pm 0.5$ & $6.7 \pm 0.4$ & $6.7 \pm 0.3$ & $6.8 \pm 0.3$ & $6.9 \pm 0.2$ \\
\hline Ratio to bw, mg/g & $0.02 \pm 0$ & $0.02 \pm 0$ & $0.02 \pm 0$ & $0.02 \pm 0$ & $0.02 \pm 0$ & $0.02 \pm 0$ & $0.02 \pm 0$ & $0.02 \pm 0$ \\
\hline
\end{tabular}


CLARITY-BPA Core Study

\begin{tabular}{|c|c|c|c|c|c|c|c|c|}
\hline Endpoint, Units & $\begin{array}{c}\text { Vehicle } \\
(18)^{b}\end{array}$ & $\begin{array}{c}2.5 \text { BPA } \\
(22)\end{array}$ & $\begin{array}{c}25 \text { BPA } \\
(18)\end{array}$ & $\begin{array}{c}250 \text { BPA } \\
(24)\end{array}$ & $\begin{array}{c}2500 \text { BPA } \\
(18)\end{array}$ & $\begin{array}{c}25000 \text { BPA } \\
(21)\end{array}$ & $\begin{array}{c}0.05 \mathrm{EE}_{2} \\
(22)\end{array}$ & $\begin{array}{c}0.5 \text { EE2 } \\
(23)\end{array}$ \\
\hline \multicolumn{9}{|l|}{ Seminal Vesicles } \\
\hline Absolute, g & $1.18 \pm 0.04$ & $1.11 \pm 0.05$ & $1.14 \pm 0.05$ & $1.17 \pm 0.05$ & $1.10 \pm 0.06$ & $1.19 \pm 0.04$ & $1.27 \pm 0.06$ & $1.12 \pm 0.04$ \\
\hline Ratio to Brain, g/g & $0.51 \pm 0.02$ & $0.48 \pm 0.02$ & $0.50 \pm 0.02$ & $0.52 \pm 0.02$ & $0.48 \pm 0.02$ & $0.53 \pm 0.02$ & $0.56 \pm 0.03$ & $0.49 \pm 0.02$ \\
\hline Ratio to bw, mg/g & $1.70 \pm 0.06$ & $1.56 \pm 0.07$ & $1.64 \pm 0.09$ & $1.66 \pm 0.08$ & $1.58 \pm 0.10$ & $1.73 \pm 0.08$ & $1.82 \pm 0.10$ & $1.60 \pm 0.05$ \\
\hline \multicolumn{9}{|l|}{ Spleen } \\
\hline Absolute, $\mathrm{g}$ & $0.92 \pm 0.03$ & $0.96 \pm 0.03$ & $0.97 \pm 0.04$ & $0.96 \pm 0.03$ & $0.96 \pm 0.04$ & $0.87 \pm 0.03$ & $0.94 \pm 0.04$ & $1.01 \pm 0.05$ \\
\hline Ratio to Brain, g/g & $0.40 \pm 0.01$ & $0.42 \pm 0.01$ & $0.42 \pm 0.02$ & $0.43 \pm 0.01$ & $0.42 \pm 0.02$ & $0.39 \pm 0.01$ & $0.41 \pm 0.02$ & $0.44 \pm 0.02$ \\
\hline Ratio to bw, mg/g & $1.33 \pm 0.05$ & $1.33 \pm 0.04$ & $1.39 \pm 0.06$ & $1.36 \pm 0.04$ & $1.36 \pm 0.05$ & $1.26 \pm 0.04$ & $1.32 \pm 0.04$ & $1.44 \pm 0.07$ \\
\hline \multicolumn{9}{|l|}{ Testes } \\
\hline Absolute, $\mathrm{g}$ & $3.59 \pm 0.12$ & $3.44 \pm 0.13$ & $3.62 \pm 0.08$ & $3.38 \pm 0.14$ & $3.56 \pm 0.08$ & $3.51 \pm 0.12$ & $3.48 \pm 0.09$ & $3.56 \pm 0.09$ \\
\hline Ratio to Brain, g/g & $1.56 \pm 0.05$ & $1.50 \pm 0.06$ & $1.58 \pm 0.04$ & $1.50 \pm 0.06$ & $1.56 \pm 0.03$ & $1.57 \pm 0.05$ & $1.52 \pm 0.04$ & $1.54 \pm 0.04$ \\
\hline Ratio to bw, mg/g & $5.16 \pm 0.18$ & $4.82 \pm 0.21$ & $5.18 \pm 0.19$ & $4.77 \pm 0.21$ & $5.12 \pm 0.13$ & $5.14 \pm 0.22$ & $4.96 \pm 0.18$ & $5.09 \pm 0.13$ \\
\hline \multicolumn{9}{|l|}{ Thymus } \\
\hline Absolute, mg & $150 \pm 11$ & $138 \pm 9$ & $125 \pm 12$ & $158 \pm 10$ & $140 \pm 10$ & $150 \pm 11$ & $124 \pm 11$ & $154 \pm 10$ \\
\hline Ratio to Brain, g/g & $65 \pm 5$ & $60 \pm 4$ & $54 \pm 5$ & $71 \pm 4$ & $62 \pm 5$ & $67 \pm 5$ & $54 \pm 5$ & $67 \pm 4$ \\
\hline Ratio to bw, mg/g & $0.22 \pm 0.02$ & $0.20 \pm 0.02$ & $0.18 \pm 0.02$ & $0.22 \pm 0.01$ & $0.20 \pm 0.02$ & $0.22 \pm 0.02$ & $0.17 \pm 0.01$ & $0.22 \pm 0.01$ \\
\hline \multicolumn{9}{|l|}{ Thyroid } \\
\hline Absolute, mg & $43.5 \pm 2.0$ & $42.7 \pm 2.4$ & $44.0 \pm 2.5$ & $42.2 \pm 1.6$ & $40.9 \pm 1.9$ & $44.5 \pm 1.8$ & $43.6 \pm 2.0$ & $43.7 \pm 1.5$ \\
\hline Ratio to Brain,mg/g & $18.8 \pm 0.8$ & $18.5 \pm 1.0$ & $19.1 \pm 1.0$ & $18.8 \pm 0.7$ & $17.8 \pm 0.7$ & $19.9 \pm 0.8$ & $19.0 \pm 0.9$ & $18.9 \pm 0.6$ \\
\hline Ratio to bw, mg/g & $0.06 \pm 0$ & $0.06 \pm 0$ & $0.06 \pm 0$ & $0.06 \pm 0$ & $0.06 \pm 0$ & $0.06 \pm 0$ & $0.06 \pm 0$ & $0.06 \pm 0$ \\
\hline
\end{tabular}

${ }^{\mathrm{a}} \mathrm{BPA}$ and $\mathrm{EE}_{2}$ doses are $\mu \mathrm{g} / \mathrm{kg}$ bw/day. The indicated organs were collected from animals at the interim (one-year) necropsy and weights recorded. BPA and EE 2 groups were analyzed separately. Paired organs are presented and were analyzed as combined weights. ANOVA was performed for absolute organ weights to determine the effect of treatment on organ weight. Separate ANOCOVA were performed to determine the effect of treatment on organ weight adjusted for brain weight or receiving weight. Comparisons of dosed groups versus vehicle control were performed using Dunnett's method to adjust for multiple comparisons. Tests of trends, increasing treatment effect with increasing dose, were also performed for vehicle and BPA groups. Tests were conducted as two-sided at the 0.05 significance level. Statistically significant effects are indicated by asterisks (*,

$p<0.05)$. There were no additional statistically significant effects in the sensitivity analysis that excluded all animals that overlapped with animals treated with $250,000 \mu \mathrm{g}$ BPA/kg bw/day (see Statistical Methods). The complete statistical report is found in Supplemental Appendix XXX.

${ }^{b}$ Numbers in parentheses are the number of animals examined. Any deviations from this number due to exclusions are indicated by footnotes.

'For adrenal, $\mathrm{n}=21$ for the $0.05 \mathrm{EE}_{2}$ dose group.

${ }^{\mathrm{d}}$ For kidneys, $\mathrm{n}=17$ for vehicle, $25 \mathrm{BPA}$, and 2,500 BPA dose groups; $\mathrm{n}=21$ for $0.05 \mathrm{EE}_{2}$ dose group. 
CLARITY-BPA Core Study

Table 49. Male Organ Weights, Vehicle and BPA Stop-Dose, Interim Sacrifice (Mean \pm S.E.M.) ${ }^{\mathrm{a}}$

\begin{tabular}{|c|c|c|c|c|c|c|}
\hline Endpoint, Units & $\begin{array}{c}\text { Vehicle } \\
(20)^{b} \\
\end{array}$ & $\begin{array}{c}2.5 \text { BPA } \\
(20) \\
\end{array}$ & $\begin{array}{c}25 \text { BPA } \\
(19)\end{array}$ & $\begin{array}{c}250 \text { BPA } \\
(19) \\
\end{array}$ & $\begin{array}{c}2500 \text { BPA } \\
(20)\end{array}$ & $\begin{array}{c}25000 \text { BPA } \\
(22)\end{array}$ \\
\hline \multicolumn{7}{|c|}{ Body Weight at Necropsy } \\
\hline $\mathrm{g}$ & $735 \pm 26$ & $787 \pm 23$ & $760 \pm 22$ & $733 \pm 21$ & $738 \pm 21$ & $743 \pm 17$ \\
\hline \multicolumn{7}{|l|}{ Adrenal } \\
\hline Absolute, mg & $63.4 \pm 2.0$ & $65.7 \pm 2.1$ & $67.9 \pm 3.3$ & $68.9 \pm 2.7$ & $65.4 \pm 3.5$ & $68.3 \pm 3.3$ \\
\hline Ratio to Brain, $\mathrm{mg} / \mathrm{g}$ & $27.3 \pm 0.8$ & $28.2 \pm 0.9$ & $29.3 \pm 1.5$ & $30.0 \pm 1.3$ & $28.7 \pm 1.4$ & $29.6 \pm 1.3$ \\
\hline Ratio to bw, mg/g & $0.09 \pm 0$ & $0.08 \pm 0$ & $0.09 \pm 0$ & $0.10 \pm 0$ & $0.09 \pm 0$ & $0.09 \pm 0$ \\
\hline \multicolumn{7}{|l|}{ Brain } \\
\hline Absolute, $\mathrm{g}$ & $2.32 \pm 0.02$ & $2.33 \pm 0.02$ & $2.32 \pm 0.03$ & $2.30 \pm 0.03$ & $2.28 \pm 0.02$ & $2.30 \pm 0.03$ \\
\hline Ratio to bw, mg/g & $3.24 \pm 0.12$ & $3.00 \pm 0.08$ & $3.10 \pm 0.09$ & $3.17 \pm 0.07$ & $3.13 \pm 0.09$ & $3.13 \pm 0.08$ \\
\hline \multicolumn{7}{|l|}{ Epididymides } \\
\hline Absolute, $\mathrm{g}$ & $1.31 \pm 0.03$ & $1.28 \pm 0.03$ & $1.30 \pm 0.03$ & $1.32 \pm 0.03$ & $1.26 \pm 0.04$ & $1.37 \pm 0.02$ \\
\hline Ratio to Brain, g/g & $0.56 \pm 0.01$ & $0.55 \pm 0.02$ & $0.56 \pm 0.01$ & $0.57 \pm 0.02$ & $0.56 \pm 0.02$ & $0.60 \pm 0.01$ \\
\hline Ratio to bw, mg/g & $1.83 \pm 0.08$ & $1.65 \pm 0.06$ & $1.73 \pm 0.05$ & $1.83 \pm 0.07$ & $1.73 \pm 0.07$ & $1.87 \pm 0.05$ \\
\hline \multicolumn{7}{|l|}{ Epididymal Fat Pad } \\
\hline Absolute, g & $13.9 \pm 0.7$ & $16.0 \pm 0.9$ & $14.9 \pm 1.0$ & $13.5 \pm 0.6$ & $14.8 \pm 1.0^{\mathrm{c}}$ & $14.6 \pm 0.7$ \\
\hline Ratio to Brain, g/g & $5.99 \pm 0.31$ & $6.85 \pm 0.38$ & $6.38 \pm 0.44$ & $5.82 \pm 0.26$ & $6.52 \pm 0.41$ & $6.38 \pm 0.33$ \\
\hline Ratio to bw, mg/g & $18.8 \pm 0.5$ & $20.3 \pm 0.9$ & $19.2 \pm 0.9$ & $18.3 \pm 0.7$ & $19.8 \pm 0.9$ & $19.6 \pm 0.7$ \\
\hline \multicolumn{7}{|l|}{ Retroperitoneal Fat Pad } \\
\hline Absolute, $\mathrm{g}$ & $25.0 \pm 2.3$ & $27.8 \pm 2.0$ & $25.6 \pm 2.3$ & $23.0 \pm 1.7$ & $23.4 \pm 2.7$ & $25.0 \pm 2.1$ \\
\hline Ratio to Brain, g/g & $10.8 \pm 1.0$ & $11.9 \pm 0.8$ & $11.0 \pm 1.0$ & $10.0 \pm 0.7$ & $10.3 \pm 1.2$ & $10.9 \pm 0.9$ \\
\hline Ratio to bw, mg/g & $33.1 \pm 2.1$ & $34.7 \pm 1.6$ & $33.0 \pm 2.3$ & $31.3 \pm 2.0$ & $31.0 \pm 2.9$ & $33.0 \pm 2.2$ \\
\hline \multicolumn{7}{|l|}{ Heart } \\
\hline Absolute, g & $2.27 \pm 0.07$ & $2.45 \pm 0.09$ & $2.40 \pm 0.08$ & $2.25 \pm 0.08$ & $2.22 \pm 0.07$ & $2.35 \pm 0.10$ \\
\hline Ratio to Brain, g/g & $0.98 \pm 0.03$ & $1.05 \pm 0.04$ & $1.03 \pm 0.04$ & $0.97 \pm 0.03$ & $0.98 \pm 0.03$ & $1.02 \pm 0.05$ \\
\hline Ratio to bw, mg/g & $3.12 \pm 0.08$ & $3.13 \pm 0.09$ & $3.18 \pm 0.11$ & $3.09 \pm 0.10$ & $3.04 \pm 0.10$ & $3.20 \pm 0.16$ \\
\hline \multicolumn{7}{|l|}{ Kidney } \\
\hline Absolute, $\mathrm{g}$ & $4.38 \pm 0.15$ & $4.43 \pm 0.15$ & $4.54 \pm 0.11$ & $4.32 \pm 0.11$ & $4.17 \pm 0.15$ & $4.33 \pm 0.10^{\mathrm{d}}$ \\
\hline Ratio to Brain, g/g & $1.89 \pm 0.07$ & $1.90 \pm 0.06$ & $1.95 \pm 0.05$ & $1.88 \pm 0.05$ & $1.83 \pm 0.06$ & $1.89 \pm 0.04$ \\
\hline Ratio to bw, mg/g & $5.98 \pm 0.09$ & $5.62 \pm 0.09$ & $6.01 \pm 0.13$ & $5.94 \pm 0.15$ & $5.64 \pm 0.08$ & $5.78 \pm 0.09$ \\
\hline \multicolumn{7}{|l|}{ Liver } \\
\hline Absolute, $g$ & $23.6 \pm 0.9$ & $24.4 \pm 0.7$ & $25.1 \pm 1.0$ & $23.2 \pm 0.8$ & $24.8 \pm 1.0$ & $25.1 \pm 0.9$ \\
\hline Ratio to Brain, g/g & $10.2 \pm 0.4$ & $10.5 \pm 0.3$ & $10.8 \pm 0.4$ & $10.1 \pm 0.3$ & $10.9 \pm 0.4$ & $10.9 \pm 0.4$ \\
\hline Ratio to bw, mg/g & $32.1 \pm 0.4^{*}$ & $31.1 \pm 0.5$ & $33.1 \pm 1.0$ & $31.8 \pm 0.8$ & $33.6 \pm 0.7$ & $33.9 \pm 1.2$ \\
\hline \multicolumn{7}{|l|}{ Pituitary } \\
\hline Absolute, mg & $15.1 \pm 0.6$ & $16.5 \pm 0.9$ & $15.3 \pm 0.3$ & $15.6 \pm 0.6$ & $14.5 \pm 0.4$ & $15.1 \pm 0.5$ \\
\hline Ratio to Brain, $\mathrm{mg} / \mathrm{g}$ & $6.5 \pm 0.2$ & $7.1 \pm 0.4$ & $6.6 \pm 0.1$ & $6.8 \pm 0.2$ & $6.4 \pm 0.2$ & $6.6 \pm 0.2$ \\
\hline Ratio to bw, mg/g & $0.02 \pm 0$ & $0.02 \pm 0$ & $0.02 \pm 0$ & $0.02 \pm 0$ & $0.02 \pm 0$ & $0.02 \pm 0$ \\
\hline
\end{tabular}


CLARITY-BPA Core Study

\begin{tabular}{|c|c|c|c|c|c|c|}
\hline Endpoint, Units & $\begin{array}{c}\text { Vehicle } \\
(20)^{b}\end{array}$ & $\begin{array}{c}2.5 \text { BPA } \\
(20)\end{array}$ & $\begin{array}{c}25 \text { BPA } \\
(19)\end{array}$ & $\begin{array}{c}250 \text { BPA } \\
(19)\end{array}$ & $\begin{array}{c}2500 \text { BPA } \\
(20)\end{array}$ & $\begin{array}{c}25000 \text { BPA } \\
(22)\end{array}$ \\
\hline \multicolumn{7}{|l|}{ Seminal Vesicles } \\
\hline Absolute, g & $1.22 \pm 0.05$ & $1.23 \pm 0.06$ & $1.27 \pm 0.09$ & $1.12 \pm 0.05$ & $1.12 \pm 0.07$ & $1.17 \pm 0.04$ \\
\hline Ratio to Brain, g/g & $0.53 \pm 0.02$ & $0.53 \pm 0.02$ & $0.54 \pm 0.04$ & $0.48 \pm 0.02$ & $0.49 \pm 0.03$ & $0.51 \pm 0.02$ \\
\hline Ratio to bw, mg/g & $1.69 \pm 0.08$ & $1.58 \pm 0.07$ & $1.70 \pm 0.13$ & $1.54 \pm 0.07$ & $1.52 \pm 0.09$ & $1.59 \pm 0.07$ \\
\hline \multicolumn{7}{|l|}{ Spleen } \\
\hline Absolute, $\mathrm{g}$ & $1.00 \pm 0.03$ & $1.04 \pm 0.04$ & $1.02 \pm 0.03$ & $1.03 \pm 0.05$ & $1.07 \pm 0.06$ & $1.06 \pm 0.06$ \\
\hline Ratio to Brain, g/g & $0.43 \pm 0.01$ & $0.45 \pm 0.02$ & $0.44 \pm 0.01$ & $0.45 \pm 0.02$ & $0.47 \pm 0.03$ & $0.46 \pm 0.02$ \\
\hline Ratio to bw, mg/g & $1.38 \pm 0.05$ & $1.33 \pm 0.06$ & $1.34 \pm 0.04$ & $1.42 \pm 0.07$ & $1.46 \pm 0.08$ & $1.44 \pm 0.10$ \\
\hline \multicolumn{7}{|l|}{ Testes } \\
\hline Absolute, $g$ & $3.64 \pm 0.07$ & $3.59 \pm 0.08$ & $3.63 \pm 0.09$ & $3.49 \pm 0.09$ & $3.55 \pm 0.15$ & $3.71 \pm 0.06$ \\
\hline Ratio to Brain, g/g & $1.57 \pm 0.03$ & $1.54 \pm 0.04$ & $1.57 \pm 0.05$ & $1.52 \pm 0.04$ & $1.56 \pm 0.07$ & $1.62 \pm 0.03$ \\
\hline Ratio to bw, mg/g & $5.05 \pm 0.17$ & $4.62 \pm 0.13$ & $4.82 \pm 0.13$ & $4.82 \pm 0.18$ & $4.84 \pm 0.18$ & $5.04 \pm 0.14$ \\
\hline \multicolumn{7}{|l|}{ Thymus } \\
\hline Absolute, mg & $137 \pm 9$ & $123 \pm 5$ & $154 \pm 10$ & $130 \pm 8$ & $134 \pm 9$ & $145 \pm 8$ \\
\hline Ratio to Brain, mg/g & $59 \pm 4$ & $53 \pm 2$ & $66 \pm 4$ & $57 \pm 3$ & $59 \pm 4$ & $63 \pm 4$ \\
\hline Ratio to bw, mg/g & $0.19 \pm 0.01$ & $0.16 \pm 0.01$ & $0.20 \pm 0.01$ & $0.18 \pm 0.01$ & $0.18 \pm 0.01$ & $0.20 \pm 0.1$ \\
\hline \multicolumn{7}{|l|}{ Thyroid } \\
\hline Absolute, mg & $43.1 \pm 1.9$ & $43.4 \pm 2.5$ & $44.9 \pm 1.8$ & $41.2 \pm 1.9$ & $42.2 \pm 2.4$ & $45.4 \pm 2.7$ \\
\hline Ratio to Brain, mg/g & $18.6 \pm 0.8$ & $18.6 \pm 1.1$ & $19.4 \pm 0.8$ & $18.0 \pm 0.9$ & $18.6 \pm 1.0$ & $19.7 \pm 1.1$ \\
\hline Ratio to bw, mg/g & $0.06 \pm 0$ & $0.06 \pm 0$ & $0.06 \pm 0$ & $0.06 \pm 0$ & $0.06 \pm 0$ & $0.06 \pm 0$ \\
\hline
\end{tabular}

${ }^{a}$ BPA doses are $\mu \mathrm{g} / \mathrm{kg}$ bw/day. The indicated organs were collected from animals at the interim (one-year) necropsy and weights recorded. Paired organs are presented and were analyzed as combined weights. ANOVA was performed for absolute organ weights to determine the effect of treatment on organ weight. Separate ANOCOVA were performed to determine the effect of treatment on organ weight adjusted for brain weight or receiving weight. Comparisons of dosed groups versus vehicle control were performed using Dunnett's method to adjust for multiple comparisons. Tests of trends, increasing treatment effect with increasing dose, were also performed for vehicle and BPA groups. Tests were conducted as two-sided at the 0.05 significance level. Statistically significant effects are indicated by asterisks $(*, p<0.05)$; asterisks in the vehicle column indicate a significant trend. There were no additional statistically significant effects in the sensitivity analysis that excluded all animals that overlapped with animals treated with $250,000 \mu \mathrm{g}$ BPA/kg bw/day (see Statistical Methods). The complete statistical report is found in Supplemental Appendix XXX.

${ }^{b}$ Numbers in parentheses are the number of animals examined. Any deviations from this number due to exclusions are indicated by footnotes.

For epididymal fat pad, $\mathrm{n}=19$ for 2,500 BPA dose group.

${ }^{\mathrm{d}}$ For kidney, $\mathrm{n}=20$ for 25,000 BPA dose group. 
CLARITY-BPA Core Study

Table 50. Sperm Analysis, Vehicle, BPA, and $\mathrm{EE}_{2}$ Continuous-Dose, Interim Sacrifice (Mean \pm S.E.M.) ${ }^{\mathrm{a}}$

\begin{tabular}{|c|c|c|c|c|c|c|c|c|}
\hline Endpoint, Units & $\begin{array}{c}\text { Vehicle } \\
(\mathbf{1 8})^{\mathrm{b}}\end{array}$ & $\begin{array}{c}2.5 \text { BPA } \\
(22)\end{array}$ & $\begin{array}{c}25 \text { BPA } \\
(18)\end{array}$ & $\begin{array}{l}250 \text { BPA } \\
(24)\end{array}$ & $\begin{array}{l}2500 \text { BPA } \\
(18)\end{array}$ & $\begin{array}{c}25000 \text { BPA } \\
(21)\end{array}$ & $\begin{array}{c}\mathbf{0 . 0 5} \mathbf{E E}_{2} \\
(22)\end{array}$ & $\begin{array}{c}0.5 \mathrm{EE}_{2} \\
(23)\end{array}$ \\
\hline $\begin{array}{l}\text { Testicular spermatid } \\
\text { heads, } 10^{6} / \mathrm{g}\end{array}$ & $83.4 \pm 9.5$ & $76.8 \pm 7.7$ & $88.5 \pm 6.1$ & $81.5 \pm 6.8$ & $85.1 \pm 7.1$ & $76.6 \pm 6.0$ & $70.0 \pm 4.7$ & $73.7 \pm 4.5$ \\
\hline $\begin{array}{l}\text { Cauda sperm counts, } \\
10^{6} / \mathrm{g}\end{array}$ & $991 \pm 78$ & $999 \pm 88$ & $1076 \pm 68$ & $998 \pm 69$ & $1062 \pm 51$ & $1027 \pm 81$ & $892 \pm 72$ & $857 \pm 53$ \\
\hline Cauda sperm, \% Motility & $65.9 \pm 4.6$ & $64.0 \pm 5.1$ & $72.4 \pm 3.1$ & $66.7 \pm 4.5$ & $69.9 \pm 3.4$ & $69.4 \pm 4.2$ & $67.2 \pm 4.8$ & $70.8 \pm 2.7$ \\
\hline $\begin{array}{l}\text { Cauda sperm, head, } \\
\text { abnormal counts per } \\
\text { animal }\end{array}$ & $0.00 \pm 0$ & $0.05 \pm 0.05$ & $0.06 \pm 0.06$ & $0.08 \pm 0.06$ & $0.11 \pm 0.08$ & $0.00 \pm 0$ & $0.23 \pm 0.10$ & $0.04 \pm 0.04$ \\
\hline $\begin{array}{l}\text { Cauda sperm, tail, } \\
\text { abnormal counts per } \\
\text { animal }\end{array}$ & $0.17 \pm 0.10$ & $0.36 \pm 0.13$ & $0.17 \pm 0.10$ & $0.08 \pm 0.06$ & $0.11 \pm 0.08$ & $0.14 \pm 0.08$ & $0.23 \pm 0.10$ & $0.13 \pm 0.08$ \\
\hline $\begin{array}{l}\text { Cauda sperm, head and } \\
\text { tail combined, abnormal } \\
\text { counts per animal }\end{array}$ & $0.17 \pm 0.10$ & $0.41 \pm 0.14$ & $0.22 \pm 0.11$ & $0.17 \pm 0.08$ & $0.22 \pm 0.11$ & $0.14 \pm 0.08$ & $0.45 \pm 0.14$ & $0.17 \pm 0.09$ \\
\hline $\begin{array}{l}\text { }{ }^{\mathrm{BPA}} \text { and } \mathrm{EE}_{2} \text { doses are } \mu \mathrm{g} / \mathrm{k} \\
\text { counts, and percent sperm mo } \\
\text { distribution and a log link fun } \\
\text { treatment effect with increasi } \\
\text { significant treatment effects. } \\
250,000 \mu \mathrm{g} \mathrm{BPA} / \mathrm{kg} \text { bw/day }\end{array}$ & $\begin{array}{l}\text { bw/day. Values } \\
\text { lity were analyz } \\
\text { ion. Pairwise c } \\
\text { dose, were als } \\
\text { ere were also } n \\
\text { e Statistical Me }\end{array}$ & $\begin{array}{l}\text { usented are mea } \\
\text { using an ANO } \\
\text { parisons to the } \\
\text { erformed for ve } \\
\text { ignificant treatr } \\
\text { ds). The full st } \\
\text { examined }\end{array}$ & $\begin{array}{l} \pm \text { S.E.M. BPA } \\
\text { model. Analys } \\
\text { icle control gro } \\
\text { le and BPA gro } \\
\text { tt effects in the } \\
\text { tical report is f }\end{array}$ & $\begin{array}{l}\mathrm{EE}_{2} \text { groups we } \\
\text { f sperm morph } \\
\text { were adjusted } \mathrm{f} \\
\text { only. All tests } \\
\text { sitivity analysi } \\
\text { d in Supplemen }\end{array}$ & $\begin{array}{l}\text { analyzed separ } \\
\text { gy data was per } \\
\text { multiple compa } \\
\text { re conducted a } \\
\text { at excluded all } \\
\text { Appendix XX }\end{array}$ & $\begin{array}{l}\text { y. Testicular sp } \\
\text { med using a ge } \\
\text { ns using Dunn } \\
\text { o-sided at the } \\
\text { mals that overla }\end{array}$ & $\begin{array}{l}\text { natid head coun } \\
\text { alized linear m } \\
\text { s method. Test } \\
\text { significance l} \\
\text { ed with animal }\end{array}$ & $\begin{array}{l}\text { auda sperm } \\
\text { with a Poisson } \\
\text { trends, increasing } \\
\text { There were no } \\
\text { ated with }\end{array}$ \\
\hline
\end{tabular}


CLARITY-BPA Core Study

Table 51. Sperm Analysis, Vehicle and BPA Stop-Dose, Interim Sacrifice (Mean \pm S.E.M.) ${ }^{\mathrm{a}}$

\begin{tabular}{|c|c|c|c|c|c|c|}
\hline Endpoint, Units & $\begin{array}{c}\text { Vehicle } \\
(20)^{b}\end{array}$ & $\begin{array}{l}2.5 \text { BPA } \\
(20)\end{array}$ & $\begin{array}{c}25 \text { BPA } \\
(19) \\
\end{array}$ & $\begin{array}{c}250 \text { BPA } \\
(19)\end{array}$ & $\begin{array}{c}2500 \text { BPA } \\
(20) \\
\end{array}$ & $\begin{array}{c}25000 \text { BPA } \\
(22) \\
\end{array}$ \\
\hline Testicular spermatid heads, $10^{6} / \mathrm{g}$ & $76.5 \pm 9.4$ & $77.7 \pm 4.8$ & $72.2 \pm 5.3$ & $72.5 \pm 6.3$ & $73.6 \pm 9.0$ & $75.8 \pm 4.7$ \\
\hline Cauda sperm counts, $10^{6} / \mathrm{g}$ & $1,059 \pm 87$ & $1,186 \pm 83$ & $1,017 \pm 66$ & $1,111 \pm 66$ & $1,020 \pm 89$ & $1,016 \pm 81$ \\
\hline Cauda sperm \% Motility & $74.8 \pm 4.3$ & $75.8 \pm 2.3$ & $72.4 \pm 3.4$ & $70.2 \pm 4.2$ & $67.9 \pm 5.6$ & $77.7 \pm 2.2$ \\
\hline $\begin{array}{l}\text { Cauda sperm, head, abnormal counts } \\
\text { per animal }\end{array}$ & $0.00 \pm 0$ & $0.00 \pm 0$ & $0.11 \pm 0.07$ & $0.05 \pm 0.05$ & $0.10 \pm 0.07$ & $0.00 \pm 0$ \\
\hline $\begin{array}{l}\text { Cauda sperm, tail, abnormal counts } \\
\text { per animal }\end{array}$ & $0.15 \pm 0.09$ & $0.15 \pm 0.09$ & $0.21 \pm 0.11$ & $0.11 \pm 0.07$ & $0.20 \pm 0.10$ & $0.00 \pm 0$ \\
\hline $\begin{array}{l}\text { Cauda sperm, head and tail combined, } \\
\text { abnormal counts per animal }\end{array}$ & $0.15 \pm 0.09$ & $0.015 \pm 0.09$ & $0.32 \pm 0.13$ & $0.16 \pm 0.09$ & $0.30 \pm 0.12$ & $0.00 \pm 0$ \\
\hline
\end{tabular}

${ }^{\mathrm{a} B P A}$ doses are $\mu \mathrm{g} / \mathrm{kg}$ bw/day. Values presented are means \pm S.E.M. Testicular spermatid head counts, cauda sperm counts, and percent sperm motility were analyzed using an ANOVA model. Analysis of sperm morphology data was performed using a generalized linear model with a Poisson distribution and a log link function. Pairwise comparisons to the vehicle control group were adjusted for multiple comparisons using Dunnett's method. Tests of trends, increasing treatment effect with increasing dose, were also performed. All tests were conducted as two-sided at the 0.05 significance level. There were no significant treatment effects. Also, there were no additional statistically significant effects in the sensitivity analysis that excluded all animals that overlapped with animals treated with $250,000 \mu \mathrm{g} \mathrm{BPA} / \mathrm{kg}$ bw/day (see Statistical Methods). The full statistical report is found in Supplemental Appendix XXXI.

bumbers in parentheses are numbers of animals examined. 
CLARITY-BPA Core Study

Table 52. Neoplastic Lesions in the Mammary Gland of Interim and Terminal Sacrifice Female Rats: Continuous-Dose BPA ${ }^{\text {a, } b}$

\begin{tabular}{|c|c|c|c|c|c|c|c|c|}
\hline Lesion & $\begin{array}{c}\text { Study Phase } \\
\text { (Interim/Terminal) }\end{array}$ & Statistic & Vehicle & 2.5 BPA & 25 BPA & 250 BPA & 2500 BPA & 25000 BPA \\
\hline \multirow[t]{7}{*}{ Adenocarcinoma } & Interim $^{\mathrm{c}}$ & Incidence & $0 / 23$ & $1 / 22(4 \%)$ & $1 / 22(4 \%)$ & $0 / 24$ & $0 / 20$ & $0 / 24$ \\
\hline & Terminal & Incidence & $4 / 50(8 \%)$ & $6 / 48(12 \%)$ & $6 / 46(13 \%)$ & $5 / 49(10 \%)$ & $9 / 50(18 \%)$ & $3 / 46(6 \%)$ \\
\hline & & Poly-3 Incidence & $4 / 34.4(12 \%)$ & $6 / 32.9(18 \%)$ & $6 / 30.4(20 \%)$ & $5 / 34.6(14 \%)$ & $9 / 31.3(29 \%)$ & $3 / 31.2(10 \%)$ \\
\hline & & Terminal Incidence & $3 / 16(19 \%)$ & $6 / 19(32 \%)$ & $1 / 14(7 \%)$ & $0 / 13(0 \%)$ & $3 / 10(30 \%)$ & $0 / 8(0 \%)$ \\
\hline & & Time-to-First & 673 & $719(\mathrm{~T})$ & 434 & 377 & 561 & 685 \\
\hline & & Poly-3 $p$-value & 0.412 & 0.336 & 0.286 & 0.504 & 0.071 & $0.555 \mathrm{~N}$ \\
\hline & & Multiple Incidence $^{\mathrm{d}}$ & $2 / 50(4 \%)$ & $3 / 48(6 \%)$ & $3 / 46(7 \%)$ & $2 / 49(4 \%)$ & $3 / 50(6 \%)$ & $1 / 46(2 \%)$ \\
\hline \multirow{7}{*}{$\begin{array}{l}\text { Adenoma or } \\
\text { Adenocarcinoma }\end{array}$} & Interim $^{\mathrm{c}}$ & Incidence & $0 / 23$ & $1 / 22(4 \%)$ & $1 / 22(4 \%)$ & $0 / 24$ & $0 / 20$ & $0 / 24$ \\
\hline & Terminal & Incidence & $6 / 50(12 \%)$ & $7 / 48(15 \%)$ & $8 / 46(17 \%)$ & $6 / 49(12 \%)$ & $10 / 50(20 \%)$ & $4 / 46(9 \%)$ \\
\hline & & Poly-3 Incidence & $6 / 34.9(17 \%)$ & $7 / 32.9(21 \%)$ & $8 / 30.4(26 \%)$ & $6 / 34.8(17 \%)$ & $\begin{array}{c}10 / 31.3 \\
(32 \%)\end{array}$ & $4 / 31.8(13 \%)$ \\
\hline & & Terminal Incidence & $4 / 16(25 \%)$ & $7 / 19(37 \%)$ & $3 / 14(21 \%)$ & $0 / 13(0 \%)$ & $4 / 10(40 \%)$ & $0 / 8(0 \%)$ \\
\hline & & Time-to-First & 564 & $719(\mathrm{~T})$ & 434 & 377 & 561 & 542 \\
\hline & & Poly-3 $p$-value & $0.513 \mathrm{~N}$ & 0.452 & 0.271 & 0.624 & 0.126 & $0.428 \mathrm{~N}$ \\
\hline & & Multiple indicence $^{\mathrm{d}}$ & $2 / 50(4 \%)$ & $3 / 48(6 \%)$ & $3 / 46(7 \%)$ & $3 / 49(6 \%)$ & $3 / 50(6 \%)$ & $1 / 46(2 \%)$ \\
\hline \multirow[t]{11}{*}{ Fibroadenoma } & Interim & Incidence & $2 / 23(9 \%)$ & $3 / 22(13 \%)$ & $3 / 22(13 \%)$ & $1 / 24(4 \%)$ & $2 / 20(10 \%)$ & $6 / 24(25 \%)$ \\
\hline & & Terminal Incidence & $2 / 21(10 \%)$ & $3 / 22(14 \%)$ & $2 / 21(10 \%)$ & $0 / 22(0 \%)$ & $2 / 20(10 \%)$ & $6 / 24(25 \%)$ \\
\hline & & Time-to-First & $361(\mathrm{~T})$ & $356(\mathrm{~T})$ & 311 & 256 & $362(\mathrm{~T})$ & $362(\mathrm{~T})$ \\
\hline & & CAFE $p$-value & 0.150 & 0.478 & 0.478 & $0.484 \mathrm{~N}$ & 0.641 & 0.136 \\
\hline & & Multiple Incidence ${ }^{\mathrm{d}}$ & $0 / 23(0 \%)$ & $0 / 22(0 \%)$ & $1 / 22(5 \%)$ & $0 / 24(0 \%)$ & $0 / 20(0 \%)$ & $1 / 24(4 \%)$ \\
\hline & Terminal & Incidence & $41 / 50(82 \%)$ & $40 / 48(83 \%)$ & $33 / 46(72 \%)$ & $39 / 49(80 \%)$ & $35 / 50(70 \%)$ & $38 / 46(83 \%)$ \\
\hline & & Poly-3 Incidence & $\begin{array}{c}41 / 47.0 \\
(87 \%)\end{array}$ & $\begin{array}{c}40 / 43.7 \\
(92 \%)\end{array}$ & $\begin{array}{c}33 / 38.4 \\
(86 \%)\end{array}$ & $\begin{array}{c}39 / 45.3 \\
(86 \%)\end{array}$ & $\begin{array}{c}35 / 42.3 \\
(83 \%)\end{array}$ & $\begin{array}{c}38 / 42.2 \\
(90 \%)\end{array}$ \\
\hline & & Terminal Incidence & $13 / 16(81 \%)$ & $17 / 19(90 \%)$ & $12 / 14(86 \%)$ & $10 / 13(77 \%)$ & $7 / 10(70 \%)$ & $7 / 8(88 \%)$ \\
\hline & & Time-to-First & 431 & 321 & 434 & 261 & 419 & 467 \\
\hline & & Poly-3 $p$-value & $0.410 \mathrm{~N}$ & 0.366 & $0.567 \mathrm{~N}$ & $0.565 \mathrm{~N}$ & $0.366 \mathrm{~N}$ & 0.457 \\
\hline & & Multiple Incidence $^{\mathrm{d}}$ & $21 / 50(42 \%)$ & $32 / 48(67 \%)$ & $20 / 46(43 \%)$ & $32 / 49(65 \%)$ & $21 / 50(42 \%)$ & $31 / 46(67 \%)$ \\
\hline
\end{tabular}

${ }^{a}$ BPA doses are $\mu \mathrm{g} / \mathrm{kg}$ bw/day. Statistical analyses were conducted for any lesion that was diagnosed in two animals in any dose group in the interim sacrifice groups or in the EE 2 terminal sacrifice groups or four animals in the control and BPA groups in the terminal sacrifice. A complete tabulation of all neoplastic lesions is found in Supplemental Appendix XXXII and complete results of the statistical analyses are found in Supplemental Appendices XXXIII (interim sacrifice) and XXXIV (terminal sacrifice). Details of the statistical methods are found in Materials and Methods and in Supplemental Appendices XXXIII (interim sacrifice) and XXXIV (terminal sacrifice). Data are presented as follows:

Incidence, lesions observed/number of animals examined microscopically with percent animals affected in parentheses; Poly-3 Incidence, Poly-3 adjusted neoplasm incidence after adjustment for intercurrent mortality in terminal sacrifice animals; Terminal Incidence, lesions observed/number of animals reaching terminal sacrifice that were microscopically examined. Time-to-First, age of animal in which lesion was first observed, $\mathrm{T}$ in parentheses indicates that the first observation occurred at terminal sacrifice. For interim sacrifice data: CAFE $p$-value, $p$-value for Cochran-Armitage trend test in vehicle column, Fisher's exact test versus vehicle control in dose columns. For terminal sacrifice data, the Poly-3 $p$-value for the trend test is given in the vehicle column and values for pairwise comparisons to vehicle control are shown in dose columns. Significant trends are shown in the 


\section{CLARITY-BPA Core Study}

vehicle column, while significant pairwise comparisons to the vehicle control are shown in BPA and EE 2 dose group columns. All $p$-values are one-sided and not corrected for multiple comparisons. " $\mathrm{N}$ " next to a $p$-value indicates a result that is a negative trend or negative relative to control. Significant effects are indicated with asterisks. $*, p<0.05 ; * *$ $p<0.01 ; * *, p<0.001$.

${ }^{b}$ There were no statistically significant effects in the female mammary gland in the continuous BPA dose groups in the interim or terminal sacrifice animals.

cStatistical analysis was not conducted since no group had 2 or more lesions diagnosed. There were no multiple adenocarcinomas in interim sacrifice animals

dProportion of animals examined that had multiple neoplasms. These animals with multiple neoplasms are included in the incidence row and were not analyzed separately. The numbers of mammary neoplasms found in each female are tabulated in Supplemental Appendix XXXII, Subappendix VII. 
CLARITY-BPA Core Study

Table 53. Neoplastic Lesions in the Mammary Gland of Interim and Terminal Sacrifice Female Rats: Continuous-Dose EE ${ }_{2}{ }^{a}$

\begin{tabular}{|c|c|c|c|c|c|}
\hline Lesion & $\begin{array}{c}\text { Study Phase } \\
\text { (Interim/Terminal) }\end{array}$ & Statistic & Vehicle $^{\text {b }}$ & $0.05 \mathbf{E E}_{2}$ & $0.5 \mathrm{EE}_{2}$ \\
\hline \multirow[t]{10}{*}{ Adenocarcinoma $^{c}$} & \multirow[t]{4}{*}{ Interim } & Incidence $^{\mathrm{d}}$ & $0 / 23(0 \%)$ & $2 / 26(8 \%)$ & $0 / 26(0 \%)$ \\
\hline & & Terminal Incidence & $0 / 21(0 \%)$ & $1 / 24(4 \%)$ & $0 / 26(0 \%)$ \\
\hline & & Time-to-First & - & 257 & - \\
\hline & & CAFE $p$-value & $0.639 \mathrm{~N}$ & 0.276 & - \\
\hline & \multirow[t]{6}{*}{ Terminal } & Incidence & $4 / 50(8 \%)$ & $2 / 26(8 \%)$ & $10 / 26(38 \%)$ \\
\hline & & Poly-3 Incidence & $4 / 34.4(12 \%)$ & $2 / 16.8(12 \%)$ & $10 / 17.6(57 \%)$ \\
\hline & & Terminal Incidence & $3 / 16(19 \%)$ & $0 / 7(0 \%)$ & $3 / 4(75 \%)$ \\
\hline & & Time-to-First & 673 & 490 & 488 \\
\hline & & Poly-3 $p$-value & $<0.001^{* * *}$ & 0.667 & $<0.001^{* * *}$ \\
\hline & & Multiple Incidence ${ }^{\mathrm{e}}$ & $2 / 50(4 \%)$ & $0 / 26$ & $4 / 26(15 \%)$ \\
\hline \multirow[t]{10}{*}{ Fibroadenoma } & \multirow[t]{4}{*}{ Interim } & Incidence $^{\mathrm{d}}$ & $2 / 23(9 \%)$ & $2 / 26(8 \%)$ & $4 / 26(15 \%)$ \\
\hline & & Terminal Incidence & $2 / 21(10 \%)$ & $2 / 24(8 \%)$ & $4 / 26(15 \%)$ \\
\hline & & Time-to-First & $361(\mathrm{~T})$ & $361(\mathrm{~T})$ & $360(\mathrm{~T})$ \\
\hline & & CAFE $p$-value & 0.297 & $0.647 \mathrm{~N}$ & 0.395 \\
\hline & \multirow[t]{6}{*}{ Terminal } & Incidence & $41 / 50(82 \%)$ & $18 / 26(69 \%)$ & $14 / 26(54 \%)$ \\
\hline & & Poly-3 Incidence & $41 / 47.0(87 \%)$ & $18 / 22.3(81 \%)$ & $14 / 20.2(69 \%)$ \\
\hline & & Terminal Incidence & $13 / 16(81 \%)$ & $5 / 7(71 \%)$ & $2 / 4(50 \%)$ \\
\hline & & Time-to-First & 431 & 460 & 360 \\
\hline & & Poly-3 $p$-value & $0.041 \mathrm{~N}^{*}$ & $0.354 \mathrm{~N}$ & $0.056 \mathrm{~N}$ \\
\hline & & Multiple Incidence $\mathrm{e}^{\mathrm{e}}$ & $21 / 50(42 \%)$ & $11 / 26(42 \%)$ & $4 / 26(15 \%)$ \\
\hline
\end{tabular}

${ }^{\mathrm{a}} \mathrm{EE}_{2}$ doses are $\mu \mathrm{g} / \mathrm{kg}$ bw/day. See legend to Table 52 for description of data presentation. Adenocarcinoma incidence was significantly increased in the $0.5 \mu \mathrm{g}$ EE $2 / \mathrm{kg}$ bw/day dose group relative to vehicle control and there was a significant dose trend.

${ }^{\text {b}}$ The continuous vehicle control group data are the same as those shown for the continuous-dose BPA data in Table 52.

${ }^{\mathrm{C} N o}$ mammary gland adenomas were diagnosed in interim or terminal sacrifice $\mathrm{EE}_{2}$ females, so no tabulation of adenoma or adenocarcinoma is included.

There were no animals with multiple mammary gland neoplasms in the interim sacrifice females.

eProportion of animals examined that had multiple neoplasms. These animals with multiple neoplasms are included in the incidence row and were not analyzed separately. The numbers of mammary neoplasms found in each female are tabulated in Supplemental Appendix XXXII, Subappendix VII. 
CLARITY-BPA Core Study

Table 54. Neoplastic Lesions in the Mammary Gland of Interim and Terminal Sacrifice Female Rats: Stop-Dose BPA ${ }^{\text {a }}$

\begin{tabular}{|c|c|c|c|c|c|c|c|c|}
\hline Lesion & $\begin{array}{c}\text { Study Phase } \\
\text { (Interim/Terminal) }\end{array}$ & Statistic & Vehicle & 2.5 BPA & 25 BPA & 250 BPA & 2500 BPA & 25000 BPA \\
\hline \multirow[t]{6}{*}{ Adenocarcinoma $^{\mathrm{b}}$} & Terminal & Incidence & $3 / 50(6 \%)$ & $11 / 50(22 \%)$ & $5 / 48(10 \%)$ & $7 / 49(14 \%)$ & $9 / 50(18 \%)$ & $5 / 46(11 \%)$ \\
\hline & & Poly-3 Incidence & $3 / 32.3(9 \%)$ & $11 / 33.3(33 \%)$ & $5 / 32.1(16 \%)$ & $7 / 35.4(20 \%)$ & $9 / 36.6(25 \%)$ & $5 / 32.0(16 \%)$ \\
\hline & & Terminal Incidence & $1 / 11(9 \%)$ & $2 / 12(17 \%)$ & $2 / 13(15 \%)$ & $0 / 13(0 \%)$ & $5 / 17(29 \%)$ & $0 / 13(0 \%)$ \\
\hline & & Time-to-First & 681 & 573 & 458 & 450 & 488 & 615 \\
\hline & & Poly-3 $p$-value & 0.453 & $0.016^{*}$ & 0.348 & 0.189 & 0.083 & 0.346 \\
\hline & & Multiple Incidence ${ }^{\mathrm{d}}$ & $1 / 50(2 \%)$ & $1 / 50(2 \%)$ & $1 / 48(2 \%)$ & $2 / 49(4 \%)$ & $1 / 50(2 \%)$ & $2 / 46(4 \%)$ \\
\hline \multirow{6}{*}{$\begin{array}{l}\text { Adenoma or } \\
\text { Adenocarcinoma }^{b}\end{array}$} & Terminal & Incidence & $4 / 50(8 \%)$ & $12 / 50(24 \%)$ & $5 / 48(10 \%)$ & $9 / 49(18 \%)$ & $9 / 50(18 \%)$ & $6 / 46(13 \%)$ \\
\hline & & Poly-3 Incidence & $4 / 33.0(12 \%)$ & $12 / 33.3(36 \%)$ & $5 / 32.1(16 \%)$ & $9 / 35.9(25 \%)$ & $9 / 36.6(25 \%)$ & $6 / 32.8(18 \%)$ \\
\hline & & Terminal Incidence & $1 / 11(9 \%)$ & $3 / 12(25 \%)$ & $2 / 13(15 \%)$ & $1 / 13(8 \%)$ & $5 / 17(29 \%)$ & $0 / 13(0 \%)$ \\
\hline & & Time-to-First & 514 & 573 & 458 & 450 & 488 & 463 \\
\hline & & Poly-3 $p$-value & 0.483 & $0.018^{*}$ & 0.482 & 0.140 & 0.149 & 0.360 \\
\hline & & Multiple Incidence $^{\mathrm{d}}$ & $1 / 50(2 \%)$ & $1 / 50(2 \%)$ & $1 / 48(2 \%)$ & $2 / 49(4 \%)$ & $1 / 50(2 \%)$ & $2 / 46(4 \%)$ \\
\hline \multirow[t]{10}{*}{ Fibroadenoma } & Interim & Incidence $^{c}$ & $4 / 20(20 \%)$ & $1 / 22(4 \%)$ & $1 / 20(5 \%)$ & $1 / 22(4 \%)$ & $1 / 20(5 \%)$ & $2 / 22(9 \%)$ \\
\hline & & Terminal Incidence & $4 / 20(20 \%)$ & $1 / 22(4 \%)$ & $1 / 20(5 \%)$ & $1 / 22(4 \%)$ & $1 / 20(5 \%)$ & $2 / 20(10 \%)$ \\
\hline & & Time-to-First & $363(\mathrm{~T})$ & $365(\mathrm{~T})$ & $365(\mathrm{~T})$ & $363(\mathrm{~T})$ & $364(\mathrm{~T})$ & $363(\mathrm{~T})$ \\
\hline & & CAFE $p$-value & $0.184 \mathrm{~N}$ & $0.144 \mathrm{~N}$ & $0.171 \mathrm{~N}$ & $0.144 \mathrm{~N}$ & $0.171 \mathrm{~N}$ & $0.286 \mathrm{~N}$ \\
\hline & Terminal & Incidence & $43 / 50(86 \%)$ & $45 / 50(90 \%)$ & $37 / 48(77 \%)$ & $42 / 49(86 \%)$ & $36 / 50(72 \%)$ & $34 / 46(74 \%)$ \\
\hline & & Poly-3 Incidence & $43 / 47.7(90 \%)$ & $45 / 47.5(95 \%)$ & $37 / 42.2(88 \%)$ & $42 / 46.2(91 \%)$ & $36 / 45.5(79 \%)$ & $34 / 40.6(84 \%)$ \\
\hline & & Terminal Incidence & 8/11(73\%) & $11 / 12(92 \%)$ & $11 / 13(85 \%)$ & $11 / 13(85 \%)$ & $12 / 17(71 \%)$ & $12 / 13(92 \%)$ \\
\hline & & Time-to-First & 385 & 400 & 339 & 448 & 390 & 383 \\
\hline & & Poly-3 $p$-value & $0.021 \mathrm{~N}^{*}$ & 0.319 & $0.489 \mathrm{~N}$ & 0.600 & $0.099 \mathrm{~N}$ & $0.257 \mathrm{~N}$ \\
\hline & & Multiple Incidence $^{\mathrm{d}}$ & $29 / 50(58 \%)$ & $32 / 50(64 \%)$ & $28 / 48(58 \%)$ & $31 / 49(63 \%)$ & $29 / 50(58 \%)$ & $28 / 46(61 \%)$ \\
\hline
\end{tabular}

${ }^{\mathrm{a}} \mathrm{BPA}$ doses are $\mu \mathrm{g} / \mathrm{kg} \mathrm{bw} / \mathrm{day}$. See legend to Table 52 for description of data presentation. There was a significant increase in adenocarcinoma, and combined adenoma or

adenocarcinoma, in the $2.5 \mathrm{BPA} \mu \mathrm{g} / \mathrm{kg}$ bw/day dose group relative to vehicle controls.

${ }^{b}$ No adenomas or adenocarcinomas were diagnosed in the mammary glands of interim sacrifice stop-dose females.

There were no interim sacrifice females with multiple fibroadenomas.

${ }^{\mathrm{d}}$ Proportion of animals examined that had multiple neoplasms. These animals with multiple neoplasms are included in the incidence row and were not analyzed separately. The numbers of mammary neoplasms found in each female are tabulated in Supplemental Appendix XXXII, Subappendix VII. 
CLARITY-BPA Core Study

Table 55. Non-Neoplastic Lesions in the Mammary Gland of Interim and Terminal Sacrifice Female Rats: Continuous-Dose BPA ${ }^{\text {a }}$

\begin{tabular}{|c|c|c|c|c|c|c|c|c|}
\hline Lesion & $\begin{array}{c}\text { Study Phase } \\
\text { (Interim/Terminal) }\end{array}$ & Statistic & Vehicle & 2.5 BPA & 25 BPA & 250 BPA & 2500 BPA & 25000 BPA \\
\hline \multirow[t]{6}{*}{ Atypical focus } & \multirow[t]{2}{*}{ Interim } & Incidence & $\begin{array}{l}0 / 23 \\
(0 \%)\end{array}$ & $\begin{array}{l}3 / 22^{\wedge} \\
(14 \%)\end{array}$ & $\begin{array}{l}2 / 22 \\
(9 \%)\end{array}$ & $\begin{array}{l}2 / 24 \\
(8 \%)\end{array}$ & $\begin{array}{l}0 / 20 \\
(0 \%)\end{array}$ & $\begin{array}{l}0 / 24 \\
(0 \%)\end{array}$ \\
\hline & & Severity Profile & - & $1|2| 0 \mid 0(1.7)$ & $0|2| 0 \mid 0(2.0)$ & $2|0| 0 \mid 0(1.0)$ & - & - \\
\hline & \multirow[t]{4}{*}{ Terminal } & Incidence & $\begin{array}{l}2 / 50 \\
(4 \%)\end{array}$ & $\begin{array}{l}7 / 48^{\wedge} \\
(15 \%)\end{array}$ & $\begin{array}{l}1 / 46 \\
(2 \%)\end{array}$ & $\begin{array}{c}5 / 49 \\
(10 \%)\end{array}$ & $\begin{array}{l}3 / 50 \\
(6 \%)\end{array}$ & $\begin{array}{l}3 / 46 \\
(6 \%)\end{array}$ \\
\hline & & Severity Profile & $0|2| 0 \mid 0(2.0)$ & $3|2| 2 \mid 0$ (1.9) & $0|1| 0 \mid 0(2.0)$ & $0|5| 0 \mid 0(2.0)$ & $1|2| 0 \mid 0(1.7)$ & $1|2| 0 \mid 0(1.7)$ \\
\hline & & Poly-3 Incidence & $2 / 34.4$ & $7 / 33.6$ & $1 / 28.2$ & $5 / 33.3$ & $3 / 29.7$ & $3 / 31.7$ \\
\hline & & & $(6 \%)$ & $(21 \%)$ & $(4 \%)$ & $(15 \%)$ & $(10 \%)$ & $(10 \%)$ \\
\hline \multirow[t]{8}{*}{ Dilatation, duct } & \multirow[t]{3}{*}{ Interim } & Incidence & $2 / 23$ & $2 / 22$ & $7 / 22^{\wedge \wedge}$ & $1 / 24$ & $2 / 20$ & $2 / 24$ \\
\hline & & & $(9 \%)$ & $(9 \%)$ & $(32 \%)$ & $(4 \%)$ & $(10 \%)$ & $(8 \%)$ \\
\hline & & Severity Profile & $1|1| 0 \mid 0(1.5)$ & $0|0| 0 \mid 2(4.0)$ & 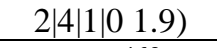 & $0|0| 1 \mid 0(3.0)$ & $1|0| 1 \mid 0(2.0)$ & $1|0| 1 \mid 0(2.0)$ \\
\hline & \multirow[t]{5}{*}{ Terminal } & Incidence & $15 / 50$ & $16 / 48$ & $7 / 46^{\wedge} \mathrm{N}$ & $9 / 49$ & $9 / 50$ & $14 / 46$ \\
\hline & & & $(30 \%)$ & $(33 \%)$ & $(15 \%)$ & $(18 \%)$ & $(18 \%)$ & $(30 \%)$ \\
\hline & & Severity Profile & $0|10| 5 \mid 0(2.3)$ & $0|12| 2 \mid 2(2.4)$ & $0|6| 1 \mid 0(2.1)$ & $0|6| 3 \mid 0(2.3)$ & $0|4| 5 \mid 0(2.6)$ & $2|9| 3 \mid 0(2.1)$ \\
\hline & & Poly-3 Incidence & $15 / 38.1$ & $16 / 39.4$ & $7 / 31.9$ & $9 / 35.7$ & 9/33.1 & $14 / 36.4$ \\
\hline & & & $(39 \%)$ & $(41 \%)$ & $(22 \%)$ & $(25 \%)$ & $(27 \%)$ & $(38 \%)$ \\
\hline \multirow{8}{*}{$\begin{array}{l}\text { Hyperplasia, } \\
\text { lobular }\end{array}$} & \multirow[t]{3}{*}{ Interim } & Incidence & $10 / 23$ & $14 / 22$ & $13 / 22$ & $15 / 24$ & $13 / 20$ & $12 / 24$ \\
\hline & & & $(44 \%)$ & $(64 \%)$ & $(59 \%)$ & $(62 \%)$ & $(65 \%)$ & $(50 \%)$ \\
\hline & & Severity Profile & $5|5| 0 \mid 0(1.5)$ & $7|7| 0 \mid 0(1.5)$ & $7|6| 0 \mid 0(1.5)$ & $11|3| 1 \mid 0$ (1.3) & $10|2| 0 \mid 1(1.4)$ & $9|1| 1 \mid 1(1.5)$ \\
\hline & \multirow[t]{5}{*}{ Terminal } & Incidence & $43 / 50$ & $41 / 48$ & $30 / 46^{\wedge \wedge} N$ & $38 / 49$ & $40 / 50^{\wedge} \mathrm{N}$ & $37 / 46$ \\
\hline & & & $(86 \%)$ & $(85 \%)$ & $(65 \%)$ & $(78 \%)$ & $(80 \%)$ & $(80 \%)$ \\
\hline & & Severity Profile & $1|8| 13 \mid 21$ (3.3) & $1|7| 11 \mid 22$ (3.3) & $1|7| 10 \mid 12(3.1)$ & $0|7| 17 \mid 14$ (3.2) & $1|14| 11 \mid 14$ (3.0) & $4|9| 5 \mid 19$ (3.1) \\
\hline & & Poly-3 Incidence & $43 / 45.4^{*} \mathrm{~N}$ & $41 / 42.5$ & $30 / 36.7^{* N}$ & $38 / 43.7$ & $40 / 43.2$ & $37 / 43.4$ \\
\hline & & & $(95 \%)$ & $(96 \%)$ & $(82 \%)$ & $(87 \%)$ & $(93 \%)$ & $(85 \%)$ \\
\hline \multirow{5}{*}{$\begin{array}{l}\text { Dilatation, } \\
\text { alveolus }{ }^{\mathrm{b}}\end{array}$} & \multirow[t]{5}{*}{ Terminal } & Incidence & $9 / 50$ & $14 / 48$ & $5 / 46$ & $7 / 49$ & $7 / 50$ & $11 / 46$ \\
\hline & & & $(18 \%)$ & $(29 \%)$ & $(11 \%)$ & $(14 \%)$ & $(14 \%)$ & $(24 \%)$ \\
\hline & & Severity Profile & $1|7| 1 \mid 0(2.0)$ & $1|11| 2 \mid 0(2.1)$ & $0|5| 0 \mid 0(2.0)$ & $0|7| 0 \mid 0(2.0)$ & $0|6| 1 \mid 0(2.1)$ & $0|9| 2 \mid 0(2.2)$ \\
\hline & & Poly-3 Incidence & $9 / 36.0$ & $14 / 38.0$ & $5 / 30.9$ & $7 / 35.2$ & $7 / 32.3$ & $11 / 34.6$ \\
\hline & & & $(25 \%)$ & $(37 \%)$ & $(16 \%)$ & $(20 \%)$ & $(22 \%)$ & $(32 \%)$ \\
\hline
\end{tabular}

aPA doses are $\mu \mathrm{g} / \mathrm{kg}$ bw/day. Statistical analyses were conducted for any lesion that was diagnosed in two animals in any dose group in the interim sacrifice groups or in the EE 2 terminal sacrifice groups or four animals in the control and BPA groups in the terminal sacrifice. A complete tabulation of all non-neoplastic lesions is found in Supplemental Appendix XXXII and complete results of the statistical analyses are found in Supplemental Appendices XXXIII (interim sacrifice) and XXXIV (terminal sacrifice). Details of the statistical methods are found in Materials and Methods and in Supplemental Appendices XXXIII and XXXIV. Selected non-neoplastic lesions are tabulated in this report. Data are presented as follows: Incidence, lesions observed/number of animals examined microscopically, with percent animals affected in parentheses; Severity Profile, number of animals diagnosed with minimal/ mild/ moderate/ marked lesions, with the average severity in affected animals given in parentheses, based upon severity scores of 1 , minimal; 2 , mild; 3 , moderate; and 4, marked.; Poly-3 Incidence, Poly-3 adjusted lesion incidence after adjustment for intercurrent mortality in the terminal sacrifice animals. Lesions in interim sacrifice animals were analyzed by the CAFE test (Cochran-Armitage trend test and Fisher's exact test to compare the incidence in each dose group to the vehicle control) and by 
the Jonckheere-Terpstra (JT) trend test/Shirley-Williams (SW) pairwise comparison test to incorporate severity scores. Because the JT/SW test enforces an assumption of a monotonic response, a relative treatment effect (RTE) analysis that also incorporates severity scores, but does not enforce monotonicity, was also conducted. Significant JT/SW results that violated the monotonicity requirement are not shown in the tables, but are reported in Supplemental Appendices XXXIII and XXXIV. Lesions in terminal sacrifice animals were analyzed by the Poly-3 test to adjust for intercurrent mortality, as well as by the JT/SW and RTE tests. All pairwise tests were one-sided and not corrected for multiple comparisons. Significant trends are shown in the vehicle column, while significant pairwise comparisons to the vehicle control are shown in BPA or EE 2 dose group columns. The CAFE or Poly-3 tests for interim and terminal sacrifice animals, respectively, were considered as the primary statistical tests and positive significant results for those tests are shown with asterisks. Pound and caret signs indicate results from the JT/SW and RTE tests, respectively. *,\#, ^, $p<0.05 ;{ }^{* *}, \# \#, \wedge \wedge, p<0.01 ; * * *, \# \# \#, \wedge \wedge \wedge, p<0.001$. "N" superscript next to significance markers indicates a result that is a negative trend or negative relative to control.

${ }^{b} \mathrm{No}$ alveolar dilatation was diagnosed in interim sacrifice females. 
CLARITY-BPA Core Study

Table 56. Non-Neoplastic Lesions in the Mammary Gland of Interim and Terminal Sacrifice Female Rats: Continuous-Dose EE$_{2}{ }^{\mathrm{a}}$

\begin{tabular}{|c|c|c|c|c|c|}
\hline Lesion & $\begin{array}{c}\text { Study Phase } \\
\text { (Interim/Terminal) }\end{array}$ & Statistic & Vehicle $^{b}$ & $0.05 \mathrm{EE}_{2}$ & $0.5 \mathrm{EE}_{2}$ \\
\hline \multirow[t]{4}{*}{ Atypical focus ${ }^{\mathrm{c}}$} & \multirow[t]{4}{*}{ Terminal } & Incidence & $\begin{array}{l}2 / 50 \\
(4 \%)\end{array}$ & $\begin{array}{l}2 / 26 \\
(8 \%)\end{array}$ & $\begin{array}{c}3 / 26 \\
(12 \%)\end{array}$ \\
\hline & & Severity Profile & $0|2| 0 \mid 0(2.0)$ & $1|1| 0 \mid 0(1.5)$ & $2|1| 0 \mid 0(1.3)$ \\
\hline & & \multirow[t]{2}{*}{ Poly-3 Incidence } & $2 / 34.4$ & $2 / 16.1$ & $3 / 16.2$ \\
\hline & & & $(6 \%)$ & $(12 \%)$ & $(19 \%)$ \\
\hline \multirow[t]{8}{*}{ Dilatation, duct } & \multirow[t]{3}{*}{ Interim } & \multirow[t]{2}{*}{ Incidence } & $2 / 23^{* * *}, \# \# \#, \wedge \wedge \wedge$ & $3 / 26$ & $22 / 26^{* * * *, \# \# \#, \wedge \wedge \wedge}$ \\
\hline & & & $(9 \%)$ & $(12 \%)$ & $(85 \%)$ \\
\hline & & Severity Profile & $1|1| 0 \mid 0(1.5)$ & $1|2| 0 \mid 0(1.7)$ & $6|12| 2 \mid 2(2.0)$ \\
\hline & \multirow[t]{5}{*}{ Terminal } & \multirow[t]{2}{*}{ Incidence } & $15 / 50^{\# \# \#, \wedge \wedge \wedge}$ & $6 / 26$ & $21 / 26^{\# \#, \wedge \wedge \wedge}$ \\
\hline & & & $(30 \%)$ & $(23 \%)$ & $(81 \%)$ \\
\hline & & Severity Profile & $0|10| 5 \mid 0(2.3)$ & $0|3| 3 \mid 0(2.5)$ & $0|10| 7 \mid 4(2.7)$ \\
\hline & & \multirow[t]{2}{*}{ Poly-3 Incidence } & $15 / 38.1^{* * *}$ & $6 / 19.0$ & $21 / 24.2^{* * *}$ \\
\hline & & & $(39 \%)$ & $(32 \%)$ & $(87 \%)$ \\
\hline \multirow[t]{8}{*}{ Hyperplasia, lobular } & \multirow[t]{3}{*}{ Interim } & \multirow[t]{2}{*}{ Incidence } & $10 / 23^{* * *, \# \#, \wedge \wedge}$ & $13 / 26$ & $23 / 26^{* * *}, \# \#, \wedge \wedge$ \\
\hline & & & $(44 \%)$ & $(50 \%)$ & $(88 \%)$ \\
\hline & & Severity Profile & $5|5| 0 \mid 0(1.5)$ & $6|6| 1 \mid 0(1.6)$ & $11|11| 1 \mid 0$ (1.6) \\
\hline & \multirow[t]{5}{*}{ Terminal } & \multirow[t]{2}{*}{ Incidence } & $43 / 50$ & $24 / 26$ & $23 / 26$ \\
\hline & & & $(86 \%)$ & $(92 \%)$ & $(88 \%)$ \\
\hline & & Severity Profile & $1|8| 13 \mid 21(3.3)$ & $1|8| 4 \mid 11(3.0)$ & $1|10| 4 \mid 8(2.8)$ \\
\hline & & \multirow[t]{2}{*}{ Poly-3 Incidence } & $43 / 45.4$ & $24 / 24.5$ & $23 / 25.1$ \\
\hline & & & $(95 \%)$ & $(98 \%)$ & $(92 \%)$ \\
\hline \multirow[t]{5}{*}{ Dilatation, alveolus ${ }^{\mathrm{d}}$} & \multirow[t]{5}{*}{ Terminal } & \multirow[t]{2}{*}{ Incidence } & $9 / 50^{\# \# \#, \wedge \wedge \wedge}$ & $5 / 26$ & $22 / 26^{\# \# \#, \wedge \wedge \wedge}$ \\
\hline & & & $(18 \%)$ & $(19 \%)$ & $(85 \%)$ \\
\hline & & Severity Profile & $1|7| 1 \mid 0(2.0)$ & $0|5| 0 \mid 0(2.0)$ & $0|18| 4 \mid 0(2.2)$ \\
\hline & & \multirow[t]{2}{*}{ Poly-3 Incidence } & $9 / 36.0^{* * *}$ & $5 / 18.8$ & $22 / 24.1^{* * *}$ \\
\hline & & & $(25 \%)$ & $(27 \%)$ & $(91 \%)$ \\
\hline
\end{tabular}

${ }^{\mathrm{a}} \mathrm{EE}_{2}$ doses are $\mu \mathrm{g} / \mathrm{kg}$ bw/day. See legend to Table 55 or description of data presentation.

${ }^{b}$ The continuous vehicle control group data are the same as those shown for the continuous-dose BPA data in Table 55.

'There were no atypical foci diagnosed in the interim vehicle, 0.05 , or $0.5 \mu \mathrm{g} \mathrm{EE} / \mathrm{kg}$ bw/day groups $(0 / 23,0 / 26$, and $0 / 26$, respectively), so there is no tabulation of data for the

interim sacrifice animals for this lesion.

dNo alveolar dilatation was diagnosed in interim sacrifice females. 
CLARITY-BPA Core Study

Table 57. Non-Neoplastic Lesions in the Mammary Gland of Interim and Terminal Sacrifice Female Rats: Stop-Dose BPA ${ }^{\mathrm{a}}$

\begin{tabular}{|c|c|c|c|c|c|c|c|c|}
\hline Lesion & $\begin{array}{c}\text { Study Phase } \\
\text { (Interim/Terminal) }\end{array}$ & Statistic & Vehicle & $2.5 \mathrm{BPA}$ & 25 BPA & 250 BPA & 2500 BPA & 25000 BPA \\
\hline \multirow[t]{5}{*}{ Atypical focus ${ }^{b}$} & \multirow[t]{5}{*}{ Terminal } & Incidence & $6 / 50$ & $2 / 50$ & $6 / 48$ & $8 / 49$ & $7 / 50$ & $5 / 46$ \\
\hline & & & $(12 \%)$ & $(4 \%)$ & $(12 \%)$ & $(16 \%)$ & $(14 \%)$ & $(11 \%)$ \\
\hline & & Severity Profile & $1|4| 1 \mid 0(2.0)$ & $0|1| 1 \mid 0(2.5)$ & $1|5| 0 \mid 0(1.8)$ & $3|4| 1 \mid 0(1.8)$ & $0|5| 0 \mid 2(2.6)$ & $2|3| 0 \mid 0(1.6)$ \\
\hline & & Poly-3 & $6 / 33.0$ & $2 / 32.0$ & $6 / 32.4$ & $8 / 36.2$ & $7 / 35.8$ & $5 / 32.6$ \\
\hline & & Incidence & $(18 \%)$ & $(6 \%)$ & $(18 \%)$ & $(22 \%)$ & $(20 \%)$ & $(15 \%)$ \\
\hline \multirow[t]{8}{*}{ Dilatation, duct } & \multirow[t]{3}{*}{ Interim } & Incidence & $4 / 20^{*}, \#, \wedge \mathrm{N}$ & $2 / 22$ & $1 / 20$ & $1 / 22^{\wedge} \mathrm{N}$ & $1 / 20$ & $1 / 22^{\#, \wedge N}$ \\
\hline & & & $(20 \%)$ & $(9 \%)$ & $(5 \%)$ & $(4 \%)$ & $(5 \%)$ & $(4 \%)$ \\
\hline & & Severity Profile & $2|1| 1 \mid 0(1.8)$ & $1|1| 0 \mid 0(1.5)$ & $0|1| 0 \mid 0(2.0)$ & $1|0| 0 \mid 0(1.0)$ & $0|1| 0 \mid 0(2.0)$ & $1|0| 0 \mid 0(1.0)$ \\
\hline & \multirow[t]{5}{*}{ Terminal } & Incidence & $16 / 50$ & $5 / 50^{\# \#, \wedge \wedge \mathrm{N}}$ & $9 / 48^{\#, \wedge N}$ & $9 / 49^{\#, \wedge N}$ & $7 / 50^{\# \#, \wedge \wedge \mathrm{N}}$ & $11 / 46^{\# N}$ \\
\hline & & & $(32 \%)$ & $(10 \%)$ & $(19 \%)$ & $(18 \%)$ & $(14 \%)$ & $(24 \%)$ \\
\hline & & Severity Profile & $0|9| 4 \mid 3$ (2.6) & $0|5| 0 \mid 0(2.0)$ & $2|4| 2 \mid 1(2.2)$ & $0|6| 3 \mid 0(2.3)$ & $0|6| 1 \mid 0(2.1)$ & $0|7| 4 \mid 0(2.4)$ \\
\hline & & Poly-3 & $16 / 37.0$ & $5 / 34.5^{* *} \mathrm{~N}$ & $9 / 33.7$ & $9 / 35.3$ & $7 / 36.4^{*} \mathrm{~N}$ & $11 / 36.2$ \\
\hline & & Incidence & $(43 \%)$ & $(14 \%)$ & $(27 \%)$ & $(26 \%)$ & $(19 \%)$ & $(30 \%)$ \\
\hline \multirow{8}{*}{$\begin{array}{l}\text { Hyperplasia, } \\
\text { lobular }\end{array}$} & \multirow[t]{3}{*}{ Interim } & Incidence & $15 / 20$ & $12 / 22$ & $8 / 20^{*}, \wedge \mathrm{N}$ & $12 / 22$ & $7 / 20^{*}, \wedge \mathrm{N}$ & $12 / 22$ \\
\hline & & & $(75 \%)$ & $(54 \%)$ & $(40 \%)$ & $(54 \%)$ & $(35 \%)$ & $(54 \%)$ \\
\hline & & Severity Profile & $10|4| 1 \mid 0(1.4)$ & $8|3| 1 \mid 0$ (1.4) & $5|1| 2 \mid 0(1.6)$ & $8|3| 1 \mid 0$ (1.4) & $4|3| 0 \mid 0(1.4)$ & $6|4| 2 \mid 0(1.7)$ \\
\hline & \multirow[t]{5}{*}{ Terminal } & Incidence & $41 / 50$ & $40 / 50$ & $39 / 48$ & $39 / 49$ & $36 / 50$ & $38 / 46$ \\
\hline & & & $(82 \%)$ & $(80 \%)$ & $(81 \%)$ & $(80 \%)$ & $(72 \%)$ & $(83 \%)$ \\
\hline & & Severity Profile & $1|8| 15 \mid 17$ (3.2) & $0|8| 12 \mid 20(3.3)$ & $\begin{array}{c}3|14| 11 \mid 11 \\
(2.8)\end{array}$ & $2|9| 13 \mid 15$ (3.1) & $3|5| 7 \mid 21(3.3)$ & $2|10| 9 \mid 17$ (3.1) \\
\hline & & Poly-3 & $41 / 45.5$ & $40 / 46.1$ & $39 / 42.7$ & $39 / 45.0$ & $36 / 45.4$ & $38 / 41.6$ \\
\hline & & Incidence & $(90 \%)$ & $(87 \%)$ & $(91 \%)$ & $(87 \%)$ & $(79 \%)$ & $(91 \%)$ \\
\hline \multirow{5}{*}{$\begin{array}{l}\text { Dilatation, } \\
\text { alveolus }^{\mathrm{c}}\end{array}$} & \multirow[t]{5}{*}{ Terminal } & Incidence & $8 / 50$ & $4 / 50$ & $4 / 48$ & $8 / 49$ & $3 / 50^{\wedge} \mathrm{N}$ & $7 / 46$ \\
\hline & & & $(16 \%)$ & $(8 \%)$ & $(8 \%)$ & $(16 \%)$ & $(6 \%)$ & $(15 \%)$ \\
\hline & & Severity Profile & $0|4| 4 \mid 0(2.5)$ & $0|4| 0 \mid 0(2.0)$ & $0|2| 2 \mid 0(2.5)$ & $0|6| 2 \mid 0(2.3)$ & $1|2| 0 \mid 0$ (1.7) & $1|6| 0 \mid 0$ (1.9) \\
\hline & & Poly-3 & $8 / 34.8$ & $4 / 33.7$ & $4 / 30.5$ & $8 / 34.9$ & $3 / 35.7$ & $7 / 34.9$ \\
\hline & & Incidence & $(23 \%)$ & $(12 \%)$ & $(13 \%)$ & $(23 \%)$ & $(8 \%)$ & $(20 \%)$ \\
\hline
\end{tabular}

${ }^{\mathrm{a} B P A}$ doses are $\mu \mathrm{g} / \mathrm{kg}$ bw/day. See legend to Table 55 for description of data presentation.

${ }^{\mathrm{b}}$ There was a $5 \%$ incidence $(1 / 20)$ of atypical focus in the mammary gland of female stop-dose vehicle control interim sacrifice animals and all BPA dose interim sacrifice dose

groups had a $0 \%$ incidence $(0 / 22,0 / 20,0 / 22,0 / 20,0 / 22$ for $2.5,25,250,2,500$, and 25,000 $\mu \mathrm{g} \mathrm{BPA} / \mathrm{kg}$ bw/day, respectively).

${ }^{\mathrm{c}}$ No alveolar dilatation was diagnosed in interim sacrifice females. 
CLARITY-BPA Core Study

Table 58. Neoplastic Lesions in the Uterus of Interim and Terminal Sacrifice Female Rats: Continuous-Dose BPA ${ }^{\mathrm{a}}$

\begin{tabular}{|c|c|c|c|c|c|c|c|c|}
\hline Lesion & $\begin{array}{c}\text { Study Phase } \\
\text { (Interim/Terminal) }\end{array}$ & Statistic & Vehicle & 2.5 BPA & 25 BPA & 250 BPA & 2500 BPA & 25000 BPA \\
\hline \multirow[t]{9}{*}{ Stromal polyps } & \multirow[t]{4}{*}{ Interim } & Incidence & $1 / 23(4 \%)$ & $0 / 22(0 \%)$ & $1 / 21(5 \%)$ & $0 / 24(0 \%)$ & $3 / 20(15 \%)$ & $3 / 24(12 \%)$ \\
\hline & & Terminal Incidence & $1 / 21(5 \%)$ & $0 / 22(0 \%)$ & $1 / 21(5 \%)$ & $0 / 22(0 \%)$ & $3 / 20(15 \%)$ & $3 / 24(12 \%)$ \\
\hline & & Time-to-First & $362(\mathrm{~T})$ & - & $360(\mathrm{~T})$ & - & $362(\mathrm{~T})$ & $362(\mathrm{~T})$ \\
\hline & & CAFE $p$-value & $0.037^{*}$ & $0.511^{\mathrm{N}}$ & 0.733 & $0.489^{\mathrm{N}}$ & 0.252 & 0.321 \\
\hline & \multirow[t]{5}{*}{ Terminal } & Incidence & $5 / 50(10 \%)$ & $3 / 48(6 \%)$ & $7 / 45(16 \%)$ & $2 / 49(4 \%)$ & $4 / 48(8 \%)$ & $3 / 46(6 \%)$ \\
\hline & & Poly-3 Incidence & $5 / 36.4(14 \%)$ & $3 / 34.6(9 \%)$ & $7 / 29.5(24 \%)$ & $2 / 33.5(6 \%)$ & $4 / 29.2(14 \%)$ & $3 / 32.2(9 \%)$ \\
\hline & & Terminal Incidence & $1 / 16(6 \%)$ & $0 / 19(0 \%)$ & $4 / 14(29 \%)$ & $0 / 13(0 \%)$ & $0 / 10(0 \%)$ & $0 / 8(0 \%)$ \\
\hline & & Time-to-First & 506 & 321 & 441 & 542 & 673 & 569 \\
\hline & & Poly-3 $p$-value & $0.333^{\mathrm{N}}$ & $0.383^{\mathrm{N}}$ & 0.231 & $0.247^{\mathrm{N}}$ & $0.638^{\mathrm{N}}$ & $0.424^{\mathrm{N}}$ \\
\hline
\end{tabular}

${ }^{\mathrm{a} B P A}$ doses are $\mu \mathrm{g} / \mathrm{kg}$ bw/day. See legend to Table 52 for description of data presentation. There were no statistically significant effects in the terminal sacrifice animals. There was a significant trend across BPA dose levels in the interim sacrifice animals.

Table 59. Neoplastic Lesions in the Uterus of Interim and Terminal Sacrifice Female Rats: Continuous-Dose $\mathbf{E E}_{2}{ }^{\mathrm{a}}$

\begin{tabular}{|c|c|c|c|c|c|}
\hline Lesion & $\begin{array}{c}\text { Study Phase } \\
\text { (Interim/Terminal) }\end{array}$ & Statistic & Vehicle $^{b}$ & $0.05 \mathrm{EE}_{2}$ & $0.5 \mathrm{EE}_{2}$ \\
\hline \multirow[t]{5}{*}{ Stromal polyps } & Terminal & Incidence & $5 / 50(10 \%)$ & $3 / 26(12 \%)$ & $1 / 26(4 \%)$ \\
\hline & & Poly-3 Incidence & $5 / 36.4(14 \%)$ & $3 / 17.4(17 \%)$ & $1 / 15.7(6 \%)$ \\
\hline & & Terminal Incidence & $1 / 16(6 \%)$ & $0 / 7(0 \%)$ & $0 / 4(0 \%)$ \\
\hline & & Time-to-First & 506 & 485 & 527 \\
\hline & & Poly-3 $p$-value & $0.383^{\mathrm{N}}$ & 0.529 & $0.389^{\mathrm{N}}$ \\
\hline
\end{tabular}

${ }^{\mathrm{a}} \mathrm{EE}_{2}$ doses are $\mu \mathrm{g} / \mathrm{kg}$ bw/day. See legend to Table 52 for description of data presentation. There were no statistically significant effects in the terminal sacrifice animals. Interim sacrifice animals are not tabulated as no group had two or more lesions.

${ }^{b}$ The continuous vehicle control group data are the same as those shown for the continuous-dose BPA data in Table 58. 
CLARITY-BPA Core Study

Table 60. Neoplastic Lesions in the Uterus of Interim and Terminal Sacrifice Female Rats: Stop-Dose BPA ${ }^{\mathrm{a}}$

\begin{tabular}{|c|c|c|c|c|c|c|c|c|}
\hline Lesion & $\begin{array}{c}\text { Study Phase } \\
\text { (Interim/Terminal) }\end{array}$ & Statistic & Vehicle & 2.5 BPA & 25 BPA & 250 BPA & 2500 BPA & 25000 BPA \\
\hline \multirow[t]{5}{*}{ Stromal polyps } & Terminal & Incidence & $7 / 49(14 \%)$ & $4 / 49(8 \%)$ & $5 / 48(10 \%)$ & $6 / 49(12 \%)$ & $4 / 49(8 \%)$ & $1 / 46(2 \%)$ \\
\hline & & Poly-3 Incidence & $7 / 33.0(21 \%)$ & $4 / 32.3(12 \%)$ & $5 / 32.5(15 \%)$ & $6 / 35.9(17 \%)$ & $4 / 35.8(11 \%)$ & $1 / 31.0(3 \%)$ \\
\hline & & Terminal Incidence & $2 / 11(18.2 \%)$ & $1 / 12(8 \%)$ & $1 / 13(8 \%)$ & $1 / 13(8 \%)$ & $1 / 17(6 \%)$ & $0 / 13(0 \%)$ \\
\hline & & Time-to-First & 467 & 446 & 461 & 497 & 539 & 706 \\
\hline & & Poly-3 $p$-value & $0.041^{*} \mathrm{~N}$ & $0.263^{\mathrm{N}}$ & $0.384^{\mathrm{N}}$ & $0.433^{\mathrm{N}}$ & $0.207^{\mathrm{N}}$ & $0.032^{*} \mathrm{~N}$ \\
\hline
\end{tabular}

${ }^{\mathrm{a} B P A}$ doses are $\mu \mathrm{g} / \mathrm{kg}$ bw/day. See legend to Table 52 for description of data presentation. Interim sacrifice animals are not tabulated as no group had two or more lesions. 
CLARITY-BPA Core Study

Table 61. Non-Neoplastic Lesions in the Uterus of Interim and Terminal Sacrifice Female Rats: Continuous-Dose BPA ${ }^{\mathrm{a}}$

\begin{tabular}{|c|c|c|c|c|c|c|c|c|}
\hline Lesion & $\begin{array}{c}\text { Study Phase } \\
\text { (Interim/Terminal) }\end{array}$ & Statistic & Vehicle & 2.5 BPA & 25 BPA & 250 BPA & 2500 BPA & 25000 BPA \\
\hline \multirow[t]{3}{*}{ Apoptosis } & \multirow[t]{3}{*}{ Interim } & Incidence & $2 / 23^{* *}, \# \#, \wedge \wedge$ & $1 / 22$ & $4 / 21$ & $5 / 24$ & $5 / 20$ & $9 / 24^{*}, \#, \wedge \wedge$ \\
\hline & & & $(9 \%)$ & $(4 \%)$ & $(19 \%)$ & $(21 \%)$ & $(25 \%)$ & $(38 \%)$ \\
\hline & & Severity Profile & $0 / 0 / 0 / 2(4.0)$ & $0 / 0 / 0 / 1(4.0)$ & $0 / 1 / 1 / 2(3.2)$ & $0 / 1 / 0 / 4(3.6)$ & $0 / 0 / 3 / 2(3.4)$ & $0 / 1 / 5 / 3(3.2)$ \\
\hline \multirow{8}{*}{$\begin{array}{l}\text { Hyperplasia, cystic, } \\
\text { endometrium }\end{array}$} & \multirow[t]{3}{*}{ Interim } & Incidence & $5 / 23$ & $1 / 22$ & $4 / 21$ & $3 / 24$ & $7 / 20$ & $4 / 24$ \\
\hline & & & $(22 \%)$ & $(4 \%)$ & $(19 \%)$ & $(12 \%)$ & $(35 \%)$ & $(17 \%)$ \\
\hline & & Severity Profile & $1|2| 1 \mid 1(2.4)$ & $1|0| 0 \mid 0(1.0)$ & $1|2| 1 \mid 0(2.0)$ & $1|1| 1 \mid 0(2.0)$ & $0|6| 1 \mid 0(2.1)$ & $0|3| 0 \mid 1(2.5)$ \\
\hline & \multirow[t]{5}{*}{ Terminal } & Incidence & $30 / 50$ & $20 / 48^{\wedge} N$ & $26 / 45$ & $23 / 49$ & $22 / 48$ & $26 / 46$ \\
\hline & & & $(60 \%)$ & $(42 \%)$ & $(58 \%)$ & $(47 \%)$ & $(46 \%)$ & $(56 \%)$ \\
\hline & & Severity Profile & $4|18| 5 \mid 3(2.2)$ & $2|13| 5 \mid 0(2.2)$ & $0|16| 7 \mid 3(2.5)$ & $4|12| 6 \mid 1(2.2)$ & $4|8| 8 \mid 2(2.4)$ & $1|14| 10 \mid 1(2.4)$ \\
\hline & & Poly-3 incidence & $30 / 41.9$ & $20 / 40.1^{* N}$ & $26 / 36.9$ & $23 / 40.6$ & $22 / 35.8$ & $26 / 39.2$ \\
\hline & & & $(72 \%)$ & $(50 \%)$ & $(70 \%)$ & $(57 \%)$ & $(61 \%)$ & $(66 \%)$ \\
\hline \multirow{8}{*}{$\begin{array}{l}\text { Hyperplasia, } \\
\text { endometrium }\end{array}$} & \multirow[t]{3}{*}{ Interim } & Incidence & $2 / 23$ & $7 / 22^{\wedge}$ & $5 / 21$ & $7 / 24^{\wedge}$ & $5 / 20$ & $2 / 24$ \\
\hline & & & $(9 \%)$ & $(32 \%)$ & $(24 \%)$ & $(29 \%)$ & $(25 \%)$ & $(8 \%)$ \\
\hline & & Severity Profile & $0|2| 0 \mid 0(2.0)$ & $3|3| 1 \mid 0(1.7)$ & $0|4| 1 \mid 0(2.2)$ & $2|4| 1 \mid 0(1.9)$ & \begin{tabular}{l|l|l|l|}
1 & 2 & 2 & $0(2.2)$ \\
\end{tabular} & $0|2| 0 \mid 0(2.0)$ \\
\hline & \multirow{5}{*}{ Terminal } & Incidence & $10 / 50$ & $15 / 48$ & $12 / 45$ & $15 / 49$ & $15 / 48$ & $12 / 46$ \\
\hline & & & $(20 \%)$ & $(31 \%)$ & $(27 \%)$ & $(31 \%)$ & $(31 \%)$ & $(26 \%)$ \\
\hline & & Severity Profile & $2|8| 0 \mid 0(1.8)$ & $5|8| 2 \mid 0(1.8)$ & $2|8| 2 \mid 0(2.0)$ & $6|7| 0 \mid 2$ (1.9) & $10|5| 0 \mid 0(1.3)$ & $2|7| 1 \mid 2(2.2)$ \\
\hline & & Poly-3 incidence & $10 / 39.1$ & $15 / 36.4$ & $12 / 31.9$ & $15 / 37.1$ & $15 / 35.9$ & $12 / 36.2$ \\
\hline & & & $(26 \%)$ & $(41 \%)$ & $(38 \%)$ & $(40 \%)$ & $(42 \%)$ & $(33 \%)$ \\
\hline \multirow{8}{*}{$\begin{array}{l}\text { Metaplasia, } \\
\text { squamous }\end{array}$} & \multirow[t]{3}{*}{ Interim } & Incidence & $1 / 23^{*}, \#, \wedge$ & $1 / 22$ & $4 / 21$ & $3 / 24$ & $3 / 20$ & $5 / 24^{b}$ \\
\hline & & & $(4 \%)$ & $(4 \%)$ & $(19 \%)$ & $(12 \%)$ & $(15 \%)$ & $(21 \%)$ \\
\hline & & Severity Profile & $0|1| 0 \mid 0(2.0)$ & $0|1| 0 \mid 0(2.0)$ & $3|1| 0 \mid 0(1.2)$ & $3|0| 0 \mid 0(1.0)$ & $2|0| 1 \mid 0(1.7)$ & $5|0| 0 \mid 0(1.0)$ \\
\hline & \multirow[t]{5}{*}{ Terminal } & Incidence & $2 / 50$ & $4 / 48$ & $4 / 45$ & $1 / 49$ & $4 / 48$ & $6 / 46$ \\
\hline & & & $(4 \%)$ & $(8 \%)$ & $(9 \%)$ & $(2 \%)$ & $(8 \%)$ & $(13 \%)$ \\
\hline & & Severity Profile & $1|1| 0 \mid 0(1.5)$ & $2|2| 0 \mid 0(1.5)$ & $1|2| 0 \mid 1(2.2)$ & $1|0| 0 \mid 0(1.0)$ & $1|3| 0 \mid 0(1.8)$ & $4|1| 1 \mid 0(1.5)$ \\
\hline & & Poly-3 incidence & $2 / 35.7$ & $4 / 35$ & $4 / 29.5$ & $1 / 32.8$ & $4 / 31.1$ & $6 / 33.9$ \\
\hline & & & $(6 \%)$ & $(11 \%)$ & $(14 \%)$ & $(3 \%)$ & $(13 \%)$ & $(18 \%)$ \\
\hline \multirow[t]{7}{*}{ Dilatation, lumen } & \multirow[t]{3}{*}{ Interim } & Incidence & $0 / 23$ & $1 / 22$ & $2 / 21$ & $2 / 24$ & $1 / 20$ & $2 / 24$ \\
\hline & & & $(0 \%)$ & $(4 \%)$ & $(10 \%)$ & $(8 \%)$ & $(5 \%)$ & $(8 \%)$ \\
\hline & & Severity Profile & - & $0|0| 1 \mid 0(3.0)$ & $0|0| 0 \mid 2(4.0)$ & $0|0| 0 \mid 2(4.0)$ & $0|0| 0 \mid 1(4.0)$ & $0|0| 0 \mid 2(4.0)$ \\
\hline & \multirow[t]{4}{*}{ Terminal } & Incidence & $\begin{array}{c}2 / 50^{\#, \wedge} \\
(4 \%)\end{array}$ & $\begin{array}{l}2 / 48 \\
(4 \%)\end{array}$ & $\begin{array}{l}3 / 45 \\
(7 \%)\end{array}$ & $\begin{array}{l}4 / 49 \\
(8 \%)\end{array}$ & $\begin{array}{c}5 / 48 \\
(10 \%)\end{array}$ & $6 / 46$ \\
\hline & & Severity Profile & $0|0| 0 \mid 2(4.0)$ & $0|0| 0 \mid 2(4.0)$ & $0|0| 0 \mid 3(4.0)$ & $0|0| 1 \mid 3(3.8)$ & $0|0| 0 \mid 54.0)$ & $0|0| 1 \mid 5(3.8)$ \\
\hline & & Poly-3 incidence & $2 / 35.6^{*}$ & $2 / 33.5$ & $3 / 28.5$ & $4 / 34.5$ & $5 / 30.1$ & $6 / 33.0^{c}$ \\
\hline & & & $(6 \%)$ & $(6 \%)$ & $(10 \%)$ & $(12 \%)$ & $(17 \%)$ & $(18 \%)$ \\
\hline
\end{tabular}

${ }^{\mathrm{a}} \mathrm{BPA}$ doses are $\mu \mathrm{g} / \mathrm{kg}$ bw/day. See legend to Table 55 for description of data presentation.

${ }^{b}$ In the sensitivity analysis that excluded all animals that overlapped with animals treated with $250,000 \mu \mathrm{g}$ BPA/kg bw/day (see Statistical Methods), there was a significant $(p=0.048)$ difference for the CAFE test for the pairwise comparison of the $25,000 \mu \mathrm{g} \mathrm{BPA} / \mathrm{kg}$ bw/day group to the vehicle control (squamous metaplasia, $5 / 20(25 \%)$ versus $0 / 15$ $(0 \%))$. 


\section{CLARITY-BPA Core Study}

'In the sensitivity analysis that excluded all animals that overlapped with animals treated with $250,000 \mu \mathrm{g}$ BPA/kg bw/day (see Statistical Methods), there was a significant Poly-3 test $(p=0.035)$ for the pairwise comparison of the $25,000 \mu \mathrm{g} \mathrm{BPA} / \mathrm{kg}$ bw/day group to the vehicle control group (lumen dilatation, Poly-3 incidences, $5 / 26.8(19 \%)$ versus $0 / 24$ $(0 \%)$. 
CLARITY-BPA Core Study

Table 62. Non-Neoplastic Lesions in the Uterus of Interim and Terminal Sacrifice Female Rats: Continuous-Dose $\mathbf{E E}_{2}{ }^{\mathrm{a}}$

\begin{tabular}{|c|c|c|c|c|c|}
\hline Lesion & $\begin{array}{c}\text { Study Phase } \\
\text { (Interim/Terminal) }\end{array}$ & Statistic & Vehicle & $0.05 \mathrm{EE}_{2}$ & $0.5 \mathrm{EE}_{2}$ \\
\hline \multirow[t]{3}{*}{ Apoptosis } & \multirow[t]{3}{*}{ Interim } & Incidence & $2 / 23^{* * *, \ldots \# \#, \wedge \wedge \wedge}$ & $6 / 25$ & $18 / 26^{* * *, \# \# \#, \wedge \wedge \wedge}$ \\
\hline & & & $(9 \%)$ & $(24 \%)$ & $(69 \%)$ \\
\hline & & Severity Profile & $0|0| 0 \mid 2(4.0)$ & $0|1| 2 \mid 3(3.3)$ & $0|2| 6 \mid 10(3.4)$ \\
\hline \multirow{8}{*}{$\begin{array}{l}\text { Hyperplasia, cystic, } \\
\text { endometrium }\end{array}$} & \multirow[t]{3}{*}{ Interim } & Incidence & $5 / 23^{*}, \#, \wedge$ & $6 / 25$ & $14 / 26^{*}, \#, \wedge$ \\
\hline & & & $(22 \%)$ & $(24 \%)$ & $(54 \%)$ \\
\hline & & Severity Profile & $1|2| 1 \mid 1(2.4)$ & $3|3| 0 \mid 0(1.5)$ & $4|6| 2 \mid 2(2.1)$ \\
\hline & \multirow[t]{5}{*}{ Terminal } & Incidence & $30 / 50$ & $14 / 26$ & $14 / 26$ \\
\hline & & & $(60 \%)$ & $(54 \%)$ & $(54 \%)$ \\
\hline & & Severity Profile & $4|18| 5 \mid 3(2.2)$ & $1|7| 4 \mid 2(2.5)$ & $1|8| 2 \mid 3(2.5)$ \\
\hline & & Poly-3 Incidence & $30 / 41.9$ & $14 / 21.8$ & $14 / 20.2$ \\
\hline & & & $(72 \%)$ & $(64 \%)$ & $(69 \%)$ \\
\hline \multirow{8}{*}{$\begin{array}{l}\text { Hyperplasia, } \\
\text { endometrium }\end{array}$} & \multirow[t]{3}{*}{ Interim } & Incidence & $2 / 23$ & $4 / 25$ & $0 / 26$ \\
\hline & & & $(9 \%)$ & $(16 \%)$ & $(0 \%)$ \\
\hline & & Severity Profile & $0|2| 0 \mid 0(2.0)$ & $0|4| 0 \mid 0(2.0)$ & - \\
\hline & \multirow[t]{5}{*}{ Terminal } & Incidence & $10 / 50$ & $10 / 26^{\wedge}$ & $2 / 26$ \\
\hline & & & $(20 \%)$ & $(38 \%)$ & $(8 \%)$ \\
\hline & & Severity Profile & $2|8| 0 \mid 0(1.8)$ & $3|6| 0 \mid 1(1.9)$ & $1|1| 0 \mid 0(1.5)$ \\
\hline & & Poly-3 Incidence & $10 / 39.1$ & $10 / 19.4^{*}$ & $2 / 16.2$ \\
\hline & & & $(26 \%)$ & $(52 \%)$ & $(12 \%)$ \\
\hline \multirow[t]{8}{*}{ Metaplasia, squamous } & \multirow[t]{3}{*}{ Interim } & Incidence & $1 / 23^{* * *, \# \# \#, \wedge \wedge \wedge}$ & $2 / 25$ & $14 / 26^{* * *, \# \# \#, \wedge \wedge \wedge}$ \\
\hline & & & $(4 \%)$ & $(8 \%)$ & $(54 \%)$ \\
\hline & & Severity Profile & $0|1| 0 \mid 0(2.0)$ & $1|0| 1 \mid 0(2.0)$ & $8|4| 2 \mid 0(1.6)$ \\
\hline & \multirow[t]{5}{*}{ Terminal } & Incidence & $2 / 50^{\#}$ & $2 / 26$ & $4 / 26$ \\
\hline & & & $(4 \%)$ & $(8 \%)$ & $(15 \%)$ \\
\hline & & Severity Profile & $1|1| 0 \mid 0(1.5)$ & $0|2| 0 \mid 0(2.0)$ & $3|0| 1 \mid 0(1.5)$ \\
\hline & & Poly-3 Incidence & $2 / 35.7^{*}$ & $2 / 16.1$ & $4 / 16.9$ \\
\hline & & & $(6 \%)$ & $(12 \%)$ & $(24 \%)$ \\
\hline \multirow[t]{8}{*}{ Dilatation, lumen } & \multirow[t]{3}{*}{ Interim } & Incidence & $0 / 23$ & $1 / 25$ & $0 / 26$ \\
\hline & & & $(0 \%)$ & $(4 \%)$ & $(0 \%)$ \\
\hline & & Severity Profile & - & $0 / 0 / 0 / 1(4.0)$ & - \\
\hline & \multirow[t]{5}{*}{ Terminal } & Incidence & $2 / 50$ & $2 / 26$ & $3 / 26$ \\
\hline & & & $(4 \%)$ & $(8 \%)$ & $(12 \%)$ \\
\hline & & Severity Profile & $0|0| 0 \mid 2(4.0)$ & $0|0| 0 \mid 2(4.0)$ & $0|0| 0 \mid 3(4.0)$ \\
\hline & & Poly-3 Incidence ${ }^{b}$ & $2 / 35.6$ & $2 / 16.9$ & $3 / 16.0^{c}$ \\
\hline & & & $(6 \%)$ & $(12 \%)$ & $(19 \%)$ \\
\hline
\end{tabular}

${ }^{\mathrm{a}} \mathrm{EE}_{2}$ doses are $\mu \mathrm{g} / \mathrm{kg}$ bw/day. See legend to Table 55 for description of data presentation.

${ }^{\text {b }}$ The continuous vehicle control group data are the same as those shown for the continuous-dose BPA data in Table 61 .

'In the sensitivity analysis that excluded all animals that overlapped with animals treated with $250,000 \mu \mathrm{g}$ BPA/kg bw/day (see Statistical Methods), there was a significant EE dose trend $(p=0.008)$ and a significant pairwise comparison $(p=0.013)$ for the $0.5 \mu \mathrm{g} \mathrm{EE} / \mathrm{kg}$ bw/day dose group versus the vehicle control group (lumen dilatation, Poly-3 incidences $3 / 10.5(29 \%)$ versus $0 / 24(0 \%))$. 
CLARITY-BPA Core Study

Table 63. Non-Neoplastic Lesions in the Uterus of Interim and Terminal Sacrifice Female Rats: Stop-Dose BPA ${ }^{\mathrm{a}}$

\begin{tabular}{|c|c|c|c|c|c|c|c|c|}
\hline Lesion & $\begin{array}{c}\text { Study Phase } \\
\text { (Interim/Terminal) }\end{array}$ & Statistic & Vehicle & 2.5 BPA & 25 BPA & 250 BPA & 2500 BPA & 25000 BPA \\
\hline \multirow[t]{2}{*}{ Apoptosis } & \multirow[t]{2}{*}{ Interim } & Incidence & $\begin{array}{l}2 / 20 \\
(10 \%)\end{array}$ & $\begin{array}{c}3 / 22 \\
(14 \%)\end{array}$ & $\begin{array}{l}2 / 20 \\
(10 \%)\end{array}$ & $\begin{array}{l}2 / 22 \\
(9 \%)\end{array}$ & $\begin{array}{l}1 / 20 \\
(5 \%)\end{array}$ & $\begin{array}{c}6 / 22 \\
(27 \%)\end{array}$ \\
\hline & & Severity Profile & $0|0| 0 \mid 2(4.0)$ & $0|1| 0 \mid 2(3.3)$ & $0|0| 1 \mid 1(3.5)$ & $0|0| 2 \mid 0(3.0)$ & $0|0| 0 \mid 1(4.0)$ & $0|1| 0 \mid 5(3.7)$ \\
\hline \multirow{7}{*}{$\begin{array}{l}\text { Hyperplasia, cystic, } \\
\text { endometrium }\end{array}$} & \multirow[t]{3}{*}{ Interim } & Incidence & $2 / 20$ & $4 / 22$ & $2 / 20$ & $2 / 22$ & $1 / 20$ & $7 / 22^{\#, \wedge}$ \\
\hline & & & $(10 \%)$ & $(18 \%)$ & $(10 \%)$ & $(9 \%)$ & $(5 \%)$ & $(32 \%)$ \\
\hline & & Severity Profile & $0|1| 0 \mid 1(3.0)$ & $0|2| 2 \mid 0(2.5)$ & $0|2| 0 \mid 0(2.0)$ & $0|2| 0 \mid 0(2.0)$ & \begin{tabular}{l|l|l|l|l|l|l}
0 & 0 & 0 & 1 & $(4.0)$ \\
\end{tabular} & $2|4| 0 \mid 1(2.0)$ \\
\hline & \multirow[t]{4}{*}{ Terminal } & Incidence & $\begin{array}{c}18 / 49^{\#, \wedge} \\
(37 \%)\end{array}$ & $\begin{array}{l}23 / 49 \\
(47 \%)\end{array}$ & $\begin{array}{l}22 / 48 \\
(46 \%)\end{array}$ & $\begin{array}{l}25 / 49 \\
(51 \%)\end{array}$ & $\begin{array}{c}28 / 49^{\#, \wedge} \\
(57 \%)\end{array}$ & $\begin{array}{l}24 / 46^{\#} \\
(52 \%)\end{array}$ \\
\hline & & Severity Profile & $3|8| 5 \mid 2(2.3)$ & $1|18| 4 \mid 0(2.1)$ & $1|15| 5 \mid 1(2.3)$ & $3|15| 6 \mid 1(2.2)$ & $0|18| 6 \mid 4(2.5)$ & $4|11| 6 \mid 3(2.3)$ \\
\hline & & Poly-3 Incidence & $18 / 38.3$ & $23 / 38.1$ & $22 / 37.1$ & $25 / 41.6$ & $28 / 42.5^{\mathrm{b}}$ & $24 / 38.6$ \\
\hline & & & $(47 \%)$ & $(60 \%)$ & $(59 \%)$ & $(60 \%)$ & $(66 \%)$ & $(62 \%)$ \\
\hline \multirow{8}{*}{$\begin{array}{l}\text { Hyperplasia, } \\
\text { endometrium }\end{array}$} & \multirow[t]{3}{*}{ Interim } & Incidence & $6 / 20$ & $9 / 22$ & $5 / 20$ & $7 / 22$ & $6 / 20$ & $9 / 22$ \\
\hline & & & $(30 \%)$ & $(41 \%)$ & $(25 \%)$ & $(32 \%)$ & $(30 \%)$ & $(41 \%)$ \\
\hline & & Severity Profile & $1|4| 1 \mid 0(2.0)$ & $1|6| 2 \mid 0(2.1)$ & $2|2| 1 \mid 0(1.8)$ & $2|4| 1 \mid 0(1.9)$ & $0|6| 0 \mid 0(2.0)$ & $0|7| 2 \mid 0(2.2)$ \\
\hline & \multirow[t]{5}{*}{ Terminal } & Incidence & $18 / 49$ & $14 / 49$ & $17 / 48$ & $14 / 49$ & $12 / 49$ & $10 / 46$ \\
\hline & & & $(37 \%)$ & $(29 \%)$ & $(35 \%)$ & $(29 \%)$ & $(24 \%)$ & $(22 \%)$ \\
\hline & & Severity Profile & $6|12| 0 \mid 0(1.7)$ & $8|6| 0 \mid 0$ (1.4) & $7|9| 0 \mid 1(1.7)$ & $6|7| 1 \mid 0(1.6)$ & $2|10| 0 \mid 0(1.8)$ & $1|8| 1 \mid 0(2.0)$ \\
\hline & & Poly-3 Incidence & $18 / 38.8^{*} \mathrm{~N}$ & $14 / 37.4$ & $17 / 37.0$ & $14 / 39.2$ & $12 / 38.5$ & $10 / 33.6$ \\
\hline & & & $(46 \%)$ & $(38 \%)$ & $(46 \%)$ & $(36 \%)$ & $(31 \%)$ & $(30 \%)$ \\
\hline \multirow[t]{8}{*}{ Metaplasia, squamous } & \multirow[t]{3}{*}{ Interim } & Incidence & $0 / 20$ & $2 / 22$ & $1 / 20$ & $1 / 22$ & $0 / 20$ & $4 / 22^{\# \#, \wedge \wedge}$ \\
\hline & & & $(0 \%)$ & $(9 \%)$ & $(5 \%)$ & $(4 \%)$ & $(0 \%)$ & $(18 \%)$ \\
\hline & & Severity Profile & - & $2|0| 0 \mid 0(1.0)$ & $0|1| 0 \mid 0(2.0)$ & $1|0| 0 \mid 0(1.0)$ & - & $1|3| 0 \mid 0(1.8)$ \\
\hline & \multirow[t]{5}{*}{ Terminal } & Incidence & $5 / 49$ & $1 / 49^{\wedge} \mathrm{N}$ & $2 / 48$ & $2 / 49$ & $4 / 49$ & $3 / 46$ \\
\hline & & & $(10 \%)$ & $(2 \%)$ & $(4 \%)$ & $(4 \%)$ & $(8 \%)$ & $(6 \%)$ \\
\hline & & Severity Profile & $3|2| 0 \mid 0(1.4)$ & $1|0| 0 \mid 0(1.0)$ & $1|1| 0 \mid 0(1.5)$ & $0|2| 0 \mid 0(2.0)$ & $3|1| 0 \mid 0(1.2)$ & $3|0| 0 \mid 0(1.0)$ \\
\hline & & Poly-3 Incidence & $5 / 33.1$ & $1 / 31.7$ & $2 / 30.8$ & $2 / 34.8$ & $4 / 36.2$ & $3 / 32.6$ \\
\hline & & & $(15 \%)$ & $(3 \%)$ & $(6 \%)$ & $(6 \%)$ & $(11 \%)$ & $(9 \%)$ \\
\hline \multirow[t]{8}{*}{ Dilatation, lumen } & \multirow[t]{3}{*}{ Interim } & Incidence & $1 / 20$ & $0 / 22$ & $1 / 20$ & $4 / 22^{\wedge}$ & $1 / 20$ & $0 / 22$ \\
\hline & & & $(5 \%)$ & $(0 \%)$ & $(5 \%)$ & $(18 \%)$ & $(5 \%)$ & $(0 \%)$ \\
\hline & & Severity Profile & $0|0| 0 \mid 1(4.0)$ & - & $0|0| 0 \mid 1(4.0)$ & $0|0| 1 \mid 3(3.8)$ & $0|0| 1 \mid 0(3.0)$ & - \\
\hline & \multirow[t]{5}{*}{ Terminal } & Incidence & $3 / 49^{\#, \wedge \mathrm{N}}$ & $6 / 49$ & $2 / 48$ & $4 / 49$ & $2 / 49$ & $0 / 46$ \\
\hline & & & $(6 \%)$ & $(12 \%)$ & $(4 \%)$ & $(8 \%)$ & $(4 \%)$ & $(0 \%)$ \\
\hline & & Severity Profile & $0|0| 1 \mid 2(3.7)$ & $0|0| 0 \mid 6$ (4.0) & $0|0| 0 \mid 2$ (4.0) & $0|0| 0 \mid 4$ (4.0) & $0|0| 1 \mid 1(3.5)$ & - \\
\hline & & Poly-3 Incidence & $3 / 32.5^{* \mathrm{~N}}$ & $6 / 33.0$ & $2 / 31.3$ & $4 / 33.8$ & $2 / 35.2$ & $0 / 30.9$ \\
\hline & & & $(9 \%)$ & $(18 \%)$ & $(6 \%)$ & $(12 \%)$ & $(6 \%)$ & $(0 \%)$ \\
\hline
\end{tabular}

BPA doses are $\mu \mathrm{g} / \mathrm{kg}$ bw/day. See legend to Table 55 for description of data presentation.

${ }^{\mathrm{b}}$ In the sensitivity analysis that excluded all animals that overlapped with animals treated with $250,000 \mu \mathrm{g}$ BPA/kg bw/day (see Statistical Methods), there was a significant Poly-3 test $(p=0.038)$ for the comparison of $2,500 \mu \mathrm{g} \mathrm{BPA} / \mathrm{kg}$ bw/day group to vehicle control (endometrial cystic hyperplasia, Poly-3 incidences, $23 / 43.3(53 \%)$ versus $12 / 28.2(43 \%)$ ). 
CLARITY-BPA Core Study

Table 64. Non-Neoplastic Lesions in the Ovary of Interim and Terminal Sacrifice Female Rats: Continuous-Dose BPA ${ }^{\mathrm{a}}$

\begin{tabular}{|c|c|c|c|c|c|c|c|c|}
\hline Lesion & $\begin{array}{c}\text { Study Phase } \\
\text { (Interim/Terminal) }\end{array}$ & Statistic & Vehicle & 2.5 BPA & 25 BPA & 250 BPA & 2500 BPA & 25000 BPA \\
\hline \multirow[t]{8}{*}{ Atrophy } & \multirow[t]{3}{*}{ Interim } & Incidence & $10 / 23$ & $7 / 22$ & $9 / 22$ & $14 / 24$ & $11 / 20$ & $11 / 24$ \\
\hline & & & $(44 \%)$ & $(32 \%)$ & $(41 \%)$ & $(58 \%)$ & $(55 \%)$ & $(46 \%)$ \\
\hline & & Severity Profile & $1|4| 0 \mid 5(2.9)$ & $0|3| 0 \mid 4(3.1)$ & $0|2| 0 \mid 7$ (3.6) & $6|4| 0 \mid 4(2.1)$ & $2|1| 0 \mid 8(3.3)$ & $0|2| 0 \mid 9(3.6)$ \\
\hline & \multirow[t]{5}{*}{ Terminal } & Incidence & $47 / 50$ & $45 / 48$ & $44 / 46$ & $46 / 49$ & $45 / 50$ & $46 / 46$ \\
\hline & & & $(94 \%)$ & $(94 \%)$ & $(96 \%)$ & $(94 \%)$ & $(90.0 \%)$ & $(100 \%)$ \\
\hline & & Severity Profile & $0|24| 15 \mid 8(2.7)$ & $1|27| 9 \mid 8(2.5)$ & $1|25| 8 \mid 10(2.6)$ & $0|27| 11 \mid 8(2.6)$ & $0|26| 7 \mid 12(2.7)$ & $0|23| 12 \mid 11(2.7)$ \\
\hline & & Poly-3 Incidence & $47 / 48.7$ & $45 / 45.1$ & $44 / 44.2$ & $46 / 47.0$ & $45 / 45.8$ & $46 / 46.0$ \\
\hline & & & $(96 \%)$ & $(100 \%)$ & $(100 \%)$ & $(98 \%)$ & $(98 \%)$ & $(100 \%)$ \\
\hline \multirow{8}{*}{ Cyst, follicle } & \multirow{3}{*}{ Interim } & Incidence & $8 / 23$ & $4 / 22$ & $10 / 22$ & $5 / 24$ & $10 / 20$ & $11 / 24$ \\
\hline & & & $(35 \%)$ & $(18 \%)$ & $(46 \%)$ & $(21 \%)$ & $(50 \%)$ & $(46 \%)$ \\
\hline & & Severity Profile & $-^{\mathrm{b}}$ & - & - & - & - & - \\
\hline & \multirow[t]{5}{*}{ Terminal } & Incidence & $3 / 50$ & $3 / 48$ & $2 / 46$ & $7 / 49$ & $6 / 50$ & $4 / 46$ \\
\hline & & & $(6 \%)$ & $(6 \%)$ & $(4 \%)$ & $(14 \%)$ & $(12 \%)$ & $(9 \%)$ \\
\hline & & Severity Profile & $-{ }^{\mathrm{b}}$ & - & - & - & - & - \\
\hline & & Poly-3 Incidence & $3 / 35.7$ & $3 / 33.4$ & $2 / 28.9$ & $7 / 34.5$ & $6 / 30.8$ & $4 / 32.3$ \\
\hline & & & $(8 \%)$ & $(9 \%)$ & $(7 \%)$ & $(20 \%)$ & $(20 \%)$ & $(12 \%)$ \\
\hline \multirow{3}{*}{$\begin{array}{l}\text { Depletion, } \\
\text { Corpus luteum }\end{array}$} & \multirow[t]{3}{*}{ Interim } & Incidence & $4 / 23^{*}$ & $4 / 22$ & $7 / 22$ & $4 / 24$ & $8 / 20$ & $9 / 24$ \\
\hline & & & $(17 \%)$ & $(18 \%)$ & $(32 \%)$ & $(17 \%)$ & $(40 \%)$ & $(38 \%)$ \\
\hline & & Severity Profile & $-^{\mathrm{b}}$ & - & - & - & - & - \\
\hline \multirow{3}{*}{$\begin{array}{l}\text { Hypertrophy, } \\
\text { Interstitial cell }\end{array}$} & \multirow[t]{3}{*}{ Interim } & Incidence & $4 / 23^{*}, \#, \wedge$ & $4 / 22$ & $6 / 22$ & $3 / 24$ & $8 / 20^{\wedge}$ & $9 / 24$ \\
\hline & & & $(17 \%)$ & $(18 \%)$ & $(27 \%)$ & $(12 \%)$ & $(40 \%)$ & $(38 \%)$ \\
\hline & & Severity Profile & $0|4| 0 \mid 0(2.0)$ & $0|3| 1 \mid 0(2.2)$ & $0|2| 4 \mid 0(2.7)$ & $0|0| 2 \mid 1(3.3)$ & $0|6| 2 \mid 0(2.2)$ & $1|7| 1 \mid 0(2.0)$ \\
\hline
\end{tabular}

${ }^{a}$ BPA doses are $\mu \mathrm{g} / \mathrm{kg}$ bw/day. See legend to Table 55 for description of data presentation.

${ }^{\mathrm{b}} \mathrm{B}$ everity scores were not assigned for this lesion. 
CLARITY-BPA Core Study

Table 65. Non-Neoplastic Lesions in the Ovary of Interim and Terminal Sacrifice Female Rats: Continuous-Dose $\mathrm{EE}_{2}{ }^{\mathrm{a}}$

\begin{tabular}{|c|c|c|c|c|c|}
\hline Lesion & $\begin{array}{c}\text { Study Phase } \\
\text { (Interim/Terminal) }\end{array}$ & Statistic & Vehicle $^{b}$ & $0.05 \mathrm{EE}_{2}$ & $0.5 \mathrm{EE}_{2}$ \\
\hline \multirow[t]{8}{*}{ Atrophy } & \multirow[t]{3}{*}{ Interim } & \multirow[t]{2}{*}{ Incidence } & $10 / 23^{* * *, \# \# \#, \wedge \wedge \wedge}$ & $9 / 25$ & $26 / 26^{* * * *}, \# \#, \wedge \wedge \wedge$ \\
\hline & & & $(44 \%)$ & $(36 \%)$ & $(100 \%)$ \\
\hline & & Severity Profile & $1|4| 0 \mid 5(2.9)$ & $0|3| 0 \mid 6(3.3)$ & $0|0| 0 \mid 26(4.0)$ \\
\hline & \multirow[t]{5}{*}{ Terminal } & \multirow[t]{2}{*}{ Incidence } & $47 / 50^{\# \# \#, \wedge \wedge \wedge}$ & $25 / 26$ & $26 / 26^{\# \#, \wedge \wedge \wedge}$ \\
\hline & & & $(94 \%)$ & $(96 \%)$ & $(100 \%)$ \\
\hline & & Severity Profile & $0|24| 15 \mid 8(2.7)$ & $0|12| 7 \mid 6(2.8)$ & $0|1| 1 \mid 24(3.9)$ \\
\hline & & \multirow[t]{2}{*}{ Poly-3 Incidence } & $47 / 48.7$ & $25 / 26.0$ & $26 / 26.0$ \\
\hline & & & $(96 \%)$ & $(96 \%)$ & $(100 \%)$ \\
\hline \multirow[t]{8}{*}{ Cyst, follicle } & \multirow[t]{3}{*}{ Interim } & \multirow[t]{2}{*}{ Incidence } & $8 / 23^{* * * *}$ & $9 / 25$ & $26 / 26^{* * *}$ \\
\hline & & & $(35 \%)$ & $(36 \%)$ & $(100 \%)$ \\
\hline & & Severity Profile & $-^{\mathrm{c}}$ & - & - \\
\hline & \multirow[t]{5}{*}{ Terminal } & \multirow[t]{2}{*}{ Incidence } & $3 / 50$ & $0 / 26$ & $3 / 26$ \\
\hline & & & $(6 \%)$ & $(0 \%)$ & $(12 \%)$ \\
\hline & & Severity Profile & $-^{c}$ & - & - \\
\hline & & \multirow[t]{2}{*}{ Poly-3 Incidence } & $3 / 35.7$ & $0 / 15.9$ & $3 / 16.1$ \\
\hline & & & $(8 \%)$ & $(0 \%)$ & $(19 \%)$ \\
\hline \multirow{3}{*}{$\begin{array}{l}\text { Depletion, Corpus } \\
\text { luteum }\end{array}$} & \multirow[t]{3}{*}{ Interim } & \multirow[t]{2}{*}{ Incidence } & $4 / 23^{* * *}$ & $6 / 25$ & $26 / 26^{* * *}$ \\
\hline & & & $(17 \%)$ & $(24 \%)$ & $(100 \%)$ \\
\hline & & Severity Profile & $-^{c}$ & - & - \\
\hline \multirow{3}{*}{$\begin{array}{l}\text { Hypertrophy, } \\
\text { Interstitial cell }\end{array}$} & \multirow[t]{3}{*}{ Interim } & \multirow[t]{2}{*}{ Incidence } & $4 / 23^{* * *, \# \# \#, \wedge \wedge \wedge}$ & $5 / 25$ & $26 / 26^{* * * *}, \# \# \#, \wedge \wedge \wedge$ \\
\hline & & & $(17 \%)$ & $(20.0 \%)$ & $(100 \%)$ \\
\hline & & Severity Profile & $0|4| 0 \mid 0(2.0)$ & $0|4| 1 \mid 0(2.2)$ & $1|13| 10 \mid 2(2.5)$ \\
\hline
\end{tabular}

${ }^{\mathrm{a}} \mathrm{EE}_{2}$ doses are $\mu \mathrm{g} / \mathrm{kg}$ bw/day. See legend to Table 55 for description of data presentation.

${ }^{\mathrm{b}}$ The continuous vehicle control group data are the same as those shown for the continuous-dose BPA data in Table 64.

'Severity scores were not assigned for this lesion. 
CLARITY-BPA Core Study

Table 66. Non-Neoplastic Lesions in the Ovary of Interim and Terminal Sacrifice Female Rats: Stop-Dose BPA ${ }^{\mathrm{a}}$

\begin{tabular}{|c|c|c|c|c|c|c|c|c|}
\hline Lesion & $\begin{array}{c}\text { Study Phase } \\
\text { (Interim/Terminal) }\end{array}$ & Statistic & Vehicle & $2.5 \mathrm{BPA}$ & 25 BPA & 250 BPA & 2500 BPA & 25000 BPA \\
\hline \multirow[t]{8}{*}{ Atrophy } & \multirow[t]{3}{*}{ Interim } & Incidence & $10 / 20$ & $9 / 22$ & $11 / 20$ & $6 / 22$ & $12 / 20$ & $15 / 22$ \\
\hline & & & $(50 \%)$ & $(41 \%)$ & $(55 \%)$ & $(27 \%)$ & $(60 \%)$ & $(68 \%)$ \\
\hline & & Severity Profile & $2|6| 0 \mid 2(2.2)$ & $1|4| 0 \mid 4(2.8)$ & $8|1| 0 \mid 2(1.6)$ & $1|3| 0 \mid 2(2.5)$ & $5|4| 0 \mid 3(2.1)$ & $4|5| 0 \mid 6(2.5)$ \\
\hline & \multirow[t]{5}{*}{ Terminal } & Incidence & $47 / 49$ & $48 / 49$ & $46 / 47$ & $50 / 50$ & $49 / 50$ & $44 / 46$ \\
\hline & & & $(96 \%)$ & $(98 \%)$ & $(98 \%)$ & $(100 \%)$ & $(98 \%)$ & $(96 \%)$ \\
\hline & & Severity Profile & $1|23| 16 \mid 7$ (2.6) & $0|34| 11 \mid 3(2.4)$ & $1|28| 11 \mid 6(2.5)$ & $1|33| 7 \mid 9(2.5)$ & $0|25| 16 \mid 8(2.7)$ & $0|23| 12 \mid 9(2.7)$ \\
\hline & & Poly-3 Incidence & $47 / 47.5$ & $48 / 48.1$ & $46 / 46.1$ & $50 / 50.0$ & $49 / 49.0$ & $44 / 44.8$ \\
\hline & & & $(99 \%)$ & $(100 \%)$ & $(100 \%)$ & $(100 \%)$ & $(100 \%)$ & $(98 \%)$ \\
\hline \multirow[t]{8}{*}{ Cyst, follicle } & \multirow[t]{3}{*}{ Interim } & Incidence & $5 / 20^{* * * *}$ & $6 / 22$ & $4 / 20$ & $7 / 22$ & $11 / 20$ & $18 / 22^{* * *}$ \\
\hline & & & $(25 \%)$ & $(27 \%)$ & $(20 \%)$ & $(32 \%)$ & $(55 \%)$ & $(82 \%)$ \\
\hline & & Severity Profile & $-^{\mathrm{b}}$ & - & - & - & - & - \\
\hline & \multirow[t]{5}{*}{ Terminal } & Incidence & $4 / 49$ & $4 / 49$ & $2 / 47$ & $1 / 50$ & $2 / 50$ & $4 / 46$ \\
\hline & & & $(8 \%)$ & $(8 \%)$ & $(4 \%)$ & $(2 \%)$ & $(4 \%)$ & $(9 \%)$ \\
\hline & & Severity Profile & $-{ }^{\mathrm{b}}$ & - & - & - & - & - \\
\hline & & Poly-3 Incidence & $4 / 32.0$ & $4 / 33.5$ & $2 / 30.2$ & $1 / 34.6$ & $2 / 35.7$ & $4 / 32.4$ \\
\hline & & & $12 \%)$ & $(12 \%)$ & $(7 \%)$ & $(3 \%)$ & $(6 \%)$ & $(12 \%)$ \\
\hline \multirow{3}{*}{$\begin{array}{l}\text { Depletion, } \\
\text { Corpus luteum }\end{array}$} & \multirow[t]{3}{*}{ Interim } & Incidence & $2 / 20$ & $4 / 22$ & $2 / 20$ & $2 / 22$ & $3 / 20$ & $6 / 22$ \\
\hline & & & $(10 \%)$ & $(18 \%)$ & $(10 \%)$ & $(9 \%)$ & $(15 \%)$ & $(27 \%)$ \\
\hline & & Severity Profile & $-^{\mathrm{b}}$ & - & - & - & - & - \\
\hline \multirow{3}{*}{$\begin{array}{l}\text { Hypertrophy, } \\
\text { Interstitial cell }\end{array}$} & \multirow[t]{3}{*}{ Interim } & Incidence & $4 / 20$ & $3 / 22$ & $1 / 20$ & $2 / 22$ & $3 / 20$ & $5 / 22$ \\
\hline & & & $(20 \%)$ & $(14 \%)$ & $(5 \%)$ & $(9 \%)$ & $(15 \%)$ & $(23 \%)$ \\
\hline & & Severity Profile & $0|1| 3 \mid 0(2.8)$ & $0|2| 1 \mid 0(2.3)$ & $0|1| 0 \mid 0(2.0)$ & $0|2| 0 \mid 0(2.0)$ & $0|3| 0 \mid 0(2.0)$ & $0|4| 1 \mid 0(2.2)$ \\
\hline
\end{tabular}

${ }^{a} \mathrm{BPA}$ doses are $\mu \mathrm{g} / \mathrm{kg}$ bw/day. See legend to Table 55 for description of data presentation.

${ }^{\mathrm{b}} \mathrm{B}$ everity scores were not assigned for this lesion. 
CLARITY-BPA Core Study

Table 67. Non-Neoplastic Lesions in the Vagina of Interim and Terminal Sacrifice Female Rats: Continuous-Dose BPA ${ }^{\mathrm{a}}$

\begin{tabular}{|c|c|c|c|c|c|c|c|c|}
\hline Lesion & $\begin{array}{c}\text { Study Phase } \\
\text { (Interim/Terminal) }\end{array}$ & Statistic & Vehicle & 2.5 BPA & 25 BPA & 250 BPA & 2500 BPA & 25000 BPA \\
\hline \multirow{8}{*}{$\begin{array}{l}\text { Hyperplasia, } \\
\text { epithelium }\end{array}$} & \multirow[t]{3}{*}{ Interim } & Incidence & $3 / 23^{* *,}, \# \#, \wedge \wedge$ & $2 / 22$ & $2 / 21$ & $4 / 24$ & $6 / 20$ & $8 / 24^{\#, \wedge}$ \\
\hline & & & $(13 \%)$ & $(9 \%)$ & $(10 \%)$ & $(17 \%)$ & $(30 \%)$ & $(33 \%)$ \\
\hline & & Severity Profile & $0|1| 2 \mid 0(2.7)$ & $0|1| 1 \mid 0(2.5)$ & $0|1| 1 \mid 0(2.5)$ & $0|0| 4 \mid 0(3.0)$ & $0|1| 5 \mid 0(2.8)$ & $0|1| 7 \mid 0(2.9)$ \\
\hline & \multirow[t]{5}{*}{ Terminal } & Incidence & $4 / 49^{\# \#, \wedge \wedge}$ & $5 / 48$ & $12 / 45^{\# \#, \wedge}$ & $10 / 49^{\#}$ & $11 / 50^{\#, \wedge}$ & $12 / 46^{\#, \wedge}$ \\
\hline & & & $(8 \%)$ & $(10 \%)$ & $(27 \%)$ & $(20 \%)$ & $(22 \%)$ & $(26 \%)$ \\
\hline & & Severity Profile & $0|1| 2 \mid 1((3.0)$ & $0|1| 4 \mid 0(2.8)$ & $1|3| 6 \mid 2(2.8)$ & $0|7| 3 \mid 0(2.3)$ & $0|3| 5 \mid 3(3.0)$ & $0|6| 4 \mid 2(2.7)$ \\
\hline & & Poly-3 Incidence & $4 / 35.2^{* *}$ & $5 / 35.3$ & $12 / 33.7^{*}$ & $10 / 36.5$ & $11 / 33.4^{*}$ & $12 / 36.8^{*}$ \\
\hline & & & $(11 \%)$ & $(14 \%)$ & $(36 \%)$ & $(27 \%)$ & $(33 \%)$ & $(33 \%)$ \\
\hline \multirow{8}{*}{$\begin{array}{l}\text { Mucification, } \\
\text { epithelium }\end{array}$} & \multirow[t]{3}{*}{ Interim } & Incidence & $10 / 23$ & $12 / 22$ & $7 / 21$ & $9 / 24$ & $7 / 20$ & $8 / 24$ \\
\hline & & & $(44 \%)$ & $(54 \%)$ & $(33 \%)$ & $(38 \%)$ & $(35 \%)$ & $(33 \%)$ \\
\hline & & Severity Profile & $0|6| 1 \mid 3(2.7)$ & $0|7| 4 \mid 1(2.5)$ & $0|0| 3 \mid 4(3.6)$ & $0|1| 5 \mid 3(3.2)$ & $0|0| 2 \mid 5(3.7)$ & $1|3| 1 \mid 3(2.8)$ \\
\hline & \multirow[t]{5}{*}{ Terminal } & Incidence & $46 / 49$ & $37 / 48$ & $34 / 45$ & $39 / 49$ & $34 / 50^{\wedge} \mathrm{N}$ & $40 / 46$ \\
\hline & & & $(94 \%)$ & $(77 \%)$ & $(76 \%)$ & $(80 \%)$ & $(68 \%)$ & $(87 \%)$ \\
\hline & & Severity Profile & $0|13| 12 \mid 21$ (3.2) & $0|7| 8 \mid 22(3.4)$ & $0|7| 11 \mid 16(3.3)$ & $0|4| 14 \mid 21(3.4)$ & $0|8| 9 \mid 17$ (3.3) & $1|10| 8 \mid 21(3.2)$ \\
\hline & & Poly-3 Incidence & $46 / 47.2$ & $37 / 42.0$ & $34 / 39.3^{* N}$ & $39 / 45.2^{*} \mathrm{~N}$ & $34 / 42.8^{* *} \mathrm{~N}$ & $40 / 43.6$ \\
\hline & & & $(97 \%)$ & $(88 \%)$ & $(87 \%)$ & $(86 \%)$ & $(79 \%)$ & $(92 \%)$ \\
\hline
\end{tabular}

${ }^{a}$ BPA doses are $\mu \mathrm{g} / \mathrm{kg}$ bw/day. See legend to Table 55 for description of data presentation. 
CLARITY-BPA Core Study

Table 68. Non-Neoplastic Lesions in the Vagina of Interim and Terminal Sacrifice Female Rats: Continuous-Dose EE $_{2}{ }^{\mathrm{a}}$

\begin{tabular}{|c|c|c|c|c|c|}
\hline Lesion & $\begin{array}{c}\text { Study Phase } \\
\text { (Interim/Terminal) }\end{array}$ & Statistic & Vehicle $^{\text {b }}$ & $0.05 \mathbf{E E}_{2}$ & $0.5 \mathrm{EE}_{2}$ \\
\hline \multirow[t]{7}{*}{$\begin{array}{l}\text { Hyperplasia, } \\
\text { epithelium }\end{array}$} & \multirow[t]{2}{*}{ Interim } & Incidence & $\begin{array}{c}3 / 23^{* * * *}, \# \#, \wedge \wedge \wedge \\
(13 \%)\end{array}$ & $\begin{array}{l}7 / 25 \\
(28 \%)\end{array}$ & $\begin{array}{c}20 / 26^{* * *}, \# \# \#, \wedge \wedge \wedge \\
(77 \%)\end{array}$ \\
\hline & & Severity Profile & $0|1| 2 \mid 0(2.7)$ & $0|4| 3 \mid 0(2.4)$ & $0|9| 11 \mid 0(2.6)$ \\
\hline & \multirow[t]{5}{*}{ Terminal } & Incidence & $4 / 49$ & $5 / 26$ & $2 / 26$ \\
\hline & & & $(8 \%)$ & $(19 \%)$ & $(8 \%)$ \\
\hline & & Severity Profile & $0|1| 2 \mid 1(3.0)$ & $0|1| 2 \mid 2(3.2)$ & $0|1| 1 \mid 0(2.5)$ \\
\hline & & Poly-3 Incidence & $4 / 35.2$ & $5 / 18.6$ & $2 / 15.8$ \\
\hline & & & $(11 \%)$ & $(27 \%)$ & $(13 \%)$ \\
\hline \multirow{8}{*}{$\begin{array}{l}\text { Mucification, } \\
\text { epithelium }\end{array}$} & \multirow[t]{3}{*}{ Interim } & Incidence & $10 / 23^{*}, \#, \wedge$ & $15 / 25$ & $18 / 26^{\#, \wedge}$ \\
\hline & & & $(44 \%)$ & $(60 \%)$ & $(69 \%)$ \\
\hline & & Severity Profile & $0|6| 1 \mid 3(2.7)$ & $0|5| 1 \mid 9(3.3)$ & $1|4| 6 \mid 7(3.1)$ \\
\hline & \multirow[t]{5}{*}{ Terminal } & Incidence & $46 / 49$ & $21 / 26$ & $23 / 26$ \\
\hline & & & $(94 \%)$ & $(81 \%)$ & $(88 \%)$ \\
\hline & & Severity Profile & $0|13| 12 \mid 21(3.2)$ & $0|4| 5 \mid 12(3.4)$ & $0|6| 8 \mid 9(3.1)$ \\
\hline & & Poly-3 Incidence & $46 / 47.2$ & $21 / 24.0$ & $23 / 24.8$ \\
\hline & & & $(97 \%)$ & $(87 \%)$ & $(93 \%)$ \\
\hline
\end{tabular}

${ }^{\mathrm{a}} \mathrm{EE}_{2}$ doses are $\mu \mathrm{g} / \mathrm{kg}$ bw/day. See legend to Table 55 for description of data presentation.

${ }^{\mathrm{b}}$ The continuous vehicle control group data are the same as those shown for the continuous-dose BPA data in Table 67. 
CLARITY-BPA Core Study

Table 69. Non-Neoplastic Lesions in the Vagina of Interim and Terminal Sacrifice Female Rats: Stop-Dose BPA ${ }^{\text {a, } b}$

\begin{tabular}{|c|c|c|c|c|c|c|c|c|}
\hline Lesion & $\begin{array}{c}\text { Study Phase } \\
\text { (Interim/Terminal) } \\
\end{array}$ & Statistic & Vehicle & 2.5 BPA & 25 BPA & 250 BPA & 2500 BPA & 25000 BPA \\
\hline \multirow[t]{5}{*}{$\begin{array}{l}\text { Hyperplasia, } \\
\text { epithelium }\end{array}$} & Interim & Incidence & $\begin{array}{c}2 / 20 \\
(10 \%)\end{array}$ & $\begin{array}{c}4 / 22 \\
(18 \%)\end{array}$ & $\begin{array}{c}2 / 20 \\
(10 \%)\end{array}$ & $\begin{array}{l}1 / 22 \\
(4 \%)\end{array}$ & $\begin{array}{c}2 / 20 \\
(10 \%)\end{array}$ & $\begin{array}{c}6 / 22 \\
(27 \%)\end{array}$ \\
\hline & & Severity Profile & $0|0| 2 \mid 0(3.0)$ & $0|0| 4 \mid 0(3.0)$ & $0|0| 2 \mid 0(3.0)$ & $0|0| 0 \mid 1(4.0)$ & $0|1| 1 \mid 0(2.5)$ & $0|1| 5 \mid 0(2.8)$ \\
\hline & Terminal & Incidence & $\begin{array}{c}6 / 49 \\
(12 \%)\end{array}$ & $\begin{array}{l}10 / 50 \\
(20 \%)\end{array}$ & $\begin{array}{l}3 / 47 \\
(6 \%)\end{array}$ & $\begin{array}{c}7 / 49 \\
(14 \%)\end{array}$ & $\begin{array}{c}7 / 49 \\
(14 \%)\end{array}$ & $\begin{array}{c}7 / 46 \\
(15 \%)\end{array}$ \\
\hline & & Severity Profile & $0|3| 2 \mid 1(2.7)$ & $0|5| 4 \mid 1(2.6)$ & $0|1| 1 \mid 1(3.0)$ & $0|2| 4 \mid 1(2.9)$ & $0|1| 5 \mid 1(3.0)$ & $0|0| 5 \mid 2(3.3)$ \\
\hline & & Poly-3 Incidence & $\begin{array}{l}6 / 33.7 \\
(18 \%) \\
\end{array}$ & $\begin{array}{c}10 / 35.4 \\
(28 \%) \\
\end{array}$ & $\begin{array}{c}3 / 31.8 \\
(9 \%) \\
\end{array}$ & $\begin{array}{l}7 / 35.9 \\
(20 \%) \\
\end{array}$ & $\begin{array}{l}7 / 37.6 \\
(19 \%) \\
\end{array}$ & $\begin{array}{l}7 / 34.6 \\
(20 \%) \\
\end{array}$ \\
\hline \multirow[t]{5}{*}{$\begin{array}{l}\text { Mucification, } \\
\text { epithelium }\end{array}$} & Interim & Incidence & $\begin{array}{c}8 / 20 \\
(40 \%)\end{array}$ & $\begin{array}{l}11 / 22 \\
(50 \%)\end{array}$ & $\begin{array}{c}9 / 20 \\
(45 \%)\end{array}$ & $\begin{array}{l}11 / 22 \\
(50 \%)\end{array}$ & $\begin{array}{l}8 / 20 \\
(40 \%)\end{array}$ & $\begin{array}{l}10 / 22 \\
(45 \%)\end{array}$ \\
\hline & & Severity Profile & $1|2| 1 \mid 4(3.0)$ & $0|3| 4 \mid 4(3.1)$ & $0|3| 4 \mid 2(2.9)$ & $0|6| 2 \mid 3(2.7)$ & $0|1| 3 \mid 4(3.4)$ & $0|2| 3 \mid 5(3.3)$ \\
\hline & Terminal & Incidence & $\begin{array}{l}40 / 49 \\
(82 \%)\end{array}$ & $\begin{array}{l}46 / 50 \\
(92 \%)\end{array}$ & $\begin{array}{l}37 / 47 \\
(79 \%)\end{array}$ & $\begin{array}{l}44 / 49 \\
(90 \%)\end{array}$ & $\begin{array}{l}39 / 49 \\
(80 \%)\end{array}$ & $\begin{array}{l}34 / 46 \\
(74 \%)\end{array}$ \\
\hline & & Severity Profile & $0|7| 12 \mid 21(3.4)$ & $1|7| 15 \mid 23(3.3)$ & $0|9| 9 \mid 19$ (3.3) & $2|12| 12 \mid 18(3.0)$ & $0|8| 9 \mid 22(3.4)$ & $1|5| 11 \mid 17(3.3)$ \\
\hline & & Poly-3 Incidence & $\begin{array}{c}40 / 45.8 \\
(87 \%)\end{array}$ & $\begin{array}{c}46 / 48.1 \\
(96 \%)\end{array}$ & $\begin{array}{c}37 / 42.4 \\
(87 \%)\end{array}$ & $\begin{array}{c}44 / 47.9 \\
(92 \%)\end{array}$ & $\begin{array}{c}39 / 44.6 \\
(88 \%)\end{array}$ & $\begin{array}{c}34 / 39.7 \\
(86 \%)\end{array}$ \\
\hline
\end{tabular}

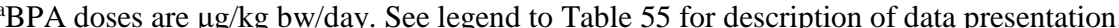

${ }^{b}$ In the sensitivity analysis that excluded all animals that overlapped with animals treated with $250,000 \mu \mathrm{g}$ BPA/kg bw/day (see Statistical Methods), there was also a significant Poly-3 dose trend $(p=0.029)$ for polymorphonuclear cellular infiltration (not shown in Table). The $p$-value for trend with all animals included was 0.136. 
CLARITY-BPA Core Study

Table 70. Neoplastic Lesions in the Pituitary Gland of Interim and Terminal Sacrifice Female Rats: Continuous-Dose BPA ${ }^{\mathrm{a}}$

\begin{tabular}{|c|c|c|c|c|c|c|c|c|}
\hline Lesion & $\begin{array}{c}\text { Study Phase } \\
\text { (Interim/Terminal) }\end{array}$ & Statistic & Vehicle & 2.5 BPA & 25 BPA & 250 BPA & 2500 BPA & 25000 BPA \\
\hline $\begin{array}{l}\text { Adenoma, pars } \\
\text { distalis }\end{array}$ & Interim $^{\mathrm{b}}$ & Incidence & $0 / 23$ & $0 / 22$ & $1 / 22$ & $1 / 24$ & $0 / 20$ & $0 / 24$ \\
\hline \multirow[t]{5}{*}{$\begin{array}{l}\text { Adenoma, pars } \\
\text { distalis }\end{array}$} & Terminal & Incidence & $\begin{array}{l}21 / 50 \\
(42 \%)\end{array}$ & $\begin{array}{l}22 / 48 \\
(46 \%)\end{array}$ & $\begin{array}{l}12 / 46 \\
(26 \%)\end{array}$ & $\begin{array}{l}20 / 49 \\
(41 \%)\end{array}$ & $\begin{array}{l}19 / 49 \\
(39 \%)\end{array}$ & $\begin{array}{l}21 / 46 \\
(46 \%)\end{array}$ \\
\hline & & Poly-3 Incidence & $\begin{array}{c}21 / 39.9 \\
(53 \%)\end{array}$ & $\begin{array}{c}22 / 37.1 \\
(59 \%)\end{array}$ & $\begin{array}{c}12 / 31.4 \\
(38 \%)\end{array}$ & $\begin{array}{c}20 / 36.6 \\
(55 \%)\end{array}$ & $\begin{array}{c}19 / 34.1 \\
(56 \%)\end{array}$ & $\begin{array}{c}21 / 35.4 \\
(59 \%)\end{array}$ \\
\hline & & Terminal Incidence & $\begin{array}{l}7 / 16 \\
(44 \%)\end{array}$ & $\begin{array}{l}12 / 19 \\
(63 \%)\end{array}$ & $\begin{array}{c}4 / 14 \\
(29 \%)\end{array}$ & $\begin{array}{l}8 / 13 \\
(62 \%)\end{array}$ & $\begin{array}{l}5 / 10 \\
(50 \%)\end{array}$ & $\begin{array}{c}4 / 8 \\
(50 \%)\end{array}$ \\
\hline & & Time-to-First & 477 & 497 & 434 & 561 & 456 & 510 \\
\hline & & Poly-3 $p$-value & 0.313 & 0.356 & $0.153 \mathrm{~N}$ & 0.523 & 0.487 & 0.356 \\
\hline \multirow{5}{*}{$\begin{array}{l}\text { Adenoma or } \\
\text { carcinoma, pars } \\
\text { distalis }\end{array}$} & Terminal & Incidence & $\begin{array}{l}22 / 50 \\
(44 \%)\end{array}$ & $\begin{array}{l}23 / 48 \\
(48 \%)\end{array}$ & $\begin{array}{l}12 / 46 \\
(26 \%)\end{array}$ & $\begin{array}{l}20 / 49 \\
(41 \%)\end{array}$ & $\begin{array}{l}19 / 49 \\
(39 \%)\end{array}$ & $\begin{array}{l}21 / 46 \\
(46 \%)\end{array}$ \\
\hline & & Poly-3 Incidence & $\begin{array}{c}22 / 40.3 \\
(55 \%)\end{array}$ & $\begin{array}{c}23 / 37.7 \\
(61 \%)\end{array}$ & $\begin{array}{c}12 / 31.4 \\
(38 \%)\end{array}$ & $\begin{array}{c}20 / 36.6 \\
(55 \%)\end{array}$ & $\begin{array}{c}19 / 34.1 \\
(56 \%)\end{array}$ & $\begin{array}{c}21 / 35.4 \\
(59 \%)\end{array}$ \\
\hline & & Terminal Incidence & $\begin{array}{c}7 / 16 \\
(44 \%)\end{array}$ & $\begin{array}{l}12 / 19 \\
(63 \%)\end{array}$ & $\begin{array}{c}4 / 14 \\
(29 \%)\end{array}$ & $\begin{array}{c}8 / 13 \\
(62 \%)\end{array}$ & $\begin{array}{c}5 / 10 \\
(50 \%)\end{array}$ & $\begin{array}{c}4 / 8 \\
(50.0 \%)\end{array}$ \\
\hline & & Time-to-First & 477 & 497 & 434 & 561 & 456 & 510 \\
\hline & & Poly-3 $p$-value & 0.408 & 0.361 & $0.115 \mathrm{~N}$ & 0.595 & 0.559 & 0.425 \\
\hline
\end{tabular}

${ }^{\mathrm{a}} \mathrm{BPA}$ doses are $\mu \mathrm{g} / \mathrm{kg}$ bw/day. See legend to Table 52 for description of data presentation. There were no significant increases relative to vehicle control.

bo statistical analysis of lesions in interim animals was conducted since no group had two or more lesions. 
CLARITY-BPA Core Study

Table 71. Neoplastic Lesions in the Pituitary Gland of Interim and Terminal Sacrifice Female Rats: Continuous-Dose EE$_{2}{ }^{\mathrm{a}}$

\begin{tabular}{|c|c|c|c|c|c|}
\hline Lesion & $\begin{array}{c}\text { Study Phase } \\
\text { (Interim/Terminal) }\end{array}$ & Statistic & Vehicle & $0.05 \mathbf{E E}_{2}$ & $0.5 \mathrm{EE}_{2}$ \\
\hline Adenoma, pars distalis & Interim $^{\mathrm{b}}$ & Incidence & $0 / 23$ & $1 / 25$ & $1 / 26$ \\
\hline \multirow[t]{5}{*}{ Adenoma, pars distalis } & Terminal & Incidence & $21 / 50(42 \%)$ & $10 / 26(38 \%)$ & $17 / 26(65 \%)$ \\
\hline & & Poly-3 Incidence & $21 / 39.9(53 \%)$ & $10 / 18.3(55 \%)$ & $17 / 23.2(73 \%)$ \\
\hline & & Terminal Incidence & $7 / 16(44 \%)$ & $4 / 7(57 \%)$ & $2 / 4(50 \%)$ \\
\hline & & Time-to-First & 477 & 484 & 360 \\
\hline & & Poly-3 $p$-value & 0.055 & 0.561 & 0.068 \\
\hline \multirow[t]{5}{*}{ Carcinoma, pars distalis } & Terminal & Incidence & $1 / 50(2 \%)$ & $0 / 26(0 \%)$ & $3 / 26(12 \%)$ \\
\hline & & Poly-3 Incidence & $1 / 34.6(3 \%)$ & $0 / 15.9(0 \%)$ & $3 / 16.1(19 \%)$ \\
\hline & & Terminal Incidence & $0 / 16(0 \%)$ & $0 / 7(0 \%)$ & $0 / 4(0 \%)$ \\
\hline & & Time-to-First & 615 & - & 610 \\
\hline & & Poly-3 $p$-value & 0.053 & $0.652 \mathrm{~N}$ & 0.084 \\
\hline \multirow{5}{*}{$\begin{array}{l}\text { Adenoma or carcinoma, } \\
\text { pars distalis }\end{array}$} & Terminal & Incidence & $22 / 50(44 \%)$ & $10 / 26(38 \%)$ & $20 / 26(77 \%)$ \\
\hline & & Poly-3 Incidence & $22 / 40.3(55 \%)$ & $10 / 18.3(55 \%)$ & $20 / 24.2(83 \%)$ \\
\hline & & Terminal Incidence & $7 / 16(44 \%)$ & $4 / 7(57 \%)$ & $2 / 4(50 \%)$ \\
\hline & & Time-to-First & 477 & 484 & 360 \\
\hline & & Poly-3 $p$-value & $0.009 * *$ & $0.615 \mathrm{~N}$ & $0.011^{*}$ \\
\hline
\end{tabular}

BPA doses are $\mu \mathrm{g} / \mathrm{kg}$ bw/day. See legend to Table 52 for description of data presentation.

bo statistical analysis of lesions in interim animals was conducted since no group had two or more lesions. 
CLARITY-BPA Core Study

Table 72. Neoplastic Lesions in the Pituitary Gland of Interim and Terminal Sacrifice Female Rats: Stop-Dose BPA ${ }^{\text {a }}$

\begin{tabular}{|c|c|c|c|c|c|c|c|c|}
\hline Lesion & $\begin{array}{c}\text { Study Phase } \\
\text { (Interim/Terminal) }\end{array}$ & Statistic & Vehicle & $2.5 \mathrm{BPA}$ & 25 BPA & 250 BPA & 2500 BPA & 25000 BPA \\
\hline \multirow[t]{5}{*}{$\begin{array}{l}\text { Adenoma or carcinoma, } \\
\text { pars distalis }\end{array}$} & Interim & Incidence & $\begin{array}{l}0 / 20 \\
(0 \%)\end{array}$ & $\begin{array}{l}0 / 22 \\
(0 \%)\end{array}$ & $\begin{array}{c}2 / 20 \\
(10 \%)^{b}\end{array}$ & $\begin{array}{l}0 / 22 \\
(0 \%)\end{array}$ & $\begin{array}{l}0 / 20 \\
(0 \%)\end{array}$ & $\begin{array}{l}0 / 22 \\
(0 \%)\end{array}$ \\
\hline & & Terminal Incidence & $0 / 20$ & $0 / 22$ & $2 / 20$ & $0 / 22$ & $0 / 20$ & $0 / 22$ \\
\hline & & & $(0 \%)$ & $(0 \%)$ & $(10 \%)$ & $(0 \%)$ & $(0 \%)$ & $(0 \%)$ \\
\hline & & Time-to-First & - & - & $364(\mathrm{~T})$ & - & - & - \\
\hline & & CAFE $p$-value & $0.408 \mathrm{~N}$ & - & 0.244 & - & - & - \\
\hline \multirow[t]{8}{*}{ Adenoma, pars distalis } & Terminal & Incidence & $23 / 49$ & $16 / 50$ & $14 / 48$ & $20 / 50$ & $20 / 50$ & $20 / 46$ \\
\hline & & & $(47 \%)$ & $(32 \%)$ & $(29 \%)$ & $(40 \%)$ & $(40 \%)$ & $(43 \%)$ \\
\hline & & Poly-3 Incidence & $23 / 38.5$ & $16 / 36.3$ & $14 / 33.5$ & $20 / 39.4$ & $20 / 38.5$ & $20 / 35.5$ \\
\hline & & & $(60 \%)$ & $(44 \%)$ & $(42 \%)$ & $(51 \%)$ & $(52 \%)$ & $(56 \%)$ \\
\hline & & Terminal Incidence & $7 / 11$ & $5 / 12$ & $8 / 13$ & $6 / 13$ & $9 / 17$ & $6 / 13$ \\
\hline & & & $(64 \%)$ & $(42 \%)$ & $(62 \%)$ & $(46 \%)$ & $(53 \%)$ & $(46 \%)$ \\
\hline & & Time-to-First & 502 & 397 & 442 & 448 & 520 & 445 \\
\hline & & Poly-3 $p$-value & 0.470 & $0.112 \mathrm{~N}$ & $0.081 \mathrm{~N}$ & $0.271 \mathrm{~N}$ & $0.308 \mathrm{~N}$ & $0.469 \mathrm{~N}$ \\
\hline \multirow{8}{*}{$\begin{array}{l}\text { Adenoma or carcinoma, } \\
\text { pars distalis }\end{array}$} & Terminal & Incidence & $23 / 49$ & $16 / 50$ & $14 / 48$ & $21 / 50$ & $20 / 50$ & $21 / 46$ \\
\hline & & & $(47 \%)$ & $(32 \%)$ & $(29 \%)$ & $(42 \%)$ & $(40 \%)$ & $(46 \%)$ \\
\hline & & Poly-3 Incidence & $23 / 38.5$ & $16 / 36.3$ & $14 / 33.5$ & $21 / 39.7$ & $20 / 38.5$ & $21 / 35.5$ \\
\hline & & & $(60 \%)$ & $(44 \%)$ & $(42 \%)$ & $(53 \%)$ & $(52 \%)$ & $(59 \%)$ \\
\hline & & Terminal Incidence & $7 / 11$ & $5 / 12$ & $8 / 13$ & $6 / 13$ & $9 / 17$ & $7 / 13$ \\
\hline & & & $(64 \%)$ & $(42 \%)$ & $(62 \%)$ & $(46 \%)$ & $(53 \%)$ & $(54 \%)$ \\
\hline & & Time-to-First & 502 & 397 & 442 & 448 & 520 & 445 \\
\hline & & Poly-3 $p$-value & 0.373 & $0.112 \mathrm{~N}$ & $0.081 \mathrm{~N}$ & $0.340 \mathrm{~N}$ & $0.308 \mathrm{~N}$ & $0.574 \mathrm{~N}$ \\
\hline
\end{tabular}

${ }^{\mathrm{a} B P A}$ doses are $\mu \mathrm{g} / \mathrm{kg}$ bw/day. See legend to Table 52 for description of data presentation. There were no significant increases relative to vehicle control.

One adenoma and one carcinoma. 
CLARITY-BPA Core Study

Table 73. Non-Neoplastic Lesions in the Pituitary of Interim and Terminal Sacrifice Female Rats: Continuous-Dose BPA ${ }^{\mathrm{a}}$

\begin{tabular}{|c|c|c|c|c|c|c|c|c|}
\hline Lesion & $\begin{array}{c}\text { Study Phase } \\
\text { (Interim/Terminal) }\end{array}$ & Statistic & Vehicle & 2.5 BPA & 25 BPA & 250 BPA & 2500 BPA & 25000 BPA \\
\hline \multirow{5}{*}{$\begin{array}{l}\text { Hyperplasia, } \\
\text { pars distalis }\end{array}$} & \multirow[t]{2}{*}{ Interim } & Incidence & $\begin{array}{l}18 / 23 \\
(78 \%)\end{array}$ & $\begin{array}{l}17 / 22 \\
(77 \%)\end{array}$ & $\begin{array}{l}18 / 22 \\
(82 \%)\end{array}$ & $15 / 24$ & $\begin{array}{l}16 / 20 \\
(80 \%)\end{array}$ & $20 / 24$ \\
\hline & & Severity Profile & $8|6| 3 \mid 1(1.8)$ & $7|6| 3 \mid 1(1.9)$ & $7|4| 5 \mid 2(2.1)$ & $8|4| 2 \mid 1(1.7)$ & $8|6| 1 \mid 1(1.7)$ & $7|8| 4 \mid 1(2.0)$ \\
\hline & \multirow[t]{3}{*}{ Terminal } & Incidence & $\begin{array}{l}27 / 50 \\
(54 \%)\end{array}$ & $\begin{array}{l}22 / 48 \\
(46 \%)\end{array}$ & $\begin{array}{l}32 / 46 \\
(70 \%)\end{array}$ & $\begin{array}{l}26 / 49 \\
(53 \%)\end{array}$ & $\begin{array}{l}29 / 49 \\
(59 \%)\end{array}$ & $\begin{array}{l}23 / 46 \\
(50 \%)\end{array}$ \\
\hline & & Severity Profile & $0|3| 12 \mid 12(3.3)$ & $1|2| 5 \mid 14(3.5)$ & $0|4| 12 \mid 16(3.4)$ & $1|5| 7 \mid 13$ (3.2) & $3|10| 4 \mid 12(2.9)$ & $1|6| 6 \mid 10(3.1)$ \\
\hline & & Poly-3 Incidence & $\begin{array}{c}27 / 43.6 \\
(62 \%)\end{array}$ & $\begin{array}{c}22 / 40.2 \\
(55 \%)\end{array}$ & $\begin{array}{c}32 / 40.6^{\mathrm{b}} \\
(79 \%)\end{array}$ & $\begin{array}{c}26 / 44.2 \\
(59 \%)\end{array}$ & $\begin{array}{c}29 / 42.7 \\
(68 \%)\end{array}$ & $\begin{array}{c}23 / 39.7 \\
(58 \%)\end{array}$ \\
\hline \multirow[t]{5}{*}{ Angiectasis } & \multirow[t]{2}{*}{ Interim $^{c}$} & Incidence & $\begin{array}{l}1 / 23 \\
(4 \%)\end{array}$ & $\begin{array}{l}0 / 22 \\
(0 \%)\end{array}$ & $\begin{array}{l}1 / 22 \\
(5 \%)\end{array}$ & $\begin{array}{l}0 / 24 \\
(0 \%)\end{array}$ & $\begin{array}{l}0 / 20 \\
(0 \%)\end{array}$ & $\begin{array}{l}0 / 24 \\
(0 \%)\end{array}$ \\
\hline & & Severity Profile & $0 / 1 / 0 / 0(2.0)$ & - & $0 / 1 / 0 / 0(2.0)$ & - & - & - \\
\hline & \multirow[t]{3}{*}{ Terminal } & Incidence & $\begin{array}{l}10 / 50 \\
(20 \%)\end{array}$ & $\begin{array}{c}8 / 48 \\
(17 \%)\end{array}$ & $\begin{array}{l}4 / 46 \\
(9 \%)\end{array}$ & $\begin{array}{c}9 / 49 \\
(18 \%)\end{array}$ & $\begin{array}{c}9 / 49 \\
(18 \%)\end{array}$ & $\begin{array}{c}9 / 46 \\
(20 \%)\end{array}$ \\
\hline & & Severity Profile & $0|1| 1 \mid 8(3.7)$ & $0|0| 0 \mid 8(4.0)$ & $0|0| 0 \mid 4(4.0)$ & $0|0| 2 \mid 7(3.8)$ & $0|2| 2 \mid 5(3.3)$ & $0|1| 1 \mid 7(3.7)$ \\
\hline & & Poly-3 Incidence & $\begin{array}{c}10 / 37.5 \\
(27 \%)\end{array}$ & $\begin{array}{l}8 / 35.4 \\
(23 \%)\end{array}$ & $\begin{array}{l}4 / 29.1 \\
(14 \%)\end{array}$ & $\begin{array}{l}9 / 34.8 \\
(26 \%)\end{array}$ & $\begin{array}{l}9 / 31.3 \\
(29 \%)\end{array}$ & $\begin{array}{l}9 / 32.2 \\
(28 \%)\end{array}$ \\
\hline
\end{tabular}

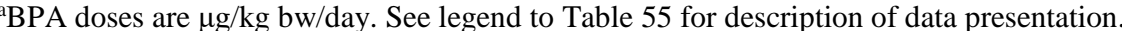

${ }^{b}$ In the sensitivity analysis that excluded all animals that overlapped with animals treated with $250,000 \mu \mathrm{g}$ BPA/kg bw/day (see Statistical Methods), there was a significant Poly-3 test $(p=0.027)$ for the pairwise comparison of the $25 \mu \mathrm{g} \mathrm{BPA} / \mathrm{kg}$ bw/day group to the vehicle control (hyperplasia of the pars distalis, Poly-3 incidences $30 / 37.0$ ( $81 \%$ ) versus $18 / 30.8(58 \%))$

cThis lesion was not statistically analyzed and is not included in Supplemental Appendix XXXIII since no dose group had two or more lesions. The data are found in Supplemental Appendix XXXII. 
CLARITY-BPA Core Study

Table 74. Non-Neoplastic Lesions in the Pituitary of Interim and Terminal Sacrifice Female Rats: Continuous-Dose $\mathbf{E E}_{2}{ }^{\mathbf{a}}$

\begin{tabular}{|c|c|c|c|c|c|}
\hline Lesion & $\begin{array}{c}\text { Study Phase } \\
\text { (Interim/Terminal) }\end{array}$ & Statistic & Vehicle $^{b}$ & $0.05 \mathrm{EE}_{2}$ & $0.5 \mathrm{EE}_{2}$ \\
\hline \multirow[t]{5}{*}{$\begin{array}{l}\text { Hyperplasia, pars } \\
\text { distalis }\end{array}$} & \multirow[t]{2}{*}{ Interim } & Incidence & $\begin{array}{c}18 / 23^{\# \#, \wedge \wedge} \\
(78 \%)\end{array}$ & $\begin{array}{l}20 / 25 \\
(80 \%)\end{array}$ & $\begin{array}{c}25 / 26^{\# \#, \wedge \wedge} \\
(96 \%)\end{array}$ \\
\hline & & Severity Profile & $8|6| 3 \mid 1(1.8)$ & $10|7| 3 \mid 0(1.6)$ & $4|11| 6 \mid 4(2.4)$ \\
\hline & \multirow[t]{3}{*}{ Terminal } & Incidence & $\begin{array}{c}27 / 50^{\#, \wedge \mathrm{N}} \\
(54 \%)\end{array}$ & $\begin{array}{l}16 / 26 \\
(62 \%)\end{array}$ & $\begin{array}{c}6 / 26^{\# \#, \wedge \wedge \mathrm{N}} \\
(23 \%)\end{array}$ \\
\hline & & Severity Profile & $0|3| 12 \mid 12(3.3)$ & $0|3| 5 \mid 8(3.3)$ & $0|1| 2 \mid 3(3.3)$ \\
\hline & & Poly-3 Incidence & $\begin{array}{c}27 / 43.6 \\
(62 \%)\end{array}$ & $\begin{array}{c}16 / 23.6 \\
(68 \%)\end{array}$ & $\begin{array}{l}6 / 16.9 \\
(36 \%)\end{array}$ \\
\hline \multirow[t]{5}{*}{ Angiectasis } & \multirow[t]{2}{*}{ Interim } & Incidence & $\begin{array}{c}1 / 23^{*, \#, \wedge} \\
(4 \%)\end{array}$ & $\begin{array}{l}2 / 25 \\
(8 \%)\end{array}$ & $\begin{array}{l}6 / 26^{\#, \wedge} \\
(23 \%)\end{array}$ \\
\hline & & Severity Profile & $0|1| 0 \mid 0(2.0)$ & $0|1| 0 \mid 1(3.0)$ & $0|4| 1 \mid 1(2.5)$ \\
\hline & \multirow[t]{3}{*}{ Terminal } & Incidence & $\begin{array}{l}10 / 50^{\# \# \#, \wedge \wedge \wedge} \\
(20 \%)\end{array}$ & $\begin{array}{c}5 / 26 \\
(19 \%)\end{array}$ & $\begin{array}{c}17 / 26^{\# \# \#, \wedge \wedge \wedge} \\
(65 \%)\end{array}$ \\
\hline & & Severity Profile & $0|1| 1 \mid 8(3.7)$ & $0|0| 0 \mid 5(4.0)$ & $0|0| 0 \mid 17$ (4.0) \\
\hline & & Poly-3 Incidence & $\begin{array}{c}10 / 37.5^{\text {*** }} \\
(27 \%)\end{array}$ & $\begin{array}{l}5 / 17.1 \\
(29 \%)\end{array}$ & $\begin{array}{c}17 / 22.0^{* * * *} \\
(77 \%)\end{array}$ \\
\hline
\end{tabular}

${ }^{a} E_{2}$ doses are $\mu \mathrm{g} / \mathrm{kg}$ bw/day. See legend to Table 55 for description of data presentation.

${ }^{\mathrm{b}}$ The continuous vehicle control group data are the same as those shown for the continuous-dose BPA data in Table 73. 
CLARITY-BPA Core Study

Table 75. Non-Neoplastic Lesions in the Pituitary of Interim and Terminal Sacrifice Female Rats: Stop-Dose BPA ${ }^{\text {a }}$

\begin{tabular}{|c|c|c|c|c|c|c|c|c|}
\hline Lesion & $\begin{array}{c}\text { Study Phase } \\
\text { (Interim/Terminal) }\end{array}$ & Statistic & Vehicle & 2.5 BPA & 25 BPA & 250 BPA & 2500 BPA & 25000 BPA \\
\hline \multirow{8}{*}{$\begin{array}{l}\text { Hyperplasia, } \\
\text { pars distalis }\end{array}$} & \multirow[t]{3}{*}{ Interim } & Incidence & $18 / 20$ & $16 / 22$ & $14 / 20$ & $20 / 22$ & $16 / 20$ & $18 / 22$ \\
\hline & & & $(90 \%)$ & $(73 \%)$ & $(70 \%)$ & $(91 \%)$ & $(80 \%)$ & $(82 \%)$ \\
\hline & & Severity Profile & $10|8| 0 \mid 0$ (1.4) & $10|4| 2 \mid 0(1.5)$ & $5|5| 4 \mid 0(1.9)$ & $10|9| 1 \mid 0$ (1.6) & $6|7| 3 \mid 0(1.8)$ & $9|8| 1 \mid 0(1.6)$ \\
\hline & \multirow[t]{5}{*}{ Terminal } & Incidence & $25 / 49$ & $32 / 50^{\wedge}$ & $34 / 48^{\wedge}$ & $26 / 50$ & $28 / 50$ & $21 / 46$ \\
\hline & & & $(51 \%)$ & $(64 \%)$ & $(71 \%)$ & $(52 \%)$ & $(56 \%)$ & $(46 \%)$ \\
\hline & & Severity Profile & $3|7| 7 \mid 8$ (2.8) & $0|5| 11 \mid 16(3.3)$ & $2|6| 14 \mid 12(3.1)$ & $0|6| 9 \mid 11(3.2)$ & $3|7| 5 \mid 13(3.0)$ & $1|3| 7 \mid 10$ (3.2) \\
\hline & & Poly-3 Incidence & $25 / 42.2$ & $32 / 43.5$ & $34 / 45.0$ & $26 / 43.3$ & $28 / 45.3$ & $21 / 39.1$ \\
\hline & & & $(59 \%)$ & $(74 \%)$ & $(76 \%)$ & $(60 \%)$ & $(62 \%)$ & $(54 \%)$ \\
\hline \multirow[t]{8}{*}{ Angiectasis } & \multirow[t]{3}{*}{ Interim } & Incidence & $0 / 20$ & $2 / 22^{\wedge}$ & $1 / 20$ & $0 / 22$ & $0 / 20$ & $0 / 22$ \\
\hline & & & $(0 \%)$ & $(9 \%)$ & $(5 \%)$ & $(0 \%)$ & $(0 \%)$ & $(0 \%)$ \\
\hline & & Severity Profile & - & $0|1| 1 \mid 0(2.5)$ & $0|1| 0 \mid 0(2.0)$ & - & - & - \\
\hline & \multirow[t]{5}{*}{ Terminal } & Incidence & $12 / 49$ & $11 / 50$ & $8 / 48$ & $12 / 50$ & $14 / 50$ & $11 / 46$ \\
\hline & & & $(24 \%)$ & $(22 \%)$ & $(17 \%)$ & $(24 \%)$ & $(28 \%)$ & $(24 \%)$ \\
\hline & & Severity Profile & $0|0| 0 \mid 12(4.0)$ & $0|0| 1 \mid 10(3.9)$ & $0|1| 2 \mid 5(3.5)$ & $0|3| 1 \mid 8(3.4)$ & $0|1| 2 \mid 11(3.7)$ & $0|2| 5 \mid 4(3.2)$ \\
\hline & & Poly-3 Incidence & $12 / 35.3$ & $11 / 34.5$ & $8 / 32.7$ & $12 / 38.4$ & $14 / 37.6$ & $11 / 33.9$ \\
\hline & & & $(34 \%)$ & $(32 \%)$ & $(24 \%)$ & $(31 \%)$ & $(37 \%)$ & $(32 \%)$ \\
\hline
\end{tabular}

aBPA doses are $\mu \mathrm{g} / \mathrm{kg}$ bw/day. See legend to Table 55 for description of data presentation. 
CLARITY-BPA Core Study

Table 76. Non-Neoplastic Lesions in the Heart of Interim and Terminal Sacrifice Female Rats: Continuous-Dose BPA ${ }^{\mathrm{a}}$

\begin{tabular}{|c|c|c|c|c|c|c|c|c|}
\hline Lesion & $\begin{array}{c}\text { Study Phase } \\
\text { (Interim/Terminal) }\end{array}$ & Statistic & Vehicle & 2.5 BPA & 25 BPA & 250 BPA & 2500 BPA & 25000 BPA \\
\hline \multirow[t]{5}{*}{ Cardiomyopathy } & Interim & Incidence & $\begin{array}{c}7 / 23 \\
(30 \%)\end{array}$ & $\begin{array}{l}10 / 22 \\
(46 \%)\end{array}$ & $\begin{array}{c}9 / 22 \\
(41 \%)\end{array}$ & $\begin{array}{c}8 / 24 \\
(33 \%)\end{array}$ & $\begin{array}{l}9 / 20 \\
(45 \%)\end{array}$ & $\begin{array}{l}7 / 24 \\
(29 \%)\end{array}$ \\
\hline & & Severity Profile & $6|1| 0 \mid 0(1.1)$ & $8|2| 0 \mid 0(1.2)$ & $7|2| 0 \mid 0(1.2)$ & $8|0| 0 \mid 0(1.0)$ & $8|1| 0 \mid 0(1.1)$ & $6|1| 0 \mid 0(1.1)$ \\
\hline & Terminal & Incidence & $\begin{array}{l}35 / 50 \\
(70 \%)\end{array}$ & $\begin{array}{l}30 / 48 \\
(62 \%)\end{array}$ & $\begin{array}{c}24 / 46^{\wedge} \mathrm{N} \\
(52 \%)\end{array}$ & $\begin{array}{l}35 / 49 \\
(71 \%)\end{array}$ & $\begin{array}{l}33 / 50 \\
(66 \%)\end{array}$ & $\begin{array}{l}33 / 46 \\
(72 \%)\end{array}$ \\
\hline & & Severity Profile & $24|10| 1 \mid 0(1.3)$ & $18|7| 4 \mid 1(1.6)$ & $18|5| 1 \mid 0(1.3)$ & $25|9| 1 \mid 0$ (1.3) & 24|7|1|1 (1.4) & $23|9| 0 \mid 1(1.4)$ \\
\hline & & Poly-3 Incidence & $\begin{array}{c}35 / 43.5 \\
(81 \%) \\
\end{array}$ & $\begin{array}{c}30 / 39.0 \\
(77 \%) \\
\end{array}$ & $\begin{array}{c}24 / 34.6 \\
(69 \%) \\
\end{array}$ & $\begin{array}{c}35 / 42.3 \\
(83 \%) \\
\end{array}$ & $\begin{array}{c}33 / 38.4 \\
(86 \%) \\
\end{array}$ & $\begin{array}{c}33 / 39.4 \\
(84 \%) \\
\end{array}$ \\
\hline
\end{tabular}

${ }^{\mathrm{a} B P A}$ doses are $\mu \mathrm{g} / \mathrm{kg}$ bw/day. See legend to Table 55 for description of data presentation. 
CLARITY-BPA Core Study

Table 77. Non-Neoplastic Lesions in the Heart of Interim and Terminal Sacrifice Female Rats: Continuous-Dose $\mathbf{E E}_{2}{ }^{\mathrm{a}}$

\begin{tabular}{|c|c|c|c|c|c|}
\hline Lesion & $\begin{array}{c}\text { Study Phase } \\
\text { (Interim/Terminal) }\end{array}$ & Statistic & Vehicle $^{\mathrm{b}}$ & $0.05 \mathrm{EE}_{2}$ & $0.5 \mathrm{EE}_{2}$ \\
\hline \multirow[t]{5}{*}{ Cardiomyopathy } & \multirow[t]{2}{*}{ Interim } & Incidence & $\begin{array}{c}7 / 23^{* *}, \# \#, \wedge \wedge \\
(30 \%)\end{array}$ & $\begin{array}{c}8 / 26 \\
(30.8 \%)\end{array}$ & $\begin{array}{c}17 / 26^{*}, \# \#, \wedge \wedge \\
(65 \%)\end{array}$ \\
\hline & & Severity Profile & $6|1| 0 \mid 0(1.1)$ & $8|0| 0 \mid 0(1.0)$ & $13|4| 0 \mid 0(1.2)$ \\
\hline & \multirow[t]{3}{*}{ Terminal } & Incidence & $\begin{array}{l}35 / 50^{\wedge} \\
(70 \%)\end{array}$ & $\begin{array}{l}19 / 26 \\
(73 \%)\end{array}$ & $\begin{array}{c}22 / 26^{\#, \wedge} \\
(85 \%)\end{array}$ \\
\hline & & Severity Profile & $24|10| 1 \mid 0$ (1.3) & $14|5| 0 \mid 0(1.3)$ & $12|9| 1 \mid 0(1.5)$ \\
\hline & & Poly-3 Incidence & $\begin{array}{c}35 / 43.5 \\
(81 \%)\end{array}$ & $\begin{array}{c}19 / 22.6 \\
(84 \%)\end{array}$ & $\begin{array}{c}22 / 24.0 \\
(92 \%)\end{array}$ \\
\hline
\end{tabular}

${ }^{\mathrm{a}} \mathrm{EE}_{2}$ doses are $\mu \mathrm{g} / \mathrm{kg}$ bw/day. See legend to Table 55 for description of data presentation.

${ }^{\mathrm{b}}$ The continuous vehicle control group data are the same as those shown for the continuous-dose BPA data in Table 76. 
CLARITY-BPA Core Study

Table 78. Non-Neoplastic Lesions in the Heart of Interim and Terminal Sacrifice Female Rats: Stop-Dose BPA ${ }^{\text {a }}$

\begin{tabular}{|c|c|c|c|c|c|c|c|c|}
\hline Lesion & $\begin{array}{c}\text { Study Phase } \\
\text { (Interim/Terminal) }\end{array}$ & Statistic & Vehicle & 2.5 BPA & 25 BPA & 250 BPA & 2500 BPA & 25000 BPA \\
\hline \multirow[t]{6}{*}{ Cardiomyopathy } & Interim & Incidence & $6 / 20$ & $8 / 22$ & $7 / 20$ & $7 / 22$ & $6 / 20$ & $7 / 22$ \\
\hline & & & $(30 \%)$ & $(36 \%)$ & $(35 \%)$ & $(32 \%)$ & $(30 \%)$ & $(32 \%)$ \\
\hline & & Severity Profile & $6|0| 0 \mid 0(1.0)$ & $8|0| 0 \mid 0(1.0)$ & $6|1| 0 \mid 0(1.1)$ & $5|2| 0 \mid 0(1.3)$ & $6|0| 0 \mid 0(1.0)$ & $5|2| 0 \mid 0(1.3)$ \\
\hline & Terminal & Incidence & $\begin{array}{c}32 / 50^{\# \#, \wedge \wedge} \\
(64 \%)\end{array}$ & $\begin{array}{l}37 / 50^{\wedge} \\
(74 \%)\end{array}$ & $\begin{array}{l}38 / 48 \\
(79 \%)\end{array}$ & $\begin{array}{c}37 / 50^{\#, \wedge} \\
(74 \%)\end{array}$ & $\begin{array}{c}35 / 50^{\#, \wedge} \\
(70 \%)\end{array}$ & $\begin{array}{c}35 / 46^{\# \#, \wedge} \\
(76 \%)\end{array}$ \\
\hline & & Severity Profile & $26|3| 3 \mid 0(1.3)$ & $22|13| 2 \mid 0(1.5)$ & $29|7| 2 \mid 0$ (1.3) & $21|14| 2 \mid 0(1.5)$ & $17|13| 5 \mid 0(1.7)$ & $16|14| 5 \mid 0(1.7)$ \\
\hline & & Poly-3 Incidence & $\begin{array}{c}32 / 42.9 \\
(74 \%)\end{array}$ & $\begin{array}{c}37 / 42.9 \\
(86 \%)\end{array}$ & $\begin{array}{c}38 / 43.1 \\
(88 \%)\end{array}$ & $\begin{array}{c}37 / 44.0 \\
(84 \%)\end{array}$ & $\begin{array}{c}35 / 44.0 \\
(80 \%)\end{array}$ & $\begin{array}{c}35 / 39.7^{\mathrm{b}} \\
(88 \%)\end{array}$ \\
\hline
\end{tabular}

${ }^{\mathrm{B} B P A}$ doses are $\mu \mathrm{g} / \mathrm{kg}$ bw/day. See legend to Table 55 for description of data presentation.

${ }^{b}$ In the sensitivity analysis that excluded all animals that overlapped with animals treated with $250,000 \mu \mathrm{g}$ BPA/kg bw/day (see Statistical Methods), there was a significant Poly-3 test $(p=0.049)$ for the comparison of the $25,000 \mu \mathrm{g} \mathrm{BPA} / \mathrm{kg}$ bw/day dose group to the vehicle controls (Poly-3 incidences $27 / 30.8(88 \%)$ versus $22 / 31.6(70 \%)$ ). 
CLARITY-BPA Core Study

Table 79. Non-Neoplastic Lesions in the Kidney of Interim and Terminal Sacrifice Female Rats: Continuous-Dose BPA ${ }^{\text {a }}$

\begin{tabular}{|c|c|c|c|c|c|c|c|c|}
\hline Lesion & $\begin{array}{c}\text { Study Phase } \\
\text { (Interim/Terminal) }\end{array}$ & Statistic & Vehicle & 2.5 BPA & 25 BPA & 250 BPA & 2500 BPA & 25000 BPA \\
\hline \multirow[t]{8}{*}{ Nephropathy } & \multirow[t]{3}{*}{ Interim } & Incidence & $6 / 23$ & $7 / 22$ & $11 / 22^{\wedge}$ & $8 / 24$ & $11 / 20^{\wedge}$ & $7 / 24$ \\
\hline & & & $(26 \%)$ & $(32 \%)$ & $(50 \%)$ & $(33 \%)$ & $(55 \%)$ & $(29 \%)$ \\
\hline & & Severity Profile & $4|1| 0 \mid 1(1.7)$ & $5|1| 1 \mid 0(1.4)$ & $6|2| 0 \mid 3(2.0)$ & $6|1| 0 \mid 1(1.5)$ & $9|0| 2 \mid 0(1.4)$ & $3|2| 1 \mid 1(2.0)$ \\
\hline & \multirow[t]{5}{*}{ Terminal } & Incidence & $19 / 50$ & $28 / 48^{\wedge \wedge}$ & $21 / 46$ & $21 / 49$ & $21 / 50$ & $25 / 46^{\#, \wedge}$ \\
\hline & & & $(38 \%)$ & $(58 \%)$ & $(46 \%)$ & $(43 \%)$ & $(42 \%)$ & $(54 \%)$ \\
\hline & & Severity Profile & $18|0| 1 \mid 0(1.1)$ & $16|9| 0 \mid 3$ (1.6) & $17|2| 1 \mid 1(1.3)$ & $12|5| 2 \mid 2(1.7)$ & $12|4| 3 \mid 2(1.8)$ & $18|3| 1 \mid 3(1.6)$ \\
\hline & & Poly-3 Incidence & $19 / 39.9$ & $28 / 40.6^{*}$ & $21 / 34.4$ & $21 / 38.5$ & $21 / 35.5$ & $25 / 38.2$ \\
\hline & & & $(48 \%)$ & $(69 \%)$ & $(61 \%)$ & $(54 \%)$ & $(59 \%)$ & $(65 \%)$ \\
\hline \multirow{8}{*}{$\begin{array}{l}\text { Cyst, renal } \\
\text { tubule }\end{array}$} & \multirow[t]{3}{*}{ Interim } & Incidence & $0 / 23$ & $7 / 22^{* *}$ & $3 / 22$ & $3 / 24$ & $3 / 20$ & $1 / 24$ \\
\hline & & & $(0 \%)$ & $(32 \%)$ & $(14 \%)$ & $(12 \%)$ & $(15 \%)$ & $(4 \%)$ \\
\hline & & Severity Profile & $-{ }^{\mathrm{b}}$ & - & - & - & - & - \\
\hline & \multirow[t]{5}{*}{ Terminal } & Incidence & $9 / 50$ & $8 / 48$ & $12 / 46$ & $15 / 49$ & $12 / 50$ & $6 / 46$ \\
\hline & & & $(18 \%)$ & $(17 \%)$ & $(26 \%)$ & $(31 \%)$ & $(24 \%)$ & $(13 \%)$ \\
\hline & & Severity Profile & $-{ }^{\mathrm{b}}$ & - & - & - & - & - \\
\hline & & Poly-3 Incidence & $9 / 36.2$ & $8 / 34.3$ & $12 / 32.1$ & $15 / 36.1^{\mathrm{c}}$ & $12 / 33.9$ & $6 / 33.4$ \\
\hline & & & $(25 \%)$ & $(23 \%)$ & $(37 \%)$ & $(42 \%)$ & $(35 \%)$ & $(18 \%)$ \\
\hline \multirow[t]{8}{*}{ Mineralization } & \multirow[t]{3}{*}{ Interim } & Incidence & $11 / 23^{*}, \# \#, \wedge \wedge$ & $5 / 22$ & $11 / 22$ & $12 / 24$ & $11 / 20$ & $16 / 24^{\#, \wedge}$ \\
\hline & & & $(48 \%)$ & $(23 \%)$ & $(50 \%)$ & $(50 \%)$ & $(55 \%)$ & $(67 \%)$ \\
\hline & & Severity Profile & $11|0| 0 \mid 0$ (1.0) & $3|1| 1 \mid 0$ (1.6) & $7|2| 1 \mid 1$ (1.6) & $11|1| 0 \mid 0$ (1.1) & $8|3| 0 \mid 0(1.3)$ & $10|3| 3 \mid 0(1.6)$ \\
\hline & \multirow[t]{5}{*}{ Terminal } & Incidence & $30 / 50$ & $23 / 48$ & $25 / 46$ & $25 / 49$ & $24 / 50$ & $26 / 46$ \\
\hline & & & $(60 \%)$ & $(48 \%)$ & $(54 \%)$ & $(51 \%)$ & $(48 \%)$ & $(57 \%)$ \\
\hline & & Severity Profile & $21|8| 1 \mid 0(1.3)$ & $20|3| 0 \mid 0(1.1)$ & $18|7| 0 \mid 0$ (1.3) & 18|7|0|0 (1.3) & $17|5| 2 \mid 0$ (1.4) & $16|7| 2 \mid 1(1.5)$ \\
\hline & & Poly-3 Incidence & $30 / 42.9$ & $23 / 37.8$ & $25 / 40.3$ & $25 / 40.6$ & $24 / 39.3$ & $26 / 38.6$ \\
\hline & & & $(70 \%)$ & $(61 \%)$ & $(62 \%)$ & $(62 \%)$ & $(61 \%)$ & $(67 \%)$ \\
\hline
\end{tabular}

${ }^{\mathrm{B} B P A}$ doses are $\mu \mathrm{g} / \mathrm{kg}$ bw/day. See legend to Table 55 for description of data presentation.

Severity scores were not assigned for this lesion.

'In the sensitivity analysis that excluded all animals that overlapped with animals treated with $250,000 \mu \mathrm{g}$ BPA/kg bw/day (see Statistical Methods), there was a significant Poly-3 test $(p=0.033)$ for the pairwise comparison of the $250 \mu \mathrm{g} \mathrm{BPA} / \mathrm{kg}$ bw/day dose group to the vehicle control group (renal tubule cyst, Poly-3 incidences $15 / 32.9$ (46\%) versus $5 / 24.8(20 \%))$. 
CLARITY-BPA Core Study

Table 80. Non-Neoplastic Lesions in the Kidney of Interim and Terminal Sacrifice Female Rats: Continuous-Dose $\mathbf{E E}_{2}{ }^{\mathbf{a}}$

\begin{tabular}{|c|c|c|c|c|c|}
\hline Lesion & $\begin{array}{c}\text { Study Phase } \\
\text { (Interim/Terminal) }\end{array}$ & Statistic & Vehicle $^{b}$ & $0.05 \mathrm{EE}_{2}$ & $0.5 \mathrm{EE}_{2}$ \\
\hline \multirow[t]{6}{*}{ Nephropathy } & \multirow[t]{2}{*}{ Interim } & Incidence & $\begin{array}{c}6 / 23^{*}, \#, \wedge \\
(26 \%)\end{array}$ & $\begin{array}{l}13 / 26 \\
(50 \%)\end{array}$ & $\begin{array}{c}15 / 26^{*}, \#, \wedge \\
(58 \%)\end{array}$ \\
\hline & & Severity Profile & $4|1| 0 \mid 1(1.7)$ & $8|5| 0 \mid 0(1.4)$ & $10|4| 1 \mid 0(1.4)$ \\
\hline & \multirow[t]{4}{*}{ Terminal } & Incidence & $\begin{array}{c}19 / 50^{\# \#, \wedge \wedge} \\
(38 \%)\end{array}$ & $\begin{array}{l}14 / 26^{\#} \\
(54 \%)\end{array}$ & $\begin{array}{c}15 / 26^{\# \#, \wedge \wedge} \\
(58 \%)\end{array}$ \\
\hline & & Severity Profile & $18|0| 1 \mid 0(1.1)$ & $10|3| 1 \mid 0$ (1.4) & $6|5| 3 \mid 1(1.9)$ \\
\hline & & Poly-3 Incidence & $19 / 39.9^{*}$ & $14 / 21.0$ & $15 / 21.6$ \\
\hline & & & $(48 \%)$ & $(67 \%)$ & $(70 \%)$ \\
\hline \multirow[t]{8}{*}{ Cyst, renal tubule } & \multirow[t]{3}{*}{ Interim } & Incidence & $0 / 23$ & $5 / 26^{*}$ & $4 / 26$ \\
\hline & & & $(0 \%)$ & $(19 \%)$ & $(15 \%)$ \\
\hline & & Severity Profile & $-^{\mathrm{c}}$ & - & - \\
\hline & \multirow[t]{5}{*}{ Terminal } & Incidence & $9 / 50$ & $5 / 26$ & $6 / 26$ \\
\hline & & & $(18 \%)$ & $(19 \%)$ & $(23 \%)$ \\
\hline & & Severity Profile & $-^{c}$ & - & - \\
\hline & & Poly-3 Incidence & $9 / 36.2$ & $5 / 18.3$ & $6 / 17.6$ \\
\hline & & & $(25 \%)$ & $(27 \%)$ & $(34 \%)$ \\
\hline \multirow[t]{8}{*}{ Mineralization } & \multirow[t]{3}{*}{ Interim } & Incidence & $11 / 23$ & $17 / 26^{\wedge}$ & $14 / 26$ \\
\hline & & & $(48 \%)$ & $(65 \%)$ & $(54 \%)$ \\
\hline & & Severity Profile & $11|0| 0 \mid 0$ (1.0) & $10|6| 1 \mid 0(1.5)$ & $7|5| 1 \mid 1(1.7)$ \\
\hline & \multirow[t]{5}{*}{ Terminal } & Incidence & $30 / 50$ & $10 / 26$ & $17 / 26$ \\
\hline & & & $(60 \%)$ & $(38 \%)$ & $(65 \%)$ \\
\hline & & Severity Profile & $21|8| 1 \mid 0$ (1.3) & $4|5| 1 \mid 0(1.7)$ & 10|7|0|0 (1.4) \\
\hline & & Poly-3 Incidence & $30 / 42.9$ & $10 / 20.7$ & $17 / 22.0$ \\
\hline & & & $(70 \%)$ & $(48 \%)$ & $(77 \%)$ \\
\hline
\end{tabular}

${ }^{a} E_{2}$ doses are $\mu \mathrm{g} / \mathrm{kg}$ bw/day. See legend to Table 55 for description of data presentation.

${ }^{b}$ The continuous vehicle control group data are the same as those shown for the continuous-dose BPA data in Table 79.

cSeverity scores were not assigned for this lesion. 
CLARITY-BPA Core Study

Table 81. Non-Neoplastic Lesions in the Kidney of Interim and Terminal Sacrifice Female Rats: Stop-Dose BPA ${ }^{\mathrm{a}}$

\begin{tabular}{|c|c|c|c|c|c|c|c|c|}
\hline Lesion & $\begin{array}{c}\text { Study Phase } \\
\text { (Interim/Terminal) }\end{array}$ & Statistic & Vehicle & 2.5 BPA & 25 BPA & 250 BPA & 2500 BPA & 25000 BPA \\
\hline \multirow[t]{8}{*}{ Nephropathy } & \multirow[t]{3}{*}{ Interim } & Incidence & $10 / 20$ & $10 / 22$ & $10 / 20$ & $11 / 22$ & $12 / 20$ & $13 / 22$ \\
\hline & & & $(50 \%)$ & $(46 \%)$ & $(50 \%)$ & $(50 \%)$ & $(60 \%)$ & $(59 \%)$ \\
\hline & & Severity Profile & $7|2| 1 \mid 0(1.4)$ & $7|2| 1 \mid 0(1.4)$ & $9|1| 0 \mid 0(1.1)$ & $7|3| 1 \mid 0(1.5)$ & $7|5| 0 \mid 0(1.4)$ & $10|1| 1 \mid 1(1.5)$ \\
\hline & \multirow[t]{5}{*}{ Terminal } & Incidence & $28 / 49^{\# \#, \wedge \wedge}$ & $25 / 50$ & $25 / 47$ & $29 / 49$ & $33 / 50$ & $30 / 46^{\wedge}$ \\
\hline & & & $(57 \%)$ & $(50 \%)$ & $(53 \%)$ & $(59 \%)$ & $(66 \%)$ & $(65 \%)$ \\
\hline & & Severity Profile & $17|8| 1 \mid 2(1.6)$ & $16|4| 2 \mid 3(1.7)$ & $17|3| 5 \mid 0(1.5)$ & $22|5| 1 \mid 1(1.3)$ & $15|11| 3 \mid 4$ (1.9) & $10|11| 6 \mid 3(2.1)$ \\
\hline & & Poly-3 Incidence & $28 / 39.2$ & $25 / 41.3$ & $25 / 38.4$ & $29 / 40.9$ & $33 / 43.3$ & $30 / 39.9$ \\
\hline & & & $(71 \%)$ & $(60 \%)$ & $(65 \%)$ & $(71 \%)$ & $(76 \%)$ & $(75 \%)$ \\
\hline \multirow{8}{*}{$\begin{array}{l}\text { Cyst, renal } \\
\text { tubule }\end{array}$} & \multirow[t]{3}{*}{ Interim } & Incidence & $4 / 20$ & $3 / 22$ & $4 / 20$ & $4 / 22$ & $6 / 20$ & $5 / 22$ \\
\hline & & & $(20 \%)$ & $(14 \%)$ & $(20 \%)$ & $(18 \%)$ & $(30 \%)$ & $(23 \%)$ \\
\hline & & Severity Profile & ${ }^{\mathrm{b}}$ & - & - & - & - & - \\
\hline & \multirow[t]{5}{*}{ Terminal } & Incidence & $7 / 49$ & $15 / 50$ & $11 / 47$ & $8 / 49$ & $16 / 50$ & $11 / 46$ \\
\hline & & & $(14 \%)$ & $(30 \%)$ & $(23 \%)$ & $(16 \%)$ & $(32 \%)$ & $(24 \%)$ \\
\hline & & Severity Profile & $-{ }^{\mathrm{b}}$ & - & - & - & - & - \\
\hline & & Poly-3 Incidence & $7 / 33.6$ & $15 / 34.9^{*}$ & $11 / 34.3$ & $8 / 36.1$ & $16 / 39.6$ & $11 / 34.4$ \\
\hline & & & $(21 \%)$ & $(43 \%)$ & $(32 \%)$ & $(22 \%)$ & $(40 \%)$ & $(32 \%)$ \\
\hline \multirow[t]{8}{*}{ Mineralization } & \multirow[t]{3}{*}{ Interim } & Incidence & $13 / 20$ & $11 / 22$ & $11 / 20$ & $14 / 22$ & $13 / 20$ & $11 / 22$ \\
\hline & & & $(65 \%)$ & $(50 \%)$ & $(55 \%)$ & $(64 \%)$ & $(65 \%)$ & $(50 \%)$ \\
\hline & & Severity Profile & $8|4| 0 \mid 1(1.5)$ & $9|2| 0 \mid 0(1.2)$ & $6|4| 0 \mid 1(1.6)$ & $8|6| 0 \mid 0(1.4)$ & $6|6| 1 \mid 0(1.6)$ & $8|3| 0 \mid 0(1.3)$ \\
\hline & \multirow[t]{5}{*}{ Terminal } & Incidence & $28 / 49$ & $22 / 50$ & $28 / 47$ & $26 / 49$ & $23 / 50$ & $23 / 46$ \\
\hline & & & $(57 \%)$ & $(44 \%)$ & $(60 \%)$ & $(53 \%)$ & $(46 \%)$ & $(50 \%)$ \\
\hline & & Severity Profile & $20|7| 0 \mid 1(1.4)$ & $14|7| 1 \mid 0$ (1.4) & $18|9| 0 \mid 1(1.4)$ & $17|8| 1 \mid 0(1.4)$ & $11|9| 3 \mid 0(1.7)$ & $13|9| 1 \mid 0(1.5)$ \\
\hline & & Poly-3 Incidence & $28 / 40.7$ & $22 / 39.1$ & $28 / 38.9$ & $26 / 42.4$ & $23 / 42.4$ & $23 / 39.1$ \\
\hline & & & $(69 \%)$ & $(56 \%)$ & $(72 \%)$ & $(61 \%)$ & $(54 \%)$ & $(59 \%)$ \\
\hline
\end{tabular}

${ }^{\mathrm{b}} \mathrm{Severity}$ scores were not assigned for this lesion. 
CLARITY-BPA Core Study

Table 82. Non-Neoplastic Lesions in the Liver of Interim and Terminal Sacrifice Female Rats: Continuous-Dose BPA ${ }^{\mathrm{a}}$

\begin{tabular}{|c|c|c|c|c|c|c|c|c|}
\hline Lesion & $\begin{array}{c}\text { Study Phase } \\
\text { (Interim/Terminal) }\end{array}$ & Statistic & Vehicle & 2.5 BPA & 25 BPA & 250 BPA & 2500 BPA & 25000 BPA \\
\hline \multirow{8}{*}{$\begin{array}{l}\text { Infiltration, } \\
\text { Mononuclear cells }\end{array}$} & \multirow[t]{3}{*}{ Interim } & Incidence & $4 / 23$ & $6 / 22$ & $4 / 22$ & $5 / 24$ & $5 / 20$ & $4 / 24$ \\
\hline & & & $(17 \%)$ & $(27 \%)$ & $(18 \%)$ & $(21 \%)$ & $(25 \%)$ & $(17 \%)$ \\
\hline & & Severity Profile & $4|0| 0 \mid 0(1.0)$ & $5|1| 0 \mid 0(1.2)$ & $4|0| 0 \mid 0(1.0)$ & $5|0| 0 \mid 0(1.0)$ & $5|0| 0 \mid 0(1.0)$ & $4|0| 0 \mid 0(1.0)$ \\
\hline & \multirow[t]{5}{*}{ Terminal } & Incidence & $37 / 50^{\# \#, \wedge \wedge N}$ & $28 / 48$ & $35 / 46$ & $29 / 49^{\#, \wedge N}$ & $26 / 50^{\# \#, \wedge \wedge}$ & $24 / 46^{\#, \wedge N}$ \\
\hline & & & $(74 \%)$ & $(58 \%)$ & $(76 \%)$ & $(59 \%)$ & $(52 \%)$ & $(52 \%)$ \\
\hline & & Severity Profile & 27|9|1|0 (1.3) & $20|8| 0 \mid 0(1.3)$ & $29|6| 0 \mid 0(1.2)$ & $24|5| 0 \mid 0(1.2)$ & $21|5| 0 \mid 0(1.2)$ & $19|4| 1 \mid 0(1.2)$ \\
\hline & & Poly-3 Incidence & $37 / 44.3^{* * N}$ & $28 / 38.1$ & $35 / 38.7$ & $29 / 41.9$ & $26 / 38.7^{* N}$ & $24 / 37.9^{* N}$ \\
\hline & & & $(83 \%)$ & $(74 \%)$ & $(90 \%)$ & $(69 \%)$ & $(67 \%)$ & $(63 \%)$ \\
\hline \multirow[t]{5}{*}{ Cystic degeneration } & \multirow[t]{5}{*}{ Terminal } & Incidence & $4 / 50$ & $3 / 48$ & $6 / 46$ & $3 / 49$ & $5 / 50$ & $1 / 46$ \\
\hline & & & $(8 \%)$ & $(6 \%)$ & $(13 \%)$ & $(6 \%)$ & $(10 \%)$ & $(2 \%)$ \\
\hline & & Severity Profile & $4|0| 0 \mid 0(1.0)$ & $3|0| 0 \mid 0(1.0)$ & $6|0| 0 \mid 0$ (1.0) & $3|0| 0 \mid 0(1.0)$ & $4|1| 0 \mid 0$ (1.2) & $1|0| 0 \mid 0(1.0)$ \\
\hline & & Poly-3 Incidence & $4 / 34.9$ & $3 / 33.3$ & $6 / 29.8$ & $3 / 33.2$ & $5 / 29.6$ & $1 / 31.2$ \\
\hline & & & $(12 \%)$ & $(9 \%)$ & $(20 \%)$ & $(9 \%)$ & $(17 \%)$ & $(3 \%)$ \\
\hline
\end{tabular}

aPPA doses are $\mu \mathrm{g} / \mathrm{kg}$ bw/day. See legend to Table 55 for description of data presentation. 
CLARITY-BPA Core Study

Table 83. Non-Neoplastic Lesions in the Liver of Interim and Terminal Sacrifice Female Rats: Continuous-Dose $\mathbf{E E}_{2}{ }^{\mathrm{a}}$

\begin{tabular}{|c|c|c|c|c|c|}
\hline Lesion & $\begin{array}{c}\text { Study Phase } \\
\text { (Interim/Terminal) }\end{array}$ & Statistic & Vehicle $^{b}$ & $0.05 \mathrm{EE}_{2}$ & $0.5 \mathrm{EE}_{2}$ \\
\hline \multirow{6}{*}{$\begin{array}{l}\text { Infiltration, } \\
\text { Mononuclear cells }\end{array}$} & Interim & Incidence & $4 / 23$ & $7 / 26$ & $2 / 26$ \\
\hline & & & $(17 \%)$ & $(27 \%)$ & $(8 \%)$ \\
\hline & & Severity Profile & $4|0| 0 \mid 0(1.0)$ & $7|0| 0 \mid 0(1.0)$ & $2|0| 0 \mid 0(1.0)$ \\
\hline & Terminal & Incidence & $\begin{array}{c}\text { 37/50\#\#, ^^^ } \mathrm{N} \\
(74 \%)\end{array}$ & $\begin{array}{l}17 / 26 \\
(65 \%)\end{array}$ & $\begin{array}{c}\text { 10/26\#\#, ^^^ } \mathrm{N} \\
(38 \%)\end{array}$ \\
\hline & & Severity Profile & $27|9| 1 \mid 0(1.3)$ & $15|2| 0 \mid 0(1.1)$ & $8|2| 0 \mid 0(1.2)$ \\
\hline & & Poly-3 Incidence & $\begin{array}{c}37 / 44.3 * * \mathrm{~N} \\
(83 \%)\end{array}$ & $\begin{array}{c}17 / 21.6 \\
(79 \%)\end{array}$ & $\begin{array}{c}10 / 18.7 * * \mathrm{~N} \\
(54 \%)\end{array}$ \\
\hline \multirow[t]{3}{*}{ Cystic degeneration } & Terminal & Incidence & $\begin{array}{l}4 / 50 \\
(8 \%)\end{array}$ & $\begin{array}{c}4 / 26 \\
(15 \%)\end{array}$ & $\begin{array}{l}2 / 26 \\
(8 \%)\end{array}$ \\
\hline & & Severity Profile & $4|0| 0 \mid 0(1.0)$ & $4|0| 0 \mid 0(1.0)$ & $1|1| 0 \mid 0(1.5)$ \\
\hline & & Poly-3 Incidence & $\begin{array}{l}4 / 34.9 \\
(12 \%)\end{array}$ & $\begin{array}{l}4 / 17.3 \\
(23 \%)\end{array}$ & $\begin{array}{l}2 / 15.2 \\
(13 \%)\end{array}$ \\
\hline
\end{tabular}

${ }^{\mathrm{a}} \mathrm{EE}_{2}$ doses are $\mu \mathrm{g} / \mathrm{kg}$ bw/day. See legend to Table 55 for description of data presentation.

${ }^{\mathrm{b}}$ The continuous vehicle control group data are the same as those shown for the continuous-dose BPA data in Table 82 . 
CLARITY-BPA Core Study

Table 84. Non-Neoplastic Lesions in the Liver of Interim and Terminal Sacrifice Female Rats: Stop-Dose BPA ${ }^{\text {a, b }}$

\begin{tabular}{|c|c|c|c|c|c|c|c|c|}
\hline Lesion & $\begin{array}{c}\text { Study Phase } \\
\text { (Interim/Terminal) }\end{array}$ & Statistic & Vehicle & 2.5 BPA & 25 BPA & 250 BPA & 2500 BPA & 25000 BPA \\
\hline \multirow[t]{6}{*}{$\begin{array}{l}\text { Infiltration, } \\
\text { Mononuclear cells }\end{array}$} & \multirow[t]{2}{*}{ Interim } & Incidence & $\begin{array}{c}2 / 20 \\
(10 \%)\end{array}$ & $\begin{array}{c}10 / 22^{*, \wedge} \\
(46 \%)\end{array}$ & $\begin{array}{c}7 / 20 \\
(35 \%)\end{array}$ & $\begin{array}{c}7 / 22 \\
(32 \%)\end{array}$ & $\begin{array}{c}2 / 20 \\
(10 \%)\end{array}$ & $\begin{array}{c}8 / 22^{*}, \#, \wedge \\
(36 \%)\end{array}$ \\
\hline & & Severity Profile & $1|1| 0 \mid 0(1.5)$ & $10|0| 0 \mid 0(1.0)$ & $7|0| 0 \mid 0(1.0)$ & $7|0| 0 \mid 0(1.0)$ & $2|0| 0 \mid 0(1.0)$ & $8|0| 0 \mid 0(1.0)$ \\
\hline & \multirow[t]{4}{*}{ Terminal } & Incidence & $\begin{array}{l}29 / 49 \\
(59 \%)\end{array}$ & $\begin{array}{l}28 / 50 \\
(56 \%)\end{array}$ & $\begin{array}{l}33 / 48 \\
(69 \%)\end{array}$ & $\begin{array}{l}25 / 50 \\
(50 \%)\end{array}$ & $\begin{array}{l}31 / 50 \\
(62 \%)\end{array}$ & $\begin{array}{l}31 / 46 \\
(67 \%)\end{array}$ \\
\hline & & Severity Profile & $22|6| 1 \mid 0(1.3)$ & $18|10| 0 \mid 0(1.4)$ & $26|7| 0 \mid 0$ (1.2) & $16|9| 0 \mid 0(1.4)$ & $25|5| 1 \mid 0(1.2)$ & $23|8| 0 \mid 0(1.3)$ \\
\hline & & Poly-3 Incidence & $29 / 39.7$ & $28 / 38.9$ & $33 / 41.5$ & $25 / 39.8$ & $31 / 41.0$ & $31 / 37.2$ \\
\hline & & & $(73 \%)$ & $(72 \%)$ & $(80 \%)$ & $(63 \%)$ & $(76 \%)$ & $(83 \%)$ \\
\hline \multirow[t]{5}{*}{ Cystic degeneration } & \multirow[t]{5}{*}{ Terminal } & Incidence & $2 / 49^{\# \#, \wedge \wedge}$ & $1 / 50$ & $6 / 48$ & $5 / 50$ & $8 / 50^{\#, \wedge}$ & $7 / 46^{\#, \wedge}$ \\
\hline & & & $(4 \%)$ & $(2 \%)$ & $(12 \%)$ & $(10 \%)$ & $(16 \%)$ & $(15 \%)$ \\
\hline & & Severity Profile & $2|0| 0 \mid 0(1.0)$ & $1|0| 0 \mid 0(1.0)$ & $6|0| 0 \mid 0(1.0)$ & $5|0| 0 \mid 0(1.0)$ & $6|2| 0 \mid 0$ (1.2) & $7|0| 0 \mid 0(1.0)$ \\
\hline & & Poly-3 Incidence & $2 / 32.0^{* *}$ & $1 / 31.7$ & $6 / 32.0$ & $5 / 35.3$ & $8 / 37.5$ & $7 / 32.3$ \\
\hline & & & $(6 \%)$ & $(3 \%)$ & $(19 \%)$ & $(14 \%)$ & $(21 \%)$ & $(22 \%)$ \\
\hline
\end{tabular}

${ }^{\mathrm{a} B P A}$ doses are $\mu \mathrm{g} / \mathrm{kg}$ bw/day. See legend to Table 55 for description of data presentation.

${ }^{b}$ In the sensitivity analysis that excluded all animals that overlapped with animals treated with $250,000 \mu \mathrm{g}$ BPA/kg bw/day (see Statistical Methods), there was a statistically significant Poly-3 dose trend ( $p=0.036$; in the analysis that included all animals, $p=0.222$ ) for mixed cell foci in terminal sacrifice animals (not shown in Table). 
CLARITY-BPA Core Study

Table 85. Non-Neoplastic Lesions in the Thyroid of Interim and Terminal Sacrifice Female Rats: Continuous-Dose BPA ${ }^{\mathrm{a}}$

\begin{tabular}{|c|c|c|c|c|c|c|c|c|}
\hline Lesion & $\begin{array}{c}\text { Study Phase } \\
\text { (Interim/Terminal) }\end{array}$ & Statistic & Vehicle & 2.5 BPA & 25 BPA & 250 BPA & 2500 BPA & 25000 BPA \\
\hline \multirow{7}{*}{$\begin{array}{l}\text { Hyperplasia, } \\
\text { C-cell }\end{array}$} & \multirow[t]{2}{*}{ Interim } & Incidence & $14 / 23$ & $11 / 22$ & $15 / 21$ & $12 / 24$ & $8 / 20$ & $16 / 24$ \\
\hline & & Severity Profile & $\begin{array}{c}(61 \%) \\
8|6| 0 \mid 0(1.4)\end{array}$ & $\begin{array}{c}(50 \%) \\
9|2| 0 \mid 0(1.2)\end{array}$ & $\begin{array}{c}(71 \%) \\
12|3| 0 \mid 0(1.2)\end{array}$ & $\begin{array}{c}(50 \%) \\
9|3| 0 \mid 0(1.2)\end{array}$ & $\begin{array}{c}(40 \%) \\
4|3| 1 \mid 0(1.6)\end{array}$ & $\begin{array}{c}(67 \%) \\
12|3| 1 \mid 0(1.3)\end{array}$ \\
\hline & \multirow[t]{5}{*}{ Terminal } & Incidence & $22 / 50$ & $17 / 48$ & $22 / 46$ & $18 / 49$ & $20 / 50$ & $17 / 46$ \\
\hline & & & $(44 \%)$ & $(35 \%)$ & $(48 \%)$ & $(37 \%)$ & $(40 \%)$ & $(37 \%)$ \\
\hline & & Severity Profile & $11|9| 2 \mid 0$ (1.6) & $12|3| 2 \mid 0(1.4)$ & $13|7| 2 \mid 0(1.5)$ & $11|7| 0 \mid 0$ (1.4) & $8|10| 2 \mid 0$ (1.7) & $7|8| 2 \mid 0(1.7)$ \\
\hline & & Poly-3 Incidence & $22 / 40.7$ & $17 / 37.3$ & $22 / 36.0$ & $18 / 38.4$ & $20 / 35.9$ & $17 / 36.6$ \\
\hline & & & $(54 \%)$ & $(46 \%)$ & $(61 \%)$ & $(47 \%)$ & $(56 \%)$ & $(46 \%)$ \\
\hline \multirow{5}{*}{$\begin{array}{l}\text { Hyperplasia, } \\
\text { Follicular cell }^{\text {b }}\end{array}$} & \multirow[t]{5}{*}{ Terminal } & Incidence & $1 / 50$ & $6 / 48^{\wedge}$ & $4 / 46$ & $3 / 49$ & $1 / 50$ & $4 / 46$ \\
\hline & & & $(2 \%)$ & $(12 \%)$ & $(9 \%)$ & $(6 \%)$ & $(2 \%)$ & $(9 \%)$ \\
\hline & & Severity Profile & $0|1| 0 \mid 0(2.0)$ & $0|2| 4 \mid 0(2.7)$ & $0|2| 1 \mid 1(2.8)$ & $0|2| 1 \mid 0(2.3)$ & $0|0| 0 \mid 1(4.0)$ & $0|2| 2 \mid 0(2.5)$ \\
\hline & & Poly-3 Incidence & $1 / 34.2$ & $6 / 34.1$ & $4 / 30.1$ & $3 / 33.3$ & $1 / 29.1$ & $4 / 32.9$ \\
\hline & & & $(3 \%)$ & $(18 \%)$ & $(13 \%)$ & $(9 \%)$ & $(3 \%)$ & $(12 \%)$ \\
\hline \multirow{8}{*}{$\begin{array}{l}\text { Ultimobranchial } \\
\text { cyst }\end{array}$} & \multirow{3}{*}{ Interim } & Incidence & $7 / 23$ & $7 / 22$ & $5 / 21$ & $8 / 24$ & $6 / 20$ & $11 / 24$ \\
\hline & & & $(30 \%)$ & $(32 \%)$ & $(24 \%)$ & $(33 \%)$ & $(30 \%)$ & $(46 \%)$ \\
\hline & & Severity Profile & $-^{\mathrm{c}}$ & - & - & - & - & - \\
\hline & \multirow[t]{5}{*}{ Terminal } & Incidence & $8 / 50$ & $2 / 48$ & $6 / 46$ & $3 / 49$ & $4 / 50$ & $7 / 46$ \\
\hline & & & $(16 \%)$ & $(4 \%)$ & $(13 \%)$ & $(6 \%)$ & $(8 \%)$ & $(15 \%)$ \\
\hline & & Severity Profile & $-^{c}$ & - & - & - & - & - \\
\hline & & Poly-3 Incidence & $8 / 36.4$ & $2 / 33.5$ & $6 / 30.2$ & $3 / 33.3$ & $4 / 30.2$ & $7 / 32.8$ \\
\hline & & & $(22 \%)$ & $(6 \%)$ & $(20 \%)$ & $(9 \%)$ & $(13 \%)$ & $(21 \%)$ \\
\hline
\end{tabular}

${ }^{\mathrm{a}} \mathrm{BPA}$ doses are $\mu \mathrm{g} / \mathrm{kg}$ bw/day. See legend to Table 55 for description of data presentation.

bata for follicular cell hyperplasia are not tabulated for interim sacrifice animals since no dose groups had two or more diagnoses of this lesion.

${ }^{\mathrm{c} N o}$ severity scores were assigned for ultimobranchial cysts. 
CLARITY-BPA Core Study

Table 86. Non-Neoplastic Lesions in the Thyroid of Interim and Terminal Sacrifice Female Rats: Continuous-Dose $\mathrm{EE}_{2}{ }^{\mathrm{a}}$

\begin{tabular}{|c|c|c|c|c|c|}
\hline Lesion & $\begin{array}{c}\text { Study Phase } \\
\text { (Interim/Terminal) }\end{array}$ & Statistic & Vehicle $^{b}$ & $0.05 \mathrm{EE}_{2}$ & $0.5 \mathrm{EE}_{2}$ \\
\hline \multirow[t]{5}{*}{ Hyperplasia, C-cell } & \multirow[t]{2}{*}{ Interim } & Incidence & $\begin{array}{l}14 / 23 \\
(61 \%)\end{array}$ & $\begin{array}{l}11 / 26 \\
(42 \%)\end{array}$ & $\begin{array}{l}13 / 26 \\
(50 \%)\end{array}$ \\
\hline & & Severity Profile & $8|6| 0 \mid 0(1.4)$ & $7|4| 0 \mid 0(1.4)$ & $11|1| 1 \mid 0(1.2)$ \\
\hline & \multirow[t]{3}{*}{ Terminal } & Incidence & $\begin{array}{l}22 / 50 \\
(44 \%)\end{array}$ & $\begin{array}{c}7 / 26 \\
(27 \%)\end{array}$ & $\begin{array}{c}9 / 25 \\
(36 \%)\end{array}$ \\
\hline & & Severity Profile & $11|9| 2 \mid 0(1.6)$ & $2|5| 0 \mid 0(1.7)$ & $6|2| 0 \mid 1(1.6)$ \\
\hline & & Poly-3 Incidence & $\begin{array}{c}22 / 40.7 \\
(54 \%)\end{array}$ & $\begin{array}{l}7 / 18.7 \\
(37 \%)\end{array}$ & $\begin{array}{l}9 / 18.1 \\
(50 \%)\end{array}$ \\
\hline \multirow[t]{5}{*}{ Hyperplasia, Follicular cell ${ }^{\mathrm{c}}$} & \multirow[t]{5}{*}{ Terminal } & Incidence & $1 / 50$ & $4 / 26^{\wedge \wedge}$ & $0 / 25$ \\
\hline & & & $(2 \%)$ & $(15 \%)$ & $(0 \%)$ \\
\hline & & Severity Profile & $0|1| 0 \mid 0(2.0)$ & $0|1| 3 \mid 0(2.8)$ & - \\
\hline & & Poly-3 Incidence & $1 / 34.2$ & $4 / 17.9^{*}$ & $0 / 14.7$ \\
\hline & & & $(3 \%)$ & $(22 \%)$ & $(0 \%)$ \\
\hline \multirow[t]{7}{*}{ Ultimobranchial cyst } & \multirow[t]{3}{*}{ Interim } & Incidence & $7 / 23$ & $7 / 26$ & $11 / 26$ \\
\hline & & & $(30 \%)$ & $(27 \%)$ & $(42 \%)$ \\
\hline & & Severity Profile & $-{ }^{\mathrm{d}}$ & - & - \\
\hline & \multirow[t]{4}{*}{ Terminal } & Incidence & $\begin{array}{c}8 / 50 \\
(16 \%)\end{array}$ & $\begin{array}{l}5 / 26 \\
(19 \%)\end{array}$ & $\begin{array}{c}8 / 25 \\
(32 \%)\end{array}$ \\
\hline & & Severity Profile & $-^{\mathrm{d}}$ & - & - \\
\hline & & Poly-3 Incidence & $8 / 36.4^{*}$ & $5 / 17.5$ & $8 / 17.4$ \\
\hline & & & $(22 \%)$ & $(29 \%)$ & $(46 \%)$ \\
\hline
\end{tabular}

${ }^{\mathrm{a}} \mathrm{EE}_{2}$ doses are $\mu \mathrm{g} / \mathrm{kg}$ bw/day. See legend to Table 55 for description of data presentation.

'The continuous vehicle control group data are the same as those shown for the continuous-dose BPA data in Table 85.

'Data for follicular cell hyperplasia are not tabulated for interim sacrifice animals since no dose groups had two or more diagnoses of this lesion.

${ }^{\mathrm{d}}$ No severity scores were assigned for ultimobranchial cysts. 
CLARITY-BPA Core Study

Table 87. Non-Neoplastic Lesions in the Thyroid of Interim and Terminal Sacrifice Female Rats: Stop-Dose BPA ${ }^{\mathrm{a}}$

\begin{tabular}{|c|c|c|c|c|c|c|c|c|}
\hline Lesion & $\begin{array}{c}\text { Study Phase } \\
\text { (Interim/Terminal) }\end{array}$ & Statistic & Vehicle & 2.5 BPA & 25 BPA & 250 BPA & 2500 BPA & 25000 BPA \\
\hline \multirow{5}{*}{$\begin{array}{l}\text { Hyperplasia, } \\
\text { C-cell }\end{array}$} & \multirow[t]{2}{*}{ Interim } & Incidence & $10 / 20$ & $16 / 22^{\wedge}$ & $11 / 20$ & $12 / 22$ & $13 / 20$ & $9 / 22$ \\
\hline & & Severity Profile & $\begin{array}{c}(50 \%) \\
10|0| 0 \mid 0(1.0)\end{array}$ & $\begin{array}{c}(73 \%) \\
11|4| 1 \mid 0(1.4)\end{array}$ & $\begin{array}{c}(55 \%) \\
8|3| 0 \mid 0(1.3)\end{array}$ & $\begin{array}{c}(54 \%) \\
8|4| 0 \mid 0(1.3)\end{array}$ & $\begin{array}{c}(65 \%) \\
11|2| 0 \mid 0(1.2)\end{array}$ & $\begin{array}{c}(41 \%) \\
8|1| 0 \mid 0(1.1)\end{array}$ \\
\hline & \multirow[t]{3}{*}{ Terminal } & Incidence & $\begin{array}{l}26 / 48 \\
(54 \%)\end{array}$ & $\begin{array}{l}29 / 49 \\
(59 \%)\end{array}$ & $\begin{array}{l}17 / 45 \\
(38 \%)\end{array}$ & $\begin{array}{l}23 / 48 \\
(48 \%)\end{array}$ & $\begin{array}{l}28 / 50 \\
(56 \%)\end{array}$ & $\begin{array}{l}24 / 46 \\
(52 \%)\end{array}$ \\
\hline & & Severity Profile & $8|15| 2 \mid 1(1.8)$ & $15|11| 1 \mid 2(1.7)$ & $11|5| 0 \mid 1(1.5)$ & $15|7| 1 \mid 0$ (1.4) & $10|12| 4 \mid 2(1.9)$ & $11|11| 2 \mid 0$ (1.6) \\
\hline & & Poly-3 Incidence & $\begin{array}{c}26 / 40.5 \\
(64 \%)\end{array}$ & $\begin{array}{c}29 / 42.0 \\
(69 \%)\end{array}$ & $\begin{array}{c}17 / 34.9 \\
(49 \%)\end{array}$ & $\begin{array}{c}23 / 39.8 \\
(58 \%)\end{array}$ & $\begin{array}{c}28 / 42.6 \\
(66 \%)\end{array}$ & $\begin{array}{c}24 / 38.9 \\
(62 \%)\end{array}$ \\
\hline \multirow{5}{*}{$\begin{array}{l}\text { Hyperplasia, } \\
\text { Follicular cell }^{\text {b }}\end{array}$} & \multirow[t]{5}{*}{ Terminal } & Incidence & $4 / 48$ & $4 / 49$ & $7 / 45$ & $6 / 48$ & $5 / 50$ & $4 / 46$ \\
\hline & & & $(8 \%)$ & $(8 \%)$ & $(16 \%)$ & $(12 \%)$ & $(10 \%)$ & $(9 \%)$ \\
\hline & & Severity Profile & $0|2| 2 \mid 0(2.5)$ & $1|3| 0 \mid 0(1.8)$ & $0|5| 2 \mid 0(2.3)$ & $0|4| 2 \mid 0(2.3)$ & $0|3| 2 \mid 0(2.4)$ & $0|3| 1 \mid 0(2.2)$ \\
\hline & & Poly-3 Incidence & $4 / 32.8$ & $4 / 32.0$ & $7 / 31.6$ & $6 / 35.4$ & $5 / 35.9$ & $4 / 33.2$ \\
\hline & & & $(12 \%)$ & $(12 \%)$ & $(22 \%)$ & $(17 \%)$ & $(14 \%)$ & $(12 \%)$ \\
\hline \multirow{6}{*}{$\begin{array}{l}\text { Ultimobranchial } \\
\text { cyst }\end{array}$} & \multirow{3}{*}{ Interim } & Incidence & $4 / 20$ & $6 / 22$ & $7 / 20$ & $4 / 22$ & $6 / 20$ & $6 / 22$ \\
\hline & & & $(20 \%)$ & $(27 \%)$ & $(35 \%)$ & $(18 \%)$ & $(30 \%)$ & $(27 \%)$ \\
\hline & & Severity Profile & $-^{\mathrm{c}}$ & - & - & - & - & - \\
\hline & \multirow[t]{3}{*}{ Terminal } & Incidence & $\begin{array}{l}2 / 48 \\
(4 \%)\end{array}$ & $\begin{array}{c}7 / 49 \\
(14 \%)\end{array}$ & $\begin{array}{l}2 / 45 \\
(4 \%)\end{array}$ & $\begin{array}{l}9 / 48 \\
(19 \%)\end{array}$ & $\begin{array}{l}11 / 50 \\
(22 \%)\end{array}$ & $\begin{array}{l}3 / 46 \\
(6 \%)\end{array}$ \\
\hline & & Severity Profile & $-^{c}$ & - & - & - & - & - \\
\hline & & Poly-3 Incidence & $\begin{array}{c}2 / 31.4 \\
(6 \%)\end{array}$ & $\begin{array}{l}7 / 33.4 \\
(21 \%)\end{array}$ & $\begin{array}{l}2 / 29.5 \\
(7 \%)\end{array}$ & $\begin{array}{l}9 / 36.1^{*} \\
(25 \%)\end{array}$ & $\begin{array}{c}11 / 38.6^{*} \\
(28 \%)\end{array}$ & $\begin{array}{c}3 / 32.1 \\
(9 \%)\end{array}$ \\
\hline
\end{tabular}

${ }^{\mathrm{a} B P A}$ doses are $\mu \mathrm{g} / \mathrm{kg}$ bw/day. See legend to Table 55 for description of data presentation.

bData for follicular cell hyperplasia are not tabulated for interim sacrifice animals since no dose groups had two or more diagnoses of this lesion.

${ }^{\mathrm{c} N o}$ severity scores were assigned for ultimobranchial cysts. 
CLARITY-BPA Core Study

Table 88. Non-Neoplastic Lesions in the Epididymis of Interim and Terminal Sacrifice Male Rats: Continuous-Dose BPA ${ }^{\mathrm{a}}$

\begin{tabular}{|c|c|c|c|c|c|c|c|c|}
\hline Lesion & $\begin{array}{c}\text { Study Phase } \\
\text { (Interim/Terminal) }\end{array}$ & Statistic & Vehicle & 2.5 BPA & 25 BPA & 250 BPA & 2500 BPA & 25000 BPA \\
\hline \multirow{8}{*}{$\begin{array}{l}\text { Exfoliated germ } \\
\text { cells }\end{array}$} & \multirow[t]{3}{*}{ Interim } & Incidence & $1 / 22^{*}, \#, \wedge$ & $1 / 22$ & $1 / 20$ & $1 / 24$ & $0 / 20$ & $6 / 22^{*}, \# \#, \wedge \wedge$ \\
\hline & & & $(4 \%)$ & $(4 \%)$ & $(5 \%)$ & $(4 \%)$ & $(0 \%)$ & $(27 \%)$ \\
\hline & & Severity Profile & $1|0| 0 \mid 0(1.0)$ & $1|0| 0 \mid 0(1.0)$ & $0|1| 0 \mid 0(2.0)$ & $1|0| 0 \mid 0(1.0)$ & - & $6|0| 0 \mid 0(1.0)$ \\
\hline & \multirow[t]{5}{*}{ Terminal } & Incidence & $10 / 49$ & $8 / 48$ & $10 / 48$ & $12 / 50$ & $13 / 50$ & $6 / 46$ \\
\hline & & & $(20 \%)$ & $(17 \%)$ & $(21 \%)$ & $(24 \%)$ & $(26 \%)$ & $(13 \%)$ \\
\hline & & Severity Profile & $5|3| 1 \mid 1(1.8)$ & $5|1| 2 \mid 0(1.6)$ & $1|9| 0 \mid 0(1.9)$ & $4|5| 2 \mid 1(2.0)$ & $4|7| 2 \mid 0(1.8)$ & $4|0| 2 \mid 0(1.7)$ \\
\hline & & Poly-3 & $10 / 36.5$ & $8 / 35.5$ & $10 / 35.4$ & $12 / 37.4$ & $13 / 38.0$ & $6 / 30.1$ \\
\hline & & Incidence & $(27 \%)$ & $(23 \%)$ & $(28 \%)$ & $(32 \%)$ & $(34 \%)$ & $(20 \%)$ \\
\hline \multirow{8}{*}{$\begin{array}{l}\text { Infiltration, cellular, } \\
\text { lymphocyte }\end{array}$} & \multirow{3}{*}{ Interim } & Incidence & $0 / 22^{*}, \#, \wedge$ & $1 / 22$ & $3 / 20$ & $2 / 24$ & $0 / 20$ & $5 / 22^{*}, \# \#, \wedge \wedge$ \\
\hline & & & $(0 \%)$ & $(4 \%)$ & $(15 \%)$ & $(8 \%)$ & $(0 \%)$ & $(23 \%)$ \\
\hline & & Severity Profile & - & $0|1| 0 \mid 0(2.0)$ & $3|0| 0 \mid 0(1.0)$ & $2|0| 0 \mid 0(1.0)$ & - & $5|0| 0 \mid 0(1.0)$ \\
\hline & \multirow[t]{5}{*}{ Terminal } & Incidence & $10 / 49$ & $12 / 48$ & $13 / 48$ & $15 / 50$ & $14 / 50$ & $15 / 46$ \\
\hline & & & $(20 \%)$ & $(25 \%)$ & $(27 \%)$ & $(30 \%)$ & $(28 \%)$ & $(33 \%)$ \\
\hline & & Severity Profile & $8|2| 0 \mid 0(1.2)$ & $12|0| 0 \mid 0(1.0)$ & $11|2| 0 \mid 0(1.2)$ & $13|2| 0 \mid 0(1.1)$ & $12|2| 0 \mid 0(1.1)$ & $13|2| 0 \mid 0(1.1)$ \\
\hline & & Poly-3 & $10 / 35.5$ & $12 / 36.5$ & $13 / 34.4$ & $15 / 36.2$ & $14 / 36.0$ & $15 / 34.1$ \\
\hline & & Incidence & $(28 \%)$ & $(33 \%)$ & $(38 \%)$ & $(41 \%)$ & $(39 \%)$ & $(44 \%)$ \\
\hline
\end{tabular}

${ }^{a}$ BPA doses are $\mu \mathrm{g} / \mathrm{kg}$ bw/day. See legend to Table 55 for description of data presentation. 
CLARITY-BPA Core Study

Table 89. Non-Neoplastic Lesions in the Epididymis of Interim and Terminal Sacrifice Male Rats: Continuous-Dose $\mathbf{E E}_{2}{ }^{\mathrm{a}}$

\begin{tabular}{|c|c|c|c|c|c|}
\hline Lesion & $\begin{array}{c}\text { Study Phase } \\
\text { (Interim/Terminal) }\end{array}$ & Statistic $^{\mathrm{b}}$ & Vehicle & $0.05 \mathbf{E E}_{2}$ & $0.5 \mathrm{EE}_{2}$ \\
\hline \multirow[t]{8}{*}{ Exfoliated germ cells } & \multirow[t]{3}{*}{ Interim } & Incidence & $1 / 22$ & $4 / 26$ & $2 / 26$ \\
\hline & & & $(4 \%)$ & $(15 \%)$ & $(8 \%)$ \\
\hline & & Severity Profile & $1|0| 0 \mid 0(1.0)$ & $3|1| 0 \mid 0(1.2)$ & $2|0| 0 \mid 0(1.0)$ \\
\hline & \multirow[t]{5}{*}{ Terminal } & Incidence & $10 / 49$ & $6 / 26$ & $4 / 26$ \\
\hline & & & $(20 \%)$ & $(23 \%)$ & $(15 \%)$ \\
\hline & & Severity Profile & $5|3| 1 \mid 1(1.8)$ & $4|1| 1 \mid 0(1.5)$ & $3|1| 0 \mid 0(1.2)$ \\
\hline & & Poly-3 Incidence & $10 / 36.5$ & $6 / 18.3$ & $4 / 20.4$ \\
\hline & & & $(27 \%)$ & $(33 \%)$ & $(20 \%)$ \\
\hline \multirow{8}{*}{$\begin{array}{l}\text { Infiltration, cellular, } \\
\text { lymphocyte }\end{array}$} & \multirow[t]{3}{*}{ Interim } & Incidence & $0 / 22^{\#, \wedge}$ & $1 / 26$ & $3 / 26^{\#, \wedge}$ \\
\hline & & & $(0 \%)$ & $(4 \%)$ & $(12 \%)$ \\
\hline & & Severity Profile & -- & $1|0| 0 \mid 0(1.0)$ & $3|0| 0 \mid 0(1.0)$ \\
\hline & \multirow[t]{5}{*}{ Terminal } & Incidence & $10 / 49^{\wedge}$ & $5 / 26$ & $10 / 26^{\wedge}$ \\
\hline & & & $(20 \%)$ & $(19 \%)$ & $(38 \%)$ \\
\hline & & Severity Profile & $8|2| 0 \mid 0(1.2)$ & $4|1| 0 \mid 0(1.2)$ & $8|2| 0 \mid 0(1.2)$ \\
\hline & & Poly-3 Incidence & $10 / 35.5$ & $5 / 18.3$ & $10 / 20.4$ \\
\hline & & & $(28 \%)$ & $(27 \%)$ & $(49 \%)$ \\
\hline
\end{tabular}

${ }^{\mathrm{a}} \mathrm{EE}_{2}$ doses are $\mu \mathrm{g} / \mathrm{kg}$ bw/day. See legend to Table 55 for description of data presentation.

${ }^{\mathrm{b}}$ The continuous vehicle control group data are the same as those shown for the continuous-dose BPA data in Table 88. 
CLARITY-BPA Core Study

Table 90. Non-Neoplastic Lesions in the Epididymis of Interim and Terminal Sacrifice Male Rats: Stop-Dose BPA ${ }^{\text {a }}$

\begin{tabular}{|c|c|c|c|c|c|c|c|c|}
\hline Lesion & $\begin{array}{c}\text { Study Phase } \\
\text { (Interim/Terminal) }\end{array}$ & Statistic & Vehicle & 2.5 BPA & 25 BPA & 250 BPA & 2500 BPA & 25000 BPA \\
\hline \multirow[t]{6}{*}{$\begin{array}{l}\text { Exfoliated germ } \\
\text { cells }\end{array}$} & \multirow[t]{2}{*}{ Interim } & Incidence & $\begin{array}{l}0 / 20 \\
(0 \%)\end{array}$ & $\begin{array}{l}3 / 20^{\wedge} \\
(15 \%)\end{array}$ & $\begin{array}{l}1 / 20 \\
(5 \%)\end{array}$ & $\begin{array}{c}2 / 19 \\
(10 \%)\end{array}$ & $\begin{array}{l}1 / 20 \\
(5 \%)\end{array}$ & $\begin{array}{l}1 / 22 \\
(4 \%)\end{array}$ \\
\hline & & Severity Profile & - & $1|2| 0 \mid 0$ (1.7) & $0|1| 0 \mid 0(2.0)$ & $2|0| 0 \mid 0(1.0)$ & $0|1| 0 \mid 0(2.0)$ & $1|0| 0 \mid 0(1.0)$ \\
\hline & \multirow[t]{4}{*}{ Terminal } & Incidence & $\begin{array}{l}12 / 49 \\
(24 \%)\end{array}$ & $\begin{array}{l}15 / 48 \\
(31 \%)\end{array}$ & $\begin{array}{l}11 / 48 \\
(23 \%)\end{array}$ & $\begin{array}{l}13 / 50 \\
(26 \%)\end{array}$ & $\begin{array}{l}17 / 50 \\
(34 \%)\end{array}$ & $\begin{array}{l}9 / 46 \\
(20 \%)\end{array}$ \\
\hline & & Severity Profile & $3|6| 3 \mid 0(2.0)$ & $5|7| 3 \mid 0(1.9)$ & $3|7| 1 \mid 0$ (1.8) & $3|9| 1 \mid 0$ (1.8) & $5|5| 6 \mid 1(2.2)$ & $4|4| 1 \mid 0(1.7)$ \\
\hline & & Poly-3 Incidence & $12 / 39.0$ & $15 / 38.3$ & $11 / 37.9$ & $13 / 36.1$ & $17 / 39.8^{b}$ & $9 / 29.5$ \\
\hline & & & $(31 \%)$ & $(39 \%)$ & $(29 \%)$ & $(36 \%)$ & $(43 \%)$ & $(30 \%)$ \\
\hline \multirow{6}{*}{$\begin{array}{l}\text { Infiltration, cellular, } \\
\text { lymphocyte }\end{array}$} & \multirow{3}{*}{ Interim } & Incidence & $1 / 20$ & $1 / 20$ & $4 / 20$ & $1 / 19$ & $2 / 20$ & $2 / 22$ \\
\hline & & & $(5 \%)$ & $(5 \%)$ & $(20 \%)$ & $(5 \%)$ & $(10 \%)$ & $(9 \%)$ \\
\hline & & Severity Profile & $1|0| 0 \mid 0(1.0)$ & $1|0| 0 \mid 0$ (1.0) & $4|0| 0 \mid 0(1.0)$ & $1|0| 0 \mid 0(1.0)$ & $2|0| 0 \mid 0(1.0)$ & $2|0| 0 \mid 0(1.0)$ \\
\hline & \multirow[t]{3}{*}{ Terminal } & Incidence & $\begin{array}{l}14 / 49 \\
(29 \%)\end{array}$ & $\begin{array}{l}16 / 48 \\
(33 \%)\end{array}$ & $\begin{array}{l}16 / 48 \\
(33 \%)\end{array}$ & $\begin{array}{l}14 / 50 \\
(28 \%)\end{array}$ & $\begin{array}{l}12 / 50 \\
(24 \%)\end{array}$ & $\begin{array}{l}13 / 46 \\
(28 \%)\end{array}$ \\
\hline & & Severity Profile & $9|5| 0 \mid 0(1.4)$ & $13|3| 0 \mid 0$ (1.2) & $14|2| 0 \mid 0$ (1.1) & $11|3| 0 \mid 0$ (1.2) & $10|2| 0 \mid 0$ (1.2) & $12|1| 0 \mid 0$ (1.1) \\
\hline & & Poly-3 Incidence & $\begin{array}{c}14 / 40.2 \\
(35 \%)\end{array}$ & $\begin{array}{c}16 / 36.8 \\
(44 \%)\end{array}$ & $\begin{array}{c}16 / 38.3 \\
(42 \%)\end{array}$ & $\begin{array}{c}14 / 35.3 \\
(40 \%)\end{array}$ & $\begin{array}{c}12 / 39.9 \\
(30 \%)\end{array}$ & $\begin{array}{c}13 / 29.6 \\
(44 \%)\end{array}$ \\
\hline
\end{tabular}

BPA doses are $\mu \mathrm{g} / \mathrm{kg}$ bw/day. See legend to Table 55 for description of data presentation.

bIn the sensitivity analysis that excluded all animals that overlapped with animals treated with $250,000 \mu \mathrm{g}$ BPA/kg bw/day (see Statistical Methods), there was a statistically significant $(p=0.046)$ difference in the pairwise comparison between the $2,500 \mu \mathrm{g} \mathrm{BPA} / \mathrm{kg}$ bw/day dose group and the vehicle control (exfoliated germ cells, Poly-3 incidences $16 / 32.4(49 \%)$ versus $7 / 27.3(26 \%))$. 
CLARITY-BPA Core Study

Table 91. Non-Neoplastic Lesions in the Dorsal/Lateral Prostate of Interim and Terminal Sacrifice Male Rats: Continuous-Dose BPA ${ }^{\mathrm{a}}$

\begin{tabular}{|c|c|c|c|c|c|c|c|c|}
\hline Lesion & $\begin{array}{c}\text { Study Phase } \\
\text { (Interim/Terminal) }\end{array}$ & Statistic & Vehicle & 2.5 BPA & 25 BPA & 250 BPA & 2500 BPA & 25000 BPA \\
\hline \multirow{6}{*}{$\begin{array}{l}\text { Infiltration, cellular, } \\
\text { lymphocyte }\end{array}$} & \multirow[t]{2}{*}{ Interim } & Incidence & $\begin{array}{c}4 / 22 \\
(18 \%)\end{array}$ & $\begin{array}{l}10 / 22^{\wedge} b \\
(46 \%)\end{array}$ & $\begin{array}{l}5 / 20 \\
(25 \%)\end{array}$ & $\begin{array}{c}6 / 24 \\
(25 \%)\end{array}$ & $\begin{array}{l}5 / 20 \\
(25 \%)\end{array}$ & $\begin{array}{c}7 / 22 \\
(32 \%)\end{array}$ \\
\hline & & Severity Profile & $4|0| 0 \mid 0(1.0)$ & $9|1| 0 \mid 0(1.1)$ & $5|0| 0 \mid 0(1.0)$ & $5|1| 0 \mid 0(1.2)$ & $5|0| 0 \mid 0$ (1.0) & $5|2| 0 \mid 0(1.3)$ \\
\hline & \multirow[t]{4}{*}{ Terminal } & Incidence & $\begin{array}{l}33 / 50 \\
(66 \%)\end{array}$ & $\begin{array}{l}26 / 48 \\
(54 \%)\end{array}$ & $\begin{array}{l}27 / 48 \\
(56 \%)\end{array}$ & $\begin{array}{l}27 / 50 \\
(54 \%)\end{array}$ & $\begin{array}{l}27 / 50 \\
(54 \%)\end{array}$ & $\begin{array}{l}20 / 46 \\
(44 \%)\end{array}$ \\
\hline & & Severity Profile & $19|11| 2 \mid 1(1.5)$ & $18|7| 1 \mid 0(1.3)$ & $21|5| 1 \mid 0(1.3)$ & $16|6| 3 \mid 2(1.7)$ & $18|8| 0 \mid 1$ (1.4) & $10|7| 1 \mid 2(1.8)$ \\
\hline & & Poly-3 Incidence & $33 / 40.4^{*} \mathrm{~N}$ & $26 / 38.2$ & $27 / 38.7$ & $27 / 39.6$ & $27 / 38.7$ & $20 / 33.0$ \\
\hline & & & $(82 \%)$ & $(68 \%)$ & $(70 \%)$ & $(68 \%)$ & $(70 \%)$ & $(61 \%)$ \\
\hline \multirow{6}{*}{$\begin{array}{l}\text { Suppurative } \\
\text { inflammation }\end{array}$} & \multirow[t]{3}{*}{ Interim } & Incidence & $18 / 22$ & $20 / 22^{\wedge}$ & $18 / 20$ & $22 / 24^{\wedge}$ & $18 / 20^{\#, \wedge}$ & $19 / 22^{\#}$ \\
\hline & & & $(82 \%)$ & $(91 \%)$ & $(90 \%)$ & $(92 \%)$ & $(90 \%)$ & $(86 \%)$ \\
\hline & & Severity Profile & $11|7| 0 \mid 0$ (1.4) & $6|14| 0 \mid 0(1.7)$ & $9|9| 0 \mid 0(1.5)$ & $9|12| 1 \mid 0(1.6)$ & $6|10| 2 \mid 0(1.8)$ & $7|11| 1 \mid 0(1.7)$ \\
\hline & \multirow[t]{3}{*}{ Terminal } & Incidence & $\begin{array}{l}41 / 50 \\
(82 \%)\end{array}$ & $\begin{array}{l}46 / 48 \\
(96 \%)\end{array}$ & $\begin{array}{l}47 / 48 \\
(98 \%)\end{array}$ & $\begin{array}{l}45 / 50 \\
(90 \%)\end{array}$ & $\begin{array}{l}43 / 50 \\
(86 \%)\end{array}$ & $\begin{array}{l}41 / 46 \\
(89 \%)\end{array}$ \\
\hline & & Severity Profile & $8|26| 4 \mid 3(2.0)$ & $10|26| 9 \mid 1(2.0)$ & $9|30| 8 \mid 0(2.0)$ & $17|24| 2 \mid 2(1.8)$ & $8|28| 6 \mid 1(2.0)$ & $12|20| 6 \mid 3(2.0)$ \\
\hline & & Poly-3 Incidence & $\begin{array}{c}41 / 45.6 \\
(90 \%)\end{array}$ & $\begin{array}{l}46 / 46.2^{*} \\
(100 \%)\end{array}$ & $\begin{array}{c}47 / 48.0 \\
(98 \%)\end{array}$ & $\begin{array}{c}45 / 47.7 \\
(94 \%)\end{array}$ & $\begin{array}{c}43 / 46.5 \\
(92 \%)\end{array}$ & $\begin{array}{c}41 / 43.3 \\
(95 \%)\end{array}$ \\
\hline
\end{tabular}

${ }^{\mathrm{a} B P A}$ doses are $\mu \mathrm{g} / \mathrm{kg}$ bw/day. See legend to Table 55 for description of data presentation.

bIn the sensitivity analysis that excluded all animals that overlapped with animals treated with 250,000 $\mu \mathrm{g}$ BPA/kg bw/day (see Statistical Methods), the lymphocyte cellular infiltration in the 2.5 BPA dose group was also significant in the CAFE analysis (lymphocyte cellular infiltration, 9/16 (56\%), in treated group versus 3/17 (18\%), in controls, $p=0.025)$. 
CLARITY-BPA Core Study

Table 92. Non-Neoplastic Lesions in the Dorsal/Lateral Prostate of Interim and Terminal Sacrifice Male Rats: Continuous-Dose EE ${ }_{2}{ }^{\mathrm{a}}$

\begin{tabular}{|c|c|c|c|c|c|}
\hline Lesion & $\begin{array}{c}\text { Study Phase } \\
\text { (Interim/Terminal) }\end{array}$ & Statistic & Vehicle $^{b}$ & $0.05 \mathrm{EE}_{2}$ & $0.5 \mathrm{EE}_{2}$ \\
\hline \multirow[t]{5}{*}{$\begin{array}{l}\text { Infiltration, cellular, } \\
\text { lymphocyte }\end{array}$} & Interim & Incidence & $\begin{array}{c}4 / 22 \\
(18 \%)\end{array}$ & $\begin{array}{c}9 / 26 \\
(35 \%)\end{array}$ & $\begin{array}{c}4 / 26 \\
(15 \%)\end{array}$ \\
\hline & & Severity Profile & $4|0| 0 \mid 0(1.0)$ & $9|0| 0 \mid 0(1.0)$ & $4|0| 0 \mid 0(1.0)$ \\
\hline & Terminal & Incidence & $\begin{array}{l}33 / 50 \\
(66 \%)\end{array}$ & $\begin{array}{l}17 / 26 \\
(65 \%)\end{array}$ & $\begin{array}{l}13 / 25 \\
(52 \%)\end{array}$ \\
\hline & & Severity Profile & $19|11| 2 \mid 1(1.5)$ & $10|6| 1 \mid 0(1.5)$ & $6|6| 0 \mid 1(1.7)$ \\
\hline & & Poly-3 Incidence & $\begin{array}{c}33 / 40.4 \\
(82 \%)\end{array}$ & $\begin{array}{l}17 / 21.3 \\
(80 \%)\end{array}$ & $\begin{array}{c}13 / 20.1 \\
(65 \%)\end{array}$ \\
\hline \multirow[t]{5}{*}{$\begin{array}{l}\text { Suppurative } \\
\text { inflammation }\end{array}$} & Interim & Incidence & $\begin{array}{l}18 / 22 \\
(82 \%)\end{array}$ & $\begin{array}{l}25 / 26 \\
(96 \%)\end{array}$ & $\begin{array}{l}25 / 26 \\
(96 \%)\end{array}$ \\
\hline & & Severity Profile & $11|7| 0 \mid 0(1.4)$ & $13|11| 0 \mid 1(1.6)$ & $12|13| 0 \mid 0(1.5)$ \\
\hline & Terminal & Incidence & $\begin{array}{l}41 / 50 \\
(82 \%)\end{array}$ & $\begin{array}{c}26 / 26 \\
(100 \%)\end{array}$ & $\begin{array}{l}22 / 25 \\
(88 \%)\end{array}$ \\
\hline & & Severity Profile & $8|26| 4 \mid 3(2.0)$ & $8|16| 1 \mid 1(1.8)$ & $6|12| 3 \mid 1(2.0)$ \\
\hline & & Poly-3 Incidence & $\begin{array}{c}41 / 45.6 \\
(90 \%)\end{array}$ & $\begin{array}{l}26 / 26.0 \\
(100 \%)\end{array}$ & $\begin{array}{c}22 / 23.1 \\
(95 \%)\end{array}$ \\
\hline
\end{tabular}

${ }^{\mathrm{a}} \mathrm{EE}_{2}$ doses are $\mu \mathrm{g} / \mathrm{kg}$ bw/day. See legend to Table 55 for description of data presentation.

${ }^{\mathrm{b}}$ The continuous vehicle control group data are the same as those shown for the continuous-dose BPA data in Table 91. 
CLARITY-BPA Core Study

Table 93. Non-Neoplastic Lesions in the Dorsal/Lateral Prostate of Interim and Terminal Sacrifice Male Rats: Stop-Dose BPA ${ }^{\mathrm{a}}$

\begin{tabular}{|c|c|c|c|c|c|c|c|c|}
\hline Lesion & $\begin{array}{c}\text { Study Phase } \\
\text { (Interim/Terminal) }\end{array}$ & Statistic & Vehicle & 2.5 BPA & 25 BPA & 250 BPA & 2500 BPA & 25000 BPA \\
\hline \multirow[t]{5}{*}{$\begin{array}{l}\text { Infiltration, cellular, } \\
\text { lymphocyte }\end{array}$} & Interim & Incidence & $\begin{array}{c}9 / 20 \\
(45 \%)\end{array}$ & $\begin{array}{l}5 / 20 \\
(25 \%)\end{array}$ & $\begin{array}{c}4 / 20 \\
(20 \%)\end{array}$ & $\begin{array}{c}8 / 18 \\
(44 \%)\end{array}$ & $\begin{array}{c}8 / 20 \\
(40 \%)\end{array}$ & $\begin{array}{c}6 / 22 \\
(27 \%)\end{array}$ \\
\hline & & Severity Profile & $9|0| 0 \mid 0(1.0)$ & $4|1| 0 \mid 0(1.2)$ & $3|1| 0 \mid 0(1.2)$ & $8|0| 0 \mid 0(1.0)$ & $5|3| 0 \mid 0(1.4)$ & $6|0| 0 \mid 0(1.0)$ \\
\hline & Terminal & Incidence & $\begin{array}{l}31 / 46 \\
(67 \%)\end{array}$ & $\begin{array}{l}30 / 48 \\
(62 \%)\end{array}$ & $\begin{array}{l}28 / 48 \\
(58 \%)\end{array}$ & $\begin{array}{l}27 / 50 \\
(54 \%)\end{array}$ & $\begin{array}{l}35 / 49 \\
(71 \%)\end{array}$ & $\begin{array}{l}22 / 45^{\#, \wedge} \mathrm{N} \\
(49 \%)\end{array}$ \\
\hline & & Severity Profile & $17|11| 1 \mid 2(1.6)$ & $20|6| 1 \mid 3(1.6)$ & $15|9| 2 \mid 2(1.7)$ & $16|8| 1 \mid 2(1.6)$ & $20|14| 1 \mid 0(1.5)$ & $14|7| 0 \mid 1(1.5)$ \\
\hline & & Poly-3 Incidence & $\begin{array}{c}31 / 41.6 \\
(75 \%)\end{array}$ & $\begin{array}{c}30 / 41.1 \\
(73 \%)\end{array}$ & $\begin{array}{c}28 / 40.0 \\
(70 \%)\end{array}$ & $\begin{array}{c}27 / 38.9 \\
(69 \%)\end{array}$ & $\begin{array}{c}35 / 42.4 \\
(83 \%)\end{array}$ & $\begin{array}{c}22 / 33.2 \\
(66 \%)\end{array}$ \\
\hline \multirow[t]{5}{*}{$\begin{array}{l}\text { Suppurative } \\
\text { inflammation }\end{array}$} & Interim & Incidence & $\begin{array}{l}18 / 20 \\
(90 \%)\end{array}$ & $\begin{array}{l}19 / 20 \\
(95 \%)\end{array}$ & $\begin{array}{l}16 / 20 \\
(80 \%)\end{array}$ & $\begin{array}{l}16 / 18 \\
(89 \%)\end{array}$ & $\begin{array}{l}19 / 20 \\
(95 \%)\end{array}$ & $\begin{array}{l}18 / 22 \\
(82 \%)\end{array}$ \\
\hline & & Severity Profile & $5|13| 0 \mid 0(1.7)$ & $12|7| 0 \mid 0(1.4)$ & $6|10| 0 \mid 0(1.6)$ & $7|9| 0 \mid 0(1.6)$ & $9|10| 0 \mid 0(1.5)$ & $5|12| 1 \mid 0(1.8)$ \\
\hline & Terminal & Incidence & $\begin{array}{l}39 / 46 \\
(85 \%)\end{array}$ & $\begin{array}{l}46 / 48 \\
(96 \%)\end{array}$ & $\begin{array}{l}41 / 48 \\
(85 \%)\end{array}$ & $\begin{array}{l}42 / 50 \\
(84 \%)\end{array}$ & $\begin{array}{l}44 / 49 \\
(90 \%)\end{array}$ & $\begin{array}{l}38 / 45 \\
(84 \%)\end{array}$ \\
\hline & & Severity Profile & $7|22| 7 \mid 3(2.2)$ & $14|21| 8 \mid 3(2.0)$ & $14|20| 5 \mid 2(1.9)$ & $10|20| 9 \mid 3(2.1)$ & $12|21| 11 \mid 0(2.0)$ & $6|25| 6 \mid 1(2.1)$ \\
\hline & & Poly-3 Incidence & $\begin{array}{c}39 / 43.3 \\
(90 \%) \\
\end{array}$ & $\begin{array}{c}46 / 46.7 \\
(98 \%) \\
\end{array}$ & $\begin{array}{c}41 / 45.4 \\
(90 \%) \\
\end{array}$ & $\begin{array}{c}42 / 45.2 \\
(93 \%) \\
\end{array}$ & $\begin{array}{c}44 / 46.3 \\
(95 \%) \\
\end{array}$ & $\begin{array}{c}38 / 41.2 \\
(92 \%) \\
\end{array}$ \\
\hline
\end{tabular}

${ }^{\mathrm{a} B P A}$ doses are $\mu \mathrm{g} / \mathrm{kg}$ bw/day. See legend to Table 55 for description of data presentation. 
CLARITY-BPA Core Study

Table 94. Neoplastic Lesions in the Ventral Prostate of Interim and Terminal Sacrifice Male Rats: Continuous-Dose BPA ${ }^{\mathrm{a}}$

\begin{tabular}{|c|c|c|c|c|c|c|c|c|}
\hline Lesion & $\begin{array}{c}\text { Study Phase } \\
\text { (Interim/Terminal) }\end{array}$ & Statistic & Vehicle & 2.5 BPA & 25 BPA & 250 BPA & 2500 BPA & 25000 BPA \\
\hline \multirow[t]{10}{*}{ Adenoma } & \multirow[t]{10}{*}{ Terminal } & \multirow[t]{2}{*}{ Incidence } & $6 / 50$ & $7 / 48$ & $2 / 48$ & $4 / 49$ & $2 / 49$ & $6 / 46$ \\
\hline & & & $(12 \%)$ & $(15 \%)$ & $(4 \%)$ & $(8 \%)$ & $(4 \%)$ & $(13 \%)$ \\
\hline & & \multirow[t]{2}{*}{ Poly-3 Incidence } & $6 / 34.4$ & $7 / 34.4$ & $2 / 32.3$ & $4 / 32.9$ & $2 / 33.1$ & $6 / 29.4$ \\
\hline & & & $(17 \%)$ & $(20 \%)$ & $(6 \%)$ & $(12 \%)$ & $(6 \%)$ & $(20 \%)$ \\
\hline & & \multirow[t]{2}{*}{ Terminal Incidence } & $3 / 15$ & $4 / 16$ & $2 / 17$ & $2 / 14$ & $2 / 16$ & $3 / 11$ \\
\hline & & & $(20 \%)$ & $(25 \%)$ & $(12 \%)$ & $(14 \%)$ & $(12 \%)$ & $(27 \%)$ \\
\hline & & Time-to-First & 603 & 683 & $726(\mathrm{~T})$ & 713 & $726(\mathrm{~T})$ & 613 \\
\hline & & Poly-3 $p$-value & $0.287 \mathrm{~N}$ & 0.499 & $0.148 \mathrm{~N}$ & $0.394 \mathrm{~N}$ & $0.139 \mathrm{~N}$ & 0.506 \\
\hline & & \multirow[t]{2}{*}{ Multiple Incidence ${ }^{\mathrm{d}}$} & $0 / 50$ & $2 / 48$ & $0 / 48$ & $0 / 49$ & $0 / 49$ & $3 / 46$ \\
\hline & & & $(0 \%)$ & $(4 \%)$ & $(0 \%)$ & $(0 \%)$ & $(0 \%)$ & $(7 \%)$ \\
\hline
\end{tabular}

${ }^{\mathrm{a} B P A}$ doses are $\mu \mathrm{g} / \mathrm{kg}$ bw/day. See legend to Table 52 for description of data presentation.

Table 95. Neoplastic Lesions in the Ventral Prostate of Interim and Terminal Sacrifice Male Rats: Continuous-Dose EE$_{2}{ }^{\mathrm{a}}$

\begin{tabular}{|c|c|c|c|c|c|}
\hline Lesion & $\begin{array}{c}\text { Study Phase } \\
\text { (Interim/Terminal) } \\
\end{array}$ & Statistic & Vehicle $^{b}$ & $0.05 \mathbf{E E}_{2}$ & $0.50 \mathrm{EE}_{2}$ \\
\hline \multirow[t]{6}{*}{ Adenoma } & Terminal & Incidence & $\begin{array}{c}6 / 50 \\
(12 \%)\end{array}$ & $\begin{array}{l}2 / 26 \\
(8 \%)\end{array}$ & $\begin{array}{l}2 / 26 \\
(8 \%)\end{array}$ \\
\hline & & Poly-3 Incidence & $\begin{array}{l}6 / 34.4 \\
(17 \%)\end{array}$ & $\begin{array}{l}2 / 17.6 \\
(11 \%)\end{array}$ & $\begin{array}{l}2 / 20.7 \\
(10 \%)\end{array}$ \\
\hline & & Terminal Incidence & $\begin{array}{c}3 / 15 \\
(20 \%)\end{array}$ & $\begin{array}{c}2 / 9 \\
(22 \%)\end{array}$ & $\begin{array}{l}0 / 12 \\
(0 \%)\end{array}$ \\
\hline & & Time-to-First & 603 & $725(\mathrm{~T})$ & 462 \\
\hline & & Poly-3 $p$-value & $0.287 \mathrm{~N}$ & $0.433 \mathrm{~N}$ & $0.344 \mathrm{~N}$ \\
\hline & & Multiple Incidence ${ }^{\mathrm{d}}$ & $\begin{array}{l}0 / 50 \\
(0 \%)\end{array}$ & $\begin{array}{l}1 / 26 \\
(4 \%)\end{array}$ & $\begin{array}{l}0 / 26 \\
(0 \%)\end{array}$ \\
\hline
\end{tabular}

${ }^{\mathrm{a}} \mathrm{EE}_{2}$ doses are $\mu \mathrm{g} / \mathrm{kg}$ bw/day. See legend to Table 52 for description of data presentation.

${ }^{\mathrm{b}}$ The continuous vehicle control group data are the same as those shown for the continuous-dose BPA data in Table 94. 
CLARITY-BPA Core Study

Table 96. Neoplastic Lesions in the Ventral Prostate of Interim and Terminal Sacrifice Male Rats: Stop-Dose BPA ${ }^{\mathrm{a}}$

\begin{tabular}{|c|c|c|c|c|c|c|c|c|}
\hline Lesion & $\begin{array}{c}\text { Study Phase } \\
\text { (Interim/Terminal) }\end{array}$ & Statistic & Vehicle & $2.5 \mathrm{BPA}$ & 25 BPA & 250 BPA & 2500 BPA & 25000 BPA \\
\hline \multirow[t]{10}{*}{ Adenoma } & \multirow[t]{10}{*}{ Terminal } & \multirow[t]{2}{*}{ Incidence } & $4 / 48$ & $4 / 47$ & $4 / 47$ & $2 / 50$ & $4 / 49$ & $6 / 45$ \\
\hline & & & $(8 \%)$ & $(8 \%)$ & $(8 \%)$ & $(4 \%)$ & $(8 \%)$ & $(13 \%)$ \\
\hline & & \multirow[t]{2}{*}{ Poly-3 Incidence } & $4 / 35.7$ & $4 / 33.0$ & $4 / 34.1$ & $2 / 30.8$ & $4 / 36.5$ & $6 / 28.7$ \\
\hline & & & $(11 \%)$ & $(12 \%)$ & $(12 \%)$ & $(6 \%)$ & $(11 \%)$ & $(21 \%)$ \\
\hline & & \multirow[t]{2}{*}{ Terminal Incidence } & $2 / 17$ & $2 / 16$ & $4 / 16$ & $2 / 13$ & $1 / 15$ & $1 / 9$ \\
\hline & & & $(12 \%)$ & $(12 \%)$ & $(25 \%)$ & $(15 \%)$ & $(7 \%)$ & $(11 \%)$ \\
\hline & & Time-to-First & 675 & 679 & $724(\mathrm{~T})$ & $724(\mathrm{~T})$ & 593 & 530 \\
\hline & & Poly-3 $p$-value & 0.273 & 0.601 & 0.619 & $0.407 \mathrm{~N}$ & $0.635 \mathrm{~N}$ & 0.233 \\
\hline & & \multirow[t]{2}{*}{ Multiple Incidence ${ }^{\mathrm{d}}$} & $1 / 48$ & $2 / 47$ & $1 / 47$ & $1 / 50$ & $2 / 49$ & $2 / 45$ \\
\hline & & & $(2 \%)$ & $(4 \%)$ & $(2 \%)$ & $(2 \%)$ & $(4 \%)$ & $(4 \%)$ \\
\hline
\end{tabular}

${ }^{a}$ BPA doses are $\mu \mathrm{g} / \mathrm{kg}$ bw/day. See legend to Table 52 for description of data presentation. 
CLARITY-BPA Core Study

Table 97. Non-Neoplastic Lesions in the Ventral Prostate of Interim and Terminal Sacrifice Male Rats: Continuous-Dose BPA ${ }^{\text {a }}$

\begin{tabular}{|c|c|c|c|c|c|c|c|c|}
\hline Lesion & $\begin{array}{c}\text { Study Phase } \\
\text { (Interim/Terminal) }\end{array}$ & Statistic & Vehicle & 2.5 BPA & 25 BPA & 250 BPA & 2500 BPA & 25000 BPA \\
\hline \multirow{6}{*}{$\begin{array}{l}\text { Infiltration, } \\
\text { cellular, } \\
\text { lymphocyte }\end{array}$} & Interim & Incidence & $\begin{array}{c}8 / 22 \\
(36 \%)\end{array}$ & $\begin{array}{l}10 / 22 \\
(45 \%)\end{array}$ & $\begin{array}{l}10 / 20 \\
(50 \%)\end{array}$ & $\begin{array}{c}8 / 24 \\
(33 \%)\end{array}$ & $\begin{array}{c}9 / 20 \\
(45 \%)\end{array}$ & $\begin{array}{c}8 / 22 \\
(36 \%)\end{array}$ \\
\hline & & Severity Profile & $8|0| 0 \mid 0$ & $8|2| 0 \mid 0$ & $9|1| 0 \mid 0$ & $7|1| 0 \mid 0$ & $8|1| 0 \mid 0$ & $7|1| 0 \mid 0$ \\
\hline & Terminal & Incidence & $\begin{array}{l}25 / 50 \\
(50 \%)\end{array}$ & $\begin{array}{c}14 / 48^{\# \#, \wedge \wedge \mathrm{N}} \\
(29 \%)\end{array}$ & $\begin{array}{c}15 / 48^{\# \#, \wedge \wedge} \mathrm{N} \\
(31 \%)\end{array}$ & $\begin{array}{c}15 / 49^{\# \#, \wedge \mathrm{N}} \\
(31 \%)\end{array}$ & $\begin{array}{c}20 / 49^{\# N} \\
(41 \%)\end{array}$ & $\begin{array}{c}15 / 46^{\#, \wedge} \mathrm{N} \\
(33 \%)\end{array}$ \\
\hline & & Severity Profile & $12|5| 5 \mid 3$ & $9|4| 1 \mid 0$ & $14|1| 0 \mid 0$ & $9|4| 0 \mid 2$ & $15|3| 1 \mid 1$ & $9|3| 1 \mid 2$ \\
\hline & & Poly-3 & $25 / 40.8$ & $14 / 38.2^{* \mathrm{~N}}$ & $15 / 39.1^{* N}$ & $15 / 39.4^{* \mathrm{~N}}$ & $20 / 39.0$ & $15 / 33.3$ \\
\hline & & Incidence & $(61 \%)$ & $(37 \%)$ & $(38 \%)$ & $(38 \%)$ & $(51 \%)$ & $(45 \%)$ \\
\hline \multirow[t]{6}{*}{$\begin{array}{l}\text { Suppurative } \\
\text { inflammation }\end{array}$} & Interim & Incidence & $\begin{array}{c}10 / 22^{* *}, \# \#, \wedge \wedge \mathrm{N} \\
(45 \%)\end{array}$ & $\begin{array}{c}3 / 22^{*}, \#, \wedge \wedge \mathrm{N} \\
(14 \%)\end{array}$ & $\begin{array}{c}4 / 20^{\#, \wedge \mathrm{N}} \\
(20 \%)\end{array}$ & $\begin{array}{c}5 / 24^{\#, \wedge N} \\
(21 \%)\end{array}$ & 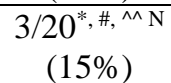 & $\begin{array}{c}1 / 22^{* *}, \# \#,{ }^{\wedge \wedge \wedge} \mathrm{N} \\
(5 \%)\end{array}$ \\
\hline & & Severity Profile & $9|1| 0 \mid 0$ & $3|0| 0 \mid 0$ & $2|2| 0 \mid 0$ & $4|1| 0 \mid 0$ & $3|0| 0 \mid 0$ & $1|0| 0 \mid 0$ \\
\hline & Terminal & Incidence & $\begin{array}{l}16 / 50 \\
(32 \%)\end{array}$ & $\begin{array}{c}5 / 48^{\# \#, \wedge \wedge} \mathrm{N} \\
(10 \%)\end{array}$ & $\begin{array}{c}5 / 48^{\# \#, \wedge \wedge \mathrm{N}} \\
(10 \%)\end{array}$ & $\begin{array}{c}6 / 49^{\# \#, \wedge \wedge} \mathrm{N} \\
(12 \%)\end{array}$ & $\begin{array}{c}5 / 49^{\# \#, \wedge \wedge} \mathrm{N} \\
(10 \%)\end{array}$ & $\begin{array}{c}11 / 46^{\# \# \mathrm{~N}} \\
(24 \%)\end{array}$ \\
\hline & & Severity Profile & $7|2| 2 \mid 5$ & $3|2| 0 \mid 0$ & $3|2| 0 \mid 0$ & $3|1| 0 \mid 2$ & $1|2| 1 \mid 1$ & $5|2| 1 \mid 3$ \\
\hline & & Poly-3 & $16 / 38.1$ & $5 / 34.6^{* * N}$ & $5 / 34.6^{* * N}$ & $6 / 35.3^{* N}$ & $5 / 34.9^{* *} \mathrm{~N}$ & $11 / 31.9$ \\
\hline & & Incidence & $(42 \%)$ & $(14 \%)$ & $(14 \%)$ & $(17 \%)$ & $(14 \%)$ & $(34 \%)$ \\
\hline \multirow{6}{*}{$\begin{array}{l}\text { Hyperplasia, } \\
\text { epithelium }\end{array}$} & Interim & Incidence & $0 / 22$ & $0 / 22$ & $0 / 20$ & $1 / 24$ & $0 / 20$ & $0 / 22$ \\
\hline & & Severity Profile & - & - & - & $0 / 1 / 0 / 0$ & - & - \\
\hline & Terminal & Incidence & $\begin{array}{l}10 / 50 \\
(20 \%)\end{array}$ & $\begin{array}{l}12 / 48 \\
(25 \%)\end{array}$ & $\begin{array}{l}10 / 48 \\
(21 \%)\end{array}$ & $\begin{array}{l}18 / 49^{\wedge} \\
(37 \%)\end{array}$ & $\begin{array}{l}12 / 49 \\
(24 \%)\end{array}$ & $\begin{array}{c}8 / 46 \\
(17 \%)\end{array}$ \\
\hline & & Severity Profile & $1|6| 3 \mid 0$ & $2|6| 3 \mid 1$ & $2|4| 4 \mid 0$ & $1|13| 3 \mid 1$ & $3|6| 3 \mid 0$ & $2|2| 2 \mid 2$ \\
\hline & & Poly-3 & $10 / 34.0$ & $12 / 36.4$ & $10 / 35.0$ & $18 / 36.3$ & $12 / 34.7$ & $8 / 30.5$ \\
\hline & & Incidence & $(29 \%)$ & $(33 \%)$ & $(28 \%)$ & $(50 \%)$ & $(35 \%)$ & $(26 \%)$ \\
\hline
\end{tabular}

${ }^{\mathrm{a} B P A}$ doses are $\mu \mathrm{g} / \mathrm{kg}$ bw/day. See legend to Table 55 for description of data presentation. 
CLARITY-BPA Core Study

Table 98. Non-Neoplastic Lesions in the Ventral Prostate of Interim and Terminal Sacrifice Male Rats: Continuous-Dose EE ${ }_{2}{ }^{\mathrm{a}}$

\begin{tabular}{|c|c|c|c|c|c|}
\hline Lesion & $\begin{array}{c}\text { Study Phase } \\
\text { (Interim/Terminal) }\end{array}$ & Statistic & Vehicle $^{b}$ & $0.05 \mathrm{EE}_{2}$ & $0.5 \mathrm{EE}_{2}$ \\
\hline \multirow[t]{5}{*}{$\begin{array}{l}\text { Infiltration, cellular, } \\
\text { lymphocyte }\end{array}$} & Interim & Incidence & $\begin{array}{c}8 / 22 \\
(36 \%)\end{array}$ & $\begin{array}{l}13 / 26 \\
(50 \%)\end{array}$ & $\begin{array}{l}13 / 26 \\
(50 \%)\end{array}$ \\
\hline & & Severity Profile & $8|0| 0 \mid 0$ & $11|2| 0 \mid 0$ & $12|1| 0 \mid 0$ \\
\hline & Terminal & Incidence & $\begin{array}{l}25 / 50 \\
(50 \%)\end{array}$ & $\begin{array}{l}6 / 26^{\wedge \wedge} \mathrm{N} \\
(23 \%)\end{array}$ & $\begin{array}{l}14 / 26 \\
(54 \%)\end{array}$ \\
\hline & & Severity Profile & $12|5| 5 \mid 3$ & $5|0| 1 \mid 0$ & $6|7| 0 \mid 1$ \\
\hline & & Poly-3 Incidence & $\begin{array}{c}25 / 40.8 \\
(61 \%)\end{array}$ & $\begin{array}{l}6 / 19.2^{* N} \\
(31 \%)\end{array}$ & $\begin{array}{c}14 / 22.3 \\
(63 \%)\end{array}$ \\
\hline \multirow[t]{5}{*}{$\begin{array}{l}\text { Suppurative } \\
\text { inflammation }\end{array}$} & Interim & Incidence & $\begin{array}{c}10 / 22^{*}, \#, \wedge \mathrm{N} \\
(45 \%)\end{array}$ & $\begin{array}{c}4 / 26^{*}, \#, \wedge \mathrm{N} \\
(15 \%)\end{array}$ & $\begin{array}{c}5 / 26^{\#, \wedge N} \\
(19 \%)\end{array}$ \\
\hline & & Severity Profile & $9|1| 0 \mid 0$ & $3|1| 0 \mid 0$ & $5|0| 0 \mid 0$ \\
\hline & Terminal & Incidence & $\begin{array}{l}16 / 50 \\
(32 \%)\end{array}$ & $\begin{array}{l}4 / 26^{\wedge} \mathrm{N} \\
(15 \%)\end{array}$ & $\begin{array}{c}8 / 26 \\
(31 \%)\end{array}$ \\
\hline & & Severity Profile & $7|2| 2 \mid 5$ & $3|0| 0 \mid 1$ & $5|2| 0 \mid 1$ \\
\hline & & Poly-3 Incidence & $\begin{array}{c}16 / 38.1 \\
(42 \%) \\
\end{array}$ & $\begin{array}{l}4 / 18.7 \\
(21 \%) \\
\end{array}$ & $\begin{array}{l}8 / 22.2 \\
(36 \%)\end{array}$ \\
\hline \multirow[t]{5}{*}{ Hyperplasia, epithelium } & Interim & Incidence & $\begin{array}{l}0 / 22 \\
(0 \%)\end{array}$ & $\begin{array}{l}0 / 26 \\
(0 \%)\end{array}$ & $\begin{array}{l}0 / 26 \\
(0 \%)\end{array}$ \\
\hline & & Severity Profile & - & - & - \\
\hline & Terminal & Incidence & $\begin{array}{l}10 / 50 \\
(20 \%)\end{array}$ & $\begin{array}{c}4 / 26 \\
(15 \%)\end{array}$ & $\begin{array}{l}7 / 26 \\
(27 \%)\end{array}$ \\
\hline & & Severity Profile & $1|6| 3 \mid 0$ & $0|1| 2 \mid 1$ & $1|4| 2 \mid 0$ \\
\hline & & Poly-3 Incidence & $\begin{array}{c}10 / 34.0 \\
(29 \%)\end{array}$ & $\begin{array}{l}4 / 17.6 \\
(23 \%)\end{array}$ & $\begin{array}{l}7 / 19.9 \\
(35 \%)\end{array}$ \\
\hline
\end{tabular}

${ }^{\mathrm{a}} \mathrm{EE}_{2}$ doses are $\mu \mathrm{g} / \mathrm{kg}$ bw/day. See legend to Table 55 for description of data presentation.

${ }^{b}$ The continuous vehicle control group data are the same as those shown for the continuous-dose BPA data in Table 97. 
CLARITY-BPA Core Study

Table 99. Non-Neoplastic Lesions in the Ventral Prostate of Interim and Terminal Sacrifice Male Rats: Stop-Dose BPA ${ }^{\text {a }}$

\begin{tabular}{|c|c|c|c|c|c|c|c|c|}
\hline Lesion & $\begin{array}{c}\text { Study Phase } \\
\text { (Interim/Terminal) }\end{array}$ & Statistic & Vehicle & 2.5 BPA & 25 BPA & 250 BPA & 2500 BPA & 25000 BPA \\
\hline \multirow[t]{6}{*}{$\begin{array}{l}\text { Infiltration, cellular, } \\
\text { lymphocyte }\end{array}$} & Interim & Incidence & $\begin{array}{c}9 / 20 \\
(45 \%)\end{array}$ & $\begin{array}{c}8 / 20 \\
(40 \%)\end{array}$ & $\begin{array}{c}9 / 20 \\
(45 \%)\end{array}$ & $\begin{array}{c}9 / 18 \\
(50 \%)\end{array}$ & $\begin{array}{c}8 / 20 \\
(40 \%)\end{array}$ & $\begin{array}{c}9 / 22 \\
(41 \%)\end{array}$ \\
\hline & & Severity Profile & $8|1| 0 \mid 0$ & $7|1| 0 \mid 0$ & $8|1| 0 \mid 0$ & $8|1| 0 \mid 0$ & $7|1| 0 \mid 0$ & $9|0| 0 \mid 0$ \\
\hline & Terminal & Incidence & $\begin{array}{l}19 / 48 \\
(40 \%)\end{array}$ & $\begin{array}{l}17 / 47 \\
(36 \%)\end{array}$ & $\begin{array}{l}15 / 47 \\
(32 \%)\end{array}$ & $\begin{array}{l}16 / 50 \\
(32 \%)\end{array}$ & $\begin{array}{l}20 / 49 \\
(41 \%)\end{array}$ & $\begin{array}{l}18 / 45 \\
(40 \%)\end{array}$ \\
\hline & & Severity Profile & $9|7| 2 \mid 1$ & $11|3| 0 \mid 3$ & $10|1| 0 \mid 4$ & $8|5| 2 \mid 1$ & $14|5| 1 \mid 0$ & $11|3| 2 \mid 2$ \\
\hline & & Poly-3 Incidence & $19 / 39.2$ & $17 / 37.7$ & $15 / 38.4$ & $16 / 34.5$ & $20 / 40.9$ & $18 / 33.2$ \\
\hline & & & $(48 \%)$ & $(45 \%)$ & $(39 \%)$ & $(46 \%)$ & $(49 \%)$ & $(54 \%)$ \\
\hline \multirow{8}{*}{$\begin{array}{l}\text { Suppurative } \\
\text { inflammation }\end{array}$} & Interim & Incidence & $3 / 20$ & $2 / 20$ & $2 / 20$ & $6 / 18$ & $4 / 20$ & $2 / 22$ \\
\hline & & & $(15 \%)$ & $(10 \%)$ & $(10 \%)$ & $(33 \%)$ & $(20 \%)$ & $(9 \%)$ \\
\hline & & Severity Profile & $2|1| 0 \mid 0$ & $2|0| 0 \mid 0$ & $2|0| 0 \mid 0$ & $6|0| 0 \mid 0$ & $4|0| 0 \mid 0$ & $2|0| 0 \mid 0$ \\
\hline & Terminal & Incidence & $10 / 48$ & $9 / 47$ & $9 / 47$ & $8 / 50$ & $10 / 49$ & $9 / 45$ \\
\hline & & & $(21 \%)$ & $(19 \%)$ & $(19 \%)$ & $(16 \%)$ & $(20 \%)$ & $(20 \%)$ \\
\hline & & Severity Profile & $5|2| 1 \mid 2$ & $5|1| 1 \mid 2$ & $3|2| 0 \mid 4$ & $4|1| 1 \mid 2$ & $5|4| 0 \mid 1$ & $4|2| 2 \mid 1$ \\
\hline & & Poly-3 Incidence & $10 / 36.8$ & $9 / 35.2$ & $9 / 37.3$ & $8 / 33.7$ & $10 / 38.0$ & $9 / 30.1$ \\
\hline & & & $(27 \%)$ & $(26 \%)$ & $(24 \%)$ & $(24 \%)$ & $(26 \%)$ & $(30 \%)$ \\
\hline \multirow{8}{*}{$\begin{array}{l}\text { Hyperplasia, } \\
\text { epithelium }\end{array}$} & Interim & Incidence & $1 / 20$ & $1 / 20$ & $0 / 20$ & $2 / 18$ & $0 / 20$ & $0 / 22$ \\
\hline & & & $(5 \%)$ & $(5 \%)$ & $(0 \%)$ & $(11 \%)$ & $(0 \%)$ & $(0 \%)$ \\
\hline & & Severity Profile & $0|1| 0 \mid 0$ & $0|1| 0 \mid 0$ & - & $2|0| 0 \mid 0$ & - & - \\
\hline & Terminal & Incidence & $17 / 48$ & $7 / 47^{\# \# \mathrm{~N}}$ & $17 / 47$ & $16 / 50$ & $14 / 49$ & $13 / 45$ \\
\hline & & & $(35 \%)$ & $(15 \%)$ & $(36 \%)$ & $(32 \%)$ & $(29 \%)$ & $(29 \%)$ \\
\hline & & Severity Profile & $0|12| 3 \mid 2$ & $2|3| 1 \mid 1$ & $4|8| 3 \mid 2$ & $4|9| 1 \mid 2$ & $1|13| 0 \mid 0$ & $1|10| 0 \mid 2$ \\
\hline & & Poly-3 Incidence & $17 / 38.9$ & $7 / 32.9^{*} \mathrm{~N}$ & $17 / 36.0$ & $16 / 35.7$ & $14 / 37.4$ & $13 / 30.2$ \\
\hline & & & $(44 \%)$ & $(21 \%)$ & $(47 \%)$ & $(45 \%)$ & $(37 \%)$ & $(43 \%)$ \\
\hline
\end{tabular}

${ }^{\mathrm{a} B P A}$ doses are $\mu \mathrm{g} / \mathrm{kg}$ bw/day. See legend to Table 55 for description of data presentation. 
CLARITY-BPA Core Study

Table 100. Non-Neoplastic Lesions in the Pituitary of Interim and Terminal Sacrifice Male Rats: Continuous-Dose BPA ${ }^{\mathrm{a}}$

\begin{tabular}{|c|c|c|c|c|c|c|c|c|}
\hline Lesion & $\begin{array}{c}\text { Study Phase } \\
\text { (Interim/Terminal) }\end{array}$ & Statistic & Vehicle & 2.5 BPA & 25 BPA & 250 BPA & 2500 BPA & 25000 BPA \\
\hline \multirow{8}{*}{$\begin{array}{l}\text { Hyperplasia, } \\
\text { pars distalis }\end{array}$} & \multirow[t]{3}{*}{ Interim } & Incidence & $4 / 22$ & $6 / 22$ & $2 / 20$ & $4 / 24$ & $2 / 20$ & $4 / 22$ \\
\hline & & & $(18 \%)$ & $(27 \%)$ & $(10 \%)$ & $(17 \%)$ & $(10 \%)$ & $(18 \%)$ \\
\hline & & Severity Profile & $3|1| 0 \mid 0(1.2)$ & $6|0| 0 \mid 0(1.0)$ & $1|1| 0 \mid 0(1.5)$ & $2|2| 0 \mid 0(1.5)$ & $2|0| 0 \mid 0(1.0)$ & $2|2| 0 \mid 0(1.5)$ \\
\hline & \multirow[t]{5}{*}{ Terminal } & Incidence & $11 / 48^{\#, \wedge}$ & $9 / 48$ & $19 / 48^{\wedge}$ & $15 / 50$ & $17 / 50$ & $19 / 45^{\#, \wedge}$ \\
\hline & & & $(23 \%)$ & $(19 \%)$ & $(40 \%)$ & $(30 \%)$ & $(34 \%)$ & $(42 \%)$ \\
\hline & & Severity Profile & $3|4| 4 \mid 0(2.1)$ & $0|4| 4 \mid 1(2.7)$ & $5|8| 2 \mid 4(2.3)$ & $4|6| 3 \mid 2(2.2)$ & $4|7| 5 \mid 1(2.2)$ & $3|9| 3 \mid 4(2.4)$ \\
\hline & & Poly-3 & $11 / 36.2^{* *}$ & $9 / 36.3$ & $19 / 37.8$ & $15 / 37.8$ & $17 / 38.5$ & $19 / 34.2^{*}$ \\
\hline & & Incidence & $(30 \%)$ & $(25 \%)$ & $(50 \%)$ & $(40 \%)$ & $(44 \%)$ & $(56 \%)$ \\
\hline
\end{tabular}

${ }^{\mathrm{a} B P A}$ doses are $\mu \mathrm{g} / \mathrm{kg}$ bw/day. See legend to Table 55 for description of data presentation.

Table 101. Non-Neoplastic Lesions in the Pituitary of Interim and Terminal Sacrifice Male Rats: Continuous-Dose EE $_{2}$ a, b

\begin{tabular}{|c|c|c|c|c|c|}
\hline Lesion & $\begin{array}{c}\text { Study Phase } \\
\text { (Interim/Terminal) }\end{array}$ & Statistic & Vehicle $^{c}$ & $0.05 \mathrm{EE}_{2}$ & $0.5 \mathrm{EE}_{2}$ \\
\hline \multirow[t]{5}{*}{$\begin{array}{l}\text { Hyperplasia, pars } \\
\text { distalis }\end{array}$} & Interim & Incidence & $\begin{array}{c}4 / 22 \\
(18 \%)\end{array}$ & $\begin{array}{c}7 / 26 \\
(27 \%)\end{array}$ & $\begin{array}{l}2 / 26 \\
(8 \%)\end{array}$ \\
\hline & & Severity Profile & $3|1| 0 \mid 0(1.2)$ & $4|2| 1 \mid 0(1.6)$ & $1|1| 0 \mid 0(1.5)$ \\
\hline & Terminal & Incidence & $\begin{array}{c}11 / 48^{\#, \wedge} \\
(23 \%)\end{array}$ & $\begin{array}{l}10 / 26 \\
(38 \%)\end{array}$ & $\begin{array}{l}13 / 26^{\#, \wedge} \\
(50 \%)\end{array}$ \\
\hline & & Severity Profile & $3|4| 4 \mid 0(2.1)$ & $2|4| 4 \mid 0(2.2)$ & $3|7| 3 \mid 0(2.0)$ \\
\hline & & Poly-3 Incidence & $\begin{array}{c}11 / 36.2^{*} \\
(30 \%)\end{array}$ & $\begin{array}{c}10 / 21.2 \\
(47 \%)\end{array}$ & $\begin{array}{c}13 / 22.6^{*} \\
(58 \%)\end{array}$ \\
\hline
\end{tabular}

${ }^{\mathrm{a} E E} 2$ doses are $\mu \mathrm{g} / \mathrm{kg}$ bw/day. See legend to Table 55 for description of data presentation.

${ }^{\mathrm{b}}$ In the sensitivity analysis that excluded all animals that overlapped with animals treated with $250,000 \mu \mathrm{g}$ BPA/kg bw/day (see Statistical Methods), there was a significant Poly-3 test $(p=0.042)$ for the pairwise comparison between the $0.05 \mu \mathrm{g} \mathrm{EE} / \mathrm{kg}$ bw/day dose group and the vehicle control group for angiectasis in the pituitary gland (Poly-3 incidences $5 / 14.2$ (35\%) versus 2/24.2 (8\%); data not shown in Table). In the analysis that included all animals, the Poly-3 incidences were $7 / 19.1$ (37\%) in the EE 2 group versus $6 / 33.5$ (18\%) in the vehicle control group.

'The continuous vehicle control group data are the same as those shown for the continuous-dose BPA data in Table 100 
CLARITY-BPA Core Study

Table 102. Non-Neoplastic Lesions in the Pituitary of Interim and Terminal Sacrifice Male Rats: Stop-Dose BPA ${ }^{\text {a, b, c }}$

\begin{tabular}{|c|c|c|c|c|c|c|c|c|}
\hline Lesion & $\begin{array}{c}\text { Study Phase } \\
\text { (Interim/Terminal) }\end{array}$ & Statistic & Vehicle & $2.5 \mathrm{BPA}$ & 25 BPA & 250 BPA & 2500 BPA & 25000 BPA \\
\hline \multirow[t]{3}{*}{$\begin{array}{l}\text { Hyperplasia, } \\
\text { pars distalis }\end{array}$} & Interim & $\begin{array}{l}\text { Incidence } \\
\text { Severity Profile }\end{array}$ & $\begin{array}{c}8 / 20 \\
(40 \%) \\
8|0| 0 \mid 0(10)\end{array}$ & $\begin{array}{c}9 / 20 \\
(45 \%) \\
711011(14)\end{array}$ & $\begin{array}{c}3 / 20 \\
(15 \%) \\
0|3| 0 \mid 0(20)\end{array}$ & $\begin{array}{c}4 / 19 \\
(21 \%) \\
4|0| 0 \mid 0(10)\end{array}$ & $\begin{array}{c}4 / 20 \\
(20 \%) \\
4|0| 0 \mid 0(10)\end{array}$ & $\begin{array}{c}7 / 22 \\
(32 \%) \\
61100(1)\end{array}$ \\
\hline & Terminal & Incidence & $\begin{array}{c}12 / 46 \\
(26 \%)\end{array}$ & $\begin{array}{c}16 / 48 \\
(33 \%)\end{array}$ & $\begin{array}{c}18 / 48 \\
(38 \%)\end{array}$ & $\begin{array}{c}15 / 49 \\
(31 \%)\end{array}$ & $\begin{array}{c}19 / 50 \\
(38 \%)\end{array}$ & $\begin{array}{c}19 / 43 \\
(44 \%)\end{array}$ \\
\hline & & $\begin{array}{l}\text { Severity Profile } \\
\text { Poly-3 Incidence }\end{array}$ & $\begin{array}{c}0|6| 3 \mid 3(2.8) \\
12 / 37.2^{*} \\
(32 \%)\end{array}$ & $\begin{array}{c}5|4| 6 \mid 1(2.2) \\
16 / 38.2 \\
(42 \%)\end{array}$ & $\begin{array}{c}4|8| 2 \mid 4(2.3) \\
18 / 40.8 \\
(44 \%)\end{array}$ & $\begin{array}{c}3|6| 3 \mid 3(2.4) \\
15 / 37.3 \\
(40 \%)\end{array}$ & $\begin{array}{c}3|9| 5 \mid 2(2.3) \\
19 / 38.9 \\
(49 \%)\end{array}$ & $\begin{array}{c}4|8| 3 \mid 4(2.4) \\
19 / 32.7^{*} \\
(58 \%)\end{array}$ \\
\hline
\end{tabular}

${ }^{\mathrm{B} B P A}$ doses are $\mu \mathrm{g} / \mathrm{kg}$ bw/day. See legend to Table 55 for description of data presentation.

bIn the sensitivity analysis that excluded all animals that overlapped with animals treated with $250,000 \mu \mathrm{g}$ BPA/kg bw/day (see Statistical Methods), there was a significant dose trend $(p=0.048)$ for pars distalis, cyst (not shown in Table). The $p$-value for the trend in the analysis including all animals was 0.056 .

${ }^{\mathrm{c}}$ Also in the sensitivity analysis mentioned in footnote $\mathrm{b}$, there was a statistically significant pairwise comparison $(p=0.041$, Poly-3 test) between the $25,000 \mu \mathrm{g}$ BPA/kg bw/day dose group and the vehicle control for hypertrophy of the pars distalis (Poly-3 incidences $4 / 21.7$ (18\%) versus 0/24.3). In the analysis that included all animals, the Poly-3 incidences were $4 / 28.5(14 \%)$ versus $1 / 35.1(3 \%), p=0.118$. This lesion is not shown in the Table. 
CLARITY-BPA Core Study

Table 103. Non-Neoplastic Lesions in the Thyroid of Interim and Terminal Sacrifice Male Rats: Continuous-Dose BPA ${ }^{\text {a }}$

\begin{tabular}{|c|c|c|c|c|c|c|c|c|}
\hline Lesion & $\begin{array}{c}\text { Study Phase } \\
\text { (Interim/Terminal) }\end{array}$ & Statistic & Vehicle & $2.5 \mathrm{BPA}$ & 25 BPA & 250 BPA & 2500 BPA & 25000 BPA \\
\hline \multirow{8}{*}{$\begin{array}{l}\text { Hyperplasia, } \\
\text { C-cell }\end{array}$} & \multirow[t]{3}{*}{ Interim } & Incidence & $10 / 22$ & $13 / 22$ & $9 / 20$ & $12 / 24$ & $7 / 18$ & $10 / 21$ \\
\hline & & & $(46 \%)$ & $(59 \%)$ & $(45 \%)$ & $(50 \%)$ & $(39 \%)$ & $(48 \%)$ \\
\hline & & Severity Profile & $3|4| 3 \mid 0(2.0)$ & $7|4| 2 \mid 0(1.6)$ & $6|2| 1 \mid 0(1.4)$ & $5|4| 3 \mid 0(1.8)$ & $2|4| 0 \mid 1(2.0)$ & $6|3| 1 \mid 0(1.5)$ \\
\hline & \multirow[t]{5}{*}{ Terminal } & Incidence & $9 / 46$ & $13 / 40$ & $15 / 47$ & $15 / 44$ & $20 / 44^{\wedge \wedge}$ & $11 / 44$ \\
\hline & & & $(20 \%)$ & $(32 \%)$ & $(32 \%)$ & $(34 \%)$ & $(46 \%)$ & $(25 \%)$ \\
\hline & & Severity Profile & $5|2| 2 \mid 0(1.7)$ & $5|7| 1 \mid 0(1.7)$ & $8|4| 2 \mid 1$ (1.7) & $8|6| 1 \mid 0(1.5)$ & $10|8| 2 \mid 0(1.6)$ & $3|7| 1 \mid 0$ (1.8) \\
\hline & & Poly-3 Incidence & 9/34.9* & $13 / 32.8$ & $15 / 35.5$ & $15 / 34.1$ & $20 / 33.8^{* *}$ & $11 / 30.5$ \\
\hline & & & $(26 \%)$ & $(40 \%)$ & $(42 \%)$ & $(44 \%)$ & $(59 \%)$ & $(36 \%)$ \\
\hline \multirow{9}{*}{$\begin{array}{l}\text { Hyperplasia, } \\
\text { follicular cell }\end{array}$} & \multirow{3}{*}{ Interim } & Incidence & $1 / 22$ & $1 / 22$ & $2 / 20$ & $2 / 24$ & $2 / 18$ & $1 / 21$ \\
\hline & & & $(4 \%)$ & $(4 \%)$ & $(10 \%)$ & $(8 \%)$ & $(11 \%)$ & $(5 \%)$ \\
\hline & & Severity profile & $0|1| 0 \mid 0(2.0)$ & $0|0| 1 \mid 0(3.0)$ & $1|0| 1 \mid 0(2.0)$ & $0|1| 1 \mid 0(2.5)$ & $0|0| 2 \mid 0(3.0)$ & $0|0| 1 \mid 0(3.0)$ \\
\hline & \multirow[t]{6}{*}{ Terminal } & Incidence & $3 / 46$ & $2 / 40$ & $9 / 47^{\wedge}$ & $6 / 44$ & $3 / 44$ & $3 / 44$ \\
\hline & & & $(6 \%)$ & $(5 \%)$ & $(19 \%)$ & $(14 \%)$ & $(7 \%)$ & $(7 \%)$ \\
\hline & & Severity Profile & $0|2| 1 \mid 0$ & $0|1| 1 \mid 0$ & $0|3| 5 \mid 1$ & $0|4| 2 \mid 0$ & $0|1| 1 \mid 1$ & $0|1| 2 \mid 0$ \\
\hline & & & $(2.3)$ & $(2.5)$ & $(2.8)$ & $(2.3)$ & $(3.0)$ & $(2.7)$ \\
\hline & & Poly-3 Incidence & $3 / 32.5$ & $2 / 31.2$ & $9 / 35.1$ & $6 / 32.0$ & $3 / 31.5$ & $3 / 28.6$ \\
\hline & & & $(9 \%)$ & $(6 \%)$ & $(26 \%)$ & $(19 \%)$ & $(10 \%)$ & $(10 \%)$ \\
\hline
\end{tabular}

${ }^{\mathrm{a} B P A}$ doses are $\mu \mathrm{g} / \mathrm{kg}$ bw/day. See legend to Table 55 for description of data presentation. 
CLARITY-BPA Core Study

Table 104. Non-Neoplastic Lesions in the Thyroid of Interim and Terminal Sacrifice Male Rats: Continuous-Dose $\mathbf{E E}_{2}{ }^{\mathrm{a}}$

\begin{tabular}{|c|c|c|c|c|c|}
\hline Lesion & $\begin{array}{c}\text { Study Phase } \\
\text { (Interim/Terminal) }\end{array}$ & Statistic & Vehicle $^{\mathrm{b}}$ & $0.05 \mathrm{EE}_{2}$ & $0.5 \mathrm{EE}_{2}$ \\
\hline \multirow[t]{6}{*}{$\begin{array}{l}\text { Hyperplasia, } \\
\text { C-cell }\end{array}$} & \multirow[t]{2}{*}{ Interim } & Incidence & $\begin{array}{l}10 / 22 \\
(46 \%)\end{array}$ & $\begin{array}{l}7 / 25 \\
(28 \%)\end{array}$ & $\begin{array}{c}9 / 24 \\
(38 \%)\end{array}$ \\
\hline & & Severity Profile & $3|4| 3 \mid 0(2.0)$ & $4|2| 1 \mid 0(1.6)$ & $4|5| 0 \mid 0$ (1.6) \\
\hline & \multirow[t]{4}{*}{ Terminal } & Incidence & $\begin{array}{c}9 / 46 \\
(20 \%)\end{array}$ & $\begin{array}{l}12 / 25^{\wedge \wedge} \\
(48 \%)\end{array}$ & $\begin{array}{c}7 / 25 \\
(28 \%)\end{array}$ \\
\hline & & Severity Profile & $5|2| 2 \mid 0(1.7)$ & $4|8| 0 \mid 0(1.7)$ & $5|1| 1 \mid 0(1.4)$ \\
\hline & & Poly-3 Incidence & $9 / 34.9$ & $12 / 20.3^{* *}$ & $7 / 20.2$ \\
\hline & & & $(26 \%)$ & $(59 \%)$ & $(35 \%)$ \\
\hline \multirow{8}{*}{$\begin{array}{l}\text { Hyperplasia, follicular } \\
\text { cell }\end{array}$} & \multirow[t]{3}{*}{ Interim } & Incidence & $1 / 22$ & $4 / 25$ & $2 / 24$ \\
\hline & & & $(4 \%)$ & $(16 \%)$ & $(8 \%)$ \\
\hline & & Severity Profile & $0|1| 0 \mid 0(2.0)$ & $0|0| 4 \mid 0(3.0)$ & $0|0| 2 \mid 0(3.0)$ \\
\hline & \multirow[t]{5}{*}{ Terminal } & Incidence & $3 / 46$ & $2 / 25$ & $3 / 25$ \\
\hline & & & $(6 \%)$ & $(8 \%)$ & $(12 \%)$ \\
\hline & & Severity Profile & $0|2| 1 \mid 0(2.3)$ & $0|1| 0 \mid 1(3.0)$ & $0|1| 2 \mid 0(2.7)$ \\
\hline & & Poly-3 Incidence & $3 / 32.5$ & $2 / 17.7$ & $3 / 19.9$ \\
\hline & & & $(9 \%)$ & $(11 \%)$ & $(15 \%)$ \\
\hline
\end{tabular}

${ }^{a} E_{2}$ doses are $\mu \mathrm{g} / \mathrm{kg}$ bw/day. See legend to Table 55 for description of data presentation.

${ }^{\mathrm{b}}$ The continuous vehicle control group data are the same as those shown for the continuous-dose BPA data in Table 103. 
CLARITY-BPA Core Study

Table 105. Non-Neoplastic Lesions in the Thyroid of Interim and Terminal Sacrifice Male Rats: Stop-Dose BPA ${ }^{\mathrm{a}}$

\begin{tabular}{|c|c|c|c|c|c|c|c|c|}
\hline Lesion & $\begin{array}{c}\text { Study Phase } \\
\text { (Interim/Terminal) }\end{array}$ & Statistic & Vehicle & $2.5 \mathrm{BPA}$ & 25 BPA & 250 BPA & 2500 BPA & 25000 BPA \\
\hline \multirow[t]{6}{*}{$\begin{array}{l}\text { Hyperplasia, } \\
\text { C-cell }\end{array}$} & Interim & Incidence & $\begin{array}{l}13 / 20 \\
(65 \%)\end{array}$ & $\begin{array}{c}9 / 20 \\
(45 \%)\end{array}$ & $\begin{array}{l}10 / 20 \\
(50 \%)\end{array}$ & $\begin{array}{l}11 / 19 \\
(58 \%)\end{array}$ & $\begin{array}{l}10 / 20 \\
(50 \%)\end{array}$ & $\begin{array}{l}12 / 22 \\
(54 \%)\end{array}$ \\
\hline & & Severity Profile & $5|7| 1 \mid 0(1.7)$ & $1|4| 4 \mid 0(2.3)$ & $6|2| 2 \mid 0(1.6)$ & $3|7| 1 \mid 0(1.8)$ & $7|2| 1 \mid 0(1.4)$ & $5|5| 2 \mid 0(1.8)$ \\
\hline & Terminal & Incidence & $\begin{array}{l}12 / 43 \\
(28 \%)\end{array}$ & $\begin{array}{l}18 / 45 \\
(40 \%)\end{array}$ & $\begin{array}{l}11 / 44 \\
(25 \%)\end{array}$ & $\begin{array}{l}19 / 45 \\
(42 \%)\end{array}$ & $\begin{array}{l}18 / 48 \\
(38 \%)\end{array}$ & $\begin{array}{l}13 / 42 \\
(31 \%)\end{array}$ \\
\hline & & Severity Profile & $4|7| 1 \mid 0(1.8)$ & $10|6| 2 \mid 0(1.6)$ & $4|5| 1 \mid 1$ (1.9) & $11|6| 2 \mid 0(1.4)$ & $10|7| 1 \mid 0(1.5)$ & $5|6| 1 \mid 1(1.8)$ \\
\hline & & Poly-3 & $12 / 36.4$ & $18 / 35.2$ & $11 / 33.8$ & $19 / 35.2$ & $18 / 39.3$ & $13 / 31.0$ \\
\hline & & Incidence & $(33 \%)$ & $(51 \%)$ & $(33 \%)$ & $(54 \%)$ & $(46 \%)$ & $(42 \%)$ \\
\hline \multirow{7}{*}{$\begin{array}{l}\text { Hyperplasia, } \\
\text { follicular cell }\end{array}$} & Interim & Incidence & $1 / 20$ & $1 / 20$ & $4 / 20$ & $1 / 19$ & $0 / 20$ & $3 / 22$ \\
\hline & & & $(5 \%)$ & $(5 \%)$ & $(20 \%)$ & $(5 \%)$ & $(0 \%)$ & $(14 \%)$ \\
\hline & & Severity Profile & $0|0| 1 \mid 0(3.0)$ & $0|0| 1 \mid 0(3.0)$ & $0|1| 3 \mid 0(2.8)$ & $0|0| 1 \mid 0(3.0)$ & - & $0|0| 3 \mid 0(3.0)$ \\
\hline & Terminal & Incidence & $\begin{array}{c}6 / 43 \\
(14 \%)\end{array}$ & $\begin{array}{c}6 / 45 \\
(13 \%)\end{array}$ & $\begin{array}{c}9 / 44 \\
(20 \%)\end{array}$ & $\begin{array}{l}10 / 45 \\
(22 \%)\end{array}$ & $\begin{array}{c}6 / 48 \\
(12 \%)\end{array}$ & $\begin{array}{c}7 / 42 \\
(17 \%)\end{array}$ \\
\hline & & Severity Profile & $0|3| 3 \mid 0(2.5)$ & $0|2| 4 \mid 0(2.7)$ & $0|2| 6 \mid 1(2.9)$ & $0|3| 7 \mid 0(2.7)$ & $0|3| 3 \mid 0(2.5)$ & $0|3| 4 \mid 0(2.6)$ \\
\hline & & Poly-3 & $6 / 34.7$ & $6 / 34.0$ & $9 / 35.5$ & $10 / 32.3$ & $6 / 36.6$ & $7 / 28.9$ \\
\hline & & Incidence & $(17 \%)$ & $(18 \%)$ & $(25 \%)$ & $(31 \%)$ & $(16 \%)$ & $(24 \%)$ \\
\hline
\end{tabular}

${ }^{a}$ BPA doses are $\mu \mathrm{g} / \mathrm{kg}$ bw/day. See legend to Table 55 for description of data presentation. 
CLARITY-BPA Core Study

Table 106. Non-Neoplastic Lesions in the Parathyroid Gland of Interim and Terminal Sacrifice Male Rats: Continuous BPA ${ }^{\mathrm{a}}$

\begin{tabular}{|c|c|c|c|c|c|c|c|c|}
\hline Lesion & $\begin{array}{c}\text { Study Phase } \\
\text { (Interim/Terminal) }\end{array}$ & Statistic & Vehicle & 2.5 BPA & 25 BPA & 250 BPA & 2500 BPA & 25000 BPA \\
\hline \multirow[t]{8}{*}{ Hyperplasia } & \multirow[t]{3}{*}{ Interim } & Incidence & $7 / 22^{*}, \#, \wedge N$ & $5 / 21$ & $7 / 19$ & $5 / 24$ & $2 / 19$ & $2 / 21^{\wedge} \mathrm{N}$ \\
\hline & & & $(32 \%)$ & $(24 \%)$ & $(37 \%)$ & $(21 \%)$ & $(10 \%)$ & $(10 \%)$ \\
\hline & & Severity Profile & $3|4| 0 \mid 0$ (1.6) & $2|3| 0 \mid 0(1.6)$ & $3|3| 1 \mid 0(1.7)$ & $1|4| 0 \mid 0$ (1.8) & $0|2| 0 \mid 0(2.0)$ & $1|1| 0 \mid 0(1.5)$ \\
\hline & \multirow[t]{5}{*}{ Terminal } & Incidence & $11 / 49$ & $11 / 46$ & $23 / 47^{\wedge \wedge}$ & $18 / 50^{\wedge}$ & $18 / 50$ & $12 / 46$ \\
\hline & & & $(22 \%)$ & $(24 \%)$ & $(49 \%)$ & $(36 \%)$ & $(36 \%)$ & $(26 \%)$ \\
\hline & & Severity Profile & $3|7| 0 \mid 1$ (1.9) & $7|3| 0 \mid 1(1.5)$ & $1|13| 6 \mid 3(2.5)$ & $2|9| 3 \mid 4(2.5)$ & $9|5| 2 \mid 2(1.8)$ & $4|5| 3 \mid 0(1.9)$ \\
\hline & & Poly-3 Incidence & $11 / 36.7$ & $11 / 33.9$ & $23 / 37.5^{* *}$ & $18 / 38.4$ & $18 / 39.0$ & $12 / 32.3$ \\
\hline & & & $(30 \%)$ & $(32 \%)$ & $(61 \%)$ & $(47 \%)$ & $(46 \%)$ & $(37 \%)$ \\
\hline
\end{tabular}

${ }^{\mathrm{a} B P A}$ doses are $\mu \mathrm{g} / \mathrm{kg}$ bw/day. See legend to Table 55 for description of data presentation.

Table 107. Non-Neoplastic Lesions in the Parathyroid Gland of Interim and Terminal Sacrifice Male Rats: Continuous-Dose EE ${ }_{2}{ }^{a}$

\begin{tabular}{|c|c|c|c|c|c|}
\hline Lesion & $\begin{array}{c}\text { Study Phase } \\
\text { (Interim/Terminal) }\end{array}$ & Statistic & Vehicle $^{b}$ & $0.05 \mathrm{EE}_{2}$ & $0.5 \mathrm{EE}_{2}$ \\
\hline \multirow[t]{3}{*}{ Hyperplasia } & Interim & $\begin{array}{l}\text { Incidence } \\
\text { Severity Profile }\end{array}$ & $\begin{array}{c}7 / 22 \\
(32 \%) \\
3|4| 0 \mid 0(1.6)\end{array}$ & $\begin{array}{c}6 / 26 \\
(23 \%) \\
0|6| 0 \mid 0(2.0)\end{array}$ & $\begin{array}{c}4 / 26 \\
(15 \%) \\
1|2| 1 \mid 0(2.0)\end{array}$ \\
\hline & Terminal & Incidence & $\begin{array}{c}11 / 49^{\#, \wedge} \\
(22 \%)\end{array}$ & $\begin{array}{c}7 / 25 \\
(28 \%)\end{array}$ & $\begin{array}{c}11 / 25^{\#, \wedge} \\
(44 \%)\end{array}$ \\
\hline & & $\begin{array}{l}\text { Severity Profile } \\
\text { Poly-3 Incidence }\end{array}$ & $\begin{array}{c}3|7| 0 \mid 1(1.9) \\
11 / 36.7^{c} \\
(30 \%)\end{array}$ & $\begin{array}{c}2|3| 1 \mid 1(2.1) \\
7 / 18.2 \\
(38 \%)\end{array}$ & $\begin{array}{c}3|5| 1 \mid 2(1.8) \\
11 / 20.9 \\
(53 \%)\end{array}$ \\
\hline
\end{tabular}

${ }^{\mathrm{a}} \mathrm{EE}_{2}$ doses are $\mu \mathrm{g} / \mathrm{kg}$ bw/day. See legend to Table 55 for description of data presentation.

${ }^{\mathrm{b}}$ The continuous vehicle control group data are the same as those shown for the continuous-dose BPA data in Table 106.

'In the sensitivity analysis that excluded all animals that overlapped with animals treated with $250,000 \mu \mathrm{g} \mathrm{BPA} / \mathrm{kg}$ bw/day (see Statistical Methods), there was a significant EE 2 dose trend $(p=0.046)$. The $p$-value for the trend in the analysis that included all animals was 0.051 . 
CLARITY-BPA Core Study

Table 108. Non-Neoplastic Lesions in the Parathyroid Gland of Interim and Terminal Sacrifice Male Rats: Stop-Dose BPA ${ }^{\mathrm{a}}$

\begin{tabular}{|c|c|c|c|c|c|c|c|c|}
\hline Lesion & $\begin{array}{c}\text { Study Phase } \\
\text { (Interim/Terminal) }\end{array}$ & Statistic & Vehicle & $2.5 \mathrm{BPA}$ & 25 BPA & 250 BPA & 2500 BPA & 25000 BPA \\
\hline \multirow[t]{5}{*}{ Hyperplasia } & Interim & Incidence & $\begin{array}{l}4 / 20 \\
(20 \%)\end{array}$ & $\begin{array}{l}9 / 20 \\
(45 \%)\end{array}$ & $\begin{array}{c}3 / 19 \\
(16 \%)\end{array}$ & $\begin{array}{c}7 / 19 \\
(37 \%)\end{array}$ & $\begin{array}{c}4 / 19 \\
(21 \%)\end{array}$ & $\begin{array}{c}8 / 22 \\
(36 \%)\end{array}$ \\
\hline & & Severity Profile & $1|2| 1 \mid 0(2.0)$ & $7|1| 1 \mid 0(1.7)$ & $1|2| 0 \mid 0$ (1.7) & $5|2| 0 \mid 0(1.3)$ & $4|0| 0 \mid 0(1.0)$ & $1|6| 1 \mid 0(2.0)$ \\
\hline & Terminal & Incidence & $\begin{array}{l}22 / 49 \\
(45 \%)\end{array}$ & $\begin{array}{l}17 / 46 \\
(37 \%)\end{array}$ & $\begin{array}{l}27 / 46 \\
(59 \%)\end{array}$ & $\begin{array}{l}23 / 49 \\
(47 \%)\end{array}$ & $\begin{array}{l}30 / 50 \\
(60 \%)\end{array}$ & $\begin{array}{l}23 / 43 \\
(54 \%)\end{array}$ \\
\hline & & Severity Profile & $1|8| 7 \mid 6(2.8)$ & $2|10| 0 \mid 5(2.5)$ & $4|15| 5 \mid 3(2.3)$ & $8|8| 5 \mid 2(2.0)$ & $3|12| 6 \mid 9(2.7)$ & $7|7| 5 \mid 4(2.3)$ \\
\hline & & Poly-3 Incidence & $\begin{array}{c}22 / 41.9^{*} \\
(52 \%)\end{array}$ & $\begin{array}{c}17 / 36.6 \\
(46 \%)\end{array}$ & $\begin{array}{c}27 / 39.4 \\
(69 \%)\end{array}$ & $\begin{array}{c}23 / 38.0 \\
(60 \%)\end{array}$ & $\begin{array}{c}30 / 44.1 \\
(68 \%)\end{array}$ & $\begin{array}{c}23 / 34.1 \\
(67 \%)\end{array}$ \\
\hline
\end{tabular}

${ }^{\mathrm{a} B P A}$ doses are $\mu \mathrm{g} / \mathrm{kg}$ bw/day. See legend to Table 55 for description of data presentation.

Table 109. Non-Neoplastic Lesions in the Kidney of Interim and Terminal Sacrifice Male Rats: Continuous-Dose BPA ${ }^{\mathrm{a}}$

\begin{tabular}{|c|c|c|c|c|c|c|c|c|}
\hline Lesion & $\begin{array}{c}\text { Study Phase } \\
\text { (Interim/Terminal) }\end{array}$ & Statistic & Vehicle & 2.5 BPA & 25 BPA & 250 BPA & 2500 BPA & 25000 BPA \\
\hline \multirow[t]{3}{*}{$\begin{array}{l}\text { Hyperplasia, } \\
\text { transitional epithelium }\end{array}$} & Terminal & Incidence & $\begin{array}{l}3 / 50 \\
(6 \%)\end{array}$ & $\begin{array}{l}4 / 48 \\
(8 \%)\end{array}$ & $\begin{array}{l}12 / 48^{\wedge \wedge} \\
(25 \%)\end{array}$ & $\begin{array}{c}7 / 50 \\
(14 \%)\end{array}$ & $\begin{array}{l}5 / 50 \\
(10 \%)\end{array}$ & $\begin{array}{l}4 / 45 \\
(9 \%)\end{array}$ \\
\hline & & Severity Profile & $1|1| 1 \mid 0(2.0)$ & $1|3| 0 \mid 0(1.8)$ & $5|6| 1 \mid 0(1.7)$ & $5|1| 0 \mid 1(1.6)$ & $2|2| 1 \mid 0$ (1.8) & $1|1| 1 \mid 1(2.5)$ \\
\hline & & Poly-3 Incidence & $\begin{array}{c}3 / 34.6 \\
(9 \%)\end{array}$ & $\begin{array}{l}4 / 34.5 \\
(12 \%)\end{array}$ & $\begin{array}{c}12 / 34.1^{* *} \\
(35 \%)\end{array}$ & $\begin{array}{l}7 / 34.8 \\
(20 \%)\end{array}$ & $\begin{array}{l}5 / 34.1 \\
(15 \%)\end{array}$ & $\begin{array}{l}4 / 28.9 \\
(14 \%)\end{array}$ \\
\hline
\end{tabular}

aBPA doses are $\mu \mathrm{g} / \mathrm{kg}$ bw/day. See legend to Table 55 for description of data presentation.

Table 110. Non-Neoplastic Lesions in the Kidney of Interim and Terminal Sacrifice Male Rats: Continuous-Dose $\mathrm{EE}_{2}{ }^{\mathrm{a}}$

\begin{tabular}{|c|c|c|c|c|c|}
\hline Lesion & $\begin{array}{c}\text { Study Phase } \\
\text { (Interim/Terminal) }\end{array}$ & Statistic & Vehicle $^{\text {b }}$ & $0.05 \mathrm{EE}_{2}$ & $0.5 \mathbf{E E}_{2}$ \\
\hline \multirow[t]{3}{*}{$\begin{array}{l}\text { Hyperplasia, } \\
\text { transitional epithelium }\end{array}$} & Terminal & Incidence & $\begin{array}{l}3 / 50 \\
(6 \%)\end{array}$ & $\begin{array}{l}2 / 26 \\
(8 \%)\end{array}$ & $\begin{array}{l}1 / 26 \\
(4 \%)\end{array}$ \\
\hline & & Severity Profile & $1|1| 1 \mid 0(2.0)$ & $1|1| 0 \mid 0(1.5)$ & $0|0| 1 \mid 0(3.0)$ \\
\hline & & Poly-3 Incidence & $\begin{array}{c}3 / 34.6 \\
(9 \%)\end{array}$ & $\begin{array}{l}2 / 17.8 \\
(11 \%)\end{array}$ & $\begin{array}{c}1 / 19.7 \\
(5 \%)\end{array}$ \\
\hline
\end{tabular}

${ }^{\mathrm{a}} \mathrm{EE}_{2}$ doses are $\mu \mathrm{g} / \mathrm{kg}$ bw/day. See legend to Table 55 for description of data presentation.

${ }^{\mathrm{b}}$ The continuous vehicle control group data are the same as those shown for the continuous-dose BPA data in Table 109. 
CLARITY-BPA Core Study

Table 111. Non-Neoplastic Lesions in the Kidney of Interim and Terminal Sacrifice Male Rats: Stop-Dose BPA ${ }^{\mathrm{a}}$

\begin{tabular}{|c|c|c|c|c|c|c|c|c|}
\hline Lesion & $\begin{array}{c}\text { Study Phase } \\
\text { (Interim/Terminal) }\end{array}$ & Statistic & Vehicle & 2.5 BPA & 25 BPA & 250 BPA & 2500 BPA & 25000 BPA \\
\hline \multirow[t]{3}{*}{$\begin{array}{l}\text { Hyperplasia, transitional } \\
\text { epithelium }\end{array}$} & Terminal & Incidence & $\begin{array}{l}12 / 50 \\
(24 \%)\end{array}$ & $\begin{array}{c}9 / 48 \\
(19 \%)\end{array}$ & $\begin{array}{l}12 / 48 \\
(25 \%)\end{array}$ & $\begin{array}{l}12 / 50 \\
(24 \%)\end{array}$ & $\begin{array}{l}20 / 50 \\
(40 \%)\end{array}$ & $\begin{array}{l}10 / 45 \\
(22 \%)\end{array}$ \\
\hline & & Severity Profile & $3|8| 0 \mid 1$ (1.9) & $4|2| 3 \mid 0(1.9)$ & $5|5| 1 \mid 1(1.8)$ & $4|7| 0 \mid 1(1.8)$ & $8|11| 1 \mid 0$ (1.6) & $3|5| 2 \mid 0$ (1.9) \\
\hline & & Poly-3 Incidence & $\begin{array}{c}12 / 40.0^{*} \\
(30 \%)\end{array}$ & $\begin{array}{l}9 / 36.7 \\
(24 \%)\end{array}$ & $\begin{array}{c}12 / 37.2 \\
(32 \%)\end{array}$ & $\begin{array}{c}12 / 34.9 \\
(34 \%)\end{array}$ & $\begin{array}{c}20 / 40.6 \\
(49 \%)\end{array}$ & $\begin{array}{l}10 / 29.4 \\
(34 \%)\end{array}$ \\
\hline
\end{tabular}

${ }^{\mathrm{a} B P A}$ doses are $\mu \mathrm{g} / \mathrm{kg}$ bw/day. See legend to Table 55 for description of data presentation. 
CLARITY-BPA Core Study

Table 112. Non-Neoplastic Lesions in the Liver of Interim and Terminal Sacrifice Male Rats: Continuous-Dose BPA ${ }^{\text {a }}$

\begin{tabular}{|c|c|c|c|c|c|c|c|c|}
\hline Lesion & $\begin{array}{c}\text { Study Phase } \\
\text { (Interim/Terminal) }\end{array}$ & Statistic & Vehicle & $2.5 \mathrm{BPA}$ & 25 BPA & 250 BPA & 2500 BPA & 25000 BPA \\
\hline \multirow[t]{8}{*}{ Fatty change } & \multirow[t]{3}{*}{ Interim } & Incidence & $0 / 22$ & $0 / 22$ & $2 / 20^{\wedge}$ & $0 / 24$ & $0 / 19$ & $0 / 22$ \\
\hline & & & $(0 \%)$ & $(0 \%)$ & $(10 \%)$ & $(0 \%)$ & $(0 \%)$ & $(0 \%)$ \\
\hline & & Severity Profile & - & - & $0|2| 0 \mid 0(2.0)$ & - & - & - \\
\hline & \multirow[t]{5}{*}{ Terminal } & Incidence & $4 / 50$ & $8 / 47$ & $5 / 48$ & $5 / 50$ & $4 / 50$ & $2 / 45$ \\
\hline & & & $(8 \%)$ & $(17 \%)$ & $(10 \%)$ & $(10 \%)$ & $(8 \%)$ & $(4 \%)$ \\
\hline & & Severity Profile & $0|1| 1 \mid 2(3.3)$ & $1|3| 1 \mid 3(2.8)$ & $2|1| 1 \mid 1(2.2)$ & $0|4| 0 \mid 1(2.4)$ & $0|3| 1 \mid 0(2.2)$ & $0|1| 0 \mid 1(3.0)$ \\
\hline & & Poly-3 incidence & $4 / 35.1$ & $8 / 36.5$ & $5 / 33.2$ & $5 / 35.4$ & $4 / 34.4$ & $2 / 29.1$ \\
\hline & & & $(11 \%)$ & $(22 \%)$ & $(15 \%)$ & $(14 \%)$ & $(12 \%)$ & $(7 \%)$ \\
\hline \multirow{8}{*}{$\begin{array}{l}\text { Hepatodiaphragmatic } \\
\text { Nodule }\end{array}$} & \multirow[t]{3}{*}{ Interim } & Incidence & $0 / 22$ & $2 / 22$ & $2 / 20$ & $3 / 24$ & $4 / 19^{*}$ & $1 / 22$ \\
\hline & & & $(0 \%)$ & $(9 \%)$ & $(10 \%)$ & $(12 \%)$ & $(21 \%)$ & $(4 \%)$ \\
\hline & & Severity Profile & $-{ }^{\mathrm{b}}$ & - & - & - & - & - \\
\hline & \multirow[t]{5}{*}{ Terminal } & Incidence & $6 / 50$ & $2 / 47$ & $4 / 48$ & $3 / 50$ & $7 / 50$ & $5 / 45$ \\
\hline & & & $(12.0 \%)$ & $(4 \%)$ & $(8 \%)$ & $(6 \%)$ & $(14 \%)$ & $(11 \%)$ \\
\hline & & Severity Profile & $-{ }^{\mathrm{b}}$ & - & - & - & - & - \\
\hline & & Poly-3 incidence & $6 / 34.6$ & $2 / 34.2$ & $4 / 32.8$ & $3 / 34.6$ & $7 / 35.4$ & $5 / 30.9$ \\
\hline & & & $(17.3 \%)$ & $(6 \%)$ & $(12 \%)$ & $(9 \%)$ & $(20 \%)$ & $(16 \%)$ \\
\hline \multirow{8}{*}{$\begin{array}{l}\text { Infiltration, cellular, } \\
\text { mononuclear cells }\end{array}$} & \multirow[t]{3}{*}{ Interim } & Incidence & $5 / 22$ & $11 / 22^{\wedge}$ & $9 / 20$ & $13 / 24^{*, \#, \wedge}$ & $11 / 19^{*}, \#, \wedge$ & $8 / 22^{\#}$ \\
\hline & & & $(23 \%)$ & $(50 \%)$ & $(45 \%)$ & $(54 \%)$ & $(58 \%)$ & $(36 \%)$ \\
\hline & & Severity Profile & $5|0| 0 \mid 0(1.0)$ & $11|0| 0 \mid 0(1.0)$ & $9|0| 0 \mid 0(1.0)$ & 12|1|0|0 (1.1) & $11|0| 0 \mid 0(1.0)$ & $8|0| 0 \mid 0(1.0)$ \\
\hline & \multirow[t]{5}{*}{ Terminal } & Incidence & $35 / 50$ & $29 / 47$ & $37 / 48$ & $36 / 50$ & $34 / 50$ & $28 / 45$ \\
\hline & & & $(70 \%)$ & $(62 \%)$ & $(77 \%)$ & $(72 \%)$ & $(68 \%)$ & $(62 \%)$ \\
\hline & & Severity Profile & $21|14| 0 \mid 0(1.4)$ & $16|13| 0 \mid 0$ (1.4) & $22|15| 0 \mid 0$ (1.4) & $26|10| 0 \mid 0(1.3)$ & $21|13| 0 \mid 0$ (1.4) & $19|9| 0 \mid 0$ (1.3) \\
\hline & & Poly-3 incidence & $35 / 42.3$ & $29 / 40.1$ & $37 / 42.6$ & $36 / 42.3$ & $34 / 40.5$ & $28 / 35.4$ \\
\hline & & & $(83 \%)$ & $(72 \%)$ & $(87 \%)$ & $(85 \%)$ & $(84 \%)$ & $(79 \%)$ \\
\hline
\end{tabular}

${ }^{\mathrm{B} B P A}$ doses are $\mu \mathrm{g} / \mathrm{kg}$ bw/day. See legend to Table 55 for description of data presentation

beverity scores were not assigned for this lesion. 
CLARITY-BPA Core Study

Table 113. Non-Neoplastic Lesions in the Liver of Interim and Terminal Sacrifice Male Rats: Continuous-Dose $\mathrm{EE}_{2}{ }^{\mathrm{a}}$

\begin{tabular}{|c|c|c|c|c|c|}
\hline Lesion & $\begin{array}{c}\text { Study Phase } \\
\text { (Interim/Terminal) }\end{array}$ & Statistic & Vehicle $^{b}$ & $0.05 \mathbf{E E}_{2}$ & $0.5 \mathrm{EE}_{2}$ \\
\hline \multirow[t]{5}{*}{ Fatty change } & Interim & Incidence & $\begin{array}{c}0 / 22^{*, \#, \wedge} \\
(0 \%)\end{array}$ & $\begin{array}{l}1 / 26 \\
(4 \%)\end{array}$ & $\begin{array}{l}4 / 26^{\#, \wedge} \\
(15 \%)\end{array}$ \\
\hline & & Severity Profile & - & $0|0| 1 \mid 0(3.0)$ & $0|1| 3 \mid 0(2.8)$ \\
\hline & Terminal & Incidence & $\begin{array}{c}4 / 50 \\
(8.0 \%)\end{array}$ & $\begin{array}{c}0 / 26 \\
(0.0 \%)\end{array}$ & $\begin{array}{c}1 / 25 \\
(4.0 \%)\end{array}$ \\
\hline & & Severity Profile & $0|1| 1 \mid 2(3.2)$ & - & $0|0| 0 \mid 1(4.0)$ \\
\hline & & Poly-3 Incidence & $\begin{array}{l}4 / 35.1 \\
(11 \%) \\
\end{array}$ & $\begin{array}{c}0 / 17.6 \\
(0 \%)\end{array}$ & $\begin{array}{c}1 / 19.9 \\
(5 \%)\end{array}$ \\
\hline \multirow{6}{*}{$\begin{array}{l}\text { Hepatodiaphragmatic } \\
\text { Nodule }\end{array}$} & Interim & Incidence & $0 / 22$ & $4 / 26$ & $4 / 26$ \\
\hline & & & $(0 \%)$ & $(15 \%)$ & $(15 \%)$ \\
\hline & & Severity Profile & $-^{\mathrm{c}}$ & - & - \\
\hline & Terminal & Incidence & $\begin{array}{l}6 / 50 \\
(12 \%)\end{array}$ & $\begin{array}{l}3 / 26 \\
(12 \%)\end{array}$ & $\begin{array}{l}1 / 25 \\
(4 \%)\end{array}$ \\
\hline & & Severity Profile & $-^{\mathrm{c}}$ & - & - \\
\hline & & Poly-3 Incidence & $\begin{array}{l}6 / 34.6 \\
(17 \%)\end{array}$ & $\begin{array}{l}3 / 17.8 \\
(17 \%)\end{array}$ & $\begin{array}{l}1 / 19.5 \\
(5 \%)\end{array}$ \\
\hline \multirow[t]{5}{*}{$\begin{array}{l}\text { Infiltration, cellular, } \\
\text { mononuclear cells }\end{array}$} & Interim & Incidence & $\begin{array}{l}5 / 22 \\
(23 \%)\end{array}$ & $\begin{array}{l}13 / 26 \\
(50 \%)\end{array}$ & $\begin{array}{c}5 / 26 \\
(19 \%)\end{array}$ \\
\hline & & Severity Profile & $5|0| 0 \mid 0(1.0)$ & $13|0| 0 \mid 0(1.0)$ & $5|0| 0 \mid 0(1.0)$ \\
\hline & Terminal & Incidence & $\begin{array}{l}35 / 50 \\
(70 \%)\end{array}$ & $\begin{array}{l}22 / 26 \\
(85 \%)\end{array}$ & $\begin{array}{l}20 / 25 \\
(80 \%)\end{array}$ \\
\hline & & Severity Profile & $21|14| 0 \mid 0(1.4)$ & $16|6| 0 \mid 0(1.3)$ & $12|8| 0 \mid 0(1.4)$ \\
\hline & & Poly-3 Incidence & $\begin{array}{c}35 / 42.3 \\
(83 \%)\end{array}$ & $\begin{array}{c}22 / 23.5^{*} \\
(94 \%)\end{array}$ & $\begin{array}{l}20 / 23.1 \\
(87 \%)\end{array}$ \\
\hline
\end{tabular}

${ }^{\mathrm{a}} \mathrm{EE}_{2}$ doses are $\mu \mathrm{g} / \mathrm{kg}$ bw/day. See legend to Table 55 for description of data presentation.

${ }^{\mathrm{b}}$ The continuous vehicle control group data are the same as those shown for the continuous-dose BPA data in Table 112.

'Severity scores were not assigned for this lesion. 
CLARITY-BPA Core Study

Table 114. Non-Neoplastic Lesions in the Liver of Interim and Terminal Sacrifice Male Rats: Stop-Dose BPA ${ }^{\mathrm{a}}$

\begin{tabular}{|c|c|c|c|c|c|c|c|c|}
\hline Lesion & $\begin{array}{c}\text { Study Phase } \\
\text { (Interim/Terminal) }\end{array}$ & Statistic & Vehicle & $2.5 \mathrm{BPA}$ & 25 BPA & 250 BPA & 2500 BPA & 25000 BPA \\
\hline \multirow[t]{8}{*}{ Fatty change } & \multirow[t]{3}{*}{ Interim } & Incidence & $0 / 20$ & $1 / 20$ & $1 / 20$ & $0 / 19$ & $2 / 20$ & $1 / 22$ \\
\hline & & & $(0 \%)$ & $(5 \%)$ & $(5 \%)$ & $(0 \%)$ & $(10 \%)$ & $(4 \%)$ \\
\hline & & Severity Profile & - & $0|1| 0 \mid 0(2.0)$ & $0|0| 1 \mid 0(3.0)$ & - & $0|0| 2 \mid 0(3.0)$ & $0|0| 1 \mid 0(3.0)$ \\
\hline & \multirow[t]{5}{*}{ Terminal } & Incidence & $5 / 50$ & $2 / 48$ & $7 / 48$ & $6 / 50$ & $1 / 50$ & $1 / 46$ \\
\hline & & & $(10 \%)$ & $(4 \%)$ & $(15 \%)$ & $(12 \%)$ & $(2 \%)$ & $(2 \%)$ \\
\hline & & Severity Profile & $1|3| 1 \mid 0(2.0)$ & $0|0| 0 \mid 2(4.0)$ & $2|2| 1 \mid 2(2.4)$ & $2|1| 1 \mid 2(2.5)$ & $0|0| 0 \mid 1(4.0)$ & $0|0| 0 \mid 1(4.0)$ \\
\hline & & Poly-3 incidence & $5 / 38.9$ & $2 / 34.4$ & $7 / 36.2$ & $6 / 33.4$ & $1 / 36.2$ & $1 / 28.1$ \\
\hline & & & $(13 \%)$ & $(6 \%)$ & $(19 \%)$ & $(18 \%)$ & $(3 \%)$ & $(4 \%)$ \\
\hline \multirow{8}{*}{$\begin{array}{l}\text { Hepatodiaphragmatic } \\
\text { Nodule }\end{array}$} & \multirow{3}{*}{ Interim } & Incidence & $1 / 20$ & $2 / 20$ & $3 / 20$ & $3 / 19$ & $2 / 20$ & $1 / 22$ \\
\hline & & & $(5 \%)$ & $(10 \%)$ & $(15 \%)$ & $(16 \%)$ & $(10 \%)$ & $(4 \%)$ \\
\hline & & Severity Profile & $-{ }^{\mathrm{b}}$ & - & - & - & - & - \\
\hline & \multirow[t]{5}{*}{ Terminal } & Incidence & $3 / 50$ & $3 / 48$ & $1 / 48$ & $5 / 50$ & $4 / 50$ & $5 / 46$ \\
\hline & & & $(6 \%)$ & $(6 \%)$ & $(2 \%)$ & $(10 \%)$ & $(8 \%)$ & $(11 \%)$ \\
\hline & & Severity Profile & $-^{\mathrm{b}}$ & - & - & - & - & - \\
\hline & & Poly-3 incidence & $3 / 37.6$ & $3 / 34.3$ & $1 / 35.2$ & $5 / 33.1$ & $4 / 37.7$ & $5 / 28.8$ \\
\hline & & & $(8 \%)$ & $(9 \%)$ & $(3 \%)$ & $(15 \%)$ & $(11 \%)$ & $(17 \%)$ \\
\hline \multirow{8}{*}{$\begin{array}{l}\text { Infiltration, cellular, } \\
\text { mononuclear cells }\end{array}$} & \multirow[t]{3}{*}{ Interim } & Incidence & $11 / 20$ & $13 / 20$ & $9 / 20$ & $13 / 19$ & $11 / 20$ & $15 / 22$ \\
\hline & & & $(55 \%)$ & $(65 \%)$ & $(45 \%)$ & $(68 \%)$ & $(55 \%)$ & $(68 \%)$ \\
\hline & & Severity Profile & $11|0| 0 \mid 0(1.0)$ & $13|0| 0 \mid 0(1.0)$ & $9|0| 0 \mid 0(1.0)$ & $13|0| 0 \mid 0(1.0)$ & $11|0| 0 \mid 0(1.0)$ & $15|0| 0 \mid 0(1.0)$ \\
\hline & \multirow[t]{5}{*}{ Terminal } & Incidence & $34 / 50$ & $40 / 48$ & $37 / 48$ & $33 / 50$ & $33 / 50$ & $29 / 46$ \\
\hline & & & $(68 \%)$ & $(83 \%)$ & $(77 \%)$ & $(66 \%)$ & $(66 \%)$ & $(63 \%)$ \\
\hline & & Severity Profile & $19|15| 0 \mid 0(1.4)$ & $27|13| 0 \mid 0(1.3)$ & $19|18| 0 \mid 0(1.5)$ & $26|7| 0 \mid 0$ (1.2) & $23|10| 0 \mid 0$ (1.3) & $15|13| 1 \mid 0(1.5)$ \\
\hline & & Poly-3 incidence & $34 / 43.3$ & $40 / 44.0$ & $37 / 43.7$ & $33 / 41.6$ & $33 / 42.3$ & $29 / 37.0$ \\
\hline & & & $(79 \%)$ & $(91 \%)$ & $(85 \%)$ & $(79 \%)$ & $(78 \%)$ & $(78 \%)$ \\
\hline
\end{tabular}

aPPA doses are $\mu \mathrm{g} / \mathrm{kg}$ bw/day. See legend to Table 55 for description of data presentation.

${ }^{\mathrm{b}} \mathrm{Severity} \mathrm{scores} \mathrm{were} \mathrm{not} \mathrm{assigned} \mathrm{for} \mathrm{this} \mathrm{lesion}$ 


\section{Year BPA Toxicity Study}

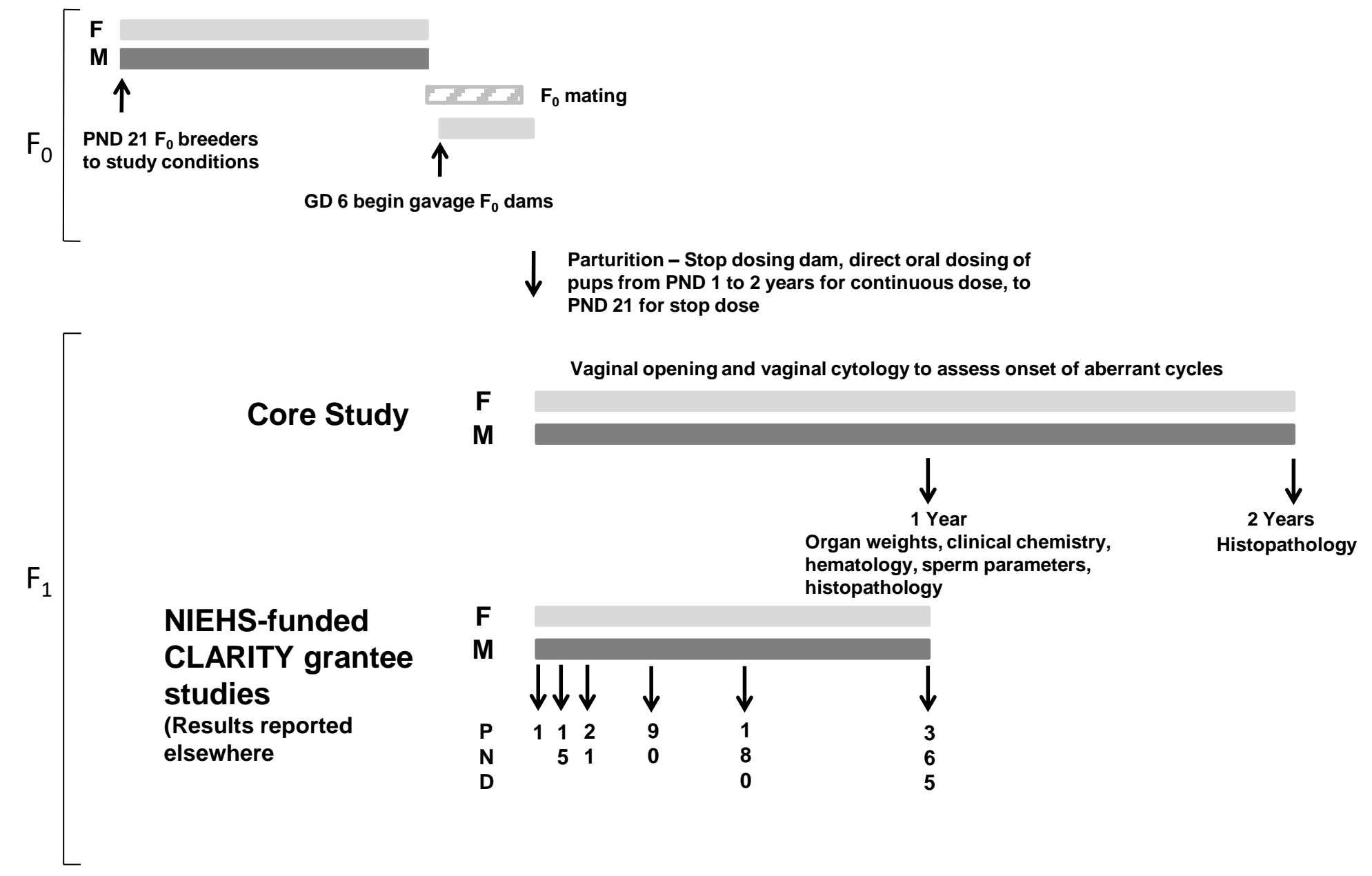

Figure 1. Scheme for Chronic BPA Toxicity Study Design 


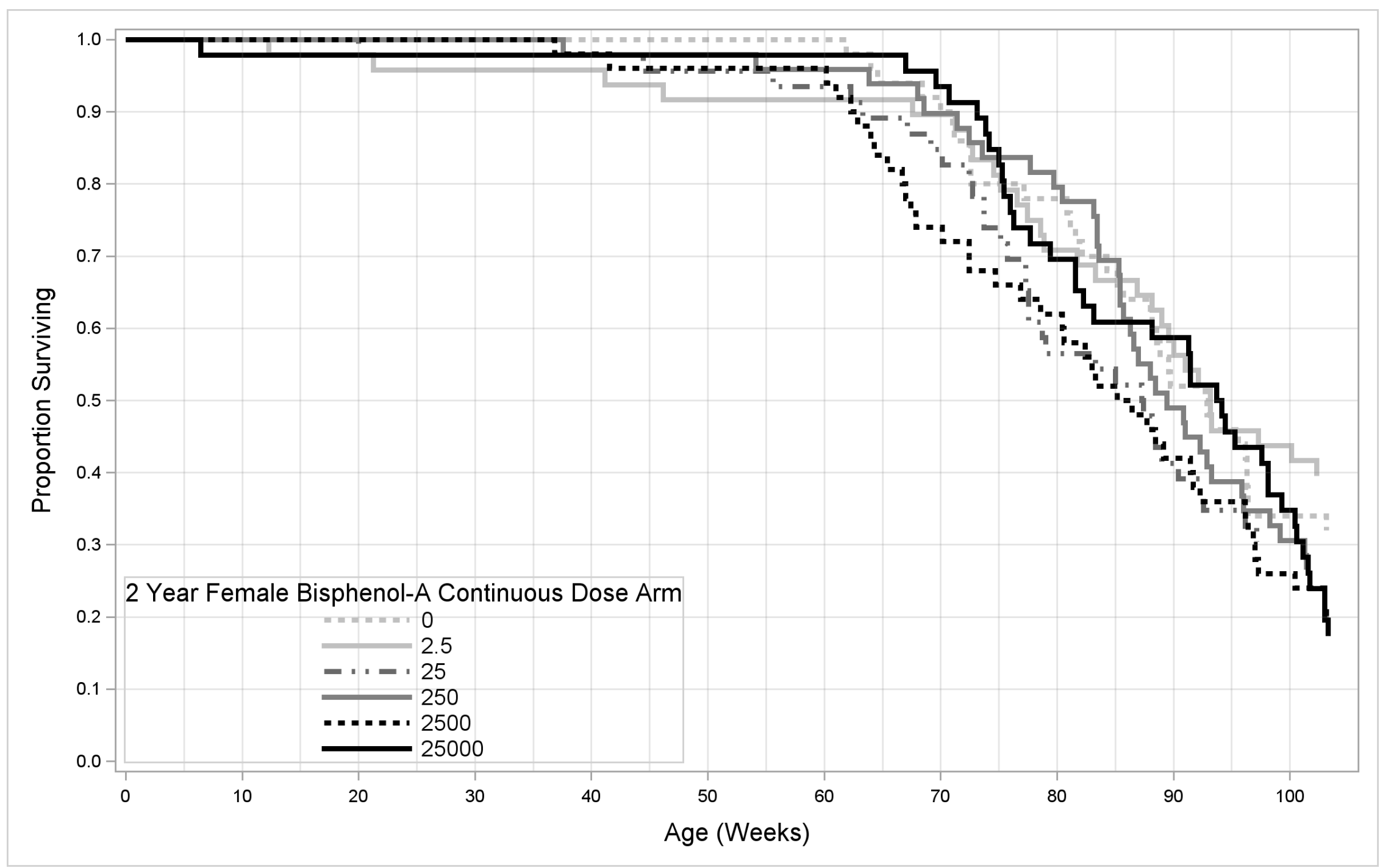

Figure 2. Kaplan-Meier Survival Curve for Terminal Sacrifice Female BPA Continuous-Dose Arm (Weeks 4-104)

See Table 18 for data analysis results. 


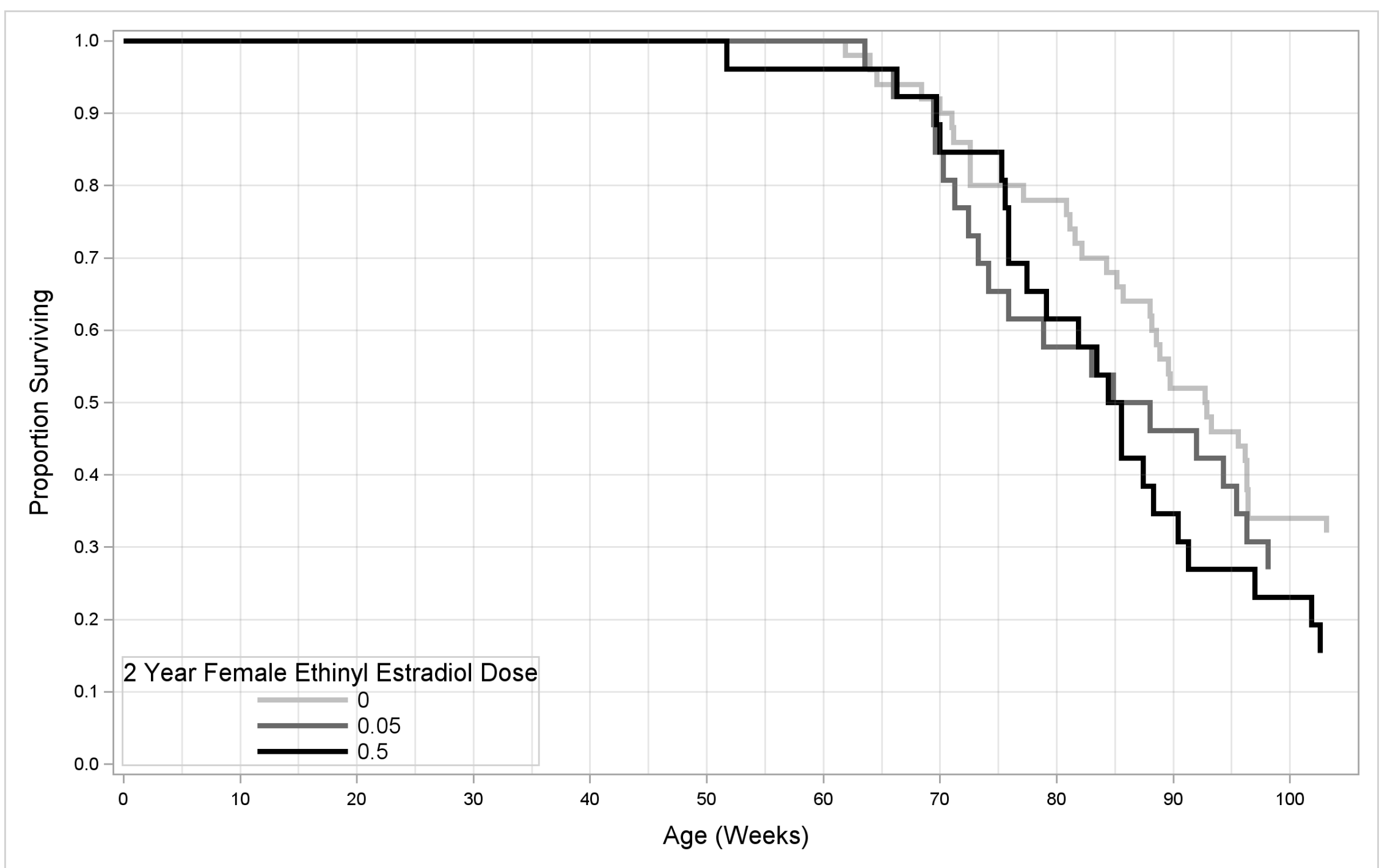

Figure 3. Kaplan-Meier Survival Curve for Terminal Sacrifice Female EE2 Continuous-Dose Arm (Weeks 4-104)

See Table 18 for data analysis results. 


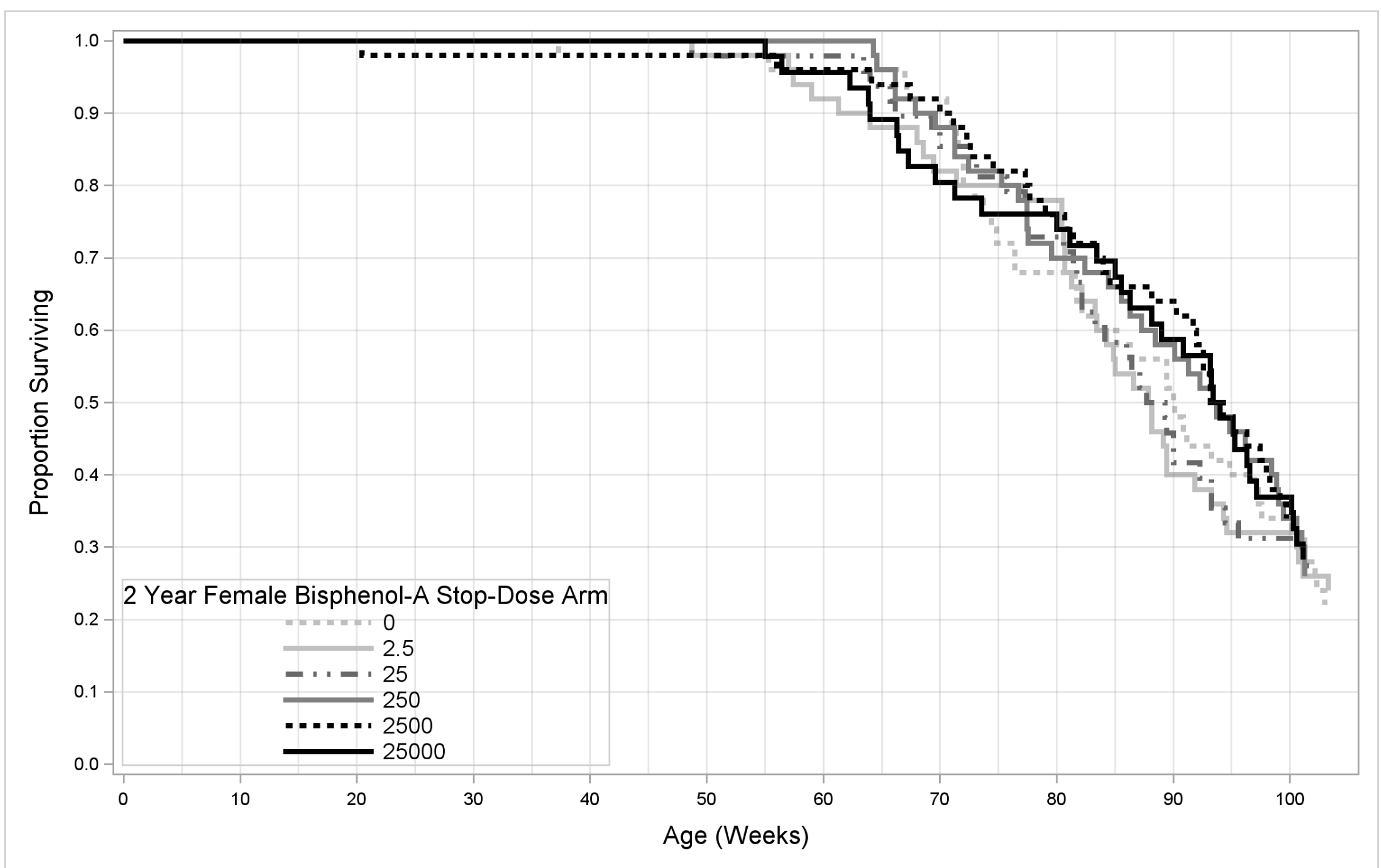

Figure 4. Kaplan-Meier Survival Curve for Terminal Sacrifice Female BPA Stop-Dose Arm (Week 4-104)

See Table 19 for data analysis results. 


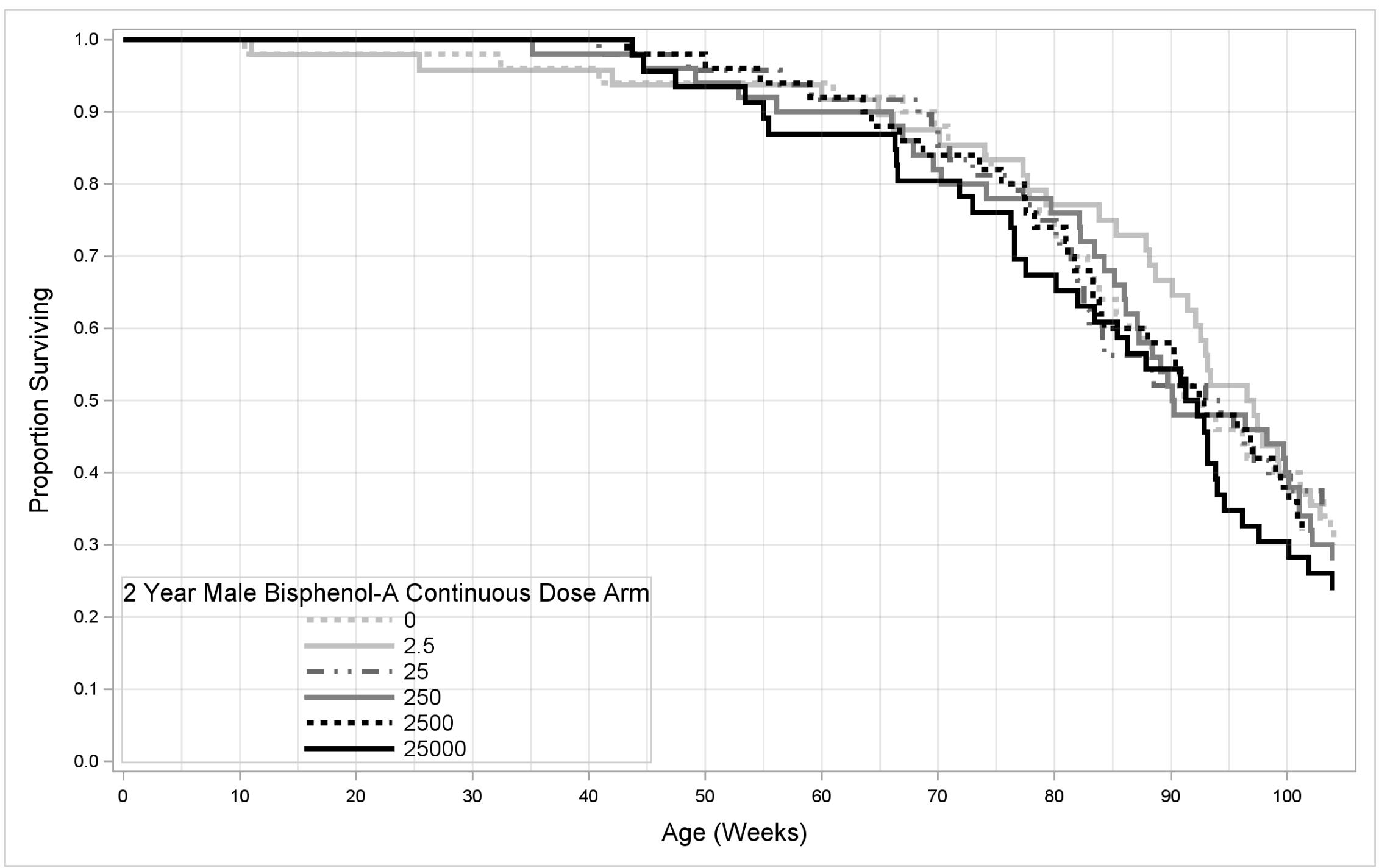

Figure 5. Kaplan-Meier Survival Curve for Terminal Sacrifice Male BPA Continuous-Dose Arm (Weeks 4-104)

See Table 20 for data analysis results. 


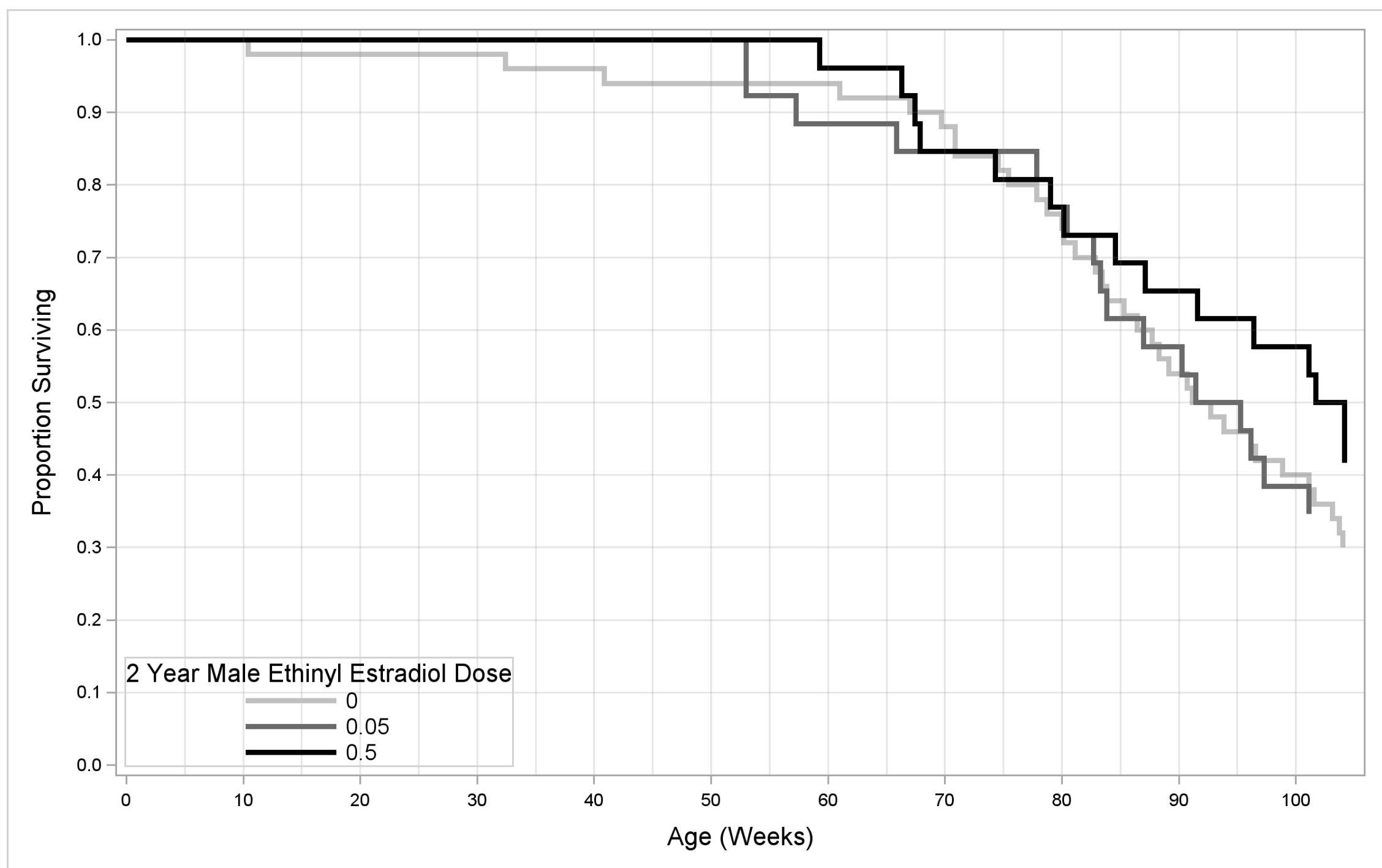

Figure 6. Kaplan-Meier Survival Curve for Terminal Sacrifice Male $\mathbf{E E}_{2}$ Continuous-Dose Arm (Weeks 4-104)

See Table 20 for data analysis results. 


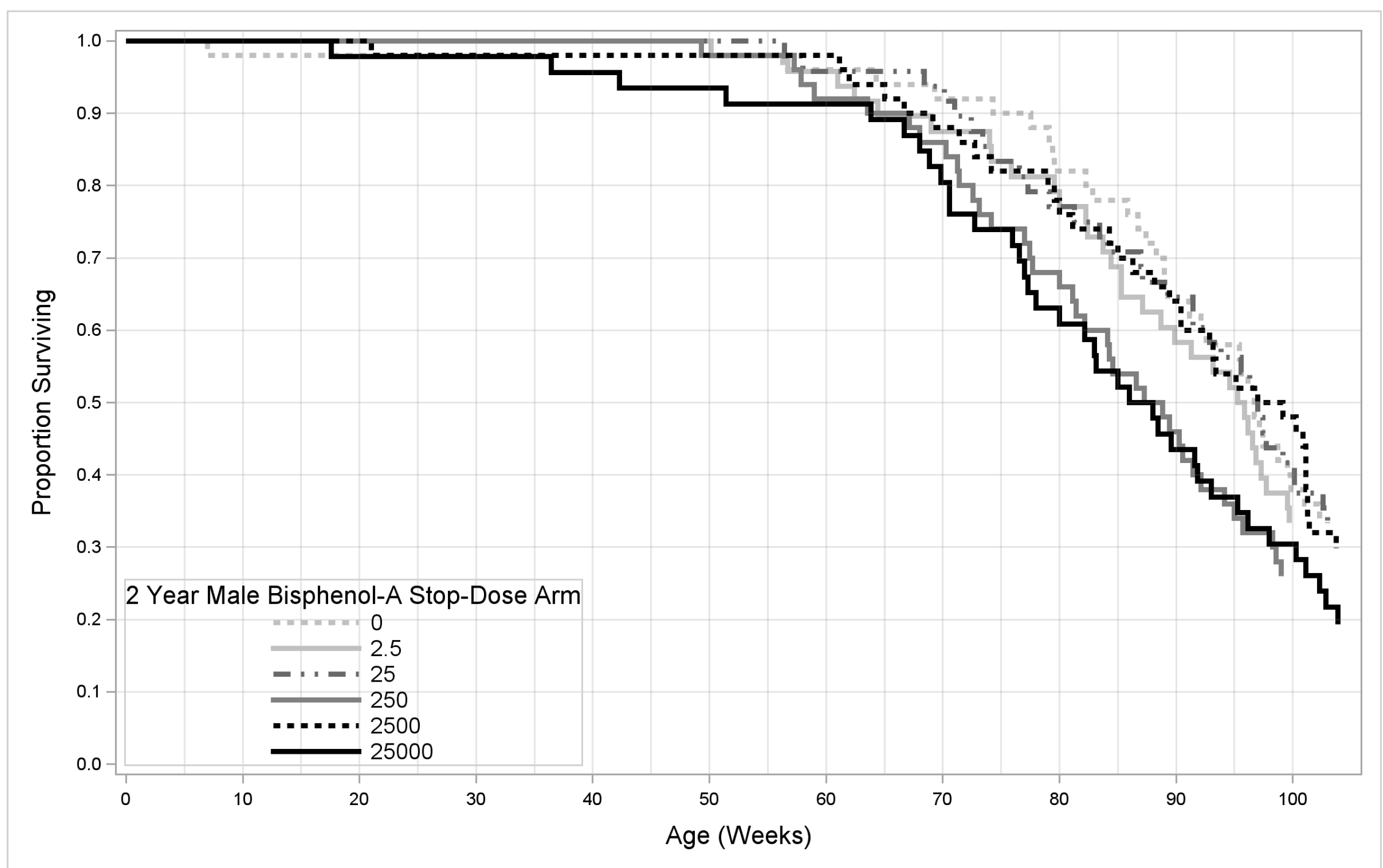

Figure 7. Kaplan-Meier Survival Curve for Terminal Sacrifice Male BPA Stop-Dose Arm (Week 4-104)

See Table 21 for data analysis results. 
CLARITY-BPA Core Study

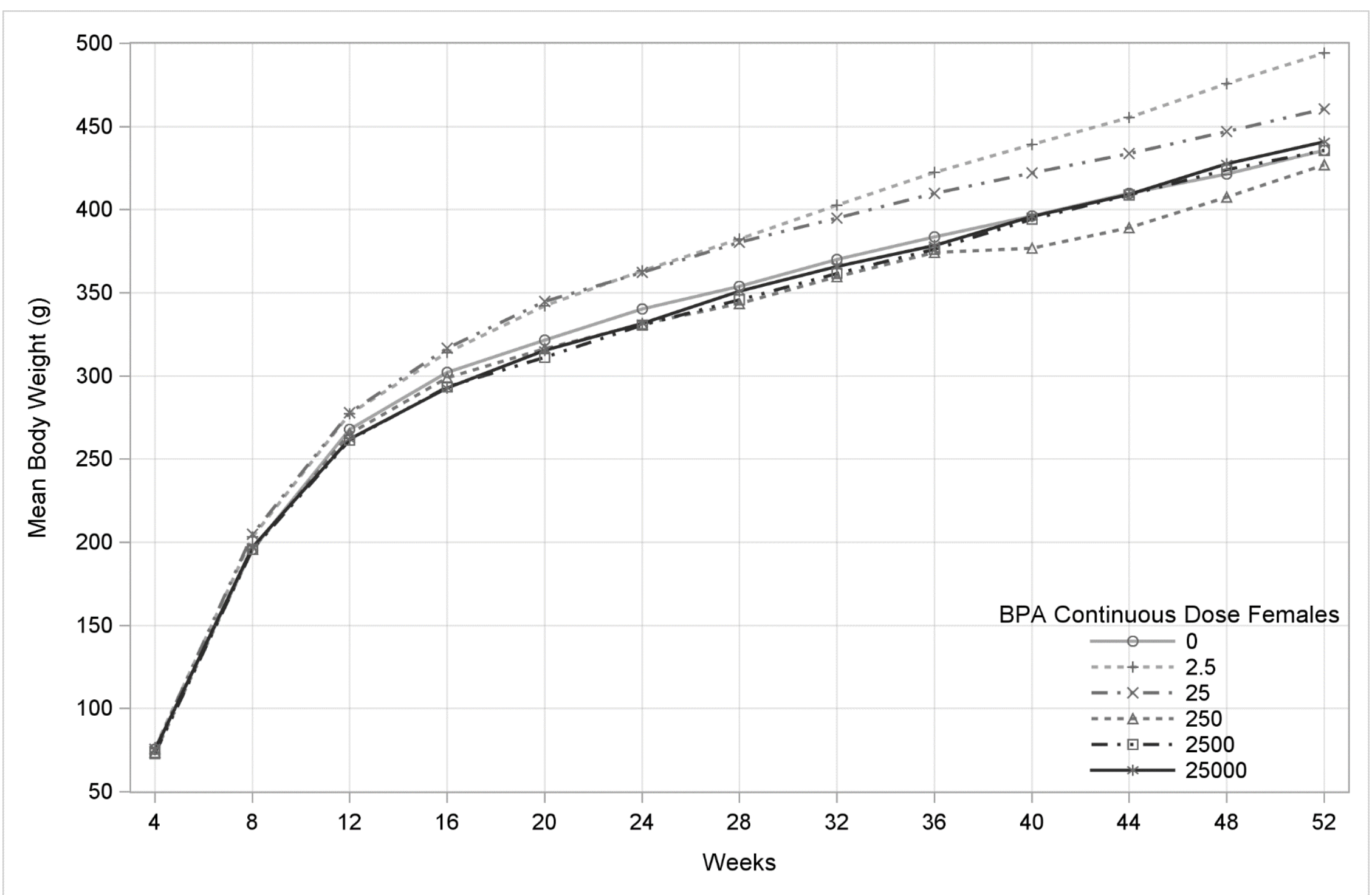

Figure 8. Body Weight for Interim Sacrifice Female BPA Continuous-Dose Arm

Data tabulated in Table 24. 
CLARITY-BPA Core Study

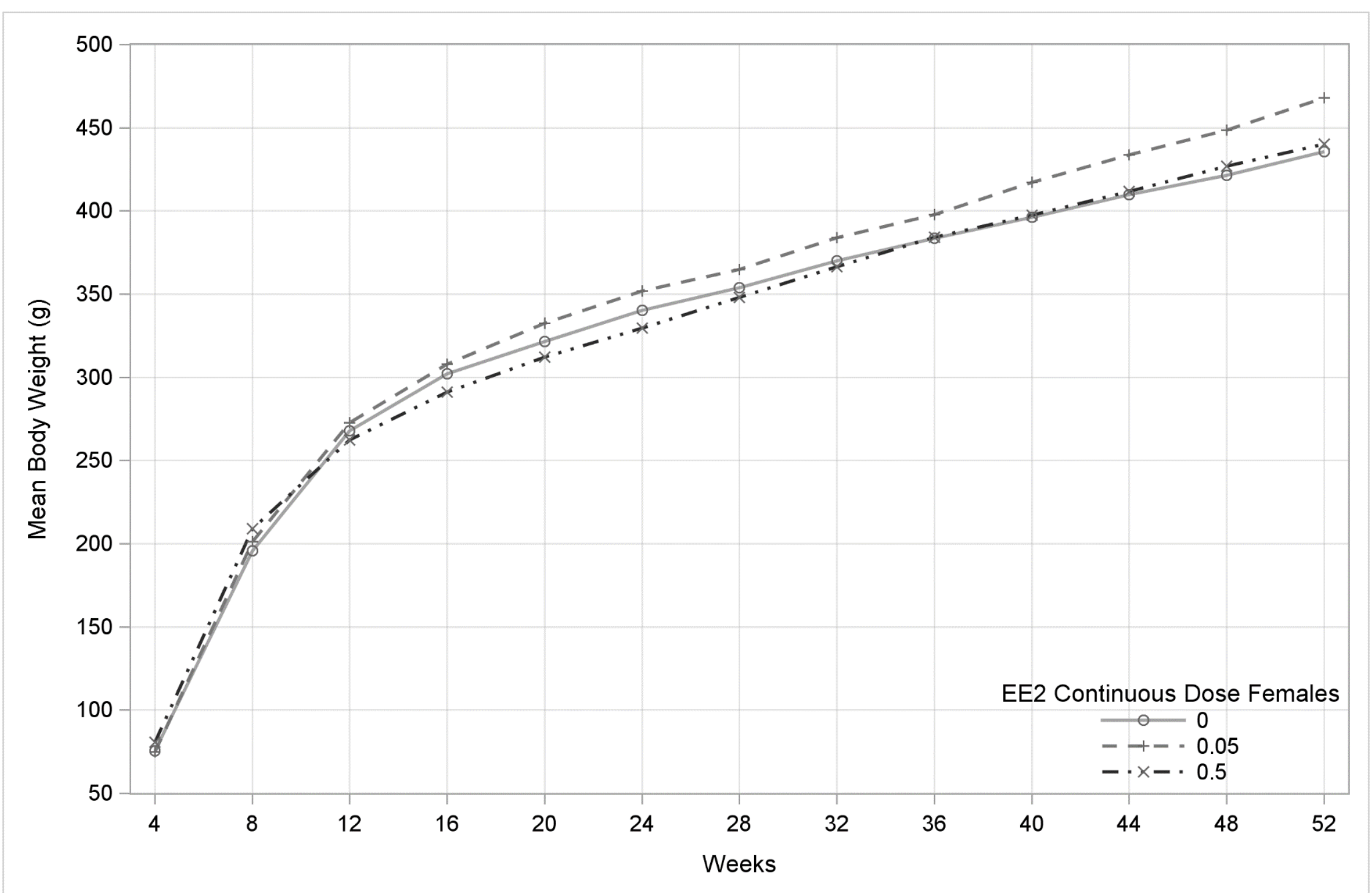

Figure 9. Body Weight for Interim Sacrifice Female $\mathbf{E E}_{2}$ Continuous-Dose Arm

Data tabulated in Table 24. 


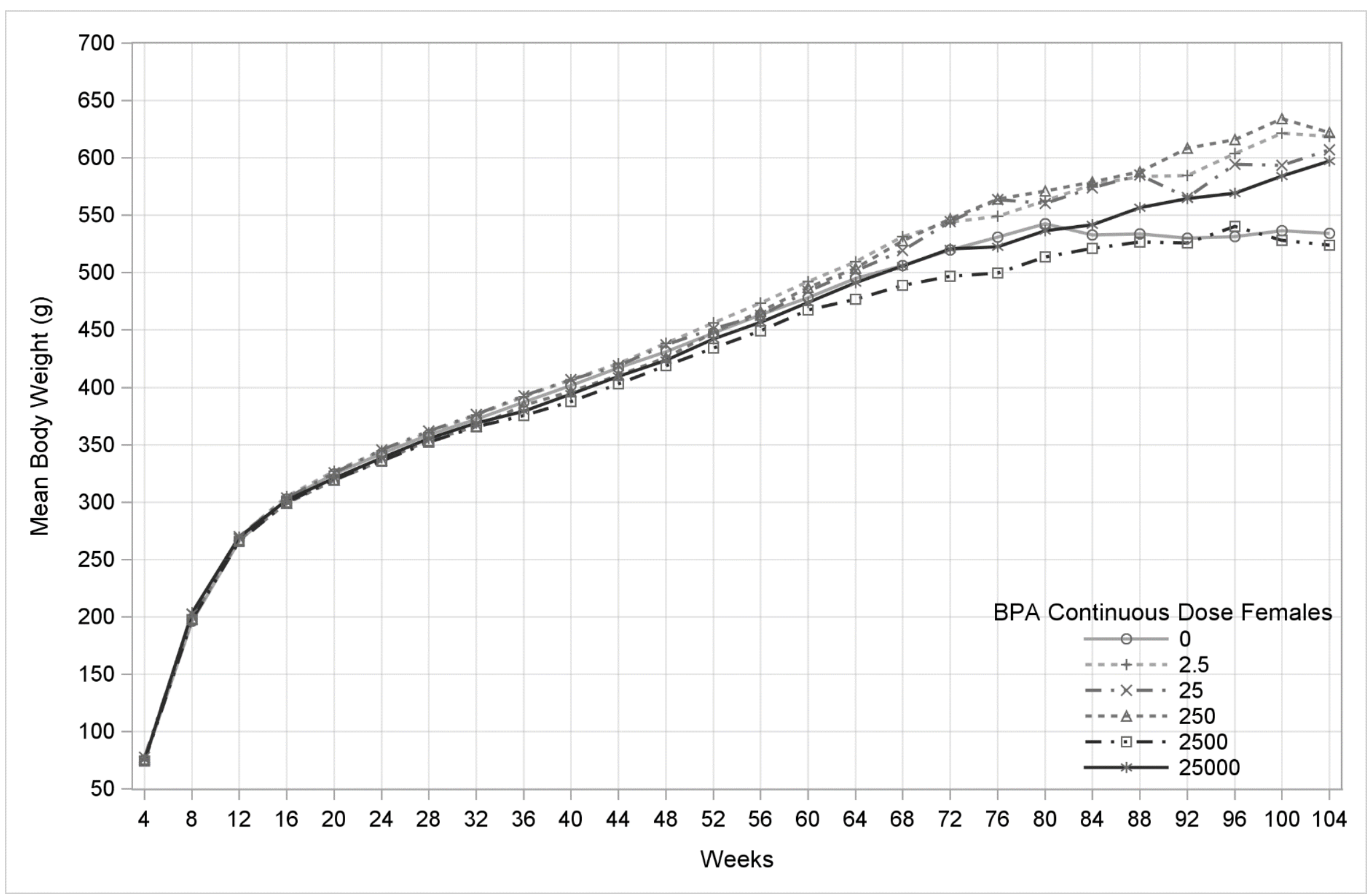

Figure 10. Body Weight for Terminal Sacrifice Female BPA Continuous-Dose Arm

Data tabulated in Table 25. 
CLARITY-BPA Core Study

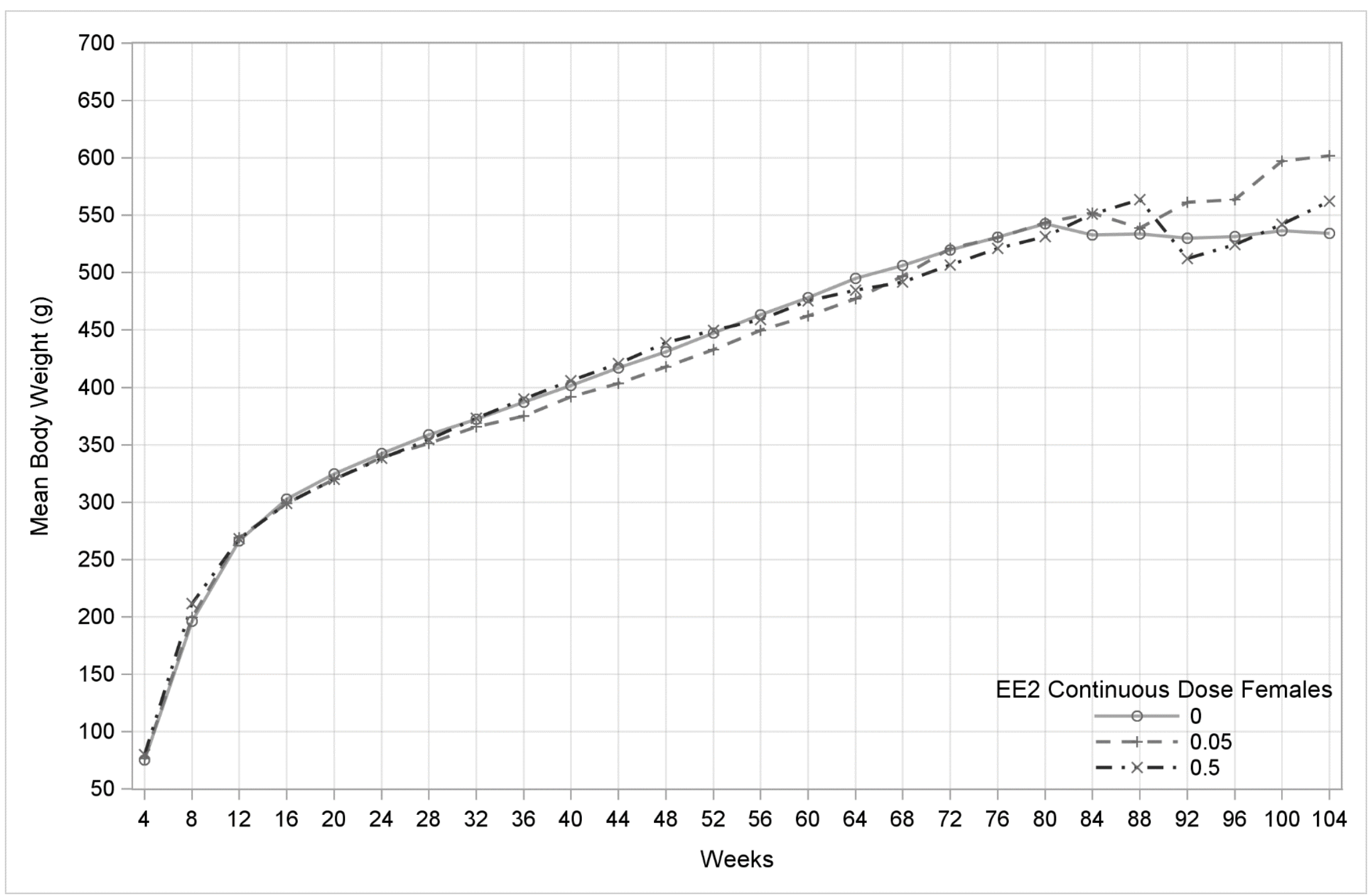

Figure 11. Body Weight for Terminal Sacrifice Female $\mathrm{EE}_{2}$ Continuous-Dose Arm

Data tabulated in Table 25. 
CLARITY-BPA Core Study

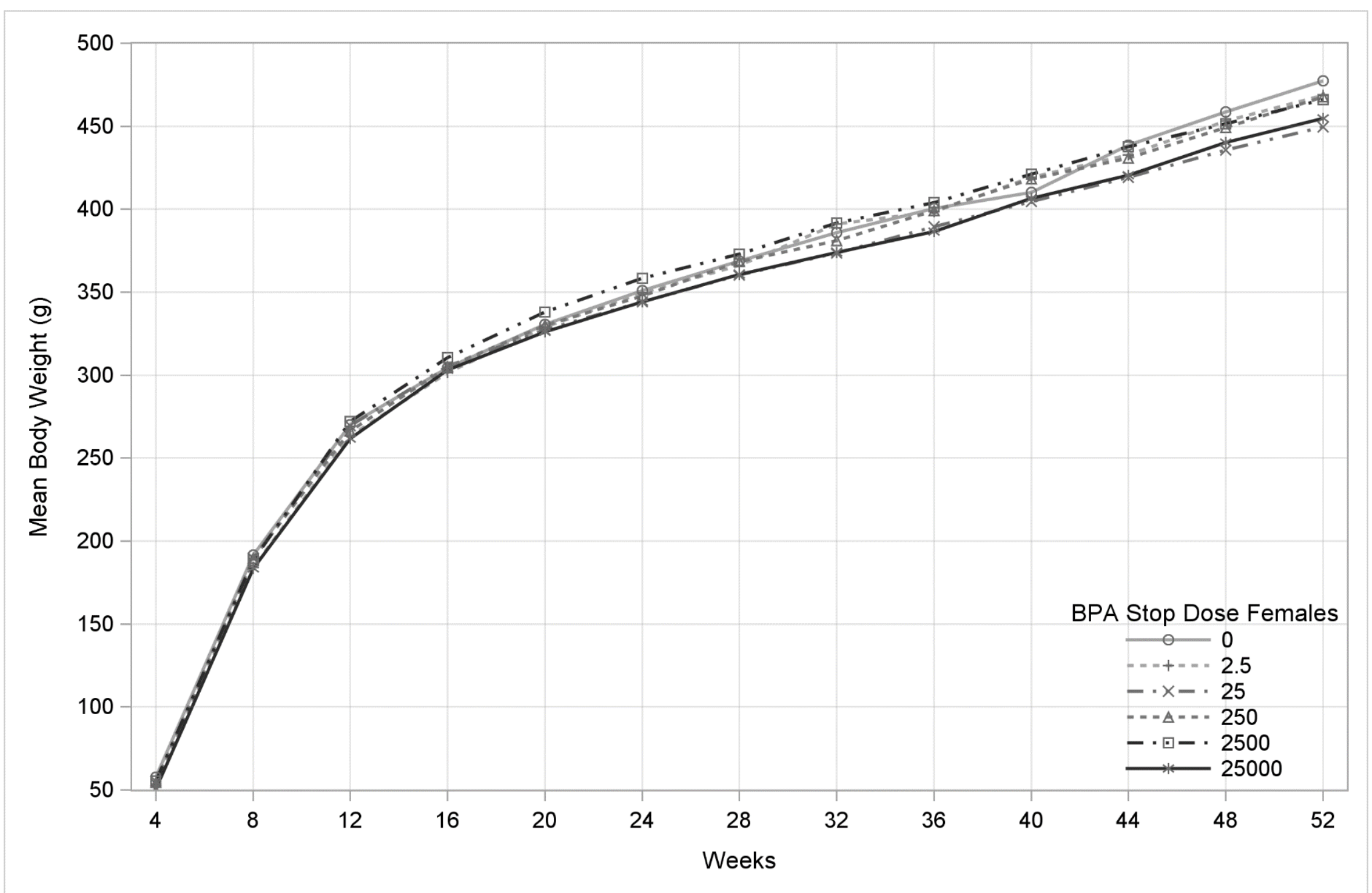

Figure 12. Body Weight for Interim Sacrifice Female BPA Stop-Dose Arm

Data tabulated in Table 26. 


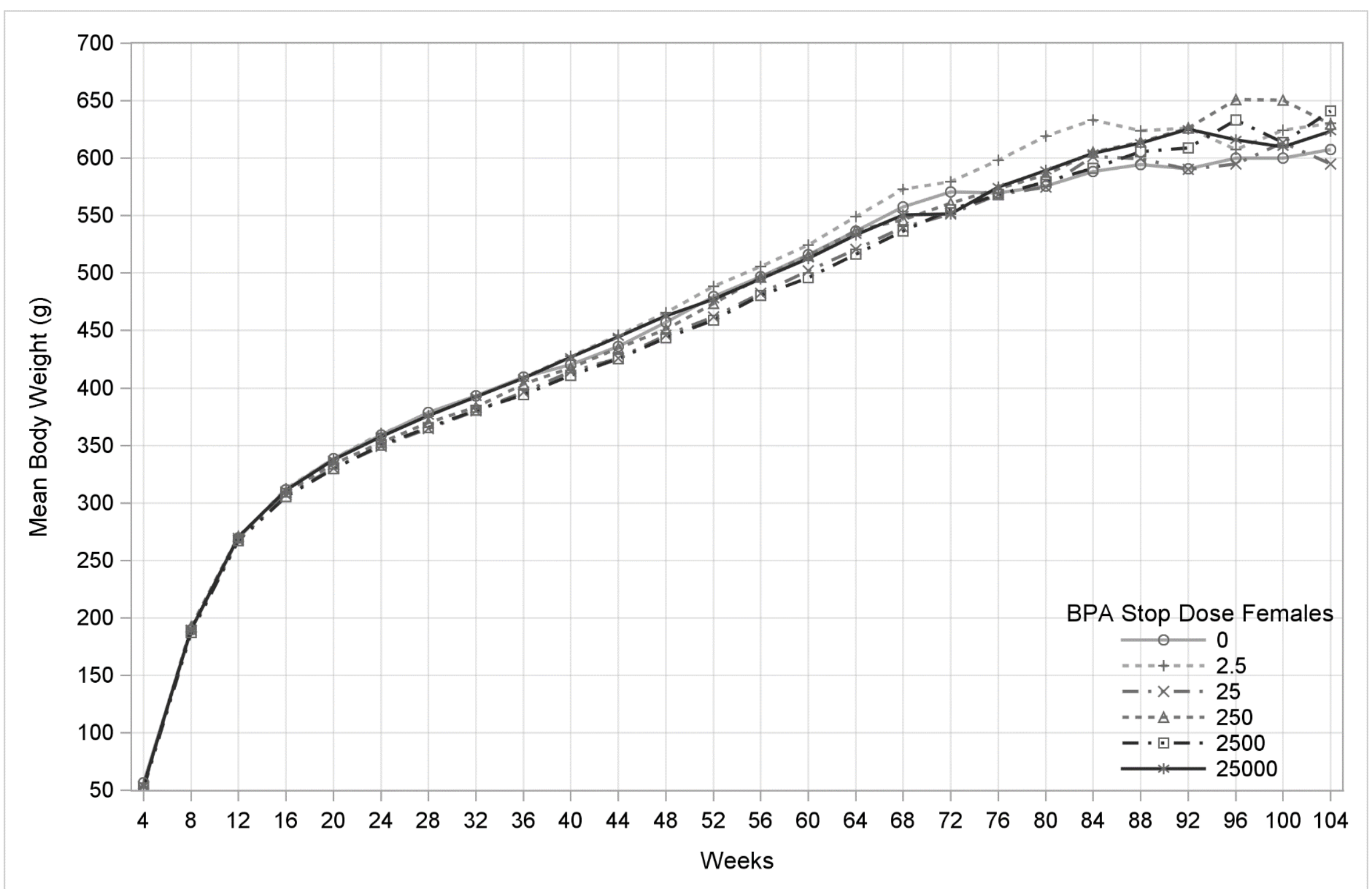

Figure 13. Body Weight for Terminal Sacrifice Female BPA Stop-Dose Arm

Data tabulated in Table 27. 
CLARITY-BPA Core Study

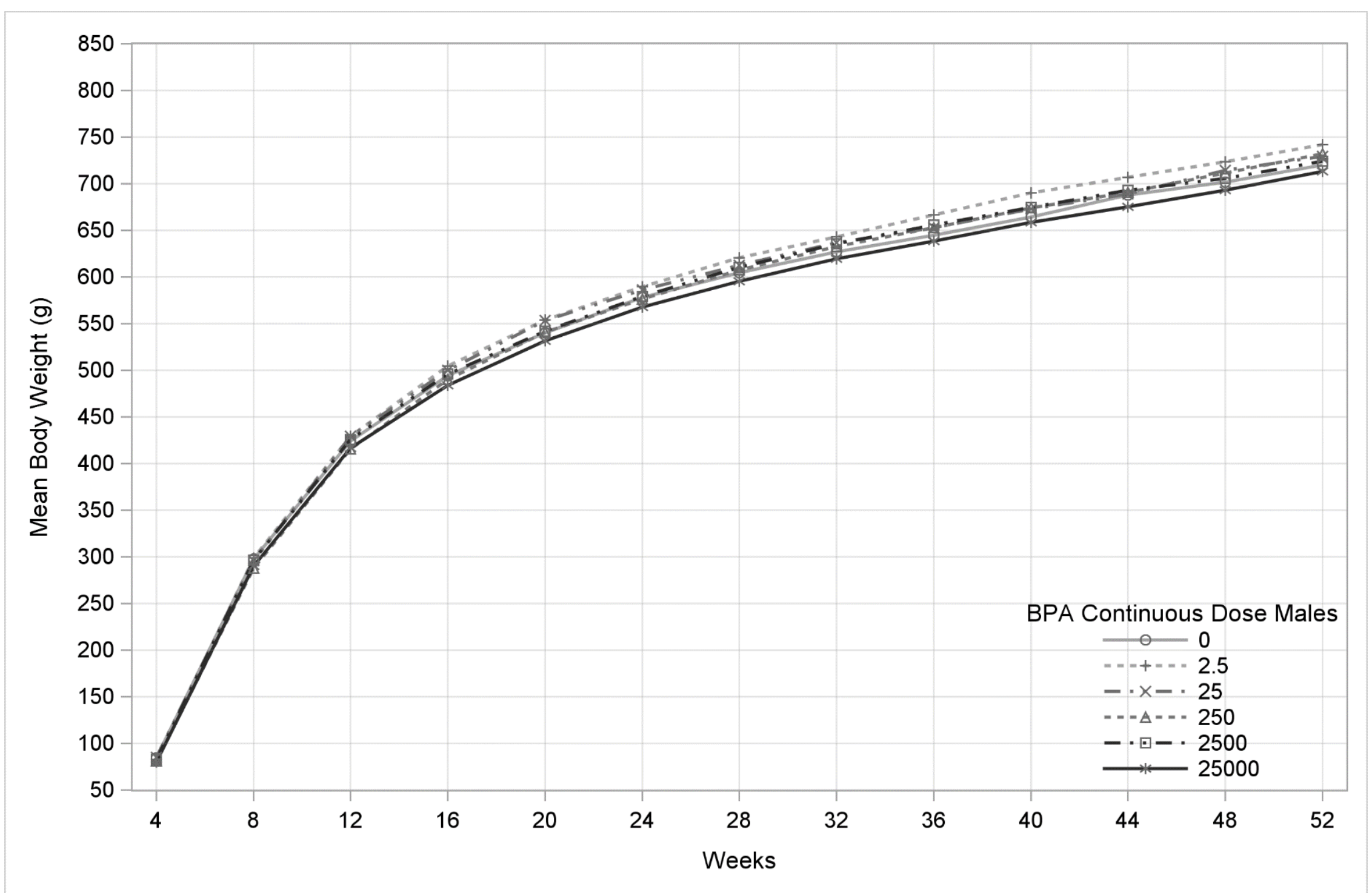

Figure 14. Body Weight for Interim Sacrifice Male BPA Continuous-Dose Arm

Data tabulated in Table 28. 
CLARITY-BPA Core Study

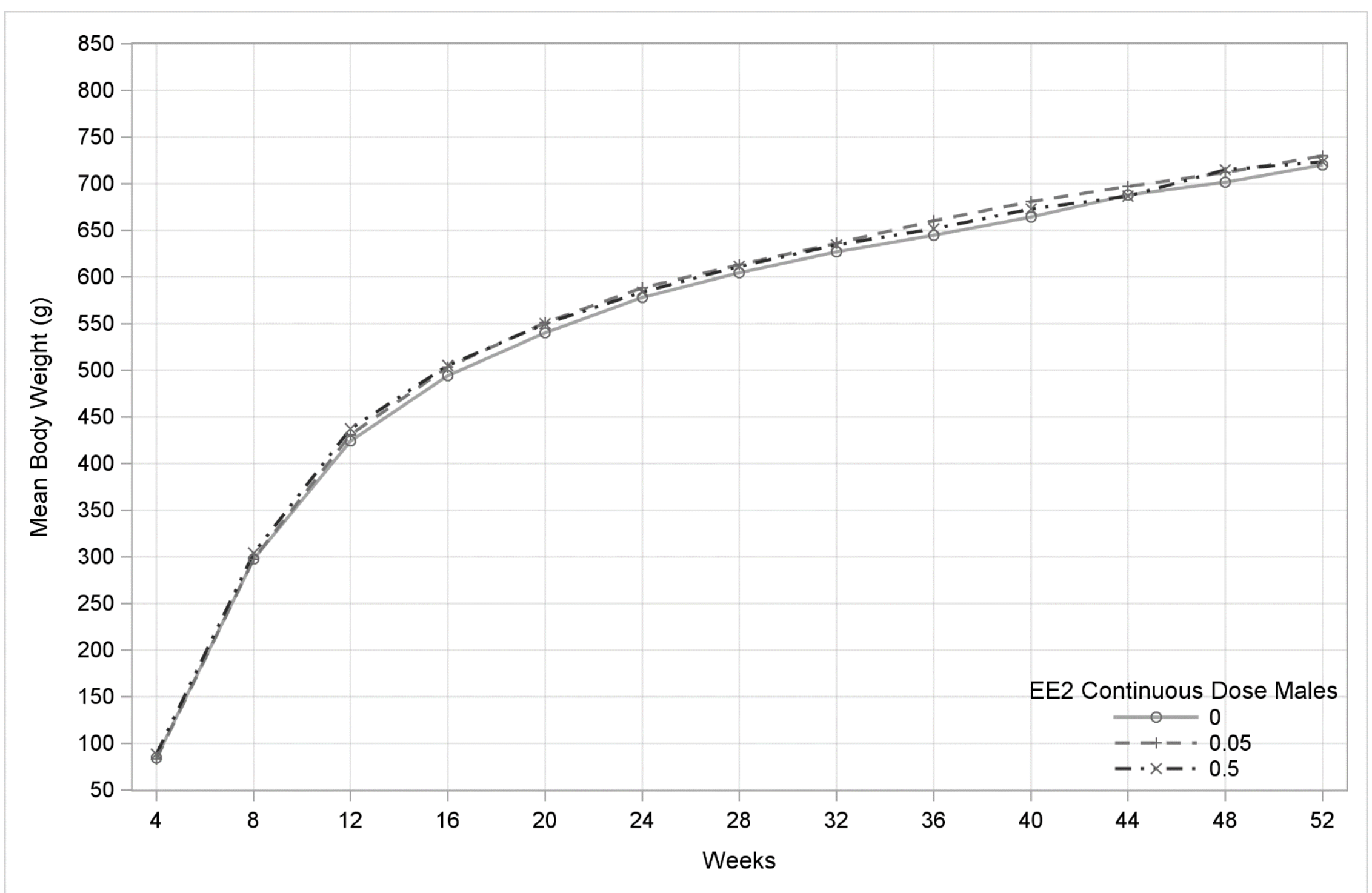

Figure 15. Body Weight for Interim Sacrifice Male $\mathbf{E E}_{2}$ Continuous-Dose Arm

Data tabulated in Table 28. 
CLARITY-BPA Core Study

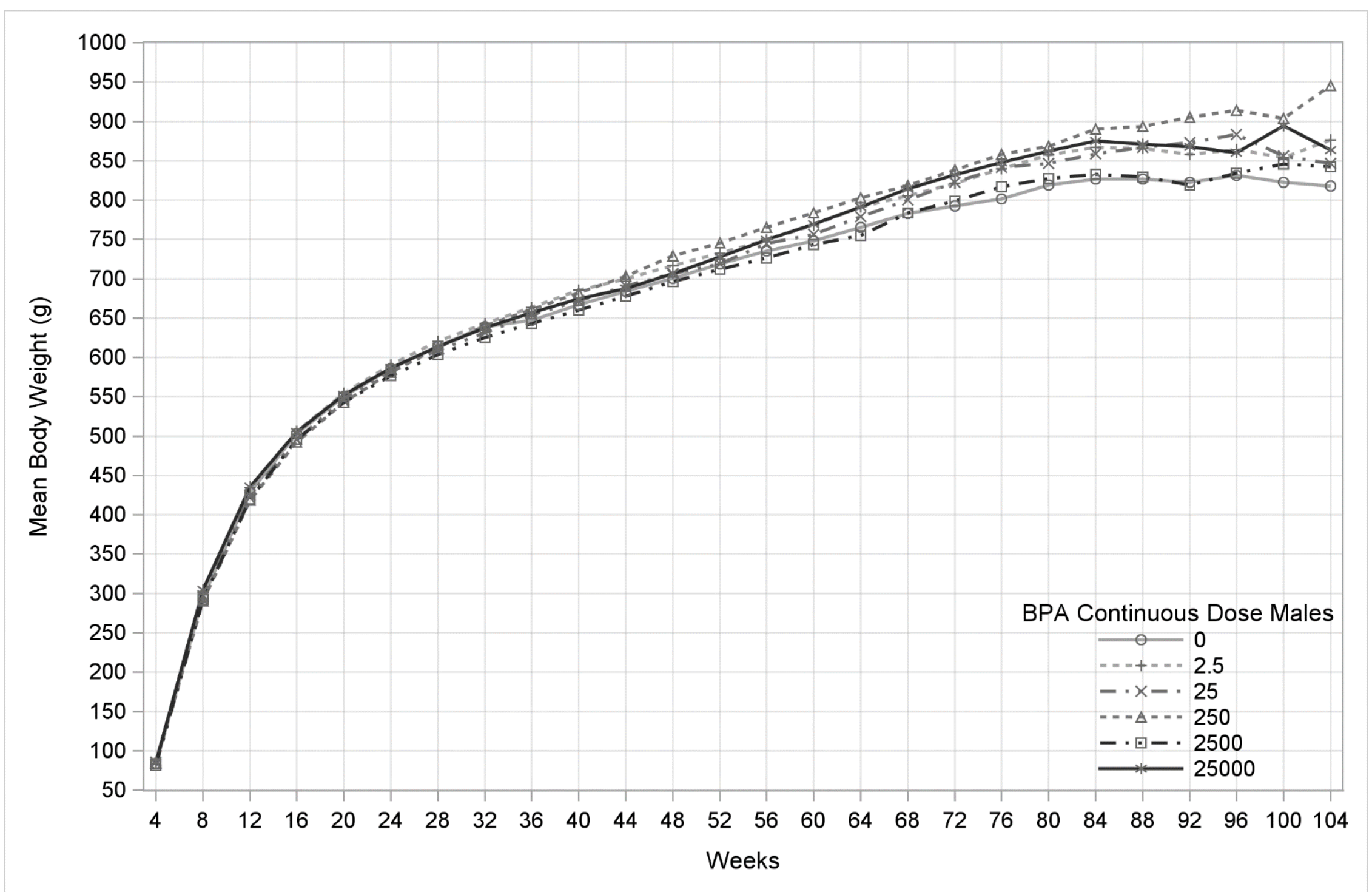

Figure 16. Body Weight for Terminal Sacrifice Male BPA Continuous-Dose Arm

Data tabulated in Table 29. 
CLARITY-BPA Core Study

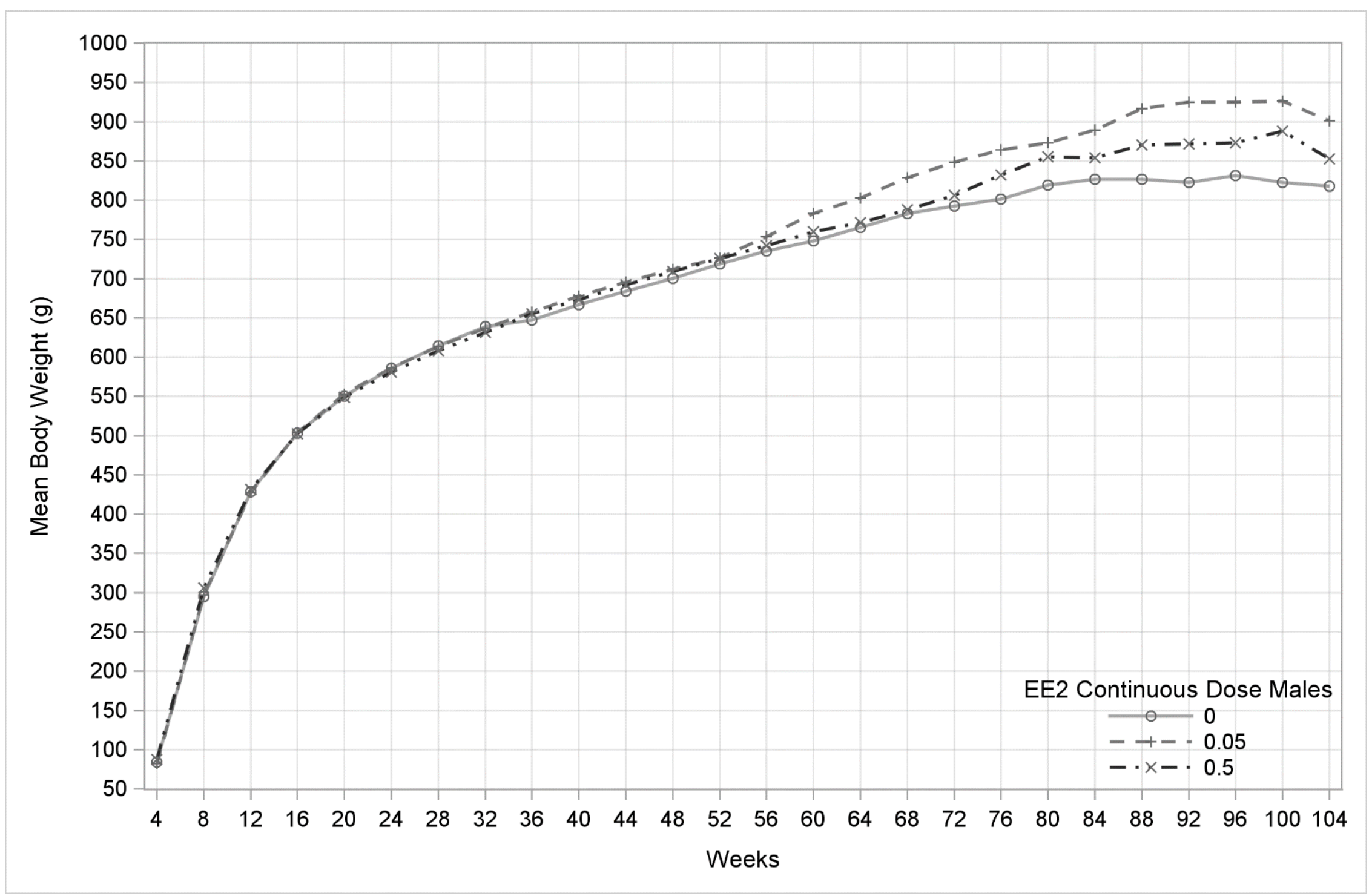

Figure 17. Body Weight for Terminal Sacrifice Male $\mathbf{E E}_{2}$ Continuous-Dose Arm

Data tabulated in Table 29. 
CLARITY-BPA Core Study

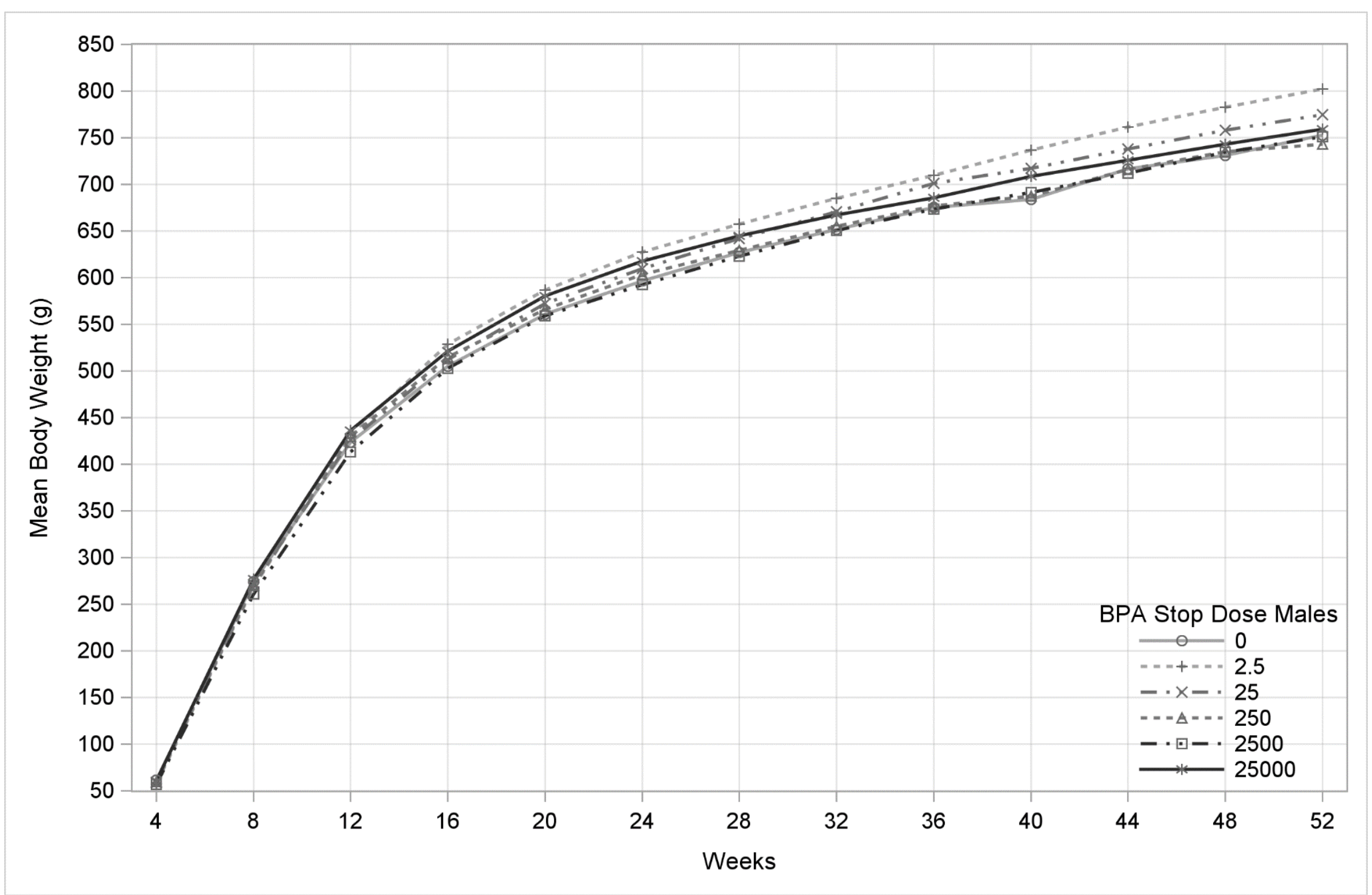

Figure 18. Body Weight for Interim Sacrifice Male BPA Stop-Dose Arm

Data tabulated in Table 30. 


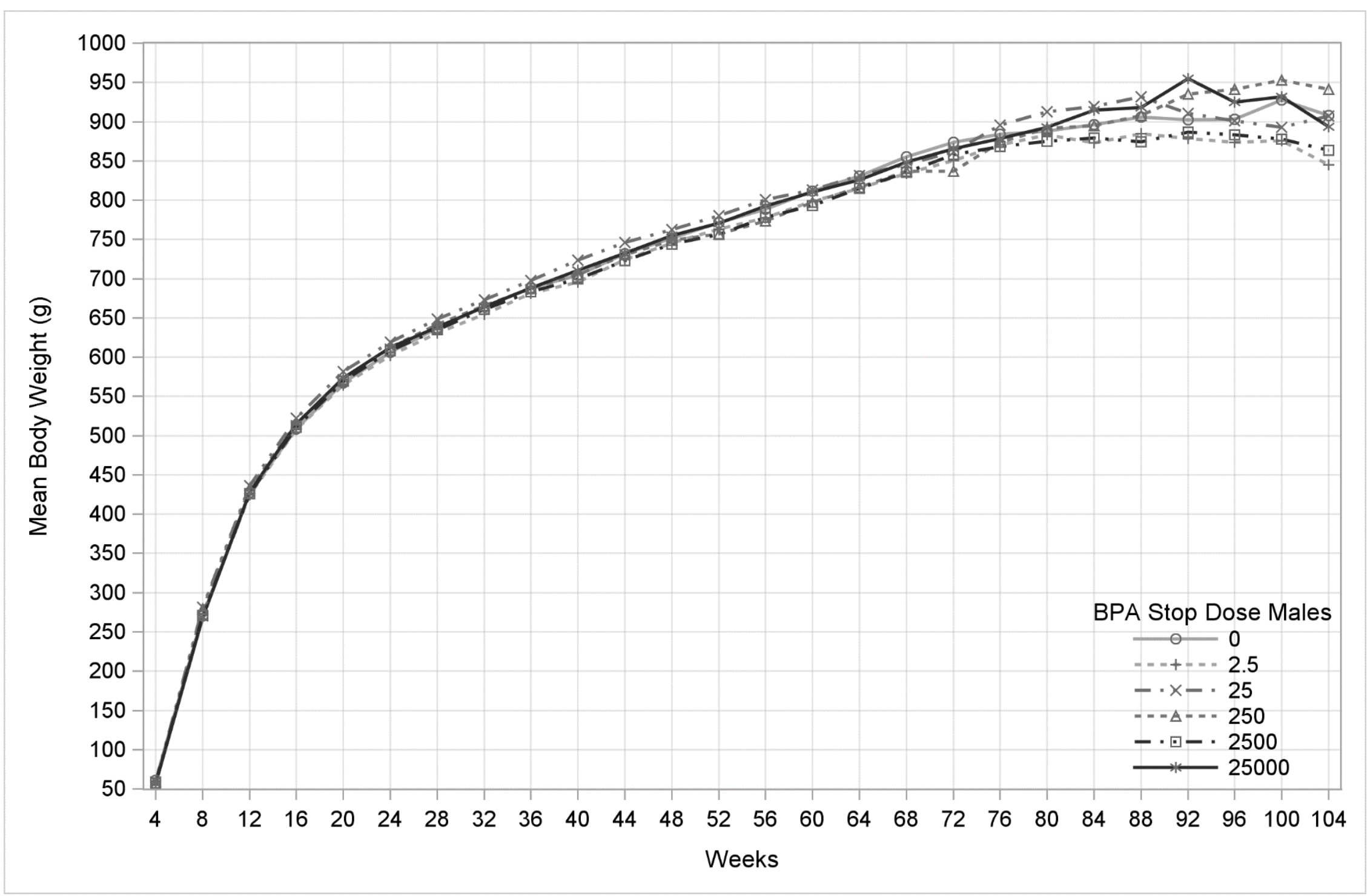

Figure 19. Body Weight for Terminal Sacrifice Male BPA Stop-Dose Arm

Data tabulated in Table 31. 


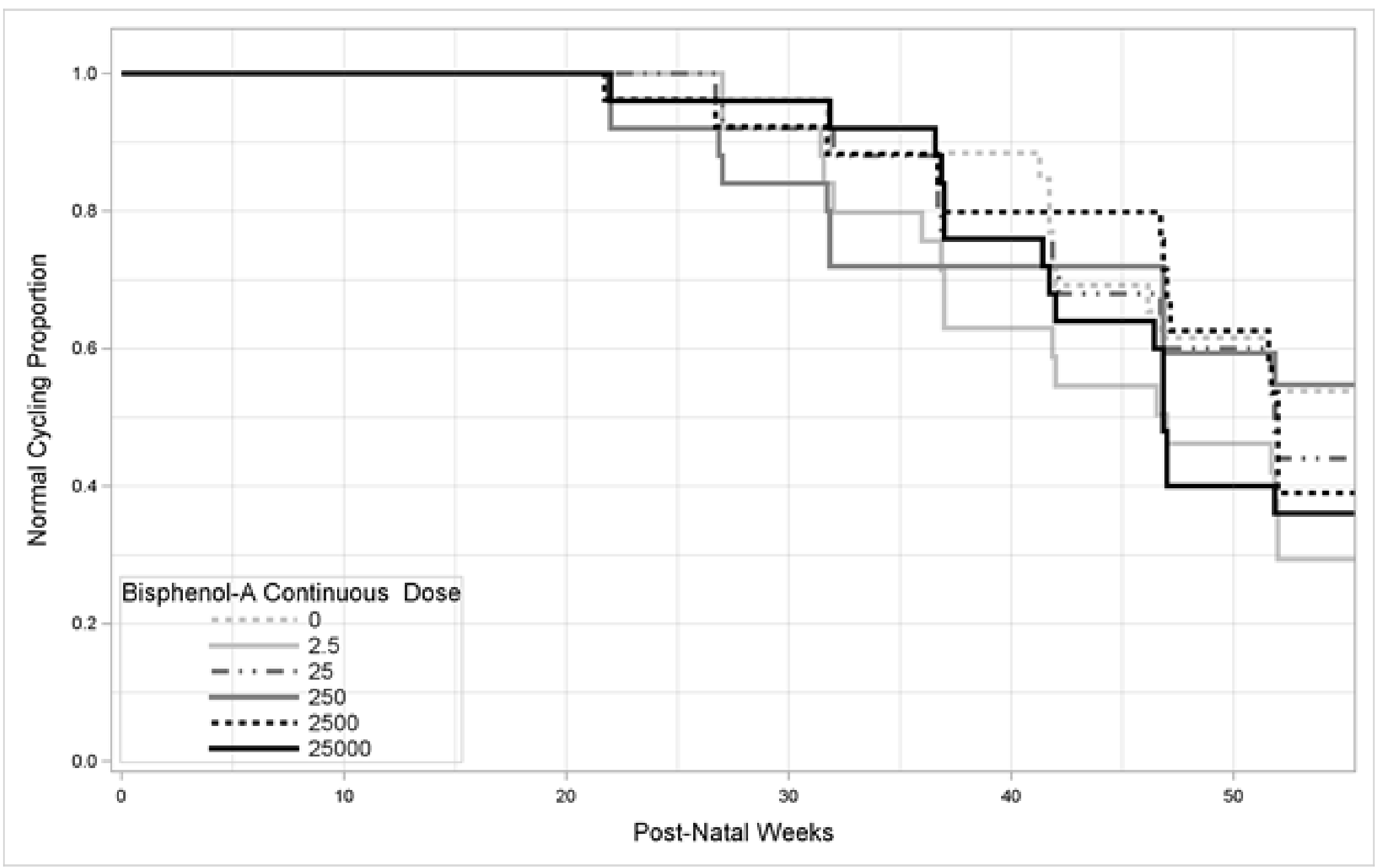

Figure 20. Kaplan-Meier Time to Aberrant Cycling Curve for BPA Continuous-Dose Arm

See Table 36 for data analysis results. 


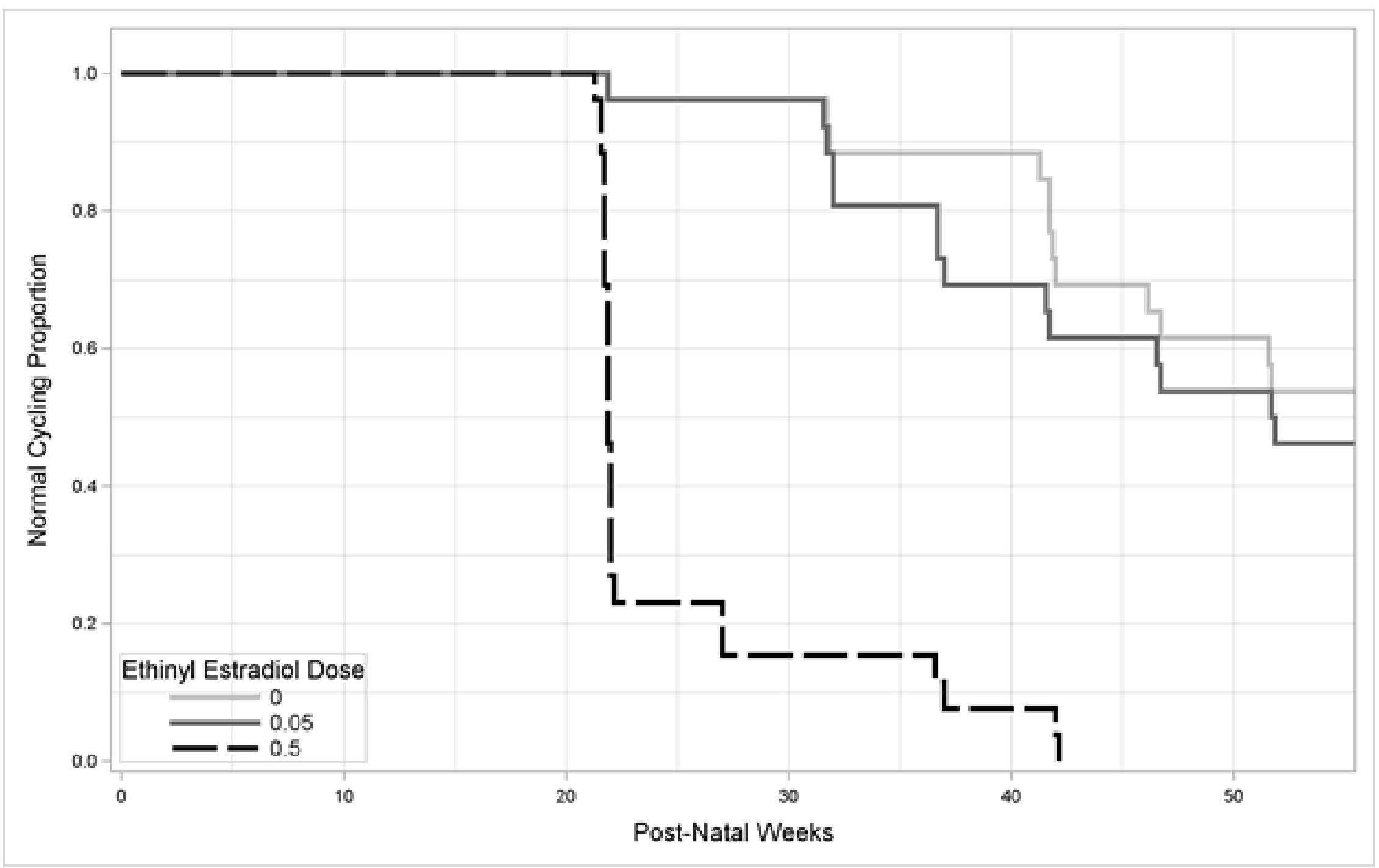

Figure 21. Kaplan-Meier Time to Aberrant Cycling Curve for $\mathbf{E E}_{2}$ Continuous-Dose Arm

See Table 36 for data analysis results. 


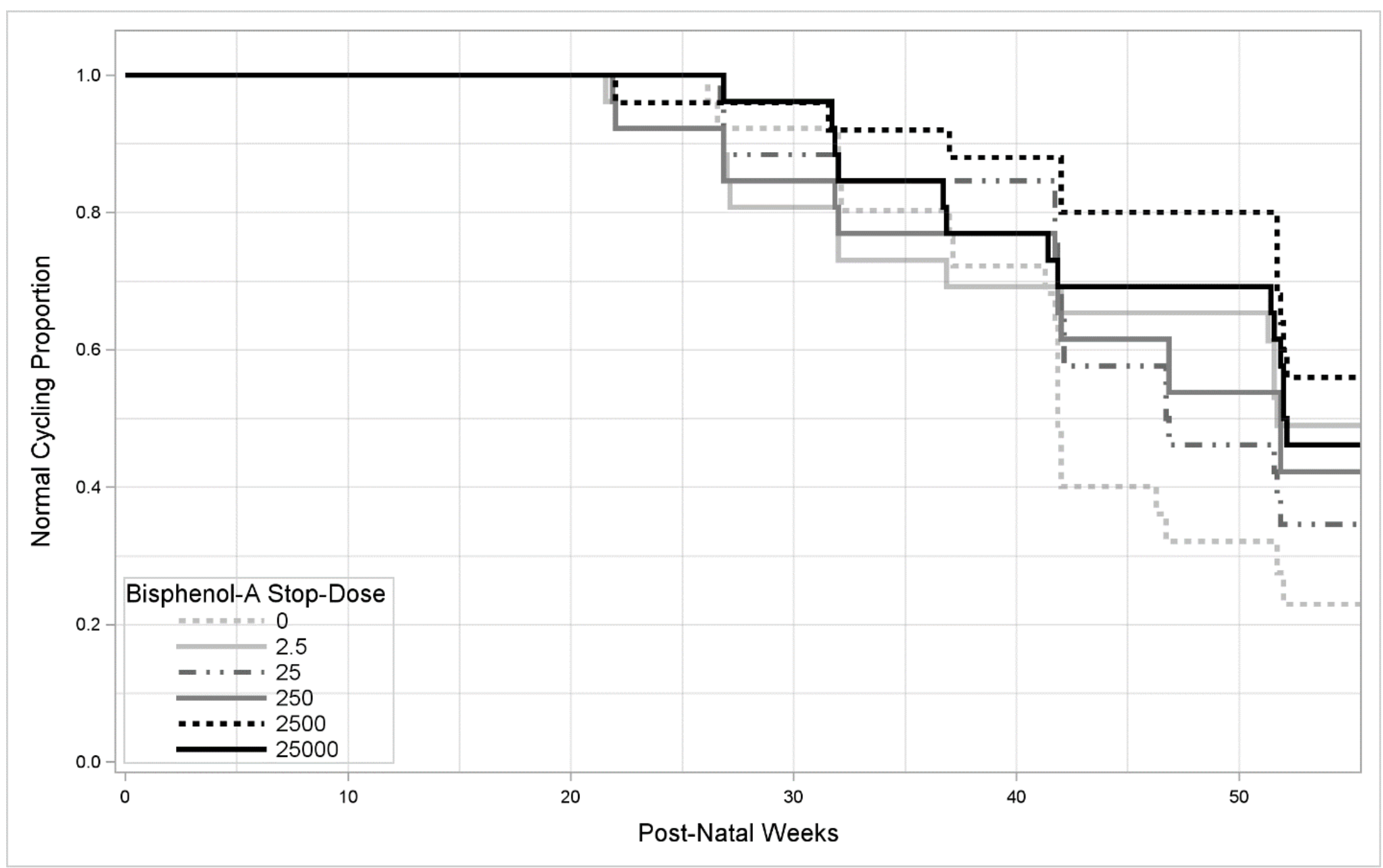

Figure 22. Kaplan-Meier Time to Aberrant Cycling Curve for BPA Stop-Dose Arm

See Table 37 for data analysis results. 


\section{Appendix A List of Supplemental Appendices}

Supplemental appendices are available at https://doi.org/10.22427/NTP-DATA-018-000150001-000-6.

\begin{tabular}{|c|c|}
\hline $\begin{array}{c}\text { Supplemental } \\
\text { Appendix }\end{array}$ & Title and Description \\
\hline I & $\begin{array}{l}\text { Protocol and Amendments* } \\
\text { Approved NCTR study protocol (E0219001), which includes description of study sponsor, testing } \\
\text { facility and responsible personnel, study objectives, background of the scientific problem being } \\
\text { addressed, and a detailed description of the study design. Follow-up amendments to the approved } \\
\text { study protocol and rationale for the changes, as well as a copy of the study protocol with all } \\
\text { amendment changes incorporated, are also included. }\end{array}$ \\
\hline II & $\begin{array}{l}\text { Protocol Deviations* } \\
\text { Documentation generated by Priority One (Animal Care contractor), Toxicologic Pathology } \\
\text { Associates (Pathology services contractor), or the Study Director to report noted deviations from } \\
\text { the procedures defined in the approved study protocol or follow-up amendments. It includes } \\
\text { description of deviations, the corrective measurements taken to prevent re-occurrence, and the } \\
\text { assessment by the Study Director on the impact of the deviation on study integrity. A summary } \\
\text { table with all deviations is also included. }\end{array}$ \\
\hline III & $\begin{array}{l}\text { Notes to Study File* } \\
\text { Documentation on various events related to the study conduct and not covered in the study } \\
\text { protocol deviations documentation. }\end{array}$ \\
\hline IV & $\begin{array}{l}\text { Study Startup Memo and Study Definition E0219002 }\left(F_{0} \text { and } \mathbf{F}_{1} \text { Preweaning)* }\right. \\
\text { Documentation generated by the Computer Support Group to describe the programming of the in- } \\
\text { life tracking system used to support the study and its validation, animal allocation and breeding } \\
\text { schemes, treatment group definitions, rack configurations used for animals of the } F_{0} \text { generation } \\
\text { and } F_{1} \text { animals prior to weaning on postnatal day } 21 \text {. Definitions of disposition and reasons for } \\
\text { removal terms are also included. }\end{array}$ \\
\hline V & $\begin{array}{l}\text { Study Startup Memo and Study Definition E0219003 (F } \mathbf{F}_{1} \text { Postweaning)* } \\
\text { Documentation generated by the Computer Support Group to describe the programming of the in- } \\
\text { life tracking system used to support the study and its validation, animal allocation scheme, } \\
\text { treatment group definitions, and rack configurations used for } F_{1} \text { animals after weaning on } \\
\text { postnatal day } 21 \text {. }\end{array}$ \\
\hline VI & $\begin{array}{l}\text { Breeder Weight Ranking, Treatment Randomization, and Pairing Schedule* } \\
\text { Documentation to define the treatment randomization and weight ranking of the } \mathrm{F}_{0} \text { breeders, the } \\
\text { breeding pair randomization, and to report the breeder pairings conducted in the study. }\end{array}$ \\
\hline VII & $\begin{array}{l}\text { Chemistry Support Report } \\
\text { Report by the Chemistry Support Group on the analyses of the study test articles, dose } \\
\text { preparations, BPA levels in study materials, and phyto/mycoestrogens in diet. The standard } \\
\text { operating procedures followed to perform these analyses are also included. }\end{array}$ \\
\hline VIII & $\begin{array}{l}\text { Microbiology Support Report } \\
\text { Report by the Surveillance/Diagnostic Program Support Group on the microbiological findings on } \\
\text { sentinel animals, animal rooms, and animal husbandry supplies. }\end{array}$ \\
\hline IX & $\begin{array}{l}\text { Diet Preparation Services Report } \\
\text { Report by the Diet Preparation Support Group describing the inventory and storage conditions of } \\
\text { the study diet and test articles, dose preparations, and the standard operating procedures followed. }\end{array}$ \\
\hline $\mathrm{X}$ & $\begin{array}{l}\text { Animal Rooms Temperature and Humidity Reports* } \\
\text { Report of the temperature and relative humidity recorded in the rooms used to house the animals } \\
\text { over the course of the study. }\end{array}$ \\
\hline
\end{tabular}




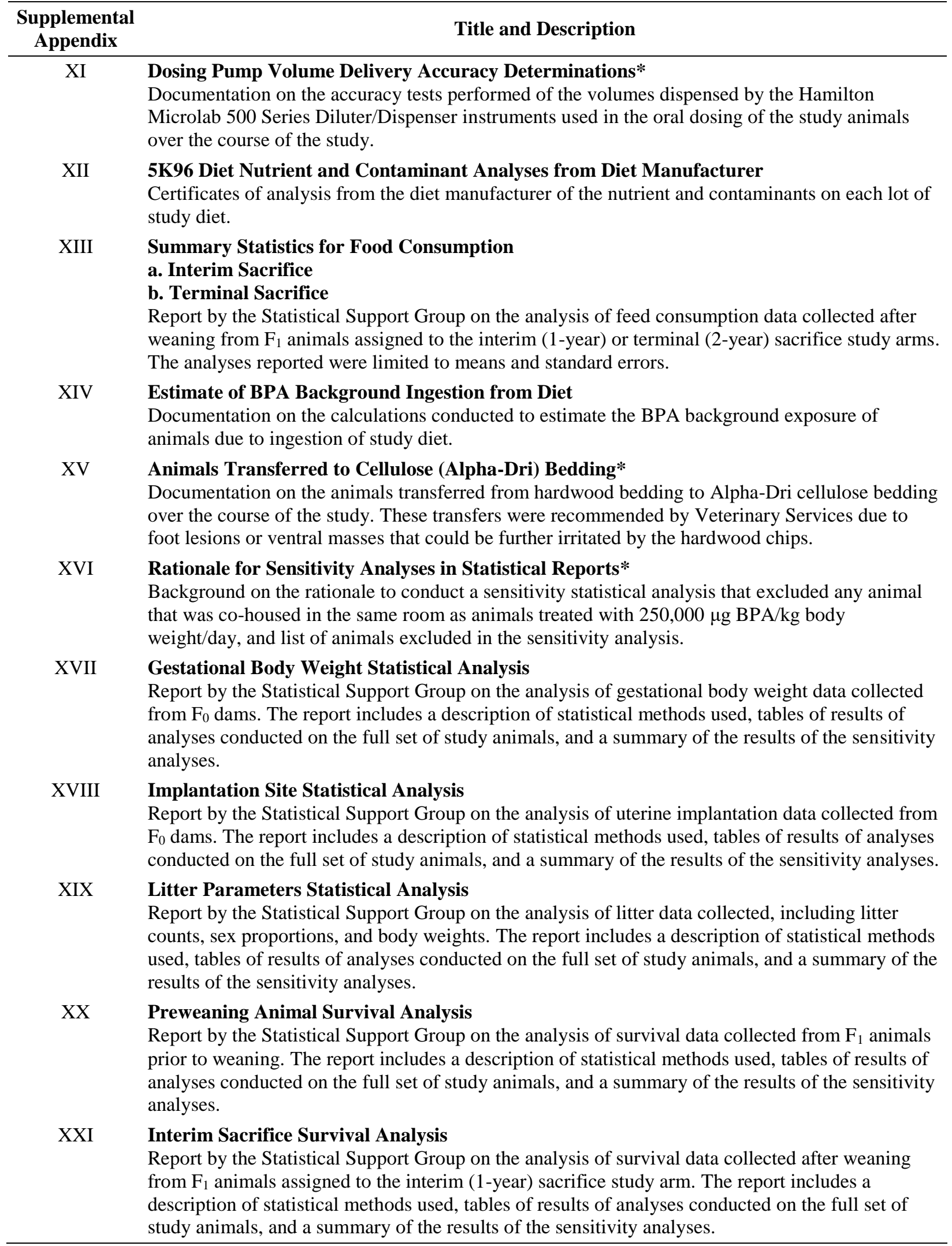




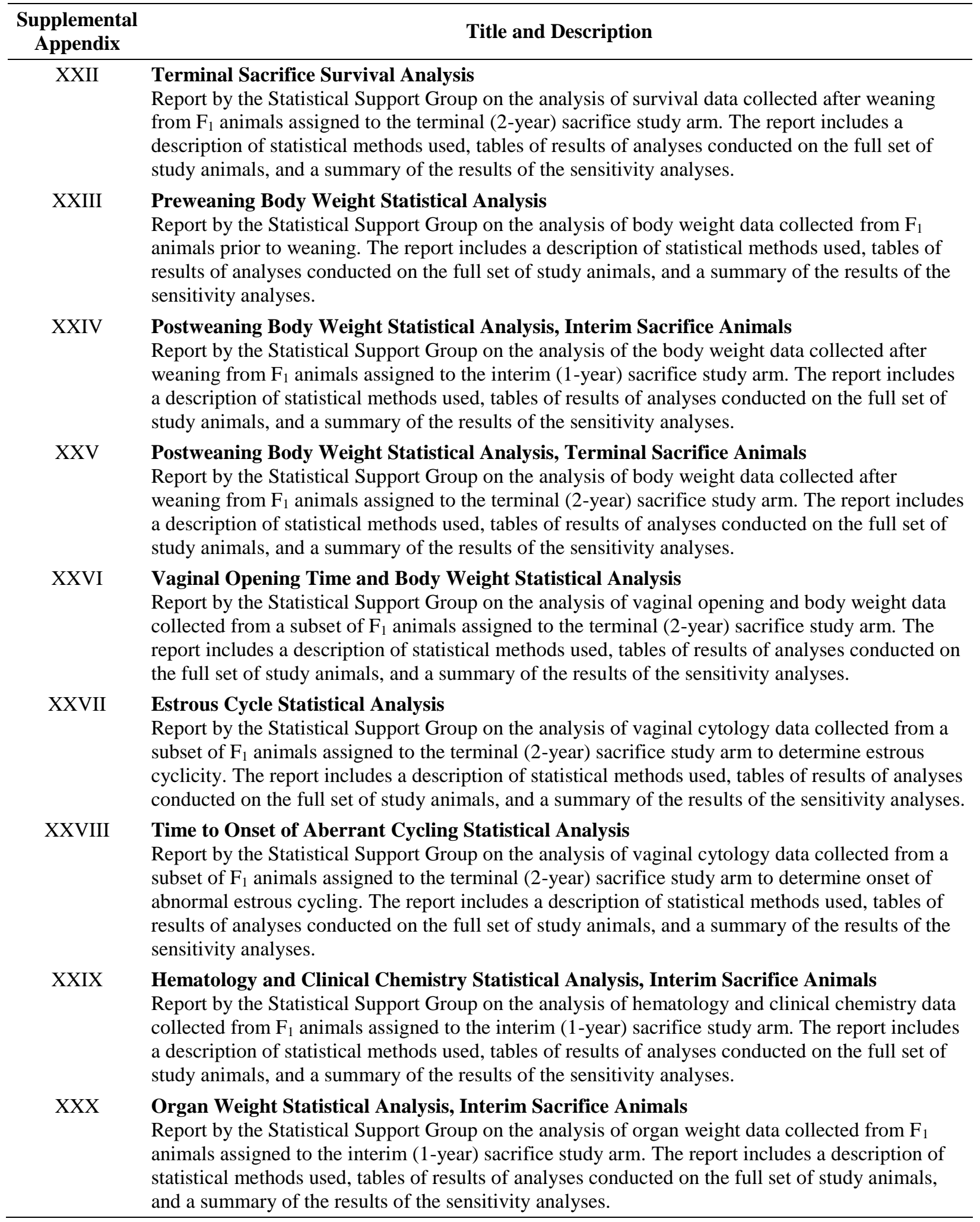




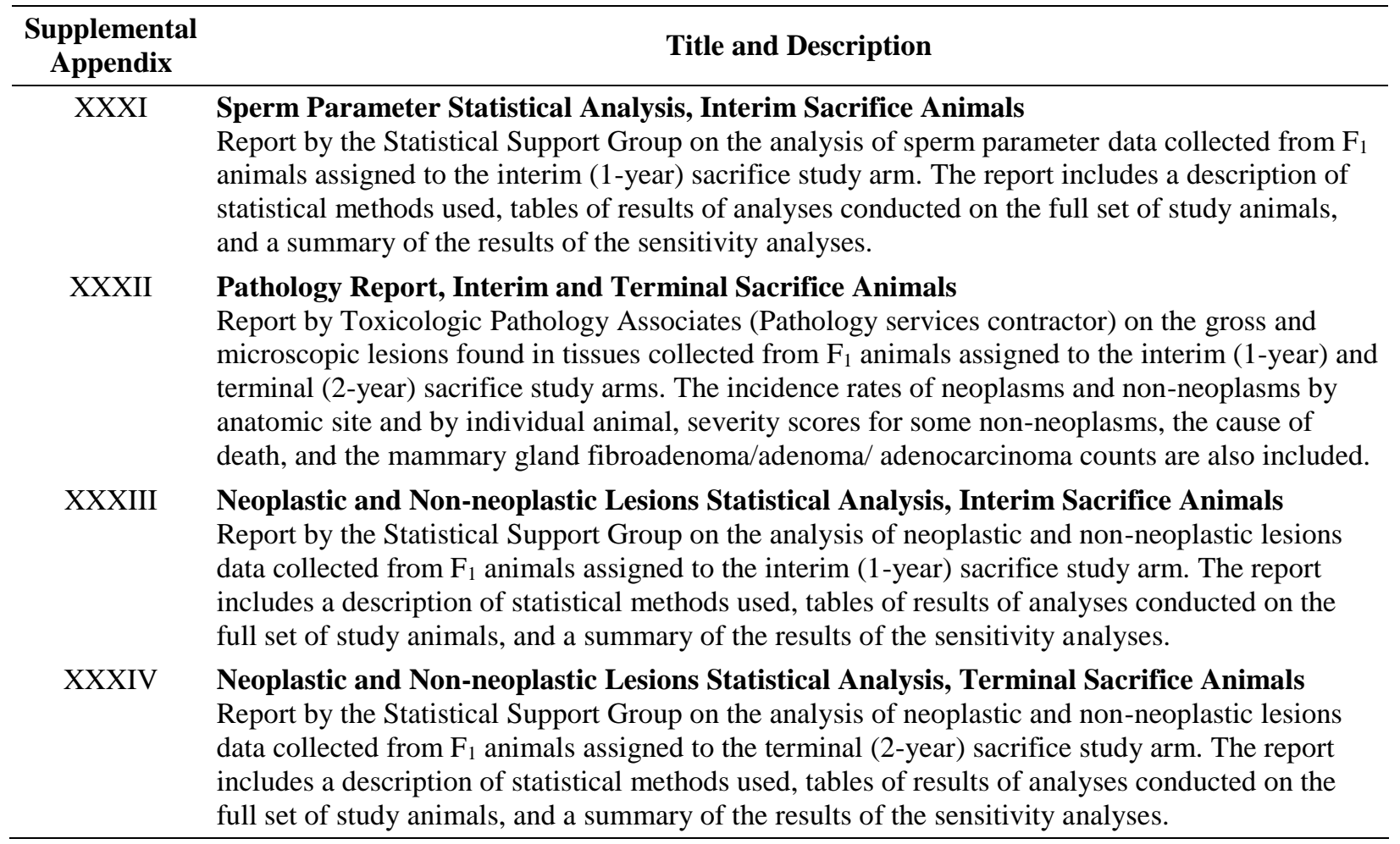

*Available upon request: $\underline{\text { cdm@ @iehs.nih.gov. }}$ 


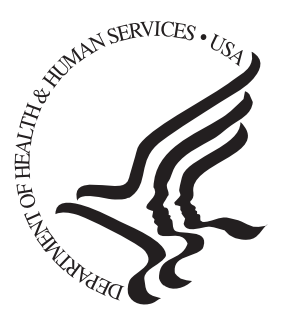

\section{National Toxicology Program}

NTP Central Data Managment, MD K2-05

National Insitute of Enviromental Health Sciences

P.O. Box 12233

Research Triangle Park, NC 27709

http://ntp.niehs.nih.gov 